Schriften zum Internationalen und

Europäischen Strafrecht

Sandra van der Stroom

Menschenrechtliche Anforderungen an den Strafvollzug

Mindeststandards und deren Implementierung im Lichte des Art. 3 EMRK 
Schriften zum Internationalen und

Europäischen Strafrecht

Herausgegeben von

Professor Dr. Martin Heger, Humboldt-Universität zu Berlin Professor Dr. Florian Jeßberger, Humboldt-Universität zu Berlin Professor Dr. Frank Neubacher, M.A., Universität zu Köln Professor Dr. Helmut Satzger, LMU München Professor Dr. Gerhard Werle, Humboldt-Universität zu Berlin

Band 56 
Sandra van der Stroom

\section{Menschenrechtliche Anforderungen an den Strafvollzug}

Mindeststandards und deren Implementierung im Lichte des Art. 3 EMRK 
Publiziert mit Unterstützung des Schweizerischen Nationalfonds zur Förderung der wissenschaftlichen Forschung.

Die Deutsche Nationalbibliothek verzeichnet diese Publikation in der Deutschen Nationalbibliografie; detaillierte bibliografische Daten sind im Internet über http://dnb.d-nb.de abrufbar.

Zugl.: Zürich, Univ., Diss., 2020

1. Auflage 2021

(c) Sandra van der Stroom

Publiziert von

Nomos Verlagsgesellschaft mbH \& Co. KG

Waldseestraße 3-5 | 76530 Baden-Baden

www.nomos.de

Gesamtherstellung:

Nomos Verlagsgesellschaft $\mathrm{mbH} \& \mathrm{Co}$. KG

Waldseestraße 3-5| 76530 Baden-Baden

ISBN (Print): 978-3-8487-8123-2

ISBN (ePDF): 978-3-7489-2540-8

ISBN (Print): 978-3-03891-377-1 (Dike Verlag, Zürich/St. Gallen)

DOI: https://doi.org/10.5771/9783748925408

Dieses Werk ist lizensiert unter einer Creative Commons Namensnennung 4.0 International Lizenz. 


\section{Meiner Familie}




\section{Vorwort}

Diese Arbeit wurde im Herbstsemester 2020 von der Rechtswissenschaftlichen Fakultät der Universität Zürich als Dissertation abgenommen. Sie entstand im Wesentlichen während meiner Tätigkeit als wissenschaftliche Mitarbeiterin am Lehrstuhl für Strafrecht und Strafprozessrecht unter Einschluss des internationalen Strafrechts von Herrn Prof. Dr. Frank Meyer. Die Fertigstellung der Arbeit erfolgte während meiner Tätigkeit am Bezirksgericht Zürich. Literatur und Rechtsprechung konnten bis einschliesslich August 2020 berücksichtigt werden.

Mit dem fertiggestellten Buch in den Händen geht ein bereichernder Lebensabschnitt zu Ende. Ohne die Menschen, welche mich auf diesem begleitet haben, wäre die vorliegende Arbeit nicht in der gleichen Form zu realisieren gewesen. Es ist deshalb an der Zeit, mich an dieser Stelle bei all jenen zu bedanken, die mir auf dem Weg zur Promotion bedingungslos zur Seite gestanden sind und die mich stets fachlich, persönlich und emotional unterstützt haben.

An vorderster Stelle danke ich meinem Doktorvater Herrn Prof. Dr. Frank Meyer. Er hat nicht nur diese Arbeit mit gehaltvollen inhaltlichen Gedanken, konstruktiver Kritik und praktischen Hilfestellungen begleitet und gefördert, sondern mein wissenschaftliches Interesse überhaupt erst geweckt, mich zur Promotion ermuntert und während der Zeit an seinem Lehrstuhl meine wissenschaftliche Arbeitsweise massgebend geprägt.

Mein Dank gebührt zudem Frau Prof. Dr. Regina Kiener für die rasche Erstellung des Zweitgutachtens und ihre inhaltlichen Anregungen. Ich kann mich glücklich schätzen, dass mit Herrn Prof. Dr. Frank Meyer und Frau Prof. Dr. Regina Kiener zwei sehr versierte Experten im vorliegenden Themenfeld aus zwei unterschiedlichen Fachgebieten diese Arbeit begutachtet haben und sich damit die Interdisziplinarität der Arbeit auch auf dieser Ebene widerspiegelt.

Weiter gilt mein Dank Herrn Prof. Dr. Oliver Diggelmann, welcher anlässlich des Doktorandenkolloquiums und auch im Rahmen eines späteren persönlichen Gesprächs grosses Interesse nicht nur an der vorliegenden Arbeit, sondern auch an meinem Werdegang gezeigt hat.

Ganz herzlich bedanken möchte ich mich auch bei Herrn Prof. Dr. Andreas Dübendorfer. Er hat das ganze Manuskript geduldig und 
kritisch gegengelesen und lektoriert. Mit seinen sehr hilfreichen Ratschlägen zu sprachlich-stilistischen Fragen hat er massgeblich zur Lesefreundlichkeit der vorliegenden Arbeit beigetragen.

Besonders bedanken möchte ich mich weiter bei meinen beiden Mitstreitern Dr. iur. Pascal Ronc und Dr. iur. des. Marta Stelzer-Więckowska. Nicht selten waren wir vor ähnliche Herausforderungen gestellt, und unser Zusammenhalt ermöglichte es uns, diese im gemeinsamen Diskurs zu meistern. Sie beide sowie Dr. iur. Lukas Staffler, MLaw Dimitrios Tsilikis, MLaw Yvonne van der Stroom und Anna Brassel haben die Zeit am Lehrstuhl zu einer unvergesslichen gemacht.

Zu Dank bin ich auch MLaw Nicole Ahoya und MLaw Thuy Xuan Truong verpflichtet für den inspirierenden Meinungsaustausch und die notwendigen technischen Kniffe, als Word an seine Grenzen zu kommen drohte.

Teil am Gelingen des vorliegenden Werkes haben auch Bezirksrichter und Vizepräsident lic. iur. Roger Harris sowie Oberrichter lic. iur. Claudio Maira. Durch ihren Einsatz und ihr Verständnis haben sie beide mir die notwendige Zeit zur kompromisslosen Fertigstellung dieses Projekts verschafft und mir dabei auch gezeigt, dass Wissenschaft und Praxis eng miteinander verwoben sind.

Innigster Dank gebührt meiner Familie, der ich diese Arbeit widme. Sie hat mich jederzeit unterstützt, mir den Rücken freigehalten und mich emotional gestärkt. Sie hat nie daran gezweifelt, dass dieses Projekt gelingen wird, und musste dabei erdulden, dass neben der Arbeit und der Dissertation wenig gemeinsame Zeit verblieb. Besonderer Dank gilt dabei meiner Schwester, MLaw Yvonne van der Stroom, mit welcher ich in dieser Zeit nicht nur wertvolle persönliche, sondern auch zahlreiche fachliche Diskussionen geführt habe und welche das Manuskript gegengelesen und auf den Prüfstand gestellt hat. 


\section{Inhaltsübersicht}

$\begin{array}{ll}\text { Abkürzungsverzeichnis } & 21\end{array}$

Abkürzungsverzeichnis Länder $\quad 29$

Abkürzungsverzeichnis Kantone 31

Teil 1: Einleitung 35

Kapitel 1: Einführung 35

Kapitel 2: Untersuchungsgegenstand 36

Kapitel 3: Gang der Untersuchung 41

Teil 2: Grundlagen 47

Kapitel 1: Der Strafvollzug - ein empirischer Überblick 47

Kapitel 2: Menschenrechte im Strafvollzug 49

Kapitel 3: Rechtsrahmen im Mehrebenensystem 67

Kapitel 4: Soft Law 102

Kapitel 5: Absolute Rechte 118

Teil 3: Mindeststandards im Strafvollzug - eine Analyse 137

Kapitel 1: Vorbemerkungen 137

Kapitel 2: Überbelegung 140

Kapitel 3: Gesundheitsversorgung im Strafvollzug 211

Teil 4: Durchsetzungsmechanismen bei unzureichenden Haftbedingungen 286

Kapitel 1: Ausgangspunkt 286

Kapitel 2: Gewährleistung eines Mindeststandards vor dem Urteilszeitpunkt? - Vorsorgliche Massnahmen 287

Kapitel 3: Mindeststandard im Urteil - Wirkung eines EGMRUrteils 301 
Kapitel 4: Die Durchsetzung des Mindeststandards im Anschluss an das Urteil

Kapitel 5: Der Einfluss des EGMR in der Durchsetzungsphase - eine Judizialisierung des Implementierungsvorgangs?

Kapitel 6: Schlussevaluation des Durchsetzungsverfahrens Optimierungsmöglichkeiten zur Gewährleistung einer effektiven Durchsetzung?

Teil 5: Schlussbetrachtung

Literaturverzeichnis

Materialienverzeichnis 


\section{Inhaltsverzeichnis}

$\begin{array}{ll}\text { Abkürzungsverzeichnis } & 21\end{array}$

Abkürzungsverzeichnis Länder $\quad 29$

Abkürzungsverzeichnis Kantone 31

Teil 1: Einleitung 35

Kapitel 1: Einführung 35

Kapitel 2: Untersuchungsgegenstand 36

I. Festlegung des Untersuchungsgegenstands 36

II. Stand der Forschung $\quad 40$

Kapitel 3: Gang der Untersuchung $\quad 41$

I. Untersuchungsablauf 41

II. Methodik 44

Teil 2: Grundlagen $\quad 47$

Kapitel 1: Der Strafvollzug - ein empirischer Überblick 47

Kapitel 2: Menschenrechte im Strafvollzug 49

I. Menschenrechtsrelevanz des Strafvollzugs 49

II. Stellung des Inhaftierten 52

1. Modifikationen zu Gunsten des Inhaftierten? 52

1.1 Konventionsrechtliche Betrachtung 52

a Materiellrechtliche Besonderheiten $\quad 52$

b Prozedurale Besonderheiten $\quad 56$

1.2 Nationale Betrachtungsweise 58

2. Modifikationen zu Lasten des Inhaftierten? 59

2.1 Konventionsrechtliche Betrachtung 59

2.2 Nationale Betrachtungsweise 60

a Anforderungen an die gesetzliche Grundlage und Gesetzesform bei Grundrechtseinschränkungen (Legalitätsprinzip) 61

b Öffentliche Interessen und Verhältnismässigkeit i.e.S. 
c Möglichkeit disziplinarischer Massnahmen 63

d Prozedurale Besonderheiten 64

III. Zwischenfazit: Menschenrechte im Strafvollzug 65

Kapitel 3: Rechtsrahmen im Mehrebenensystem 67

I. Vereinte Nationen (UN) 67

II. Europarat 75

1. Die Europäische Menschenrechtskonvention 76

2. Europäisches Übereinkommen zur Verhütung von Folter und unmenschlicher oder erniedrigender Behandlung oder Strafe

3. Von den Europäischen Mindestgrundsätzen für die Behandlung der Gefangenen (ESMR) zu den Europäischen Strafvollzugsgrundsätzen (EPR) $\quad 87$

4. Weitere Empfehlungen des Europarates 91

III. Nationale Ebene / Schweiz 93

1. Bundesebene 94

1.1 Bundesverfassung 94

1.2 Bundesgesetze 96

1.3 Nationale Kommission zur Verhütung von Folter (NKVF)

2. Interkantonale Ebene 98

3. Kantonale Ebene 100

IV. Zwischenfazit zum Rechtsrahmen im Mehrebenensystem 102

Kapitel 4: Soft Law 102

I. Begriff, Charakter und normative Einordnung 102

II. Praktische Wirksamkeit 106

1. Praktische Wirksamkeit durch Implementierungskontrolle / Implementierungshilfe $\quad 106$

2. Praktische Wirksamkeit durch Rechtssetzung 108

3. Praktische Wirksamkeit in der Rechtsprechung 109

3.1 Im Allgemeinen 109

3.2 Bei Konventionsrechten 111

a Auf Rechtsebene 111

b Auf Sachverhaltsebene $\quad 114$

III. Zwischenfazit 117

Kapitel 5: Absolute Rechte 118

I. Vorbemerkungen 118 
II. Merkmale und Kernelemente eines absoluten Rechts am Beispiel von Art. 3 EMRK

1. Hinsichtlich der Anwendbarkeit

1.1 Kriterien

1.2 Prüfungsmethodisches Vorgehen

2. Bei der Bestimmung des Schutzbereichs und des Eingriffs

2.1 Relativität der Schutzbereichsbestimmung und des Eingriffsbegriffs

2.2 Umgang mit konfligierenden Interessen und Rechten

2.3 Bestimmtheitserfordernis

III. Absolutes Recht im Sonderrechtsverhältnis

IV. Absolute Rechte und Soft Law?

V. Zwischenfazit

Teil 3: Mindeststandards im Strafvollzug - eine Analyse

I. Untersuchungsgegenstand und Vorgehen

II. Mindeststandard und das "no more harm principle“

III. Mindeststandard und die Ressourcen der Konventionsstaaten

I. Überbelegung - Einführung und Definition

II. Überbelegung - Auswirkungen

III. Regelungsinstrumente zur Überbelegung - vorgeschlagene Mindeststandards

1. UN-Mindestgrundsätze für die Behandlung von Gefangenen und Nelson-Mandela-Regeln

2. Europäisches Komitee zur Verhütung von Folter und unmenschlicher oder erniedrigender Behandlung oder Strafe (CPT)

3. Europäische Mindestgrundsätze für die Behandlung der Gefangenen (ESMR) und Europäische Strafvollzugsgrundsätze (EPR)

4. European Recommendation R (99) 22

IV. Rechtsprechung des EGMR - Analyse und Kritik

1. Genese der Rechtsprechung - von Ananyev zu Muršić materieller Kern des Standards

2. Prüfungsmethodik des EGMR - Analyse

2.1 Vermutungswirkungen - Widerlegbarkeit -

Beweismass und -last 
2.2 Vermutungswirkungen, Voraussetzungen an die Kompensation zur Widerlegung

2.3 Vermutungswirkung und Grenze der Widerlegbarkeit - Element der Dauer

2.4 Prüfungsmethodisches Vorgehen des Gerichtshofs und Auswirkungen auf den Grundrechtsschutz

a Kumulation und Menschenrechtsschutz

b Kompensation und Menschenrechtsschutz

3. Dogmatische Einordnung der Überbelegung durch den EGMR

3.1 Überbelegung - unmenschlich oder erniedrigend?

3.2 Kriterien der Standardbildung

4. Notwendigkeit einer revidierten Prüfungsmethodik? - Ein

Lösungsvorschlag

5. EGMR im Verhältnis zu anderen Standards und zu anderen Institutionen

5.1 Einfluss anderer Institutionen auf die Rechtsprechung des EGMR bei der Überbelegung

a Rechtsebene

b Sachverhaltsebene

5.2 Zukunftsperspektiven des Soft Law in Bezug auf prekäre Platzbedingungen

6. Zwischenfazit

V. Situation in der Schweiz

1. Materieller Mindeststandard - Analyse der Gesetzeslage in der Schweiz

2. Rechtsprechung in der Schweiz - Analyse 200

2.1 Materieller Kern des Standards

2.2 Prüfungsmethodik des Schweizerischen Bundesgerichts und Vergleich mit dem EGMR

2.3 Dogmatische Einordnung der Überbelegung durch das Bundesgericht und Kriterien der Standardbildung

3. Bundesgerichtliche Rechtsprechung und das Soft Law 208

3.1 Rechtsebene

3.2 Sachverhaltsebene

4. Zwischenfazit

Kapitel 3: Gesundheitsversorgung im Strafvollzug

I. Gesundheitsversorgung im Strafvollzug - Einführung, Definition und Auswirkungen 
II. Gesundheitsversorgung im Strafvollzug - Prinzipien

1. Äquivalenzprinzip

2. Freiwilligkeitsprinzip im Strafvollzug - das Prinzip der Einwilligung (informed consent)

3. Vertrauensprinzip und Vertraulichkeit im Strafvollzug Arzt-Patienten-Beziehung

4. Zwischenfazit

III. Regelungsinstrumente zur Gesundheitsversorgung vorgeschlagene bzw. gesetzte Standards

1. Internationaler Pakt über wirtschaftliche, soziale und kulturelle Rechte (UNO-Pakt I)

2. UN-Mindestgrundsätze für die Behandlung von Gefangenen und Nelson-Mandela-Regeln

2.1 UN-Mindestgrundsätze für die Behandlung von Gefangenen

2.2 Die Nelson-Mandela-Regeln

3. Europäisches Komitee zur Verhütung von Folter und unmenschlicher oder erniedrigender Behandlung oder Strafe (CPT)

4. Europäische Mindestgrundsätze für die Behandlung der Gefangenen (ESMR) und Europäische Strafvollzugsgrundsätze (EPR)

5. Weitere Europaratsempfehlungen

6. Zwischenfazit

IV. Rechtsprechung des EGMR - Analyse und Kritik

1. Genese der Rechtsprechung - materieller Kern des Standards

2. Dogmatische Einordnung der Gesundheitsversorgung durch den EGMR und Kriterien der Standardbildung

3. Prüfungsmethodik des EGMR

3.1 Prüfungstiefe und -dichte und Beweislast

3.2 Äquivalenzprinzip vs. Angemessenheitsprüfung

a Bedeutung in der Rechtsprechung des Gerichtshofs

b Äquivalenz oder Angemessenheit als geeigneter Mindeststandard?

c Zwischenfazit

3.3 Wechselwirkungen - Kumulation und Kompensation? 254 
4. EGMR im Verhältnis zu anderen Standards und zu anderen Institutionen

4.1 Einfluss anderer Institutionen auf die Rechtsprechung des EGMR bei der Gesundheitsversorgung im Strafvollzug

a Rechtsebene

b Sachverhaltsebene

4.2 Zukunftsperspektiven des Soft Law in Bezug auf eine angemessene Gesundheitsversorgung im Strafvollzug

5. Zwischenfazit

V. Situation in der Schweiz

1. Materieller Mindeststandard - Analyse der Regulierung in der Schweiz

2. Rechtsprechung in der Schweiz - Analyse 278

2.1 Materieller Kern des Standards

2.2 Prüfungsmethodik des Schweizerischen Bundesgerichts und Vergleich mit dem EGMR

3. Bundesgerichtliche Rechtsprechung und das Soft Law

3.1 Rechtsebene

3.2 Sachverhaltsebene 284

4. Zwischenfazit

Teil 4: Durchsetzungsmechanismen bei unzureichenden Haftbedingungen

Kapitel 1: Ausgangspunkt

Kapitel 2: Gewährleistung eines Mindeststandards vor dem Urteilszeitpunkt? - Vorsorgliche Massnahmen

I. Bedeutung der vorsorglichen Massnahmen für die Durchsetzung von EGMR-Urteilen

II. Vorsorgliche Massnahmen bei unmenschlichen und erniedrigenden Haftbedingungen i.S.v. Art. 3 EMRK

1. Anordnung vorsorglicher Massnahmen nach Regel 39 VerfO

2. Befolgungspraxis der Konventionsstaaten bei vorsorglichen Massnahmen und die Rolle von Art. 34 EMRK

III. Zwischenfazit

Kapitel 3: Mindeststandard im Urteil - Wirkung eines EGMRUrteils

I. Feststellungsurteil 
II. Bindungswirkung und Orientierungswirkung - ein Überblick 302

III. Befolgungspflicht 304

1. Beendigungspflicht 304

2. Anordnung bestimmter Abhilfemassnahmen 305

2.1 Anordnung individueller Massnahmen bei unmenschlichen und erniedrigenden Haftbedingungen 309

2.2 Anordnung genereller Massnahmen bei unmenschlichen und erniedrigenden Haftbedingungen 311

IV. Piloturteilstechnik

1. Möglichkeit von Piloturteilsverfahren - im Allgemeinen

1.1 Entstehungsgeschichte und Überblick

1.2 Funktionsweise und Begriff des Piloturteilsverfahrens

2. Piloturteile bei unmenschlichen und erniedrigenden

Haftbedingungen i.S.v. Art. 3 EMRK

2.1 Vorbemerkungen

2.2 Vorliegen eines strukturellen oder systemischen Problems

a Die Piloturteile im Einzelnen 318

b Analyse der Piloturteile

aa Numerische und prognostizierende Betrachtung

bb Bedeutung der Kooperationsbereitschaft der Konventionsstaaten

cc Geographische Verteilung

dd Themenbereiche unmenschlicher und erniedrigender Haftumstände

ee Zeitlicher Verlauf der Anwendung der Piloturteilsverfahrenstechnik

ff Zwischenfazit

2.3 Anordnung konkreter individueller und genereller Massnahmen in den Piloturteilen

a Die Piloturteile im Einzelnen

b Weiterführende Analyse

aa Grundsätze des Gerichtshofs und Vorgehensweise

bb Themenbereiche unmenschlicher und erniedrigender Haftumstände

cc Art und Zeithorizont der vorgeschlagenen Massnahmen

dd Möglichkeit der Übergangslösungen 
ff Umsetzungsfrist 349

gg Parallelfälle $\quad 352$

2.4 Zwischenfazit 353

V. Gerechte finanzielle Entschädigung i.S.v. Art. 41 EMRK 355

Kapitel 4: Die Durchsetzung des Mindeststandards im Anschluss an das Urteil 357

I. Arbeitsweise und Rolle des Ministerkomitees bei der Durchsetzung von EGMR-Urteilen 358

1. Ausgangspunkt, Überwachungsgegenstand und Grundsätze 358

2. Entwicklung und Funktionsweise des Monitoringprozesses 359

2.1 Änderungen infolge des 14. Zusatzprotokolls 360

2.2 Priorisierungsverfahren des Ministerkomitees $\quad 362$

a Twin-track supervision - Zuteilung der Fälle 363

b Twin-track supervision - das Monitoringverfahren im Einzelnen 364

2.3 Überwachung der Zahlungsverpflichtung 368

2.4 Zusammenarbeit des Ministerkomitees mit weiteren Akteuren

II. Umsetzung der Piloturteile 371

1. Status der Umsetzung der angeordneten Massnahmen 371

2. Umsetzungsverfahren der Piloturteile 382

3. Analyse der Umsetzung der Piloturteile 387

3.1 Priorisierungsverfahren und Stand der Umsetzung $\quad 387$

3.2 Implementierungsdauer 389

3.3 Strukturelles Problem und Art der ergriffenen

Massnahmen

3.4 Generelle Implementierungsmassnahmen und Folgerechtsprechung

3.5 Bedeutung weiterer Akteure im Implementierungsverfahren

3.6 Stellungnahmen von NGOs, nationalen

Menschenrechtsinstitutionen und Beschwerdeführern $\quad 395$

3.7 Bedeutung des infringement proceeding 396

3.8 Zwischenfazit 396

III. Umsetzung übriger Urteile 397

1. Parallelfälle vs. Fälle ohne Konnex zu Piloturteilen und die Bedeutung des Priorisierungsverfahrens 398

2. Status der Umsetzung und die Art ergriffener Massnahmen 399

2.1 Umsetzungsverfahren und Notwendigkeit genereller Massnahmen 
2.2 Konsequenz der Gesamtbetrachtung bei der Durchsetzung des Urteils

Kapitel 5: Der Einfluss des EGMR in der Durchsetzungsphase - eine Judizialisierung des Implementierungsvorgangs?

Kapitel 6: Schlussevaluation des Durchsetzungsverfahrens Optimierungsmöglichkeiten zur Gewährleistung einer effektiven Durchsetzung?

Literaturverzeichnis

Materialienverzeichnis 


\section{Abkürzungsverzeichnis}

a.A.

Abs.

AEMR

AJIL

allg.

AöR

APA

APuZ

Art.

Aufl.

ausf.

BAG

Beschl.

betr.

BGE

BGer

BGG

BG KVF

BIG

BJ

BMC Public Health

BP

BSK

bspw.

BV

BVerfG

bzgl.

bzw. anderer Ansicht

Absatz

Allgemeine Erklärung der Menschenrechte

American Journal of International Law (Zeitschrift)

allgemein

Archiv des öffentlichen Rechts (Zeitschrift)

American Psychological Association

Aus Politik und Zeitgeschichte (Zeitschrift)

Artikel

Auflage

ausführlich

Bundesamt für Gesundheit (Schweiz)

Beschluss

betreffend

Leitentscheid des Bundesgerichts (Schweiz)

Bundesgericht (Schweiz) bzw. Entscheid des Schweizerischen Bundesgerichts

Bundesgerichtsgesetz vom 17. Juni 2005 (SR 173.110)

Bundesgesetz über die Kommission zur Verhütung von Folter vom 20. März 2009 (SR 150.1)

Projekt Bekämpfung von Infektionskrankheiten im Gefängnis

Bundesamt für Justiz (Schweiz)

BioMed Central Public Health (Zeitschrift)

Ban Public (Zeitschrift)

Basler Kommentar

beispielsweise

Bundesverfassung der Schweizerischen Eidgenossenschaft vom 18. April 1999 (SR 101)

Bundesverfassungsgericht (Deutschland)

bezüglich

beziehungsweise 
c.

CAT

CDPC

CEDH

CEPEJ

CESCR

CPT

d.h.

diesbzgl.

diss. op.

ECHR / ECtHR

ECOSOC

EDA

EDI

EDI-VO

EGMR

EJCPR

EJIL

EJPD

EJProb

EMRK

ENDIPP

Entsch. contre / gegen

Committee Against Torture / UN-Antifolterausschuss

European Committee on Crime Problems / Europäischer

Ausschuss für Strafrechtsfragen

Convention européenne des droits de l'homme / Europäische Menschenrechtskonvention

Commission européenne pour l'efficacité de la justice / Europäische Kommission für die Effizienz der Justiz

Committee on Economic, Social and Cultural Rights / Ausschuss für wirtschaftliche, soziale und kulturelle Rechte

European Committee for the Prevention of Torture and Inhuman or Degrading Treatment or Punishment / Europäisches Komitee zur Verhütung der Folter und der unmenschlichen und erniedrigenden Behandlung oder Strafe

das heisst

diesbezüglich

dissenting opinion / abweichendes Votum

European Court of Human Rights / Europäischer Gerichtshof für Menschenrechte

Economic and Social Council / Wirtschafts- und Sozialrat der Vereinten Nationen

Eidgenössisches Departement für auswärtige Angelegenheiten

Eidgenössisches Departement des Innern

Verordnung des EDI über die Meldung von Beobachtungen übertragbarer Krankheiten des Menschen vom 1. Dezember 2015 (SR 818.101.126)

Europäischer Gerichtshof für Menschenrechte

European Journal on Criminal Policy and Research (Zeitschrift)

European Journal of International Law (Zeitschrift)

Eidgenössisches Justiz- und Polizeidepartement

European Journal of Probation (Zeitschrift)

Konvention zum Schutze der Menschenrechte und Grundfreiheiten, abgeschlossen in Rom am 4. November 1950 (SR 0.101)

European Network on Drugs an Infections Prevention in Prison

Entscheidung 
EpG

EPR

$\mathrm{EpV}$

ERS

ESMR

et al.

etc.

ETS

EU

EuGRZ

Eur J Crim Policy

Res

EYHR

f./ff.

Fn.

FS

gem.

GG

GVG

HMG

HRLR

HRTF

HRRS

Hrsg.

HR\&ILD

HUDOC

HUDOC-Exec
Bundesgesetz über die Bekämpfung übertragbarer Krankheiten des Menschen (Epidemiengesetz) vom 28. September 2012 (SR 818.101)

European Prison Rules / Europäische Strafvollzugsgrundsätze

Verordnung über die Bekämpfung übertragbarer Krankheiten des Menschen (Epidemienverordnung) vom 29. April 2015 (SR 818.101.1)

Satzung des Europarates, angenommen in London am

5. Mai 1949 (SR 0.192.030)

European Standard Minimum Rules for the Treatment of Prisoners / Europäische Mindestgrundsätze für die Behandlung Gefangener

et alii / et aliae / und andere

et cetera

European Treaty Series

Europäische Union

Europäische Grundrechte-Zeitschrift (Zeitschrift)

European Journal on Criminal Policy and Research (Zeitschrift)

European Yearbook on Human Rights

und folgende

Fussnote

Festschrift

gemäss

Grundgesetz für die Bundesrepublik Deutschland

Gerichtsverfassungsgesetz (Deutschland)

Bundesgesetz über Arzneimittel und Medizinprodukte (Heilmittelgesetz) vom 15. Dezember 2000 (SR 812.21)

Human Rights Law Review (Zeitschrift)

Human Rights Trust Fund

Höchstrichterliche Rechtsprechung zum Strafrecht (OnlineZeitschrift)

Herausgeber

Human Rights \& International Legal Discourse (Zeitschrift)

Human Rights Documentation

Human Rights Documentation of the Department for the

Execution of Judgments of the ECHR 
ICPC

ICRC

i.d.R.

i.e.S.

IGH-Statut

IK

IKRK

insb.

IPbpR

i.S.d.

i.S.e.

i.S.v.

i.V.m.

JVV ZH

JZ

$\mathrm{KJ}$

KKJPD

KOK

KVG

KVV

lit.

LS

$\mathrm{m}^{2}$

Mio.

MLR

m.V.z.

m.w.H.
International Classification of Primary Care / medizinische Klassifikation

International Committee of the Red Cross / Internationales Komitee vom Roten Kreuz

in der Regel

im engeren Sinne

Statut des Internationalen Gerichtshof vom 26. Juni 1945, von der Bundesversammlung genehmigt am 12. März 1948 (SR 0.193.501)

Internationaler Kommentar zur EMRK

Internationales Komitee vom Roten Kreuz

insbesondere

Internationaler Pakt über bürgerliche und politische Rechte, abgeschlossen in New York am 16. Dezember 1966

(SR 0.103.2)

im Sinne des / der

im Sinne einer

im Sinne von

in Verbindung mit

Justizvollzugsverordnung des Kantons Zürich vom 6. Dezember 2006 (LS 331.1)

Juristen Zeitung (Zeitschrift)

Kritische Justiz (Zeitschrift)

Konferenz der Kantonalen Justiz- und Polizeidirektorinnen und -direktoren

Koordinationskonferenz Justizvollzug

Bundesgesetz über die Krankenversicherung vom 18. März 1994 (SR 832.10)

Verordnung über die Krankenversicherung vom 27. Juni 1995 (SR 832.102)

litera

Loseblattsammlung (Zürich)

Quadratmeter

Millionen

The Modern Law Review (Zeitschrift)

mit Verweis zu

mit weiteren Verweisen 
m.w.N.

$\mathrm{N}$

NGO

NJECL

NJW

NKVF

No.

$\mathrm{Nr}$.

NStZ

NVwZ

NWI

ONU

OPCAT

OSK

$\mathrm{Pl}$

PMC

resp.

Rn.

RRIP

RTDH

s.

S.

SAMW

SJZ

SK

SKJV

sog. mit weiteren Nachweisen

Note

Non-Governmental Organization / Nichtregierungsorganisation

New Journal of European Criminal Law (Zeitschrift)

Neue Juristische Wochenschrift (Zeitschrift)

Nationale Kommission zur Verhütung von Folter

Number / Nummer

Nummer

Neue Zeitschrift für Strafrecht (Zeitschrift)

Neue Zeitschrift für Verwaltungsrecht (Zeitschrift)

Strafvollzugskonkordat der Nordwest- und Innerschweiz, Konkordatsvereinbarung vom 5. Mai 2006 (SSED 01.0)

Organisation des Nations Unies / Vereinte Nationen

Optional Protocol to the Convention Against Torture / Fakultativprotokoll zum Übereinkommen gegen Folter und andere grausame, unmenschliche oder erniedrigende Behandlung oder Strafe, abgeschlossen in New York am 18. Dezember 2002

Konkordat der ostschweizerischen Kantone über den Vollzug von Strafen und Massnahmen vom 29. Oktober 2004 (LS 334)

Plenary / Plenum

Public Monitoring Committee

respectively / respektive

Randnummer

Règlement sur le régime intérieur de la prison et le statut des personnes incarcérées de Genève du 30 septembre 1985 (législation genevoise F 1 50.04)

Revue Trimestrielle des Droits de l'Homme (Zeitschrift) siehe

Seite

Schweizerische Akademie der Medizinischen Wissenschaften Schweizerische Juristen-Zeitung (Zeitschrift)

Systematischer Kommentar zur Strafprozessordnung mit GVG und EMRK

Schweizerisches Kompetenzzentrum für den Justizvollzug

sogenannt 
SPACE

SPS

SPT

sq. m

SR

SSED

StGB

StJVG ZH

StPO

StV

SZK

TLR

u.

u.a.

UCL JLJ

$\mathrm{UN}$

UNCAT

UNCCPCJ

UN-Charta
Statistiques Pénales Annuelles du Conseil de l'Europe / jährliche Statistiken des Europarates

Santé Prison Suisse

Subcommittee on Prevention of Torture and other Cruel, Inhuman or Degrading Treatment or Punishment (UN) / Unterausschuss zur Verhütung von Folter und anderer grausamer, unmenschlicher oder erniedrigender Behandlung oder Strafe der Vereinten Nationen

square meter / Quadratmeter

Systematische Rechtssammlung des Bundes

Systematische Sammlung der Erlasse und Dokumente

Schweizerisches Strafgesetzbuch vom 21. Dezember 1973

(SR 311.0)

Straf- und Justizvollzugsgesetz des Kantons Zürich vom 19. Juni 2006 (LS 331)

Schweizerische Strafprozessordnung vom 5. Oktober 2007 (SR 312.0)

Strafverteidiger (Zeitschrift)

Schweizerische Zeitschrift für Kriminologie (Zeitschrift)

Tulane Law Review (Zeitschrift)

und

und andere / unter anderem

UCL Journal of Law and Jurisprudence (Zeitschrift)

United Nations / Vereinte Nationen

Convention against Torture and Other Cruel, Inhuman or Degrading Treatment or Punishment / Übereinkommen gegen Folter und andere grausame, unmenschliche oder erniedrigende Behandlung oder Strafe, abgeschlossen in New York am 10. Dezember 1986 (SR 0.105)

Commission on Crime Prevention and Criminal Justice / UN-Kommission für Verbrechensverhütung und Strafrechtspflege

Charta der Vereinten Nationen, abgeschlossen in San Francisco am 26. Juni 1945 (SR 0.120) 
UNCPCTO

UNO

UNODC

UNO-Pakt I

UNO-Pakt II

UN-SMR

Urt.

v/vs.

$\mathrm{v}$.

v.a.

VerfO

vgl.

WSK-Ausschuss

WVK

ZaöRV

z.B.

ZEK

ZEuS

ZfStrVO

ZGB

Ziff.
United Nations Congress on the Prevention of Crime and the Treatment of Offenders resp. since 2005 United Nations Congress on Crime Prevention and Criminal Justice / Kongress der Vereinten Nationen für Verbrechensverhütung und die Behandlung Straffälliger resp. seit 2005 Kongress der Vereinten Nationen zur Verbrechensverhütung und Strafrechtspflege

United Nations Organization / Vereinte Nationen

United Nations Office on Drugs and Crime / Büro der Vereinten Nationen für Drogen- und Verbrechensbekämpfung Internationaler Pakt über wirtschaftliche, soziale und kulturelle Rechte, abgeschlossen in New York am 16. Dezember 1966 (SR 0.103.1)

Internationaler Pakt über bürgerliche und politische Rechte, abgeschlossen in New York am 16. Dezember 1966 (SR 0.103.2)

United Nations Standard Minimum Rules for the Treatment of Prisoners / Mindestgrundsätze für die Behandlung der Gefangenen

Urteil

versus

vom

vor allem

Verfahrensordnung des Europäischen Gerichtshofs für Menschenrechte vom 4. November 1998 (SR 0.101.2)

vergleiche

Ausschuss für wirtschaftliche, soziale und kulturelle Rechte

Wiener Übereinkommen über das Recht der Verträge, abgeschlossen in Wien am 23. Mai 1969 (SR 0.111)

Zeitschrift für ausländisches öffentliches Recht und Völkerrecht (Zeitschrift)

zum Beispiel

Zentrale Ethikkommission

Zeitschrift für Europarechtliche Studien (Zeitschrift)

Zeitschrift für Strafvollzug und Straffälligenhilfe (Zeitschrift)

Schweizerisches Zivilgesetzbuch vom 10. Dezember 1907

(SR 210)

Ziffer 


\section{Abkürzungsverzeichnis}

ZIS

zit.

ZÖR

$\mathrm{ZP}$

ZSR

ZStW
Zeitschrift für Internationale Strafrechtsdogmatik (Zeitschrift)

zitiert

Zeitschrift für öffentliches Recht (Zeitschrift)

Zusatzprotokoll

Zeitschrift für Schweizerisches Recht (Zeitschrift)

Zeitschrift für die gesamte Strafrechtswissenschaft (Zeitschrift) 


\section{Abkürzungsverzeichnis Länder}

\begin{tabular}{ll} 
ALB & Albanien \\
ARM & Armenien \\
AUT & Österreich \\
AZE & Aserbaidschan \\
BEL & Belgien \\
BIH & Bosnien u. Herzegowina \\
BUL & Bulgarien \\
CRO & Kroatien \\
CYP & Zypern \\
CZE & Tschechische Republik \\
DEN & Dänemark \\
ESP & Spanien \\
EST & Estland \\
FIN & Finnland \\
FRA & Frankreich \\
GBR & Grossbritannien \\
GEO & Georgien \\
GER & Deutschland \\
GRE & Griechenland \\
HUN & Ungarn \\
IRL & Irland \\
ITA & Italien \\
LAT & Lettland \\
LTU & Litauen \\
MDA & Moldawien \\
MKD & Nordmazedonien \\
MNE & Montenegro \\
NED & Niederlande \\
POL & Polen \\
ROM & Rumänien \\
RUS & Russland \\
& \\
\hline
\end{tabular}


Abkürzungsverzeichnis Länder

$\begin{array}{ll}\text { SLO } & \text { Slowenien } \\ \text { SUI } & \text { Schweiz } \\ \text { TUR } & \text { Türkei } \\ \text { UKR } & \text { Ukraine }\end{array}$




\section{Abkürzungsverzeichnis Kantone}

$\begin{array}{ll}\text { AG } & \text { Aargau } \\ \text { AI } & \text { Appenzell Innerrhoden } \\ \text { AR } & \text { Appenzell Ausserrhoden } \\ \text { BE } & \text { Bern } \\ \text { BL } & \text { Basel-Landschaft } \\ \text { BS } & \text { Basel-Stadt } \\ \text { FR } & \text { Freiburg } \\ \text { GE } & \text { Genf } \\ \text { GL } & \text { Glarus } \\ \text { GR } & \text { Graubünden } \\ \text { JU } & \text { Jura } \\ \text { LU } & \text { Luzern } \\ \text { NE } & \text { Neuenburg } \\ \text { NW } & \text { Nidwalden } \\ \text { OW } & \text { Obwalden } \\ \text { SG } & \text { St. Gallen } \\ \text { SH } & \text { Schaffhausen } \\ \text { SO } & \text { Solothurn } \\ \text { SZ } & \text { Schwyz } \\ \text { TG } & \text { Thurgau } \\ \text { TI } & \text { Tessin } \\ \text { UR } & \text { Uri } \\ \text { VD } & \text { Waadt } \\ \text { VS } & \text { Wallis } \\ \text { ZG } & \text { Zug } \\ \text { ZH } & \text { Zürich }\end{array}$


"The mood and temper of the public with regard to the treatment of crime and criminals is one of the most unfailing tests of the civilization of any country."

Winston Churchill 


\section{Teil 1: Einleitung}

\section{Kapitel 1: Einfübrung}

„Human rights instruments assume that prisoners are sent to prison as punishment not for punishment, so prisoners remain all their human rights except the right to liberty. "1

Wenn der Strafprozess in einer Verurteilung der beschuldigten Person mündet, ist es die Aufgabe des Richters, im gesetzlich vorgegebenen Rahmen eine dem Verschulden entsprechende Strafe auszusprechen. Das formelle Strafverfahren endet mit dem richterlichen Urteil; es folgt, sofern die Sanktion unbedingt ausgesprochen wurde, die Phase des Vollzugs der gefällten Strafe oder Massnahme. ${ }^{2}$ Eine mögliche Strafart ist die Freiheitsstrafe. Die Freiheitsstrafe ist heute zumindest in europäischen Strafrechtsordnungen die aus menschenrechtlicher Sicht sensibelste Sanktionsform. ${ }^{3}$ Dabei bestimmt nicht nur die Länge der Freiheitsstrafe über deren Eingriffsintensität. Massgeblichen Einfluss haben auch die Modalitäten

1 Charles/Draper Journal of Medical Ethics 2012, $215 \mathrm{ff}$.; zur rechtlichen Einordnung des „no more harm principle“ vgl. insbesondere Teil 3 Kap. 1 II.

2 Vgl. auch Laubenthal S. 13 ff.: Das Strafvollzugsrecht bilde neben dem materiellen Strafrecht und dem Strafverfahrensrecht eine eigenständige Rechtsmaterie innerhalb des gesamten Kriminalrechts. Laubenthal führt dabei zu Recht weiter aus, dass die „Drei-Säulen-Theorie“, gemäss welcher den verschiedenen Instanzen je unterschiedliche Aufgaben zukommen (generalpräventive Gesetzgebung, vergeltende Rechtsprechung und resozialisierender Strafvollzug), aber veraltet sei, zumal die Dynamik der Strafrechtsrealisierung durch diese vernachlässigt werde.

3 Dünkel/Lappi-Seppälä/Morgenstern/van ZyL Smit in: Dünkel/Lappi-Seppälä/ Morgenstern/van Zyl Smit, S. 1023 (1023f.): Freiheitsstrafe als "ultima ratio“ im europäischen Sanktionensystem; vgl. auch van ZYL SMIT/SNACKen S. 86 ff.: „imprisonment as a last resort"; DünKel/van ZyL Smit in: van Zyl Smit/Dünkel, S. 796. Vgl. aber auch Morgenstern/van ZyL SMIt in: Bruinsma/Weisburd, S. $1614 \mathrm{ff}$., welche sich mit den menschenrechtlichen Implikationen anderer Strafarten auseinandersetzen. Siehe auch Morgenstern Internationale Mindeststandards für ambulante Strafen und Massnahmen S. 8: Bei ihrer Einführung sei die Freiheitsstrafe allerdings ein Schritt zur Humanisierung des Strafens gewesen; sie war der Todesstrafe, den Körperstrafen, der Sklaverei oder der Verbannung vorzuziehen. Vgl. ferner Gräfenstein ZfStrVO 2003, 10 ff.; Snacken Eur J Crim Policy Res 2006, 143 (143, $150 \mathrm{ff}$.$) .$ 
des Strafvollzugs, insbesondere die materiellen Haftbedingungen ${ }^{4}$ Mindeststandards im Strafvollzug und deren effektive Implementierung sind Thema der vorliegenden Arbeit.

Der Arbeit liegt dabei die These zugrunde, dass ein effektiver Menschenrechtsschutz im Strafvollzug einerseits einen klaren Mindeststandard im Sinne einer normativen Erwartung, und andererseits eine umfassende Umsetzung dieses Standards in der Praxis erfordert. An diesen beiden Prämissen setzt die Untersuchung der Arbeit an. Die vorliegende Arbeit bezweckt, damit einen Beitrag hin zu einem rechtsstaatlichen, menschenrechtsgewährleistenden, aber auch effizienten und praktikablen Strafvollzug zu leisten. Unter der weiteren Prämisse, dass humanitäre ${ }^{5}$, einem Mindeststandard genügende Haftbedingungen Grundvoraussetzung für die Erfüllung des Vollzugsziels der Resozialisierung sind ${ }^{6}$ bezweckt die vorliegende Arbeit letztlich auch die Erreichung dieses Ziels. In einen grösseren Kontext gesetzt, soll die Arbeit durch die Thematisierung von Mindeststandards letztlich auch zur Sensibilisierung der eingriffsintensiven Menschenrechtsthematik des Strafvollzugs allgemein beitragen. In diesem Sinne verbindet die Arbeit die genuin strafrechtliche Thematik des Strafvollzugs mit ihrem menschenrechtlichen Wesensgehalt.

\section{Kapitel 2: Untersuchungsgegenstand}

\section{Festlegung des Untersuchungsgegenstands}

Der in der vorliegenden Arbeit verwendete Begriff des Strafvollzugs beschränkt sich auf den Vollzug einer rechtskräftigen Freiheitsstrafe. Präventive Haftformen, wie die Untersuchungs- oder Sicherheitshaft, und strafrechtliche Massnahmen sind damit, soweit für diese andere Grundsätze gelten, von vornherein ausgenommen. Auch die ausländerrechtliche Administrativhaft, wie die Ausschaffungs- oder Durchsetzungshaft, werden ausdrücklich nicht vom hier verwendeten Begriff umfasst. Diese Vollzugs-

4 Drenkhahn/Dudeck/Dünkel S. 4; Morgenstern in: Dünkel/Drenkhahn/Morgenstern, S. 35 ff.; vgl. auch Drenkhahn/Morgenstern in: Reeves, S. 137 ff.

$5 \mathrm{Zu}$ ethischen Grundlagen der Gefängnisverwaltung vgl. auch: CoYLE/FAIR/JACOBSON/WalmsLey S. $69 \mathrm{f}$.

6 Vgl. etwa Maelicke Neue Kriminalpolitik 2003, 143; Neale in: Muncie/Sparks, S. 203 (206f.); Kretschmer NStZ 2005, 251 (255); Keller/Schädler ZSR 2013, 195 (209f.); zum Zusammenhang der Überbelegung und der Rückfallgefahr vgl. bereits Farrington/Nuttall Journal of Criminal Justice 1980, 221 (229 f.). 
arten verfolgen je einen anderen Zweck, weshalb für sie in mancher Hinsicht andere Standards anzusetzen sind. ${ }^{7}$

Der Begriff der Menschenrechte wird im Kontext der vorliegenden Arbeit als Überbegriff verwendet und umfasst einklagbare Rechtsansprüche Privater gegenüber dem Staat. Sie dienen dem Schutz grundlegender Aspekte der Person und ihrer Würde, unabhängig davon, ob sie nun von der Verfassung gewährt (Grundrechte ${ }^{8}$ ) oder vom Völkerrecht garantiert (Menschenrechte i.e.S. ${ }^{9}$ ) werden. Im Kontext der vorliegenden Arbeit ist es nämlich nicht zwingend notwendig, terminologisch durchgehend zwischen Grundund Menschenrechten i.e.S. zu differenzieren, ist es doch nur deren rechtliche Grundlage, welche jeweils ausdrücklich zu benennen ist, und nicht Inhalt oder Funktion, welche sie voneinander unterscheidet. ${ }^{10}$

Der Strafvollzug ist Aufgabe der einzelnen Nationalstaaten. Die anzuwendenden Vollzugsmodalitäten sind denn auch primär im nationalen Recht normiert. ${ }^{11}$ Mindestanforderungen - im Sinne von Untergrenzen an materielle Haftbedingungen beim Vollzug einer Freiheitsstrafe lassen sich aus Grund- bzw. Menschenrechten ableiten. Vorgaben an das innerstaatliche Recht können sich demnach nicht nur aus den nationalen Grundrechten, sondern insbesondere auch aus abgeschlossenen (Menschenrechts-)Verträgen ergeben. Auch europäisches resp. internationales Soft Law ${ }^{12}$ kann Einfluss auf das innerstaatliche Recht und die nationale Rechtsprechung nehmen. Vor diesem Hintergrund scheint ein rein nationaler Ansatz als ungeeignet, auch wenn sich ein Mindeststandard für Vollzugsbedingungen immer erst auf nationaler Ebene manifestiert. Geeigneter erscheint eine internationale oder aber eine europäische Betrachtungsweise. Im Vergleich zur internationalen Betrachtung hat eine europäische Betrachtung zwei Vorteile: Erstens hat sich der EGMR als einer der Hauptakteure bei der Festsetzung von Untergrenzen im Bereich

$7 \mathrm{Zu}$ internationalen Mindeststandards für ambulante Strafen und Massnahmen vgl. etwa Morgenstern Internationale Mindeststandards für ambulante Strafen und Massnahmen; zu verfassungs- und europarechtlichen Vorgaben für den Untersuchungshaftvollzug vgl. Morgenstern StV 2013, 529 ff.; zu der ausländerrechtlichen Administrativhaft vgl. etwa ausf. Catak Kanber S. $1 \mathrm{ff} ., 247 \mathrm{ff}$.

8 Kiener in: Biaggini/Gächter/Kiener, S. 425.

9 Kiener in: Biaggini/Gächter/Kiener, S. 425; KäLIn/KünZli S. 35 f.

10 KäLIN/KüNZLi S. $35 \mathrm{f}$.

11 Vgl. auch Riegel/Speicher StV 2016, $250 \mathrm{ff}$.

12 Zum Begriff des Soft Law vgl. Teil 2 Kap. 4 ff. 
der Haftbedingungen ${ }^{13}$ etabliert, und die herausragende Stellung des EGMR findet auf internationaler Ebene kein Pendant. Die Möglichkeit der Individualbeschwerde i.S.v. Art. 34 EMRK, mit welcher es jeder natürlichen Person offensteht, sich an den Gerichtshof zu wenden und eine Verletzung der in der Konvention oder in den Protokollen anerkannten Rechte zu rügen, ist international einzigartig. ${ }^{14}$ Die Kasuistik des EGMR eignet sich deshalb in besonderem Masse als Ausgangspunkt für eine normativ belegte Herauskristallisierung von Mindeststandards im Bereich der Haftbedingungen. Sie wird vorliegend als Erkenntnisquelle genutzt. Zweitens bringt der Blick auf einen internationalen Mindestkonsens aus einer menschenrechtlichen Optik aus schweizerischer Perspektive keinen Mehrwert, da dieser auch im europäischen Standard implementiert ist und damit keine weiterführende Anforderungen an den Strafvollzug stellt, sondern sogar umgekehrt: Je mehr Staaten involviert sind, desto mehr unterscheiden sich die Grundgegebenheiten und umso schwieriger ist es entsprechend, diese Diskrepanzen konsensuell zu überwinden, die bestehenden Spannungsverhältnisse aufzulösen und sich auf einen allgemein gültigen Mindeststandard zu einigen, welcher in jedem der beteiligten Staaten nationalrechtlich umzusetzen ist. ${ }^{15}$ Ein konventionsrechtlicher Ausgangspunkt scheint für die vorliegende Arbeit damit angezeigt. Internationale Übereinkommen und andere internationale Regelwerke werden als Erkenntnisquelle hinzugezogen, soweit sie Auswirkungen auch auf Europaratsebene zeitigen. Aufgrund der Verbindlichkeiten der auf Europaratsebene ausgearbeiteten Standards, auch für die Schweiz, scheint es angezeigt, auch einen Blick auf nationale Bestimmungen und Rechtsprechung zu werfen.

Aufgrund der fundamentalen Bedeutung und der überragenden Relevanz des Art. 3 EMRK bezüglich Mindestanforderungen an den Strafvollzug in der Rechtsprechung der Strassburger Organe werden das Verbot

13 Vgl. etwa SK-StPO/MeYer Art. 3 EMRK Rn. 44. Im Allgemeinen ist der Europarat im Bereich der Menschenrechte federführend: vgl. etwa schon LALumière in: Macdonald/Matscher/Petzold, S. XV: „No international organization, whether regional or global, is able to parallel the unique achievements of the Council of Europe in the human-rights field."

14 Meyer-Ladewig/Nettesheim/von Raumer Einleitung Rn. 2; vgl. auch Stone Sweet/Keller in: Keller/Stone Sweet, S. 3: „most effective human rights regime in the world“; Nawparwar S. $100 \mathrm{ff} ., 281$.

15 Hinzu kommen auch grössere kulturelle Unterschiede, was sich sowohl im Menschenbild als solchem, aber in concreto auch im Strafzweck und dem Vollzugsziel niederschlägt. 
der Folter sowie die Verbote der unmenschlichen und erniedrigenden Behandlung und Bestrafung in der vorliegenden Arbeit als menschenrechtlicher Ankerpunkt genommen. Dies im Bewusstsein, dass darüber hinaus weitere menschenrechtliche Garantien Vorgaben an die materiellen Haftbedingungen stellen.

Anknüpfend an die beiden der vorliegenden Arbeit zugrunde liegenden Thesen, dass ein effektiver Menschenrechtsschutz im Strafvollzug einerseits einen klaren Mindeststandard im Sinne einer normativen Erwartung und andererseits eine umfassende Umsetzung dieses Standards in der Praxis erfordert, wird zunächst der Frage nachgegangen, welche europäischen Mindeststandards aus Art. 3 EMRK hergeleitet werden können. Es geht dabei nicht darum, eine Enzyklopädie eines Mindeststandards zu verfassen. Vielmehr werden zwei strafvollzugsspezifische und menschenrechtliche bezüglich Art. 3 EMRK relevante Themen materieller Haftbedingungen herausgegriffen: (1) Fragen rund um die Überbelegung und (2) rund um die Gesundheitsversorgung im Strafvollzug. Dies wiederum im Bewusstsein, dass ein mit Art. 3 EMRK konformer Strafvollzug darüber hinaus weit mehr Anforderungen an den Strafvollzug bzw. an die Haftbedingungen stellt: $\mathrm{Zu}$ erwähnen seien bloss etwa Fragen hinsichtlich einer notwendigen Möglichkeit von Outdoor-Aktivitäten, Beschäftigungs- und Arbeitsmöglichkeiten als solche, Schutz vor Witterung, Anforderungen an die hygienischen Verhältnisse im Strafvollzug, Zugang zu sanitären Einrichtungen und genügend Privatsphäre bei deren Nutzung, Anforderungen an die Licht-, Luft- und Temperaturverhältnisse in der Zelle sowie ausreichende und angemessene Ernährung während des Vollzugs. Die Auswahl der beiden oben genannten Themen basiert auf einer Analyse der EGMRUrteile der letzten Jahre. Diese verdeutlicht, dass diese beiden Themenfelder in der Praxis vor dem EGMR von besonderer Relevanz sind und gerade die Überbelegungsthematik als Ankerpunkt für die Beurteilung auch der weiteren angeführten Haftbedingungen fungiert. Wie zu zeigen ist, sind die beiden ausgewählten Themenkreise auch geeignet, repräsentative Aussagen zur Vorgehensweise bei der Standardbestimmung und damit zur Methodik des Gerichtshofs zu treffen. Resultate dieser Analyse können deshalb in der Folge auch auf die weiteren genannten Haftbedingungen angewandt werden.

Der eruierte europäische Mindeststandard ist von sämtlichen durch die EMRK verpflichteten Staaten umzusetzen, weshalb in der Folge die Durchsetzungsmechanismen bei unzureichenden Haftbedingungen analysiert werden und ein allfälliger Handlungsbedarf aufgezeigt und nach Optimierungsmöglichkeiten bei der Durchsetzung gesucht wird. Aufgrund des in 
der EMRK statuierten Günstigkeitsprinzips (Art. 53 EMRK) bleiben durch die vorliegende Arbeit, welche den Fokus auf zu fordernde Untergrenzen legt, sämtliche Praktiken der Konventionsstaaten, welche einen über den Mindeststandard hinausgehenden Schutz gewähren, unberührt. Die vorliegende Arbeit ist folglich ausdrücklich nicht als Anreiz zu sehen, einen nationalrechtlichen Standard, der über die menschenrechtlich zwingende Grundlage hinausgeht, in irgendeiner Weise in Frage zu stellen.

\section{Stand der Forschung}

Eine detaillierte systematische Auseinandersetzung mit Mindestanforderungen an den Strafvollzug, insbesondere bezüglich Überbelegung und der Gesundheitsversorgung, welche auch die Methodik des EGMR bei der Standardbestimmung miteinbezieht, findet sich in der Literatur trotz ihrer immensen Praxisrelevanz nicht.

Die Literatur behandelt zwar einzelne für Art. 3 EMRK relevante Aspekte des Strafvollzugs; eine eingehende Analyse etwa der Rechtsprechungslinie des EGMR in Bezug auf die genannten Themen bleibt dabei allerdings aufgrund anderer Zielsetzungen der jeweiligen Projekte aus. ${ }^{16}$ Auch das Wesensmerkmal der Absolutheit des Art. 3 EMRK war in der Vergangenheit bereits mehrfach Anknüpfungspunkt für philosophische und rechtstheoretische Ausführungen; ${ }^{17}$ eine systematische Verknüpfung der Ergebnisse dieser Analysen mit der vorliegenden Fragestellung wurde bisher allerdings noch nicht vorgenommen. Gleich verhält es sich mit der Rolle

16 Vgl. etwa die Dissertation von van DER BERG, deren Untersuchung es bezweckt, ein vollständiges Bild des Art. 3 EMRK zu schaffen: S. 19 ff., 22. Siehe auch die Dissertation von von Schwichow S. $1 \mathrm{ff}$., der die Menschenwürde ins Zentrum der Untersuchung rückt. Vgl. auch die Dissertation von Enengel S. 1 ff., welche Art. 3 EMRK und Art. 8 EMRK zum Ausgangspunkt der Untersuchung nimmt.

17 Vgl. etwa Addo/Grief EJIL 1998, 510 ff.; Addo/Grief European Law Review Human Rights Survey 1998, $17 \mathrm{ff}$.; Mavronicola HRLR 2012, $723 \mathrm{ff}$.; SMEt HRLR 2013, 469 ff.; Greer HRLR 2015, $101 \mathrm{ff}$; Mavronicola HRLR 2015, 721 ff.; Mavronicola HRLR 2017, 479 ff.; vgl. auch van Der Berg S. 29 ff. 
des Soft Law für Menschenrechte ${ }^{18}$ oder gar für Art. 3 EMRK, ${ }^{19}$ welche verschiedentlich bereits Anknüpfungspunkt für Untersuchungen war. Eine eingehende systematische Untersuchung des Einflusses des Soft Law spezifisch in Bezug auf die vorliegenden Themen wurde allerdings noch nicht durchgeführt. ${ }^{20}$ Ebenso mangelt es in der Literatur an einer eingehenden Analyse der effektiven Nutzung der vorhandenen Durchsetzungsmechanismen spezifisch im Bereich materieller Haftbedingungen. Gleichwohl waren die nachfolgend zu analysierenden Durchsetzungsmechanismen im Allgemeinen schon früher Untersuchungsgegenstand. ${ }^{21}$

Insgesamt fügt sich die vorliegende Arbeit damit in den bestehenden wissenschaftlichen Diskurs ein und ergänzt diesen mit neuen, bislang noch nicht systematisch untersuchten Facetten.

\section{Kapitel 3: Gang der Untersuchung}

\section{Untersuchungsablauf}

Die Arbeit gibt zunächst einen empirischen Überblick über die Fakten des Strafvollzuges in den Europaratsstaaten. Die Übersicht dient dazu, die Rahmenbedingungen des Strafvollzugs aufzuzeigen, und ist dabei gleichzeitig

18 Vgl. etwa die Beiträge in Gammeltoft-Hansen/Lagoutte/Cerone (Hrsg.), Tracing the Roles of Soft Law in Human Rights; vgl. insbesondere auch GLAS HRLR 2017, 97 ff.: GLAs führte eine repräsentative Analyse durch, indem sie eine Stichprobe von 795 EGMR-Urteilen analysierte. Dabei untersuchte sie Anzahl und Bedeutung der Urteile, in denen der Gerichtshof ein Europaratsdokument zitierte, sowie die Organe und zitierten Dokumente. Weiter eruierte sie auch die Relevanz der zitierten Dokumente für die Argumentation des Gerichtshofs.

19 Etwa Ronc ex ante 2017, 67 ff. (betr. lebenslangen Freiheitsentzug); SјöHolm S. $387 \mathrm{ff} ., 418 \mathrm{f}$. (betr. häusliche Gewalt).

20 Vgl. insb. aber auch folgende Beiträge: Morgenstern in: Dünkel/Drenkhahn/ Morgenstern, S. 35 ff.; Murdoch S. 212 ff. Vgl. auch die Dissertation von Cernко, welche sich allerdings auf die Untersuchung der Umsetzung von CPT-Empfehlungen im deutschen Strafvollzug beschränkte.

21 Vgl. insb. Keller/Marti EJIL 2015, 829 ff.; zu den Urteilswirkungen und zum Dialog zwischen Mitgliedstaaten, EGMR und Ministerkomitee insb. GLaS EYHR 2018, 287 ff.; zum Potential des Dialogs zwischen den beteiligten Akteuren in der Durchsetzungsphase auch GLas S. 207 ff.; vgl. auch die weiteren einschlägigen Beiträge von Glas: Glas HRLR 2014, 671 ff.; Glas HR\&ILD 2019, 73 ff.: GLAs untersuchte unter anderem den Erfolg des Piloturteilsverfahrens im Hinblick auf die Umsetzung der Piloturteile im Allgemeinen. Zum Piloturteilsverfahren vgl. insb. auch die Dissertationen von Eschment, Haider und Kindt. 
ein erster Schritt bei der Absteckung der faktischen Möglichkeiten der Mindeststandards. Da der Mindeststandard aus Art. 3 EMRK abgeleitet wird, ist die Verdeutlichung der Menschenrechtsrelevanz des Strafvollzugs notwendiger nächster Schritt. Besonderes Augenmerk wird auf die Rechtsstellung des Inhaftierten gelegt, wobei die Modifikationen der Rechtsstellung bzw. prozessuale Vor- und Nachteile durch den Umstand der Inhaftierung den Schwerpunkt der Abhandlung bilden. Hierbei werden die verschiedenen Dimensionen der Menschenrechte, die Argumentationsfigur des Sonderstatusverhältnisses bzw. des besonderen Gewaltverhältnisses sowie die besondere Fürsorgepflicht durchleuchtet.

Die Normierung der materiellen Haftbedingungen im Mehrebenensystem verlangt, auf dieses im Folgenden näher einzugehen. Dabei werden die für Mindeststandards bei Haftbedingungen wichtigsten Regulatorien auf internationaler, europäischer und national schweizerischer Ebene näher betrachtet; sie sind der Ausgangspunkt bei der Ausarbeitung der Mindeststandards.

Es wird später in der Arbeit weiter konstatiert, dass Soft Law bisweilen einen bedeutenden Einfluss sowohl auf die nationale Legislative und Judikative als auch auf die Rechtsprechung des EGMR in Bezug auf materielle Haftbedingungen hat, weshalb vorab auf den Begriff des Soft Law, seine normative Einordnung und seine Wirkungsmechanismen eingegangen wird. Weitere Grundvoraussetzung für die Untersuchung von Mindeststandards als Ausfluss einer Untergrenze gemäss Art. 3 EMRK ist ein präzises Verständnis der Charakteristika dieses absoluten Rechts, weshalb in einem nächsten Schritt seine Wesensgehalte ausgearbeitet werden. Damit sind schliesslich alle Grundlagen geschaffen, so dass in der Folge die beiden Themengebiete der Überbelegung und der Gesundheitsversorgung im Strafvollzug zum Ausgangspunkt der weiteren Untersuchung gemacht werden können. Für diese ausgewählten Themengebiete erfolgt in einem ersten Schritt eine genaue Analyse der für dieses Gebiet gemachten Vorgaben resp. Empfehlungen in den einschlägigen Regelwerken. Der Schwerpunkt wird hier auf die Regulatorien der Europaratsebene gelegt; internationale Normierungen werden ergänzend hinzugezogen. In einem zweiten Schritt wird die Rechtsprechung des EGMR unter dem Gesichtspunkt von Art. 3 EMRK untersucht. Dabei richtet sich zum einen der Fokus auf inhaltliche Angaben, aus denen ein Mindeststandard betreffend eine konkrete Frage abgeleitet werden kann. Zum anderen sind auch das Vorgehen des EGMR bei der Herleitung seiner inhaltlichen Aussagen und seine allgemeinen Argumentationsformen Zentrum der Betrachtung. Auch die Einhaltung des absoluten Charakters von Art. 3 EMRK wird hier einer 
Prüfung unterzogen. Sowohl der eruierte Inhalt des Mindeststandards als auch die methodische Vorgehensweise des EGMR werden in der Folge kritisch gewürdigt und mit den Vorschlägen der analysierten Regulatorien abgeglichen, wobei der Stellenwert des Soft Law ergründet wird. Im Anschluss wird schliesslich jeweils die Situation in der Schweiz betrachtet.

Es folgt eine Übersicht über die Möglichkeiten der Durchsetzung des menschenrechtlich zu garantierenden Standards. Hierzu werden die Phase vor dem Urteilszeitpunkt, die Phase des Urteils selbst sowie die Phase nach dem Urteilszeitpunkt unterschieden. ${ }^{22}$ In der Phase vor dem Urteil werden der Stellenwert und das Potential des vorsorglichen Rechtsschutzes bei unmenschlichen bzw. erniedrigenden Haftbedingungen analysiert. Dabei wird nicht nur die Anordnungspraxis des Gerichtshofes, sondern auch die Befolgungspraxis der Konventionsstaaten näher betrachtet. In der Phase des Urteilszeitpunktes sind die Urteilswirkungen selbst Ausgangspunkt der Untersuchung. Die klassischen Urteilswirkungen haben durch die weiterentwickelte Verfahrenstechnik - des Piloturteilsverfahrens - Modifikationen erfahren. Neben den allgemeinen Urteilswirkungen und deren Bedeutung für die Umsetzung des Mindeststandards ist für die vorliegende Arbeit deshalb insbesondere die Praxis der Piloturteilsverfahren bei unmenschlichen bzw. erniedrigenden Haftbedingungen von Interesse. Es werden dabei sowohl individuelle als auch generelle Massnahmemöglichkeiten und deren Potential in Bezug auf die Durchsetzung des Mindeststandards bei unzureichenden Haftbedingungen austariert. Auch für die Phase nach dem Urteilsspruch, d.h. für die Implementierungsphase, sind die Urteilswirkungen der Ausgangspunkt, wobei insbesondere die Verpflichtungen des Art. 46 EMRK Anknüpfungspunkt der Untersuchung sind. Es wird weiter aufgezeigt, dass in dieser Phase das Ministerkomitee zu einem zentralen Akteur wird, Ministerkomitee, Gerichtshof und weitere Akteure jedoch zusammenwirken, so dass es die Wechselwirkungen auszumachen gilt. Sodann werden mögliche Umsetzungsschwächen im Bereich inadäquater Haftbedingungen und deren Ursachen eruiert sowie Optimierungsvorschläge ausgearbeitet. Die Arbeit schliesst mit einer Schlussbetrachtung.

22 So auch bereits: Keller/Marti EJIL 2015, 829 (831). 


\section{Methodik}

Die vorliegende Arbeit macht sich bei der Ausarbeitung des vom EGMR postulierten Mindeststandards zu Nutze, dass dem EGMR neben seiner Rechtsschutzfunktion auch die Aufgabe der Rechtsfortbildung zukommt. ${ }^{23}$ Selbst wenn die jeweiligen herangezogenen konkreten Urteile des EGMR nur eine inter partes-Wirkung entfalten, und die Urteile damit nur zwischen den Verfahrensparteien Rechtskraft entfalten, ${ }^{24}$ kommt ihnen darüber hinaus auch eine Orientierungswirkung zu, welche auch als Steuerungsinstrument bezeichnet wird. ${ }^{25}$ Von dieser indirekten Wirkung resp. der Präjudizienwirkung wird gerade erhofft oder erwartet, dass sowohl der betroffene Mitgliedstaat als auch alle weiteren Mitgliedstaaten in

23 Vgl. die Präambel der EMRK: „In der Erwägung, dass es das Ziel des Europarats ist, eine engere Verbindung zwischen seinen Mitgliedern herzustellen, und dass eines der Mittel zur Erreichung dieses Zieles die Wahrung und Fortentwicklung der Menschenrechte und Grundfreiheiten ist"; vgl. auch bereits Mahoney/PreBENSEN in: Macdonald/Matscher/Petzold, S. 621 (621 f.): „[...] the Court's judgments also serve the collective purpose of establishing a ,European common law' in the field of human rights"; vgl. auch Stone Sweet/Keller in: Keller/ Stone Sweet, S. 3 (3 f.): „Today, the Court is an important, autonomous source of authority on the nature and content of fundamental rights in Europe. In addition to providing justice in individual cases, it works to identify and to consolidate universal standards of rights protection [...]“; vgl. auch HeINE S. 191: EGMR als „Motor des Europäischen Menschenrechtsschutzes“ und „Quasi-Verfassungsgericht sui generis“ mit der Aufgabe der dynamischen Auslegung mit Hilfe der Rechtsfortbildung; kritisch zur richterlichen Rechtsfortbildung des EGMR: Breuer ZÖR 2013, $729 \mathrm{ff}$. Ausgangspunkt von Breuers Kritik ist die Gesetzesbindung des Richters, deren demokratische Legitimation sowie die Gewaltenteilung. BREUER sieht auf der anderen Seite aber auch das Bedürfnis eines effektiven Schutzes durch eine „dynamischen Interpretation“ der EMRK. Die Zulässigkeit der Rechtsfortbildung habe folglich durch eine Austarierung dieser verschiedenen Interessen zu erfolgen: BREuER ZÖR 2013, 729 (762) mit Verweisen zur diss. op. von Richter Myjer. Auch Myjer weist auf die Grenzen der Rechtsfortbildung durch den EGMR hin: „[...] the Court's jurisdiction cannot extend to the creation of rights not enumerated in the Convention, however expedient or even desirable such new rights might be. In interpreting the Convention in such a way, the Court may ultimately forfeit its credibility among the Contracting States as a court of law“, Richter Myjer, diss. op., EGMR - Muñoz Díaz/ESP, Urt. v. 08.12.2009, 49151/07; kritisch zur Rolle des Gerichtshofs vgl. auch BAADE S. 359 ff.; zur Auseinandersetzung mit der Kritik an der dynamischen Auslegung aus schweizerischer Sicht: Dummermuth SJZ 2014, 597 (598).

24 Die formelle und materielle Rechtskraft betrifft nur die Verfahrensparteien und den konkreten Sachverhalt.

25 Vgl. etwa Grabenwarter/Pabel $\$ 16$ Rn. 2, 8 f. 
der Zukunft ihr Verhalten danach richten werden. ${ }^{26}$ Insbesondere durch die Analyse einer Vielzahl von Urteilen können denn auch allgemeingültige Aussagen herausdestilliert werden.

Die Abstraktion verallgemeinerungsfähiger Aussagen aus dem Fallrecht wird dabei in erster Linie durch die Begründungspflicht der Urteile (Art. 45 Abs. 1 EMRK) ermöglicht. Durch die besondere Stellung des EGMR als internationales Gericht und Menschenrechtsgerichtshof unterliegt die Art und Weise der Begründung nämlich Besonderheiten: Der Begründungsstil ist einerseits durch die Entscheidung des Einzelfalls geprägt, enthält ,in jüngerer Vergangenheit“ stellenweise aber auch „sehr allgemeine Aussagen ". ${ }^{27}$ Insbesondere bei den Rechtsausführungen ${ }^{28}$ rezipiert der EGMR zum Teil seine frühere Judikatur, anhand derer er bisweilen selbst seine eigenen Rechtsprechungslinien aufarbeitet, bevor er diese in der Folge auf den konkreten, aktuell zu beurteilenden Einzelfall anwendet. ${ }^{29}$ Diese erste eigene Systematisierung des EGMR widerspiegelt insbesondere die Rechtsfortbildungsfunktion des Gerichtshofs. ${ }^{30}$

Soweit vorhanden wird in der vorliegenden Arbeit an dieser eigenen Systematisierung des EGMR angeknüpft und diese für die ausgewählten

26 Gemäss des Wortlauts der Interlaken Deklaration werden die Vertragsstaaten aufgefordert, die EGMR-Rechtsprechung zu berücksichtigen ("taking into account"): Interlaken Declaration v. 19.02.2010; zu dieser Terminologie auch Keller/Marti Justice - Justiz - Giustizia 2015/1, 1 (5, 9 ff.); zur Intensität der Präzedenzwirkung und deren massgebenden Faktoren vgl. Keller/Marti Justice - Justiz - Giustizia 2015/1, 1 (10).

27 Grabenwarter/Pabel $\mathbb{1} 14$ Rn. 4; vgl. auch Glas EYHR 2018, 287 (295). Vgl. indes noch Matscher FS Bernhardt, S. 503 (509, 518 ff.), welcher festhielt, dass die Rechtsprechung des Gerichtshofs auf weiten Strecken kasuistisch geblieben sei und die Aussagen der Urteile des EGMR selten generalisierungsfähig seien. Eine zusammenfassende Schau sei der EGMR-Judikatur kaum zu entnehmen.

28 Urteile des EGMR beginnen gewöhnlich mit einer Erläuterung zu den einzelnen bisherigen Verfahrensschritten „Procedure“ gefolgt von einer Darlegung des Sachverhalts „The Facts“, bevor zu den Rechtsausführungen „The Law“ gelangt wird.

29 Grabenwarter/Pabel $\mathbb{1} 14$ Rn. 6. Dieses Vorgehen ist bei Urteilen der Grossen Kammer besonders ausgeprägt. Eindrückliches Beispiel im vorliegend zu behandelnden Themengebiet: EGMR (GK) - Muršić/COR, Urt. v. 20.10.2016, 7334/13. In Muršić arbeitet der EGMR seine ganze bisherige Judikatur bezüglich eines Mindeststandards hinsichtlich des jedem Einzelnen zu gewährenden persönlichen Platzes auf und weist dabei auf verschiedene bisherige Rechtsprechungslinien hin, bevor er den konkreten Einzelfall entscheidet (im Einzelnen vgl. dazu Teil 3 Kap. 2 IV.).

30 BREUER erkennt eine signifikante „Häufung rechtsfortbildender EGMR-Urteile in den letzten Jahren“: Breuer ZÖR 2013, 729 (761). 
Themengebiete einer Analyse unterzogen. Sofern der EGMR selbst noch keine Systematisierungsversuche unternommen hat, werden in der vorliegenden Arbeit eigene Fallgruppen gebildet, wobei diese sich einerseits an themenspezifischen Kriterien resp. Prinzipien orientieren und andererseits soweit angezeigt den Strafvollzug chronologisch in unterschiedliche Phasen (Hafteintrittsphase, eigentliche Strafvollzugsphase und Haftaustrittsphase) unterteilen.

Im Rahmen dieser tiefergreifenden Strukturierung werden Typologien ausgebildet sowie die Hintergründe und die Methodik des EGMR untersucht. Die theoretisch erarbeiteten Grundsätze etwa zur Charakteristik eines absoluten Rechts oder der praktischen Wirksamkeitserlangung des Soft Law werden dazu mit der Rechtsprechungspraxis des EGMR verknüpft. Da der Mindeststandard in aller Regel nicht exakt berechenbar ist, ${ }^{31}$ wird es auch in der vorliegenden Arbeit vielmehr darum gehen, die objektiven bzw. zumindest objektivierbaren Kriterien auszumachen, nach welchen die Menschenrechtskonformität des Strafvollzugs zu beurteilen ist.

Obgleich die vorliegende Analyse der bestehenden Fallpraxis zu einer retrospektiven Betrachtung führt, indem vergangene Missstände als Ausgangspunkt der Untersuchung genommen werden, erfolgt die vorliegende Analyse vor dem Hintergrund der dynamisch-evolutiven Auslegungspraxis des EGMR. In diesem Sinne steht der aus dieser Untersuchung resultierende Massstab auch jederzeit einer Fortentwicklung offen.

Die Untersuchung der konventionsrechtlichen Durchsetzungsmechanismen und der effektiv erfolgten Implementierung des durch den EGMR im vorliegenden Themengebiet festgesetzten Standards erfolgt anhand einer analytisch strukturierten Auswertung. Bei dieser wird in doppelter Hinsicht chronologisch vorgegangen. Zum einen werden die Durchsetzungsmechanismen und die Implementierung der EGMR-Rechtsprechung in drei Phasen unterteilt: die Phase vor dem Urteilszeitpunkt, die Phase des Urteils selbst sowie die Phase nach dem Urteil. Zum anderen wird der Implementierungsstand einzelner ergangener (Pilot-)Urteile nach deren zeitlicher Abfolge analysiert. Letzteres ermöglicht es, Entwicklungstendenzen und Perspektiven sichtbar zu machen. Zu weiteren Ausführungen gibt die in diesem Teil der Arbeit angewandte Methode keinen Anlass.

31 Vgl. bereits SK-StPO/MeYer Art. 3 EMRK Rn. 51. 


\section{Teil 2: Grundlagen}

\section{Kapitel 1: Der Strafvollzug - ein empirischer Überblick}

Gemäss der World-Prison-Population-Liste befinden sich weltweit rund 10,74 Mio., europaweit rund 1,57 Mio. und schweizweit rund 6900 Personen im Freiheitsentzug. ${ }^{32}$ Die Anzahl der Inhaftierten ist damit weltweit seit dem Jahr 2000 um rund 25\% gestiegen. ${ }^{33}$ Setzt man die Anzahl Inhaftierter ins Verhältnis zur Bevölkerungszahl, befinden sich am Stichtag des 30. September 2018 in Europa 187 Personen pro 100'000 Einwohner im Freiheitsentzug. ${ }^{34}$ Setzt man weiter jeweils die Anzahl der für den Freiheitsentzug zur Verfügung stehenden Plätze in den europäischen Staaten mit der Anzahl Inhaftierter ins Verhältnis, so ergibt sich, dass von 100 zur Verfügung stehenden Plätzen durchschnittlich deren 87,6 tatsächlich belegt sind. ${ }^{35}$ Haftanstalten in Nordmazedonien, Rumänien, Frankreich, Italien, Moldawien, Serbien, Portugal, Tschechien, Griechenland, Österreich, der Slowakei und Dänemark haben am stärksten mit Überbelegungen zu kämpfen (bis 122,3\%). ${ }^{36}$

32 Walmsley S. 2, 13, 17: Für die Länder Eritrea, Somalia und Nordkorea sind allerdings keine Zahlen verfügbar und die Angaben von China und GuineaBissau sind unvollständig. Ferner fehlen auch Angaben von einigen international nicht vollständig anerkannten Jurisdiktionen. Die Gefangenenpopulation dürfte deshalb noch grösser sein und könnte wohl sogar 11 Mio. betragen. Vgl. auch Lines, International Journal of Prisoner Health 2006, 269 (270), welcher darauf hinweist, dass die jährliche Zahl der vom Strafvollzug betroffenen Individuen nochmals deutlich höher ist, zumal die genannten Zahlen immer nur die Gefängnispopulation zu einem Stichtag wiedergeben.

33 WalmsLey S. 2.

34 Walmsley S. 17.

35 Aebi/Tiago Key-Findings S. 7: Der Median liegt bei 91,4. Der Median legt fest, bei welchem Wert 50\% der Daten unterhalb und 50\% der Daten über diesem liegen. Der Median ist gegenüber dem Durchschnitt insofern aussagekräftiger, als er durch extrem hohe bzw. tiefe Werte weniger stark beeinflusst wird.

36 Aebi/Tiago Key-Findings S. 7, 12: Die Länder Albanien, Belgien und Ungarn gehörten im Jahr 2016 auch zu den von der Überbelegung stark betroffenen Ländern. Im Jahr 2018 lieferten sie für diese Statistik jedoch keine Daten mehr. In diesem Zusammenhang bleibt jedenfalls vorwegzunehmen, dass evidenterweise eine Korrelation zwischen dem Problem der Überbelegung und der generellen Auslastung der Vollzugsanstalten besteht; dennoch spielen bei der Überbelegung 
Der transnationale Charakter des Strafvollzugs manifestiert sich im Ausländeranteil der Inhaftierten. Am Stichtag des 31. Januar 2018 besassen durchschnittlich knapp 25\% der Gefangenen in europäischen Gefängnissen eine ausländische Staatsangehörigkeit. ${ }^{37}$ Rund ein Drittel der ausländischen Inhaftierten sind dabei im Besitz einer Staatsangehörigkeit eines anderen europäischen Landes. ${ }^{38}$ In Osteuropa ist der Ausländeranteil in den Haftanstalten mit normalerweise unter 5\% sehr niedrig; in Zentralund Westeuropa variiert der Ausländeranteil der Gefangenen zwischen 1\% und 71\%. ${ }^{39}$ Die höchsten Ausländeranteile europaweit hatten am Stichtag Monaco (100\%), Andorra (80\%), Liechtenstein (75\%), Luxemburg $(72,1 \%)$ die Schweiz (71,4\%) und Österreich $(54,7 \%)$ zu verzeichnen. ${ }^{40}$ Mit $1,1 \%$ ist der Ausländeranteil im Strafvollzug europaweit in Polen am niedrigsten. ${ }^{41}$

Hinsichtlich der Dauer der zu verbüssenden Freiheitsstrafe hatten europaweit rund $16 \%$ der Inhaftierten eine Freiheitsstrafe von weniger als einem Jahr, 24\% eine Freiheitsstrafe zwischen einem und drei Jahren, 16\% eine Freiheitsstrafe von drei bis fünf Jahren, 17\% eine Freiheitsstrafe von fünf bis zehn Jahren und 1,2\% eine lebenslange Freiheitsstrafe zu verbüssen. ${ }^{42}$ Weniger als $1 \%$ der Inhaftierten unterlagen Sicherungsmassnahmen. ${ }^{43}$ Die übrigen rund $25 \%$ waren nicht rechtskräftig verurteilt und befanden sich in Untersuchungs- resp. Sicherheitshaft. ${ }^{44}$ Die rechtskräftig verurteilten Insassen verbüssten am häufigsten Strafen wegen Betäubungsmitteldelikten (17\%), wegen Diebstahls (16\%), wegen (versuchter) Tötungsdelikte (13\%) und wegen Raubs (11\%).45

weitere Faktoren wie die effektive Verteilung der Inhaftierten auf die verschiedenen Haftanstalten resp. auf die einzelnen Haftzellen eine bedeutende Rolle. Überbelegungssituationen in gewissen Strafvollzugsanstalten sind damit auch dann denkbar, wenn die Auslastungsrate europaweit oder auch national unter $100 \%$ liegt.

37 Aebi/Tiago Key-Findings S. 3: Der Median liegt bei knapp 16\%.

38 Aebi/Tiago Key-Findings S. 2; zu (EU-)Ausländern in europäischen Gefängnissen vgl. auch Morgenstern Neue Kriminalpolitik 2007, $139 \mathrm{ff}$.

39 Aebi/Tiago Key-Findings S. 2, wobei nur Staaten mit mindestens einer Million Einwohnern berücksichtigt wurden. In kleineren Staaten variiert die Ausländerrate in Zentral- und Westeuropa von $72 \%$ bis $100 \%$ (100\% in Monaco).

40 Aebi/Tiago Key-Findings S. 3.

41 Aebi/Tiago Key-Findings S. 3.

42 Aebi/Tiago Key-Findings S. $3 \mathrm{f}$.

43 Aebi/Tiago Key-Findings S. 3 f.

44 Aebi/Tiago Key-Findings S. 3 f.

45 Aebi/Tiago Key-Findings S. 4 f. 
Die durchschnittlichen Ausgaben pro inhaftierter Person pro Tag variieren innerhalb Europa stark: etwa $€ 2.50$ in Russland, € 6.- in Aserbaidschan, $€ 9.40$ in Moldawien oder $€ 778.4$ im Vereinigten Königreich, $€$ 380.- in Schweden und $€ 346.20$ in San Marino. ${ }^{46}$ Die durchschnittlichen Ausgaben liegen in Europa bei $€ 128$.- pro Inhaftiertem pro Tag (europäischer Median € 66.50). ${ }^{47}$ Obwohl die unterschiedlichen Lebenshaltungskosten und die wirtschaftlichen Indikatoren der einzelnen Länder sowie der Umwechslungskurs in Euro hier unberücksichtigt bleiben, sprechen die immensen Unterschiede für sich.

\section{Kapitel 2: Menschenrechte im Strafvollzug}

\section{Menschenrechtsrelevanz des Strafvollzugs}

Wird einer Person mit hoheitlichem Urteil die Freiheit entzogen, beschlägt dies die Menschenrechte der betroffenen Person. Zu unterscheiden sind die prinzipielle Menschenrechtsgeltung und der Geltungsumfang der Menschenrechte. ${ }^{48}$ Heute ist unbestritten, dass eine Person bei Antritt der Freiheitsstrafe ihre Menschenrechtsträgerschaft nicht verliert. ${ }^{49} \mathrm{Im}$ Gegenteil verlangt das allgemeine menschenrechtliche Postulat des „no more harm principle“, auch Entgegenwirkungsprinzip oder Prinzip des nil nocere genannt, dem Inhaftierten während des Vollzugs nicht mehr Leid zuzufügen, als durch den Freiheitsentzug an sich hervorgerufen wird. ${ }^{50}$ Als positive Pflicht des Staates wird demnach eine so menschenrechtsschonende Ausgestaltung des Strafvollzugs wie nur möglich verlangt. ${ }^{51}$ Dieser

46 Aebi/Tiago SPACE I S. 112.

47 Aebi/Tiago SPACE I S. 112.

48 MÜLLER S. $45 \mathrm{ff}$.

49 Historisch war dies allerdings nicht immer so: vgl. dazu BAEChtold/Weber/ Hostettler S. 204: Im Jahr 1871 bezeichnete das Oberste Gericht der USA Inhaftierte noch als sog. „Outlaws“ resp. „Staatssklaven“, welche keinerlei Rechte hätten und der Willkür der Obrigkeit ausgeliefert seien. Vgl. ferner CoYLE/FAIR/ Jacobson/Walmsley S. 68; Barisch KJ 2008, 425 (432); Cornille/Devos/Mahieu BP 2006, $1 \mathrm{ff}$.

50 Grabenwarter/Pabel $\mathbb{2} 20$ Rn. 61; Karpenstein/Mayer/Sinner Art. 3 Rn. 22; Schilling Rn. 188; IK/Kau Art. 3 EMRK Rn. 54; Peters/Altwicker $\mathbb{} 6$ Rn. 15; Ligthart/van Oploo/Meijers/Meynen/Kooijmans NJECL 2019, 287 (289f.); Riegel/Speicher StV 2016, 250 ff.; im Einzelnen vgl. auch Coyle/Fair/Jacobson/ WaLMSLEY S. $68 \mathrm{f} ., 71 \mathrm{f}$.

51 EGMR (GK) - Paladi/MDA, Urt. v. 10.03.2009, 39806/05, Rn. 71. 
Ausgestaltung des Vollzugs kommt umso grössere Bedeutung zu, als dass der Strafvollzug eine Vielzahl von Menschenrechten betrifft. ${ }^{52}$ Der Strafvollzug tangiert nämlich nicht nur das Recht auf Freiheit und Sicherheit i.S.v. Art. 5 EMRK und den in dieser Arbeit behandelten Art. 3 EMRK (Verbot der Folter, der unmenschlichen und erniedrigenden Strafe oder Behandlung). So hat der Staat etwa aufgrund von Art. 2 EMRK (Recht auf Leben) im Sinne einer positiven Pflicht nicht nur lebenssichernde Leistungen bereitzustellen, sondern aufgrund seiner Schutzpflicht auch zu gewährleisten, dass Inhaftierte vor Lebensbedrohungen, beispielsweise durch andere Gefangene, geschützt werden. Dazu gehört beispielsweise, dass bekanntermassen gefährliche Inhaftierte nicht mit anderen Inhaftierten untergebracht werden dürfen, wenn sie auch für diese eine Gefahr sind. ${ }^{53}$ Auch Art. 8 EMRK (Recht auf Achtung des Privat- und Familienlebens) zeitigt vielfältige Auswirkungen auf die Ausgestaltung des Vollzugs. So fliessen aus ihm Mindestanforderungen beispielsweise an die zu gewährenden Besuchsrechte, ${ }^{54}$ an die Aussenkontakte über andere Kommunikationsmittel (bspw. Telefonate oder Briefverkehr) ${ }^{55}$ oder an die die Privatsphäre sichernde Ausgestaltung der Haftzelle ${ }^{56}$. Bei Letzterer ist etwa an eine genügende Abtrennung des sanitären Bereichs zu denken, wenn mehrere Personen in einer Haftzelle untergebracht sind. Aufgrund von Art.9 EMRK (Gedanken-, Gewissens- und Religionsfreiheit) kann der Staat sodann beispielsweise verpflichtet sein, Besuche ei-

52 Der Freiheitsentzug und die damit einhergehende Beschränkung der Bewegungsfreiheit hat Reflexwirkungen auf andere Menschenrechte. Von einer Reflexwirkung wird ausgegangen, wenn neben der Bewegungsfreiheit ein anderes Menschenrecht vom Freiheitsentzug faktisch zumindest partiell mitbetroffen ist:

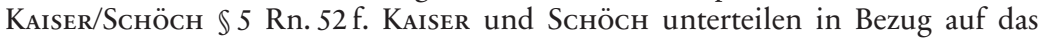
deutsche Grundgesetz die Grundrechte in zwei Kategorien; solche, die vom Freiheitsbezug zumindest faktisch mitbetroffen sind und solche, die sowohl aus rechtlicher als auch aus faktischer Perspektive auch während des Strafvollzugs weiter unbeschränkte Wirkung entfalten. Vgl. auch Keller/Schädler ZSR 2013, 195 ff., welche auf die komplexe Aufgabe eines Rechtsstaats hinweisen, die Anforderungen an das staatliche Strafvollzugswesen zu erfüllen und dabei die Freiheitsrechte der Inhaftierten nicht übermässig zu beschränken.

53 EGMR - Paul u. Audrey Edwards/GBR, Urt. v. 14.03.2002, 46477/99, Rn. 63 f.

54 EGMR - Polyakova u.a./RUS, Urt. v. 07.03.2017, 35090 u.a.; EGMR - Istvan Gabor Kovacs/HUN, Urt. v. 17.01.2012, 15707/10, Rn. 35 ff.; EGMR - Gradek/POL, Urt. v. 08.06.2010, 39631/06, Rn. 40 ff.; vgl. auch Keller/Schädler ZSR 2013, 195 (213 f. m.w.H.).

55 EGMR - Lebois/BUL, Urt. v. 19.10.2017, 67482/14; vgl. auch Keller/SchädLER ZSR 2013, 195 (213 f. m.w.H.).

56 EGMR - Szafrański/POL, Urt. v. 15.12.2015, 17249/12, Rn. 39 f. 
nes Priesters oder Pastors in der Strafvollzugsanstalt zuzulassen ${ }^{57}$ und religionskonforme Nahrung ${ }^{58}$ bereitzustellen. Auch das Recht auf freie Meinungsäusserung i.S.v. Art. 10 EMRK endet nicht mit der Inhaftierung, weshalb aus Art. 10 EMRK auch während des Strafvollzugs entsprechende abwehr- und gewährleistungsrechtliche Verpflichtungen fliessen. ${ }^{59} \mathrm{Zu}$ mal die persönliche Freiheit keine notwendige Voraussetzung für die Ausübung des Rechts auf Eheschliessung ist, hat der Staat weiter aufgrund von Art. 12 EMRK (Recht auf Eheschliessung und Familienbegründung) auch Inhaftierten zu ermöglichen, eine eheliche Beziehung ihrer Wahl einzugehen. ${ }^{60}$ Art. 14 EMRK gebietet schliesslich, die Konventionsrechte und Freiheiten ohne Diskriminierung zu gewährleisten. Im Kontext des Strafvollzugs hatte sich der Gerichtshof aufgrund dieser Bestimmung etwa mit (angeblichen) Diskriminierungen im Zusammenhang mit der Anwendung eines bestimmten Gefängnisregimes oder vorzeitiger Entlassungen auseinanderzusetzen. ${ }^{61}$

57 EGMR - Poltoratski/UKR, Urt. v. 29.04.2003, 38812/97, Rn. 163 ff.; EGMR Mozer/MDA u. RUS, Urt. v. 23.02.2016, 11138/10, Rn. $197 \mathrm{ff}$.

58 EGMR - Jakobski/POL, Urt. v. 07.12.2010, 18429/06, Rn. 42 ff.: Anspruch eines buddhistischen Strafgefangenen, im Strafvollzug fleischlose Mahlzeiten zu erhalten; zur Gewährleistung der Glaubens- und Gewissensfreiheit im Strafvollzug im Einzelnen: KELLER/SCHÄDLER ZSR 2013, 195 (217 ff.).

59 Vgl. etwa EGMR - Yankov/BUL, Urt. v. 11.12.2003, 39084/97: Feststellung einer Verletzung von Art. 10 EMRK, nachdem der Beschwerdeführer für „mässig“ anstössige Äusserungen gegen das Justiz- und Strafvollzugssystem in einem persönlichen Manuskript mit sieben Tagen in einer Disziplinarzelle bestraft worden war. Vgl. auch Keller/Schädler ZSR 2013, 195 (219f.), welche die Kommunikationsgarantien für die Realisierung des Normalisierungsgrundsatzes und die Resozialisierung als besonders wichtig erachten.

60 Vgl. EGMR - Frasik/POL, Urt. v. 05.01.2010, 22933/02, Rn.95; vgl. im Einzelnen sowie zu zulässigen Einschränkungen auch Keller/SchäDLER ZRSR 2013, 195 (215 ff.).

61 Vgl. etwa EGMR - Chaldayev/RUS, Urt. v. 28.05.2019, 33172/16, Rn. 76 ff.; EGMR - Varnas/LTU, Urt. v. 09.07.2013, 42615/06, Rn. 116 ff.: Unterschiedliche Besuchsregelung im gewöhnlichem Strafvollzug und während der Untersuchungshaft; EGMR - Gülay Cetin/TUR, Urt. v. 05.03.2013, 44084/10: Unterschiedliche Anforderungen an die Entlassungsmöglichkeit bei finaler Krankheit im gewöhnlichen Strafvollzug und während der Untersuchungshaft; EGMR (GK) - Kafkaris/CYP, Urt. v. 12.02.2008, 21906/04, Rn. 162 ff.: Beschwerde eines Inhaftierten, welcher eine lebenslange Strafe verbüsste, bzgl. der Begnadigungspraxis; EGMR - Clift/GBR, Urt. v. 13.07.2010, 7205/07, Rn. 73 ff.: Abhängigkeit der prozessualen Voraussetzungen der vorzeitigen Entlassung vom zu verbüssenden Strafmass; EGMR - X/TUR, Urt. v. 09.10.2012, 24626/09, Rn. 51 ff.: Totale 
Es sind denn auch diese Rechte, welche Untergrenzen an den Haftstandard stellen und so in ihrem Geltungsbereich die konkrete Ausgestaltung des Freiheitsentzuges mit all seinen Modalitäten prägen. Wie in dieser Arbeit gezeigt wird, nimmt die EMRK heute bei der Gewährleistung und Durchsetzung menschenrechtskonformer Haftbedingungen eine wegweisende Funktion ein. ${ }^{62}$ Dies, obwohl bei ihrer Verabschiedung kein besonderer Fokus auf die Rechte der Strafgefangenen gelegt worden war. ${ }^{63}$

\section{Stellung des Inhaftierten}

Das besondere Näheverhältnis zwischen Staat und Inhaftierten (Sonderstatusverhältnis) verpflichtet den Staat im Sinne einer „besonderen Fürsorgepflicht" ${ }^{\text {"64 }}$ bzw. im Sinne einer „besonderen Obhuts- und Sorgepflicht" zu weitergehenden (insbesondere positivrechtlichen) Gewährleistungen. Andererseits führte resp. führt dieses besondere Näheverhältnis, in seiner Ausprägung als „,besonderes Gewaltverhältnis“, auch dazu, dass dem Staat weitere Einschränkungsmöglichkeiten eingeräumt wurden resp. bisweilen immer noch werden. Auf diese Besonderheiten wird in der Folge näher eingegangen; dies sowohl aus einem konventionsrechtlichen als auch aus einem nationalen Blickwinkel.

1. Modifikationen zu Gunsten des Inhaftierten?

\subsection{Konventionsrechtliche Betrachtung}

a Materiellrechtliche Besonderheiten

Es ist anerkannt, dass inhaftierte Personen sich aufgrund des Freiheitsentzugs, welcher die direkte Unterstellung unter die staatliche Kontrolle bewirkt, in einer missbrauchsanfälligen Lebenssituation befinden und somit eine besonders verletzliche Personengruppe bilden, welche es in

Isolierung eines homosexuellen Inhaftierten, um ihn vor den Mitinhaftierten zu schützen.

62 Vgl. insb. Teil 3 und Teil 4.

63 Van Zyl SMit/SNacken S. 10.

64 Grabenwarter/Pabel $\$ 20$ Rn. 70. 
besonderem Masse zu schützen gilt. ${ }^{65}$ Mit anderen Worten hat der Staat gegenüber Strafgefangenen aufgrund des Sonderstatusverhältnisses eine besondere Obhuts- und Sorgepflicht. ${ }^{66}$ Im Einzelnen bedeutet dies, dass die Konventionsrechte als subjektive Rechte ${ }^{67}$ den Staat nicht bloss zu einem negativen Verhalten, sprich zu einem Unterlassen (,abwehrrechtliche Dimension der Menschenrechte"), verpflichten, sondern die Konventionsrechte des Strafgefangenen dem Staat auch in besonderem Masse eine Verpflichtung zu einem positiven Tätigwerden auferlegen. ${ }^{68}$ Diese positiven Verpflichtungen beinhalten eine schutz- und eine leistungs- resp.

65 Vgl. etwa SK-StPO/Meyer Art. 2 EMRK Rn. 37, Art. 3 EMRK Rn. 14; IK/KaU Art. 3 EMRK Rn. 54; Karpenstein/Mayer/Sinner Art. 3 Rn. 22; MeYer-Ladewig/ Lehnert Art. 3 Rn. 11, 26; Kiener/Kälin/Wyttenbach $\$ 4$ Rn. 33; Hohl-Chirazi forumpoenale 2018, 187 (189); zur „vulnerability“ als konzeptionelles Instrument für die Beurteilung von Menschenrechtsfragen weitergehend vgl. Furusho UCL JLJ 2016, 175 ff.; vgl. ferner EGMR (GK) - Salman/TUR, Urt. v. 27.06.2000, 21986/93, Rn.99; EGMR - Keenan/GBR, Urt. v. 03.04.2001, 27229/95, Rn.91; EGMR - Trubnikov/RUS, Urt. v. 05.07.2005, 49790/99, Rn.68; EGMR - Renolde/FRA, Urt. v. 16.10.2008, 5608/05, Rn. 83.

66 SK-StPO/Meyer Art. 3 EMRK Rn. 161; Keller/Schädler ZSR 2013, 195 $(209,211)$.

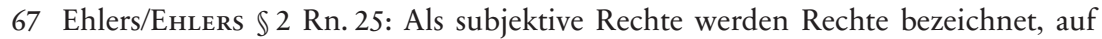
die sich der Menschenrechtsträger zur Befriedigung seiner Individualinteressen jederzeit berufen kann und welche von ihm auch auf dem Gerichtsweg durchgesetzt werden können. Im Umkehrschluss verpflichten subjektive Rechte des Menschenrechtsträgers auch unmittelbar den Staat. Vgl. auch SK-StPO/MeYer Methodik Rn. 60: Vom subjektiven Gehalt der Konventionsrechte sei deren „objektiver Wertordnungskern" zu unterscheiden, welcher überindividuelle Zwecke verfolge. Zur Menschenwürde als objektiv-rechtlicher Grundsatz, der in der ganzen Konvention zum Tragen komme vgl. Ronc S. 336; zur Frage des Bestehens einer objektiv-rechtlichen Menschenrechtsdimension bei Konventionsrechten vgl. eingehend Holoubek S. $78 \mathrm{ff}$.

68 SK-StPO/Meyer Art. 3 EMRK Rn. 14; Meyer-Ladewig/Lehnert Einleitung Rn. 30, Art. 3 Rn. 7, 11; vgl. auch Harris/O'Boyle/Warbrick S. 24 f.; implizit auch: IK/Kau Art. 3 EMRK Rn. 7; Peters/Altwicker \6 Rn. 14. Positive Verpflichtungen treffen den Staat selbstverständlich nicht nur beim Vorliegen von Sonderstatusverhältnissen. Der EGMR hat vielmehr schon früh Menschenrechtswirkungen anerkannt, welche weit über eine rein abwehrrechtliche Dimension der Konventionsrechte hinausgehen. Zur allgemeinen Konventionsrechtsdogmatik vgl. im Einzelnen etwa HoloubeK S. 45 ff., 54 ff.; vgl. auch Kiener/KäLin/ Wyttenbach $\ 4$ Rn. 4, 14: Leistungsansprüche bestünden bei Freiheitsrechten insbesondere dann, wenn ohne staatliche Unterstützung eine Freiheit nicht genutzt werden könnte. Vgl. bspw. EGMR (GK) - Kudta/POL, Urt. v. 26.10.2000, 30210/96, Rn. 94; EGMR - Pakhomov/RUS, Urt. v. 30.09.2010, 44917/08, Rn. 61; EGMR - Kalashnikov/RUS, Urt. v. 15.07.2002, 47095/99, Rn. 95. 
gewährleistungsrechtliche Komponente („schutzrechtliche- und leistungsrechtliche Dimension der Menschenrechte“). ${ }^{69}$ Weiter umfassen die positiven Verpflichtungen gewisse prozedurale Untersuchungspflichten, wenn Hinweise für eine Konventionsrechtsverletzung vorliegen oder eine solche von einem Betroffenen glaubhaft vorgebracht wird. ${ }^{70}$

Veranschaulichen lässt sich die „Verpflichtungstrias“ der abwehr-, schutz- und leistungsrechtlichen Dimension der Konventionsrechte am Beispiel der Gesundheitsversorgung im Strafvollzug. ${ }^{71}$ Der Staat hat aus der abwehrrechtlichen Dimension des Art. 3 EMRK im Allgemeinen Handlungen zu unterlassen, welche den Zugang zu Gesundheitsgütern erschweren oder verunmöglichen, wenn dies zu einer unmenschlichen oder erniedrigenden Behandlung der Betroffenen führt. ${ }^{72}$

Nimmt der Staat dem Strafgefangenen mit dessen Inhaftierung nun aber die Möglichkeit, sich selbst um einen angemessenen Gesundheitsschutz resp. eine angemessene Gesundheitsversorgung zu kümmern, reicht es nicht mehr aus, dass der Staat sich nur passiv verhält und bloss nicht interveniert. Die Inhaftnahme hat vielmehr zur Folge, dass der Staat aufgrund seiner positiven Verpflichtung nun umfassend für den Gesundheitsschutz und die Gesundheitsversorgung des Strafgefangenen verantwortlich wird. ${ }^{73}$ Die schutzrechtliche Dimension von Art. 3 EMRK verpflichtet nun

69 SK-StPO/Meyer Art. 3 EMRK Rn. 14; Ehlers/Ehlers $\$ 2$ Rn. 26 ff., wobei Ehlers bei den Leistungsrechten weiter unterscheidet, ob sie auf originäre oder derivative Teilhabe gerichtet sind. Bei Ersterem führe die Leistungspflicht dazu, dass noch nicht vorhandene staatliche Massnahmen ergriffen werden müssen. Bei Zweiterem folge aus ihr, dass Zugang zu den schon bestehenden Leistungen geschaffen werden müsse. $\mathrm{Zu}$ den Schutzwirkungen der Konventionsrechte im Einzelnen vgl. auch Holouber S. 45 ff.

70 Zum Inhalt dieser Untersuchungspflichten im Einzelnen Meyer-Ladewig/ LeHNert Einleitung Rn. 30; SK-StPO/MeYer Art. 3 EMRK Rn. 14; EGMR (GK) - Labita/ITA, Urt. v. 06.04.2000, 26772/95, Rn.131; EGMR - Stoica/ROM, Urt. v. 04.03.2008, 42722/02, Rn. 67; EGMR - Assenov u.a./BUL, Urt. v. 28.10.1998, 24760/94, Rn. 102.

71 Zur „Verpflichtungstrias“ der Abwehr-, Schutz- und Gewährleistungspflichten hinsichtlich Gesundheitsfragen bei Strafgefangenen vgl. auch KüNZLi/AcherMANN S. $14 \mathrm{ff}$.

72 KünZli/Achermann S. 15.

73 Künzli/Achermann S. 15. Diese umfassende Verpflichtung betrifft aber selbstverständlich lediglich das Ergreifen von geeigneten Mitteln („obligation of means") und nicht ein konkretes Resultat. Vgl. auch Schilling Rn. 188; Meyer-Ladewig/Lehnert Art. 3 Rn. 11, 42 ff.; Peters/Altwicker $\$ 6$ Rn. 15; EGMR (GK) - Kudta/POL, Urt. v. 26.10.2000, 30210/96, Rn. 94; EGMR - Mozer/ MDA u. RUS, Urt. v. 23.02.2016, 11138/10, Rn. 178; EGMR (GK) - Idalov/RUS, 
nämlich den Staat, Inhaftierte auch vor Gefahren zu schützen, welche von Dritten (nicht staatlichen Akteuren) oder vom betroffenen Strafgefangenen selber ausgehen, sofern der Staat um die Gefahr wusste oder wissen musste und sofern ihm zumutbare Mittel zur Intervention zur Verfügung standen. ${ }^{74}$ Konkret kann dies beispielsweise bedeuten, dass der Staat Massnahmen zu ergreifen hat, damit gesunde Inhaftierte nicht mit Inhaftierten mit ansteckenden Krankheiten untergebracht werden. ${ }^{75}$ Dies hat in präventiver Hinsicht wiederum zur Konsequenz, dass der Staat zumindest bei Verdachtsfällen verpflichtet ist, eine Untersuchung der potentiell kranken Inhaftierten zu veranlassen oder andere präventive Massnahmen zum Schutz der übrigen Inhaftierten zu ergreifen. ${ }^{76}$ Die positive Verpflichtung des Staates, auch dann tätig zu werden, wenn der Inhaftierte seine gesundheitlich prekäre Situation selbst herbeigeführt hat, hat beispielsweise bei einem sich im Hungerstreik befindenden Inhaftierten zur Folge, dass der Staat eine Zwangsernährung zu prüfen und gegebenenfalls auch zu veranlassen hat. ${ }^{77}$ Zusammenfassend trifft den Staat aus der schutzrechtlichen Dimension des Art. 3 EMRK also die Pflicht, Risikoanalysen vorzunehmen und bei absehbaren und bekannten Gefahren vorhandene und zumutbare reaktive Massnahmen zu ergreifen. ${ }^{78}$

Urt. v. 22.05.2012, 5826/03, Rn.93; EGMR - Popov/RUS, Urt. v. 13.07.2006, 26853/04, Rn. 208.

74 EGMR - Paul u. Audrey Edwards/GBR, Urt. v. 14.03.2002, 46477/99, Rn. 55; EGMR - D.F./LAT, Urt. v. 29.10.2013, 11160/07, Rn. 81 ff.; SK-StPO/MeYer Art. 3 EMRK Rn.14, 146; zum Schutz vor sich selbst vgl. SK-StPO/Meyer Art. 2 EMRK Rn. 77, 80 ff.; EGMR - Coselav/TUR, Urt. v. 09.10.2012, 1413/07, Rn. 52 ff. (Verpflichtung zur erhöhten Suizidprävention); EGMR - Rappaz/SUI, Entsch. v. 26.03.2013, 73175/10 (Pflicht zum Tätigwerden bei Hungerstreikenden in der Vollzugsanstalt).

75 Gemeint sind hier Übertragungen ohne Mitwirkung des Angesteckten, wie dies etwa bei über Tröpfcheninfektion übertragbaren Infektionskrankheiten häufig der Fall sein dürfte (bspw. COVID-19 etc.). Differenziert auch zu Ansteckungen mit Zutun des Angesteckten vgl. Künzli/Achermann S. 18 f. (Übertragungen etwa durch Spritzenaustausch oder über einvernehmlichen Sexualverkehr).

76 Die Untersuchung von potentiell Kranken hat gegenüber den weiteren Inhaftierten eine schutzrechtliche Dimension; gegenüber dem Betroffenen selbst basiert die Untersuchung vornehmlich auf dem leistungsrechtlichen Gehalt seiner Menschenrechte.

77 SK-StPO/Meyer Art. 2 EMRK Rn. 77, 80 ff.; EGMR - Rappaz/SUI, Entsch. v. 26.03.2013, 73175/10. Diese positive Verpflichtung beinhaltet hier sowohl eine schutz- als auch eine leistungsrechtliche Komponente.

78 Vgl. auch SK-StPO/Meyer Art. 3 EMRK Rn. 136, 146; Kiener/Kälin/WyttenBACH $\$ 4$ Rn. 33 . 
Aufgrund des leistungs- resp. gewährleistungsrechtlichen Gehalts der Menschenrechte hat der Staat sodann sicherzustellen, dass dem Strafgefangenen während seiner Inhaftierung eine angemessene präventive und therapeutische Gesundheitsversorgung zuteil wird. ${ }^{79}$ Dieser Verpflichtung kann der Staat entweder nachkommen, indem er innerhalb der Strafvollzugsanstalt personelle und infrastrukturelle Massnahmen ergreift und so Inhaftierten in der Strafvollzugsanstalt eine angemessene Gesundheitsversorgung zur Verfügung stellt oder indem er in organisatorischer Hinsicht sicherstellt, dass der Strafgefangene prompten Zugang zu einer angemessenen Gesundheitsversorgung ausserhalb des Strafvollzuges erhält. ${ }^{80}$

Die positiven Verpflichtungen des Staates, sei es aufgrund der schutzrechtlichen oder gewährleistungsrechtlichen Dimension der Menschenrechte, enden dabei nicht mit dem Ergreifen von Massnahmen auf administrativer Ebene. Besonders missbrauchsanfällige Lebenssituationen können darüber hinaus auch legislatorische Massnahmen im Sinne einer „menschenrechtsschützenden“ Regulierung notwendig machen. ${ }^{81}$ Aufgrund des gewährleistungsrechtlichen Gehalts ist der Staat dabei auch verpflichtet, gewisse Menschenrechtsverletzungen wie Verletzungen von Art. 3 EMRK unter Strafe zu stellen. ${ }^{82}$

\section{b Prozedurale Besonderheiten}

Der Staat hat nicht nur eine Sorge- und Obhutspflicht gegenüber den Strafgefangenen, sondern verfügt in der Regel auch über eine Informationsherrschaft, wenn es um Vorgänge oder Unterbringungsmodalitäten im

79 EGMR - Pakhomov/RUS, Urt. v. 30.09.2010, 44917/08, Rn. 61; EGMR - Yevgeniy Alekseyenko/RUS, Urt. v. 27.01.2011, 41833/04, Rn. 99; EGMR (GK) - Kudta/RUS, Urt. v. 26.10.2000, 30210/96, Rn.93; EGMR - Khudobin/RUS, Urt. v. 26.10.2006, 59696/00, Rn.93; EGMR - Kalashnikov/RUS, Urt. v. 15.07.2002, 47095/99, Rn. 95; EGMR - Mouisel/FRA, Urt. v. 14.11.2002, 67263/01, Rn. 40; EGMR - Kaprykowski/POL, Urt. v. 03.02.2009, 23052/05, Rn. 69; vgl. auch Peters/AltwickeR $\$ 6$ Rn. 14; in Bezug auf Art. 2 EMRK auch KäLIN/KüNZLI S. $345 \mathrm{f}$.

80 In der Terminologie von Ehlers/EhLers $₫ 2$ Rn. 28 (vgl. bereits Fn. 69) würde es sich bei Ersterem um originäre und bei Letzterem um derivative Teilhaberechte handeln.

81 SK-StPO/Meyer Art. 3 EMRK Rn. 14, 136; EGMR - Opuz/TUR, Urt. v. 09.06.2009, 33401/02, Rn. $158 \mathrm{ff}$.

82 Die Strafrechtsbestimmungen im nationalen Recht sind entsprechend auszugestalten: Meyer ZStW 123 (2011), 1 (13); SK-StPO/Meyer Art. 3 EMRK Rn. 14, 136. 
Strafvollzug geht. ${ }^{83}$ Dies bringt den Inhaftierten regelmässig in Beweisnot, wenn er die Haftumstände beanstanden möchte. ${ }^{84}$ Im Sinne der Gewährleistung einer effektiven Durchsetzung der garantierten Rechte trägt der EGMR diesem Umstand durch die Einführung gewisser beweisrechtlicher Erleichterungen Rechnung. ${ }^{85}$ Es liegt zwar zunächst am Beschwerdeführer, einen „Beweis des ersten Anscheins“ für menschenrechtswidrige Haftbedingungen zu erbringen. Gelingt dieser, kehrt allerdings die Beweisführungslast um, und es ist in der Folge am Konventionsstaat, die Vorbringen des Beschwerdeführers zu widerlegen. ${ }^{86}$ Massnahmen gegenüber dem Strafgefangenen und Vollzugsmodalitäten sind durch die Vollzugsbehörde deshalb stets vollständig, präzise und zeitnah zu dokumentieren.

Die Anforderungen an den „Beweis des ersten Anscheins“ sind für Verletzungen in staatlicher Obhut nochmals reduziert: ${ }^{87}$ Betrifft die Beschwerde des Inhaftierten einen Eingriff in seine körperliche Integrität während des Strafvollzugs, muss dieser zunächst nur die Verletzungen belegen; 88 es liegt dann am Staat zu erklären, woher die Verletzungen stammen. ${ }^{89}$ Dabei kann der Staat nur dann den Einwand vorbringen, die Verletzungen hätten bereits bei Antritt der Freiheitsstrafe bestanden, wenn dies durch eine ärztliche Eintrittsuntersuchung festgestellt wurde. ${ }^{90}$

83 EGMR (GK) - Salman/TUR, Urt. v. 27.06.2000, 21986/93, Rn. 100: Bei Inhaftierungssituationen: „exclusive knowledge of the authorities“; EGMR (GK) - Bouyid/ BEL, Urt. v. 28.09.2015, 23380/09, Rn. 83; SK-StPO/Meyer Art. 3 EMRK Rn. 161; Ehlers/Uerpmann-Wittzack $₫ 3$ Rn. 42 (in Bezug auf Polizeigewahrsam).

84 Grabenwarter/Pabel $\$ 20$ Rn. 70; SK-StPO/Meyer Art. 3 EMRK Rn. 160; IK/Kau Art. 3 EMRK Rn. 28; Peters/Altwicker $₫ 6$ Rn. 15.

85 SK-StPO/Meyer Art. 3 EMRK Rn. 161; Ehlers/Uerpmann-Wittzack $\mathbb{3}$ Rn. 42 (in Bezug auf Polizeigewahrsam); vgl. auch Meyer-Ladewig/Lehnert Art. 3 Rn. 54.

86 EGMR - Ribitsch/AUT, Urt. v. 04.12.1995, 18896/91, Rn. 34; SK-StPO/MeYer Art. 3 EMRK Rn. 161; Rudolf EuGRZ 1996, 497, 500 f.

87 SK-StPO/Meyer Art. 3 EMRK Rn. 161.

88 Peters/Altwicker $\$ 6$ Rn. 15; SK-StPO/Meyer Art. 3 EMRK Rn. 161; Grabenwarter/Pabel $\$ 20$ Rn. 70.

89 Grabenwarter/Pabel $\ 20$ Rn. 70: Mögliche Erklärungen seien etwa, dass die Verletzungen zum Zeitpunkt der Inhaftnahme bereits bestanden haben, dass die Verletzungen im „Gefängnisalltag“ entstanden sind oder dass der Inhaftierte die Verletzungen selbst hervorgerufen hat. Vgl. auch MeYer-Ladewig/LehnerT Art. 3 Rn. 54; Karpenstein/Mayer/SInNer Art. 3 Rn. 31; EGMR (GK) - Salman/ TUR, Urt. v. 27.06.2000, 21986/93, Rn. 99; EGMR (GK) - Selmouni/FRA, Urt. v. 28.07.1999, 25803/94, Rn. 87.

90 Karpenstein/Mayer/Sinner Art. 3 Rn. 31; SK-StPO/Meyer Art. 3 EMRK Rn. 161. 
Gelingt dem Beschwerdeführer der Beweis des ersten Anscheins und bleiben Beweise seitens des Staates aus, entscheidet der EGMR auf der Grundlage der verfügbaren Unterlagen „beyond reasonable doubt“. Kohärenz und Stringenz der Ausführungen des Beschwerdeführers sind deshalb wesentlich. ${ }^{91}$ Regelmässig dürfte dann in solchen Fällen eine Verletzung von Art. 3 EMRK festgestellt werden. ${ }^{92}$

\subsection{Nationale Betrachtungsweise}

Auch auf nationaler Ebene wird erkannt, dass die Vollzugsbehörden gegenüber den Strafgefangenen eine besondere Fürsorgepflicht (vollzugsrechtliche Fürsorgepflicht) trifft. ${ }^{93}$ Diese stellt weitergehende Anforderungen an die Ausgestaltung des Strafvollzugs. Im Einzelnen wird in der Lehre etwa die Errichtung und Zurverfügungstellung von Gesundheitsund Sozialdiensten, der Beizug von Fachpersonen sowie die Gewährleistung einer Gefängnisseelsorge gefordert. ${ }^{94}$ Wesentliche Unterschiede zur konventionsrechtlichen Betrachtungsweise bestehen weder in materieller noch in prozessualer Hinsicht. Es ist deshalb auf die konventionsrechtlichen Ausführungen zu verweisen. ${ }^{95}$

91 SK-StPO/Meyer Art. 3 EMRK Rn. 162.

92 IK/Kau Art. 3 EMRK Rn. 28.

93 BGE 139 I 180, E.1.3 (Art. 75 Abs. 1 StGB als positivrechtliche Verankerung einer besonderen Fürsorgepflicht/Betreuungspflicht); BAECHTOLD/WeBER/HostettLeR S. 32; vgl. auch BGer 6B_421/2019, Urt. v. 11.07.2019, E. 2.8; BGer 6B_1075/2018, Urt. v. 15.11.2018, E. 3.3.3.f. (Verlegung in eine psychiatrische Klinik aufgrund der vollzugsrechtlichen Fürsorgepflicht).

94 Baechtold/Weber/Hostettler S. 32.

95 Baechtold/Weber/Hostettler S. 211: Das Bundesgericht berücksichtige und antizipiere zunehmend die Rechtsprechung der Organe der EMRK. Zum Einfluss der EMRK auf das Verfassungsrecht der Schweiz vgl. auch KiEner in: Jaag/ Kaufmann, S. 53 ff., 69 ff.: KIENER weist im Allgemeinen auch auf die transformative Funktion des Bundesgerichts hin, welches die Rechtsprechung des EGMR nachvollzogen und in das schweizerische Verfassungsrecht inkorporiert habe. 
2. Modifikationen zu Lasten des Inhaftierten?

\subsection{Konventionsrechtliche Betrachtung}

Schon kurz nach der Inkraftsetzung der EMRK im Jahre 1953 machten Beschwerden von Strafgefangenen einen Grossteil der Arbeit der Strassburger Organe aus. ${ }^{96}$ Während langer Zeit war die Rechtsprechung von Kommission und Gerichtshof allerdings von der Annahme geprägt, dass mit dem Strafvollzug ein inhärenter Verlust weiterer Rechte einhergehe. ${ }^{97}$ Mit und infolge des Urteils Golder/GBR wurden für die prozessualen Gehalte der EMRK besondere Einschränkungsmöglichkeiten bei Inhaftierten schrittweise abgeschafft. ${ }^{98}$ Die Kehrtwende bezüglich der besonderen Einschränkungsmöglichkeiten der materiellen Gehalte der EMRK kam erst deutlich später. Das Schutzniveau der EMRK für Personen im Strafvollzug war bis in die 1980er Jahre sehr tief. ${ }^{99}$ Ungenügende Haftbedingungen infolge Überbelegungssituationen oder unangemessener Medikation wurden von Kommission und Gerichtshof zu dieser Zeit nur bei politischen Gefangenen als Verletzungen von Art. 3 EMRK erkannt. ${ }^{100}$ Die Weigerung der Strassburger Organe, ungenügende Haftbedingungen konsequent als Verstösse gegen Art. 3 EMRK zu beurteilen, führte denn auch seit Mitte der 80er Jahre vermehrt zu Kritik. ${ }^{101}$ In den 90er Jahren wurden auf europäischer Ebene verschiedene Vorschläge gemacht, welche die Rechtsstellung der Inhaftierten verbessern sollten, insbesondere hinsichtlich der Gewährleistung einer angemessenen Unterbringung und der medizinischen Versorgung. ${ }^{102}$ Diese mündeten in eine Europäische Strafvollzugspolitik. ${ }^{103}$

96 Van ZyL SMit/SNACKen S. 10.

97 VAN ZyL SMIT/SNACKen S. 10.

98 Vgl. insb. EGMR (Pl) - Golder/GBR, Urt. v. 21.02.1975, 4451/70; van ZyL SMIT/ SNACKen S. $11 \mathrm{f}$.

99 Van ZyL Smit/Snacken S. 12.

100 Vgl. etwa EKMR (Pl) - DEN u.a./GRE, Report v. 05.11.1969, 3321/67 u.a., Rn. 8 (1. Griechenland-Fall); van ZyL Smit/Snacken S. 12; Livingstone Punishment \& Society 2000, 309 (314).

101 Vgl. auch Jung S. 91 ff.: Das Zögern des Gerichtshofes, sich auf eine Bewertung der konkreten Lebensbedingungen im Alltag des Strafvollzuges einzulassen, sei mit der umfassenden Schutzrichtung von Art. 3 EMRK nur schwerlich vereinbar. Zur menschenrechtlichen Situation Inhaftierter auch kritisch: TreCHSEL, Human Rights, S. 30 ff.; van ZyL Smit/SNaCKen S. 12 f.

102 Van Zyl Smit/Snacken S. 12.

103 Van Zyl Smit/Snacken S. 12. 
Im Urteil Kudta/RUS ${ }^{104}$ hielt der Gerichtshof dann erstmals fest, dass gestützt auf Art. 3 EMRK allen Strafgefangenen das Recht zukäme, unter menschenwürdigen Haftbedingungen untergebracht zu sein. ${ }^{105}$ Besondere Möglichkeiten zur Einschränkung der Rechte des Inhaftierten gehören nun seit längerem der Vergangenheit an. ${ }^{106}$ Heute prägen deshalb nicht mehr besondere Möglichkeiten zur Einschränkung der Rechtsstellung des Inhaftierten, sondern vielmehr das Bewusstsein über die Menschenrechtssensibilität des Freiheitsentzugs, die Rechtsprechung des EGMR. ${ }^{107}$

\subsection{Nationale Betrachtungsweise}

Die ältere Terminologie auf nationaler Ebene verwendete den Begriff des „besonderen Gewaltverhältnisses“, um das Verhältnis zwischen Staat und Inhaftiertem zu beschreiben. ${ }^{108}$ Das besondere Gewaltverhältnis war dabei dem Innenraum des Staates zugeordnet, während das allgemeine Gewaltverhältnis zwischen Bürger und Staat dem Aussenverhältnis zugeordnet war. ${ }^{109}$ Der Begriff des Gewaltverhältnisses wurde auch auf nationaler Ebene von den Begriffen des „Sonderstatusverhältnisses“ bzw. des „besonderen Rechtsverhältnisses“ abgelöst. ${ }^{110}$ Auch das Sonderstatusverhältnis führt

104 EGMR (GK) - Kudta/RUS, Urt. v. 26.10.2000, 30210/96.

105 EGMR (GK) - Kudta/RUS, Urt. v. 26.10.2000, 30210/96, Rn.94; vgl. auch Sudre FS Cohen-Jonathan, S. 1499 (1503): Durch die konstruktive Auslegung der Konvention im Urteil Kudta/POL sei unter Art. 3 EMRK ein neues Recht entstanden, das Recht auf menschenwürdige Haftbedingungen.

106 Keller/SChÄDler ZSR 2013, 195 (205). Anders noch GräFenstein ZfStrVO 2003, 10 (10 f., 15), welche noch im Jahr 2003 feststellte, dass hinsichtlich des Fallmaterials zu Strafvollzugsfragen zwar eine zunehmende Tendenz zu beobachten sei, die Analyse der inhaltlichen Substanz der Spruchpraxis zu Art. 3 EMRK aber noch ernüchternd ausfalle. Die Konkretisierung und Realisierung von Art. 3 EMRK sei in vielen Fällen noch durch institutionsbedingte und zum Teil auch strafzweckbedingte Vorbehalte behindert.

$107 \mathrm{Zu}$ den Einschränkungsvoraussetzungen der Konventionsrechte im Strafvollzug im Einzelnen vgl. KeLLER/SCHÄDLER ZSR 2013, 195 (206 ff.).

108 BGE 68 I 73, E. 1; Griffel N 147; Baechtold/Weber/Hostettler S. 205; Baechtold S. 9; Keller/Schädler ZSR 2013, 195 (198); zur dogmengeschichtlichen Herkunft der Rechtsfigur des besonderen Gewaltverhältnisses im deutschen Kontext vgl. von KielmansegG Juristische Arbeitsblätter 2012, 881 (881 f.); ausf. von Kielmansegg S. 15 ff.

109 GRIfFel N 147.

110 BGE 99 Ia 262, E. III.; Baechtold S.9; für Deutschland vgl. BverfG Urt. v. 14.03.1972 - 41/7, NJW 1972, 811 (812); zur Parallelität dieser Entwick- 
aber noch heute zu mehreren Besonderheiten, welche die Möglichkeit eröffnen, Freiheitsrechte des Betroffenen in besonderem Masse einzuschränken. ${ }^{111}$

a Anforderungen an die gesetzliche Grundlage und Gesetzesform bei Grundrechtseinschränkungen (Legalitätsprinzip)

In der Vergangenheit wurde davon ausgegangen, dass Personen im besonderen Gewaltverhältnis zwar ihre Grundrechtsträgerschaft behielten, Einschränkungen ihrer Grundrechte jedoch keiner gesetzlichen Grundlage bedürften, weil sich die Möglichkeit zur Einschränkung der Grundrechte mithin direkt aus der Natur des jeweiligen besonderen Gewaltverhältnisses ergebe. ${ }^{112}$ Im Leitentscheid Minelli $I^{113}$ erwog dann aber das Bundesgericht, dass es aus rechtsstaatlichen Gründen unerlässlich erscheine, die wichtigsten mit Untersuchungshaft oder Strafvollzug verbundenen Freiheitsbeschränkungen durch einen allgemeinen Erlass zu regeln, um den Gefangenen vor Willkür zu schützen. ${ }^{114}$

lung in Deutschland und in der Schweiz sowie zu eigenen Nuancen in der Schweiz vgl. ausf. von Kielmansegg S. 97 ff. Sowohl vom Begriff des Gewaltverhältnisses als auch vom Begriff des Sonderstatusverhältnisses werden nicht nur inhaftierte Personen, sondern eine sehr heterogene Personengruppe umfasst: Militärpersonen, das Personal des öffentlichen Dienstes oder etwa Schüler: KELLER/SCHÄdLER ZSR 2013, 195 (198); HäFELIN/Müller/UhlmanN N 451.

111 Häfelin/Müller/Uhlmann N 450. Im Vergleich zur früheren Rechtsprechung des Bundesgerichts zum „besonderen Gewaltverhältnis“ sind diesen besonderen Einschränkungsmöglichkeiten aber enge Schranken gesetzt: BAECHTOLD S. 9 ff.; vgl. auch kritisch Keller/Schädler ZSR 2013, 195 (196): Die Schweizer Staatsrechtsdoktrin habe die Figur des Sonderstatusverhältnisses aus dem deutschen Recht übernommen. Während das Konzept des Sonderstatusverhältnisses in Deutschland von Lehre und Rechtsprechung mittlerweile aufgegeben worden sei, werde in der Schweiz nach wie vor an diesem festgehalten (vgl. auch Fn. 108). Bei der Beurteilung, ob Grundrechtsbeschränkungen im Vollzugswesen rechtmässig erfolgt seien oder nicht, biete der Sonderstatus allerdings kaum noch einen zeitgemässen juristischen Lösungsansatz.

112 Vgl. auch BGE 98 I b 305, E. 2 a; BGE 97 I 52; Baechtold/Weber/HostettleR S. 205; Keller/SCHÄdLER ZSR 2013195 (198).

113 BGE 99 Ia 262.

114 BGE 99 Ia 262, E. III. Im Jahr zuvor hatte das deutsche Bundesverfassungsgericht in seinem Entscheid BVerfG, Urt. v. 14.03.1972 - 41/7, NJW 1972, 811 (812) bereits festgehalten, dass Grundrechte von Strafgefangenen nur durch Gesetz oder aufgrund eines Gesetzes eingeschränkt werden können. 
Zur notwendigen Bestimmtheit des Rechtssatzes im Sonderstatusverhältnis führte das Bundesgericht aus, dass wesentliche Einzelheiten in einem allgemeinen Erlass zu regeln seien, sie dürften nicht dem freien Ermessen der Anstaltsbeamten überlassen werden (Normdichte). ${ }^{115}$ Im Freiheitsentzug könne jedoch nicht nach dem Prinzip verfahren werden, dass alles, was nicht ausdrücklich verboten sei, gestattet sei. Nicht alle sich stellenden Konstellationen seien voraussehbar, weshalb gewisse Generalklauseln im Einzelfall ausreichend sein müssten. ${ }^{116}$

Zur notwendigen Gesetzesform erwog das Bundesgericht im Leitentscheid Minelli I weiter, die Anordnung der Untersuchungshaft oder der Strafe erfordere eine klare Grundlage in einem formellen Gesetz (Normstufe). ${ }^{117}$ Einer Grundlage in einem formellen Gesetz bedürften darüber hinaus insbesondere auch die Art und die mögliche Höchstdauer des Freiheitsentzugs. ${ }^{118}$ Die Regelung der Einzelheiten der Vollzugsordnung dürfe jedoch vom Gesetzgeber an die Exekutive delegiert werden, weil es dabei nicht um Grundsatzfragen gehe, deren Entscheidung nur auf dem Wege der formellen Gesetzgebung zulässig sei. ${ }^{119}$ Letzteres gelte, obwohl das Mass der Freiheitsbeschränkung wesentlich von der Ausgestaltung des Vollzugs betroffen sei. ${ }^{120}$

115 BGE 99 Ia 262, E. III.

116 BGE 99 Ia 262, E. III; vgl. auch Häfelin/Müller/Uhlmann N 453; vgl. auch Müller S. 33 f.: Mindestvoraussetzung an die Bestimmtheit der Haftordnung sei aber, dass sowohl die Strafgefangenen als auch das Personal der Strafvollzugsbehörde die Konsequenzen ihres Handelns mit dem erforderlichen Grad der Gewissheit abschätzen können und ihre Handlungen und ihr Verhalten daran orientieren können. Vgl. kritisch Keller/SchädleR ZSR 2013195 (205): geringe Anforderungen an die Bestimmtheit als potentieller rechtsstaatlicher Stolperstein.

117 BGE 99 Ia 262, E. III.

118 BGE 99 Ia 262, E. III.

119 BGE 99 Ia 262, E. III.

120 BGE 99 Ia 262, E. III; dazu kritisch Kellek/SchädLER ZSR 2013195 (200 f., 205): Eine Delegation von Rechtssetzungsbefugnissen sei immer dann möglich, wenn keine schwerwiegenden Grundrechtseinschränkungen vorliegen. Für die Beurteilung, ob es sich um schwerwiegende Einschränkungen handele oder nicht, sei der Zweck des Sonderstatusverhältnisses massgebend. So lasse sich eine eingeschränkte Bewegungsfreiheit im Strafvollzug etwa aus dem Zweck des Strafvollzugs ableiten, weshalb diese Beschränkung vorhersehbar und damit nicht schwerwiegend sei. Für das Erfordernis der formellen Gesetzesgrundlage sei folglich entscheidend, wie weit der Zweck des entsprechenden Rechtsverhältnisses verstanden werde. Diese Abgrenzung sei aber unpräzise und deshalb problematisch. 
Diese Grundsätze gelten im Wesentlichen noch heute. ${ }^{121}$ Zusammengefasst sind beim Sonderstatusverhältnis demnach die Anforderungen an Normdichte geringer, und dem formellen Gesetzgeber ist eine grosszügigere Gesetzesdelegation erlaubt. ${ }^{122}$

b Öffentliche Interessen und Verhältnismässigkeit i.e.S.

In der älteren Rechtsprechung wurde a priori von einer Vorrangstellung des öffentlichen Interesses gegenüber den individuellen Interessen der Inhaftierten ausgegangen. ${ }^{123}$ Dem ist heute nicht mehr so: Das Bundesgericht geht heute bei Güterabwägungsfragen sehr grundrechtssensibel vor, wobei die individuellen Interessen der Strafgefangenen gleichberechtigt berücksichtigt werden wie die öffentlichen Interessen; sie treten mithin nicht mehr von vorneherein in den Hintergrund. ${ }^{124}$

Damit führt das Sonderstatusverhältnis heute hinsichtlich der Verhältnismässigkeitsprüfung im engeren Sinne nicht mehr zu Besonderheiten zu Lasten des Inhaftierten.

\section{c Möglichkeit disziplinarischer Massnahmen}

Im Sonderstatusverhältnis verfügt die Behörde über Disziplinargewalt, welche den Erlass von Disziplinarmassnahmen ermöglicht. ${ }^{125}$ Disziplinarmassnahmen als administrative Sanktion mit pönalem Charakter können

121 Das Bundesgericht hatte sich mit diversen Einzelfragen zu beschäftigen, so dass es Gelegenheit hatte, seine Rechtsprechung zum Sonderstatusverhältnis und zu den Anforderungen an die gesetzliche Grundlage weiter auszudifferenzieren. An seiner grundsätzlichen Haltung änderte sich dabei allerdings nichts.

122 Griffel N 149: Griffel wendet diesbezüglich allerdings ein, dass die Anforderungen an Normdichte und Normstufe auch „im Normalfall“ nicht eindeutig feststünden und von verschiedenen Faktoren abhängen würden. Zwischen Sonderstatusverhältnis und normalen Verhältnissen bestünde heute kein kategorialer Unterschied, es bestünden vielmehr nur graduelle Unterschiede, weshalb die Rechtsfigur des Sonderstatusverhältnisses heute entbehrlich sei. Zur Abkehr vom Sonderstatus vgl. auch Keller/Schädler ZSR 2013, 195 (195 ff.): Die Sonderstatuskonzeption sei eine veraltete Rechtsfigur und weise erhebliche dogmatische Mängel auf.

123 Müller S. $60 \mathrm{f}$.

124 Müller S. 60 f., 123 f.; vgl. auch Keller/Schädler ZSR 2013195 (203).

125 Häfelin/Müller/Uhlmann N 1505; Griffel N 586. 
etwa bei der Verletzung einer Anstaltsordnung angeordnet werden, dienen der Wahrung oder Wiederherstellung der Ordnung und gewähren dabei die ordnungsgemässe Aufgabenerfüllung sowie das Ansehen und die Vertrauenswürdigkeit der Verwaltungsbehörde. ${ }^{126}$ Die Anordnung einer Disziplinarmassnahme setzt eine entsprechende gesetzliche Grundlage voraus. ${ }^{127}$ An die Bestimmtheit dieser gesetzlichen Grundlagen werden allerdings keine allzu hohen Anforderungen gestellt; den Behörden wird nicht selten ein Entschliessungs- bzw. ein Auswahlermessen zugebilligt. ${ }^{128}$ Der Behörde steht es sodann auch frei, ganz auf die Anordnung der Disziplinarmassnahme zu verzichten (Opportunitätsprinzip). ${ }^{129}$

\section{d Prozedurale Besonderheiten}

In der Vergangenheit wurde auf nationaler Ebene, dem materiellen Charakter des besonderen Gewaltverhältnisses entsprechend, auf prozessualer Ebene gefolgert, dass dem Inhaftierten kein Recht auf gerichtlichen Rechtsschutz zusteht. ${ }^{130}$ Durch die materielle Fortentwicklung der Rechtsfigur des Sonderstatusverhältnisses folgten auch aus prozessualer Hinsicht Anpassungen. ${ }^{131}$ Gewisse prozessuale Besonderheiten bestehen allerdings fort: ${ }^{132}$

Bei Beschwerden im Rahmen des Strafvollzugs ist zunächst das kantonale Recht zu beachten. In einigen Kantonen sind besondere Beschwerdeinstanzen vorgesehen, etwa die Anstaltsdirektion oder das ihr übergeordnete Departement. ${ }^{133}$ Abweichungen zum allgemeinen Verwaltungsrecht können auch z.B. bei den Beschwerdefristen vorgesehen sein. ${ }^{134}$ Sind für den Strafvollzug keine besonderen, abweichenden Vorschriften erlassen worden, ist subsidiär das allgemeine Verwaltungsrecht anwendbar. ${ }^{135}$ Seit

126 Griffel N 585; Häfelin/Müller/Uhlmann N 1506.

127 Griffel N 587; Häfelin/Müller/UhlmanN N 1514.

128 Griffel N 587; Häfelin/Müller/UhlmanN N 1514, 1517.

129 Häfelin/Müller/Uhlmann N 1517; Griffel N 587.

130 Müller S. 69.

131 MÜLler S. 69, 124.

132 MÜLLER S. 69, 124.

133 Baechtold/Weber/Hostettler S. 212: Im Kanton Jura ist die Vollzugsbehörde, im Kanton Glarus der Verhörrichter und im Wallis die Kantonsregierung zuständig.

134 Baechtold/Weber/Hostettler S. 212, diese würden von 24 Stunden bis zu 30 Tagen reichen.

135 Baechtold/Weber/Hostettler S. 212. 
der Einführung der Einheitsbeschwerde auf Bundesebene (BGG) hat der Inhaftierte die Möglichkeit, Entscheide im Bereich des Strafvollzugs auf Bundesebene anzufechten, wobei es keine Rolle spielt, welche kantonale Instanz letztinstanzlich entschieden hat. ${ }^{136}$ Gemäss Art. 95 BGG $e$ contrario ist die Verletzung von kantonalem Recht vor Bundesgericht allerdings kein Beschwerdegrund. Je nach Kanton befinden sich aber konkrete strafvollzugsrechtliche Vorschriften gerade auf kantonaler Ebene. Vor Bundesgericht gerügt werden kann aber immerhin die Verletzung kantonaler verfassungsmässiger Rechte (Art. 95 lit. c BGG) und von Bundesrecht (Art. 95 lit. a BGG), was auch die Rüge der willkürlichen Anwendung kantonalen Rechts umfasst. Schliesslich ist vor Bundesgericht auch die Rüge von Völkerrecht zulässig (Art. 95 lit. b BGG).

Für den Rechtsschutz entscheidend ist nicht nur die generelle Beschwerdemöglichkeit, sondern auch Kontrollumfang und Kontrolldichte. ${ }^{137}$ Die Gerichte sind dabei verpflichtet, die ihnen zugewiesene Kognition auszunutzen. ${ }^{138}$ Materiellrechtliche Besonderheiten des Sonderstatusverhältnisses hinsichtlich der Normdichte wirken sich hier aus: Offen formulierte Rechtssätze und Generalklauseln führen zu einem weiten Ermessen der Strafvollzugsbehörde. Selbst bei voller Kognition auferlegen sich die Gerichte bei Fragen, welche besonderes Fachwissen erfordern oder organisatorische Belange betreffen - was gerade beim Strafvollzug der Fall sein dürfte -, bei der Überprüfung der Streitfrage nämlich eine besonders grosse Zurückhaltung. ${ }^{139}$

Zusammenfassend ist der Rechtsschutz von Personen im Sonderstatusverhältnis leicht eingeschränkt. Im Umfang des bestehenden Rechtsschutzes kommen allerdings insbesondere auf beweisrechtlicher Ebene auch die Besonderheiten zu Gunsten des Inhaftierten zur Anwendung.

\section{Zwischenfazit: Menschenrechte im Strafvollzug}

Das besondere Näheverhältnis zwischen Staat und Inhaftiertem prägt die Rechtsstellung des Gefangenen. Während früher sowohl von konventionsrechtlicher als auch von nationaler Seite Personen im Freiheitsvollzug nur eine minderwertige Menschenrechtsstellung zugesprochen wurde, stehen

136 Baechtold/Weber/Hostettler S. 213.

137 MÜller S. 107.

138 MüLlER S. 107 f.: Ansonsten läge ein Fall formeller Rechtsverweigerung vor.

139 Müller S. 110. 
heute die Obhuts- und die Fürsorgepflicht des Staates im Vordergrund, selbst wenn nach wie vor gewisse Nachteile fortbestehen.

Hinsichtlich der abwehrrechtlichen Dimension der Grundrechte ist der Inhaftierte auf nationaler Ebene auch heute noch mit der Situation konfrontiert, dass geringere Anforderungen an die Normdichte bzw. an die Bestimmtheit der gesetzlichen Grundlage gestellt werden und dem formellen Gesetzgeber eine grosszügigere Gesetzesdelegation erlaubt ist. Inhaftierte unterstehen weiter der Disziplinargewalt der Strafvollzugsbehörden, wobei Disziplinarmassnahmen aber einer gesetzlichen Grundlage bedürfen.

Ganz anders wirkt sich das Sonderstatusverhältnis auf die leistungs-, schutz-, und gewährleistungsrechtliche Dimension der Menschenrechte aus. Sowohl auf konventionsrechtlicher als auch auf nationaler Ebene werden aus dem Umstand, dass der Betroffene durch den Freiheitsentzug nicht mehr in der Lage ist, selbst für seine Grundbedürfnisse zu sorgen, $b e$ sondere positive Leistungspflichten des Staates gefolgert. Die Inhaftierung bürdet dem Staat auch gesteigerte Schutzpflichten auf, sowohl vor anderen, etwa Mitinhaftierten, als auch vor sich selbst. Damit wird der besonders verletzlichen Position des Inhaftierten Rechnung getragen.

Die materiellrechtliche Entwicklung korreliert mit den prozessualen Folgen eines Sonderstatusverhältnisses: Der Rechtsschutz der Inhaftierten wurde sukzessive ausgebaut. Auf nationaler Ebene sind in einigen Kantonen gleichwohl nach wie vor besondere Beschwerdeinstanzen und kürzere Beschwerdefristen vorgesehen. Hinsichtlich des Kontrollumfangs wirkt sich das Zugeständnis eines weiten Ermessens der Strafvollzugsbehörden aus. Insbesondere bei Fragen, welche grosses Fachwissen erfordern, oder bei organisatorischen Fragen des Strafvollzugs, ist weiter von einer reduzierten Kontrolldichte auszugehen, da Gerichte sich bei der Überprüfung solcher Fragen selbst bei voller Kognition besondere Zurückhaltung auferlegen. Nicht nur die Sorge- und Obhutspflicht des Staates gegenüber dem Gefangenen, sondern auch die Informationsherrschaft des Staates betreffend Vorgänge und Unterbringungsmodalitäten, haben beweisrechtliche Erleichterungen für den Strafgefangenen zur Folge: Der Beschwerdeführer hat den Beweis des ersten Anscheins zu erbringen; gelingt dieser, kehrt die Beweisführungslast um, und es ist am Konventionsstaat, die Vorbringen des Beschwerdeführers zu widerlegen. Nochmals reduziert sind die Anforderungen an den Anscheinsbeweis, wenn der Beschwerdeführer geltend macht, er habe im Strafvollzug Verletzungen erlitten. Die Durchsetzung eines effektiven Mindeststandards in der Haft wird damit massgeblich auf beweisrechtlicher Ebene abgesichert. 
Vereinfacht gesagt, fand ein umfassender Wandel der Menschenrechtsstellung der Inhaftierten statt: Von der ursprünglichen Annahme eines dem Freiheitsentzug innewohnendem immanenten Entzugs der Menschenrechte hin zum menschenrechtlichen Postulat, dem Inhaftierten während des Vollzugs nicht mehr Leid zuzufügen, als durch den Freiheitsentzug an sich hervorgerufen wird („no more harm principle“).

\section{Kapitel 3: Rechtsrahmen im Mehrebenensystem}

Vorgaben an den Strafvollzug finden sich auf sämtlichen Stufen des Mehrebenensystems. Soweit diese für die konkrete Ausgestaltung des Strafvollzugs für menschenrechtliche Mindeststandards bei den Haftbedingungen von Relevanz sind, wird ein Überblick über die wichtigsten Rechtsnormen auf internationaler, europäischer und nationaler Ebene gegeben. Um ein Verständnis für Wirkungsweise und normativen Geltungsanspruch der einzelnen Regelwerke zu erhalten, drängt es sich auf, nicht nur auf die Regelwerke als solche, sondern auch auf Zweck, Aufbau und Organisation der massgeblichen regelsetzenden Institutionen einzugehen. Die einzelnen Ebenen werden dabei nicht für sich allein betrachtet. Der Blick wird vielmehr auch auf die Interaktion der verschiedenen Ebenen gerichtet. Sofern die hier übersichtsartig dargelegten Regelwerke themenspezifische Anforderungen an den Strafvollzug stellen, werden sie in den jeweiligen Kapiteln wieder aufgegriffen.

\section{Vereinte Nationen (UN)}

Hauptziel der Vereinten Nationen ist die Wahrung des Weltfriedens und der internationalen Sicherheit (Art. 1 Ziff. 1 UN-Charta). Weiter bezweckt die UN die Herbeiführung einer internationalen Zusammenarbeit, um internationale Probleme wirtschaftlicher, sozialer, kultureller und humanitärer Art zu lösen und die Achtung vor den Menschenrechten und Grundfreiheiten für alle ohne Unterschied der Rasse, des Geschlechts, der Sprache oder der Religion zu fördern und zu festigen (Art. 1 Ziff. 3 UNCharta). Die Gewährung und Förderung eines menschenrechtskonformen Strafvollzugs ist damit vom Aufgabengebiet der Vereinten Nationen ohne Weiteres umfasst. ${ }^{140}$

140 In diesem Sinne vgl. auch Meyer S. 104. 
Akteure der UN sind u.a. die Generalversammlung, der Sicherheitsrat, der Wirtschafts- und Sozialrat (ECOSOC), die Sonderorganisationen sowie der Internationale Gerichtshof.

Die Generalversammlung wird in Art. 10 i.V.m. Art. 11 UN-Charta dazu ermächtigt, sich mit allen Fragen, welche von der UN-Charta umfasst sind, zu befassen und Empfehlungen an die Mitgliedstaaten oder an den Sicherheitsrat auszusprechen. ${ }^{141}$ Zudem ist der Abschluss völkerrechtlicher Verträge eines ihrer wichtigsten Handlungsinstrumente. ${ }^{142}$

Konkrete Einzelrechte, welche sich auch auf die Gestaltung des Strafvollzugs auswirken können, wurden etwa in der Allgemeinen Erklärung der Menschenrechte (AEMR) ${ }^{143}$ festgehalten. ${ }^{144}$ Diese schaffte ursprünglich allerdings keine durchsetzbaren verbindlichen Rechte; vielmehr handelt es sich um eine nicht bindende Entschliessung der UN-Generalversammlung, welche aber von Gerichten bei der Auslegung von subjektiven Rechten beigezogen wird. ${ }^{145}$ Heute hat die AEMR allerdings den Status von Völkergewohnheitsrecht erlangt. ${ }^{146}$ Einschlägige völkerrechtliche Verträge wurden beispielsweise mit dem Internationalen Pakt über wirtschaft-

141 Vorbehalten bleibt der Vorrang des Sicherheitsrates: Zu Angelegenheiten, mit welchen sich der Sicherheitsrat befasst, darf die Generalversammlung keine Empfehlungen machen, es sei denn, sie wird vom Sicherheitsrat darum ersucht (Art. 11 Abs. 2 i.V.m. Art. 12 Abs. 1 UN-Charta).

142 Meyer S. 140.

143 A/RES/217(III)[A], Universal Declaration of Human Rights.

144 Vgl. auch SK-StPO/Meyer Einleitung (EMRK) Rn. 3.

145 Vgl. auch SK-StPO/Meyer Einleitung (EMRK) Rn. 3; Brownlie S. 612, insbesondere der EGMR ziehe die AEMR als Auslegungshilfe hinzu.

146 Vgl. SK-StPO/MeYer Einleitung (EMRK) Rn. 3: Im Einzelnen bestehe Uneinigkeit darüber, welche Rechte der AEMR tatsächlich den Status des Völkergewohnheitsrechts erlangt hätten. Vgl. ferner Fassbender APuZ 46/2008, 3 (4): Viele der Artikel der AEMR würden als Ausdruck des Völkergewohnheitsrechts anerkannt. Vgl. auch bereits Buergenthal Studien zu Grund- und Menschenrechten 1999, 19 (27): Immer mehr Rechte der AEMR würden den Status von Völkergewohnheitsrecht erlangen. Die Frage, ob es sich bei den Rechten der AEMR um Völkergewohnheitsrecht handle oder ob in dieser nur allgemeine Rechtsprinzipien verbrieft seien, verliere aber immer mehr an Bedeutung, zumal die meisten Staaten den internationalen Menschenrechtspakten beigetreten seien und viele Staaten auch weitere wichtige Menschenrechtsverträge ratifiziert hätten, in denen dieselben Rechte verbrieft seien. Vgl. dazu auch KäLIN/KüNZLI S. 15: Es sei von Beginn weg geplant gewesen, die AEMR in verbindliches Vertragsrecht zu überführen, wozu etwa die Verabschiedung der beiden Menschenrechtspakte habe dienen sollen. Zur Debatte der völkergewohnheitsrechtlichen Verbindlichkeit der AEMR auch ausf. Richter S. $141 \mathrm{ff}$. 
liche, soziale und kulturelle Rechte (UNO-Pakt I ${ }^{147}$, dem Internationalen Pakt über bürgerliche und politische Rechte (UNO-Pakt II) ${ }^{148}$ und dem Übereinkommen gegen Folter und andere grausame, unmenschliche oder erniedrigende Behandlung oder Strafe (UNCAT) geschaffen. ${ }^{149}$

Das UNCAT enthält in Art. 1 Abs. 1 eine Definition der Folter, auf welche sich auch der EGMR bezieht. ${ }^{150}$ Überwacht wird die Durchsetzung dieses Übereinkommens durch den UN-Ausschuss gegen Folter (Committee against Torture, CAT). Für die Staaten bindende Entscheide kann das CAT allerdings nicht fällen. ${ }^{151}$ Am 18. Dezember 2002 wurde das Übereinkommen sodann durch das Fakultativprotokoll zum Übereinkommen gegen Folter und andere grausame, unmenschliche oder erniedrigende

147 Der UNO-Pakt I enthält keine Vorschrift, wie er innerstaatlich durchgesetzt werden soll. Es ist am nationalen Recht, wie die staatsvertragliche Verpflichtung zu erfüllen ist und ob bzw. inwiefern der UNO-Pakt I resp. einzelne seiner Normen vom Einzelnen im Rahmen eines Individualbeschwerdeverfahrens direkt angerufen werden können sollen (self-executing). Es gibt auch kein Vertragsorgan, welchem die Kompetenz zukäme, die aufgeführten Verpflichtungen verbindlich zu konkretisieren. Durchsetzungssystem sei lediglich das Berichtssystem: vgl. etwa bereits BGer 2P.273/1999, Urt. v. 22.09.2000, E. 1.

148 Vgl. insb. Art. 7 UNO-Pakt II, der festhält, dass niemand der Folter oder grausamer, unmenschlicher oder erniedrigender Behandlung oder Strafe unterworfen werden darf und insbesondere niemand ohne seine freiwillige Zustimmung medizinischen oder wissenschaftlichen Versuchen unterworfen werden darf, und Art. 10 Abs. 1 und Abs. 3 UNO-Pakt II, welche festhalten, dass jedem, dem seine Freiheit entzogen wurde, menschlich und mit Achtung vor der dem Menschen innewohnende Würde behandelt werden muss. Der Strafvollzug schliesse eine Behandlung der Gefangenen mit ein, die vornehmlich auf ihre Besserung und gesellschaftliche Wiedereingliederung hinzielt.

149 MeYer S. 198: Diese würden nicht nur dazu dienen, „das menschenrechtliche Bewusstsein der internationalen Gemeinschaft" zu steigern, sondern würden die Anerkennung universeller Regeln statuieren, welche Staaten auch gegen ihren Willen binden würden, und seien so wichtige Eckpfeiler. Zur Entstehungsgeschichte des UNCAT vgl. etwa Evans International and Comparative Law Quarterly 2002, 365 ff.

150 Vgl. unter vielen: EGMR (GK) - Selmouni/FRA, Urt. v. 28.07.1999, 25803/94, Rn.97; EGMR - Ilascu u.a./MDA, Urt. v. 08.07.2004, 48787/99, Rn. 426; zur Definition im Einzelnen vgl. auch Evans International and Comparative Law Quarterly 2002, 365 (369, 375 ff.); vgl. zum Folterbegriff auch Voyame in: Matscher, S. 109 (110 f.). Eine Definition der Begriffe der anderen grausamen, unmenschlichen oder erniedrigenden Behandlungen oder Strafen findet sich im Übereinkommen demgegenüber nicht.

151 Vgl. zum Ganzen auch SK-StPO/Meyer Art. 3 EMRK Rn. 4; zum Durchsetzungsmechanismus vgl. auch van ZYL SMIT Crime and Justice 2010, 503 (511 ff.). 
Behandlung oder Strafe (Optional Protocol to the Convention Against Torture, OPCAT) ergänzt. Das OPCAT enthält keine neuen materiellrechtlichen Bestimmungen. Es bezweckt vielmehr die Durchsetzung des UNCAT. ${ }^{152}$ So ist in Art. 2 OPCAT die Schaffung eines Unterausschusses des CAT vorgesehen (Subcommittee on Prevention of Torture and other Cruel, Inhuman or Degrading Treatment or Punishment, SPT). ${ }^{153}$ Dem SPT sind Besuchs- und Kontrollrechte in Haftanstalten der Vertragsstaaten zu gewähren. Art. 3 OPCAT verpflichtet die Vertragsstaaten weiter, auf innerstaatlicher Ebene mindestens ein Gremium zu schaffen, welches seinerseits Besuche von Orten der Freiheitsentziehung vornimmt (nationaler Präventionsmechanismus; zur Schweiz vgl. NKVF). ${ }^{154}$

Weiter kommt dem Wirtschafts- und Sozialrat (ECOSOC, Art. $61 \mathrm{ff}$. UN-Charta) eine breite Befassungs- und Empfehlungskompetenz zu (vgl. insb. Art. 62 Abs. 2 UN-Charta). ${ }^{155}$ Die ihm in Art. 62 UN-Charta zugewiesenen Aufgaben der wirtschaftlichen sozialen Entwicklung stehen in einem engen Konnex zum Schutz der körperlichen Integrität des Einzelnen. Die Gewährleistung dieser ist bei Verfolgungs- und Vollzugsmassnahmen entscheidend, ${ }^{156}$ weshalb dem ECOSOC im Bereich des Strafvollzugs eine wichtige Rolle zukommt, wenn es um Fragen rund um die Behandlung Strafgefangener oder Gefängnisstandards geht (vgl. insbesondere United Nations Standards and Norms in Crime Prevention and Criminal Justice). ${ }^{157}$

Mit der Resolution 1992/1 hat der ECOSOC die Commission on Crime Prevention and Criminal Justice (UNCCPCJ) errichtet. ${ }^{158}$ Im Jahr 2006

152 Vgl. auch van ZyL Smit Crime and Justice 2010, 503 (516).

153 Das europäische Modell in Form des CPT stand hierzu Pate: vgl. dazu auch Murdoch Eur J Crim Policy Res 2006, 121 (122); van ZyL Smit Crime and Justice 2010, 503 (516).

154 Es resultiert folglich ein Nebeneinander von internationalem und nationalem Gremium. Dies als Kompromisslösung langjähriger Verhandlung der Vertragsstaaten: vgl. van ZyL SMIT Crime and Justice 2010, 503 (516); Nowak/Ammer/ Birk/Monina/Krisper Art. 3 OPCAT Rn. 2: Das „Zwei-Säulen-System“ gelte als Ausdruck des Subsidiaritätsprinzips. Die Primärverpflichtung eines effektiven Menschenrechtsschutzes liege bei den Vertragsstaaten, währenddem den internationalen Monitoringgremien eine ergänzende Aufgabe zukäme. Zum „ZweiSäulen-System" und zur Zusammenarbeit der beiden Präventionsmechanismen vgl. auch HungerbüHLer S. 35 ff., S. 43 f.

155 Meyer S. 104.

156 Ausf. Meyer S. $104 \mathrm{f}$.

157 Meyer S. 105; Clark S. $11 \mathrm{ff} ., 147 \mathrm{ff}$.

158 E/RES/1992/1, Concerning the Establishment of the Commission on Crime Prevention and Criminal Justice; vgl. auch A/RES/46/152, Concerning the Cre- 
wurden die Aufgaben des UNCCPCJ durch die Resolution 61/252 ausgeweitet: Die UNCCPCJ wurde dabei befähigt, als Lenkungsorgan des United Nations Office on Drugs and Crime (UNODC) zu fungieren. ${ }^{159}$ Weiter ist die UNCCPCJ vorbereitendes Gremium für die United Nations Congresses on the Prevention of Crime and Treatment of Offenders (UNCPCTO) ${ }^{160}$, welche vor allem in ihrer ersten Zeit bedeutende Arbeit hinsichtlich des Strafvollzugs leisteten. ${ }^{161}$ So verabschiedete der UNCPCTO nämlich im Jahr 1955 die Mindestgrundsätze für die Behandlung der Gefangenen. Dieses Standardwerk wurde durch den ECOSOC mit Resolution 663 C (XXIV) angenommen. Da diese später unter anderem als Vorbild für die European Standard Minimum Rules for the Treatment of Prisoners ${ }^{162}$ (Europäische Mindestgrundsätze für die Behandlung von Gefangenen) dienten, ${ }^{163}$ wird im Folgenden auch auf diese eingegangen.

Wichtige Hinweise betreffend Auslegung und eigenen Geltungsanspruch der Mindestgrundsätze für die Behandlung der Gefangenen lassen sich aus den Vorbemerkungen zu den Mindestgrundsätzen entnehmen. In der ersten Vorbemerkung wird festgehalten, dass nicht bezweckt wird, ein detailliertes Modellsystem für Strafvollzugsanstalten aufzustellen. Ziel sei vielmehr, bewährte Grundsätze und Praktiken bei der Behandlung der Gefangenen und der Führung einer Anstalt zu erkennen und an ihnen festzuhalten. In der zweiten Vorbemerkung weisen sie auf die grosse Varietät in den Mitgliedstaaten hin. Angesichts der grossen Unterschiede bei den Entwicklungsstandards, den finanziellen Möglichkeiten der Mitgliedstaaten und den weiteren sozioökonomischen Gegebenheiten räumen sie in diesem Sinne selbst ein, dass die statuierten Regelungen nicht zu jeder Zeit an jedem Ort angewendet werden können. Die dritte Vorbemerkung

ation of an Effective United Nations Crime Prevention and Criminal Justice Programme.

159 A/RES/61/252, Questions Relating to the Programme Budget for the Biennium 2006-2007, XI.

160 Der United Nations Congress on the Prevention of Crime and the Treatment of Offenders (UNCPCTO) bzw. seit dem Jahr 2005 der United Nations Congress on Crime Prevention and Criminal Justice ist aus Politikern, Praktikern, Wissenschaftlern und Vertretern der Mitgliedstaaten und der Gesellschaft zusammengesetzt und findet seit dem Jahr 1995 im Fünf-Jahres-Rhythmus statt.

161 Meyer S. 106.

162 Recommendation R (87) 3 on the European Prison Rules 1987.

163 Vgl. auch Morgenstern in: Dünkel/Drenkhahn/Morgenstern, S. 35 (37): Mindeststandards der Vereinten Nationen zur Behandlung Strafgefangener als „Mutter aller Strafvollzugsgrundsätze“. 
weist auf den evolutiven Charakter der Mindestgrundsätze hin: Weiterentwicklung, Ausbau und Fortschritt im Bereich der Behandlung Gefangener und Führung von Strafvollzugsanstalten sollen nicht gebremst werden. Die Mindestgrundsätze halten den Weg für neue Versuche und Praktiken offen, sofern diese die Mindestgrundsätze wahren und ihrem Geist gesamthaft entsprechen.

In Bezug auf die Höhe bzw. Ausgereiftheit des in den Mindestgrundsätzen für die Behandlung der Gefangenen festgesetzten Standards ist mit Blick auf die genannten Vorbemerkungen einerseits zu vermuten, dass die Erzielung eines Konsenses aufgrund der immensen Varietät in den Mitgliedstaaten nur bei einem nicht allzu hohen Standard gelingen kann. Andererseits dürften sich die den Mitgliedstaaten zugestandenen situativen Relativierungsmöglichkeiten positiv auf die Konsensfähigkeit und damit auf die Höhe des Standards auswirken. Es wird jedenfalls schon aus den Vorbemerkungen deutlich, mit welchen Herausforderungen man bei der Erarbeitung dieser internationalen Mindeststandards konfrontiert war und um was für ein ambitioniertes Projekt es sich handelt. Die dritte Vorbemerkung verdeutlicht schliesslich die „Motorfunktion“, welche den vereinbarten Grundsätzen zur Gewährleistung eines menschenrechtswahrenden Mindeststandards zukommen soll.

Systematisch sind die Mindestgrundsätze von 1955 in zwei Teile gegliedert. Der erste Teil befasst sich mit den allgemein anzuwendenden Grundsätzen, welche für alle Kategorien von Inhaftierten gelten. Sie befassen sich mit der Führung einer Vollzugsanstalt und legen diesbezüglich Grundprinzipien fest, bspw. hinsichtlich Fragen der Unterbringung, der Hygiene, der Gesundheitsfürsorge, der Disziplinarstrafen, der Zwangsmittel oder der Verlegung. Im zweiten Teil der Mindestgrundsätze von 1955 wird zwischen verschiedenen Kategorien von Inhaftierten differenziert, wobei Sonderfragen zu jeder Kategorie Regelungsgegenstand sind. In der Fassung von 1955 wurde im zweiten Teil zwischen „Strafgefangene[n]“, „Geisteskranke[n] und geistig abnorme[n] Gefangene[n]“, „Festgenommene[n] oder Untersuchungsgefangene[n]“ sowie „Zivilgefangene[n]“ unterschieden. Im Jahr 1977 wurde durch den neu beigefügten Art. 95 eine weitere Kategorie „Personen, die festgenommen oder in Haft gehalten werden, ohne dass sie einer strafbaren Handlung beschuldigt sind“ hinzugefügt. ${ }^{164}$

164 Dies führte nicht nur im Teil zwei zu einer weiteren Kategorie, sondern erstreckt auch den Anwendungsbereich des ersten Teils der Mindestgrundsätze auf diese Personengruppe. 
Ganz im Sinne ihrer „Motorfunktion“ haben sich die Mindestgrundsätze von 1955 seither weiterentwickelt: Im Jahr 2011 entschied die Generalversammlung, eine intergouvernementale Expertengruppe mit der Überprüfung und, wenn notwendig, mit der Überarbeitung der UN-Mindestgrundsätze von 1955 zu beauftragen. Die Generalversammlung gab der Expertengruppe dabei vor, dass die Revision nicht zu einer Senkung, sondern nur zu einem Ausbau des Standards führen darf. Auch der Geltungsbereich der UN-Mindestgrundsätze sei beizubehalten. Unter Berücksichtigung dieser Vorgaben identifizierte die Expertengruppe bei drei Treffen zwischen den Jahren 2012 und 2014 in enger Zusammenarbeit mit dem UNODC Themengebiete und Regeln, welche einer Revision zu unterziehen sind. Anlässlich eines vierten Treffens im März 2015 einigte sich die Expertengruppe schliesslich auf eine überarbeitete Version der Mindestgrundsätze: Die „Nelson-Mandela-Regeln“ ${ }^{165}$ Diese revidierten Regeln wurden im Dezember 2015 einstimmig von der UN-Generalversammlung verabschiedet, nachdem der ECOSOC diese Regeln zuvor angenommen hatte. Damit wurde nach 60 Jahren das Regelwerk der Mindestgrundsätze umfassend revidiert. Während die zweigeteilte Grundstruktur und die einzelnen Kategorien im zweiten Teil bestehen blieben, ${ }^{166}$ gab es inhaltliche Änderungen und Anpassungen sowohl bei den generellen Regelungen und Grundprinzipien als auch bei den einzelnen themenspezifischen Ausführungen. Ziel war es, neuen Entwicklungen im Bereich der Menschenrechte und der Grundsätze des Strafrechts Rechnung zu tragen und diese in eine neue, zeitgemässe Fassung zu integrieren. Präzisierungen und zahlreiche neue Regelungen führten insgesamt zu einem Ausbau des Schutzstandards. Sofern die Änderungen bei den themenspezifischen Fragen relevant werden, wird auf diese im jeweiligen Kapitel näher eingegangen.

Neben diesem „Standardwerk“ der Mindestgrundsätze für die Behandlung der Gefangenen finden sich auf Ebene der Vereinten Nationen zahlreiche weitere einschlägige Regelwerke. So wurden am 14. Dezember

165 A/RES/70/175, United Nations Standard Minimum Rules for the Treatment of Prisoners („The Nelson Mandela Rules“); vgl. auch: Short Guide to the Revised United Nations Standard Minimum Rules for the Treatment of Prisoners (Nelson Mandela Rules), zuletzt abgerufen am 06.08.2020. Der Kongress fand in Südafrika statt. Das revidierte Regelwerk wurde als Ehrung von Nelson Mandela, welcher sich während 27 Jahren im Strafvollzug in Südafrika befand und sich für die Rechte der Gefangenen einsetzte, nach diesem benannt.

166 In den Kategorienüberschriften kam es lediglich zu zeitgemässen terminologischen Anpassungen etwa von "Geisteskranke und geistig abnorme Gefangene“ hin zu „Gefangene mit psychischen Behinderungen und/oder Erkrankungen“. 
1990 etwa die Grundprinzipien für die Behandlung der Gefangenen (Basic Principles for the Treatment of Prisoners ${ }^{167}$ ) verabschiedet, welche elf Grundprinzipien statuieren. Von ihrer Konzeption her handelt es sich bei diesen um grundsätzliche Werte und weniger um präzis ausformulierte Normen, aus denen sich direkte Vorschriften betreffend die materiellen Haftbedingungen ergeben würden. ${ }^{168}$ Auch die Formulierungen der einzelnen Prinzipien („shall“) weisen auf einen programmatischen Charakter hin. Im Rahmen des vorliegenden Untersuchungsgegenstands ist weiter etwa der Grundsatzkatalog für den Schutz aller Personen einschlägig, die irgendeiner Form von Haft oder Strafgefangenschaft unterworfen sind (Body of Principles for the Protection of All Persons under Any Form of Detention or Imprisonment ${ }^{169}$ ). Neben diesen allgemeinen Regelwerken finden sich auf Ebene der Vereinten Nationen auch je Kategorie der Inhaftierten weitere spezifische Regelwerke, so etwa die Regeln der Vereinten Nationen für die Behandlung weiblicher Gefangener und Massnahmen ohne Freiheitsentzug für weibliche Straffällige (Rules for the Treatment of Women Prisoners and Non-custodial Measures for Women Offenders [„The Bangkok Rules“] ${ }^{170}$ ) oder die Mindeststandards der Vereinten Nationen für die Verwaltung der Jugendgerichtsbarkeit (United Nations Standard Minimum Rules for the Administration of Juvenile Justice [„The Beijing Rules" ${ }^{171}$ ).

167 A/RES/45/111, Concerning the Basic Principles for the Treatment of Prisoners.

168 Es wird etwa festgehalten, dass die Menschenwürde der Strafgefangenen zu wahren ist, es nicht zu Diskriminierungen kommen darf oder dass die Religion und Kultur im Vollzug zu berücksichtigen sind, wenn dies die Umstände erlauben. Weiter wird statuiert, dass die Menschenrechte im Vollzug nicht weiter eingeschränkt werden dürfen, wie es durch den Freiheitsentzug an sich unerlässlich ist.

169 A/RES/43/173, Concerning the Body of Principles for the Protection of All Persons under Any Form of Detention or Imprisonment.

170 A/RES/65/229, Concerning the United Nations Rules for the Treatment of Women Prisoners and Non-Custodial Measures for Women Offenders („The Bangkok Rules").

171 A/RES/40/33, Concerning the United Nations Standard Minimum Rules for the Administration of Juvenile Justice („The Beijing Rules“). 


\section{Europarat}

Der Europarat nimmt als älteste internationale Organisation Europas ${ }^{172}$ und als normativer Akteur ${ }^{173}$ die Aufgabe wahr, eine engere Verbindung zwischen seinen Mitgliedstaaten zu schaffen, die Ideale und Grundsätze zu fördern, die das gemeinsame Erbe der Mitgliedstaaten bilden, sowie den wirtschaftlichen und sozialen Fortschritt zu fördern (Art. 1 lit. a ERS). Zur Aufgabenerfüllung berät der Europarat Fragen von gemeinsamem Interesse, schliesst Abkommen oder geht gemeinschaftlich auf wirtschaftlichen, sozialen, kulturellen und wissenschaftlichen Gebieten und auf Gebieten des Rechts und der Verwaltung vor und schützt die Fortentwicklung der Menschenrechte und Grundfreiheiten (Art. 1 lit. b ERS). Durch sein grosses Engagement im Bereich der Menschenrechte wird dem Europarat auch die Funktion eines „rechtsstaatlichen-menschenrechtlichen Gewissens Europas" zugeschrieben. ${ }^{174}$

Handlungsorgan des Europarates ist das Ministerkomitee (Art. 13 ERS). Mögliche Handlungsformen sind der Abschluss von Abkommen und Vereinbarungen und die Annahme einer gemeinsamen Politik durch die Regierungen (Art. 15 lit. a ERS) ${ }^{175}$ sowie der Erlass von Beschlüssen in Form von Empfehlungen an die Regierungen der Mitgliedstaaten (Art. 15 lit. b Satz 1 ERS). ${ }^{176}$ Über vertikale Rechtssetzungsbefugnisse verfügt der Europarat mangels Übertragung von Hoheitsrechten der Mitgliedstaten auf den Europarat jedoch gerade nicht. ${ }^{177}$

Die Empfehlungen des Europarates sind Ausdruck der gemeinsamen Werteordnung und beinhalten eine normativ-autoritäre Überzeugung zu einem Vorgehen bei einer bestimmten Sachfrage. ${ }^{178}$ Aus ihnen können

172 Meyer S. 254; vgl. auch Oesch S. 9: Europarat als erste Organisation im Nachkriegseuropa, welche sich auch mit politischen Fragen beschäftigte.

173 Meyer S. 858.

174 Meyer S. 261; Ehlers/Walter $₫ 1$ Rn. 2: Europarat als „Hüter von Menschenrechten“; vgl. auch Erklärung des Deutschen Bundestags „50 Jahre Europarat: 50 Jahre europäischer Menschenrechtsschutz“, BT-Drs 14/1568 v. 09.09.1999, 2.

175 Die Entscheidungsautonomie zum Abschluss der Abkommen verbleibt dabei aber bei den Mitgliedstaaten: vgl. MeYer S. 263; vgl. auch JAAG/HäNNi Rn. 309, Oesch S. 9; Ehlers/Walter $\$ 1$ Rn. 5.

176 Vgl. zu diesen auch JaAg/Hänni Rn.310; Grabenwarter ZaöRV 2014, 419 (431): Erlass von Soft Law im Zuge des Standardsettings als typische Tätigkeit des Europarats schlechthin.

177 Meyer S. 254, 262: Nicht möglich seien deshalb bindende supranationale Beschlüsse des Europarates.

178 Meyer S. 263. 
zwar niemals unmittelbare rechtliche Pflichten erwachsen, gleichwohl sind sie eine bedeutende Rechtserkenntnisquelle. ${ }^{179}$

Neben seiner legislativen Tätigkeit ist das Ministerkomitee in der Implementierungsphase mit Monitoringaufgaben betraut: Es kann Mitgliedstaaten dazu auffordern, über Massnahmen zu berichten, welche sie aufgrund einer Empfehlung des Ministerkomitees ergriffen haben (Art. 15 lit.b Satz 2 ERS). Darüber hinausgehende Befugnisse, etwa zur Ersatzvornahme bei Nichteinhaltung der rechtlich verbindlichen Konventionen oder zur weitergehenden Durchsetzung der Empfehlungen, kommen dem Ministerkomitee aber nicht zu. ${ }^{180}$

Hervorzuheben ist im vorliegenden Themenbereich auch die Arbeit des Europäischen Ausschusses für Strafrechtsfragen (CDPC). ${ }^{181}$ Dieser wurde durch das Ministerkomitee geschaffen, setzt sich als nicht politisches Gremium aus Sachverständigen zusammen und unterstützt durch sein Fachwissen die Vorarbeiten zu Übereinkommen, Resolutionen und Empfehlungen massgeblich. ${ }^{182}$ Auch in der Implementierungsphase kommt dem CDPC eine bedeutende Rolle zu.

Zum Zwecke seiner Aufgabenerfüllung hat der Europarat eine Vielzahl für den Untersuchungsgegenstand der vorliegenden Arbeit relevanter Regelwerke und Normen in Form von Abkommen und Empfehlungen erlassen, auf welche in der Folge näher eingegangen wird.

\section{Die Europäische Menschenrechtskonvention}

Die EMRK wurde am 4. November 1950 in Rom als erstes rechtlich verbindliches Menschenrechtsabkommen unterzeichnet. ${ }^{183}$ Heute ist ihre überragende Bedeutung nicht von der Hand zu weisen. ${ }^{184}$ Mit der

179 Zur rechtlichen Relevanz der Empfehlungen ausf. vgl. auch Wittinger S. 206 ff.; Meyer S. 263; vgl. auch Jung FS Ress, S. 519 (523, 526).

180 Meyer S. 543; Grabenwarter ZaöRV 2014, 419 (431).

181 KLARE The British Journal of Criminology 1961, 377 ff.; WilkitZKi ZStW 105 (1993), 821 (824f.).

182 Ausf. Meyer S. 256f.; zu den historischen und politischen Hintergründen vgl. auch BATES S. 5 ff., 33 ff., 77 ff.

183 Grabenwarter/Pabel $\$ 1$ Rn. 1; Meyer S. 265.

184 Meyer S. 265 f.: Meyer hebt insbesondere die Ausstrahlungswirkung der EMRK hervor, sowohl auf den nationalen Gesetzgeber und die nationalen Gerichte als auch auf die Europäische Union. Die EMRK konstituiere dabei eine „objektive Werteordnung mit supranationalen Werten“. 
Möglichkeit, nach Erschöpfung des nationalen Instanzenzugs (Art. 35 Abs. 1 EMRK) ${ }^{185}$ bei einer unabhängigen Instanz - dem EGMR - Individualbeschwerde einzureichen (Art.34 EMRK), besteht ein effektiver rechtlicher Durchsetzungs- und Kontrollmechanismus der in der EMRK gewährleisteten Garantien. ${ }^{186}$ Nachdem bereits aufgezeigt wurde, dass bei der Durchführung des Strafvollzugs eine ganze Reihe an Garantien der EMRK zu beachten ist, wird nun ein Überblick über die Wesensmerkmale der Fundamentalgarantie von Art. 3 EMRK gegeben, auf dessen Begriffsmerkmale eingegangen und der Bezug zu den materiellen Haftbedingungen hergestellt.

Art. 3 EMRK schützt als Fundamentalnorm den Einzelnen vor Folter, unmenschlicher oder erniedrigender Behandlung oder Strafe. Die Garantien in Art. 3 EMRK gelten absolut. ${ }^{187}$ Der absolute Charakter von Art. 3 EMRK unterstreicht die Bedeutung des von Art. 3 EMRK zu schützenden Schutzgutes für die „freiheitlich-rechtsstaatlichen Grundlagen demokratischer Gesellschaften“.188 Die Begriffe „Folter“, „unmenschliche“ oder „erniedrigende Behandlung“ sind unbestimmter Natur und damit auslegungsbedürftig. ${ }^{189}$

185 Zum Erfordernis der Rechtswegerschöpfung im Einzelnen vgl. etwa SK-StPO/ MeYer Verfahrensrecht (EMRK) Rn. $88 \mathrm{ff}$.

186 Vgl. auch Meyer-Ladewig/Nettesheim/von Raumer Einleitung Rn. 2; Meyer S. 590; zur historischen Entwicklung des Individualbeschwerdeverfahrens vgl. ausf. Ehlers/Walter $\mathbb{1} 1 \mathrm{Rn} .8 \mathrm{ff}$.; Grabenwarter/Pabel $\ 1$ Rn. $3 \mathrm{ff}$.

187 SK-StPO/MeYer Art. 3 EMRK Rn. 8; EGMR - Öcalan/TUR, Urt. v. 12.03.2003, 46221/99, Rn. 218; EGMR (GK) - Labita/ITA, Urt. v. 06.04.2000, 26772/95, Rn. 119.

188 SK-StPO/Meyer Art. 3 EMRK Rn. 9; Demko HRRS 2005, 94; EGMR (GK) Selmouni/FRA, Urt. v. 28.07.1999, 25803/94, Rn. 95; EGMR (GK) - Bouyid/BEL, Urt. v. 28.09.2015, 23380/09, Rn. 81; EGMR - Öcalan/TUR, Urt. v. 12.03.2003, 46221/99, Rn. 218; EGMR (Pl) - Soering/GBR, Urt. v. 07.07.1989, 14038/88, Rn. 88.

189 Meyer-Ladewig/Lehnert Art. 3 Rn. 6; Frowein/Peukert/Frowein Art. 3 Rn. 1; Deмко HRRS 2005, 94 (95). 
Der EGMR legt die Konvention autonom aus. ${ }^{190}$ Er verfolgt dabei keine statische Auslegung ${ }^{191}$, vielmehr orientiert er sich an der „europäischen öffentlichen Ordnung “. ${ }^{192}$ Er folgt einer effektivitätssichernden und einer dynamisch-teleologischen Auslegung, bei welcher er andere internationale Übereinkommen berücksichtigt und im Einklang mit der gemeineuropäischen Rechtsüberzeugung agiert. ${ }^{193}$ Dieser dynamische Auslegungsansatz kann im Kontext materieller Haftbedingungen etwa dann zentral werden, wenn neue wissenschaftliche Erkenntnisse etwa zu Auswirkungen spezifischer Praktiken oder Umstände auf den Einzelnen gewonnen werden. ${ }^{194}$

Art. 3 EMRK bezweckt den Schutz der physischen und psychischen Integrität des Einzelnen vor Eingriffen, welche eine „Missachtung der Person in ihrem Menschsein“ erkennen lassen. ${ }^{195}$ Die Begriffe der "Folter“, der "unmenschlicher Behandlung“ und der „erniedrigenden Behandlung“" sind in einem Stufenverhältnis angeordnet. ${ }^{196}$ Die Folter bildet gemeinhin die schwerste Form und die erniedrigende Behandlung die schwächste Form einer Verletzung von Art. 3 EMRK, weshalb die Schwere des Eingriffs nicht nur darüber entscheidet, ob eine Verletzung von Art. 3 EMRK vorliegt; vielmehr kommt ihr auch innerhalb von Art. 3 EMRK qualifizierende Wirkung zu. ${ }^{197}$ Eine Verletzung von Art. 3 EMRK setzt aber stets ein Mindestmass an Schwere der Beeinträchtigung voraus. ${ }^{198}$ Die erforderliche

190 Zum Begriff der autonomen Auslegung etwa Schilling Rn. 44: Die Begriffe der EMRK würden nur nach ihrem Sinn und ihrer Funktion im Rahmen der Konvention selbst ausgelegt. Ein Rückgriff auf die technische Bedeutung der Begriffe im innerstaatlichen Recht habe damit zu unterbleiben. Zur Figur der autonomen Auslegung und deren methodischer Einordnung auch BAADE S. $153 \mathrm{ff}$.

191 Vgl. EGMR - Tyrer/GBR, Urt. v. 25.04.1978, 5856/72, Rn.31; EGMR (GK) Selmouni/FRA, Urt. v. 28.07.1999, Rn. 101; vgl. auch Schilling Rn. 45.

192 Frowein/Peukert/Frowein Art. 3 Rn. 1; Mahoney in: Widmer, S. 143 ff.; zum Ganzen vgl. auch Holoubek S. 49 ff.; kritisch zur Annahme eines europäischen Standards vgl. Grabenwarter EuGRZ 2011, 229 (231 f.).

193 Ausf. SK-StPO/Meyer Methodik der Grundrechtsanwendung (EMRK) Rn. 74; vgl. auch Bernhardt FS Wiarda, S. 65 (67, 69 ff.); zur Effektivitätssicherung auch BATES S. $321 \mathrm{f} ., 341 \mathrm{ff}$.

194 SK-StPO/MeYer Art. 3 EMRK Rn. 12.

195 Grabenwarter/Pabel $\mathbb{} 20$ Rn. 41; Meyer-Ladewig/Lehnert Art. 3 Rn. 1.

196 Grabenwarter/Pabel $\mathbb{2} 20$ Rn. 41; SK-StPO/Meyer Art. 3 EMRK Rn. 10.

197 SK-StPO/Meyer Art. 3 EMRK Rn. 10: Bedeutung habe die Differenzierung für Fragen der Verwertbarkeit und der Entschädigung. Vgl. ferner GrabenwarTER/

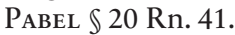

198 SK-StPO/Meyer Art. 3 EMRK Rn. 10; EGMR (GK) - Selmouni/FRA, Urt. v. 28.07.1999, 25803/94, Rn. 100. 
Eingriffsintensität beurteilt sich dabei nach einem relativen Massstab, welcher sich nach allen Umständen des jeweiligen Einzelfalles richtet. ${ }^{199}$ Die Art und der Zusammenhang der Behandlung oder Strafe, die Art und Methode der Durchführung, die Dauer, die körperlichen und seelischen Auswirkungen und je nach dem auch das Geschlecht, das Alter, der Gesundheitszustand sowie auch die besondere Verletzlichkeit des Opfers sollen bei der Frage des Erreichens der erforderlichen Schwelle des Mindestmasses an Schwere eine Rolle spielen. ${ }^{200}$ Das Eingriffsverständnis des EGMR ist dabei ein kumulatives.

In der vorliegenden Arbeit stehen Fragen zu Überbelegungssituationen und rund um eine angemessene Gesundheitsversorgung im Zentrum, weshalb im Folgenden der Fokus auf die Begriffe der unmenschlichen und erniedrigenden Behandlung oder Strafe gelegt wird. Für das Vorliegen von Folter i.S.v. Art. 3 EMRK dürfte es in diesem Zusammenhang nämlich regelmässig an der Voraussetzung des subjektiven Moments des „probibited purpose" fehlen. ${ }^{201}$

Während unter Strafe i.S.v. Art. 3 EMRK sämtliche hoheitlichen Massnahmen mit Sanktionencharakter verstanden werden, umfasst die Behandlung als Auffangbegriff sämtliches sonstiges staatliches Verhalten gegenüber dem Betroffenen, sei dies in Form eines staatlichen Tuns oder Unterlassens. ${ }^{202}$ Die Verbüssung der Freiheitsstrafe als solche ist aufgrund ihres Sanktionencharakters damit evidenterweise der Strafe zuzuordnen. Geht es allerdings um die Beurteilung der Haftbedingungen, sind diese am Massstab einer unmenschlichen resp. erniedrigenden Behandlung zu prüfen. Der Sanktionencharakter der Haftstrafe bezieht sich nämlich gerade nicht auf die Bedingungen ihrer Verbüssung, sondern nur auf den Freiheitsentzug als solchen.

199 Grabenwarter/Pabel $\$ 20$ Rn. 41; EGMR (GK) - Selmouni/FrA, Urt. v. 28.07.1999, 25803/94, Rn. 100; EGMR (GK) - Ilascu u.a./MDA u. RUS, Urt. v. 08.07.2004, 48787/99, Rn. 427; EGMR (GK) - A. u.a./GBR, Urt. v. 19.02.2009, 3455/05, Rn. 127.

200 EGMR (GK) - Labita/ITA, Urt. v. 06.04.2000, 26772/95, Rn. 120; EGMR (GK) Ilascu u.a./MDA u. RUS, Urt. v. 08.07.2004, 48787/99, Rn. 427; EGMR (GK) A. u.a./GBR, Urt. v. 19.02.2009, 3455/05, Rn. 127; EGMR (GK) - Selmouni/FRA, Urt. v. 28.07.1999, 25803/94, Rn. 100; Grabenwarter/Pabel $\mathbb{2 0}$ Rn. 43; van ZyL SMIT/SNACKen S. 127.

201 Vgl. dazu auch die Definition des Art. 1 Abs. 1 UNCAT, auf welche der EGMR immer wieder Bezug nimmt; vgl. etwa EGMR (GK) - Selmouni/FRA, Urt. v. 28.07.1999, 25803/94, Rn. 97.

202 SK-StPO/MeYer Art. 3 EMRK Rn. 22; Villiger $\ 15$ Rn. 274; Pösl S. 57. 
Unmenschlich ist jede Behandlung oder Strafe, welche längere Zeit andauert, absichtlich erfolgt und entweder eine tatsächliche Körperverletzung oder intensive psychische resp. physische Schmerzen hervorruft. ${ }^{203}$ Das Vorliegen der Absicht ist für eine Verletzung von Art. 3 EMRK im Einzelfall allerdings nicht konstitutiv. ${ }^{204}$ Für die Qualifikation der unmenschlichen Behandlung oder Strafe ist vielmehr das Erleiden erheblicher seelischer und körperlicher Qualen massgeblich. ${ }^{205}$ Soweit bisweilen weiter darauf abgestellt wird, dass beim Betroffenen bei einer unmenschlichen Behandlung auch Gefühle der Furcht und Erniedrigung ausgelöst werden, ${ }^{206}$ führt dies zu einer Verwischung der Abgrenzung der unmenschlichen und erniedrigenden Behandlung, sind diese Kriterien doch gerade Wesensmerkmal der Erniedrigung. ${ }^{207}$

Im Unterschied zur unmenschlichen Behandlung setzt die erniedrigende Behandlung nämlich nicht voraus, dass es zu einer Verursachung von Schmerzen resp. Leid kommt; zentrale Voraussetzung ist vielmehr das Vorliegen eines Demütigungsmoments. ${ }^{208}$ Insbesondere bei einer Herabsetzung oder Entwürdigung, bei mangelndem Respekt, bei einem Hervorrufen von Gefühlen der Angst, Ohnmacht oder der Minderwertigkeit ist damit von einer erniedrigenden Behandlung auszugehen. ${ }^{209}$ Ausreichend kann dabei sein, dass der Betroffene sich subjektiv, d.h. in seinen eigenen Augen, gedemütigt fühlt. ${ }^{210}$ Der Gerichtshof berücksichtigt bei seiner Prü-

203 Meyer-Ladewig/Lehnert Art. 3 Rn. 22; SK-StPO/Meyer Art. 3 EMRK Rn. 23; EGMR (GK) - Labita/ITA, Urt. v. 06.04.2000, 26772/95, Rn. 120; EGMR (GK) Kudta/POL, Urt. v. 26.10.2000, 30210/96, Rn. 92; EGMR (GK) - A. u.a./GBR, Urt. v. 19.02.2009, 3455/05, Rn. 127.

204 Grabenwarter/Pabel $\$ 20$ Rn. 43.

205 SK-StPO/Meyer Art. 3 EMRK Rn. 23.

206 Vgl. etwa Grabenwarter/Pabel $\$ 20$ Rn. 43.

207 Kritisch auch: SK-StPO/MeYer Art. 3 EMRK Rn. 24.

208 EGMR (GK) - A. u.a./GBR, Urt. v. 19.02.2009, 3455/05, Rn. 127; EGMR (GK) Labita/ITA, Urt. v. 06.04.2000, 26772/95, Rn. 120; Grabenwarter/Pabel $\$ 20$ Rn. 48.

209 SK-StPO/Meyer Art. 3 EMRK Rn. 24; Grabenwarter/Pabel $\$ 20$ Rn. 48; EGMR (GK) - Labita/ITA, Urt. v. 06.04.2000, 26772/95, Rn.120; EGMR (GK) Kudta/POL, Urt. v. 26.10.2000, 30210/96, Rn.92; EGMR (GK) - A. u.a./GBR, Urt. v. 19.02.2009, 3455/05, Rn. 127; EGMR - Vasyukov/RUS, Urt. v. 05.04.2011, 2974/05, Rn. 59; EGMR (GK) - Bouyid/BEL, Urt. v. 28.09.2015, 23380/09, Rn. 87.

210 Vgl. etwa EGMR - Tyrer/GBR, Urt. v. 25.04.1978, 5856/72, Rn. 32; EGMR Raninen/FIN, Urt. v. 16.12.1997, 20972/92, Rn. 55. 
fung zwar, ob eine Erniedrigungsabsicht vorliegt oder nicht, konstitutive Voraussetzung ist eine solche aber nicht. ${ }^{211}$

Der Gerichtshof betont weiter, dass, obgleich sich die Konvention nicht ausdrücklich auf die Menschenwürde beziehe, die Achtung der Menschenwürde zum Wesen der Konvention gehöre. ${ }^{212}$ Eine besonders enge Verbindung der Menschenwürde bestünde hinsichtlich des Konzepts einer erniedrigenden Behandlung i.S.v. Art. 3 EMRK. ${ }^{213}$ Gleichwohl scheint die Menschenwürderelevanz für sich genommen kein geeignetes Abgrenzungskriterium zur unmenschlichen Behandlung zu sein, ist doch sämtlichen Einzelgarantien des Art. 3 EMRK ein Menschenwürdekern inhärent. ${ }^{214}$

Die Begriffsmerkmale einer „unmenschlichen“ resp. einer „erniedrigenden Behandlung" verdeutlichen, dass bei deren Unterscheidung nicht allein auf die Intensität des Verstosses abgestellt werden kann. Obwohl die beiden Garantien grundsätzlich - zumindest was die messbaren gesundheitlichen Auswirkungen betrifft - in einem Stufenverhältnis angeordnet sind, unterscheiden sie sich gerade auch aufgrund ihrer Einwirkungsweisen auf den Betroffenen. Damit sind die Unterschiede auch normativer Natur. ${ }^{215}$ Ihnen gemein ist allerdings ihr Menschenwürdebezug.

211 Vgl. etwa EGMR (GK) - Jalloh/GER, Urt. v. 11.07.2006, 54810/00, Rn. 68; im Einzelnen auch SK-StPO/MeYer Art. 3 EMRK Rn. 73 f.: Erniedrigungsabsicht sei für den EGMR aber wichtiger Indikator.

212 EGMR (GK) - Bouyid/BEL, Urt. v. 28.09.2015, 233380/09, Rn. 89: Der Gerichtshof weist dabei daraufhin, dass sich die Menschenwürde immerhin in der Präambel des 13. ZP der Konvention finde.

213 EGMR (GK) - Bouyid/BEL, Urt. v. 28.09.2015, 233380/09, Rn.90; vgl. etwa auch EGMR (GK) - Kudta/POL, Urt. v. 26.10.2000, 30210/96, Rn. 94; EGMR Valasinas/LTU, Urt. v. 24.07.2001, 44558/98, Rn. 102; EGMR - Yankov/BUL, Urt. v. 11.12.2003, 39084/97, Rn. 114.

214 Vgl. etwa EGMR (GK) - Bouyid/BEL, Urt. v. 28.09.2015, 233380/09, Rn. 81: „[...] the prohibition of torture and inhuman or degrading treatment or punishment is a value of civilisation closely bound up with respect for human dignity."; vgl. auch Bergmann S. $120 \mathrm{ff}$.

215 Vermeulen/Battjes in: van Dijk/van Hoof u.a., S. 386, 399; SK-StPO/Meyer Art. 3 EMRK Rn. 71. Vgl. auch Prosenjak S. 9 ff., 171, der zunächst auf die Abgrenzung der Einzelgarantien durch das Kriterium der Schwere eingeht, schliesslich aber mit Blick auf die Kriterien der Folter und der unmenschlichen Behandlung zum Schluss gelangt, dass diese beiden Tatbestände sich nicht durch ein "Mebr" oder "Weniger" unterscheiden lassen, sondern ihnen eine andere Art von Vorwerfbarkeit innewohne, womit Folter ein aliud sei. Dies gilt nicht nur im Verhältnis zwischen Folter und unmenschlicher Behandlung, sondern auch im Verhältnis unmenschlicher und erniedrigender Behandlung. Vgl. so auch bereits Addo/Grief EJIL 1998, 510 (511): „The distinction between the 
Weder den Begriffsmerkmalen der erniedrigenden noch denen der unmenschlichen Behandlung i.S.v. Art. 3 EMRK lässt sich eindeutig entnehmen, inwieweit die Menschenrechtsprüfung an normativen oder soziomedizinischen resp. empirischen Kriterien zu bestimmen ist bzw. inwiefern diese beiden Elemente jeweils gar miteinander zu kombinieren sind. ${ }^{216}$ Der Rechtsprechung zu Art. 3 EMRK lassen sich jedenfalls sowohl soziomedizinische resp. empirische als auch normativ wertende Elemente entnehmen. So resümiert der Gerichtshof auch in seinem Urteil Keenan/GBR, dass, selbst wenn der Nachweis der Schwere des einer bestimmten Massnahme zuzuschreibenden physischen oder psychischen Leidens in vielen der von ihm nach Art. 3 EMRK entschiedenen Fällen eine wichtige Rolle gespielt hat, ${ }^{217}$ Situationen denkbar sind, in denen der Nachweis der tatsächlichen Auswirkungen auf den Betroffenen kein wesentliches Kriterium für die Feststellung eines Verstosses gegen Art. 3 EMRK ist. ${ }^{218}$ So könne die Behandlung einer psychisch kranken Person mit den durch Art. 3 EMRK auferlegten Normen zum Schutz der grundlegenden Menschenwürde unvereinbar sein, auch wenn diese Person möglicherweise nicht in der Lage sei, auf irgendwelche spezifische Beeinträchtigungen hinzuweisen. ${ }^{219}$ Ähnlich argumentierte der Gerichtshofs bereits bei der Beurteilung von Gewaltanwendungen an Personen, denen die Freiheit entzogen ist. Anstatt auf ein Mindestmass an erlittenem Leiden abzustellen, prüft der Gerichtshof hier, ob die Gewaltanwendung unbedingt erforderlich war. War sie dies nicht, stellt er fest, dass die Würde des Opfers verletzt wurde und ein Verstoss

three categories of infringement identified in Article 3 is useful for the purpose of applying the appropriate label to a particular form of abuse“; vgl. auch GREER HRLR 2018, 297 (299): „very different form of harmful conduct“.

216 Vgl. auch die Überlegungen von SALIGER ZStW (116) 2004, 35 (37, 41 f.); in Bezug auf die erniedrigende Behandlung vgl. auch WeBster S. $64 \mathrm{ff.}$

217 Der EGMR forderte insbesondere bei der Prüfung einer unmenschlichen Behandlung immer wieder einen konkreten Nachweis der seelisch oder körperlich erlittenen Qualen. Konnte der Beschwerdeführer solche nicht vorbringen, verneinte der Gerichtshof in diesen Fällen das Vorliegen einer unmenschlichen Behandlung i.S.v. Art. 3 EMRK: vgl. etwa EGMR - Kaprykowski/POL, Urt. v. 03.02.2009, 23052/05, Rn.75; EGMR - Hummatov/AZE, Urt. v. 29.11.2007, 9852/03 u. 13413/04, Rn. 121; EGMR - Sarban/MDA, Urt. v. 04.10.2005, 3456/05, Rn. 86.

218 EGMR - Keenan/GBR, Urt. v. 03.04.2001, 27229/95, Rn. 113 ff.: Der Gerichtshof stellte vorliegend sowohl eine unmenschliche als auch eine erniedrigende Behandlung i.S.v. Art. 3 EMRK fest. Vgl. auch Dörr/Grote/Marauhn/Bank Kap. 11 Rn. 21.

219 EGMR - Keenan/GBR, Urt. v. 03.04.2001, 27229/95, Rn. 113. 
gegen Art. 3 EMRK vorliegt. ${ }^{220}$ Der Gerichtshof legt demnach im Rahmen seiner autonomen Auslegung die Beurteilungskriterien sowohl bei unmenschlichen als auch bei erniedrigenden Behandlungen je Fallgruppe fest und stellt anhand dieser für die einzelnen Fallgruppen je spezifische Prüfprogramme auf.

Für die folgende Art. 3 EMRK-Prüfung von Überbelegungssituationen und der Gesundheitsversorgung bedeutet dies zusammenfassend, dass jeweils Klarheit über die konkret zu beurteilende Einzelgarantie (unmenschliche oder erniedrigende Behandlung) sowie über die jeweiligen Beurteilungskriterien und das spezifische Prüfprogramm zu schaffen ist.

\section{Europäisches Übereinkommen zur Verhütung von Folter und} unmenschlicher oder erniedrigender Behandlung oder Strafe

Das Europäische Übereinkommen zur Verhütung von Folter und unmenschlicher oder erniedrigenden Behandlung vom 26. November $1987^{221}$, welches unter der Aufsicht des CDPC ausgearbeitet wurde, ist neben der EMRK die einzige Konvention, welche für sämtliche Mitgliedstaaten bindend ist und gemeinsame, transnationale Werte konsolidiert. ${ }^{222}$ Es verstärkt das UN-Übereinkommen zur Verhütung von Folter und unmenschlicher oder erniedrigender Behandlung. ${ }^{223}$

Das Übereinkommen bezweckt, schon auf der Stufe der Prävention ansetzend, den Schutz von Personen, welche sich im Freiheitsentzug befinden, vor Folter, unmenschlicher oder erniedrigender Behandlung oder Strafe (vgl. Präambel).224 Mit einem nichtjustiziellen Kontrollmechanismus bezweckt es die Verhütung von Verletzungen des Art. 3 EMRK. ${ }^{225}$ Dies unter ausdrücklichem Hinweis darauf, dass Personen, die sich durch eine

220 EGMR - Keenan/GBR, Urt. v. 03.04.2001, 27229/95, Rn. 113; EGMR - Ribitsch/ AUT, Urt. v. 04.12.1995, 18896/91, Rn. 38; vgl. auch Dörr/Grote/Marauhn/BAnK Kap. 11 Rn. 21.

221 Europäisches Übereinkommen zur Verhütung von Folter und unmenschlicher oder erniedrigender Behandlung oder Strafe vom 26.11.1987.

222 MeYer S. 275.

223 SK-StPO/Meyer Art. 3 EMRK Rn. 5.

224 Zum verwendeten Begriff der Folter und der unmenschlichen und erniedrigenden Behandlung im Einzelnen: Evans International and Comparative Law Quarterly 2002, 365 (369, 373 ff.); van ZyL SMIT Crime and Justice 2010, 503 (519).

225 SK-StPO/Meyer Art. 3 EMRK Rn. 5 mit Verweis auf Art. 3 des Abkommens. 
Verletzung des Art. 3 EMRK beschwert fühlen, die in der Konvention vorgesehenen Verfahren in Anspruch nehmen können (Präambel). Art. 17 Abs. 2 des Abkommens hält fest, dass das Übereinkommen so auszulegen ist, dass die Befugnisse der Organe der Europäischen Menschenrechtskonvention oder die von den Vertragsparteien nach jener Konvention eingegangenen Verpflichtungen nicht eingeschränkt würden.

Art. 1 des Abkommens sieht die Bildung eines Ausschusses zur Verhütung von Folter und unmenschlicher oder erniedrigender Behandlung oder Strafe vor („European Committee for the Prevention of Torture and Inhuman or Degrading Treatment or Punishment" / „CPT-Ausschuss" / „Ausschuss“). ${ }^{226}$ Der Ausschuss stattet Orten, an welchen Personen durch eine öffentliche Behörde die Freiheit entzogen wurde, regelmässige Besuche ab (Art. 7 Abs. 1 des Abkommens). Die Vertragsparteien erklären sich mit der Unterzeichnung des Übereinkommens bereit, dem Ausschuss in ihrem Hoheitsgebiet Zugang zu diesen Orten zu gewähren (Art. 2 des Abkommens). Ferner verpflichtet sich der unterzeichnende Staat, sicherzustellen, dass dem Ausschuss gewisse Erleichterungen zuteil werden (Zugang zum Hoheitsgebiet, Informationen und Zugang zu den Orten, in denen sich Personen im Freiheitsentzug befinden; Art. 8 Abs. 2 des Abkommens). Es ist dem Ausschuss ferner auch zu erlauben, sich mit jeder Person, der die Freiheit entzogen wurde, und mit Personen, welche sachdienliche Auskünfte geben können, zu unterhalten (Art. 8 Abs. 3 und Abs. 4 des Abkommens). Die Mitglieder des Ausschusses und die Sachverständigen geniessen Vorrechte und Immunitäten (Art. 16 des Abkommens i.V.m. Anlage).

Nach den Besuchen verfasst der Ausschuss einen Bericht, welcher grundsätzlich vertraulich zu behandeln ist. ${ }^{227}$ Dieser enthält die beim Besuch festgestellten Tatsachen unter Berücksichtigung von Äusserungen der betreffenden Vertragspartei. ${ }^{228}$ Dieser Bericht wird samt erforderlichen Emp-

226 Zur Entwicklung und Arbeit des CPT eingehend Murdoch Eur J Crim Policy Res 2006, $121 \mathrm{ff}$.

227 Art. 11 des Abkommens sieht vor, dass der Ausschuss den Bericht grundsätzlich nur dann (gegebenenfalls mit einer Stellungnahme der betreffenden Vertragspartei) veröffentlicht, wenn die Vertragspartei darum ersucht.

228 Vgl. auch Koeppel S. 185 ff.: Die präventiv ausgerichtete Arbeit lasse sich in der Praxis nicht eindeutig von einer nachträglichen Kontrolle abgrenzen. Dies habe aber nur vordergründig ein Kompetenzproblem zur Folge. Eine effektive Prävention setze das Wissen über eventuelle Missstände voraus. Die Berichte hätten sich allerdings nicht auf einzelne Menschenrechtsverletzungen zu konzentrieren, sondern mehr auf potentielle Gefahrenquellen hinzuweisen. 
fehlungen der Vertragspartei übermittelt. Neben der Zustellung dieses Berichts kann der Ausschuss Konsultationen mit der Vertragspartei führen, um Verbesserungen des Schutzes von Personen, denen die Freiheit entzogen ist, vorzuschlagen (Art. 10 Abs. 1 des Abkommens). Lehnt die Vertragspartei ab, die Lage im Sinne der Empfehlungen des Ausschusses zu verbessern, resp. verweigert die Vertragspartei die Zusammenarbeit mit dem Ausschuss, kann der Ausschuss mit Zweidrittelmehrheit seiner Mitglieder beschliessen, eine öffentliche Erklärung abzugeben (Art. 10 Abs. 2 des Abkommens).229 Damit kommt der öffentlichen Erklärung eine sanktionierende Wirkung zu. ${ }^{230}$ In der Praxis stimmen allerdings fast alle Staaten ohnehin einer Veröffentlichung der Berichte zu. ${ }^{231}$ Öffentliche Erklärungen gegen den Willen der Staaten sind äusserst selten. ${ }^{232}$ Neben diesen Länderberichten verfasst der Ausschuss jährlich einen allgemeinen Bericht über seine Tätigkeit (Art. 12 des Abkommens). Die Veröffentlichung dieser Jahresberichte ermöglicht eine externe Kontrolle der Arbeit des CPT. 233

Das Europäische Übereinkommen zur Verhütung von Folter und unmenschlicher oder erniedrigender Behandlung oder Strafe wird durch einen erläuternden Bericht ergänzt. ${ }^{234}$ Anders als der Name es vermuten liesse, dient dieser nicht dazu, „unklare Begriffe oder Punkte“ des Übereinkommens bloss „zu erläutern“.235 Vielmehr legt er die Art und Weise der Auslegung und Anwendung der Regeln der Konvention fest, wobei das CPT selbst den erläuternden Bericht als „massgebliche Interpretation“ des Übereinkommens erachtet. 236

229 Allgemein zur Monitoringfunktion des CPT im Nachgang seiner Besuche vgl. Murdoch Eur J Crim Policy Res 2006, 121 (131 f.).

230 Vóкó S. 259; Murdoch S. 44: Öffentliche Erklärungen seien denn auch das einzige Sanktionsmittel, welches dem CPT zur Verfügung stehe. Vgl. ferner Morgenstern in: Dünkel/Drenkhahn/Morgenstern, S. 35 (47): Öffentliche Erklärung als Ausübung politischen Drucks.

231 Vgl. etwa Murdoch S. 43.

232 Bislang gegen die Türkei (1992 und 1996), Russland (2001, 2003, 2007 und 2019), Griechenland (2011), Bulgarien (2015) und Belgien (2017): Vollständige aktuelle Liste abrufbar auf der Seite des CPT: CPT - Public Statements, zuletzt abgerufen am 07.07.2020; vgl. dazu auch Thiele in: Beichelt/Choluj/ Rowe/Wagener S. 247 (259 f.); Murdoch Eur J Crim Policy Res 2006, 121 (134); Murdoch S. 44; Brummer S. 210.

233 Vóкó S. 259.

234 Explanatory Report to the European Convention for the Prevention of Torture and Inhuman or Degrading Treatment or Punishment v. 26.11.1087.

235 Vóкó S. 264.

236 Vgl. dazu Vókó S. 264 f.: Fraglich sei allerdings, ob das Ministerkomitee überhaupt befugt ist, eine „massgebliche Interpretation“ des Übereinkommens zu 
Der erläuternde Bericht hält fest, dass der Ausschuss nicht bezweckt, Staaten zu verurteilen, sondern sich im Geiste der Zusammenarbeit und der Beratung um Verbesserungen beim Schutz von Personen, denen die Freiheit entzogen wurde, zu bemühen (Abs. 20 des erläuternden Berichts). ${ }^{237}$ Es sei nicht Aufgabe des Ausschusses, justizielle Funktionen auszuüben oder zu erklären, dass Verletzungen der einschlägigen internationalen Übereinkünfte begangen worden seien. Der Ausschuss werde deshalb weder in abstracto noch bezüglich konkreter Tatsachen Stellungnahmen zur Auslegung dieser Übereinkünfte abgeben (Abs. 17 des erläuternden Berichts). Für die Beurteilung, ob der Ausschuss der Vertragspartei Empfehlungen zu geben habe, müsse der Ausschuss allerdings die Fakten, die er während der Besuche festgestellt habe, bewerten. Wenn die Fakten unklar seien, habe er keine ausreichende Grundlage, um Empfehlungen auszusprechen, da er gerade nicht dafür zuständig sei, Zeugen gemäss den allgemeinen Grundsätzen des rechtlichen Verfahrens anzuhören. Das weitere Vorgehen sei deshalb, die Vertragspartei über diesen Umstand zu informieren und weitere Ermittlungen auf nationaler Ebene vorzuschlagen, mit dem Ersuchen, über die Ergebnisse der nationalen Untersuchung informiert zu werden (Abs. 18 des erläuternden Berichts). Weitere Besuche an bereits besuchten Orten können als Folgemassnahme organisiert werden (Abs. 19 des erläuternden Berichts).

Das CPT zieht aus dem ihm zugesprochenen präventiven Charakter selbst vier Hauptschlussfolgerungen. ${ }^{238}$ Erstens: Gegenstand der Betrachtung des CPT sind die allgemeinen Bedingungen, welche es während seinen Besuchen vorfindet. Dabei konzentriert sich das CPT aber nicht nur auf bestehende Verletzungen, sondern richtet seine Aufmerksamkeit auch auf Umstände, welche Indikatoren für zukünftige Verletzungen sein

liefern. Die zur Prüfung dieser Frage hinzugezogene Gruppe von Rapporteuren habe einstimmig die Meinung vertreten, dass das Ministerkomitee nicht zur massgeblichen Interpretation befugt sei. Gleichwohl habe der erläuternde Bericht für die Auslegung des Übereinkommens grossen Wert, wenngleich er nicht denselben Wert habe wie der Text als solcher.

237 Vgl. dazu etwa auch Murdoch Eur J Crim Policy Res 2006, 121 (125, $131 \mathrm{f}$.): Dem CPT gehe es um Kooperation und nicht um Konfrontation. Ziel sei ein ständiger Dialog mit den Staaten anstelle einer Verurteilung. So auch GräFENSTEIN ZfStrVO 2003, 10 (11); vgl. auch Koeppel S. 208: Es bestehe ein Zusammenhang der vom CPT verwendeten Sprache in den Berichten mit seiner Zielsetzung der Kooperation mit den Mitgliedstaaten. Empfehlungen und Fragen des CPT seien sehr höflich, wenngleich die Berichte durchaus kritisch seien. CPT - 1st General Report [CPT/Inf (91) 3], Rn. 47. 
könnten. ${ }^{239}$ Zweitens: Das CPT bezieht methodologisch die den Problemen zugrundeliegenden Ursachen mit ein: ${ }^{240}$ Der soziale und wirtschaftliche Kontext und der Hintergrund der verschiedenen Länder wird vom CPT dabei berücksichtigt. ${ }^{241}$ Die Menschenwürde müsse aber in allen Mitgliedstaaten der Konvention effektiv respektiert werden. ${ }^{242}$ Drittens: Die durch das CPT vorzuschlagenden Verbesserungsmöglichkeiten haben aufgrund seines präventiven Handlungsauftrags vor allem langfristige Massnahmen zu sein. ${ }^{243}$ Sofortige oder kurzfristige Massnahmen hält das CPT für inadäquat. ${ }^{244}$ Viertens: Der anzuwendende bzw. der zu fordernde Massstab hat zur Erfüllung der Funktion des CPT ein höherer zu sein als derjenige, der von der Europäischen Kommission und vom EGMR bei der Prüfung einer Verletzung von Art. 3 EMRK angewendet werde. ${ }^{245}$

Zusammenfassend bedeutet dies, dass dem CPT hauptsächlich eine Präventionsfunktion zukommt. Gemäss erläuterndem Bericht soll es insbesondere nicht Aufgabe des CPT sein, die Konformität mit Art. 3 EMRK zu prüfen bzw. diese gar sicherzustellen. Ein Bestreben nach einem einheitlichen Massstab mit den Konventionsorganen der EMRK ist dem erläuternden Bericht auch nicht zu entnehmen. Unterschiedliche Standards lassen sich gemäss CPT vielmehr durch die unterschiedlichen Aufgaben und Rollen der verschiedenen Regelwerke und Gremien legitimieren. ${ }^{246}$

3. Von den Europäischen Mindestgrundsätzen für die Behandlung der Gefangenen (ESMR) zu den Europäischen Strafvollzugsgrundsätzen (EPR)

Den Empfehlungen der European Standard Minimum Rules for the Treatment of Prisoners des Ministerkomitees von 1973 (Europäische Mindest-

239 CPT - 1st General Report [CPT/Inf (91) 3], Rn. 48.

240 CPT - 1st General Report [CPT/Inf (91) 3], Rn. 49.

241 CPT - 1st General Report [CPT/Inf (91) 3], Rn. 49.

242 CPT - 1st General Report [CPT/Inf (91) 3], Rn. 49.

243 CPT - 1st General Report [CPT/Inf (91) 3], Rn. 50: In Frage kämen etwa Bildungsstrategien.

244 CPT - 1st General Report [CPT/Inf (91) 3], Rn. 50.

245 CPT - 1st General Report [CPT/Inf (91) 3], Rn. 51.

246 Vgl. dazu auch Morgan in: van Zyl Smit/Dünkel, S. 717 (719 f., 729), der dabei aber auch auf potentielle Probleme der unterschiedlichen Verwendung der Begriffe hinweist. Zum Verhältnis des Standards der EMRK und des CPT vgl. auch Murdoch S. $46 \mathrm{ff}$; Morgenstern in: Dünkel/Drenkhahn/Morgenstern, S. $47 \mathrm{f}$. 
grundsätze für die Behandlung Gefangener, ESMR) ${ }^{247}$, welche unter der Aufsicht des CDPC ausgearbeitet wurden, kommt auf Europaratsebene eine herausragende Bedeutung zu. Sie sind Ausgangspunkt weiterer themenspezifischer Empfehlungen. Inhaltlich orientieren sich die ESMR stark an den Mindestgrundsätzen für die Behandlung der Gefangenen der Vereinten Nationen. ${ }^{248}$

Aufgrund der engen Anlehnung der ESMR an die UN-Mindestgrundsätze für die Behandlung Gefangener stellt sich die Frage, welcher Mehrwert den ESMR zukommt. Die Europäischen Mindestgrundsätze sind eine auf europäische Verhältnisse angepasste Empfehlung (vgl. Präambel der ESMR), mit welcher sich europäische Staaten unter Umständen besser identifizieren können als mit der globalen Empfehlung der Vereinten $\mathrm{Na}$ tionen. ${ }^{249}$ Gleichwohl bleibt einzuwenden, dass auch Europa weder im Jahr 1955 noch heute ein homogener Raum ist - weder was den allgemeinen Lebensstandard betrifft, noch was die materiellen Haftbedingungen in Strafvollzugsanstalten angeht. ${ }^{250}$ Grundsätzlich ähnlichere Wertvorstellungen und Bedingungen lassen innerhalb Europas dennoch eine weitergehende Konsensfähigkeit vermuten als auf globaler Ebene. Damit sind die ESMR grundsätzlich ein geeignetes Instrument, auf europäischer Ebene auf Empfehlungsbasis eine inhaltliche Weiterentwicklung des Standards zu erzielen. Eine vergleichende Analyse kam jedoch zum Schluss, dass die ESMR zumindest keine revolutionären Neuerungen gegenüber den Mindestgrundsätzen der Vereinten Nationen beinhalten. Der Achtung der Menschenwürde der Strafgefangenen komme in den ESMR gegenüber den UN-Mindestgrundsätzen allerdings eine akzentuierte Bedeutung zu. ${ }^{251}$

Selbst wenn die ESMR gegenüber den UN-Mindestgrundsätzen keine revolutionären Neuerungen enthält, führt sie jedenfalls zu einer Rezeption der UN-Mindestgrundsätze, welche geeignet ist, zu deren Konsolidierung beizutragen. ${ }^{252}$ In der Präambel der ESMR wird allerdings von einem

247 Resolution (73) 5, Standard Minimum Rules for the Treatment of Prisoners.

248 Im Einzelnen vgl. Teil 3 Kap. 2 III. 1. u. 3.; Teil 3 Kap. 3 III. 2. u. 4.

249 Vgl. auch van ZyL Smit Eur J Crim Policy Res 2006, 107 (110).

250 Vgl. dazu auch div. länderspezifische Monitoringberichte etwa des CPT.

251 Reynaud S. 34: „[...] le texte européenne ne contient pas de modifications révolutionnaires par rapport au texte de l'ONU, on peut toutefois relever quelque notions nouvelles ou de précisions supplémentaires qui y ont été introduites afin de mieux assurer le respect de la dignité humaine des personnes incarcérées“; vgl. auch van ZyL Smit Eur J Crim Policy Res 2006, 107 (110).

252 In diesem Sinne vgl. auch van ZyL SMIt/SNacken S. 21; zum Effekt der Konsolidierung einer Soft Law-Bestimmung für deren „Grad der Normativität“ vgl. 
differenzierteren Verhältnis zu den UN-Mindestgrundsätzen ausgegangen (vgl. Präambel der ESMR). Die Promotion der UN-Mindestgrundsätze ist dabei nur eines der angestrebten Zwecke. ${ }^{253}$ Im Selbstverständnis der ESMR stehen die Modernisierung ${ }^{254}$ und Fortentwicklung der UN-Mindestgrundsätze im europäischen Kontext im Vordergrund (vgl. Präambel). Der in diesem Sinne weiterentwickelte Standard ist jener, der verbreitet werden soll. Die ESMR sehen sodann einen Reporting-Mechanismus vor: Die Mitgliedstaaten sind eingeladen, alle fünf Jahre den Generalsekretär über Massnahmen zu informieren, welche sie im Sinne der ESMR ergriffen haben (vgl. schon Präambel II.).

Die Frage, ob die Zielsetzung der ESMR erreicht wurde, eine effektive Fortentwicklung des UN-Standards zu bewirken, lässt sich nicht mit einem Vergleich der beiden Ausgangsdokumente der UN-Mindestgrundsätze von 1955 und der ESMR von 1973 beantworten, weil beide Regelwerke unabhängig reformierbar sind. Auf Europaratsebene ist aufgrund der geringeren Anzahl der beteiligten Vertragsstaaten und aufgrund des ähnlicheren Werteverständnisses eine höhere Konsensfähigkeit zu erwarten als auf UN-Ebene. ${ }^{255}$ Es erstaunt deshalb nicht, dass bereits im Jahr 1987 vom Ministerkomitee ein neu ausformulierter Entwurf der ESMR, die

etwa Richter Pinto de Albuquerque partly diss. op., EGMR (GK) - Muršić/CRO, Urt. v. 20.10.2016, 7334/13, Rn. 28: „[...] the repetition of soft law by the same or different public authorities hardens its normative claim. While the mere accumulation of non-law instruments cannot per se create international law, the emergence of an opinio juris, if accompanied by other hardening factors, can transmute such instruments into international legal norms, by sliding them up the scale of international normativity and thus integrating them into the Council of Europe's binding normative system."

253 Ansonsten hätte sich meines Erachtens ernstlich die Frage gestellt, ob die Propagierung einer bereits bestehenden Empfehlung als alleinige Zielsetzung einer neuen Empfehlung eine erneute Legiferierung legitimiert hätte. Dies insbesondere, da der Geltungsbereich der ESMR ein kleinerer ist als bei den UN-Mindestgrundsätzen und sich auch hinsichtlich des Verpflichtungsgrades nichts Wesentliches ändert, da sowohl die UN-Mindestgrundsätze als auch die ESMR Empfehlungscharakter haben. Aufgrund des Empfehlungscharakters hätte aber auch argumentiert werden können, dass die Anforderungen an die Legitimation des entsprechenden Vorhabens geringer sein dürften. Wenn es lediglich um die Propagierung der UN-Mindestgrundsätze gegangen wäre, dann hätte es aber diverse andere Möglichkeiten gegeben, um diese Zielsetzung zu erreichen. $\mathrm{Zu}$ denken wäre etwa an die Verbesserung der Zugänglichkeit der UN-Regelwerke sowohl für die Inhaftierten als auch für Behörden.

254 Zwischen der Verabschiedung der UN-Mindestgrundsätzen und den ESMR liegen immerhin 18 Jahre.

255 Vgl. auch Neale in: Muncie/Sparks, S. 203 (207). 
Europäischen Strafvollzugsgrundsätze von 1987 bzw. die European Prison Rules von 1987 (EPR) 256 , angenommen wurde. Die Europäischen Strafvollzugsgrundsätze von 1987 führten zu einer erneuten Europäisierung des Regelwerks. Auch die Bedeutung der Gewährleistung der Menschenwürde der Strafgefangenen rückte noch mehr in den Vordergrund. ${ }^{257}$ Knapp 20 Jahre später wurden auch diese Europäischen Strafvollzugsgrundsätze von 1987 vollständig überarbeitet und aktualisiert (Europäische Strafvollzugsgrundsätze von 2006 bzw. die European Prison Rules from 2006 $\left.{ }^{258}\right) .{ }^{259}$ Dieser erneute Reformprozess brachte grundsätzlich detailreichere Bestimmungen hervor. ${ }^{260}$

Aus der Präambel der Europäischen Strafvollzugsgrundsätze von 2006 geht hervor, dass sowohl die EMRK und die Rechtsprechung des EGMR als auch die Arbeit des CPT im Reformprozess der Europäischen Strafvollzugsgrundsätze berücksichtigt wurden. ${ }^{261}$ Auch wurden gemeinsame Grundsätze des Strafvollzugs der einzelnen Mitgliedstaaten beobachtet. Dies im Bewusstsein, dass gemeinsame Grundsätze geeignet sind, die internationale Kooperation auszubauen. Daneben flossen frühere themenspezifische Europaratsempfehlungen in den Reformprozess der Europäischen Strafvollzugsgrundsätze ein. Obgleich auch hier die Wechselwirkung zwischen den einzelnen Regelwerken deutlich wird, geht auch die EPR nicht so weit, einen einheitlichen Standard der einzelnen Regelwerke zu fordern.

Der Natur einer Empfehlung entsprechend halten die Strafvollzugsgrundsätze hinsichtlich ihres Geltungsanspruchs fest, dass sich die Mit-

256 Recommendation R (87) 3 on the European Prison Rules 1987.

257 Van ZyL Smit/Snacken S. 23; zu den Neuerungen im Einzelnen vgl. auch NeaLE in: Muncie/Sparks, S. 203 (208 ff.).

258 Recommendation Rec (2006) 2 on the European Prison Rules 2006.

259 Dünkel/Morgenstern/Zolondek Neue Kriminalpolitik 2006, 86, wobei die Autoren das Bedürfnis nach einer Aktualisierung der Strafvollzugsgrundsätze von $1987 \mathrm{im}$ Wesentlichen auf vier Faktoren zurückführen: die Verabschiedung des Übereinkommens zur Verhütung von Folter und unmenschlicher oder erniedrigenden Behandlung und die Arbeit des CPT, die Entwicklung der Rechtsprechung des EGMR, die europäische Erweiterung nach den gesellschaftlichen Umwälzungen in Mittel- und Osteuropa sowie die Aktivität des Europäischen Parlaments und der Parlamentarischen Versammlung, welche die Zustände in Gefängnissen zahlreicher Länder beklagt hätten.

$260 \mathrm{Zu}$ den Faktoren, welche eine weitergehende Standardsetzung erleichterten, im Einzelnen: van ZyL Smit Eur J Crim Policy Res 2006, 107 (111 ff.).

261 Zum Verhältnis der Arbeit des CPT und der Europäischen Strafvollzugsgrundsätze vgl. Murdoch S. 35 ff. 
gliedstaaten in ihrer Gesetzgebung, ihren Richtlinien und ihrer Praxis von den in der Empfehlung zu Grunde gelegten Regelungen leiten zu lassen haben. ${ }^{262}$ Der Geltungsanspruch der Europäischen Strafvollzugsgrundsätze geht folglich nicht so weit, eine jederzeitige akribische Einhaltung des festgehaltenen Standards zu fordern. ${ }^{263}$ Internationale und nationale Gerichte können sich aber selbstverständlich auf die Europäischen Strafvollzugsgrundsätze berufen, auch um auf andere nationale Behörden und die Regierung Druck auszuüben, die derzeitige Strafvollzugspraxis nach Massgabe der europäischen Standards zu verbessern. ${ }^{264}$ Sicherzustellen ist durch die Mitgliedstaaten jedenfalls, dass die Europäischen Strafvollzugsgrundsätze und deren begleitender Kommentar übersetzt und soweit als nur möglich verbreitet werden; dies insbesondere bei Inhaftierten, Strafvollzugsbeamten und gerichtlichen Instanzen. Der begleitende Kommentar zu den Europäischen Strafvollzugsgrundsätzen hält fest, dass die Bedeutung der EPR seit 1987 gestiegen sei; sowohl EGMR als auch CPT würden die EPR nun regelmässig heranziehen, was in Zukunft in noch ausgeprägterem Masse erfolgen solle. ${ }^{265}$ Die Nichteinhaltung der Strafvollzugsgrundsätze bedeutet aber nicht zwingend eine Verletzung von Art. 3 EMRK.

\section{Weitere Empfehlungen des Europarates}

Die inhaltlich umfassenden ESMR bzw. EPR werden auf Europaratsebene durch eine Vielzahl weiterer spezifischer Empfehlungen ergänzt. Auch diese wurden unter der Aufsicht des CDPC ausgearbeitet. Empfehlungen, welche vor der umfassenden Revision der Europäischen Strafvollzugsgrundsätze im Jahr 2006 erlassen wurden, wurden bisweilen in diesem Reformprojekt wieder aufgegriffen und fanden so teilweise Eingang auch in dieses allgemeine Standardregelwerk. Das CDPC liefert zu den Empfehlungen meist auch gleich eigene Kommentare (Explanatory Reports /

262 Zum Geltungsanspruch und der Wirkungsweise der Strafvollzugsgrundsätze von 1987 im Einzelnen auch Neale in: Muncie/Sparks, S. 203 (210 ff.).

263 Zum Geltungsanspruch der Europäischen Strafvollzugsgrundsätze vgl. auch Murdoch S. $34 \mathrm{f}$.

264 Koeppel S. 220.

265 Commentary to Recommendation Rec (2006) 2 on the European Prison Rules, Introduction. 
Explanatory Memorandum), ${ }^{266}$ welche den zuständigen Behörden als Auslegungsrichtlinien dienen.

Regelungssubjekt der spezifischen Empfehlungen ist jeweils eine konkrete Personengruppe im Strafvollzug; Regelungsobjekt ein konkretes Gebiet des Strafvollzugs. So finden sich etwa Empfehlungen zum Umgang mit gefährlichen Strafgefangenen, ${ }^{267}$ zu ausländischen Strafgefangenen ${ }^{268}$ oder zu jugendlichen Strafgefangenen ${ }^{269}$ sowie Empfehlungen zur Arbeit oder zur Ausbildung während des Strafvollzugs, ${ }^{270}$ zum Transfer von verurteilten Strafgefangenen, ${ }^{271}$ zum Umgang mit ansteckenden Krankheiten im Strafvollzug, 272 Empfehlungen für Personen, welche mit der Implementierung von Sanktionen und Massnahmen betraut sind, ${ }^{273}$ oder etwa Empfehlungen zu ethischen und organisatorischen Fragen der Gesundheitsversorgung in Strafanstalten, ${ }^{274}$ Empfehlungen zum Problem der Überbelegung, ${ }^{275}$ zur lebenslangen Freiheitsstrafe bzw. zum Langzeitstraf-

266 Vgl. etwa Recommendation R (99) 22 Concerning Prison Overcrowding and Prison Population Inflation and Explanatory Memorandum; Recommendation R (98) 7 Concerning the Ethical and Organisational Aspect of Health Care in Prison and Explanatory Memorandum etc.

267 Recommendation R (82) 17 Concerning Custody and Treatment of Dangerous Prisoners; Recommendation CM/Rec (2014) 3 Concerning Dangerous Offenders.

268 Recommendation R (84) 12 Concerning Foreign Prisoners; Recommendation $\mathrm{CM} / \operatorname{Rec}$ (2012) 12 Concerning Foreign Prisoners.

269 Recommendation CM/Rec (2008) 11 on the European Rules for Juvenile Offenders Subject to Sanctions or Measures.

270 Recommendation R (89) 12 on Education in Prison.

271 Recommendation R (92) 18 Concerning the Practical Application of the Convention on the Transfer of Sentenced Persons: Dabei geht es insbesondere darum, das Verfahren zu vereinfachen, damit die Strafgefangenen in ihrem Heimatstaat ihre Strafe absitzen können.

272 Recommendation R (93) 6 Concerning Prison and Criminological Aspects of the Control of Transmissible Diseases Including AIDS and Related Health Problems in Prisons.

273 Recommendation R (97) 12 on Staff Concerned with the Implementation of Sanctions and Measures.

274 Recommendation R (98) 7 Concerning the Ethical and Organisational Aspect of Health Care in Prison.

275 Recommendation R (99) 22 Concerning Prison Overcrowding and Prison Population Inflation. 
vollzug, ${ }^{276}$ zur Untersuchungshaft, ${ }^{277}$ zu Fragen der Bewährungshilfe ${ }^{278}$ und zu einem ethischen Code für das Aufsichtspersonal. ${ }^{279}$

\section{Nationale Ebene / Schweiz}

In der Schweiz finden sich strafvollzugsrelevante Normen auf der Ebene des Bundes, der interkantonalen und der kantonalen Ebene. Was die Normhierarchie betrifft, sind Modalitäten zum Strafvollzug auf Verfassungs-, Konkordats-, Gesetzes- und Verordnungsebene normiert.

Völkerrechtliche Verträge sind dabei zu beachten ${ }^{280}$ : Auch wenn die völkerrechtliche Verantwortlichkeit zur Umsetzung der völkerrechtlichen Verträge beim Bund liegt, bleiben die Kantone innerstaatlich dennoch in der Pflicht (vgl. auch Art. 5 Abs. 4 BV). ${ }^{281}$ Das föderalistische System der Schweiz, welches Autonomie und ein daraus abgeleitetes Recht auf Selbstgesetzgebung und Selbstverwaltung der Kantone vorsieht, kann allerdings dazu führen, dass völkerrechtliche Vorgaben von den Kantonen auf je

276 Recommendation Rec (2003) 23 on the Management by Prison Administrations of Life Sentence and Other Long-Term Prisoners.

277 Recommendation Rec (2006) 13 on the Use of Remand in Custody, the Conditions in which it Takes Place and the Provision of Safeguards against Abuse.

278 Recommendation CM/Rec (2010) 1 on the Council of Europe Probation Rules.

279 Recommendation CM/Rec (2012) 5 on the European Code of Ethics for Prison Staff.

280 Zum Beitritt der Schweiz zur EMRK im Besonderen vgl. KLEY/SIGRIST in: Jaag/ Kaufmann, S. 17 (35 ff.); zur Rezeption der EMRK und zum Verhältnis von nationalem und internationalem Recht in der Schweiz im Einzelnen etwa THuRNHERR in: Keller/Stone Sweet, S. 311 (316 ff., 329 ff.). Art. 190 BV äussert sich nicht zum Verhältnis von Bundesgesetz und Völkerrecht. Das Bundesgericht hielt zunächst fest, dass Völkerrecht zwar grundsätzlich Gesetzesrecht vorgeht, dieser Grundsatz gelte allerdings dann nicht, wenn die Bundesversammlung bewusst ein völkerrechtswidriges Gesetz verabschiedet hat, in diesem Fall sei das spätere völkerrechtswidrige Gesetz (lex posterior) massgebend („Schubert-Praxis“: BGE 99 Ib 39 ff.). Diese Rechtsprechungspraxis relativierte das Bundesgericht später, indem es festhielt, dass internationale Menschenrechtsgarantien dem Landesrecht im Konfliktfall stets vorgingen („PKK-Praxis“: BGE 125 II 417).

281 Vgl. ausf. Kiener in: Fink/Arnold/Genillod-Villard/Oberholzer, S. 3 ff.: Es sei denn auch am Bund, soweit im völkerrechtlichen Vertrag vorgesehen, Rechenschaftsberichte zuhanden von dessen Implementierungsorganen zu verfassen. Auch allfällige Rügen einer mangelhaften Umsetzung würden an den Bund gerichtet, selbst wenn die Umsetzungsverantwortung innerstaatlich bei den Kantonen liegen würde. 
unterschiedliche Art und Weise umgesetzt werden. ${ }^{282}$ Grenze der kantonalen Vielfalt sind nicht nur menschenrechtliche völkerrechtliche Vorgaben, sondern auch massgebendes Bundesrecht (vgl. Art. 49 BV, Bundesrecht geht entgegenstehendem kantonalem Recht vor).

\section{Bundesebene}

\subsection{Bundesverfassung}

Auf Verfassungsstufe sind zunächst die Grundrechte zu beachten. Der Strafvollzug tangiert nämlich nicht nur die in der EMRK verankerten Menschenrechte, sondern eben auch diverse in der Bundesverfassung verankerte Grundrechte: ${ }^{283}$ beispielsweise Art. 7 BV (Menschenwürde), Art. 8 Abs. 1 BV (Rechtsgleichheit), Art. 8 Abs. 2 BV (Diskriminierungsverbot), Art. 9 BV (Willkürverbot), Art. 10 Abs. 1 BV (Recht auf Leben), Art. 10 Abs. 2 BV (Recht auf persönliche Freiheit), Art. 10 Abs. 3 BV (Folterverbot), Art. 12 BV (Recht auf Hilfe in Notlagen), Art. 13 BV (Schutz der Privatsphäre), Art. 14 BV (Recht auf Ehe und Familie), Art. 15 BV (Glaubensund Gewissensfreiheit) und Art. 16 BV (Meinungs- und Informationsfreiheiten). Auch Art. 31 BV und Art. 32 BV halten Rechte einer Person im Freiheitsentzug bzw. im Strafverfahren fest, betreffen im Unterschied zu den vorgenannten Grundrechten aber nicht die Modalitäten materieller Haftbedingungen. Art. 35 BV sieht schliesslich vor, dass die Grundrechte umfassend zu verwirklichen sind.

Die Gesetzgebungskompetenz auf dem Gebiet des Straf-und Strafprozessrechts liegt gemäss Art. 123 Abs. 1 BV beim Bund. Art. 123 Abs. 1 BV ist eine Bundeskompetenz mit nachträglich derogatorischer Kraft; die Kanto-

282 Zum Spannungsverhältnis der völkerrechtlichen Verantwortlichkeit des Bundes und der innerstaatlichen Kompetenzordnung ausf.: KIENER in: Fink/Arnold/ Genillod-Villard/Oberholzer, S. 3 ff.; vgl. auch Thurnherr in: Keller/Stone Sweet, S. 311 (335).

283 Zum Einfluss der EMRK auf das Verfassungsrecht der Schweiz im Allgemeinen Kiener in: Jaag/Kaufmann, S. 53 ff.; vgl. auch Thurnherr in: Keller/Stone Sweet, S. 311 (364 ff.); Keller/Marti Justice - Justiz - Giustizia 2015/1, 1 (6): Innerstaatliche Richter hätten auch dann die Rechtsprechung des EGMR zu berücksichtigen, wenn von den Parteien nur die entsprechenden Bestimmungen der BV angeführt würden. Dies, da es für das Erfordernis der Ausschöpfung des innerstaatlichen Instanzenzugs genüge, dass der Beschwerdeführer die Rüge einer EMRK-Verletzung der Sache nach vorgebracht habe. 
ne bleiben solange zuständig, bis der Bund legiferiert hat (konkurrierende Kompetenz). ${ }^{284}$ Für den Straf- und Massnabmenvollzug sind gemäss Art. 123 Abs. 2 BV hingegen die Kantone zuständig, soweit das Gesetz nichts anderes vorsieht. Gemäss Art. 123 Abs. 3 Satz 1 BV ist der Bund allerdings befugt, eigene Vorschriften zum Straf- und Massnahmenvollzug zu erlassen. Art. 123 Abs. 3 Satz 1 BV wird in dem Sinn ausgelegt, dass der Bund einen „Systemwechsel vom kantonalen zu einem einheitlichen Bundesvollzugsrecht vornehmen kann" ${ }^{285}$ Bislang hat der Bund diese Möglichkeit nicht wahrgenommen und (noch) kein besonderes Strafvollzugsgesetz geschaffen. ${ }^{286}$ Bundesrechtliche Rahmenbestimmungen finden sich allerdings im Strafgesetzbuch.

Der Bund kann den Kantonen weiter Beiträge für die Errichtung von Anstalten (Art. 123 Abs. 3 lit. a BV) resp. für Verbesserungen im Straf- und Massnahmenvollzug (Art. 123 Abs. 3 lit. b BV) gewähren. ${ }^{287}$ Diese Bundesförderung ist durch die finanzielle Entlastung ein geeignetes Mittel, Anreize für die Kantone zur Umsetzung von menschenrechtlichen Vorgaben zu schaffen, ohne dass dies zu Kompetenzeinbussen zulasten der Kantone führen würde. ${ }^{288}$ Der Bund kann an die Gewährung dieser Beiträge allerdings Bedingungen knüpfen, womit er zumindest indirekten Einfluss auf die Modalitäten des Strafvollzugs nehmen kann.289

Systematisch unter dem Abschnitt „Zusammenwirken von Bund und Kantonen" sieht Art. 48 BV vor, dass Kantone im Rahmen ihrer Zuständigkeiten miteinander Verträge schliessen können. Namentlich können sie Aufgaben von regionalem Interesse gemeinsam wahrnehmen. Auf An-

284 Der Bund hat die Kompetenz mit der Verabschiedung des StGB und der StPO wahrgenommen, im Bereich des materiellen Strafrechts verbleiben den Kantonen in engem Umfang weiterhin Gesetzgebungskompetenzen (vgl. etwa Art. 335 Abs. 1 StGB und Art. 3 BV): BSK BV/GöKsu Art. 123 BV Rn. 3.

285 Kritisch: BSK BV/GöKsu Art. 123 BV Rn. 22: Dies vertrage sich nur schlecht mit der verfassungsmässigen Kompetenzausscheidung, die Kantone stünden gem. Art. 3 BV im Genuss der Kompetenzvermutung. GöKsu fordert daher, Art. 123 Abs. 3 BV so auszulegen, dass dieser dem Bund lediglich punktuelle Eingriffe in die kantonale Gesetzgebungshoheit erlaube, wenn eine bundesrechtliche Ordnung sich gegenüber einer föderalistischen Ordnung aufdränge.

286 Vgl. auch Kiener in: Fink/Arnold/Genillod-Villard/Oberholzer, S. 3 (7 f.).

287 Nicht umfasst von dieser Norm sind Leistungen für die Verbesserung der menschenrechtlichen Situation in Polizeigefängnissen: vgl. KIENER in: Fink/Arnold/ Genillod-Villard/Oberholzer, S. 3 (16).

288 Vgl. KIENER in: Fink/Arnold/Genillod-Villard/Oberholzer, S. 3 (16).

289 BSK BV/GöKsu Art. 123 BV Rn. 23 f. mit Verweis auf das Bundesgesetz über die Leistungen des Bundes für den Straf- und Massnahmenvollzug (SR 341); vgl. auch KIENER in: Fink/Arnold/Genillod-Villard/Oberholzer, S. 3 (16). 
trag interessierter Kantone kann der Bund die Kantone zur Zusammenarbeit und insb. auch zur Harmonisierung des Vollzugsrechts verpflichten (Art. 48a Abs. 1 lit. a BV). ${ }^{290}$

Wenngleich dem Bund auch im Bereich des Strafvollzugs gewisse Gesetzgebungskompetenzen zugesprochen wurden und er diese teilweise auch wahrgenommen hat, bleiben die Kantone betreffend Modalitäten des Strafvollzugsrechts wichtiger Gesetzgeber.

\subsection{Bundesgesetze}

Auf Gesetzesstufe finden sich auf Bundesebene sowohl im Strafgesetzbuch (StGB) als auch in der Strafprozessordnung ( $\mathrm{StPO}$ ) strafvollzugsspezifische Vorschriften. Gemäss Art. 372 Abs. 1 StGB sind die Kantone zum Strafvollzug verpflichtet, wenn dieser von ihren Strafgerichten gestützt auf das StGB ausgefällt wurde. Die Kosten des Straf- und Massnahmenvollzugs tragen die Kantone, wobei die Verurteilten in angemessener Weise an den Kosten des Vollzugs beteiligt werden können (Art. 380 StGB). Gegen Ersatz der Kosten sind die Kantone weiter verpflichtet, Urteile der Bundesstrafbehörden zu vollziehen.

Art. 372 Abs. 3 StGB verpflichtet die Kantone, einen einheitlichen Vollzug strafrechtlicher Sanktionen zu gewährleisten. Über die gemeinsame Errichtung und den gemeinsamen Betrieb von Anstalten und Einrichtungen können die Kantone Vereinbarungen treffen oder sich das Mitbenutzungsrecht an Anstalten und Einrichtungen anderer Kantone sichern (Art. 378 Abs. 1 StGB).

Grundsätze betreffend den Vollzug einer Freiheitsstrafe finden sich auf Bundesebene insbesondere in den Art. $74 \mathrm{ff}$. StGB. In grundlegender Weise hält Art. 74 StGB fest, dass die Menschenwürde des Gefangenen zu achten ist und dass seine Rechte nur so weit zu beschränken sind, als der Freiheitsentzug und das Zusammenleben in der Vollzugseinrichtung es erfordern. Als allgemeines Vollzugsziel postuliert Art. 75 Abs. 1 StGB sodann, dass das soziale Verhalten des Gefangenen - insbesondere die Fähigkeit, straffrei zu leben (Resozialisierung) - zu fördern ist. Der Strafvollzug hat dabei den allgemeinen Lebensverhältnissen soweit als möglich zu entsprechen (Normalisierungsprinzip), die Betreuung des Gefangenen zu gewährleisten und schädlichen Folgen des Freiheitsentzugs entgegenzuwirken (Betreuungsprinzip und Entgegenwirkungsprinzip / Prinzip des nil

290 Vgl. auch BSK BV/Brägger Art. 74 BV Rn. 2. 
nocere) sowie dem Schutz der Allgemeinheit, des Vollzugspersonals und der Mitgefangenen Rechnung zu tragen (Sicherungsprinzip).

Zusammenfassend normiert der Bund Grundsätze und Leitlinien für den Vollzug, ohne in die konkrete Organisation des Strafvollzugs einzugreifen. Soweit weitere Bestimmungen des StGB oder andere bundesrechtliche Erlasse für die themenspezifischen Fragen relevant werden, werden diese an dortiger Stelle aufgegriffen.

\subsection{Nationale Kommission zur Verhütung von Folter (NKVF)}

Zur Überwachung der Einhaltung der Verpflichtungen der Schweiz aus dem Übereinkommen der Vereinten Nationen vom 10. Dezember 1984 gegen Folter und andere grausame, unmenschliche oder erniedrigende Behandlung oder Strafe wurde im Jahr 2010 die Nationale Kommission zur Verhütung von Folter (NKVF) geschaffen. ${ }^{291}$ Die NKVF ist eine von Bund und Kantonen unabhängige nationale Kommission. ${ }^{292}$ Der Kommission gehören 12 Mitglieder an. Sie setzt sich aus Fachleuten zusammen, die über die erforderlichen beruflichen und persönlichen Kompetenzen und Kenntnisse verfügen, insbesondere im medizinischen, psychiatrischen, juristischen oder interkulturellen Bereich oder im Bereich des Freiheitsentzugs und des Besuchs von Orten des Freiheitsentzugs (Art. 5 BG KVF ${ }^{293}$ ).

Gemäss Art. 2 lit. a BG KVF überprüft die Kommission regelmässig die Situation von Personen, denen die Freiheit entzogen ist, und besucht regelmässig alle Orte, an denen sich diese Personen befinden. ${ }^{294}$ Mit den Zielen, die Behandlung und die Situation der Inhaftierten zu verbessern sowie Folter und andere grausame, unmenschliche oder erniedrigende Behandlung oder Strafe zu verhüten, gibt sie Empfehlungen an die zuständigen Behörden ab (Art. 2 lit. b Ziff. 1 und Ziff. 2 BG KVF). Weiter unter-

291 Eingehend zur NKVF HungerbüHLER S. 69 ff.; zum Verhältnis des SPT und den nationalen Präventionsmechanismen vgl. Nowak/Ammer/Birk/Monina/KrISPER Art. 3 OPCAT Rn. 3: Nationale Präventionsmechanismen seien grundsätzlich besser in der Lage, regelmässige Besuche in Haftanstalten und Follow-upBesuche durchzuführen und mit den Behörden in einen Dialog zu treten. In diesem Sinne wurden sie auch als "the front line of torture prevention" bezeichnet.

292 OmLIN in: Brägger, S. 319.

293 Bundesgesetz über die Kommission zur Verhütung von Folter vom 20. März 2009, SR 150.1.

294 Zur Besuchstätigkeit der NKVF im Einzelnen vgl. etwa HungerbüHLER S. 274, $277 \mathrm{ff}$. 
breitet sie Vorschläge und Bemerkungen zu geltenden Erlassen oder zu Erlassentwürfen (Art. 2 lit.c BG KVF). Die Kommission verfasst sodann einen jährlichen Bericht über ihre Tätigkeit, welcher der Öffentlichkeit zugänglich ist (Art. 2 lit.d BG KVF). Die Kommission unterhält schliesslich Kontakte mit dem Unterausschuss für Prävention und dem CPT. Sie übermittelt diesen beiden Gremien Informationen und stimmt ihre Tätigkeit mit ihnen ab (Art. 2 lit. e BG KVF).

\section{Interkantonale Ebene}

Die Kantone machten im Bereich des Strafvollzugs von der Möglichkeit Gebrauch, Verträge miteinander zu schliessen (vgl. insb. Art. 123 BV i.V.m. Art. 48 BV und Art. 372 und Art. 378 StGB). Im Sinne regionaler Vollzugsgemeinschaften ${ }^{295}$ haben sie drei regionale Konkordate geschlossen: das Konkordat der Nordwest- und Innerschweiz (AG, BS, BL, BE, LU, OW, NW, SZ, SO, UR, ZG), das Ostschweizer Konkordat (AI, AR, GL, GR, SH, SG, TG, ZH) und das Konkordat der lateinischen Schweiz (FR, GE, JU, NE, VD, VS, TI). Sämtliche Kantone der Schweiz sind damit Teil eines der drei Strafvollzugskonkordate. ${ }^{296}$

Gründungsvertrag und damit Ausgangsdokument ist bei allen drei Strafvollzugskonkordaten die jeweilige Konkordatsvereinbarung. Diese enthält Bestimmungen zu den verfolgten Zielen, den Organen und deren Kompetenzen. Die Harmonisierungsbemühungen werden durch die Verabschiedung von interkantonalen Vollzugsbestimmungen wahrgenommen. ${ }^{297}$ Dies in Form von Reglementen, Richtlinien, Beschlüssen und Empfehlungen. Art. 48 Abs. 4 BV sieht vor, dass Kantone interkantonale Organe zum Erlass rechtssetzender Bestimmungen ermächtigen können, die einen interkantonalen Vertrag umsetzen. Dies unter der Voraussetzung, dass der Vertrag nach dem gleichen Verfahren, welches für die Gesetzgebung gilt, genehmigt worden ist und die inhaltlichen Grundzüge der Bestimmungen selbst festlegt (Art. 48 Abs. 4 lit. a und lit. b BV). Das Strafvollzugskonkordat der Nordwest- und Innerschweiz sowie das Strafvollzugskonkordat

295 SCHÄrer in: Brägger, S. 251 (252).

296 Historisch sind die Strafvollzugskonkordate darauf zurückzuführen, dass in kleinen Kantonen für eigene Vollzugsanstalten kein Bedarf bestand und auch grosse Kantone nicht in der Lage waren, alle gemäss StGB erforderlichen Anstaltstypen zu betreiben: Baechtold S. 7; vgl. auch Baechtold in: van Zyl Smit/ Dünkel, S. 653 (656f.).

297 Vgl. auch Schärer in: Brägger, S. 251 (252). 
der Ostschweiz enthalten zwar eine Kompetenznorm zu Gunsten der Konkordatskonferenz bzw. zu Gunsten der Strafvollzugskommission, welche sie zum Erlass von strafvollzugsrechtlichen Bestimmungen ermächtigt. $\mathrm{Zu}$ einer Übertragung von Rechtssetzungsbefugnissen kam es aufgrund der fehlenden materiellen Ausgestaltung der Konkordatsvereinbarung hingegen nicht. ${ }^{298}$ Ihre Richtlinien und Reglemente sind damit Soft Law und somit rechtlich nicht verbindlich. ${ }^{299}$ Eine Verbindlicherklärung der Bestimmungen bleibt möglich, setzt allerdings die Zustimmung aller Konkordatskantone voraus (Art. 2 lit.c OSK bzw. Art. 3 lit.f NWI ${ }^{300}$ ). Anders im Strafvollzugskonkordat der lateinischen Schweiz; ihre Konkordatskonferenz ist ermächtigt, verbindliches Recht zu verabschieden. ${ }^{301}$ Die Beschlüsse der Konkordatskonferenz richten sich an bestimmte Personen und regeln deren Rechtsstellung (Rechte und Pflichten), womit sie verbindlich sind. Nicht verbindlich sind aber auch im Geltungsbereich des Strafvollzugskonkordats der lateinischen Schweiz ihre Richtlinien und Empfehlungen. Diese dienen als Auslegungsdirektive und geben Hinweise dafür, wie der Ermessensspielraum sowohl bundesrechtlicher als auch kantonaler Bestimmungen genutzt werden sollte.

Regelungsgegenstand sind konkrete Einzelfragen des Strafvollzugs wie beispielsweise der Vollzugsplan, das Disziplinarrecht, das Arbeitsentgelt oder der Urlaub während des Strafvollzugs. Sieht das anwendbare Strafvollzugskonkordat eine bestimmte Regelung vor, tritt diese an die Stelle der kantonalen Bestimmung. ${ }^{302}$ Halten kantonale Erlasse nur die Grundsätze einer Materie fest und verweisen im Einzelnen auf die anwendbaren Richtlinien oder das Reglement, ergänzen sie die kantonalen Erlasse. ${ }^{303}$

Eine gesamtschweizerische Harmonisierung findet durch die Konkordate damit nicht statt. Auf institutioneller Ebene finden sich allerdings verschiedene Gremien, welche auf unterschiedliche Weise auf eine weitere

298 SCHÄrer in: Brägger, S. 251 (254).

299 SснӓвеR in: Brägger, S. 251 (254); eingehend zur Rechtsnatur der Richtlinien in der NWI: SCHÄRER SZK 2/2012, 51 (51, 53 ff.): SCHÄrER stellt fest, dass Richtlinien durch die breite Implementierung und ihre konsequente Anwendung einer Rechtsverordnung sehr nahekämen.

300 SCHÄrer SZK 2/2012, 51 (55): Von dieser Möglichkeit sei aufgrund der grossen Akzeptanz und der breiten Einhaltung der Richtlinien in den Konkordatskantonen bis anhin kein Gebrauch gemacht worden. Die Akzeptanz beruhe vor allem darauf, dass die Richtlinien gemeinsam erarbeitet und beschlossen worden seien, weshalb sie auf einem breiten Konsens beruhen würden.

301 SCHÄrER in: Brägger, S. 251 (254).

302 Vgl. auch SchärER in: Brägger, S. 251 (253).

303 Vgl. auch Schärer in: Brägger, S. 251 (253). 
Harmonisierung hinwirken. So etwa die Regierungskonferenz der Kantonalen Justiz- und Polizeidirektoren (KKJPD), welche beispielsweise im November 2014 eine gemeinsame Grundlage für den schweizerischen Sanktionenvollzug verabschiedet hat. ${ }^{304}$ Auch die Koordinationskonferenz Justizvollzug (KOK) bezweckt als gesamtschweizerisches Organ einen Austausch zwischen den Kantonen und trägt so zu einer gewissen weiteren Harmonisierung unter den Kantonen bei. ${ }^{305}$ Auf eine gesamtschweizerische Zusammenarbeit bei Fragen rund um den Strafvollzug wirkt auch das Schweizerische Kompetenzzentrum für den Justizvollzug (SKJV) hin. ${ }^{306}$

Zusammenfassend findet in normativer Hinsicht durch die drei regionalen Strafvollzugskonkordate zumindest innerhalb des jeweiligen Konkordatsgebiets eine Harmonisierung zentraler Vollzugsfragen statt. Daneben bestehen verschiedene weitere Gremien, namentlich die KKJPD, die KOK und das SKJV, welche auf eine gewisse gesamtschweizerische Harmonisierung hinwirken.

\section{Kantonale Ebene}

Art. 51 BV verpflichtet die Kantone, sich eine demokratische Verfassung zu geben. Die meisten Kantonsverfassungen enthalten Grundrechts- und Aufgabenbestimmungen. ${ }^{307}$ Relevant werden diese vor allem dann, wenn sie über die in der Bundesverfassung gewährleisteten Grundrechte hinausgehen. ${ }^{308}$ Spezifische strafvollzugsrechtliche Grundrechte finden sich soweit ersichtlich jedoch keine in den Kantonsverfassungen.

Sowohl Gesetzesstufe als auch Regelungsbreite und Regelungstiefe des kantonalen Strafvollzugsrechts variieren stark. ${ }^{309}$ Auf Gesetzesstufe haben die Kantone Bern, Solothurn, Zürich, Graubünden und Neuenburg im Be-

304 Grundlagen für den strafrechtlichen Sanktionenvollzug in der Schweiz, zuletzt abgerufen am 15.01.2020.

305 Konferenz der Kantonalen Justiz- und Polizeidirektorinnen und -direktoren, Straf- und Massnahmenvollzug, zuletzt abgerufen am 13.02.2020.

306 Schweizerisches Kompetenzzentrum für den Justizvollzug - Ziele und Aufgaben, zuletzt abgerufen am 06.08.2020; WaLter in: Fink/Arnold/GenillodVillard/Oberholzer, S. 241 (247): Das SKJV als potentielle Drehscheibe für eine mögliche weitere gesamtschweizerische Angleichung.

307 JAAG in: Biaggini/Gächter/Kiener $\mathbb{1 1}$ Rn. 22.

308 Als verfassungsmässige Rechte sind sie auch vor Bundesgericht möglicher Beschwerdegrund (Art. 95 lit. c BGG).

309 Zum Ganzen: Schärer in: Brägger, S. 251 (253); Schärer SZK 2/2012, 51 ff.; Baechtold in: van Zyl Smit/Dünkel, S. 653 (656f.). 
reich des Strafvollzugsrechts umfassend legiferiert (Gesetze im formellen Sinn). Auf Verordnungsstufe haben die Kantone Bern, Solothurn, Zürich, Neuenburg, Aargau, St. Gallen, Tessin, Waadt und Wallis umfassende Bestimmungen zum Strafvollzugsrecht verabschiedet. In den übrigen Kantonen wurde das Strafvollzugsrecht gar nicht oder nur rudimentär normiert; Verwaltungsvorschriften übernehmen hier die Regelung des Strafvollzugs. ${ }^{310}$ Beispielhaft wird im Folgenden näher auf die Bestimmungen des Kantons Zürich eingegangen.

Einschlägige Erlasse im Kanton Zürich sind das Straf- und Justizvollzugsgesetz (StJVG ZH) und die Justizvollzugsverordnung (JVV ZH). Das StJVG ZH regelt zunächst in den $\mathbb{\$} 14 \mathrm{ff}$. die Zuständigkeiten im Justizvollzug. Der für den Justizvollzug zuständigen Direktion des Regierungsrates obliegen alle im Zusammenhang mit dem Vollzug strafrechtlicher Sanktionen anfallenden Aufgaben und Entscheide, welche nicht ausdrücklich anderen Instanzen übertragen wurden ( $\$ 14$ Abs. 1 StJVG ZH). Vollzugsbestimmungen finden sich in den $\$ \$ 20 \mathrm{ff}$. StJVG ZH. Gemäss $\$ 31$ lit. b StJVG ZH liegt die Regelungskompetenz für den Vollzug freiheitsentziehender Sanktionen, insbesondere für die Rechte und Pflichten der Verurteilten im Anstaltsalltag, beim Regierungsrat. Der Regierungsrat erliess unter anderem gestützt auf diese Bestimmung die Justizvollzugsverordnung (JVV ZH).

$\$ 32$ StJVG ZH sieht schliesslich vor, dass der Regierungsrat mit anderen Kantonen Vereinbarungen treffen kann. Regelungsgegenstand dieser Vereinbarungen können etwa der Vollzug von Strafen und Massnahmen sowie die Bewährungshilfe durch ausserkantonale Anstalten und Dienste, die Übernahme des Vollzugs ausserkantonaler Sanktionen und die dabei zur Anwendung gelangenden Tarife sein ( $\$ 32$ Abs. 1 lit. a StJVG ZH). Zweck dieser Vereinbarungen kann etwa die Vereinheitlichung des Vollzugs von freiheitsentziehenden Sanktionen in staatlichen Einrichtungen oder die gemeinsame Planung eines bedarfsgerechten Platzangebots für freiheitsentziehende Sanktionen sein ( $\$ 32$ Abs. 1 lit. c und d StJVG ZH).

Zusammenfassend zeigt sich in den Kantonen ein sehr heterogenes Bild. Die Harmonisierungsbemühungen durch die Konkordate sind vor diesem Hintergrund zu würdigen.

310 Vgl. auch Schärer in: Brägger, S. 251 (253); eindrücklich zur Heterogenität und „Unübersichtlichkeit“ des kantonalen Strafvollzugsrechts: BAECHTOLD SZK 1/2004, $61 \mathrm{ff}$. 


\section{Zwischenfazit zum Rechtsrahmen im Mehrebenensystem}

Freiheitsstrafen werden durch den Nationalstaat vollzogen. Der Rahmen, in welchem der Strafvollzug zu erfolgen hat, wird allerdings nicht nur durch die nationale Verfassung, nationale Gesetze und nationale Verordnungen vorgegeben. Vielmehr greifen internationale und europäische Institutionen den Strafvollzug als Regelungsgegenstand auf. Insbesondere der Europarat hat sich diesbezüglich als bedeutender Akteur hervorgetan. Die Tendenz geht sowohl auf UN-Ebene als auch auf Europaratsebene dahin, dass betreffend das Strafvollzugsrecht die Normdichte und Normtiefe im Verlaufe der Zeit zunahm. Es ist auch zu erwarten, dass sich diese Tendenz in der Zukunft fortsetzen wird. Die erlassenen Bestimmungen unterscheiden sich sowohl hinsichtlich ihrer rechtlichen Bindungswirkung gegenüber den Mitgliedstaaten als auch in ihrem Selbstverständnis. Während völkerrechtliche Verträge von den Mitgliedstaaten zwingend einzuhalten sind, wirken Empfehlungen als Soft Law auf eine andere Art und Weise auf die mitgliedstaatliche Rechtsanschauung ein.

\section{Kapitel 4: Soft Law}

I. Begriff, Charakter und normative Einordnung

Eine universell anerkannte Definition von Soft Law gibt es nicht, ${ }^{311}$ weshalb zu definieren ist, was in der vorliegenden Arbeit unter Soft Law verstanden wird. Dazu ist zunächst festzulegen, ob einem binären Ansatz

311 Zum Spektrum dessen, was als Soft Law verstanden werden könnte vgl. Cerone in: Lagoutte/Gammeltoft-Hansen/Cerone, S. 15 ff.: Aus völkerrechtlicher Sicht seien bestehende Definitionen des Begriffs Soft Law vielfältig, uneinheitlich und zuweilen inkohärent. Vgl. auch Zemanek FS Seidl-Hohenveldern, S. 843 (856): „It is the undifferentiated use of the term Soft Law for quite different phenomena which is confusing, since observations which are pertinent to one type are not relevant to the other. A more subtly differentiating evaluation would, therefore, help to clarify the presently fluid notion"; noch weiter gehend Thürer ZSR 1985, 429 (433): Eine exakte wissenschaftliche Definition sei aufgrund des Untersuchungsgegenstandes gar nicht möglich. Soft Law sei kein Rechtsbegriff mit einem dogmatisch bestimmbaren Inhalt und Umfang. Es sei vielmehr eine Formulierung, welche sich aufgrund ihres diffusen und gezielten "Schlagwortcharakters" einer begrifflichen Abgrenzung von vorneherein zu entziehen scheine. Zum Soft Law-Begriff vgl. weiter auch Gammeltoft-Hansen/ Lagoutte/Cerone in: Lagoutte/Gammeltoft-Hansen/Cerone, S. 1 (2 ff.). 
zwischen Recht und Nicht-Recht gefolgt wird oder ob Recht vielmehr als Kontinuum zu verstehen ist. ${ }^{312}$ Wird ersterem positivistischen Ansatz gefolgt, sind Soft Law-Regeln entweder Bestandteil des positiven Rechts - d.h. nach den etablierten Quellen bestehendes internationales Recht -, welche aber in ihrer Handhabung „weich“ sind, da beispielsweise die verwendete Formulierung nicht justiziabel ist ${ }^{313}$, oder sie sind überhaupt nicht als Recht zu betrachten (sog. "Non-Law"314). Distanziert man sich demgegenüber von einem binären Ansatz und wird der Dichotomie von Verbindlichkeit und Nichtverbindlichkeit weniger Bedeutung zugemessen und „Recht" weiter begriffen, als von Art. 38 IGH-Statut umfasst, sind als Soft Law jene Bestimmungen zu verstehen, welche zwar nicht rechtsverbindlich im technischen Sinne sind, welche aber gleichwohl eine normative, verhaltensbeeinflussende Wirkung entfalten. ${ }^{315}$ Soft Law-Normen erzeugen damit Rechtswirkungen, ohne dass ihnen Rechtsverbindlichkeit im technischen Sinne zukommt. ${ }^{316}$ Dem Soft Law-Begriff, wie er in der vorliegenden Arbeit verwendet wird, liegt der zweite Ansatz zugrunde. ${ }^{317}$

Im Sinne der Begriffskomponente „Soft“ begründen diese Regelwerke damit zwar keine verbindlichen subjektiven Rechte und Pflichten und

312 Cerone in: Lagoutte/Gammeltoft-Hansen/Cerone, S. 15 (16).

313 Cerone in: Lagoutte/Gammeltoft-Hansen/Cerone, S. 15 (16): Weil die „Softness“ bei diesem Definitionsansatz nicht auf den Charakter des Instruments zurückzuführen ist, gäbe es in diesem Verständnis gar kein Soft Law-Instrument.

314 So Cerone in: Lagoutte/Gammeltoft-Hansen/Cerone, S. 15 (16).

315 Cerone in: Lagoutte/Gammeltoft-Hansen/Cerone, S. 15 (16f.); zum Verständnis von Art. 38 IGH in diesem Kontext vgl. auch Zemanska FS Seidl-Hohenveldern, S. 843 (844).

316 ThÜRER ZSR 1985, 429 (434).

317 Anders etwa der Arbeitsbegriff von Cerone in: Lagoutte/Gammeltoft-Hansen/ Cerone, S. 16 ff., welcher einem binären Ansatz folgt, wobei er Soft Law zur Kategorie "Nicht-Recht“ zählt. Konstitutives Element von Soft Law sei dann aber, dass ihm eine gewisse "Zugkraft" zukomme. Als Soft Law seien damit alle Regeln zu verstehen, welche eine gewisse autoritative Akzeptanz hätten, aber nicht von Art. 38 IGH umfasst seien. Schliesst der binäre Ansatz, so wie er von Cerone verstanden wird, ein Spektrum relativer Weichheit resp. Härte nicht aus, scheint es meines Erachtens für die vorliegende Fragestellung auch nicht passend, einem binären Ansatz zu folgen. Es geht nicht nur um die Frage, ob Soft oder Hard Law vorliegt, sondern eben gerade auch um die Frage, wie es sich mit der Normativität des Soft Law verhält bzw. wie sich diese entwickeln kann (vgl. hardened Soft Law). Die reine Unterscheidung zwischen Recht und Nicht-Recht rückt damit in den Hintergrund. Vgl. in diesem Sinne ZemaneK FS Seidl-Hohenveldern, S. 843 (844ff., 858), welcher die Dichotomie von „bindend“ und „nicht bindend“ zur Bestimmung der Natur von Soft Law für wenig hilfreich hält. 
wenden sich auch nicht primär an die Betroffenen. Sie sehen denn auch keine direkten Durchsetzungsmassnahmen vor und stehen keiner direkten gerichtlichen Überprüfung offen. ${ }^{318}$ Auch formale oder verfahrensrechtliche Anforderungen an den Entstehungsprozess von Soft Law gibt es in diesem Sinne nicht; im Unterschied zu Hard Law haben sie den für die Rechtserzeugung vorgesehenen Weg gerade nicht oder zumindest nicht ganz durchlaufen. ${ }^{319}$ Im Sinne des Wortbestandteils „Law" sehen sie aber gleichwohl Verhaltensweisen vor resp. bringen Verhaltenserwartungen zum Ausdruck, welche sich auf materielle bzw. rechtliche Erwägungen stützen. ${ }^{320} \mathrm{Im}$ Unterschied zu blossen Akten der Politik oder der Moral ist ihnen damit ein normativer Gehalt inhärent, womit sie eine gewisse Rechtsnähe aufweisen und gewisse Rechtswirkungen erzeugen. ${ }^{321}$ Der Grad der Normativität der einzelnen Regelwerke bzw. der betreffenden Bestimmungen kann allerdings variieren. ${ }^{322}$

Ausgearbeitet wird Soft Law in der Regel durch Ausschüsse internationaler Organisationen, durch NGOs oder überstaatliche Expertengremien. ${ }^{323}$ Damit sind im Sinne einer "Expertokratie“ meist Sachverständige mit der Schaffung von Soft Law-Regelwerken befasst. Soft Law kann dabei ergänzend zu hartem, kodifiziertem Recht etwa in Form zusätzlicher Absichtserklärungen der Verfasser oder aber ohne Konnex zu kodifiziertem

$318 \mathrm{Zu}$ diesen Kriterien kritisch Cerone in: Lagoutte/Gammeltoft-Hansen/Cerone, S. 15 (18): Mangelnde Justiziabilität oder das Fehlen von Rechtsbehelfen mache eine vertragliche Verpflichtung noch nicht rechtlich unverbindlich. Dem ist meines Erachtens zwar zuzustimmen; gleichwohl bedeutet dies e contrario nicht, dass diese Merkmale für Soft Law nicht zutreffen und für dieses nicht gerade auch bezeichnend sind.

319 Thürer ZSR 1985, 429 (434); differenziert auch Richter Pinto de Albuquerque partly diss. op., EGMR (GK) - Muršić/CRO, Urt. v. 20.10.2016, 7334/13, Rn. 28. Richter Pinto de Albuquerque bringt vor, dass die Existenz von travaux préparatoires, erläuternden Berichten und Kommentaren mit einer gründlichen Diskussion der Ursachen und Folgen der Entscheidungen Hinweis auf die normative Dichte des Soft Law-Textes seien. Auch die Komplexität des Beratungsverfahrens, einschliesslich des Abstimmungsmusters, seien ein Härtefaktor.

320 Thürer ZSR 1985, 429 (434); vgl. auch Richter Pinto de Albuquerque partly diss. op., EGMR (GK) - Muršić/CRO, Urt. v. 20.10.2016, 7334/13, Rn. 6.

321 Vgl. Thürer ZSR 1985, 429 (434).

322 Vgl. auch Richter Pinto de Albuquerque partly diss. op., EGMR (GK) - Muršićl CRO, Urt. v. 20.10.2016, 7334/13, Rn. 28, welcher den Grad der Normativität anhand verschiedener Faktoren, die sich entweder auf das Verfahren der Regelsetzung oder auf das Verfahren der Regelanwendung beziehen, bestimmt sehen will.

323 Meyer S. 30. 
Recht geschaffen werden. ${ }^{324}$ Häufig zeichnet sich das Soft Law durch detaillierte Bestimmungen aus, ${ }^{325}$ was darauf zurückzuführen sein dürfte, dass Mitgliedstaaten eher einem (zumindest scheinbar) unverbindlichen Instrumentarium zustimmen als einem rechtlich verbindlichem, bei welchem die Nichteinhaltung für den Mitgliedstaat zu direkten Konsequenzen wie etwa einer (finanziellen) Sanktionierung führt. ${ }^{326}$

Was die Legitimität von Soft Law anbelangt, sind die Meinungen im Schrifttum ambivalent. Während Soft Law von den einen als „Bereicherung“327 angesehen wird, wird es von anderen als „unerwünschtes Phänomen" 328 betitelt, wobei es aufgrund seiner Wirkungsweise bisweilen als Deckmantel der Macht einzelner staatlicher und nichtstaatlicher Akteure betrachtet wird. ${ }^{329}$ Sogar die Demokratie und die Souveränität des Staates werden durch das Bestehen von Soft Law in Gefahr gesehen. ${ }^{330}$ Vorliegend wird jedoch schwerpunktmässig nicht der Frage der Legitimität nachgegangen. Vielmehr wird aufgrund der grossen praktischen Relevanz des Soft Law auf dem Gebiet des Strafvollzugs untersucht, welche konkrete Rolle Soft Law hinsichtlich menschenrechtlicher Anforderungen an den Strafvollzug tatsächlich zukommt bzw. inwieweit es auf die Standardsetzung tatsächlich Einfluss nimmt. Es stellt sich insbesondere die Frage, ob sich einschlägiges Soft Law zu „hardened soft law" resp. gar zu Hard Law verhärtet resp. transformiert; ob also der Grad der Normativität im Laufe der Zeit zunimmt. Zur Einordnung dieser Frage wird zunächst auf die Mechanismen eines solchen Transformationsprozesses eingegangen: das Erlangen praktischer Wirksamkeit von Soft Law durch Implementierungskontrollen,

324 Hillgenberg EJIL 1999, 499 (515); vgl. auch Richter Pinto de Albuquerque partly diss. op., EGMR (GK) - Muršić/CRO, Urt. v. 20.10.2016, 7334/13, Rn. 8 f.

325 Meyer S. 263: Meyer sieht im Zusammenhang mit den Handlungsinstrumenten einen Vorteil des Erlasses von Empfehlungen gegenüber dem Abschluss von völkerrechtlichen Abkommen gerade darin, dass mit Empfehlungen nicht nur eine „Minimallösung“, sondern „ausgefeilte Regelungen“ erzielt werden könnten, was zu einem grösseren „Annäherungseffekt“ führen könne. Vgl. ferner HiRsCh S. 51.

326 Vgl. auch Hillgenberg EJIL 1999, 499.

327 Vgl. etwa Popovic S. 54; vgl. Mavronicola HRLR 2015, 721 (730).

328 Vgl. etwa Klabbers Nordic Journal of International Law 1998, 381 ff.; vgl. auch WeIL AJIL 1983, $413 \mathrm{ff}$.

329 KLabbers Nordic Journal of International Law 1998, 381 (391).

330 Klabbers Nordic Journal of International Law 1998, 381 (391): „[...] we need to insist in a degree of formalism, because it is precisely this formalism that protects us from arbitrariness on the part of the powers that be." 
durch Einflussnahme auf die Rechtssetzung und durch Einflussnahme auf die Rechtsanwendung von Hard Law-Normen.

\section{Praktische Wirksamkeit}

1. Praktische Wirksamkeit durch Implementierungskontrolle / Implementierungshilfe

Im Soft Law sind bisweilen nachgelagerte Implementierungskontrollen resp. Monitorings durch speziell dafür eingesetzte Ausschüsse oder Sachverständige vorgesehen. ${ }^{331}$ Dazu werden Befugnisse zur Auslegung und Konfliktlösung an ein unabhängiges drittes Gremium übertragen, welches Follow-up-Mechanismen vorsieht. ${ }^{332}$ Die stetige Kontrolle des Umsetzungsstandes und die entsprechende (öffentliche) Berichterstattung sollen sanften Druck auf die Vertragsparteien ausüben und so die Erfüllungsverpflichtung der Vertragsparteien verstärken. ${ }^{333}$

Solche Implementierungskontrollen finden sich in der Praxis sowohl auf internationaler und europäischer als auch auf nationaler Ebene. Auf der Ebene der Vereinten Nationen hat beispielsweise der ECOSOC Verfahren zur effektiven Implementierung der UN-Mindestgrundsätze für die Behandlung der Gefangenen angenommen. ${ }^{334}$ Es werden etwa regelmässig Fragebogen an die Mitgliedstaaten versandt und deren Antworten evaluiert. ${ }^{335}$ Zum Voranbringen der Implementierung der UN-Mindestgrundsätze setzen die Vereinten Nationen weiter etwa auf die Zurverfügungstel-

331 Meyer S. 285.

332 Vgl. etwa Directorate General Human Rights and Rule of Law, Practical Impact of the Council of Europe Monitoring Mechanisms, zuletzt abgerufen am 23.07.2020; vgl. auch Richter Pinto de Albuquerque partly diss. op., EGMR (GK) - Muršíc/CRO, Urt. v. 20.10.2016, 7334/13, Rn. 28 m.w.N.

333 Meyer S. 285, 923: Monitoring, Evaluierung und ggf. begleitende Stellungnahmen als sanfte Überzeugungsarbeit zur Steuerung der Rechtsentwicklung und Kontrolle der geschaffenen Standards; vgl. auch Richter Pinto de Albuquerque partly diss. op., EGMR (GK) - Muršić/CRO, Urt. v. 20.10.2016, 7334/13, Rn. 28: Auch die „kontrafaktische Kraft des normativen Textes“ würde durch Mechanismen der Rechenschaftspflicht verstärkt.

334 Vgl. E/RES/1984/47, Annex to the Procedures for the Effective Implementation of the Standard Minimum Rules for the Treatment of Prisoners; im Einzelnen dazu Abels S. $36 \mathrm{ff}$.

335 Kritisch zu dieser Evaluationsmethode: Abels S. 36: Die geringe Anzahl an Antworten nationaler Behörden liesse keine aussagekräftigen generellen Schlussfol- 
lung eines Trainingsangebots, auf technische Unterstützung, auf Hilfe bei der Verbreitung der UN-Mindestgrundsätze unter den Strafgefangenen und dem Personal von Strafvollzugsanstalten sowie auf das Erstellen periodischer Berichte über die erzielten Fortschritte. ${ }^{336}$

Auf Europaratsebene ist etwa das CDPC bei der Implementierungskontrolle bedeutender Akteur. Es nimmt schon durch die Ausarbeitung von Explanatory Reports resp. Explanatory Memoranda, welche es mit den Entwürfen der Regelwerke gleich mitliefert, entscheidenden Einfluss auf die Handhabung der Regelwerke in der Praxis - mithin auf ihre effektive Implementierung. 337 Weiter kann das CDPC (permanente) Unterausschüsse bilden, wie etwa die CDPC Sub-group on Prison Overcrowding, und diese mit Monitoringaufgaben betrauen. Konkret wurde unter der Aufsicht des CDPC beispielsweise das White Paper on Prison Overcrowding (Weissbuch) ausgearbeitet, in welchem die Situation der Überbelegung der Gefängnisse analysiert und spezifische Massnahmen empfohlen werden, die von den Behörden zu ergreifen sind. ${ }^{338}$ Zur Förderung der im Weissbuch vorgesehenen Massnahmen beschloss das CDPC weiter die Organisation einer High Level Conference.

Diese sanfte Druckausübung kann im Ermessensbereich des bestehenden Hard Law direkte Wirkung auf deren Auslegung und damit auf praktische Handhabungsfragen haben. Auch eine breite Öffentlichkeitswirkung und eine allgemeine Aufmerksamkeit entfalten allein schon praktische Wirkung. ${ }^{339}$ Ferner kann die Implementierungskontrolle eine Art Reflexwirkung auf die Legislative und Judikative haben (zur praktischen Wirksamkeit durch Rechtssetzung und Rechtsprechung vgl. sogleich). In der Praxis kommt der Implementierungskontrolle damit bisweilen eine derart wichtige Bedeutung zu, dass sie - sogar im Bereich des Strafrechts - als "alternatives Mittel zur Rechtssetzung" bezeichnet wird. ${ }^{340}$

gerungen zu. Weiter sei die Vertrauenswürdigkeit der Informationen fraglich, da diese von den Nationalstaaten selbst kämen.

336 Vgl. Procedures 1 to 13 inkl. Commentaries: E/RES/1984/47, Annex to the Procedures for the Effective Implementation of the Standard Minimum Rules for the Treatment of Prisoners; zum Ganzen vgl. auch Abels S. $36 \mathrm{f}$.

337 Vgl. auch Meyer S. 285.

338 White Paper on Prison Overcrowding, zuletzt abgerufen am 15.01.2020.

339 Richter Pinto de Albuquerque partly diss. op., EGMR (GK) - MuršićlCRO, Urt. v. 20.10.2016, 7334/13, Rn. 28.

340 Meyer S. 285; vgl. auch Jung FS Ress, S. 519 (524). 


\section{Praktische Wirksamkeit durch Rechtssetzung}

Auf legislativer Ebene kann Soft Law praktische Wirkung entfalten, indem es etwa im nationalen Gesetzgebungsprozess berücksichtigt wird bzw. gar den Anstoss für einen solchen bildet, insbesondere wenn Empfehlungen Anknüpfungspunkte für nationales Strafvollzugsrecht werden. ${ }^{341}$ Weiter kann Soft Law auch Ausgangspunkt der Ausarbeitung eines rechtlich verbindlichen Regelwerks durch dieselbe Organisation resp. durch dasselbe Gremium sein, sofern dieses auch dazu ermächtigt ist. ${ }^{342}$ Wenn zuvor noch kein Konsens bestand, kann Soft Law als Instrument der Überzeugungsbildung dienen. ${ }^{343}$ Denkbar ist allerdings auch, dass bei den Vertragsparteien grundsätzlich bereits ein Konsens bestand und die Entscheidung, in einem ersten Schritt Soft Law zu verabschieden, rein auf einem zeitlichen Faktor basierte, beansprucht bei Internationalen Organisationen die Verabschiedung von rechtlich verbindlichen Verträgen in aller Regel doch ungleich mehr Zeit. Eine Rezeption des Soft Law kann weiter auch in der Weise erfolgen, dass dieses von einer anderen Organisation oder einem anderen Gremium aufgegriffen wird und für ein anderes Soft LawRegelwerk Pate steht. So kann der Wirkungsbereich des übernommenen Soft Law ausgedehnt und bzw. oder die Dringlichkeit der Umsetzung untermauert werden. ${ }^{344}$

341 Hirsch S. 51; vgl. auch Cerone in: Lagoutte/Gammeltoft-Hansen/Cerone, S. 15 (17); Ligthart/van Oploo/Meijers/Meynen/Kooijmans NJECL 2019, 287 (290); zum Beispiel des Einflusses der Europäischen Mindestgrundsätze auf die deutschen Gesetzgebungsarbeiten zum Jugendstrafvollzug vgl. DünKeL/BAECHTOLD/van ZyL SMIT in: Goerdeler/Walkenhorst, S. 114 (138): Die Grundsätze würden zum unmittelbaren Prüfungsmassstab der Gesetzgebungsarbeiten.

342 Mit Beispielen auf der Ebene der Vereinten Nationen vgl. Villeneuve in: Lagoutte/Gammeltoft-Hansen/Cerone, S. 213 (216 ff.); für die Europaratsebene vgl. Meyer S. 284, 542: Der Europarat stecke mit Empfehlungen zunächst Themenfelder $a b$ und schaffe für diese zunächst allgemeine Sensibilität, um die Empfehlung dann als Ausgangspunkt zur Ausarbeitung eines völkerrechtlichen Abkommens zu nehmen. Eine Empfehlung könne aber auch eine bereits bestehende Konvention zum Ausgangspunkt nehmen und diese fortentwickeln. Das inhaltlich über die Konvention Hinausgehende hat dann (vorerst) rechtlich unverbindlichen Charakter, bis es allenfalls später in einer weiteren Konvention aufgenommen wird.

343 Zemanex FS Seidl-Hohenveldern, S. 843 (858): „shaping a consensus for future multilateral conventions“; im Zusammenhang mit den Empfehlungen des Europarates: MEYER S. 542.

344 Vgl. etwa die Wiederaufnahme der UN-Mindestgrundsätze für die ESMR auf Europaratsebene. 
Die Wiederaufnahme des Soft Law durch die Legislative ist mit Blick auf die kritischen Stimmen, welche ein Demokratiedefizit oder ein Untergraben der staatlichen Souveränität erkennen, grundsätzlich unproblematisch. Der demokratisch legitimierte Gesetzgebungsprozess, bzw. das gewöhnliche Vorgehen zum Abschluss völkerrechtlicher Übereinkommen, wird wie gewöhnlich durchlaufen. Das Soft Law-Regelwerk selbst bleibt von diesem Prozess unberührt; dieses behält nach wie vor den rechtlich unverbindlichen Charakter. Wenn es um die Durchsetzung geht, ist denn auch nicht das Soft Law-Regelwerk, sondern das neu verabschiedete Gesetz oder das neue Übereinkommen einzuklagen. ${ }^{345}$ Nicht mehr gleich unproblematisch wäre die Situation erst dann, wenn das Soft Law dem Gesetzgeber nicht mehr als blosse Inspirationsquelle diente und damit nicht mehr nur eine Motorfunktion innehätte, sondern die „sanfte“ Druckausübung durch die Implementierungskontrolle des Soft Law quasi zu einem Befolgungszwang führen würde. Der formell demokratisch legitimierte Gesetzgebungsprozess würde in dieser Konstellation Gefahr laufen, blosser Schein zu sein.

3. Praktische Wirksamkeit in der Rechtsprechung

\subsection{Im Allgemeinen}

Auf die Rechtsprechung kann Soft Law grundsätzlich auf zwei verschiedene Arten Einfluss nehmen. Zum einen kann sich eine rechtsanwendende Behörde bei der Auslegung von Hard Law an einer Soft Law-Bestimmung orientieren; ${ }^{346}$ die Rezeption von Soft Law erfolgt dann auf der Rechtsebene. Zum anderen kann Soft Law indirekt durch seine nachgelagerte Implementierungskontrolle, deren Ergebnisse in Berichten festgehalten werden, bei der Sachverhaltserstellung von Relevanz sein. Im Allgemeinen dürfte Letzteres umso bedeutsamer sein, je schwieriger die Beweiserhebung und je tiefer das Beweismass ist. Genügt beispielsweise die Erbringung eines Anscheinsbeweises, ist von einem Monitoringbericht, der die allgemeine

345 Vorausgesetzt, dass das Übereinkommen genügend bestimmt und damit „selfexecuting" ist.

346 Villeneuve in: Lagoutte/Gammeltoft-Hansen/Cerone, S. 213 (218); in diesem Sinne vgl. auch Ligthart/van Oploo/Meijers/Meynen/Kooijmans NJECL 2019, 287 (290); ZемANeK in: FS Seidl-Hohenveldern, S. 843 (860). 
Situation in einem Land schildert, mehr zu erwarten, als wenn ein Einzelnachweis zu erbringen ist.

Die Orientierung der Rechtsprechung an einer Soft Law-Bestimmung ist es, welche zur Kritik des Demokratiedefizits bzw. des Souveränitätsmangels geführt haben dürfte. Als primär rechtsanwendende Behörde hat ein Gericht rechtlich verbindliche Normen auszulegen, anzuwenden und im Einzelfall durchzusetzen. Moniert wird, dass durch die Aufnahme des Soft Law in der Rechtsprechung die Grenze zwischen der zulässigen richterlichen Rechtsfortbildung und der Rechtssetzung verwischt und die Grenze üblicher (verfassungs-)gerichtlicher Befugnisse überschritten würden. ${ }^{347}$ Dem ist entgegenzuhalten, dass die Soft Law-Bestimmung, welche über den Weg der Auslegung einer rechtlich verbindlichen Norm Eingang in die Rechtsprechung findet, zwar indirekt im Einzelfall durchgesetzt würde, das Urteil aber individuell-konkreter Natur ist, womit eine allgemeine rechtliche Verbindlichkeit der Soft Law-Bestimmung weiterhin nicht existiert. Gleichwohl muss selbst ohne rechtlich durchsetzbare generellabstrakte Wirkung der Bestimmung eingestanden werden, dass nicht mehr von einer rechtlichen Unverbindlichkeit im ursprünglichen Sinne auszugehen ist, wenn die Soft Law-Bestimmung regelmässig gerichtlich durchgesetzt werden kann. Setzt die Vertragspartei die Soft Law-Bestimmung nicht um, drohen nämlich weitere Beschwerden und Verurteilungen und der normative Anspruch der Soft Law-Bestimmung steigt. ${ }^{348}$ Es ist dieser Vorgang, welcher als Transformationsprozess oder als „hardening Soft Law" bezeichnet wird. ${ }^{349}$ Rechtspolitische Zielbestimmungen können sich auf diese Weise nämlich zu rechtsverbindlichen, subjektiven Rechten formen. ${ }^{350}$ Die Grenze zulässiger richterlicher Rechtsfortbildung ist für jede auszulegende Norm nach Massgabe des für sie anwendbaren Auslegungskanons separat zu bestimmen.

347 Grabenwarter/Pabel $₫ 5$ Rn. 12.

348 Richter Pinto de Albuquerque partly diss. op., EGMR (GK) - MuršićlCRO, Urt. v. 20.10.2016, 7334/13, Rn. 28.

349 Richter Pinto de Albuquerque partly diss. op., EGMR (GK) - MuršićlCRO, Urt. v. 20.10.2016, 7334/13; vgl. etwa auch Villeneuve in: Lagoutte/Gammeltoft-Hansen/Cerone, S. 213 (218 f.).

350 SK-StPO/MeYer Art. 3 EMRK Rn. 44. 


\subsection{Bei Konventionsrechten}

\section{a Auf Rechtsebene}

Als völkerrechtlicher Vertrag ist für die EMRK die Wiener Vertragsrechtskonvention (WVK) massgebend. ${ }^{351}$ Gemäss Art. 31 Abs. 1 WVK ist ein völkerrechtlicher Vertrag nach Wortlaut und Systematik sowie im Sinne seiner Ziele und Zwecke auszulegen. Aus der Präambel der EMRK geht hervor, dass die EMRK die universelle und wirksame Anerkennung und Einhaltung der in ihr aufgeführten Rechte bezweckt. Weiter wird erwogen, dass es Ziel des Europarates sei, eine engere Verbindung zwischen seinen Mitgliedern herzustellen, und dass eines der Mittel zur Erreichung dieses Zieles die Wahrung und Fortentwicklung der Menschenrechte und Grundfreiheiten sei. Damit wird deutlich, dass die EMRK sich in ihrem Selbstverständnis in den Gesamtkontext der Arbeiten des Europarates stellt. Art. 31 Abs. 3 lit. c WVK hält fest, dass jeder in den Beziehungen zwischen den Vertragsparteien anwendbare einschlägige Völkerrechtssatz für die Auslegung des Vertrags zu berücksichtigen ist. ${ }^{352}$ Nach wie vor nicht abschliessend geklärt ist allerdings, ob Soft Law aufgrund seiner rechtlich nicht bindenden Natur von dieser Bestimmung umfasst ist oder nicht. ${ }^{353}$

351 EGMR (Pl) - Golder/GBR, Urt. v. 21.02.1975, 4451/70, Rn. 29 f. (obwohl die Normen der WVK zu diesem Zeitpunkt noch gar nicht in Kraft getreten waren); vgl. etwa auch EGMR (GK) - Mamatkulov u. Abduraslovic/TUR, Urt. v. 06.02.2003, 46827/99 u. 46951/99, Rn. 99; Benavides Casals S. 70: die Auslegungsregelungen der WVK als Ausgangspunkt für die Interpretation der EMRK; Matscher FS Bernhardt, S. 503 (504 m.w.H.): Die in der WVK verankerten Auslegungsprinzipien seien zu beachten; allerdings seien auch die Besonderheiten des internationalen Menschenrechtsschutzes zu berücksichtigen. In diesem Sinne auch Bernhardt FS Wiarda, S. 65 (65 f.): Die Besonderheit von Menschenrechtsabkommen sei, dass es nicht um gegenseitige Beziehungen und den Austausch von Vorteilen zwischen souveränen Staaten gehe, sondern um das Einräumen von Rechten für die der Jurisdiktion unterliegenden Menschen. Vgl. auch Peters/Altwicker $\$ 2$ Rn. 40; Villiger FS Ress, S. 317 ff.; Flauss in: Widmer, S. 159 (160).

352 Vgl. dazu auch Benavides Casals S. 75 f.; EGMR - Bankovic u.a./BEL u.a., Entsch. v. 12.12.2001, 52207/99.

353 Dörr/Schmalenbach/Dörr Art. 31 WVK Rn.97: „Notwithstanding the fact ,rules' would imply that only legally binding instruments can play a role under lit. c, parts of international judicial practice seem to apply this condition somewhat less restrictively and also consider non-binding documents as material relevant for interpretation"; ablehnend auch VILLIGER Art. 31 WVK Rn.25: „The term ,applicable‘ leaves no room for doubt: non-binding rules cannot be 
Der Gerichtshof selbst scheut sich im Einzelfall jedenfalls nicht, auch rechtlich nicht verbindliche Regelwerke des Europarates für die Auslegung eines Konventionsrechts hinzuzuziehen. ${ }^{354}$ Es wurde bereits ausgeführt, dass der EGMR keine statische Auslegung vornimmt. Vielmehr orientiert er sich an der europäischen öffentlichen Ordnung und entscheidet auf diese Weise im Einklang mit der gemeineuropäischen Rechtsüberzeugung. ${ }^{355}$ Für die Auslegung der EMRK sind damit nicht nur die Konvention und ihre Zusatzprotokolle massgebend; ${ }^{356}$ vielmehr greift der EGMR auch internationale und europäische Regelwerke auf. Ob er dies in Anwendung von Art. 31 Abs. 1 WVK oder Art. 31 Abs. 3 lit.c WVK tut, geht aus der EGMR-Rechtsprechung nicht immer eindeutig hervor. ${ }^{357}$ Unabhängig von

relied upon. [...] the applicable rules are those in force at the time of the interpretation of the treaty“; zumindest kritisch Grabenwarter/Pabel $₫ 5$ Rn. 12; vgl. auch Ronc ex ante, 67 (74); Lagoutte S. 28; Forowicz S. 43 ff. Auch die Konventionsstaaten argumentieren vor dem Gerichtshof immer wieder, dass der Einbezug nicht bindender Regelwerke von Art. 31 Abs. 3 lit. c WVK nicht umfasst sei: vgl. etwa die Türkei in EGMR (GK) - Demir u. Baykara/TUR, Urt. v. 12.11.2008, 34503/97, Rn. 61.

354 EGMR (GK) - Demir u. Baykara/TUR, Urt. v. 12.11.2008, 34503/97, Rn. 65, 74: „In a number of judgments the Court has used, for the purpose of interpreting the Convention, intrinsically non-binding instruments of Council of Europe organs, in particular recommendations and resolutions of the Committee of Ministers and the Parliamentary Assembly“; EGMR (GK) - Öneryildiz/TUR, Urt. v. 30.11.2004, 48939/99, Rn. 59, 71, 90, 93; dieses Vorgehen befürwortend vgl. Rozakis TLR 2005, 257 (274); vgl. auch Ronc ex ante 67 (74).

355 Zur rechtsvergleichenden Methodik des EGMR ausf. auch Mahoney/KondaK in: Andenas/Fairgrieve, S. $119 \mathrm{ff}$., welche betonen, dass das Konsensergebnis weder bindend noch ausschlaggebend für das Ergebnis sei, es werde heute aber als einer der indikativen oder überzeugenden Faktoren anerkannt, welche Interpretationssprünge rechtfertigen können. Vgl. auch Mowbray in: Brems/Gerards, S. 17 (35); Mahoney in: Widmer, S. 143 ff.; Wildhaber/HJartarson/Donnelly HRLJ 2013, 248 (256).

356 Vgl. SK-StPO/MeYer Einleitung (EMRK) Rn. 63; EGMR - Rantsev/CYP u. RUS, Urt. v. 07.01.2010, 25965/04, Rn. 273.

357 Wird die Soft Law-Bestimmung in Anwendung von Art. 31 Abs. 1 WVK zur Auslegung eines Konventionsrechts herangezogen, führt dies meines Erachtens im Sinne der teleologischen Auslegung zu einer verstärkten Gewichtung europäischer Soft Law-Regelwerke. Würden nicht bindende Regelwerke, welche zwischen den Vertragsparteien anwendbar sind, demgegenüber als solche von Art. 31 Abs. 3 lit. c WVK umfasst gesehen, wären sowohl europäische als auch internationale Regelwerke bei der Auslegung der Konventionsrechte grundsätzlich gleichermassen zu berücksichtigen. Da auch bei dieser Auslegung Art. 31 Abs. 1 WVK im Allgemeinen weiterhin massgebend bliebe, weshalb eine verstärkte Berücksichtigung europäischer Regelwerke auch dann möglich bliebe, 
der konkreten Rechtsgrundlage des Einbezugs des Soft Law verdeutlicht dieses Vorgehen als solches den evolutiven Gehalt der Konventionsrechte („Konvention als living instrument ${ }^{\text {"358). }}$.

Zusammengefasst bedeutet dies, dass die Berücksichtigung von Soft Law-Bestimmungen bei der Auslegung eines Konventionsrechtes aufgrund ihres Auslegungskanons vorgesehen ist, weshalb die Grenze der zulässigen Rechtsfortbildung dadurch nicht per se überschritten wird. Dieser Auslegungsweise haben die Mitgliedstaaten bei ihrem Beitritt zur EMRK denn auch zugestimmt, weshalb nicht vorgebracht werden kann, dass sie durch den Beizug von Soft Law zu etwas verpflichtet würden, dem sie nicht zugestimmt hätten. Gleichwohl kann auf diese Weise ein Transformationsprozess im oben genannten Sinn (,hardening Soft Law“) einsetzen. Im Bereich des Strafvollzugsrechts wird darin bisweilen eine Verwischung zwischen europäischer Vollzugspolitik und Menschenrechtsschutz erkannt. 359

Das Aufgreifen von Soft Law durch die rechtsanwendenden Behörden darf nach der hier vertretenen Auffassung jedenfalls nicht leichtfertig geschehen. ${ }^{360}$ In diesem Sinn ist eine vertiefte Auseinandersetzung der rechtsanwendenden Behörde zur Wertigkeit der konkreten Soft Law-Bestimmung in Bezug auf den Schutzgehalt der Konventionsnorm zu fordern. Für die Auslegung von Art. 3 EMRK bedeutet dies, dass die rechtsanwendende Behörde eine Soft Law-Bestimmung grundsätzlich heranziehen darf; sie hat aber deren Gehalt in Bezug auf eine unmenschliche oder erniedrigende Behandlung genau zu prüfen.

hat die Rechtsgrundlage des Einbezugs des Soft Law meines Erachtens nur graduelle Auswirkungen. Zum Verhältnis der einzelnen Interpretationsmittel von Art. 31 WVK im Allgemeinen vgl. auch Villiger FS Ress, S. 317 (327).

358 Vgl. bereits EGMR - Tyrer/GBR, Urt. v. 25.04.1978, 5856/72: In Tyrer verwendete der Gerichtshof erstmals das Leitmotiv der „Konvention als living instrument“, welches besagt, dass die Auslegung der Konvention vor dem Hintergrund der sich entwickelnden Normen des nationalen und internationalen Rechts zu erfolgen hat. Richter Pinto de Albuquerque partly diss. op., EGMR (GK) Muršić/CRO, Urt. v. 20.10.2016, 7334/13, Rn. 20: Die Formulierung des europäischen Konsenses in Tyrer zeige die Vision einer deliberativen internationalen Demokratie, in der eine Mehrheit oder ein repräsentativer Anteil der Vertragsparteien des Übereinkommens als im Namen aller sprechend angesehen werde und somit berechtigt sei, den anderen Parteien ihren Willen aufzuzwingen. Der Konsens als volonté générale könne auch dann bestehen, wenn nicht alle Vertragsparteien derselben Lesart der Konvention folgen würden.

359 SK-StPO/Meyer Art. 3 EMRK Rn. 44; Ronc S. 110.

360 Vgl. auch Wildhaber/HJartarson/Donnelly HRLJ 2013, 248 (256), welche eine vertiefte Auseinandersetzung des Gerichtshofs zur Frage fordern, welche Bedeutung Soft Law für die Bildung eines europäischen Konsenses zukommt. 
Nur wenn die rechtsanwendende Behörde die konventionsrechtlichen Gehalte nach ihrem eigenen Verständnis bei Nichtanwendung der Soft Law-Bestimmung als verletzt erachtet, ist die Soft Law-Bestimmung im Einzelfall anzuwenden; sie ist dann nämlich von deren Schutzgehalt umfasst. Im konkreten Einzelfall diente das Soft Law der rechtsanwendenden Behörde dann als Inspirationsquelle, womit ihr auch im Sinne eines effektiven Menschenrechtsschutzes eine Motorfunktion zukommt. Weiter ist durch die rechtsanwendende Behörde dann besondere Vorsicht bei der Annahme eines europäischen Konsenses geboten, wenn im Soft Law selbst Vorbehalte oder Ausnahmemöglichkeiten vorgesehen sind; zum Beispiel, wenn es seinen Geltungsanspruch situationsabhängig selbst einschränkt. Einzuwenden bleibt jedenfalls, dass dieses Vorgehen der Rechtssicherheit abträglich ist; dies insbesondere aufgrund der beachtlichen Normdichte des Soft Law im Mehrebenensystem. Es bleibt kaum vorhersehbar, bei welchen Soft Law-Bestimmungen der EGMR sich veranlasst sehen könnte, diese aufzugreifen. ${ }^{361}$

\section{b Auf Sachverhaltsebene}

Da das Potential von Soft Law-Monitoringberichten zur Sachverhaltserstellung entscheidend von beweisrechtlichen Grundsätzen abhängt, werden diese vorab überblicksartig dargelegt.

Der Gerichtshof ermittelt gem. Art. 38 EMRK den entscheiderheblichen Sachverhalt ex officio (Amtsermittlungsgrundsatz). Er entscheidet dabei frei über die Notwendigkeit, den Umfang und die Form der zu ergreifenden Ermittlungen. ${ }^{362}$ An Parteivorbringen und Beweisanträge ist er nicht gebunden. ${ }^{363}$ Aufgrund mangelnder Ressourcen und seiner subsidiären Rolle entspricht es allerdings nicht seiner Konzeption, als Tatsacheninstanz zu amten. ${ }^{364}$ Dies ist dann kein Problem, wenn - wie es überwiegend der Fall

361 SK-StPO/Meyer Art. 3 EMRK Rn. 44; Ronc S. 110; in diese Richtung auch Breuer ZÖR 2013, 729 ff.: Der EGMR beziehe sich auf internationale Rechtstexte ohne erkennbares System und unabhängig davon, ob der betroffene Staat Vertragsstaat sei oder nicht.

362 SK-StPO/Meyer Verfahrensrecht (EMRK) Rn. 226.

363 Meyer-Ladewig/Ebert Art. 38 Rn. 2; SK-StPO/Meyer Verfahrensrecht (EMRK) Rn. 226.

364 SK-StPO/Meyer Verfahrensrecht (EMRK) Rn. 228; vgl. auch Schabas S. 807: Es erfordere normalerweise zwingende Elemente, um den Gerichtshof dazu zu veranlassen, von der Sachverhaltserstellung der nationalen Gerichte abzu- 
ist - der Sachverhalt schon im nationalen Instanzenzug genügend erstellt oder unbestritten ist. ${ }^{365}$ In diesen Fällen nimmt der Gerichtshof nämlich nur noch eine Schlüssigkeitsprüfung des dargelegten Sachverhalts vor. ${ }^{366}$ Eine besonders genaue Prüfung der Schlüsse der nationalen Gerichte erfolgt bei vorgebrachten Verstössen gegen Art. 2 und Art. 3 EMRK. ${ }^{367}$

Ist der Sachverhalt nicht genügend erstellt, beispielsweise weil der Mitgliedstaat über eine Informationsherrschaft der Beweismittel verfügt und eine effektive Aufklärung des Sachverhaltes verweigert, hat der Gerichtshof als Tatsacheninstanz zu fungieren. ${ }^{368}$ Gemäss Regel A1 VerfOAnnex kann der Gerichtshof jede sachdienliche Ermittlungshandlung ergreifen. Die Mitgliedstaaten sind ihrerseits zur Mitwirkung verpflichtet (vgl. Art. 34 EMRK und Art. 38 EMRK). ${ }^{369}$ Der EGMR dürfte selbst etwa Augenscheine vornehmen oder Sachverständige befragen ${ }^{370}$; in der Praxis hat sich dieses Vorgehen allgemein allerdings nicht etabliert. ${ }^{371}$ Dem EGMR steht es gemäss Regel A1 Abs. 2 VerfO-Annex weiter frei, jede Institution seiner Wahl um Bewertungen und Informationen zu ersuchen. Schliesslich steht es dem Gerichtshof im Sinne eines ,faktischen Outsourcings" offen, Berichte anderer Institutionen beizuziehen. ${ }^{372}$ Konkret

weichen, da der Gerichtshof der Tatsachenfeststellung der nationalen Gerichte grosse Achtung beimesse. Vgl. auch EGMR - Edwards/GBR, Urt. v. 16.12.1992, 13071/87, Rn.34; EGMR (GK) - Giuliani u. Gaggio/ITA, Urt. v. 24.03.2011, 23458/02, Rn. 180; EGMR - Barbu Anghelescu/ROM, Urt. v. 05.10.2004, 443/99, Rn. 52; EGMR - Avsar/TUR, Urt. v. 10.07.2001, 25657/94, Rn.283; EGMR Klaas/GER, Urt. v. 22.09.1993, 15473/89, Rn. 29.

365 Jacobs/White/Ovey S. 27; SK-StPO/Meyer Verfahrensrecht (EMRK) Rn. 228.

366 Schabas S. 807; SK-StPO/Meyer Verfahrensrecht (EMRK) Rn. 228; vgl. etwa EGMR - Avsar/TUR, Urt. v. 10.07.2001, 25657/94, Rn. 283.

367 Vgl. etwa EGMR - Avsar/TUR, Urt. v. 10.07.2001, 25657/94, Rn. 284; EGMR Ribitsch/AUT, Urt. v. 04.12.1995, 18896/91, Rn. 32.

368 SK-StPO/Meyer Verfahrensrecht (EMRK) Rn. 229 f.; Jacobs/White/Ovey S. 27; hinsichtlich der Praxis des EGMR kritisch vgl. Sснürer EuGRZ 2014, 512 ff.

369 Ausf. SK-StPO/Meyer Verfahrensrecht (EMRK) Rn. 237 ff.; zum Problem unkooperativer Konventionsstaaten vgl. auch Leach/Paraskeva/Uzelac S. 74.

370 Vgl. etwa EGMR - Peers/GRE, Urt. v. 19.04.2001, 28524/95, Rn. 4 (Fact-FindingBesuch des Gefängnisses Koridallos).

$371 \mathrm{Zu}$ einer detaillierten Analyse von Fact-Finding Missionen der Kommission und des Gerichtshofs vgl. Leach/Paraskeva/Uzelac S. 4 ff.; vgl. auch Jacobs/ White/Ovey S. 27; SK-StPO/Meyer Verfahrensrecht (EMRK) Rn. 232; Karpenstein/Mayer/Wenzel Art. 38 Rn. 6.

372 SK-StPO/Meyer Verfahrensrecht (EMRK) Rn. 236: Der Gerichtshof greife auf Berichte von NGOs, den UN-Organen und Nebenorganen, UN-Sonderorganisationen, Europaratsgremien, der EU oder gar auf Feststellungen anderer justizieller Menschenrechtsorgane zurück. Dieses faktische Outsourcing ist dabei vom 
bedeutet dies, dass Soft Law-Regelwerke bzw. deren Implementierungsberichte selbst dann der Sachverhaltserstellung vor dem Gerichtshof dienen können, wenn sie keine der Parteien als Beweismittel vorgebracht hat. Greift der Gerichtshof allerdings in Form von Evaluationen und Berichten fremde Tatsachenfeststellungen auf, liegt es fortan am Gerichtshof, die Qualitätsanforderungen sicherzustellen ${ }^{373}$ resp. deren Beweiswert bei der Beweiswürdigung zu berücksichtigen. Bei der Bewertung des Beweiswertes können sowohl formelle als auch materielle Kriterien massgeblich werden. Entscheidend kann beispielsweise sein, ob die Berichte von unabhängigen, etablierten Expertengremien wie etwa dem CPT stammen, ob die Berichte aktuell sind resp. ob sie mit dem in Frage stehenden Beurteilungszeitraum übereinstimmen, wie konkret sie verfasst sind und inwieweit die festgestellten Tatsachen weiter dokumentiert wurden resp. wie nachvollziehbar diese sind.

Wie bereits ausgeführt, ist zu vermuten, dass Implementierungsberichten bei der Sachverhaltserstellung eine umso grössere Bedeutung zukommt, je tiefer das Beweismass ist. Da die Informationsherrschaft des Staates betreffend Vorgänge im Strafvollzug und Unterbringungsmodalitäten $\mathrm{zu}$ beweisrechtlichen Erleichterungen für den Strafgefangenen führt, hat der Beschwerdeführer zunächst lediglich den Beweis des ersten Anscheins zu erbringen. Da die Implementierungsberichte in der Praxis geeignet sein dürften, die Vorbringen des Beschwerdeführers zu untermauern, ist zu erwarten, dass der Anscheinsbeweis mit deren Hilfe regelmässig erbracht werden kann.

Die Wirkungsweise des Beizugs von Implementierungsberichten der Soft Law-Regelwerke auf der Ebene der Sachverhaltserstellung unterscheidet sich in seinen Wirkungen massgeblich vom Beizug internationaler Regelungsinstrumentarien auf der Rechtsebene. Ein Transformationsprozess im engeren Sinn (Wandlung von Soft Law zu Hard Law) findet von vorneherein nicht statt. Auch wenn zum Zeitpunkt der Verabschiedung des Soft Law-Regelwerks sicherlich nicht bezweckt war, dass ein darauf basierender Implementierungsbericht später als Beweismittel in

Vorgehen nach Regel A 1 Abs. 2 VerfO (Möglichkeit der Kammer, eine Person oder Institution um Bewertung und Informationen zu ersuchen) und von der Anwendung der Regel 44 Abs. 3 lit. a VerfO (Einladung von Institutionen zu einer motion to intervene) zu unterscheiden. Vgl. auch Jacobs/White/Ovey S. 27; vgl. unter vielen EGMR - Ostrovar/MDA, Urt. v. 13.09.2005, 35207/03, Rn. 58 (Aufgreifen von CPT-Berichten).

373 SK-StPO/Meyer Verfahrensrecht (EMRK) Rn. 236; Meyer FS Paeffgen, S. 793 (804 ff.); Bergsmo (Hrsg.), Quality Control in Fact-Finding. 
einem gerichtlichen Verfahren dient, ist darin kein relevantes Problem zu sehen. Dies umso weniger, als die Mitgliedstaaten vor dem Gerichtshof ohnehin zur Mitwirkung bei der Sachverhaltserstellung verpflichtet wären. Der Konventionsstaat wäre damit nämlich ohnehin verpflichtet, die in den Implementierungsberichten offengelegten Informationen dem Gerichtshof zur Verfügung zu stellen, zumindest wenn der Gerichtshof darum ersucht.

\section{Zwischenfazit}

Soft Law bezeichnet rechtlich unverbindliche Regelwerke. Sie zeichnen sich oft durch besonders detaillierte Bestimmungen aus, gewähren dem Einzelnen aber keine subjektiven Rechte. Trotz ihres damit primär nicht durchsetzbaren Charakters erlangen sie oft eine grosse praktische Wirksamkeit. Diese basiert auf drei interagierenden Hauptpfeilern: (1) Das Soft Law selbst führt eine effektive Implementierungskontrolle durch und übt auf diese Weise (sanften) Druck auf die Vertragsparteien zur effektiven Umsetzung aus. (2) Das Soft Law dient als Inspirationsquelle für neue Rechtssetzungs- bzw. Reformprojekte. (3) Das Soft Law findet Eingang in die Rechtsprechung und nimmt auf diese Weise Einfluss auf die Rechtsfortbildung.

Die Implementierungskontrolle entfaltet eine Art Reflexwirkung, indem auf diese Weise Rechtssetzungsprojekte angestossen oder die rechtsanwendenden Behörden dazu veranlasst werden, die Soft Law-Regelwerke aufzugreifen und umzusetzen, oder indem der Beschwerdeführer bei der Sachverhaltsdarstellung Monitoringberichte als Beweismittel vorbringt.

Unter dem Demokratiegesichtspunkt ist das Aufgreifen von Soft Law in der Rechtssetzung unproblematisch, solange dieses als blosse Inspirationsquelle fungiert und nicht von einem eigentlichen Befolgungszwang auszugehen ist. Erfolgt die Rezeption in der Rechtsprechung, ist insbesondere die Grenze der richterlichen Rechtsfortbildung zu wahren. Die EMRK verfolgt grundsätzlich einen evolutiven Auslegungsansatz („EMRK als living instrument ${ }^{\text {“374); }}$; die Berücksichtigung von Soft Law bei der Schutzgehaltsbestimmung bleibt damit und auch mit Blick auf den telos der EMRK grundsätzlich zulässig, solange diese nicht leichtfertig erfolgt, son-

374 Vgl. EGMR - Tyrer/GBR, Urt. v. 25.04.1978, 5856/72, Rn.31; EGMR (GK) Selmouni/FRA, Urt. v. 28.07.1999, Rn. 101; vgl. auch Bates S. 326 ff.; MaHONEY in: Widmer, S. 143 (147 ff.). 
dern mit Blick auf die Wertigkeit der jeweiligen Bestimmung für die anzuwendende Norm. Zurückhaltung der rechtsanwendenden Behörde ist weiter insbesondere dann zu fordern, wenn das Soft Law selbst Vorbehalte resp. Ausnahmemöglichkeiten vorsieht; auf diese Weise ist sicherzustellen, dass seitens der rechtsanwendenden Behörde nicht von einem inhaltlich weitergehenden als dem tatsächlich bestehenden europäischen Konsens ausgegangen wird. Der Beizug von Soft Law-Implementierungsberichten internationaler Gremien auf der Ebene der Sachverhaltserstellung führt zu keinem Transformationsprozess; relevante rechtliche Probleme stellen sich hier nicht. Stützt die rechtsanwendende Behörde sich bei der Sachverhaltserstellung auf solche Berichte, hat sie aber eine Qualitätskontrolle vorzunehmen; der Beweiswert der Berichte haben sich in der Beweiswürdigung niederzuschlagen.

Das Aufgreifen von Soft Law, ob gerechtfertigt oder nicht, bringt jedenfalls eine gewisse Rechtsunsicherheit mit sich. Für die Verhandlungsparteien scheinen die Folgen des Soft Law in Bezug auf dessen Verbindlichkeit damit oft nicht mehr vorhersehbar. Wenn in der nachfolgenden Untersuchung im Bereich des Strafvollzugsrechts tatsächlich ein Transformationsprozess zum "hardening Soft Law" festgestellt werden sollte, wird sich in der Zukunft zeigen, ob und wie sich dies auf die Bereitschaft der Mitgliedstaaten in Bezug auf die Erarbeitung weiterer Soft Law-Regelwerke auswirken wird. 375

Kapitel 5: Absolute Rechte

I. Vorbemerkungen

Während im ethischen und philosophischen Kontext über die Frage debattiert wird, ob absolute moralische Rechte existieren, ${ }^{376}$ geht es in dieser

375 So wurde bereits festgestellt, dass Unsicherheiten betreffend fortschreitende Verbindlichkeit von Soft Law bei den Vertragsstaaten in Einzelfällen zu Widerwillen führen kann. „Hardening Soft Law" birgt damit zumindest die Gefahr einer Gegenreaktion und vermehrter Abneigung der Staaten, womit Soft Law letztlich Opfer seines eigenen Erfolges würde. Am Beispiel der Erklärung zu den Rechten indigener Völker der Vereinten Nationen: Villeneuve in: Lagoutte/ Gammeltoft-Hansen/Cerone, S. $213 \mathrm{ff}$.

376 Vgl. etwa schon: Gewirth The Philosophical Quarterly 1981, 1 ff.; Levinson The Philosophical Quarterly 1982, 73 ff.; GewIrTh The Philosophical Quarterly $1982,348 \mathrm{ff}$. 
Arbeit darum, was ein absolutes Recht im rechtlichen Sinn ausmacht. ${ }^{377}$ Es wird nämlich gemeinhin davon ausgegangen, dass Art. 3 EMRK absolut gilt. ${ }^{378} \mathrm{Da}$ alle Garantien von Art. 3 EMRK - das Folterverbot und das Verbot der unmenschlichen und der erniedrigenden Behandlung oder Strafe - als absolut anerkannt werden, ${ }^{379}$ können diese Gehalte in diesem

377 Ausf. Mavronicola HRLR 2012, 723 (724f.): Die Frage nach den Merkmalen absoluter Rechte gehe auch der Frage voraus, ob das Gesetz absolute Rechte vorsehen sollte.

378 SK-StPO/Meyer Art. 3 EMRK Rn. 8: Art. 3 EMRK unterliege als einzige Bestimmung der EMRK keinerlei Ausnahmen, Einschränkungen oder Vorbehalten. Vgl. auch Karpenstein/Mayer/Sinner Art. 3 Rn. 1. Trotz des klaren Wortlautes führte die Einschränkungslosigkeit von Art. 3 EMRK in der Vergangenheit immer wieder zu Diskussionen. Vgl. zu dieser Debatte im Einzelnen Grabenwar-

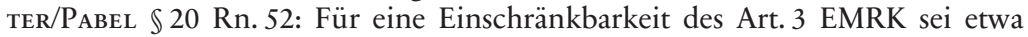
vorgebracht worden, dass Art. 2 EMRK (Recht auf Leben) unter besonderen Voraussetzungen zum Schutz des Lebens eines anderen die gezielte Tötung vorsehe, was zu einem Wertungswiderspruch führen würde (der Staat dürfe zur Rettung eines Lebens töten, hingegen nicht foltern, eine unmenschliche oder erniedrigende Behandlung anwenden). Gegen dieses Argument spricht zum einen der klare Wortlaut von Art. 3 EMRK und die Tatsache, dass zwischen Art. 2 EMRK und Art. 3 EMRK auch im Rahmen von Art. 15 EMRK differenziert wird. So darf von Art. 3 EMRK in keinem Fall, das heisst auch nicht bei einer Gefährdung des „Lebens der Nation“ durch Krieg oder einen anderen öffentlichen Notstand, abgewichen werden (Art. 15 EMRK). Eine Abweichung von Art. 2 EMRK ist hingegen bei Todesfällen infolge rechtmässiger Kriegshandlungen zulässig (Art. 15 Abs. 2 EMRK). Vgl. ausf. auch STEIN S. $334 \mathrm{ff}$. Gemäss STEIN bleibe im Wege der Auslegung von Art. 3 EMRK jedenfalls kein Raum für eine Analogie zu Art. 2 EMRK. Eine solche Fortbildung wäre als eine schöpferische Rechtsfindung contra legem zu verstehen, welche gegen die fundamentalen Grundsätze der Rechtsstaatlichkeit verstossen würde. Vgl. ferner Gebauer NVwZ 2004, 1405 ff.: Gebauer ist der Ansicht, dass die Absolutheit des Folterverbots weder durch formale Verweise auf Folterverbotsnormen noch mit Verweisen auf die Menschenwürde zu begründen sei, vielmehr liege das Erfordernis der Absolutheit des Folterverbots in der dieser Sache inhärenten Missbrauchsgefahr. Die Absolutheit von Art. 3 EMRK durch die Einführung einer Rechtfertigungsmöglichkeit in Frage zu stellen, ist vor den genannten Argumenten meines Erachtens jedenfalls klar abzulehnen.

379 SK-StPO/Meyer Art. 3 EMRK Rn. 8: Art. 3 EMRK differenziere intern nicht zwischen den Arten der unzulässigen Beeinträchtigung, so dass es keine normimmanente Möglichkeit der Rechtfertigung niedrigschwelligerer Beeinträchtigungen gebe. So auch Addo/Grief EJIL 1998, 510 (512). Vgl. aber MavronicoLA HRLR 2012, 723 (755), welche einwendet, dass sich bei Beweisverwertungsfragen, welche sich im Rahmen von Art. 6 EMRK stellen, eine Hierarchie unter den Garantien von Art. 3 EMRK abzuzeichnen scheine. Dies werfe die Frage auf, ob dies den Parameter der Anwendbarkeit untergrabe. Vgl. zur Bedeutung 
Kontext gemeinsam abgehandelt werden. Es geht hier denn auch nicht um die Konturierung der Grenzen zwischen diesen Garantien, sondern um Art. 3 EMRK als solchen - also um die Frage der Wesensmerkmale eines absoluten Rechts. ${ }^{380}$

II. Merkmale und Kernelemente eines absoluten Rechts am Beispiel von Art. 3 EMRK

Bei der Frage, welche Merkmale die Absolutheit ausmachen, wird auf rechtstheoretischer Ebene auf zwei unterschiedliche Parameter zurückgegriffen: die Anwendbarkeit des absoluten Rechts („applicability criterion“) und die methodische Bestimmung des zu schützenden Inhalts des absoluten Rechts („specification criterion“). ${ }^{381}$ Diese beiden Parameter sind miteinander verbunden. ${ }^{382}$ Das Anwendbarkeitskriterium geht der Frage nach, ob und wann die Anwendung eines Standards aufgrund konkurrierender oder konfligierender Interessen rechtmässig verdrängt werden kann, ${ }^{383}$ während das Spezifitätskriterium die Konkretisierung des gewährleisteten Massstabes betrachtet und den Fokus auf die Definition und Umgrenzung des zu schützenden Standards legt. ${ }^{384}$ Mit anderen Worten bestimmt das Spezifitätskriterium den Inhalt des Rechtes und das Anwendbarkeitskriterium legt dann fest, ob von diesem Standard abgewichen werden darf.

1. Hinsichtlich der Anwendbarkeit

\subsection{Kriterien}

Hinsichtlich des Anwendbarkeitskriteriums basiert die Absolutheit auf drei kumulativen Merkmalen: (1) keine Rechtfertigung durch ein anderes legitimes in der demokratischen Gesellschaft notwendiges Ziel, ${ }^{385}$ (2) kei-

des Art. 3 EMRK im Rahmen von Art. 6 EMRK auch ausf. SK-StPO/Meyer Art. 3 EMRK Rn. $131 \mathrm{ff}$.

380 Mavronicola HRLR 2012, 723 (725).

381 Mavronicola HRLR 2012, 723 (724 f., S. 729).

382 Mavronicola HRLR 2012, 723 (724 f., S. 729).

383 Mavronicola HRLR 2012, 723 (724f.).

384 Mavronicola HRLR 2012, 723 (724f.).

385 Vgl. etwa Riegel/Speicher StV 2016, 520 ff.: Ein Abwiegen des Rechtes mit den Zwecken, welche die Massnahme verfolgt, erfolgt nicht. 
ne Abweichung im Falle eines Notstandes (vgl. auch Art. 15 EMRK) und (3) Irrelevanz des Verhaltens des Menschenrechtsträgers. ${ }^{386}$

Das Missbrauchsverbot gemäss Art.17 EMRK bleibt hingegen - als Grundvoraussetzung für die Anwendbarkeit des Rechts als solches - unberührt von der Absolutheit des Rechts.

\subsection{Prüfungsmethodisches Vorgehen}

Das prüfungsmethodische Vorgehen variiert je nach Funktion des Menschenrechts. Bei Abwehrrechten wird im Allgemeinen eine dreistufigen Prüfung ${ }^{387}$ vorgenommen: Zunächst werden der Schutzbereich selbst, sodann der Eingriff in den Schutzbereich und in einem letzten Schritt die Rechtfertigung des Eingriffs geprüft. ${ }^{388}$ Der Schutzbereich ist der Anwendungsbereich des Menschenrechts; er legt den „potentiellen Gewährleistungsbereich"389 fest. ${ }^{390}$ Geprüft wird in diesem Schritt, ob das fragliche Verhalten überhaupt von einem bestimmten Menschenrecht erfasst ist. Bei der Prüfung des Eingriffs wird ermittelt, ob eine Einschränkung der gewährleisteten Menschenrechtsposition - also des Schutzbereichs - vorliegt. ${ }^{391}$ Wird ein Eingriff in den Schutzbereich festgestellt, hat dies im Allgemeinen die Frage nach der Rechtfertigung des Eingriffs zur Folge. ${ }^{392}$ Obwohl eine Wesensgehaltsgarantie vom EGMR grundsätzlich nicht ausdrücklich vorgesehen ist, prüft er, ob ein Eingriff den Kerngehalt eines Rechts betrifft. ${ }^{393}$

386 Vgl. Mavronicola HRLR 2012, 723 (737).

387 Der EGMR handelt die ersten beiden Prüfungsschritte oft zusammen ab, so dass praktisch eine zweistufige Prüfung erfolgt: SK-StPO/Meyer Methodik der Grundrechtsanwendung (EMRK) Rn. 2: Zu Gunsten einer exakten Begriffsbildung und einer differenzierten Eingriffsdogmatik sei allerdings das dreistufige Vorgehen zu bevorzugen. Kritisch auch Papien FS Wildhaber, S. 523 (525): Verwischung von Schutzbereichsbestimmung und Beschränkung, selbst dogmatisch sei eine Unterscheidung oftmals nur schwer möglich.

388 Grabenwarter/Pabel $\mathbb{S} 18$ Rn. 1 .

389 SK-StPO/MeYer Methodik der Grundrechtsanwendung (EMRK) Rn. 13.

390 Grabenwarter/Pabel $\mathbb{1} 18$ Rn. 2.

391 Schilling Rn. 88 ff.; Grabenwarter/Pabel $₫ 18$ Rn. 5.

392 Grabenwarter/Pabel $\mathbb{1} 18$ Rn. 5; zur Prüfungsmethodik der Rechtfertigung bei Abwehrrechten ausf. SK-StPO/Meyer Methodik der Grundrechtsanwendung (EMRK) Rn. 31 ff. Vgl. auch Schilling Rn.93, welcher in diesem Zusammenhang von der Prüfung der "Zulässigkeit" spricht.

393 SK-StPO/MeYer Methodik der Grundrechtsanwendung (EMRK) Rn. 53. 
Auch wenn es um die Frage der Beachtung positiver Gewährleistungsoder Schutzpflichten geht ${ }^{394}$, wird in der Regel eine dreistufige Prüfung durchlaufen. Im ersten Prüfungsschritt wird ermittelt, ob dem in Frage stehenden Menschenrecht überhaupt eine leistungsrechtliche Dimension innewohnt, ob der Staat die Möglichkeit hat, dieser zu entsprechen und ob ein individueller Anspruch auf Erfüllung der positiven Pflicht besteht. ${ }^{395}$ Als Korrelat zur Eingriffsprüfung - wie sie bei einem Abwehrrecht durchgeführt wird - wird bei der positiven Gewährleistungspflicht in einem zweiten Schritt geprüft, ob eine Beeinträchtigung durch das Unterlassen einer Pflicht verursacht wird. ${ }^{396}$ Bei der Erfüllung einer positiven Verpflichtung wird dem Staat vor allem hinsichtlich der zu ergreifenden Mittel ein grosser Spielraum zugestanden. ${ }^{397}$ Ist der Staat einer solchen Pflicht nicht nachgekommen, ist in einem dritten Schritt die Rechtmässigkeit des Unterlassens zu prüfen. ${ }^{398}$ Anders als bei Abwehrrechten, bei denen sich mögliche Rechtfertigungsgründe häufig bereits dem Wortlaut entnehmen lassen, finden sich bezüglich der Gewährleistungspflichten keine solchen textlich verankerten Gründe. Der EGMR greift bei den positiven Verpflichtungen vielmehr auf eine Kriteriensammlung zurück, welche er in seinem Fallrecht für jedes Menschenrecht einzeln entwickelt hat. ${ }^{399}$

394 Zur Entwicklung positiver Schutzpflichten durch den EGMR im Allgemeinen: Mowbray S. 1 ff.; Starmer in: Jowell/Cooper, S. 139 ff.: Starmer sieht die Grundlage der positiven Rechte in der Anerkennung des Umstands, dass ein rein abwehrrechtlicher Ansatz für sich alleine nicht geeignet ist, einen effektiven Schutz der gewährleisteten Menschenrechte zu bieten.

395 SK-StPO/Meyer Methodik der Grundrechtsanwendung (EMRK) Rn.6; vgl. auch Grabenwarter/Pabel $\$ 19$ Rn. 5.

396 Grabenwarter/Pabel $\mathbb{1} 19$ Rn. 5; SK-StPO/Meyer Methodik der Grundrechtsanwendung (EMRK) Rn. 9, 22: Die Unterlassung habe bei einer positiven Verpflichtung zwar keinen Eingriffscharakter im technischen Sinne, nichtsdestotrotz könne sie aber eine Beeinträchtigung des gewährleisteten Menschenrechts sein.

397 SK-StPO/Meyer Methodik der Grundrechtsanwendung (EMRK) Rn. 10; zu den Beurteilungsspielräumen der Mitgliedstaaten bei positiven Verpflichtungen differenziert Grabenwarter/Pabel $\mathbb{1} 19$ Rn. 7.

398 SK-StPO/MeYer Methodik der Grundrechtsanwendung (EMRK) Rn. 10; vgl. aber Dörr/Grote/Marauhn/KrIEger Kap. 6 Rn. 45: KRIEger befürwortet zwar eine dreistufige Prüfung kritisiert aber, dass die Konventionsorgane bei der Prüfung von Schutzpflichten nicht immer zwischen der Frage, ob der Schutzbereich durch staatliches Unterlassen berührt sei, und der Rechtfertigung dieses Unterlassens differenzieren würden.

399 SK-StPO/Meyer Methodik der Grundrechtsanwendung (EMRK) Rn. 10; vgl. auch Harris/O'Boyle/Warbrick S. 26: Die für die Abwehrrechte ausdrücklich genannten Kriterien können dabei auch bei positiven Verpflichtungen zumin- 
Im Allgemeinen kann ein Unterlassen durch die Wahrung der Rechte Dritter, zu Gunsten gegenläufiger Interessen der Allgemeinheit, aufgrund politischer Richtungsentscheidungen demokratisch legitimierter Organe, zu Gunsten konfligierender Ziele oder aufgrund einer Knappheit an Ressourcen gerechtfertigt sein. ${ }^{400}$ Für die Rechtmässigkeit des Unterlassens wird jedenfalls keine gesetzliche Grundlage gefordert. 401

Der absolute Charakter - mithin die Vorbehaltslosigkeit des Art. 3 EMRK - führt aufgrund des Anwendbarkeitskriteriums dazu, dass bloss mehr eine zweistufige Prüfung ${ }^{402}$ vorzunehmen ist. Im ersten Schritt wird wie bis anhin geprüft, ob der Schutzbereich greift. Geht es um die abwehrrechtliche Dimension des Art. 3 EMRK, folgt in einem zweiten Schritt die Prüfung, ob ein Eingriff vorliegt und, bei der gewährleistungsrechtlichen Dimension, ob eine Beeinträchtigung des Schutzgehaltes durch ein Unterlassen einer bestehenden positiven Verpflichtung vorliegt. Der dritte Prüfungsschritt einer möglichen Rechtfertigung des Eingriffs bzw. der Beeinträchtigung entfällt. ${ }^{403}$ Die Menschenrechtsverletzung liegt damit grundsätzlich per se vor, wenn es zu einer Beeinträchtigung des Schutzgehaltes kommt. Eingriffe in den Schutzgehalt sind damit grundsätzlich nicht zulässig. 404

dest von einer gewissen Relevanz sein: EGMR - Powell u. Rayner/GBR, Urt. v. 21.02.1990, 9310/81, Rn. 40; EGMR (GK) - Hatton u.a./GBR Urt. v. 08.07.2003, 36022/97, Rn. 98; EGMR - Demir u. Baykara/TUR, Urt. v. 12.11.2008, 34503/97, Rn. 111; vgl. auch Dörr/Grote/Marauhn/Krieger Kap. 6 Rn. 45: Gemäss KrieGER würde eine Schutzpflichtverletzung nicht unmittelbar an den Schranken von Abs. 2 geprüft, die Abwägung erfolge vielmehr im Rahmen von Abs. 1, wobei allerdings die „Wertung“ von Abs. 2 beigezogen würde.

400 SK-StPO/MeYER Methodik der Grundrechtsanwendung (EMRK) Rn. 10.

401 Dörr/Grote/Marauhn/Krieger Kap. 6 Rn. 45; SK-StPO/Meyer Methodik der Grundrechtsanwendung (EMRK) Rn. 11.

402 Wird bei Abwehrrechten durch die Zusammenfassung der ersten beiden Prüfungsschritte von einer zweistufigen Prüfung ausgegangen, führt der absolute Charakter des Art. 3 EMRK analog dazu bei Zusammenfassung der ersten beiden Prüfungsschritte zu einer einstufigen Prüfung. Vgl. etwa SMEt in: Brems/ Gerards, S. 273.

403 Mavronicola HRLR 2012, 723 (736); vgl. auch schon Hassemer FS Maihofer, S. 183 (183f.): „[...] Wer sich auf eine Abwägung zwischen den drohenden Schäden und den bedrohten Prinzipien einlässt, hat die Prinzipien schon an diesem Punkt der Überlegung aufgegeben“; Grabenwarter/Pabel $\mathbb{} 20$ Rn. 51; vgl. auch Meyer-Ladewig/Lehnert Art. 3 Rn. 3.

404 Grabenwarter/Pabel $\mathbb{2} 20$ Rn. 40; SK-StPO/Meyer Methodik der Grundrechtsanwendung (EMRK) Rn. 4; vgl. auch SMET HRLR 2017, 499 (508); STEIN S. 333: Jeder Eingriff in das geschützte Fundamentalrecht bedeute eine Verletzung der Vorschrift. Vgl. ferner Möhlenbeck S. 53 f. 
Vorbehaltlos garantierte Rechte schliessen allerdings die Annahme immanenter bzw. impliziter Schranken nicht zwingend aus. Auch der EGMR anerkennt bisweilen immanente bzw. implizite Schranken. ${ }^{405} \mathrm{Da}$ die EMRK, obwohl ihr ein Verfassungscharakter innewohnt, keine „vollintegrierte Verfassung“ ist, ${ }^{406}$ bleibt der EGMR bei der Anerkennung immanenter Schranken jedoch zurückhaltend. Für Art. 3 EMRK hat der EGMR (bislang) soweit ersichtlich auch keine eindeutigen verfassungsimmanenten Schranken anerkannt. ${ }^{407}$ Schutzbereich und Kerngehalt von Art. 3 EMRK sind damit deckungsgleich. ${ }^{408}$ Anerkennt man im Allgemeinen bei vorbehaltlos garantierten Rechten immanente Schranken, ist jedenfalls die Verhältnismässigkeit zu wahren. ${ }^{409}$ Die Verhältnismässigkeitsprüfung unterliegt dann gewissen Besonderheiten. ${ }^{410}$

Aus dem absoluten Charakter von Art. 3 EMRK folgt zusammenfassend hinsichtlich des Anwendbarkeitskriteriums, dass kein noch so grosses

405 SK-StPO/MeYer Methodik der Grundrechtsanwendung (EMRK) Rn. 4: Der EGMR habe bei gewissen schrankenlos gewährleisteten Rechten wie beim Recht auf Zugang zum Gericht gem. Art. 6 Abs. 1 EMRK oder dem Recht auf freie Wahlen gem. Art. 3 ZP I zur EMRK immanente bzw. implizite Schranken anerkannt. Vgl. auch Zimmermann in: Böllmann/Hemme/Korkmaz/Kühn/ Sinn, S. 63 (69). Vgl. weiter Peters/Alwicker $\$ 3$ Rn. 20, welche allerdings dafür plädieren, impliziten Schranken enge Grenzen zu setzen; ein Verweis auf die Verwaltungspraktikabilität oder eine angespannte Haushaltslage müssten etwa von vorneherein als taugliche implizite Schranken ausscheiden.

406 Breuer in: Zimmermann, S. 51 ff.: Die EMRK sei eine „kupierte“ Verfassung; es handle sich um eine Verfassung mit Grundrechtsteil, jedoch ohne Staatsorganisationsteil; SK-StPO/MeYer Methodik der Grundrechtsanwendung (EMRK) Rn. 4: Die EMRK gewähre mit ihrem Grundrechtskatalog vielmehr Einzelrechte mit spezifischen Einschränkungsvorbehalten. Vgl. auch Alleweldt in: Zimmermann, S. 75: Die EMRK inkl. Rechtsprechung des EGMR sei kein klassisches Völkerrecht. Wenn man den Terminus der Verfassung in einem weiten Sinne verstehe, könne man sie aber als Teil der europäischen Verfassungsordnung bezeichnen.

407 Vgl. ausf. SK-StPO/Meyer Art. 3 EMRK Rn. 9 m.w.N.: Es würden aber immanente Schranken diskutiert, welche Ausnahmen im vitalen Interesse staatlicher Selbstbehauptung und zum Schutz der Menschenwürde und des Lebens von (potentiellen) Opfern (Schutzpflicht) rechtfertigen können sollen.

408 Bei nicht absolut gewährleisteten Rechten stellen sich hinsichtlich des absoluten Kerngehaltes im Allgemeinen denn auch dieselben Fragen wie bei Art. 3 EMRK. Zum „absolut geschützten, aber abwägungsoffenen Kernbereich“ vgl. etwa BALDus JZ 2008, 218 (224 ff.).

409 SK-StPO/Meyer Methodik der Grundrechtsanwendung (EMRK) Rn. 5, 44.

410 SK-StPO/MeYer Methodik der Grundrechtsanwendung (EMRK) Rn. 45: Das Kriterium des dringenden sozialen Bedürfnisses spiele als Ausgangspunkt der Überlegungen etwa keine Rolle. 
entgegenstehendes Interesse Folter, unmenschliche oder erniedrigende Behandlung oder Strafe rechtfertigen kann. Eine Interessenabwägung findet in diesem Sinn nicht statt. Von den Garantien des Art. 3 EMRK darf mit anderen Worten selbst dann nicht abgewichen werden, wenn das Leben der Nation bedroht ist. Unter Umständen bleibt allerdings eine Berücksichtigung konfligierender Rechte bei der Bestimmung des Schutzbereichs möglich. ${ }^{411}$

2. Bei der Bestimmung des Schutzbereichs und des Eingriffs

Nach welchen Grundsätzen die Verpflichtung festzulegen ist, ist eine Frage des Spezifitätskriteriums. ${ }^{412}$ Aufgrund der Vorbehaltslosigkeit von Art. 3 EMRK ist die Bestimmung des Schutzbereichs von umso grösserer Relevanz ${ }^{413}$ da die positive oder negative Verpflichtung, einmal ausgelöst, nicht mehr rechtmässig ausser Kraft gesetzt werden kann. Deshalb legt auch der Gerichtshof bei Art. 3 EMRK besonderen Wert auf die Schutzbereichsbestimmung. ${ }^{414}$

\subsection{Relativität der Schutzbereichsbestimmung und des Eingriffsbegriffs}

Eine unmenschliche und erniedrigende Behandlung oder Bestrafung im Sinne von Art. 3 EMRK setzt ein Mindestmass an Schwere voraus. ${ }^{415}$ Entscheidend ist damit die Festlegung dieses Mindestmasses. Bereits im Urteil IRL/GBR hielt der EGMR fest, dass die Ermittlung der Mindestmassschwelle schon aufgrund der Natur der Dinge relativ zu erfolgen habe. ${ }^{416}$ $\mathrm{Zu}$ berücksichtigen sind alle Umstände des Einzelfalls wie die Dauer der Behandlung, die physischen und mentalen Folgen, welche die Behandlung mit sich bringt, in gewissen Fällen auch das Geschlecht, das Alter sowie

411 SK-StPO/MeYer Methodik der Grundrechtsanwendung (EMRK) Rn. 4.

412 Mavronicola HRLR 2012, 723 (738).

413 Siehe auch Mavronicola HRLR 2012, 723 (738f.).

414 Grabenwarter/Pabel $\$ 18$ Rn. 3.

415 SK-StPO/MeYer Art. 3 EMRK Rn. 10.

416 EGMR - IRL/GBR, Urt. v. 18. 01.1978, 5310/71, Rn. 162. Es handelt sich dabei auch um das erste Urteil des EGMR, in welchem dieser eine Verletzung von Art. 3 EMRK feststellte. Zur Relativität der Anwendung des Begriffs der unmenschlichen oder erniedrigenden Behandlung auch Feldman S. 242. 
der Gesundheitszustand des von der Behandlung oder Bestrafung Betroffenen. Auch Natur und Kontext der Behandlung werden berücksichtigt. ${ }^{417}$

Vor dem Hintergrund, dass der EGMR bei der Bestimmung des Schutzbereichs bezüglich des Erreichens des Mindestmasses an Schwere eine relative Betrachtung zuzulassen scheint, stellt sich die Frage, welche Faktoren bei der Schutzbereichsbestimmung legitimerweise berücksichtigt werden dürfen, ohne dass unter dem Deckmantel der Ermittlung des notwendigen Mindestmasses an Schwere die Essenz des Anwendbarkeitskriteriums untergraben wird. ${ }^{418}$ Dieses zielt nämlich gerade darauf $a b$, dass besondere Merkmale oder Verhaltensweisen des Opfers unberücksichtigt bleiben. ${ }^{419}$ Bei der Schutzbereichsbestimmung sollten nur jene relativen Kriterien berücksichtigt werden, welche sich auf den Grad des erlittenen Leidens bzw. die Schwere der zugefügten Behandlung auswirken. ${ }^{420}$ Auf materielle Haftbedingungen angewendet, bedeutet dies, dass der Gesundheitszustand des Inhaftierten berücksichtigt werden muss, wenn sich dieser auf die potentielle Leidzufügung auswirkt. ${ }^{421}$ So ist etwa anzunehmen, dass schlechte hygienische Zustände in einer Zelle bei einem kranken Inhaftierten in

417 EGMR - IRL/GBR, Urt. v. 18. 01.1978, 5310/71, Rn. 162; EGMR (GK) - Labita/ ITA, Urt. v. 06.04.2000, 26772/95, Rn. 120; EGMR (GK) - Ilascu u.a./MDA u. RUS, Urt. v. 08.07.2004, 48787/99, Rn. 427; EGMR (GK) - A. u.a./ GBR, Urt. v. 19.02.2009, 3455/05, Rn. 127; EGMR (GK) - Selmouni/FRA, Urt. v. 28.07.1999, 25803/94, Rn. 100; vgl. auch etwa van ZyL SMIt/SNACKeN S. 127; Mavronicola MLR 2013, 589 (593); Mavronicola HRLR 2012, 723 (749): Die Bestimmung des Schutzbereichs von Art. 3 EMRK basiere damit auf zwei Elementen: der Schwere der Behandlung, welche sich auf die Handlung des Ausführenden beziehe, und der Intensität des Leidens, welche den von der Handlung Betroffenen ins Zentrum rücke. Vgl. ferner Grabenwarter/Pabel $\$ 20$ Rn. 53.

418 Vgl. auch Smet in: Brems/Gerards, S. 273 (275 ff.).

419 Vgl. auch Mavronicola HRLR 2012, 723 (750); zur Relativität der Schutzbereichsbestimmung bei „absoluten“ Rechten kritisch Greer HRLR 2015, 101 (115); kritisch auch Harris/O'Boyle/Warbrick S. 238.

420 Mavronicola HRLR 2012, 723 (750); mit Beispielen siehe auch Mavronicola MLR 2013, 589 (593 f.); vgl. auch PösL S. 50, 53: Die Prüfung der Mindestschwere dürfe nicht zu einer „verkappten Verhältnismässigkeitsprüfung“ führen. Es dürfe nur darum gehen, „Bagatellbehandlungen“ auszuscheiden.

421 Vgl. etwa EGMR - Dybeku/ALB, Urt. v. 18.12.2007, 41153/06, Rn. 47: „The Court accepts that the very nature of the applicant's psychological condition made him more vulnerable than the average detainee and that his detention may have exacerbated to a certain extent his feelings of distress, anguish and fear. In this connection, the Court considers that the fact that the Government admitted that the applicant was treated like the other inmates, notwithstanding his particular state of health, shows the failure of their commitment to improv- 
potenzierter Weise negative Auswirkungen entfalten. Diese Relativität untergräbt gerade nicht das Anwendbarkeitskriterium. Etwa renitentes Verhalten eines Inhaftierten oder der Umstand, dass dieser wegen einer schweren Straftat verurteilt wurde, haben aber keinen Einfluss auf das Mass des erlittenen Leidens oder die Schwere der zugefügten Behandlung 422; es kann folglich nicht davon ausgegangen werden, dass ein gefährlicher Straftäter weniger unter schlechten hygienischen Bedingungen leidet. Würde man Letzteres annehmen, würde das starre Anwendbarkeitskriterium durch eine falsch verstandene Relativität bei der Bestimmung des Schutzbereichs ausgehebelt werden. Die Gefährlichkeit des Betroffenen ist in dieser Konstellation unerheblich für die Bestimmung des Schutzbereichs. ${ }^{423}$

\subsection{Umgang mit konfligierenden Interessen und Rechten}

Die Berücksichtigung konfligierender Rechte bleibt bei der Bestimmung des Schutzbereichs grundsätzlich möglich. ${ }^{424}$ Aufgrund der Interaktion zwischen Anwendbarkeitsforderung und Schutzbereichsbestimmung ist allerdings auch hier in besonderer Weise darauf zu achten, dass die Schutzbereichsbestimmung die Anwendbarkeitsforderung nicht untergräbt.

Schon auf rechtstheoretischer Ebene wird argumentiert, dass das Konzept eines absoluten Rechts eine Hierarchie verlangt: Die Gewährung des absoluten Rechts hat dem nicht absolut zu gewährendem Recht in jedem

ing the conditions of detention in compliance with the recommendations of the Council of Europe."

422 Vgl. etwa EGMR - Dybeku/ALB, Urt. v. 18.12.2007, 41153/06, Rn. 35, 47: „It prohibits in absolute terms torture or inhuman or degrading treatment or punishment, irrespective of the circumstances and the victim's behaviour. [...] The Court considers that the fact that the applicant was found criminally responsible for an offence of homicide and sentenced to life imprisonment is not decisive for the question whether the authorities fulfilled their obligation under Article 3 to protect him from treatment or punishment contrary to this provision“; EGMR (GK) - Labita/ITA, Urt. v. 06.04.2000, 26772/95, Rn. 119.

423 Anders könnte hinsichtlich der Gefährlichkeit dann zu argumentieren sein, wenn es um Massnahmen geht, welche etwa bezwecken eine mögliche Flucht zu verhindern resp. Drittpersonen zu schützen, wenn konkrete Anhaltspunkte für eine solche Gefahr vorliegen. Dazu kritisch Harris/O'Boyle/Warbrick S. 238. Vgl. aber SMEt in: Brems/Gerards, S. 273 (275 ff.), welcher argumentiert, dass die Berücksichtigung der Gefährlichkeit des Betroffenen in diesen Situationen vom absoluten Charakter des Art. 3 EMRK gedeckt sei.

424 SK-StPO/MeYer Methodik der Grundrechtsanwendung (EMRK) Rn. 4. 
Fall vorzugehen. ${ }^{425}$ Weiter wird argumentiert, dass keine absolute positive Pflicht bestehen kann, wenn diese eine negative absolute Pflicht verletzen würde. ${ }^{426}$ Es wird in dieser Konstellation folglich schon gar keine positive Verpflichtung erkannt und auf diese Weise die Notwendigkeit einer Abwägung zwischen zwei absoluten Rechten verhindert. ${ }^{427}$

In Bezug auf den leistungsrechtlichen Gehalt von Art. 3 EMRK, welchem insbesondere bei Sonderstatusverhältnissen besondere Bedeutung zukommt, stellt sich bei der Spezifizierung des Schutzbereichs die Frage, inwieweit fiskalische Interessen des Staates bei der Bestimmung eines angemessenen Standards berücksichtigt werden dürfen. ${ }^{428}$ Auch dies ist mit Blick auf das Zusammenwirken der Schutzbereichsbestimmung und des Anwendbarkeitskriteriums zu beantworten. Die Verlagerung von Elementen, welche in ihrer Essenz zu einer Verhältnismässigkeitsprüfung führen, ${ }^{429}$ vom dritten Prüfungsschritt der Rechtfertigung in den ersten Prüfungsschritt der Schutzbereichsbestimmung scheint mit Blick auf das absolut geltende Anwendbarkeitskriterium zu einer gewissen Widersprüch-

425 Mavronicola HRLR 2012, 723 (734).

426 Mavronicola HRLR 2012, 723 (732); am Beispiel von Gäfgen/GER: MavroniCOLA HRLR 2017, 479 (482 ff.): Die positive Verpflichtung des Staates, eine Person vor Folter, unmenschlicher und erniedrigender Behandlung durch eine Drittperson zu schützen, umfasse das Ergreifen sämtlicher möglicher und zulässiger Mittel. Kein solch zulässiges Mittel - und damit nicht von der positiven Pflicht umfasst - sei aber die Ausübung von Folter, unmenschliche oder erniedrigende Behandlung des Staates gegenüber dem Gefährder. Vielmehr greife hier die negative absolute Pflicht gegenüber dem Gefährder. Vgl. dazu EGMR (GK) - Gäfgen/GER, Urt. v. 01.06.2010, 22978/05.

427 A.A. Greer HRLR 2015, 101 (122 ff.); vgl. auch Greer HRLR 2018, 297 (300, $302 \mathrm{f}$.): Diese Betrachtungsweise würde das Dilemma mit dem Umgang konfligierender Interessen bei absoluten Rechten nicht lösen, sondern nur umgestalten. Eine überzeugende Lösung sei hingegen noch nicht gefunden. Vgl. auch SALIger ZStW 116 (2004), 35 (47), welcher ausführt, dass eine abwägende Verrechnung der Würde eines Geiselnehmers mit der Würde des Opfers nicht überzeuge. Die Verpflichtung des Staates, die Menschenwürde aktiv nicht zu verletzen, sei stärker als die Verpflichtung, sie gegen andere zu schützen. Im Allgemeinen wiege die Verletzung eines Handlungsverbotes tendenziell schwerer als die Nichterfüllung eines Handlungsgebots. Es sei nicht zu sehen, wie der Staat seine Schutzpflichten rechtsstaatlich erfüllen soll.

428 Mavronicola HRLR 2012, 723 (757).

429 Diesbzgl. unkritisch Grabenwarter/Pabel $\$ 20$ Rn. 53, welcher die Prüfung der Mindestschwere des Eingriffs sogar gerade als Interessenabwägung bezeichnet. Eine kritische Differenzierung von Umständen, welche einbezogen werden dürfen, und solchen, die unbeachtet zu bleiben haben, wird in der Folge auch nicht vorgenommen. 
lichkeit oder zumindest zu einer gewissen Aufweichung der absoluten Geltung zu führen. ${ }^{430}$ So erkennt der Gerichtshof in der Berücksichtigung fiskalischer Interessen zwar nicht per se einen Verstoss gegen das Absolutheitsgebot, ${ }^{431}$ hält gleichzeitig aber auch fest, dass mangelnde Ressourcen keine Umstände rechtfertigen dürfen, die Art. 3 EMRK verletzen. 432

Zusammenfassend lässt der Gerichtshof die Berücksichtigung konfligierender Interessen und Rechte unter gewissen Voraussetzungen auch bei absoluten Rechten zu. Konfligierende Interessen können dazu führen, dass das Mindestmass an Schwere des Eingriffs nicht als gegeben erachtet oder dass eine ergriffene Massnahme als sozialadäquat und angemessen angesehen wird. ${ }^{433}$

\subsection{Bestimmtheitserfordernis}

Der absolute Charakter führt allgemein gesagt dazu, dass Beurteilungsspielräume sich auf die Schutzbereichsebene begrenzen. ${ }^{434}$ Mit Blick auf die aufgezeigte Interaktion zwischen Bestimmung der Schutzbereichsebene und der absoluten Anwendbarkeitsforderung kommt allgemein der Bestimmtheit des Massstabes auf Schutzbereichsebene eine umso grössere Bedeutung zu. Die Berücksichtigung von Variablen bei der Schutzbereichsbestimmung führt direkt auch zu einer Variabilität des Ergebnisses. ${ }^{435}$ Dies führt letztlich zu einer gewissen Rechtsunsicherheit, was genau nun abso-

430 Betreffend die absolute Geltung der Menschenwürde und Abwägungsfragen vgl. auch BaLdus AöR 2011, 529 (536 ff.).

431 Vgl. etwa EGMR (GK) - N./GBR, Urt. v. 27.05.2008, 26565/05, Rn. 44; MavroNICOLA HRLR 2012, 723 (757): Die Ursache der Zurückhaltung des Gerichtshofs liege darin, dass er eine zu weite Verlagerung der Pflichten der Konventionsstaaten in den sozioökonomischen Bereich verhindern wolle. Vgl. auch Vermeulen/Battjes in: van Dijk/van Hoof u.a., S. 409.

432 EGMR - Mamedova/RUS, Urt. v. 01.06.2006, 7064/05, Rn. 63; EGMR - Orchowski/POL, Urt. v. 22.10.2009, 17885/04, Rn.153; EGMR - Neshkov u.a./ BUL, Urt. v. 27.01.2015, 36925/10 u.a., Rn. 229; EGMR - Varga u.a./HUN, Urt. v. 10.03.2015, 14097/12 u.a., Rn. 103; vgl. dazu auch Smet in: Brems/Gerards, S. 273 (291 f.).

433 SK-StPO/Meyer Art. 3 EMRK Rn. 8.

434 Vgl. auch SK-StPO/MeYER Methodik der Grundrechtsanwendung (EMRK) Rn. 115.

435 Mavronicola HRLR 2012, 723 (753). 
lut ist. ${ }^{436}$ Eine Weigerung, einen harten Massstab festzulegen, ist deshalb mit Blick auf den absoluten Charakter, der die fundamentale Bedeutung der ihr innewohnenden Garantien unterstreicht, nicht unproblematisch. Der absolute Charakter darf auf der anderen Seite aber auch nicht dazu führen, dass eine Weiterentwicklung des Schutzstandards gebremst oder die Flexibilität der Einzelfallbetrachtung ausgeschlossen würde. ${ }^{437}$

Zusammenfassend ist im Lichte des absoluten Charakters von Art. 3 EMRK für jedes Themengebiet eine möglichst grosse Rechtssicherheit durch einen möglichst harten Massstab zu fordern. Gleichwohl dürfen dadurch die „Living instrument"-Konzeption der EMRK und die notwendige Flexibilität für eine Einzelfallbetrachtung nicht verhindert werden. Mit anderen Worten hat die Rechtsprechung bei absoluten Rechten in besonderer Weise auf die Grenze zwischen einer zu spezifischen und einer zu allgemeinen Rechtsprechung zu achten. Es ist ein ausreichendes Mass an Allgemeingültigkeit und Klarheit zu gewährleisten. ${ }^{438}$

\section{Absolutes Recht im Sonderrechtsverhältnis}

Die Inhaftierung des Betroffenen führt - wie bereits aufgezeigt - zu einem besonderen Näheverhältnis zum Staat, was sich wiederum auf das Rechtsverhältnis zwischen Inhaftiertem und Staat auswirkt, indem dem Inhaftierten einerseits besondere Rechte eingeräumt werden, er andererseits aber auch gewisse Einschränkungen in Kauf zu nehmen hat. Soweit das Sonderstatusverhältnis weitergehende positivrechtliche Garantien oder prozessuale Vorteile zur Folge hat, ist dies mit Blick auf den absoluten Charakter von Art. 3 EMRK von vorneherein unproblematisch. Führt das Sonderstatusverhältnis nämlich im Rahmen der schutzrechtlichen oder gewährleistungsrechtlichen Dimension der Konventionsrechte etwa zur Notwendigkeit einer besonders ausgeprägten "menschenrechtsschützenden“ Regulierung, entspricht dies gleichzeitig auch den Anforderungen des Spezifitätskriteriums bei der Bestimmung des Schutzbereichs absoluter Rechte. Demgegenüber bleiben die aus dem Sonderstatusverhältnis fliessenden

436 Mavronicola HRLR 2012, 723 (751); vgl. auch bereits Addo/Grief EJIL 1998, 510 (522); vgl. auch Pösl S. 50 f.

437 Sinngemäss Mavronicola HRLR 2012, 723 (755); vgl. auch bereits Addo/Grief EJIL 1998, 510 (522).

438 Mavronicola HRLR 2012, 723 (743). 
Nachteile im Lichte der Kernelemente eines absoluten Rechts mit besonderem Bedacht zu würdigen.

Auf nationaler Ebene ist im Sonderstatusverhältnis bei der Einschränkbarkeit eines Grundrechts die Anforderung an die Normdichte der einschränkenden Norm geringer, und dem formellen Gesetzgeber ist eine grosszügigere Gesetzesdelegation erlaubt. ${ }^{439}$ Aufgrund der Anwendbarkeitsforderung des absoluten Rechts, die eine gesetzliche Einschränkung ohnehin ausschliesst, besteht bei absoluten Rechten dieser Nachteil im Sonderstatusverhältnis prima vista nicht. Aufgrund der Wechselwirkungen zwischen Anwendbarkeitskriterium und Spezifitätskriterium ist bei absoluten Rechten allerdings insofern Vorsicht geboten, als im Sinne der positiven Verpflichtung des Staates und mit Blick auf das Spezifitätskriterium stets auf eine genügende menschenrechtsschützende Regulierung des Schutzbereichs zu achten ist. Mit anderen Worten dürfen bei absoluten Rechten im Sonderstatusverhältnis eine Gesetzesdelegation oder unbestimmte Normen auf Schutzbereichsebene nicht zu Unsicherheiten führen, was nun absolut geschützt sei.

Im Allgemeinen führt das Sonderstatusverhältnis heute nicht mehr zur Annahme, dass das öffentliche Interesse jenes des Inhaftierten grundsätzlich überwiegt, vielmehr hat immer eine menschenrechtssensible Güterabwägung zu erfolgen, bei welcher sich das öffentliche Interesse und das Privatinteresse des Inhaftierten grundsätzlich gleichberechtigt gegenüberstehen. Auch dieser allgemeine Grundsatz kann nicht ohne Weiteres auf absolute Rechte übertragen werden. Eine Interessenabwägung findet auch im Sonderstatusverhältnis bei absoluten Rechten in diesem Sinne nicht statt; es ist vielmehr grundsätzlich von einem Vorrang des Interessen desjenigen auszugehen, der ein absolutes Recht für sich beansprucht. Beim leistungsrechtlichen Gehalt bleibt das öffentliche Interesse hinsichtlich des Spezifitätskriteriums bei der Frage, welcher Standard als angemessen angesehen wird, jedoch nicht gänzlich unberücksichtigt.

Das Sonderstatusverhältnis führt weiter dazu, dass die Behörde grundsätzlich über Disziplinargewalt verfügt, welche den Erlass von Disziplinarmassnahmen ermöglicht. ${ }^{440}$ Der abwehrrechtliche Gehalt absoluter Rechte ist hier jedenfalls als Grenze der zulässigen Disziplinarmassnahmen zu sehen.

Soweit das Sonderstatusverhältnis in prozessrechtlicher Hinsicht auf kantonaler Ebene zu besonderen Beschwerdeinstanzen (etwa die Anstalts-

439 Griffel N 149.

440 HäFelin/Müller/UhlmanN N 1505; Griffel N 586. 
direktion oder das ihr übergeordnete Departement) oder abweichenden Beschwerdefristen führt, verlangen absolute Rechte, wie alle Menschenrechte, nach einer effektiven Durchsetzungsmöglichkeit. Solange diese auch bei abweichenden Beschwerdeinstanzen und Beschwerdefristen gewährleistet bleiben, stellen sich auch hier keine Schwierigkeiten. Im Sinne eines effektiven Rechtsschutzes ist bei absoluten Rechten in inhaltlicher Sicht sodann in besonderer Weise auf die Grenze einer zu spezifischen und einer zu allgemeinen Rechtsprechung zu achten. ${ }^{441}$ Auch dieser Grundsatz absoluter Rechte hat im Sonderstatusverhältnis nämlich gleichermassen zu gelten.

\section{Absolute Rechte und Soft Law?}

Die in Art. 3 EMRK verbürgten Garantien, der Verbote von Folter, unmenschlicher und erniedrigenden Behandlung, werden von verschiedenen weiteren (Soft Law-)Regelwerken und Gremien aufgegriffen. Wie aufgezeigt, kann Soft Law grundsätzlich durch seine Rezeption in der Rechtsprechung praktische Wirksamkeit entfalten. Es stellt sich deshalb die Frage, ob auch absolute Rechte die Berücksichtigung von Soft Law bzw. der Arbeit von Gremien, welche die Einhaltung des Soft Law überwachen, zulassen und ob aufgrund des absoluten Charakters ein einheitlicher Massstab zu erwarten resp. anzustreben ist.

Als Kernelemente des absoluten Rechts wurden die Forderung nach dessen unbedingter Anwendbarkeit, ein gewisses Konkretisierungserfordernis bei der Bestimmung des Schutzbereichs und des Eingriffs sowie der Grundsatz des Vorrangs absoluter Rechte vor nicht absoluten Rechten ausgemacht. Gleichwohl wurde erkannt, dass auch absolute Rechte es zulassen, den Schutzbereich relativ zu bestimmen, konfligierende Interessen und Rechte bei der Bestimmung des Schutzbereichs oder des Eingriffsbegriffs zumindest zu berücksichtigen und auch einen evolutiven Auslegungsansatz anzuwenden.

Gehen Forderungen des Soft Law über das hinaus, was bislang als harte Garantien unter einem absoluten Recht erkannt wird, ist eine Berücksichtigung des Soft Law zumindest insofern unproblematisch, als es dadurch in dieser Konstellation zu keinerlei Einschränkungen der zu gewährleistenden Garantien kommt. Da auch die Inhalte absoluter Rechte nicht starr sind, ist die Berücksichtigung von Soft Law-Regelwerken auch bei absolu-

441 Mavronicola HRLR 2012, 723 (743). 
ten Rechten grundsätzlich nach den allgemein ausgearbeiteten Kriterien der richterlichen Rechtsfortbildung zulässig. Wird durch das Aufgreifen bestehenden Soft Law der Schutzbereich eines absoluten Rechts aber ausgedehnt, also einem evolutiven Ansatz gefolgt, wirkt sich dies aufgrund der unbedingten Anwendbarkeitsforderung wiederum direkt auch auf das Ergebnis aus, kommen doch Ausgleichsmöglichkeiten auf der Rechtfertigungsebene von vorneherein nicht in Betracht. Die Auseinandersetzung zur Wertigkeit der konkreten Soft Law-Bestimmung in Bezug auf den Gehalt des Schutzbereichs der absoluten Norm hat deshalb mit nochmals gesteigertem Bedacht zu erfolgen.

Ist der im Soft Law verankerte Standard aber tiefer als jener, welcher unter einem absoluten Recht bereits gewährleistet ist, darf das Soft LawRegelwerk jedenfalls nicht in der Weise hinzugezogen werden, dass aus ihm Rechtfertigungsgründe oder Ausnahmetatbestände abgeleitet würden. Grenze der Berücksichtigung von Soft Law ist damit jedenfalls die unbedingte Anwendbarkeitsforderung des absoluten Rechts: Sähe zum Beispiel das Soft Law vor, dass der vom EGMR unter Art. 3 EMRK gewährleistete Standard in einer Notsituation unterschritten werden dürfte, und würde der EGMR dem folgen, stünde die Notstandsfestigkeit und damit die Natur des absoluten Rechts in Frage. ${ }^{442}$ Unabhängig davon, ob es sich um ein absolutes hartes Recht oder um ein "gewöhnliches hartes Recht“443 handelt, wird aufgrund der Konstitutionalisierung der europäischen Rechtsordnung zudem eine Aufweichung eines etablierten harten Rechts durch

442 EGMR (GK) - Muršić/CRO, Urt. v. 20.10.2016, 7334/13, Rn. 65: Hinweis auf das IKRK, welches einen tieferen Unterbringungsstandard für Notstandssituationen vorsieht: „The ICRC also made a distinction between the general accommodation requirements and accommodation requirements in emergency situations (such as political crisis, natural disasters, fire, riots, health crises in which large numbers of detainees needed to be separated from the others or events which required the transfer of detainees from a prison that had been damaged to another prison). The ICRC's initial indication that in such situations 2 sq. $m$ per prisoner should be provided had been subsequently criticized by the experts. The emphasis was thus placed not on the indication of minimum requirement of personal space but the necessity of returning a prison to normal conditions (including minimum space specifications) as soon as possible. In particular, it was emphasized that in such instances it is necessary to avoid a situation in which the restrictions introduced to deal with the emergency situation developed into a chronic deficiency."

443 Gemeint sind sämtliche Konventionsrechte, welchen kein absoluter Charakter zukommt. 
Soft Law generell als unzulässig betrachtet. ${ }^{444}$ Für absolute Rechte würde dies bedeuten, dass der vormals festgelegte Schutzbereich durch neues Soft Law keinesfalls mehr eingeschränkt werden dürfte. Einzuwenden bleibt, dass die Beurteilung, was durch die Auslegung der Konventionsrechte im Einzelfall als harte Garantie, welche nicht mehr aufgeweicht werden darf, beurteilt werden kann, gerade vor dem Hintergrund des „Living instrument-Charakters" nicht immer eindeutig sein dürfte.

Auch bei der Frage, ob ein einheitlicher Massstab anzustreben ist, ist bei der Essenz dessen, was absolute Rechte ausmachen, anzusetzen. Das besondere Bestimmtheitserfordernis absoluter Rechte könnte zwar dahingehend ausgelegt werden, dass ein einheitlicher Massstab anzustreben ist. Allerdings ergibt sich schon aus dem evolutiven Charakter der EMRK und dem Umstand, dass Soft Law blosse Rechtserkenntnisquelle für die Auslegung von Konventionsrechten ist, dass ein einheitlicher Standard der EMRK und anderer Regelwerke nicht zu fordern sein kann. Das würde unter Umständen die Weiterentwicklung des Standards gefährden oder die Geschwindigkeit der Reaktion auf neue Entwicklungen verlangsamen. Gegen ein absolutes Erfordernis, einen einheitlichen Standard zu verlangen, spricht auch, dass die EMRK und das jeweilige Soft Law eine andere Zielsetzung resp. einen anderen Auftrag verfolgen können. Der besonderen Expertise von etablierten internationalen Gremien im Bereich des Soft Law ist gleichwohl besondere Beachtung zu schenken; eine unterschiedliche Zielsetzung darf deshalb nicht bloss als vorgeschobenes Argument für die Rechtfertigung nach unten abweichender Standards dienen.

Zusammenfassend darf bzw. muss aufgrund des Auslegungskanons der EMRK Soft Law auch bei absoluten Rechten berücksichtigt werden. Ein einheitlicher Standard zwischen EMRK und den verschiedenen Soft LawRegelwerken ist allerdings auch bei absoluten Rechten keinesfalls zwingend.

\section{Zwischenfazit}

Die Methodik der Bestimmung des Schutzbereichs und die Frage der Anwendbarkeit interagieren. Der absolute Charakter basiert prima vista auf der absoluten Anwendbarkeit des einmal bestimmten Schutzbereichs.

444 Richter Pinto de Albuquerque partly diss. op., EGMR (GK) - MuršićlCRO, Urt. v. 20.10.2016, 7334/13, Rn. 30 ff.: Dies sei nämlich gleichbedeutend mit der Umgehung verbindlicher internationaler Verpflichtungen. 
Jeder Eingriff in den Schutzbereich ist damit eine Konventionsverletzung oder mit anderen Worten sind der Schutzbereich und der Kerngehalt des Konventionsrechts deckungsgleich.

Die Bestimmung des Schutzbereichs erfolgt demgegenüber relativ. Um die absolute Anwendbarkeit nicht zu untergraben, ist bei der relativen Bestimmung des Schutzbereichs besonders darauf zu achten, welche Kriterien in die Schutzbereichsbestimmung einbezogen werden. Es ist nur dann legitim, ein relatives Kriterium einzubeziehen, wenn es sich auf den Grad des Leidens resp. die Schwere der zugefügten Behandlung auswirkt. Die Berücksichtigung anderer Kriterien würde zu einer Aufweichung der absoluten Anwendbarkeitsforderung führen.

$\mathrm{Zu}$ einer gewissen Aufweichung der unbedingten Anwendbarkeitsforderung kommt es auch, wenn der Gerichtshof bei absoluten Rechten unter gewissen Voraussetzungen die Berücksichtigung konfligierender Interessen und Rechte zulässt. Konfligierende Rechte können dazu führen, dass das Mindestmass an Schwere des Eingriffs nicht als gegeben oder dass eine Massnahme als sozialadäquat und angemessen erachtet wird. Im Allgemeinen ist allerdings davon auszugehen, dass absolute und nicht absolute Rechte in einem Hierarchieverhältnis stehen, wobei absolute Rechte grundsätzlich vorgehen. Eine absolut positive Verpflichtung wird weiter dann nicht erkannt, wenn ihr eine absolute negative Verpflichtung gegenüberstehen würde. Eine gewisse Aufweichung des Anwendbarkeitskriteriums scheint der EGMR weiter bei positiven leistungsrechtlichen Verpflichtungen zuzulassen, indem er den Konventionsstaaten aus sozioökonomischen Überlegungen einen gewissen Spielraum bei der Frage der Angemessenheit des Standards zuzugestehen scheint.

Obwohl, wie gezeigt, eine gewisse Relativität des Standards nicht ausgeschlossen ist, führt die Abhängigkeit des Standards von mehreren Variablen des Einzelfalls auch zu einer grossen Variabilität des Ergebnisses. Ein fehlender harter Massstab gefährdet die Rechtssicherheit der Betroffenen, weshalb hier in besonderem Masse Vorsicht geboten ist. Das Zugeständnis, auch bei absoluten Rechten gewisse relative Kriterien zu berücksichtigen, ist jedoch auch als Ausdruck eines progressiven, einem stetigen Wandel unterliegenden Schutzstandards zu sehen, welcher Raum für Eventualitäten und die Weiterentwicklung des Standards lässt (EMRK als living instrument"). Zum einen bleibt damit für jedes Themengebiet genau zu analysieren, welche Kriterien legitimerweise in die Schutzbereichsbestimmung miteinbezogen werden dürfen resp. müssen, ohne dass die absolute Anwendbarkeitsforderung untergraben wird. Zum anderen ist für jedes Themengebiet auszutarieren, wie konkret der unter Art. 3 EMRK 
zu garantierende Standard zu formulieren ist, dass sowohl der der EMRK zugrundeliegenden Konzeption des stetigen Wandels und der Einzelfallbetrachtung als auch dem Umstand, dass gerade der absolute Charakter nach einer gesteigerten Rechtssicherheit verlangt, Rechnung getragen wird. Das Bestehen eines Sonderrechtsverhältnisses hat prima vista keinen entscheidenden Einfluss auf die Gewährung absoluter Rechte. Absolute Rechte sind vielmehr Schranken für Einschränkungsmöglichkeiten im Sonderstatusverhältnis. Aufgrund der Wechselwirkungen zwischen Anwendbarkeitskriterium und Spezifitätskriterium ist jedoch bei der Schutzbereichsbestimmung in besonderem Masse darauf zu achten, dass Gesetzesdelegationen oder unbestimmte Normen den Anforderungen einer menschenrechtsschützenden Regulierung im Sinne der positiven Verpflichtung des Staates nicht zuwiderlaufen.

Die Kernelemente absoluter Rechte führen weiter nicht dazu, dass Soft Law bei absoluten Rechten nicht berücksichtigt werden dürfte. Im Gegenteil sind auch absolute Rechte nicht starr. Soft Law darf damit jedenfalls dann berücksichtigt werden, wenn es dadurch zu einer Weiterentwicklung des Standards kommt. Vorsicht ist aber dann geboten, wenn der Beizug von Soft Law zu einer Aufweichung eines bereits etablierten harten Standards eines absoluten Rechts führt. Die Berücksichtigung von Soft Law darf bei absoluten Rechten insbesondere nicht zu einer Aufweichung der Anwendbarkeitsforderung führen. Trotz des besonderen Bestimmtheitserfordernisses führt die Absolutheit eines Rechts schliesslich nicht dazu, dass ein einheitlicher Standard zwischen der EMRK und des Soft Law zu verlangen ist. Der besonderen Expertise etablierter Standards und Gremien hat der EGMR allerdings gerade bei absoluten Rechten gebührend Rechnung zu tragen und darf eine mögliche unterschiedliche Zielsetzung der Standards und der EMRK nicht bloss vorschieben, um ein Abweichen vom etablierten Soft Law-Standard nach unten zu begründen. 


\section{Teil 3: Mindeststandards im Strafvollzug - eine Analyse}

\section{Kapitel 1: Vorbemerkungen}

\section{Untersuchungsgegenstand und Vorgehen}

Es werden zwei für Art. 3 EMRK relevante strafvollzugsspezifische und menschenrechtliche Themen materieller Haftbedingungen herausgegriffen: (1) Fragen rund um die Überbelegung und (2) Fragen rund um die Gesundheitsversorgung im Strafvollzug. Diese sind in der Praxis des EGMR von besonderer Bedeutung, und gerade die Überbelegungsthematik fungiert - wie aufgezeigt wird - als Ankerpunkt für die Beurteilung weiterer Haftbedingungen wie etwa der Möglichkeit von Outdoor-Aktivitäten, der Beschäftigungs- und Arbeitsmöglichkeiten, des Zugangs zu sanitären Einrichtungen und des Vorhandenseins von genügend Privatsphäre bei deren Nutzung, der Anforderungen an die Licht-, Luft- und Temperaturverhältnisse in der Zelle sowie ausreichender und angemessener Ernährung während des Vollzugs. Die Analyse dieser beiden ausgewählten Themenkreise ist weiter geeignet, repräsentative Aussagen zur Standardbestimmung bei Haftbedingungen und zur Methodik des Gerichtshofs zu machen. Resultate dieser Analyse können deshalb in der Folge auch auf die weiteren genannten Haftbedingungen übertragen werden.

Zunächst wird jeweils auf das Problem als solches eingegangen, wobei insbesondere dessen Auswirkungen auf den Einzelnen aufgezeigt werden. In einem nächsten Schritt werden die in den einschlägigen Regelungsinstrumentarien vorgeschlagenen Standards dargelegt. Darauf folgt eine Analyse der Rechtsprechung der Strassburger Organe zu Art. 3 EMRK. Es wird dabei nicht nur untersucht, welcher Mindeststandard in materieller Hinsicht festgesetzt wird, sondern es wird auch das prüfungsmethodische Vorgehen des Gerichtshofs gewürdigt. Die Absolutheit von Art. 3 EMRK, die Bedeutung als Fundamentalgarantie und die Prämisse eines effektiven Menschenrechtsschutzes werden bei der Analyse auf den Prüfstand gestellt. Auch die Bedeutung des Soft Law in der Rechtsprechung des EGMR wird durchleuchtet, wobei insbesondere der Frage nachgegangen wird, inwieweit Soft Law-Standards durch den EGMR konsolidiert werden und ob ein Transformationsprozess dieser Standards stattfindet. In einem 
letzten Schritt wird der Blick jeweils auf die nationale Ebene der Schweiz gewandt.

\section{Mindeststandard und das „no more harm principle“}

Es stellt sich vorab die Frage, welcher Gehalt dem menschenrechtlichen Postulat des „no more harm principle“ innewohnt und wie dieses in Bezug auf die Setzung des Mindeststandards rechtlich einzuordnen ist.

Das „no more harm principle" besagt, dass die Haftbedingungen des Strafvollzugs auf eine Weise auszugestalten sind, dass sie zu keinem physischen oder psychischen Leid führen, welches dem Freiheitsentzug als solchem nicht von vorneherein inhärent ist. ${ }^{445}$ Mit Blick auf die Definition eines Mindeststandards für Haftbedingungen kommt dem „no more harm principle" wohl kein eigenständiger konkreter materieller Gehalt zu. Eine Bestimmung des „unvermeidlichen Masses“ dürfte sich in der Praxis als sehr schwierig erweisen und hätte auch nur sehr geringes Beschränkungspotential, ist doch theoretisch betrachtet wohl bei jeglichen geltenden Haftbedingungen weiteres Verbesserungspotential vorhanden. ${ }^{446}$ Gleichwohl kann das Prinzip bei der Ausarbeitung des Standards eine wichtige Auslegungsdirektive sein.

445 Vgl. zur ständigen Rechtsprechung des Gerichtshofs bspw.: EGMR (GK) Kudta/POL, Urt. v. 26.10.2000, 30210/96, Rn.94; EGMR - Kalashnikov/RUS, Urt. v. 15.07.2002, 47095/99, Rn.95; EGMR - Poltoratskiy/UKR, Urt. v. 29.04.2003, 38812/97, Rn. 132; EGMR - Yankov/BUL, Urt. v. 11.12.2003, 39084/97, Rn. 107; EGMR (GK) - Ramirez Sanchez/FRA, Urt. v. 04.07.2006, 59459/00, Rn. 119; EGMR (GK) - Paladi/MDA, Urt. v. 10.03.2009, 39806/05, Rn.71; EGMR (GK) - Idalov/RUS, Urt. v. 22.05.2012, 5826/03, Rn.93; EGMR (GK) - Svinarenko u. Slyadnev/RUS, Urt. v. 17.07.2014, 32541/08 u. 43441/08, Rn. 116; EGMR (GK) - Mozer/MDA u. RUS, Urt. v. 23.02.2016, 11138/10, Rn. 178: „[...] do not subject him [the detainee] to distress or hardship of an intensity exceeding the unavoidable level of suffering inherent in detention". Auch die schweizerische bundesgerichtliche Rechtsprechung rezipiert diesen Grundsatz, vgl. etwa BGE 140 I 125, E. 3.5; BGer 1B_335/2013, Urt. v. 26.02.2014, E. 3.5; vgl. ferner SK-StPO/Meyer Art. 3 EMRK Rn. 43: Die dem Vollzug inhärente Beschränkung bestimme sich nach der Art der Freiheitsentziehung und mit Blick auf die Menschenwürde.

446 Dörr/Grote/Marauhn/Bank Kap. 11 Rn. 22. 


\section{Mindeststandard und die Ressourcen der Konventionsstaaten}

Der fortdauernde unterschiedliche Entwicklungsstand der Konventionsstaaten und die empirischen Daten zum Strafvollzug (Ukraine mit Ausgaben von 2.68 Euro pro Tag und Inhaftiertem im Vergleich zu San Marino mit 685 Euro pro Tag und Inhaftiertem) lassen vermuten, dass Strafvollzugsanstalten in den verschiedenen Konventionsstaaten auf tatsächlicher Ebene sehr verschiedene Grundvoraussetzungen haben. ${ }^{447}$ Grundsätzlich ist allerdings der vom EGMR festgesetzte Massstab ein einheitlicher. Dieser basiert auf dem europäischen Konsens und findet auf alle Konventionsstaaten gleichermassen Anwendung. ${ }^{448}$ In Kenntnis um die Kostenintensität ${ }^{449}$ des Strafvollzugs hält der Gerichtshof auf rechtlicher Ebene immer wieder fest, dass finanzielle und logistische Schwierigkeiten bei der Setzung und Einhaltung des Mindeststandards grundsätzlich keine validen Argumente sind, unzureichende Haftbedingungen zu begründen. ${ }^{450}$ In Anbetracht

447 Der Kostenintensität der Massnahmen zur Verbesserung des Mindeststandards sind aber auch die sozialen und finanziellen Kosten, welche gerade durch inadäquate Bedingungen entstehen, entgegenzuhalten: vgl. etwa Richter Sajó, López Guerra u. Wojtyczek, joint partly diss. op., EGMR (GK) - Muršić/CRO, Urt. v. 20.10.2016, 7334/13, Rn. 7: ,[...] the economic analysis of law applied to prison condition questions has to take into account not only the costs of implementing the most fundamental human rights standards but also the enormous social and financial costs of a penitentiary system which does not ensure adequate space in prison."

448 Vgl. auch Helgesen HRLJ 2011, 275 (280): Die Strassburger Organe hätten von Beginn weg versucht, den gemeinsamen europäischen Standard zu ergründen. Die steigende Anzahl Konventionsstaaten und das gehobene zu gewährleistende Schutzniveau stelle das Konzept der Gewährung eines gemeinsamen europäischen Standards aber vor Herausforderungen. Es sei sich zu fragen, ob an diesem auch in Zukunft unverändert festgehalten werden könne.

449 Gerade die Lösung struktureller bzw. systemischer Defizite benötige dabei die Mobilisation der notwendigen Ressourcen: EGMR - Orchowski/POL, Urt. v. 22.10.2009, 17885/04, Rn. 153.

450 EGMR - Mamedova/RUS, Urt. v. 01.06.2006, 7064/05, Rn. 63; EGMR - Orchowski/POL, Urt. v. 22.10.2009, 17885/04, Rn.153; EGMR - Neshkov u.a./ BUL, Urt. v. 27.01.2015, 36925/10 u.a., Rn. 229; EGMR - Varga u.a./HUN, Urt. v. 10.03.2015, 14097/12 u.a., Rn. 103; zur Umsetzung abweichender Urteile, welche mit Kostenfolgen einen höheren Standard begründen vgl. kritisch Richter Sajó, López Guerra u. Wojtyczek, joint partly diss. op., EGMR (GK) Muršić/CRO, Urt. v. 20.10.2016, 7334/13, Rn. 4 f. (Notwendigkeit der Gewährung einer Übergangsperiode); zum Erfordernis der Ressourcenbereitstellung zur Implementierung von Menschenrechten im Allgemeinen instruktiv: SKOGLY HRLR 2012, $393 \mathrm{ff}$. 
des Fundamentalcharakters von Art. 3 EMRK und vor dem Hintergrund seiner absoluten Geltung kann hinsichtlich dieses Grundsatzes zu keinem anderen Ergebnis gekommen werden. Dies auch dann nicht, wenn man berücksichtigt, dass der Gerichtshof sich selbst bei absoluten Rechten in sozioökonomischen Bereichen eine gewisse Zurückhaltung auferlegt.

Im Unterschied zum Standard der EMRK ist nichts dagegen einzuwenden, wenn das Soft Law nicht für alle Mitgliedstaaten einen einheitlichen Standard proklamiert oder fiskalisch bedingte Relativierungsmöglichkeiten einräumt. Solche sind etwa in der zweiten einleitenden Bemerkung zu den UN-Mindestgrundsätzen für die Behandlung Gefangener vorgesehen - sowohl in ihrer Version von 1955 als auch in der aktuellen Version von 2015 (Nelson Mandela Rules ${ }^{451}$ ). Der grossen Varietät der rechtlichen, sozialen, wirtschaftlichen und geographischen Umstände weltweit sei es gerade geschuldet, dass nicht alle in den UN-SMR niedergelegten Regeln jederzeit überall zur Anwendung gelangen können (,it is evident that not all of the rules are capable of application in all places and at all times"). Auch Soft Law kann allerdings Relativierungsmöglichkeiten begrenzen. In diesem Sinn statuieren die Europäischen Strafvollzugsgrundsätze von $2006^{452}$ ausdrücklich, dass ein Ressourcenmangel keineswegs Grund für menschenrechtswidrige Haftbedingungen sei (Rule 4 EPR). Die Europäischen Strafvollzugsgrundsätze grenzen somit bezüglich ihres Geltungsanspruchs die bei den UN-Mindestgrundsätzen zulässige, durch wirtschaftliche Umstände begründete Relativierungsmöglichkeit ein. Dies dürfte nicht zuletzt darauf zurückzuführen sein, dass die Gruppe der Unterzeichnerstaaten bei den Europäischen Strafvollzugsgrundsätzen im Vergleich zu jener der UN-Mindestgrundsätze eine homogenere ist.

\section{Kapitel 2: Überbelegung}

I. Überbelegung - Einführung und Definition

Die weltweit steigende Anzahl Personen, welche sich im Freiheitsentzug befinden, ${ }^{453}$ und die hohe Auslastungsrate der Strafvollzugsanstalten in

451 A/RES/70/175, United Nations Standard Minimum Rules for the Treatment of Prisoners („The Nelson Mandela Rules“).

452 Recommendation Rec (2006) 2 on the European Prison Rules 2006.

453 Walmsley S. 2. 
Europa $^{454}$ lassen das Problem der Überbelegung bereits vermuten. Es handelt sich bei der Überbelegung auch nicht nur um ein äusserst aktuelles Phänomen, sondern um ein schon lange bestehendes, viel diskutiertes und sich verschärfendes ${ }^{455}$ Problem. Auch wenn die Überbelegung im Bereich der präventiven Haft besonders akut ist, betrifft sie alle Bereiche des Strafvollzugs. ${ }^{456}$ Zurückgeführt wird das sich zuspitzende Überbelegungsproblem bisweilen auf einen „Trend zur gesteigerten Punitivität“ in Form einer Strafschärfungsmentalität. ${ }^{457}$

Der für diese Arbeit zu verwendende Begriff der Überbelegung 458 bedarf zunächst einer Definition. In der Literatur wird der Begriff je nach wissenschaftlicher Perspektive und Fragestellung nämlich unterschiedlich definiert. Beim sozialpsychologischen Überfüllungsbegriff steht meist die subjektive Wahrnehmung der Betroffenen im Vordergrund.459 Von einer kriminalpolitischen Überfüllung ist demgegenüber dann die Rede, wenn sich ein besonders grosser Anteil der Bevölkerung im Strafvollzug befindet. ${ }^{460}$ Die vollzugswissenschaftliche Definition der Überfüllung knüpft wiederum in aller Regel an die Kapazitätsgrenze an. ${ }^{461}$ Diese kann sich dabei an unterschiedlichen Kriterien orientieren: Die Personalkapazität legt etwa fest, wie viele Inhaftierte durch die beschäftigten Strafvollzugsbeamten be-

454 Aebi/Tiago Key-Findings S. 7.

455 Vgl. etwa die Rechtsprechung des EGMR unter vielen: EGMR - Sukachov/UKR, Urt. v. 30.01.2020, 14057/17; EGMR - Rezmiveș u.a./ROM, Urt. v. 25.04.2017, 61467/12 u.a.; EGMR (GK) - Muršić/CRO, Urt. v. 20.10.2016, 7334/13; vgl. auch Barisch KJ 2008, 425 (427); Junger-Tas Eur J Crim Policy Res 2006, 79 f.; DüNKel/LAPpI-SeppäLä/Morgenstern/van ZyL Smit in: Dünkel/Lappi-Seppälä/Morgenstern/van Zyl Smit, S. 1023 (1037): DüNKel et al. wenden aber ein, dass nicht sämtliche europäischen Länder von Überbelegungssituationen betroffen seien, von einem gesamteuropäischen Problem könne deshalb keine Rede sein. Gleichwohl sind zahlreiche Strafvollzugsanstalten in Europa und vielen weiteren Ländern weltweit von Überbelegungssituationen betroffen. Allen voran die USA: vgl. Karstedt in: Dünkel/Lappi-Seppälä/Morgenstern/van Zyl Smit, S. 935 (935 f.); in Bezug auf die USA vgl. auch Pitts/Griffin/Johnson Contemporary Justice Review 2014, $124 \mathrm{ff}$.

456 CPT - 7th General Report [CPT/Inf (97) 10], Rn. 12.

457 Barisch KJ 2008, 425 (435); vgl. auch DünkeL/van ZyL Smit in: van Zyl Smit/Dünkel, S. 796 (813): Viele Länder würden die vermehrte Nutzung der Freiheitsstrafe als dermassen wichtiges Mittel der sozialen Kontrolle befinden, dass sie Überbelegungssituationen als notwendiges Übel akzeptieren würden.

458 In der Terminologie des Gerichtshofs: „overcrowding“.

459 Oberheim S. 11.

460 OBERHEIM S. 13.

461 Oberheim S. 13; vgl. auch Kromrey H. S. 194; vgl. auch bereits Ostfeld/Kasl/ D’Atri/Fitzgerald S. 23. 
aufsichtigt und betreut werden können, ${ }^{462}$ während sich die Haftplatzkapazität an den vorhandenen Haftplätzen orientiert. ${ }^{463}$ In der Praxis dürften aber Personalkapazität und Haftplatzkapazität regelmässig korrelieren.

Ausgehend von der Haftplatzkapazität ist evidenterweise dann von einer Überbelegung auszugehen, wenn die Auslastung 100\% übersteigt. ${ }^{464}$ Die Bestimmung der Auslastungsrate setzt allerdings voraus, dass die Kapazität mit einem Belegungsplan eindeutig definiert ist. ${ }^{465}$ Die errechnete Kapazität ist dann mit der tatsächlichen Belegung ins Verhältnis zu setzen. Wiederum kann die Kapazitätsberechnung ihrerseits erst erfolgen, wenn festgelegt wurde, wie viel persönlicher Platz jedem Einzelnen aus menschenrechtlicher Sicht zur Verfügung stehen muss. Die vollzugswissenschaftliche Definition der Überbelegung ist damit durch eine menschenrechtliche Fragestellung geprägt.

Die menschenrechtliche Herangehensweise verlangt auch, dass sich der Begriff der Überbelegung im Sinne von Art. 3 EMRK am Einzelnen orientiert. Eine Subsumtion der Überbelegungssituation unter Art. 3 EMRK setzt im Einzelfall nämlich das Vorliegen einer unmenschlichen oder erniedrigenden Behandlung voraus. Eine menschenrechtsrelevante Überbelegung basierend auf der Haftplatzkapazität liegt deshalb nicht automatisch vor, wenn die nationale Auslastungsrate oder auch die Auslastungsrate der konkreten Haftanstalt die 100\%-Grenze überschreitet. ${ }^{466}$ Ausschlaggebend ist vielmehr die Wirkung auf den Einzelnen. Aus einer menschenrechtlichen Perspektive relevant wird die Überbelegung folglich etwa dann, wenn die Haftzelle, in welcher die betroffene Person untergebracht ist, von der Überbelegung betroffen ist und der Betroffene dadurch physischem oder psychischem Leid ausgesetzt ist oder in seiner Persönlichkeit

462 Zur Personalpräsenz bei einem Mindeststandard vgl. Kromrey H. S. 200 f.: Eine mangelnde Personalpräsenz könne bei einer Menschenwürdeverletzung als erschwerender Umstand hinzukommen, sei aber weniger geeignet eine eigenständige Menschenwürdeverletzung zu begründen.

463 Oberheim S. 13.

464 Vgl. auch Кromrey H. S. 194. Unter Vollzugspraktikern wird allerdings auch die Ansicht vertreten, dass Strafvollzugsanstalten bei einer Belegung von $85 \%$ als voll ausgelastet anzusehen seien. Dies, weil behandlerische Aspekte eine gewisse Flexibilität voraussetzen würden: DünKel/LAPPI-SEPpäLä/MorGenstern/van ZyL Smit in: Dünkel/Lappi-Seppälä/Morgenstern/van Zyl Smit, S. 1023 (1037).

$465 \mathrm{Zu}$ den Problemen bei der Erstellung solcher Belegungspläne und der Manipulationsgefahr vgl. Kromrey H. S. 194.

466 Gleichwohl kann eine solche Feststellung auch im Einzelfall beweisrechtlich relevant werden. 
herabgesetzt wird. Die Überbelegungssituation kann darüber hinaus aber auch dann menschenrechtsrelevant werden, wenn zwar die Haftplatzkapazität der konkreten Zelle nicht überschritten wird, der ganze Haftbetrieb und -alltag aber von der allgemeinen Überbelegungssituation (bestimmt nach der Haftplatzkapazität der Strafvollzugsanstalt) geprägt ist und es deshalb zu einer unmenschlichen oder erniedrigenden Behandlung i.S.v. Art. 3 EMRK kommt. Zu denken wäre an ein aus der Überbelegung resultierendes Fehlen von Gemeinschaftsräumen, unzureichende Bewegungsmöglichkeiten ausserhalb der Zelle oder ungenügenden Zugang zu sanitären Einrichtungen.

Im Rahmen der vorliegenden Arbeit wird der vollzugswissenschaftliche Überbelegungsbegriff, welcher sich an der Haftplatzkapazität orientiert und durch menschenrechtliche Elemente geprägt ist, angewendet.

\section{II. Überbelegung - Auswirkungen}

Die Überbelegung wirkt sich in erster Linie auf die Position des Strafgefangenen selbst aus, sind es doch die Inhaftierten, die ihr direkt ausgesetzt sind. Objektiv führt die Überbelegung zu sich verknappenden Platzverhältnissen. Aus dem sich zuspitzenden Platzmangel können sich weitere, kaum abwendbare Folgen ergeben. Der zur Verfügung stehende Platz hat Auswirkungen auf die Zellengestaltung (etwa die Zelleneinrichtung, Sitz-, Schlaf- und Bewegungsmöglichkeiten), auf die klimatischen Verhältnisse (Temperatur), auf das Lüftungssystem (frische Luft), unter Umständen auch auf die Lichtverhältnisse (Tageslicht: zu denken ist an durch Überbelegung verstellte Fenster), auf die Möglichkeit von und den Zugang zu Aktivitäten inner- und ausserhalb der Zelle (körperliche Aktivitäten, Zugang zu Büchern, Zeitungen, Radio etc.) und nicht zuletzt auch auf die hygienischen Bedingungen (etwa Zugang zu sanitären Einrichtungen). Ein Missverhältnis von Vollzugspersonal und Insassen (vgl. Korrelation Haftplatzkapazität und Personalkapazität) kann sich weiter auch auf die Resozialisierungsprognose der Insassen niederschlagen. ${ }^{467}$ Vollzugslockerungen und damit ein sinnvoller Übergang zu vermehrter Eigenverantwortung lassen sich in überbelegten Strafvollzugsanstalten kaum realisieren. ${ }^{468}$

467 Kromrey H. S. 200.

468 Kromrey H. S. 200. 
Subjektiv kann die Überbelegung sowohl psychische als auch physische Folgen für einen Betroffenen zeitigen. ${ }^{469}$ Die Überbelegungssituation kann zu einem Gefühl der Hilfslosigkeit führen; der Strafgefangene kann dabei das Gefühl haben, dass das eigene Verhalten keine Auswirkungen mehr auf die allgemeine Situation hat. ${ }^{470}$ Ferner kann sie die Erhöhung des Stresspegels zur Folge haben, ${ }^{471}$ was wiederum zu einem Anstieg des Blutdrucks und zu einem gesteigerten Bedürfnis nach medizinischer Versorgung führen kann. ${ }^{472}$ Stress kann nach dem aktuellen Stand der medizinischen Wissenschaften weiter unter anderem Herz-Kreislauf-Probleme begünstigen. ${ }^{473}$

Auch die Wahrscheinlichkeit, an parasitären oder Infektionskrankheiten zu erkranken, wird durch Überbelegungssituationen erhöht - nicht nur durch die Steigerung des Stresspegels, sondern auch durch die grössere „soziale Dichte“474 und die prekären hygienischen Umstände. ${ }^{475}$ Die Überbelegung gilt deshalb als Hindernis bei der Prävention und Bekämpfung von HIV bzw. AIDS in Strafvollzugsanstalten. ${ }^{476}$ Ferner wird von einem Zusammenhang zwischen Überbelegungssituationen und Selbstverletzungshandlungen ausgegangen; ${ }^{477}$ in der Folge von Überbelegungssitua-

469 Vgl. etwa van Zyl Smit/Snacken S. 131; Garcia-Guerrero/Marco Revista Española de Sanidad Penitenciaria 2012, 106 (110); Haney in: Cutler/Zapf, S. 415 (426).

470 Van Zyl Smit/Snacken S. 131.

471 Oberheim S. 146; Haney in: Cutler/Zapf, S. 415 (422, 426).

472 Van Zyl Smit/Snacken S. 131; Haney in: Cutler/Zapf, S. 415 (426); Schmidt S. 140 ff. m.w.N.; zum Zusammenhang betr. Stress, Blutdruck, Gesundheit und Überbelegung in Strafvollzugsanstalten vgl. auch die Studie von OstFeLD/KaSL/ D'Atri/Fitzgerald S. 22 ff., 184 m.w.H.: Ostfeld et al. identifizieren in ihrer Studie neben Überbelegungssituationen weitere massgebliche Faktoren betr. Stressniveau der Inhaftierten.

473 Anisman S. $180 \mathrm{ff}$., $206 \mathrm{ff}$.

474 Van Zyl Smit/Snacken S. 131: Zu denken sei etwa an grosse Schlafräume ohne Unterteilung im Vergleich zu Einzelzellen.

475 Garcia-Guerrero/Marco Revista Española de Sanidad Penitenciaria 2012, $106(110)$.

476 Stöver/MacDonald/Atherton S. 123: Die Überbelegung sei dabei aber nur eine der Schwierigkeiten. Auch die Gefangenenpopulation als solche sei beim Kampf gegen HIV und AIDS eine besondere Herausforderung. In vielen Ländern bestehe diese nämlich aus einem signifikanten Anteil an wegen Verstössen gegen das Betäubungsmittelgesetz Verurteilten. Zurückhaltender: GarciaGuerrero/Marco Revista Española de Sanidad Penitenciaria 2012, 106 (110).

477 Wolff/Casillas/Perneger/Heller/Golay/Mouton/Bodenmann/GetaZ International Journal of Prisoner Health 2016, 39 ff.: WolfF et al. differenzieren zwischen nichttödlichen und tödlichen (suizidale) Selbstverletzungshandlungen. 
tionen kann es zu einem signifikanten Anstieg von suizidalen Handlungen kommen. ${ }^{478}$

Verschiedene Erklärungsmodelle setzen sich mit den aufgezeigten negativen Auswirkungen der Überbelegung auseinander. Das Stimulus-overloadModell geht von einer durch die Überbelegung verursachten Reizüberlastung aus, ${ }^{479}$ während das Verbaltensbeschränkungs-Modell annimmt, dass die negativen Folgen der Überbelegung dadurch hervorgerufen werden, dass individuelle "physische, psychisch-emotionale und soziale Verhaltensmöglichkeiten" vereitelt werden. ${ }^{480}$ Das ökologische Modell [recte: ökonomische Modell] erkennt die Ursache der negativen Konsequenzen in der durch die Überbelegung verursachten Verknappung der Ressourcen und dem damit einhergehenden Kampf um die entsprechenden Sachmittel. ${ }^{481}$

Keines der Erklärungsmodelle vermag für sich alleine zu überzeugen; der Komplexität der Auswirkungen der Überbelegung kann nur eine Kombination der verschiedenen Modelle gerecht werden. Für die Ausarbeitung eines Mindeststandards spielt es aber letztlich keine Rolle, welcher Aspekt für die negativen Auswirkungen bei den Betroffenen verantwortlich ist, solange Einigkeit darüber besteht, dass die Überbelegung kausal für die genannten negativen Folgen ist.

Neben den Inhaftierten sind von der Überbelegung auch die Strafvollzugsbeamten betroffen. Die Überbelegungssituation wirkt sich automa-

478 Wolff/Casillas/Perneger/Heller/Golay/Mouton/Bodenmann/Getaz International Journal of Prisoner Health 2016, 39 (42): Die Studie bezog sich auf das Schweizer Untersuchungsgefängnis Champ-Dollon im Kanton Genf im Zeitraum 2006-2014. Relativierend ist jedoch anzumerken, dass es sich bei Selbstverletzungshandlungen um ein komplexes Phänomen handelt, welches nicht auf eine einzige Ursache zurückzuführen ist; sie basieren vielmehr auf einer grundsätzlich individuellen Verletzlichkeit, welche aber durch Stressfaktoren verstärkt werden kann. Vgl. auch: Garcia-Guerrero/Marco Revista Española de Sanidad Penitenciaria 2012, 106 (110 f.); vgl. in diesem Zusammenhang aber auch die Untersuchung von Frühwald/Frottier/Eher/Benda/Ritter Psychiatrische Praxis 2001, 326 ff.: Hinsichtlich der von ihnen untersuchten Suizide in österreichischen Gefängnissen vom 01.01.1975 bis zum 31.12.1999 hatte sich ergeben, dass die Überbelegung nur eine untergeordnete Rolle gespielt hatte (71,9\% der untersuchten Suizide wurden in Einzelzellen verübt, 23\% der Suizide in Zellen mit zwei bis drei Inhaftierten, und nur 4,5\% der Suizide fanden in Zellen mit mehr als drei Strafgefangenen statt).

479 Oberheim S. 147.

480 Oberheim S. 147.

481 Oberheim S. 147; in diese Richtung implizit auch Wolff/Casillas/Perneger/ Heller/Golay/Mouton/Bodenmann/Getaz, International Journal of Prisoner Health 2016, $39 \mathrm{ff}$. 
tisch auch auf die Organisation des Haftbetriebs und somit auf den Arbeitsalltag des Personals aus. Dies gilt insbesondere dann, wenn zum Anstieg der Anzahl Inhaftierter nicht proportional weitere Strafvollzugsbeamte eingestellt werden; die Betreuungssituation verschärft sich (Korrelation Haftplatz- und Personalkapazität). ${ }^{482}$ Auch das durch die Überbelegung gesteigerte Kriminalitätsrisiko innerhalb der Haftanstalt ${ }^{483}$ betrifft nicht nur die Inhaftierten selbst, sondern direkt auch die Vollzugsbeamten, liegt es doch an ihnen, diese Situationen wieder aufzulösen. Die Überbelegung kann ferner auch Folgen für die Allgemeinheit haben, zum Beispiel als erhöhtes Sicherheits- oder Krankheitsrisiko ausserhalb der Strafvollzugsanstalten. ${ }^{484} \mathrm{Im}$ Folgenden stehen aber die Auswirkungen der Überbelegung auf die Inhaftierten im Vordergrund.

Die gravierenden Auswirkungen der Überbelegung insbesondere für die Inhaftierten machen aus einer menschenrechtlichen Sichtweise eine eingehende Analyse zu einem Mindeststandard an persönlichem Platz notwendig.

482 Barisch KJ 2008, 425 (426); Oberheim S. 143; Kromrey H. S. 200.

483 So etwa auch das CPT: CPT - 7th General Report [CPT/Inf (97) 10], Rn. 13: Gewaltzunahme zwischen den Inhaftierten sowie zwischen den Inhaftierten und dem Gefängnispersonal; vgl. auch EGMR - Canali/FRA, Urt. v. 25.04.2013, 40119/09, Rn. 26 mit Verweis zum Artikel „Surpopulation, vétusté, problème d'accès aux soins... état des lieux sans concession de la prison Charles III“ erschienen in: L'Est Républicain v. 23.12.2008: „[...] Cette surpopulation n’est pas sans incidence sur les risques de violences au sein de l'établissement puisque les tensions s'accroissent inévitablement à raison de la promiscuité et du manque de place engendrés dans les cellules [...]." Im betreffenden Gefängnis Nancy Charles III waren am Stichtag 320 Strafgefangene bei einer Kapazitätsgrenze von 260 Haftplätzen untergebracht. Vgl. auch BARISCH KJ 2008, 425 ff.: Das Risikopotential von Gemeinschaftsunterbringungen werde unterschätzt. Unruhen, Gewalt und sexuelle Übergriffe innerhalb von Haftanstalten seien auf die durch die Überbelegung entstehende räumliche Enge zurückzuführen. Vgl. ferner Garcia-Guerrero/Marco Revista Española de Sanidad Penitenciaria 2012, 106 (110); Stöver/MacDonald/Atherton S. 123; Kromrey H. S. 200.

484 So betonen etwa Garcia-Guerrero und Marco, dass das erhöhte Krankheitsrisiko im Vollzug auch Auswirkungen auf das Krankheitsrisiko ausserhalb des Vollzugs habe. Bei Gefängnissen handle es sich nämlich nicht um in sich geschlossene Systeme. Es würden vielmehr immer wieder Personen (Vollzugsbeamte, Rechtsanwälte, NGO-Mitarbeiter etc.) in Gefängnisse gehen und diese wieder verlassen. Auf diese Weise würden Krankheiten auch nach „aussen“ verschleppt werden: Garcia-Guerrero/Marco Revista Española de Sanidad Penitenciaria 2012, 106 (110); vgl. auch ОвеRнeim S. 142 ff. 
III. Regelungsinstrumente zur Überbelegung - vorgeschlagene Mindeststandards

Das Problem der Überbelegung und der dem Einzelnen zur Verfügung zu stellende Platz sind sowohl auf internationaler als auch auf europäischer und nationaler Ebene schon seit geraumer Zeit Gegenstand von Regulierungsmechanismen und Empfehlungen. Schwerpunktmässig werden die auf Europaratsebene vorgeschlagenen Standards in diesem Kapitel näher betrachtet, wobei Entwicklungstendenzen herausdestilliert und Gemeinsamkeiten und Unterschiede ausgearbeitet werden. Besonderes Augenmerk verdienen auch die Begründungen der vorgeschlagenen Standards; weshalb also gerade der jeweils vorgeschlagene Mindeststandard anzustreben sein soll.

1. UN-Mindestgrundsätze für die Behandlung von Gefangenen und Nelson-Mandela-Regeln

Sowohl die UN-Mindestgrundsätze für die Behandlung Gefangener als auch die revidierten Nelson-Mandela-Regeln sehen hinsichtlich der Unterbringungsform, der Überbelegungssituationen und des jedem Inhaftierten zu gewährenden persönlichen Platzes in rudimentärer Weise nur Folgendes vor: Wenn die Unterbringung in Zellen erfolge, solle jeder Inhaftierte über Nacht seine eigene Zelle haben. Wenn aussergewöhnliche Umstände, wie etwa Überbelegungssituationen, es notwendig machen würden, könne von dieser Regel abgewichen werden, wenngleich dies nicht erstrebenswert sei (Regel 9.1 UN-SMR; Regel 12.1 der Nelson-Mandela-Regeln). Wenn zur Unterbringung Schlafsäle genutzt würden, sei sorgfältig zu prüfen, welche Strafgefangenen für diese Unterbringung geeignet seien und wer unter diesen Umständen zusammenpasse. Nachts habe weiter eine regelmässige Überwachung stattzufinden (Regel 9.2 UN-SMR; Regel 12.2 der Nelson-Mandela-Regeln). Bei allen Unterbringungsformen seien Anforderungen an den Gesundheitsschutz zu erfüllen, wozu neben den klimatischen Bedingungen und der Beleuchtung und Belüftung auch das Zur-Verfügung-Stellen eines Mindestmasses an Platz gehöre (Regel $10 \mathrm{UN}$ SMR; Regel 13 der Nelson-Mandela-Regeln). Eine nähere Definition, was dies konkret bedeutet oder aber nach welchen Kriterien dies zu bestimmen sein soll, folgt nicht. Wie gezeigt sind die Nelson-Mandela-Regeln unverändert verglichen mit jenen der UN-SMR, was bedeutet, dass die intergouvernementale Expertengruppe, welche mit der Revision der UN- 
SMR betraut wurde, bei diesen Regelungen keinen Reformbedarf erkannte oder hinsichtlich einer Präzisierung keine Einigung gefunden werden konnte. Dies erstaunt vor dem Hintergrund, dass es sich um eine relativ umfassende Reform handelte, welche insgesamt durch die Verabschiedung zahlreicher detaillierterer Bestimmungen einen höheren Schutzstandard zur Folge hatte.

2. Europäisches Komitee zur Verhütung von Folter und unmenschlicher oder erniedrigender Behandlung oder Strafe (CPT)

Bereits im zweiten General Report (Berichtszeitraum: 01.01.199131.12.1991) hält das CPT die Relevanz der Überbelegung für sein Tätigkeitsgebiet und das ihm übertragene Mandat fest. ${ }^{485}$ Das Komitee weist auf die Auswirkungen der Überbelegung auf den Haftbetrieb und die Haftumstände hin. ${ }^{486}$ Insbesondere ein Zusammentreffen von Überbelegung, raren Aktivitätsmöglichkeiten und ungenügendem Zugang zu den sanitären Anlagen sieht das CPT als besonders belastend. Es stellt aber gleichwohl schon in diesem zweiten General Report fest, dass die Überbelegung für sich alleine betrachtet schon unmenschlich oder erniedrigend sein kann. ${ }^{487}$

Die direkte Bedeutung der Überbelegung für die Tätigkeit des CPT widerspiegelt sich in zahlreichen General Reports, in denen die Überbelegung immer wieder thematisiert und bemängelt wird. ${ }^{488}$ Der jedem Einzelnen zu gewährleistende persönliche Platz („living space“), nach welchem

485 CPT - 2nd General Report [CPT/Inf (92) 3], Rn. 46.

486 CPT - 2nd General Report [CPT/Inf (92) 3], Rn. 46.

487 CPT - 2nd General Report [CPT/Inf (92) 3], Rn. 46.

488 Die Thematik der Überbelegung findet sich beispielsweise in den folgenden General Reports: 2nd General Report [CPT/Inf (92) 3], CPT - 3rd General Report [CPT/Inf (93) 12], CPT - 7th General Report [CPT/Inf (97) 10], CPT 8th General Report [CPT/Inf (98) 12], CPT - 11th General Report [CPT/Inf (2001) 16], CPT - 13th General Report [CPT/Inf (2003) 35], CPT - 15th General Report [CPT/Inf (2005) 17], CPT - 17th General Report [CPT/Inf (2007) 39], CPT - 18th General Report [CPT/Inf (2008) 25], CPT - 19th General Report [CPT/Inf (2009) 27], CPT - 20th General Report [CPT/Inf (2010) 28], CPT - 21st General Report [CPT/Inf (2011) 28], CPT - 22nd General Report [CPT/Inf (2012) 25], CPT - 23th General Report [CPT/Inf (2013) 29], CPT 24th General Report [CPT/Inf (2015) 1], CPT - 25th General Report [CPT/Inf (2016) 10], CPT - 26th General Report [CPT/Inf (2017) 5], CPT - 27th General Report [CPT/Inf (2018) 4], CPT - 28th General Report [CPT/Inf (2019) 9], CPT - 29th General Report [CPT/Inf (2020) 17]. 
die Belegung beurteilt werden sollte, wird zu diesem Zeitpunkt aber noch nicht einheitlich definiert, wenngleich immer wieder konkrete Quadratmeterzahlen ins Feld geführt wurden. ${ }^{489}$ Vereinzelte Angaben zum Minimum an zu gewährendem persönlichen Platz finden sich auch in diversen Länderberichten. ${ }^{490}$

Diese versprengten und fragmentarischen Ansätze zur Überbelegung und zum zu fordernden persönlichen Platz führt das CPT erst im Jahr 2015 mit dem Dokument „Living Space per Prisoner in Prison Establishments" zusammen. ${ }^{491}$ Vor dem Hintergrund eines zunehmenden Interesses für das Problem der Überbelegung, und damit einhergehend eines gesteigerten Interesses auch der Mitgliedstaaten an den Berichten und Arbeiten des CPT, strebte das CPT die Benennung eindeutiger Standards an, was mit diesem Dokument umgesetzt werden sollte. 492

Um eine klare Positionierung bemüht, muss sich das CPT Herausforderungen stellen: ${ }^{493}$ Die Art der freiheitsentziehenden Einrichtung (Polizei-

489 Beispielsweise hält schon der 2. General Report fest, dass die Notwendigkeit besteht, Angaben zu den ungefähren Grössen einer Polizeizelle zur Unterbringung einer einzelnen Person zu machen („need for a rough guideline“): Erstrebenswert seien $7 \mathrm{~m}^{2}$ Platz, wenn der Aufenthalt länger als einige Stunden dauere: CPT - 2nd General Report [CPT/Inf (92) 3], Rn. 43. Im 23. General Report wurden für die Unterbringung in einer Strafvollzugsanstalt konkret $4 \mathrm{~m}^{2}$ persönlicher Platz gefordert (Mehrbettzellen): CPT - 23th General Report [CPT/Inf (2013) 29], Rn. 45. Auch im 24. General Report forderte das CPT bei Mehrbettzellen die Gewährung von $4 \mathrm{~m}^{2}$ persönlichem Platz eines jeden Inhaftierten: CPT - 24th General Report [CPT/Inf (2015)], Rn. 76. Auch ausserhalb der konkreten Überbelegungsthematik wurde das Erfordernis eines genügenden "living space immer wieder thematisiert. Im Kontext ausländerrechtlicher Haft: CPT - 7th General Report [CPT/Inf (97) 10], Rn. 29; im Zusammenhang mit in psychiatrischen Einrichtungen untergebrachten Patienten: CPT - 8th General Report [CPT/Inf (98) 12], Rn. 34: Aufforderung, genügend Platz zur Verfügung zu stellen, wobei hier keine Konkretisierung erfolgte.

490 Vgl. bspw.: CPT - Report to the Albanian Government [CPT/Inf (2006) 24], Rn.93; CPT - Report to the Azerbaijani Government [CPT/Inf (2004) 36], Rn. 87; CPT - Report to the Bulgarian Government [CPT/Inf (2008) 11], Rn. 55, 77; CPT - Report to the Croatian Government [CPT/Inf (2008) 29], Rn. 56, 71; CPT - Report to the Latvian Government [CPT/Inf (2005) 8], Rn. 65; CPT - Report to the Hungarian Government [CPT/Inf (2010) 16], Rn. 65 und 80.

491 CPT - Living Space per Prisoner [CPT/Inf (2015) 44].

492 CPT - Living Space per Prisoner [CPT/Inf (2015) 44], Rn. 3.

493 Das CPT betont selbst, dass es nicht so einfach sei, einen Standard zu definieren, wie es zunächst erscheinen möge: CPT - Living Space per Prisoner [CPT/Inf (2015) 44], Rn. 6. 
zelle, psychiatrische Institution, Untersuchungshaft oder "gewöhnliche“ Strafvollzugsanstalt), die unterschiedlichen Unterbringungskonzepte (Einzelzellen oder Mehrbettzellen mit weiteren Unterscheidungsnotwendigkeiten [etwa: Zweierzelle vs. Grossraumschlafräume $\left.{ }^{494}\right]$ ) und die Notwendigkeit der Berücksichtigung des jeweiligen Haftregimes ${ }^{495}$ würden Differenzierungen beim zu fordernden Mindeststandard notwendig machen. ${ }^{496}$

Wegen der Abhängigkeit des zu gewährenden persönlichen Platzes vom Haftregime sieht sich das CPT veranlasst, sich von der Proklamierung eines absolut geltenden Anspruchs zu distanzieren. ${ }^{497}$ Das CPT folgert weiter, dass bei einer geringfügigen Abweichung (minor deviation), d.h. bei einer geringfügigen Unterschreitung des vorgeschlagenen Standards, nicht automatisch von einer unmenschlichen oder erniedrigenden Behandlung auszugehen sei. ${ }^{498}$ Die Bedeutung der "Geringfügigkeit“ als offener Rechtsbegriff wird zunächst allerdings nicht näher umschrieben. Erleichternde Umstände, wie etwa die Tatsache, dass der Strafgefangene viel Zeit ausserhalb der Zelle verbringen kann, könnten kompensierend wirken. ${ }^{499}$ Trotzdem empfiehlt das CPT auch beim Vorliegen kompensierender Faktoren die Einhaltung des vom CPT erarbeiteten Mindeststandards. ${ }^{500}$ Mit Blick auf das bei absoluten Rechten geltende Konkretisierungserfordernis sowie der Verkündung, einen eindeutigen Standard setzen zu wollen ${ }^{501}$, scheint die Anerkennung kompensierender Elemente durch das CPT prima vista nicht unproblematisch, zumindest wenn dieser Standard vom EGMR zur Auslegung eines absoluten Konventionsrechts herangezogen wird. Neben extremen Überbelegungssituationen, welche für sich alleine schon als unmenschliche oder erniedrigende Behandlung angesehen werden, anerkennt das CPT weiter eine Kumulationswirkung zusätzlicher negativer Faktoren wie beispielsweise einer ungenügenden Anzahl Betten, einer schlechten hygienischen Versorgung, eines Ungezieferbefalls,

494 Das CPT kritisiert dabei bereits von vorneherein das Konzept der GrossraumSchlafräume und befürwortet eine Entwicklung hin zu Unterbringungsmodalitäten mit einer kleineren Anzahl Personen, welche sich eine Zelle teilen: CPT Living Space per Prisoner [CPT/Inf (2015) 44], Rn. 7; vgl. zuvor auch schon CPT 11th General Report [CPT/Inf (2001) 16], Rn. 29.

495 CPT - Living Space per Prisoner [CPT/Inf (2015) 44], Rn. 8, 21.

496 CPT - Living Space per Prisoner [CPT/Inf (2015) 44], Rn. 6 f.

497 CPT - Living space per Prisoner [CPT/Inf (2015) 44], Rn. 21.

498 CPT - Living Space per Prisoner [CPT/Inf (2015) 44], Rn. 21.

499 CPT - Living Space per Prisoner [CPT/Inf (2015) 44], Rn. 21.

500 CPT - Living Space per Prisoner [CPT/Inf (2015) 44], Rn. 21.

501 CPT - Living Space per Prisoner [CPT/Inf (2015) 44], Rn. 3. 
schlechter Belüftungs- oder Lichtverhältnisse oder weniger oder keiner Outdoor-Aktivitäten. ${ }^{502}$ Das CPT nimmt damit eine Gesamtbetrachtung vor, welche sowohl Kumulations- als auch Kompensationswirkungen berücksichtigt.

In seinem konsolidierten Dokument „Living Space per Prisoner in Prison Establishments" von 2015 legt das CPT den Mindeststandard wie folgt fest: Als Faustregel soll für Einzelzellen ein Minimum von $6 \mathrm{~m}^{2}$ Wohnplatz (exkl. sanitären Anlagen) gewährt werden, während in einer Mehrbettzelle (multiple-occupancy cell ${ }^{503}$ ) jedem Inhaftierten mindestens $4 \mathrm{~m}^{2}$ Wohnplatz zur Verfügung zu stellen seien (exkl. sanitären Anlagen). ${ }^{504}$ Für die sanitären Anlagen sei mit zusätzlichen 1-2 $\mathrm{m}^{2} \mathrm{zu}$ rechnen. ${ }^{505}$ Für Einrichtungsgegenstände wird jedoch kein zusätzlicher Platz gefordert. Die Mindestdeckenhöhe legt das CPT einheitlich auf 2,5 m fest. ${ }^{506}$ Darüber hinaus macht das CPT auch Vorgaben an die Ausgestaltung der Zellen. So fordert es bei Mehrbettzellen eine vollständige Abtrennung des Sanitärbereichs vom Wohnplatz. ${ }^{507}$ Der Zellenraum dürfe sodann in keiner Dimension $2 \mathrm{~m}$ unterschreiten. ${ }^{508}$

Zur Begründung des Mindeststandards von $4 \mathrm{~m}^{2}$ persönlichen Platzes in Mehrbettzellen fügt das CPT selbst das Folgende an:

"When devising the standard of $4 \mathrm{~m}^{2}$ of living space, the CPT had in mind on the one hand the trend observed in a number of western European coun-

502 CPT - Living Space per Prisoner [CPT/Inf (2015) 44], Appendix. Als kumulierende Elemente werden damit hauptsächlich Faktoren innerhalb der Zelle aufgeführt. Gewisse Faktoren, welche nicht direkt die Zelle, wohl aber die Situation des Strafvollzugs betreffen, finden aber auch Berücksichtigung. Das CPT hatte zuvor schon in seinem 2. General Report auf die Wirkung kumulativer Elemente hingewiesen: CPT - 2nd General Report [CPT/Inf (92) 3], Rn. 35.

503 Das CPT hat den Terminus "multiple occupancy“ soweit ersichtlich nie näher definiert, die Länderberichte wiesen aber darauf hin, dass das CPT damit eine Belegung von zwei bis vier Strafgefangenen meint: so CPT - Living Space per Prisoner [CPT/Inf (2015) 44], Rn. 14.

504 CPT - Living Space per Prisoner [CPT/Inf (2015) 44], Rn. 9.

505 CPT - Living Space per Prisoner [CPT/Inf (2015) 44], Rn. 10.

506 CPT - Living Space per Prisoner [CPT/Inf (2015) 44], Rn. 11.

507 CPT - Living Space per Prisoner [CPT/Inf (2015) 44], Rn. 10. Das CPT hält dies aufgrund seines Kompetenzbereiches und Handlungsauftrags im Zusammenhang mit der unmenschlichen und erniedrigenden Behandlung fest. Meines Erachtens liegt diese Thematik aus menschenrechtlicher Sicht zumindest im Schnittstellenbereich auch zu Fragen der Privatsphäre, so dass solche Forderungen insbesondere auch aus Art. 8 EMRK abzuleiten wären.

508 CPT - Living Space per Prisoner [CPT/Inf (2015) 44], Rn. 11. 
tries of doubling up 8 to $9 \mathrm{~m}^{2}$ cells that were originally designed for single occupancy, and on the other hand the existence of large-capacity dormitories in prison establishments (colonies) in various central and eastern European countries."509

Da das CPT schon zuvor das Bestehen von Grossraumschlafräumen kritisiert hatte, ${ }^{510}$ gedenkt das CPT hier wohl erneut, seine Präferenz zu einer Belegung mit weniger Inhaftierten auszudrücken. Gestützt auf die Beobachtung, dass in mehreren westlichen Ländern in Zellen mit 8-9 $\mathrm{m}^{2}$ Fläche zwei Personen untergebracht werden, führt die Festlegung des Mindeststandards bei $4 \mathrm{~m}^{2}$ zudem dazu, dass in solchen bestehenden Zellen nicht mehr als zwei Personen untergebracht werden. Gleichwohl vermag diese Begründung für sich alleine noch nicht ausreichend $\mathrm{zu}$ belegen, weshalb die Gewährung von $4 \mathrm{~m}^{2}$ persönlichem Platz auch aus einer menschenrechtlichen Perspektive genügt. Die Tatsache allein, dass das Modell der westlichen europäischen Länder gegenüber dem Modell der zentralen und östlichen europäischen Länder zu bevorzugen ist, bedeutet nämlich noch lange nicht, dass der typischerweise im westlichen Modell gewährte Standard dem Mindeststandard entspricht, der aus einer menschenrechtlichen Sicht erforderlich scheint. ${ }^{511}$ Der Mindeststandard ist vielmehr unabhängig von vorbestehenden baulichen und infrastrukturellen Gegebenheiten dort festzulegen, wo die Platzverhältnisse keine unmenschlichen oder erniedrigenden Auswirkungen auf den Einzelnen im Sinne des Europäische Übereinkommens zur Verhütung von Folter und unmenschlicher oder erniedrigenden Behandlung haben. In diesem Sinne hatte das CPT zuvor auch selbst das Folgende festgestellt:

„The consequences of overcrowding have been highlighted repeatedly by the CPT in its visit reports: cramped and unbygienic accommodation; constant lack of privacy; reduced out of cell activities, due to demand outstripping the staff and facilities available; overburdened health-care services; increased tension and hence more violence between prisoners and between prisoners

509 CPT - Living Space per Prisoner [CPT/Inf (2015) 44], Rn. 13.

510 CPT - Living Space per Prisoner [CPT/Inf (2015) 44], Rn. 7; CPT - 11th General Report [CPT/Inf (2001) 16], Rn. 29.

511 Wäre dies die einzige Begründung des CPT zur Proklamation von $4 \mathrm{~m}^{2}$ persönlichem Platz, läge ein "Sein-Sollen-Fehlschluss“ vor. Vom tatsächlichen Umstand des „Seins" kann nämlich noch nicht auf das "Sollen“ geschlossen werden. Dies auch dann nicht, wenn es mitunter gerade Aufgabe des CPT ist, den Zustand in den Mitgliedstaaten - somit den „Ist-Zustand“ - zu ermitteln. Das „Sollen“ ist auf eine andere Weise herzuleiten. 
and staff. The CPT considers that the question of minimum living space per inmate is intrinsically linked to the commitment of every Council of Europe member state to respect the dignity of persons sent to prison. "512

Dies verdeutlicht, dass sich das CPT bei seiner Empfehlung des Mindeststandards von $4 \mathrm{~m}^{2}$ nicht nur auf vorgegebene infrastrukturelle Gegebenheiten abstützte, sondern mit Blick auf die Kriterien einer unmenschlichen oder erniedrigenden Behandlung auch eine von der Menschenwürde her gedachte, wertende Beurteilung vornahm, welche sich auf soziomedizinische Faktoren abstützt. ${ }^{513}$ Nur eine solche Analyse und entsprechende Begründung erlaubt es denn meines Erachtens, überhaupt Aussagen betreffend einen erforderlichen menschenrechtlichen Mindeststandard zu treffen.

Das CPT räumt in einigen Länderberichten selbst gewisse Einwände hinsichtlich seines festgesetzten Standards ein. So stellte es in einigen Berichten fest, dass Zellen von 8-9 $\mathrm{m}^{2}$ Fläche „preferably“514 bzw. „idéalement" ${ }^{\text {"515 }}$ nur für einen Strafgefangenen genutzt werden sollten, dass 8-10 $\mathrm{m}^{2}$ grosse Zellen bei einer Zweifachbelegung "not without discom-

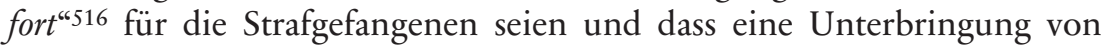
zwei Personen in einer $8 \mathrm{~m}^{2}$ grossen Zelle zu vermeiden sei ${ }^{517}$. Ausgehend vom Mindeststandard von $6 \mathrm{~m}^{2}$ für eine Einzelzelle erscheine es auch nicht von vorneherein klar, dass eine Zelle von $8 \mathrm{~m}^{2}$ Fläche für zwei Personen genüge. ${ }^{518}$

Wohl durch die Zweifel am eigenen Mindeststandard veranlasst, legt das CPT - neben dem weiterhin geltenden Mindeststandard - einen erstrebenswerten Standard (desirable standard) fest, welcher ganz im Sinn der präventiven Konzeption des CPT vor allem im Hinblick auf den Neu-

512 CPT - Living Space per Prisoner [CPT/Inf (2015) 44], Rn. 5.

513 Es sei hier erwähnt, dass das CPT wichtige Analysen zu verschiedenen Strafvollzugssystemen insbesondere zu den Zellengrössen und den Aufschlusszeiten gemacht hat.

514 CPT - Living Space per Prisoner [CPT/Inf (2015) 44], Rn. 14 m.V.z. CPT Report to the Slovenian Government [CPT/Inf (2008) 7] und CPT - Report to the Hungarian Government [CPT/Inf (2014) 13].

515 CPT - Living Space per Prisoner [CPT/Inf (2015) 44], Rn. 14 m.V.z. CPT Rapport au gouvernement de la Belgique [CPT/Inf (2010) 24].

516 CPT - Living Space per Prisoner [CPT/Inf (2015) 44], Rn. 14 m.V.z. CPT Report to the Government of the Netherlands [CPT/Inf (2012) 21].

517 CPT - Living Space per Prisoner [CPT/Inf (2015) 44], Rn. 14 m.V.z. CPT Report to the Government of Ireland [CPT/Inf (2011) 3].

518 CPT - Living Space per Prisoner [CPT/Inf (2015) 44], Rn. 15. 
bau von Strafvollzugsanstalten von Bedeutung sein soll. ${ }^{519}$ Der desirable standard legt für Zellen mit einer Belegung von zwei bis vier Inhaftierten einen im Vergleich zum Mindeststandard höheren Standard fest, welchem der Standard für Einzelzellen $\left(6 \mathrm{~m}^{2}\right)$ als Ausgangspunkt dient. ${ }^{520} \mathrm{Zu}$ den sechs Quadratmetern für die erste Person werden beim desirable standard pro weitere Person $4 \mathrm{~m}^{2}$ addiert (wiederum exkl. des sanitären Bereichs). ${ }^{521}$ Bei zwei Personen ergibt dies einen desirable standard von $10 \mathrm{~m}^{2}$, bei drei Personen von $14 \mathrm{~m}^{2}$ und bei 4 Personen von $18 \mathrm{~m}^{2}$. Keine Modifizierung nimmt das CPT für Zellen mit fünf und mehr Personen vor, so dass es bei fünf Personen bei $20 \mathrm{~m}^{2}$ bleibt (Mindeststandard $5 \times 4 \mathrm{~m}^{2}$ ). Auch wenn sich die Differenz zwischen dem Mindeststandard und dem desirable standard pro zusätzlichen Gefangenen verkleinert und somit der Effekt dieser modifizierten Rechenmethode bei steigender Anzahl Personen pro Zelle insgesamt abnimmt, ${ }^{522}$ ist nicht von vorneherein einleuchtend, warum die Notwendigkeit der Proklamierung eines zusätzlichen desirable standard gerade bei einer Zellenbelegung von vier Inhaftierten aufhört. Es könnte nämlich analog zur vormaligen Argumentation des CPT vorgebracht werden, dass das Zurverfügungstellen von $20 \mathrm{~m}^{2}$ für fünf Personen nicht einleuchtet, wenn für vier Personen $18 \mathrm{~m}^{2}$ vorgesehen sind. Wahrscheinlich scheint allerdings, dass das CPT überhaupt nur Zellen mit einer Belegung von zwei bis vier Personen als zu fördernd betrachtet und deshalb auch nur für diese Unterbringungsweise einen desirable standard festlegt (allg. Präferenz für Unterbringungsmodelle mit wenigen Inhaftierten pro Zelle).

Die Festsetzung eines höheren desirable standard lässt gerade in Anbetracht der Präventionsfunktion des CPT die Vermutung zu, dass das CPT den eigenen Mindeststandard nicht (mehr) wirklich für ausreichend erachtet und langfristig einen höheren Standard anzustreben gedenkt. Die zeitlich nach der Ausarbeitung des desirable standard verfassten jährlichen General Reports, welche ihrerseits hauptsächlich auf den Mindeststandard verweisen, verdeutlichen allerdings, dass der desirable standard (zumindest vorerst) nichts an der Weitergeltung des festgesetzten Mindeststandards zu ändern vermochte. ${ }^{523}$ So hält das CPT etwa auch im 26. General Report grundsätzlich an den $4 \mathrm{~m}^{2}$ fest und geht davon aus, dass ein Unter-

519 CPT - Living Space per Prisoner [CPT/Inf (2015) 44], Rn. 18.

520 CPT - Living Space per Prisoner [CPT/Inf (2015) 44], Rn. 16: Für Einzelzellen wird kein besonderer Standard festgelegt.

521 CPT - Living Space per Prisoner [CPT/Inf (2015) 44], Rn. 16.

522 Die prozentuale Differenz wird immer kleiner, da die zusätzlichen $2 \mathrm{~m}^{2}$ für die erste Person von immer mehr Insassen geteilt werden.

523 CPT - 25th General Report [CPT/Inf (2016) 10], Rn. 83 ff. 
schreiten der 4-m²-Grenze die Wahrscheinlichkeit einer Verletzung von Art. 3 EMRK wesentlich erhöht. ${ }^{524}$

Zusammenfassend kann festgehalten werden, dass die Überbelegung und die damit verbundenen engen Platzverhältnisse in Strafvollzugsanstalten vom CPT seit Langem als zentrale Probleme erkannt wurden. Seine versprengten Ansätze und Äusserungen konsolidierte das CPT allerdings erst im Jahr 2015 im Dokument „Living Space per Prisoner in Prison Establishments: CPT Standards" ${ }^{\text {25 }}$, in welchem es sowohl einen Mindeststandard als auch einen desirable standard festsetzte. Es wurde aufgezeigt, dass dem Mindeststandard des CPT eine von der Menschenwürde her gedachte normative Wertung zugrunde liegt, welche als Bewertungsgrundlage auch soziomedizinische Schlussfolgerungen aufgreift. Das Ziel, eine klare Position zu beziehen, erreichte das CPT nicht vollumfänglich, da es selbst Relativierungen etwa durch Kompensationsmöglichkeiten bei nicht weiter definierten „geringfügigen Abweichungen“ ${ }^{\circ 26}$ vom Standard zulässt. Auf diese Weise weicht es seinen Standard auf.

3. Europäische Mindestgrundsätze für die Behandlung der Gefangenen (ESMR) und Europäische Strafvollzugsgrundsätze (EPR)

Die ursprüngliche Version der Europäischen Mindestgrundsätze für die Behandlung der Gefangenen vom 19.01.1973 (ESMR) ${ }^{527}$ thematisiert die Überbelegung bzw. die Unterbringungsform sowie den zu gewährenden persönlichen Platz nur am Rande. In Regel 8 ESMR wird lediglich - wie schon in den UN-Mindestgrundsätzen - der Grundsatz festgehalten, dass Strafgefangene über Nacht in eigenen Zellen untergebracht werden sollten, sofern die Umstände nicht andere Lösungen gebieten würden. Zu den Platzverhältnissen hält Regel 9 ESMR lediglich fest, dass in allen Unterbringungsräumen und insbesondere in den Schlafräumen ein Minimum an Platz („minimum floor space") zu gewähren sei. Auch hier wird in den

524 CPT - 26th General Report [CPT/Inf (2017) 5], Rn. 56: Das CPT verweist dabei auch auf das Urteil EGMR (GK) - Muršić/CRO, Urt. v. 20.10.2016, 7334/13 und wiederholt dabei im Wesentlichen die Kernpunkte des EGMR, ohne sich dazu näher zu positionieren.

525 CPT - Living Space per Prisoner [CPT/Inf (2015) 44].

526 Den offenen Rechtsbegriff der „geringfügigen Abweichungen“ gilt es durch das CPT näher zu konkretisieren.

527 Resolution (73) 5, Standard Minimum Rules for the Treatment of Prisoners. 
ESMR die gleiche schwammige Terminologie wie in den Mindestgrundsätzen für die Behandlung Gefangener der Vereinten Nationen verwendet.

Die Europäischen Strafvollzugsgrundsätze vom 12.02.1987 ${ }^{528}$ sehen eine ähnliche Regelung vor. Am Grundsatz der Einzelunterbringung über Nacht wird festgehalten (Regel 14). Ausnahmen von diesem Grundsatz sollen nur noch möglich sein, wenn eine Unterbringung mit weiteren Strafgefangenen Vorteile mit sich bringt (Regel 14 EPR). Aus dem Wortlaut der Regel 14 geht noch nicht eindeutig hervor, wem die Vorteile dienen sollen, dem Inhaftierten oder dem Staat (vgl. zur revidierten Fassung sogleich Regel 18.5). Die neue Regel 15 hält zu den Platzverhältnissen jetzt fest, dass ein angemessenes Mass an Fläche („reasonable amount of space") zu garantieren sei.

Ein Vergleich des Wortlautes der beiden Bestimmungen (ESMR und EPR) - die Modifikation von einem Minimum an Platz hin zu angemessenem Platz - erlaubt die Interpretation, dass die Anforderungen an den zur Verfügung zu stellenden Platz zu Gunsten der Strafgefangenen gesteigert werden sollten. Dennoch benutzen auch die EPR einen unbestimmten Rechtsbegriff, der auslegungsbedürftig und mit weiteren konkreten Gehalten zu füllen ist.

Auch nach der vollständigen Revision der Europäischen Strafvollzugsgrundsätze vom Jahr $2006^{529}$, welche verschiedene europaratliche themenspezifische Empfehlungen wieder aufnimmt - so insbesondere die Recommendation R (99) 22 Concerning Prison Overcrowding and Prison Population Inflation -, wird weiterhin am Grundsatz der Einzelunterbringung der Strafgefangenen über Nacht festgehalten (Regel 18.5). Ausnahmen sind nun allerdings nur noch dann möglich, wenn eine Unterbringung mit Mitinhaftierten für den Strafgefangenen vorzuziehen ist (Regel 18.5). Für eine gemeinsame Unterbringung wird zudem eine prinzipielle Eignung der Strafgefangenen für eine gemeinsame Unterbringung sowie das Zusammenpassen ${ }^{530}$ der betroffenen Inhaftierten gefordert (Regel 18.6). Eine gemeinsame Unterbringung ist den Strafgefangenen soweit als möglich zur Wahl zu stellen (Regel 18.7). Bezüglich des spezifisch zu gewährenden persönlichen Platzes und der Unterbringungsform finden sich in den Europäischen Strafvollzugsgrundsätzen in der Version von 2006

528 Recommendation R (87) 3 on the European Prison Rules 1987.

529 Recommendation Rec (2006) 2 on the European Prison Rules 2006.

$530 \mathrm{Im}$ erläuternden Bericht wird etwa festgehalten, dass Nicht-Raucher nicht mit Rauchern unterzubringen sind: Commentary to Recommendation Rec (2006) 2 on the European Prison Rules, Rule 18.5. 
gegenüber jener von 1987 keine echten Neuerungen. Die Unterbringung (insbesondere auch die Unterbringung über Nacht) habe die Menschenwürde und soweit als möglich auch die Privatsphäre zu schützen, und es sei insbesondere den Platzverhältnissen [...] gebührend Beachtung zu schenken (Regel 18.1). Nationales Recht habe dabei einen konkreten Mindeststandard vorzusehen (Regel 18.3) und zu garantieren, dass die vorgesehenen Minimalanforderungen nicht durch Überbelegungssituationen unterlaufen würden (Regel 18.4).

Zu Regel 18.3 wird im erläuternden Bericht zu den Empfehlungen von $2006^{531}$ ausgeführt, woran sich das nationale Recht bei der Festlegung eines Mindeststandards zu orientieren habe. $\mathrm{Zu}$ berücksichtigen seien Überlegungen bezüglich Menschenwürde, Gesundheit und Hygienegesichtspunkten. ${ }^{532}$ Die Mitgliedstaaten sind folglich durch die Europäischen Strafvollzugsgrundsätze aufgefordert, den gesetzlich vorgesehenen Mindeststandard auf eine von der Menschenwürde her gedachte, normative Wertung zu stützen, bei welcher auch soziomedizinische Faktoren (wie eben Gesundheits- und Hygieneaspekte) zu berücksichtigen sind. Weiter verweist der erläuternde Bericht auf die CPT-Massstäbe und die EGMR-Rechtsprechung und gelangt zum Schluss, dass die Mitgliedstaaten gut beraten seien, den CPT-Standard umzusetzen, wobei in diesem Zusammenhang auch darauf hingewiesen wird, dass sich die Anforderungen an die Menschenwürde in diesem Bereich ständig weiterentwickeln. ${ }^{533}$

Vor dem Hintergrund der Überbelegungsproblematik erstaunt der Grundsatz der Einzelunterbringung über Nacht, an welchem seit den ersten Europäischen Mindestgrundsätzen für die Behandlung der Gefangenen von 1973 festgehalten wird. ${ }^{534}$ Abweichungen von diesem Prinzip wurden durch die Revisionen im Laufe der Zeit sogar nur noch restriktiver zugelassen. Lässt der Wortlaut der ESMR (1973) noch sämtliche Gründe für eine Abweichung genügen (denkbar wären wohl etwa auch logistische Gründe), darf gemäss dem klaren Wortlaut der EPR (2006) nur noch

531 Commentary to Recommendation Rec (2006) 2 on the European Prison Rules 2006.

532 Commentary to Recommendation Rec (2006) 2 on the European Prison Rules 2006, Rule 18.3: „Such standards would have to meet wider considerations of human dignity as well as practical ones of health and hygiene."

533 Commentary to Recommendation Rec (2006) 2 on the European Prison Rules 2006, Rule 18.3.

534 Der gleiche Grundsatz wird aber etwa auch in den Nelson Mandela Rules statuiert: A/RES/70/175, United Nations Standard Minimum Rules for the Treatment of Prisoners („The Nelson Mandela Rules“), Rule 12. 
dann von einer Einzelunterbringung abgesehen werden, wenn dies für den Inhaftierten selbst vorteilhaft erscheint, wobei ihm vor der Unterbringung mit weiteren Strafgefangenen soweit möglich gar eine Mitsprachemöglichkeit eingeräumt werden soll. Das Spannungsverhältnis zwischen der Überbelegung und dem Grundsatz der Einzelunterbringung über Nacht wird denn auch im erläuternden Bericht zu den Empfehlungen EPR (2006) erkannt. Eine Notwendigkeit, diesen Grundsatz zu überdenken, wird darin allerdings nicht gesehen. Vielmehr wird der Grundsatz bekräftigt und statuiert, dass es auf lange Sicht keine Option sei, sich aufgrund herrschender Überbelegung vom Einzelunterbringungsgrundsatz zu distanzieren. ${ }^{535} \mathrm{Be}-$ gründet wird der Grundsatz vor allem mit Blick auf Langzeitinhaftierte, für welche die Zelle ein „Zuhause“ sei. ${ }^{536}$ So wird im erläuternden Bericht zu den Empfehlungen EPR (2006) an die Nationalstaaten appelliert, bei der Planung und dem Bau von neuen Strafvollzugsanstalten den Grundsatz der Einzelunterbringung zu berücksichtigen. Vor dem Hintergrund, dass sich die von den EPR propagierte Unterbringungsform damit doch wesentlich von jener des CPT, welches keine Präferenz hin zu einer Einzelunterbringung vorsieht, unterscheidet, erstaunt, dass der erläuternde Bericht bezüglich des mindestens zu gewährenden persönlichen Platzes Anhaltspunkte beim CPT sucht.

Zusammenfassend ist festzuhalten, dass die ESMR, und später die Europäischen Strafvollzugsgrundsätze, das Prinzip der Einzelunterbringung über Nacht empfehlen. Möglichkeiten zur Abweichung von diesem Grundsatz wurden durch die Revisionen immer weiter eingeschränkt. Bei der Frage des konkret zu gewährenden Platzes nimmt der Europarat im Rahmen der Europäischen Strafvollzugsgrundsätze eine sehr zurückhaltende Position ein und überlässt die Festlegung eines konkreten Mindeststandards den Nationalstaaten. Dem erläuternden Bericht zu den Empfehlungen von 2006 ist aber immerhin zu entnehmen, nach welchen Kriterien die Nationalstaaten den Standard festzusetzen haben und welche Rolle dabei dem CPT-Standard zuzukommen hat.

535 Commentary to Recommendation Rec (2006) 2 on the European Prison Rules 2006, Rule 18.5.

536 Commentary to Recommendation Rec (2006) 2 on the European Prison Rules 2006, Rule 18.5. 


\section{European Recommendation R (99) 22}

Die Europaratsempfehlung R (99) $22^{537}$, welche auch Eingang in die überarbeiteten Europäischen Strafvollzugsgrundsätze vom Jahr 2006 gefunden hat, setzt sich, auf die Inflation der Gefangenenpopulation eingehend, spezifisch mit der Überbelegungsproblematik auseinander. ${ }^{538}$ In Regel 7 wird festgehalten, dass im Falle einer Überbelegung den Platzverhältnissen besondere Beachtung zu schenken sei. Ein konkretes Minimum an zu gewährleistendem Platz wird jedoch nicht festgelegt. Eingehend setzt sich die Empfehlung demgegenüber mit möglichen Abhilfemassnahmen zur Überbelegung auseinander. ${ }^{539}$ Regel 8 greift dazu den Kompensationsgedanken auf (vgl. dazu auch die CPT-Empfehlungen). Den negativen Konsequenzen der Überbelegung sei durch ausgebaute Kontaktmöglichkeiten des Strafgefangenen mit seiner Familie und durch so viel Unterstützung als möglich zu begegnen. Konsequenzen betreffend die Höhe des zu gewährleistenden Mindeststandards lassen sich diesen vorgesehenen Kompensationsmechanismen hingegen nicht entnehmen.

Die Empfehlung R (99) 22 bezweckt in erster Linie die Sensibilisierung für die Überbelegung und die Ausarbeitung von Abhilfemassnahmen, ${ }^{540}$ weshalb es nicht erstaunt, dass hier kein konkreter Massstab hinsichtlich des zu gewährleistenden Platzes festgesetzt wurde. Für die Festsetzung eines Mindeststandards ist das Heranziehen dieser Empfehlung damit nur begrenzt geeignet. Die Empfehlung kann jedoch Lösungsansätze für festgestellte Mängel liefern.

Zusammenfassend ist festzuhalten, dass diese themenspezifische Europaratsempfehlung keinen konkreten Standard zum zu gewährleistenden persönlichen Platz festlegt. Kompensationsmechanismen sind in der Empfehlung im Sinne einer Möglichkeit zur Abmilderung der negativen Aus-

537 Recommendation R (99) 22 Concerning Prison Overcrowding and Prison Population Inflation.

538 Diese Empfehlung wurde ausgearbeitet, nachdem sich in den 1990er Jahren das Problem der Überbelegung in vielen europäischen Ländern verschärft hat: DüNKel/Geng Neue Kriminalpolitik 2003, 146; Dünkel/Morgenstern FS MüllerDietz 2001, S. $133 \mathrm{ff}$.

539 Beispielhaft: Die Festlegung von Kapazitätsgrenzen (Regel 6), Propagierung von zur Freiheitsstrafe alternativen Strafen (Regel 15), Anordnung von Untersuchungshaft nur wenn notwendig (Regel 11).

540 Vgl. auch Snacken Eur J Crim Policy Res 2006, 143 (150): In der Empfehlung R (99) 22 seien sämtliche Elemente einer reduktionistischen Strafrechtspolitik enthalten. Zustimmend zu den ausgearbeiteten Abhilfemassnahmen etwa DüNKEL/GENG Neue Kriminalpolitik 2003, 146 (149). 
wirkungen der Überbelegung vorgesehen; Schlussfolgerungen hinsichtlich der Höhe des zu gewährleistenden Standards werden aus diesen aber nicht gezogen. Wichtige Ansätze kann die Empfehlung jedoch bezüglich zu ergreifender Abhilfemassnahmen in Überbelegungssituationen liefern.

\section{Rechtsprechung des EGMR - Analyse und Kritik}

Die Auswirkungen der Überbelegung auf den Einzelnen und die daraus resultierende Relevanz von Art. 3 EMRK erfordern eine Analyse der EGMRRechtsprechung. Der aus menschenrechtlicher Sicht jedem Inhaftierten während des Freiheitsentzuges zur Verfügung zu stellende Platz wird deshalb im Folgenden anhand der Rechtsprechung der Strassburger Organe und vor dem Hintergrund der bereits analysierten Regelungsinstrumente analysiert.

Die Notwendigkeit einer Unterscheidung beim Mindeststandard zwischen einer Unterbringung in Einzelzellen oder in Mehrbettzellen wurde schon festgestellt. Die folgenden Ausführungen beziehen sich hier, sofern nicht ausdrücklich anders vermerkt, immer auf den Mindeststandard bei einer Unterbringung in einer Zelle mit mehreren Personen.

\section{Genese der Rechtsprechung - von Ananyev zu Muršić - materieller Kern des Standards}

Im Piloturteil Ananyev u.a./RUS griff der Gerichtshof seine bisherige Rechtsprechung zu ungenügenden Platzverhältnissen resp. Überbelegungssituationen auf ${ }^{541}$ und legte aufgrund dieser schliesslich drei Krite-

541 Der Gerichtshof hatte vor dem Urteil Ananyev u.a./RUS in zahlreichen Urteilen festgehalten, dass das Unterschreiten von $3 \mathrm{~m}^{2}$ persönlichem Platz für sich genommen zu einem Verstoss gegen Art. 3 EMRK führen kann: vgl. unter vielen etwa EGMR - Trepashkin/RUS (Nr.2), Urt. v. 16.12.2010, 14248/05, Rn. 113; EGMR - Kozhokar/RUS, Urt. v. 16.12.2010, 33099/08, Rn. 96. Weiter hatte der Gerichtshof in anderen Fällen schon zuvor bei mangelnden Schlafgelegenheiten die Relevanz von Art. 3 EMRK erkannt: vgl. bspw. EGMR - Gusev/RUS, Urt. v. 15.05.2008, 67542/01, Rn. 57; EGMR - Bagel/RUS, Urt. v. 15.11.2007, 37810/03, Rn. 56 ff.; EGMR - Dorokhov/RUS, Urt. v. 14.02.2008, 66802/01, Rn. 53 ff. Schliesslich hatte der Gerichtshof bei der Prüfung von Art. 3 EMRK schon früher festgestellt, dass bei kleinen Zellen, in welchen nur wenige Inhaftierte untergebracht werden konnten, die verbleibende Bodenfläche nach Abzug des Platzes, welcher für das Mobiliar gebraucht würde, bisweilen kaum ausreiche, 
rien fest, mit deren Hilfe die Frage der Konventionskonformität nach Art. 3 EMRK zu beantworten ist (im Folgenden „Ananyev-Test“). ${ }^{542}$ Es wird festgehalten, dass (1) jeder Inhaftierte einen individuellen Schlafplatz haben muss, (2) jedem Strafgefangenen mindestens $3 \mathrm{~m}^{2}$ persönlicher Platz zur Verfügung stehen müssen und (3) jeder Inhaftierte sich frei zwischen den Einrichtungsgegenständen bewegen können muss. ${ }^{543}$ Ist eine dieser drei kumulativen Voraussetzungen nicht erfüllt, greift eine starke Vermutung, dass die Unterbringung eine erniedrigende Behandlung i.S.v. Art. 3 EMRK ist. ${ }^{544}$ Diese Vermutung kann - so der Gerichtshof durch kompensatorische Effekte widerlegt werden. Der Wortlaut des Urteils Ananyev u.a./RUS lässt Kompensationsmöglichkeiten bei allen drei aufgestellten Kriterien zu. Eine Analyse der Folgerechtsprechung lässt allerdings die Annahme zu, dass eine Kompensationsmöglichkeit zumindest für das Fehlen des individuellen Schlafplatzes nicht ernsthaft in Betracht kommen kann. ${ }^{545}$ Ein individueller Schlafplatz muss bei einer Unterbringung in einer Strafvollzugsanstalt unter dem Gesichtspunkt von Art. 3 EMRK folglich absolut gewährleistet werden. ${ }^{546}$ Mit anderen Worten: Das Fehlen eines individuellen Schlafplatzes erreicht immer die erforderliche Mindestschwelle an Schwere, weshalb dies zwingend als Verstoss gegen Art. 3 EMRK zu qualifizieren ist. In der Folgerechtsprechung führte das zweite Kriterium des Ananyev-Tests dazu, dass der EGMR beim Unterschreiten von $3 \mathrm{~m}^{2}$ persönlichem Platz regelmässig davon ausging, dass die Überbelegung ohne weitere erschwerende Faktoren für sich alleine dermassen schwer wiegt, dass ein Verstoss gegen Art. 3 EMRK vorliegt. ${ }^{547}$

Im Urteil der Grossen Kammer Muršić/CRO arbeitet der Gerichtshof im Oktober 2016 seine bisherige Rechtsprechung auf und zeigt dabei seine bisherigen unterschiedlichen Rechtsprechungslinien auf. ${ }^{548} \mathrm{Im}$ Wesentlichen erkennt die Grosse Kammer, dass in einer Vielzahl der bisherigen

um sich innerhalb der Zelle noch bewegen zu können: vgl. etwa EGMR - Yevgeniy Alekseyenko/RUS, Urt. v. 27.01.2011, 41833/04, Rn. 87; EGMR - Gladkiy/ RUS, Urt. v. 21.12.2010, 3242/03, Rn. 68.

542 EGMR - Ananyev u.a./RUS, Urt. v. 10.01.2012, 42525/07 u. 60800/08, Rn. 143 ff.

543 EGMR - Ananyev u.a./RUS, Urt. v. 10.01.2012, 42525/07 u. 60800/08, Rn. 148.

544 EGMR - Ananyev u.a./RUS, Urt. v. 10.01.2012, 42525/07 u. 60800/08, Rn. 148.

545 Vgl. Kromrey H. S. 199. Auch im Urteil Ananyev u.a./RUS bezog sich die weitere Begründung der Kompensationsmöglichkeit nur auf die Haftraumgrösse.

546 So auch Kromrey H. S. 199, 202.

547 EGMR (GK) - MuršićlCRO, Urt. v. 20.10.2016, 7334/13, Rn. 105 mit Verweisen etwa zu EGMR - Orchowski/POL, Urt. v. 22.10.2009, 17885/04, Rn. 122; EGMR - Varga u.a./HUN, Urt. v. 10.03.2015, 14097/12 u.a., Rn. 75.

548 EGMR (GK) - Muršić/CRO, Urt. v. 20.10.2016, 7334/13, Rn. 103 ff. 
Urteile ein Verstoss gegen Art. 3 EMRK allein aufgrund der Platzverhältnisse und ohne weitere erschwerende Umstände bei einem Unterschreiten von $3 \mathrm{~m}^{2}$ persönlichem Platz festgestellt wurde. ${ }^{549}$ Standen zwischen $3 \mathrm{~m}^{2}$ und $4 \mathrm{~m}^{2}$ persönlicher Platz zur Verfügung, stellte der EGMR jedoch mehrheitlich nur dann eine Verletzung von Art. 3 EMRK fest, wenn weitere negative Faktoren zu den Platzverhältnissen hinzukamen. ${ }^{550}$ In Frage kommen etwa zusätzlich inadäquater Zugang zu Outdoor-Aktivitäten, ungenügende Belichtung, inadäquate Belüftung, ungenügende Möglichkeiten, die Toilette im Privaten zu nutzen oder die Nichteinhaltung genügender sanitärer oder hygienischer Voraussetzungen. ${ }^{551}$ In einer Minderheit der Fälle ging der EGMR aber auch bereits bei einer Unterschreitung von $4 \mathrm{~m}^{2}$ Platz von einer Verletzung von Art. 3 EMRK aus, ohne dass weitere negative Umstände zur Argumentation hinzugezogen worden wären. ${ }^{552}$ In Anbetracht und im Bewusstsein der mäandernden bisherigen Rechtsprechungslinien hielt der Gerichtshof in Muršić/CRO zunächst fest, dass an den früheren Piloturteilen (und der Mehrzahl der Urteile) festzuhalten sei und $3 \mathrm{~m}^{2}$ im Sinne von Art. 3 EMRK der relevante Mindeststandard seien. ${ }^{553}$

549 EGMR (GK) - Muršić/CRO, Urt. v. 20.10.2016, 7334/13, Rn. 105; vgl. die zitierten Entscheide in EGMR - Orchowski/POL, Urt. v. 22.10.2009, 17885/04, Rn.122; sowie die zitierten Entscheide in EGMR - Ananyev u.a./RUS, Urt. v. 10.01.2012, 42525/07 u. 60800/08, Rn. 145; vgl. unter vielen etwa EGMR Lind/RUS, Urt. v. 06.12.2007, 25664/05, Rn. 59; EGMR - Kantyrev/RUS, Urt. v. 21.06.2007, 37213/02, Rn. 50 f.; EGMR - Andrey Forlov/RUS, Urt. v. 29.03.2007, Rn. 47 ff.; EGMR - Labzov/RUS, Urt. v. 16.06.2005, 62208/00, Rn. 44; EGMR Melnik/UKR, Urt. v. 28.03.2006, 72286/01, Rn. 102 f.; EGMR - Dmitriy Sazonov/ RUS, Urt. v. 01.03.2012, Rn. 31 f.; EGMR - Nieciecki/GRE, Urt. v. 04.12.2012, 11677/11, Rn. 49 ff.; EGMR - Kanakis/GRE (Nr. 2), Urt. v. 12.12.2013, 40146/11, Rn.106f.; EGMR - Tatishvili/GRE, Urt. v. 31.07.2014, 26452/11, Rn.43; EGMR - Tereshchenko/RUS, Urt. v. 05.06.2014, 33761/05, Rn. $83 \mathrm{f}$.

550 Unter anderen: EGMR - Orchowski/POL, Urt. v. 22.10.2009, 17885/04, Rn. 122; EGMR - Ananyev u.a./RUS, Urt. v. 10.01.2012, 42525/07 u. 60800/08, Rn. 149; EGMR - Torreggiani u.a./ITA, Urt. v. 08.01.2013, 43517/09 u.a., Rn.69; EGMR - Vasilescu/BEL, Urt. v. 25.11.2014, 64682/12, Rn. 88; EGMR - Varga u.a./HUN, Urt. v. 10.03.2015, 14097/12 u.a., Rn. 78; EGMR - Jirsák/CZE, Urt. v. 05.04.2012, 8968/08, Rn. 64 ff.; EGMR - Culev/MDA, Urt. v. 17.04.2012, $60179 / 09$, Rn. $35 \mathrm{ff}$.

551 EGMR (GK) - Muršić/CRO, Urt. v. 20.10.2016, 7334/13, Rn. 106 m.w.N.

552 EGMR - Cotlet/ROM, Urt. v. 01.10.2013, 49549/11, Rn. 34, 36; EGMR - Apostu/ ROM, Urt. v. 03.02.2015, 22765, Rn. 79.

553 EGMR (GK) - Muršić/CRO, Urt. v. 20.10.2016, 7334/13, Rn. 109 f.: Der Gerichtshof argumentiert, im Bewusstsein dessen, dass er nicht durch seine früheren Urteile gebunden ist, mit der Rechtssicherheit, der Gleichbehandlung und 
Die Berechnungsmodalitäten konkretisiert der EGMR dahingehend, dass die $3 \mathrm{~m}^{2}$ exklusiv des Platzes, welcher innerhalb der Zelle für sanitäre Einrichtungen verwendet wird, zu verstehen sind. ${ }^{554}$ Anders wird bezüglich der übrigen Einrichtungsgegenstände vorgegangen. Der Platz, der für diese verwendet wird, ist nicht zu den $3 \mathrm{~m}^{2}$ hinzuzuzählen. ${ }^{555}$ Diesbezüglich wird aber immerhin weiter am dritten Kriterium des $A n$ anyev -Tests festgehalten, so dass auf jeden Fall sichergestellt sein muss, dass der Inhaftierte sich in der Zelle zwischen den Einrichtungsgegenständen frei bewegen kann. 556

Hat die Grosse Kammer im Urteil Muršić/CRO zunächst festgelegt, dass im Sinne von Art. 3 EMRK jedem Inhaftierten mindestens $3 \mathrm{~m}^{2}$ persönlicher Platz gewährt werden müssen, befasste sie sich in einem zweiten Schritt auch mit der Frage der Rechtsfolgen, welche beim Unterschreiten der 3- $\mathrm{m}^{2}$-Grenze eintreten sollen, also ob aus einem Unterschreiten der massgeblichen $3 \mathrm{~m}^{2}$ aus Sicht des EGMR der Verstoss gegen Art. 3 EMRK als Rechtsfolge automatisch eintritt (d.h. ohne Berücksichtigung weiterer Faktoren) oder ob das Unterschreiten der 3- $\mathrm{m}^{2}$-Grenze vorerst bloss $\mathrm{zu}$ einer Vermutung einer Verletzung von Art. 3 EMRK führt, welche widerlegt werden kann. Wie bereits ausgeführt, hatte der Gerichtshof in der Vergangenheit bisweilen im Unterschreiten der 3- $\mathrm{m}^{2}$-Grenze für sich alleine einen Verstoss gegen Art. 3 EMRK festgestellt: „in itself led to the conclusion that there had been a violation of Article 3 “. ${ }^{557}$ In die gleiche Richtung zielten auch die Fälle, bei denen der Gerichtshof beim Unterschreiten der $3 \mathrm{~m}^{2}$ eine Verletzung von Art. 3 EMRK festgestellt und in der Folge zur blossen

der Vorhersehbarkeit. Er wolle deshalb nicht ohne Grund die Rechtsprechungslinie früherer Pilot- und Leiturteile aufgeben.

554 EGMR (GK) - MuršićlCRO, Urt. v. 20.10.2016, 7334/13, Rn. 114.

555 EGMR (GK) - Muršić/CRO, Urt. v. 20.10.2016, 7334/13, Rn. 114. Hinsichtlich der Berechnungsmodalitäten stimmen EGMR und CPT folglich überein.

556 EGMR (GK) - Muršić/CRO, Urt. v. 20.10.2016, 7334/13, Rn. 114 m.V.z. EGMR - Ananyev u.a./RUS, Urt. v. 10.01.2012, 42525/07 u. 60800/08, Rn. 147 f.; EGMR - Vladimir Belyayev/RUS, Urt. v. 17.10.2013, 9967/06, Rn. 34.

557 EGMR (GK) - Muršić/CRO, Urt. v. 20.10.2016, 7334/13, Rn. 117 m.V.z. EGMR - Sulejmanovic/ITA, Urt. v. 16.07.2009, 22635/03, Rn. 43; EGMR - Trepashkin/RUS, Urt. v. 16.12.2010, 14248/05, Rn. 113; EGMR - Mandic u. Jovic/ SLO, Urt. v. 20.10.2011, 5774/10 u. 5985/10, Rn. 80; EGMR - Lin/GRE, Urt. v. 06.11.2012, 58158/10, Rn. 53 f.; EGMR - Blejuşcă/ROM, Urt. v. 19.03.2013, 7910/10, Rn. 43 ff; EGMR - Ivakhnenko/RUS, Urt. v. 04.04.2013, 12622/04, Rn. 35; EGMR - A.F./GRE, Urt. v. 13.06.2013, 83709/11, Rn. 77 f.; EGMR Kanakis/GRE, Urt. v. 12.12.2013, 40146/11, Rn. 106 f.; EGMR - Gorbulya/RUS, Urt. v. 06.03.2014, 31535/09, Rn. 64 ff.; EGMR - T. u. A./TUR, Urt. v. 21.10.2014, 47146/11, Rn. 96 ff. 
Untermauerung weitere erschwerende Elemente hinzugezogen hat ,[...] and then examined other conditions of detention only as further aggravating circumstances". ${ }^{558}$ Demgegenüber hatte der Gerichtshof in zahlreichen anderen Fällen die Unterschreitung auch der 3- $\mathrm{m}^{2}$-Grenze lediglich als starke Vermutung („strong presumption“) für das Vorliegen einer Verletzung von Art. 3 EMRK gewürdigt (vgl. bereits Ananyev-Test ${ }^{559}$ ). ${ }^{560}$ Über das Vorliegen einer Verletzungvon Art. 3 EMRK entschied in jenen Fällen dann letztlich, ob die aufgestellte Vermutung durch andere Faktoren (im Sinne einer Kompensation) widerlegt werden konnte: „rebutted by other cumulative effects ${ }^{561 “ .562}$ In zahlreichen "Post-Ananyev-Urteilen" nahm der Gerichtshof eine Gesamtbetrachtung der Haftumstände vor, um in der Folge über das Vorliegen einer Verletzung von Art. 3 EMRK zu entscheiden. ${ }^{563}$ Nach der Darlegung dieser grundsätzlichen Optionen bezüglich der Rechtsfolge eines Unterschreitens der $3 \mathrm{~m}^{2}$ hält die Grosse Kammer im Urteil MuršićlCRO in prüfungsmethodischer Hinsicht an einem „Strong presumption-Test“ fest. ${ }^{564}$ Auf diese Weise bleibe nämlich auch gewährleistet, dass der Schutzbereich von Art. 3 EMRK weiterhin relativ bestimmt

558 EGMR (GK) - Muršić/CRO, Urt. v. 20.10.2016, 7334/13, Rn. 117; EGMR Torreggiani u.a./ITA, Urt. v. 08.01.2013, 43517/09 u.a., Rn. 77; EGMR - Vasilescu/ BEL, Urt. v. 25.11.2014, 64682/12, Rn. $100 \mathrm{ff}$.

559 EGMR - Ananyev u.a./RUS, Urt. v. 10.01.2012, 42525/07 u. 60800/08, Rn. 148.

560 EGMR - Ananyev u.a./RUS, Urt. v. 10.01.2012, 42525/07 u. 60800/08, Rn. 148; ähnlich siehe auch bereits: EGMR - Orchowski/POL, Urt. v. 22.10.2009, 17885/04, Rn. 123. Der „Strong presumption-Test“ wurde in der Folge auch von mehreren Piloturteilen im Zusammenhang mit der Überbelegung wieder aufgenommen: vgl. EGMR - Neshkov u.a./BUL, Urt. v. 27.01.2015, 36925/10 u.a., Rn. 232; EGMR - Varga u.a./HUN, Urt. v. 10.03.2015, 14097/12 u.a., Rn. 74, 77.

561 In der Terminologie des Gerichtshofs ist der Begriff der „cumulative effects“ als Oberbegriff sowohl für erschwerende als auch für kompensatorische Faktoren zu verstehen. Da es hier um die Widerlegung der Vermutung einer Verletzung von Art. 3 EMRK geht, sind hier in concreto kompensatorische Elemente gemeint.

562 EGMR (GK) - Muršić/CRO, Urt. v. 20.10.2016, 7334/13, Rn. 121 m.V.z. EGMR - Ananyev u.a./RUS, Urt. v. 10.01.2012, 42525/07 u. 60800/08, Rn. 166; EGMR - Orchowski/POL, Urt. v. 22.10.2009, 17885/04, Rn. 135; EGMR - Lind/ RUS, Urt. v. 06.12.2007, 25664/05, Rn. 59 ff.; EGMR - Kokoshkina/RUS, Urt. v. 28.05.2009, 2052/08, Rn. 62 f.

563 Vgl. bspw. EGMR (GK) - Idalov/RUS, Urt. v. 22.05.2012, 5826/03, Rn.101; EGMR - Iacov Stanciu/ROM, Urt. v. 24.07.2012, 35972/05, Rn. 176 ff.; EGMR Asyanov/RUS, Urt. v. 09.10.2012, 11677/11, Rn. 43; EGMR - Neshkov u.a./BUL, Urt. v. 27.01.2015, 36925/10 u.a., Rn. 246 ff.; EGMR - Varga u.a./HUN, Urt. v. 10.03.2015, 14097/12 u.a., Rn. 88.

564 EGMR (GK) - Muršić/CRO, Urt. v. 20.10.2016, 7334/13, Rn. 122 ff. 
werde, da die Umstände des Einzelfalls wie etwa die Dauer der entsprechenden Unterbringung, die physischen und psychischen Auswirkungen, in gewissen Konstellationen auch das Geschlecht, das Alter oder der Gesundheitszustand, nach wie vor zu berücksichtigen sind. ${ }^{565}$ Eine Reduktion auf einen rein numerischen Standard würde gemäss EGMR zu kurz greifen und auch der gelebten Wirklichkeit im Strafvollzug nur ungenügend gerecht werden. ${ }^{566}$

Folglich gilt: Stehen einem Inhaftierten im konkreten Fall weniger als $3 \mathrm{~m}^{2}$ persönlicher Platz zur Verfügung, soll fortan die starke Vermutung (,strong presumption“) für das Vorliegen einer Verletzung von Art. 3 EMRK greifen.

Im Bewusstsein um das Abweichen vom durch das CPT vorgeschlagenen Standard von $4 \mathrm{~m}^{2}$ behält sich der Gerichtshof zusätzlich auch in Zukunft vor, den vom CPT gesetzten Standard im Auge zu behalten, und gibt an, die Fälle, die unter die vom CPT vorgeschlagenen $4 \mathrm{~m}^{2}$ fallen, besonders genau zu betrachten:

"[...] it (the Court) gives careful scrutiny to cases where the particular conditions of detention fall below the CPT's standard of $4 \mathrm{sq} . \mathrm{m}$."

Was genau der Gerichtshof unter einer „besonders genauen Betrachtung“ - insbesondere in Abgrenzung zum Gelten einer starken Vermutung versteht, ist nicht abschliessend geklärt. Wahrscheinlich ist, dass der Gerichtshof sich in diesen Fällen selbst eine erhöhte Prüfungsdichte und -tiefe auferlegt.

2. Prüfungsmethodik des EGMR - Analyse

2.1 Vermutungswirkungen - Widerlegbarkeit - Beweismass und -last

Nachdem das Gelten einer starken Vermutung bei der Prüfung eines Verstosses gegen Art. 3 EMRK als zentrales Kriterium identifiziert wurde, wird im Folgenden näher auf die konkreten weiteren Wirkungen des Vermutungseintritts eingegangen.

565 EGMR (GK) - Muršić/CRO, Urt. v. 20.10.2016, 7334/13, Rn. 122.

566 EGMR (GK) - Muršić/CRO, Urt. v. 20.10.2016, 7334/13, Rn. 123. Die damit einhergehenden relativierten Rechtswirkungen der Unterschreitung des 3- $\mathrm{m}^{2}$ Standards (prüfungsmethodisches Vorgehen des Gerichtshofs) haben direkte Wirkungen auf den Menschenrechtsschutz des Betroffenen. 
Die Vermutungsregelung hat direkte Auswirkungen auf die Frage der Beweislast. Die Vermutung soll eine gewichtige, nicht aber eine unwiderlegbare sein (,a weighty but not irrebuttable presumption“).567 Das Unterschreiten der $3 \mathrm{~m}^{2}$ und damit der Eintritt der Vermutung einer Verletzung von Art. 3 EMRK führt dazu, dass es fortan an der Regierung liegt, überzeugend darzulegen, dass andere positive Umstände die knappen Platzverhältnisse adäquat kompensieren. ${ }^{568}$ Gelingt dies der Regierung, gilt die starke Vermutung als widerlegt; gelingt es der Regierung jedoch nicht, stellt der Gerichtshof einen Verstoss gegen Art. 3 EMRK fest. ${ }^{569}$ Die Anforderungen an die vorzubringenden kompensierenden Elemente variieren dabei; sie sind umso höher, je gravierender die 3- $\mathrm{m}^{2}$-Grenze unterschritten wird. ${ }^{570}$

Die vom Gerichtshof aufgestellte Vermutungsregelung ist im Lichte der allgemein gültigen Beweisregeln in Strafvollzugssituationen zu beurteilen. Schon im Allgemeinen hat der Gerichtshof in seiner Rechtsprechung prozessuale Besonderheiten zu Gunsten des Inhaftierten mit Blick auf seine besondere Verletzlichkeit und die aus dem Freiheitsentzug resultierende Beweisnot aufgestellt. Der Inhaftierte hat folglich von vorneherein nur einen Anscheinsbeweis, also einen Prima facie-Beweis ${ }^{571}$ (Beweismass) zu erbringen, dass er im Vollzug erniedrigenden oder unmenschlichen Bedingungen ausgesetzt ist. Dieser Anscheinsbeweis verlangt dennoch ein detailliertes und konsistentes Vorbringen zu der erlittenen Behandlung. ${ }^{572}$ Gelingt ein solcher Anscheinsbeweis, ist es fortan an der Regierung, welche Zugang zu sämtlichen relevanten Informationen zur Situation des Strafgefangenen hat, diesen Anscheinsbeweis wieder auszuräumen (Exkul-

567 EGMR (GK) - Muršić/CRO, Urt. v. 20.10.2016, 7334/13, Rn. 125.

568 EGMR (GK) - Muršić/CRO, Urt. v. 20.10.2016, 7334/13, Rn. 126.

569 EGMR (GK) - Muršić/CRO, Urt. v. 20.10.2016, 7334/13, Rn. 126.

570 Implizit: EGMR (GK) - Murší́/CRO, Urt. v. 20.10.2016, 7334/13, Rn. 125.

571 Zum Prima facie-Beweis vgl. auch etwa: SK-StPO/MeYer Verfahrensrecht (EMRK) Rn. 254.

572 Vgl. auch EGMR (GK) - Muršić/CRO, Urt. v. 20.10.2016, 7334/13, Rn. 127: Es können Beweise wie etwa schriftliche Aussagen von Mitgefangenen oder gar Fotografien beigelegt werden. 
pationsbeweis). ${ }^{573}$ Die Beweislast ${ }^{574}$ trägt dann die Regierung: „[T]he burden of proof is shifted to the respondent Government. " 575

Die spezifisch für Überbelegungssituationen konstruierte Vermutungsregelung bringt mit Blick auf die allgemeingültigen Beweisregeln für den Inhaftierten den Vorteil, dass das Vorbringen der knappen Platzverhältnisse (weniger als $3 \mathrm{~m}^{2}$ ) für sich alleine den Anforderungen des Anscheinsbeweises für eine Verletzung von Art. 3 EMRK genügt. Eine weitere Substantiierung zu weiteren inadäquaten Bedingungen ist in diesen Fällen nicht mehr notwendig, um die Vermutungswirkung eintreten zu lassen.

Betrachtet man die allgemeinen Konsequenzen eines gelungenen Anscheinsbeweises, unterscheiden sich diese nicht von der hier aufgestellten Vermutungsregel der „strong presumption“. Sowohl Vermutungseintritt als auch das Gelingen des allgemeinen Anscheinsbeweises führen durch die Widerlegungsmöglichkeit resp. durch die Notwendigkeit des Erbringens eines Exkulpationsbeweises zu einer Umkehr der objektiven Beweislast; die Regierung trägt das Risiko der Folgen der Beweislosigkeit. Gelingt die Widerlegung resp. der Exkulpationsbeweis nicht, kann der Gerichtshof daraus Schlüsse zu Ungunsten der Regierung ziehen. Die konkrete Anforderung an die Widerlegung der Vermutung und die Anforderung an einen erfolgreichen Exkulpationsbeweis scheinen sich dabei nicht voneinander zu unterscheiden. Die Regel der „strong presumption“, dass an die Widerlegung umso höhere Anforderungen zu stellen sind, je gravierender die Umstände sind, entspricht nämlich auch den allgemeinen Anforderungen an den Exkulpationsbeweis. Mit Blick auf die Widerlegbarkeit der Vermutung ist dem Inhaftierten in der Praxis deshalb wohl weiterhin zu raten, über das Vorbringen des Unterschreitens der 3- $\mathrm{m}^{2}$-Grenze hinaus sämtliche Rügen und Beweise zu weiteren inadäquaten Bedingungen vorzubringen, obwohl die Vermutungswirkung auch ohne diese eintreten würde. Denn je prekärer die vorgetragenen Haftumstände, desto stärker ist die Vermutung und desto höher werden die Voraussetzungen zu de-

573 EGMR (GK) - Muršić/CRO, Urt. v. 20.10.2016, 7334/13, Rn. 128: Der Gerichtshof hat sich nicht nur auf die von den Parteien vorgetragenen Beweise zu stützen. Er kann vielmehr auch weitere Quellen wie etwa Informationen anderer internationaler Institutionen (wie etwa des CPT) beiziehen, wenn er zu beurteilen hat, ob Art. 3 EMRK verletzt wurde.

574 Gemeint ist immer die objektive Beweislast, d.h. das Tragen des Risikos der Folgen der Beweislosigkeit. Eine subjektive Beweislast (eine Beweisführungslast) gibt es vor dem Gerichtshof nicht, da der Gerichtshof von Amtes wegen ermittelt. Vgl. dazu SK-StPO/Meyer Verfahrensrecht (EMRK) Rn. 252.

575 EGMR (GK) - Muršić/CRO, Urt. v. 20.10.2016, 7334/13, Rn. 128. 
ren Widerlegung. Alleine das Gelingen des Anscheinsbeweises nützt dem Inhaftierten nämlich wenig, wenn die Vermutung vom Staat widerlegt werden kann. Dies dürfte umso wichtiger sein, je geringer die Unterschreitung der 3- $\mathrm{m}^{2}$-Grenze im konkreten Fall ist, weil die Anforderungen an die Widerlegung der Vermutung dann umso geringer sind.

Vor dem Hintergrund der allgemeinen Regelungen zu Beweismass und Beweislast in Strafvollzugskonstellationen ist der Mehrwert der aufgestellten Vermutungsregelung in Überbelegungskonstellationen bei weniger als $3 \mathrm{~m}^{2}$ Platz relativ gering. Einziger Vorteil ist, dass die Hürden für den Eintritt der Umkehr der Beweislast insofern noch einmal gesenkt wurden (Vorbringen kann sich auf die Platzverhältnisse beschränken). Da die Hürden eines Anscheinsbeweises aber ohnehin nicht hoch waren (Beweismass) und dem Inhaftierten in der Praxis mit Blick auf die Widerlegbarkeit der Vermutung dennoch geraten ist, sämtliche inadäquaten Umstände neben den prekären Platzverhältnissen vorzutragen, sind die Auswirkungen in der Praxis wohl nur von geringer Bedeutung. Es handelt sich mehr um eine Spezifizierung der allgemeinen Regelung als um eine Anpassung. Anders wäre die Situation zu beurteilen gewesen, wenn die $3 \mathrm{~m}^{2}$ absolut gelten würden, die Vermutung also eine Unwiderlegbare wäre; eine Exkulpation käme dann nicht mehr in Frage, so dass der Inhaftierte sich bei seinem Vorbringen auch tatsächlich allein auf die mangelhaften Platzverhältnisse stützten könnte.

\subsection{Vermutungswirkungen, Voraussetzungen an die Kompensation zur Widerlegung}

Im Sinne einer Kompensation kommen im Falle der grundsätzlichen Widerlegbarkeit der starken Vermutung gemäss dem Gerichtshof Faktoren wie genügende („,sufficient") Bewegungsmöglichkeiten, adäquate („adequate") Outdoor-Aktivitäten als auch die Unterbringung in einer insgesamt angemessenen Haftanstalt (,confinement in, viewed generally, an appropriate detention facility") in Betracht. ${ }^{576}$ Widerlegt werden kann - so der Gerichtshof - die Vermutung in der Regel („,normally“) nur, wenn die genannten kompensierenden Faktoren kumulativ vorliegen. Zwingend ist das kumulative Vorliegen dieser Voraussetzungen folglich nicht; auch hier wird also Raum für Ausnahmen geöffnet.

576 EGMR (GK) - Muršić/CRO, Urt. v. 20.10.2016, 7334/13, Rn. 132. 
Wurden die Kompensationsfaktoren benannt, stellt sich in einem nächsten Schritt die Frage nach den Voraussetzungen an diese Faktoren. Konkret ist zu fragen, was der Gerichtshof unter „sufficient", „adequate“ und „appropriate“ versteht. Das Vorliegen genügender Bewegungsmöglichkeiten und adäquater Outdoor-Aktivitäten beurteilt der Gerichtshof mit Blick auf den CPT-Standard. ${ }^{577}$ Das CPT sieht vor, dass jeder Inhaftierte täglich die Möglichkeit haben sollte, mindestens eine Stunde an der frischen Luft zu verbringen, wenn möglich mit einem genügenden Angebot an OutdoorAktivitäten. Zudem muss das Platzangebot an der frischen Luft ausreichen (,reasonably spacious") sowie Schutz vor schlechter Witterung bieten. Der Gerichtshof stellt dann ohne weitere Spezifikation fest, dass internationale Standards vorsehen, dass Gefangenen die Möglichkeit zukommen sollte, einen angemessenen Teil des Tages (,reasonable part of the day") ausserhalb der Zelle zu verbringen und unterschiedlichen Aktivitäten nachzugehen (Arbeit, Erholung, Bildung). 578

Die Umschreibung der Anforderungen an die Kompensationskriterien „sufficient" und „adequate" bleibt zumindest im Urteil der Grossen Kammer Muršić/CRO wenig konkret. Ist im allgemeinen Sprachgebrauch bereits dem Begriff der Kompensation inhärent, dass höhere, d.h. über dem Mindeststandard liegende Umstände gewährt werden müssen, scheint dies für die Grosse Kammer zur Erzielung der Kompensationswirkungen nicht zwingend erforderlich zu sein. Schon die Gewährung der Mindeststandards in anderen Bereichen der Haftbedingungen (genügend Bewegungsmöglichkeiten und adäquate Outdoor-Aktivitäten) soll eine kompensierende Wirkung erzielen können. ${ }^{579}$ Diese tiefen Anforderungen des Gerichtshofs an die Kompensation lassen vermuten, dass der Gerichtshof der Annahme folgt, beim Unterschreiten der 3- $\mathrm{m}^{2}$-Grenze seien in der Regel auch die weiteren Haftbedingungen ungenügend resp. inadäquat, so dass er es für besonders positiv zu werten hält, wenn dies wider Erwarten nicht der Fall sein sollte.

Die weitere Voraussetzung der Unterbringung in einer insgesamt angemessenen Haftanstalt („overall appropriateness of the detention facility") beurteilt der Gerichtshof vor dem Hintergrund seines eigenen Fallrechts,

577 EGMR (GK) - Muršić/CRO, Urt. v. 20.10.2016, 7334/13, Rn. 133.

578 EGMR (GK) - Muršić/CRO, Urt. v. 20.10.2016, 7334/13, Rn. 133.

579 Kritisch dazu Richter Sajó, López Guerra u. Wojtyczek, joint partly diss. op., EGMR (GK) - Muršić/CRO, Urt. v. 20.10.2016, 7334/13, Rn. 8; Richter Lazarova Trajkovska, de Gaetano u. Grozev, joint partly diss. op., EGMR (GK) - Muršićl CRO, Urt. v. 20.10.2016, 7334/13, Rn. 10. 
insbesondere seiner bisherigen Piloturteile und des internationalen Standards. ${ }^{580}$ Die Unterbringung und die gesamten Umstände dürfen - in Anlehnung an das "no more harm principle - keine weiteren zusätzlichen erschwerenden Elemente beinhalten „Accordingly, in addition to sufficient freedom of movement and adequate out-of-cell activities, no violation of Article 3 would be found where no other aggravating circumstance arises with regard to general conditions of an applicant's detention." 581

Diese letzte Voraussetzung zur Widerlegung der Vermutung beinhaltet folglich von vorneherein keine kompensierenden Elemente im Sinne des allgemeinen Sprachgebrauchs, sondern ist vielmehr als Negativ-Voraussetzung zu verstehen, welche die kompensierenden Faktoren flankieren soll, damit sich deren kompensierende Wirkung überhaupt erst entfalten kann. Auch hier gilt allerdings, dass eine Widerlegung auch beim Fehlen dieser Voraussetzung nicht zwingend ausgeschlossen ist (vgl. „normally“). ${ }^{582}$

Nach Feststellung der grundsätzlichen Widerlegbarkeit und deren Anforderungen wird nun auf mögliche Grenzen der Widerlegbarkeit eingegangen. Besondere Bedeutung kommt hier dem Element der Dauer der Behandlung zu.

2.3 Vermutungswirkung und Grenze der Widerlegbarkeit - Element der Dauer

Widerlegbar soll die Vermutung in der Regel („normally“) von vorneherein nur dann sein, wenn die Unterschreitung des Mindestmasses an Platz nur für eine kurze Dauer erfolgt und es sich nur um eine gelegentliche und geringe Unterschreitung der notwendigen Platzerfordernisse handelt: „only short, occasional and minor reductions in the required personal space ${ }^{\text {"5 } 583}$.

580 EGMR (GK) - Muršić/CRO, Urt. v. 20.10.2016, 7334/13, Rn. 134 m.V.z.; EGMR - Alver/EST, Urt. v. 08.11.2005, 64812/01, Rn. 53; EGMR - Andrei Georgiev/BUL, Urt. v. 26.07.2007, 61507/00, Rn. 61; EGMR - Dolenec/CRO, Urt. v. 26.11.2009, 25282/06, Rn. 134.

581 EGMR (GK) - Muršić/CRO, Urt. v. 20.10.2016, 7334/13, Rn. 134.

582 Zur Kritik zu diesem Kriterium vgl. auch Richter Pinto de Albuquerque partly diss. op., EGMR (GK) - Muršić/CRO, Urt. v. 20.10.2016, 7334/13, Rn. 53.

583 EGMR (GK) - Muršić/CRO, Urt. v. 20.10.2016, 7334/13, Rn. 130; vgl. auch EGMR - Fetisov u.a./RUS, Urt. v. 17.01.2012, 43710/07 u.a., Rn. 134 ff.; EGMR Dmitriy Rozhin/RUS, Urt. v. 23.10.2012, 4265/06, Rn. 52 f.; EGMR - Vladimir Belyayev/RUS, Urt. v. 17.10.2013, 9967/06, Rn. 33 ff.; EGMR - Kurkowski/POL, Urt. v. 09.04.2013, 36228/06, Rn. $66 \mathrm{f}$. 
Dies deutet darauf hin, dass die Vermutung nur beschränkt widerlegbar sein soll, wobei der Gerichtshof sich auch hier von einer absoluten Geltung dieses Grundsatzes distanziert und wiederum Raum für Ausnahmen schafft („normally“). Die kurze Dauer für sich alleine genügt allerdings nicht, die Vermutung zu widerlegen; das zeitliche Element ist vielmehr kumulativ zu den kompensierenden Massnahmen und den insgesamt angemessenen Umständen. Das zeitliche Element enthält für sich alleine denn auch keine kompensierende Komponente.

Betreffend die Dauer der Behandlung - als wichtiger Faktor der Art. 3 EMRK-Beurteilung ${ }^{584}$ - stellen sich im Wesentlichen zwei Fragen: (1) Ab welcher Zeitdauer geht der Gerichtshof nicht mehr von einer kurzen Dauer aus und (2) wie sind zeitliche Unterbrechungen der Situation (zu wenig Platz, genügend Platz, zu wenig Platz) zu würdigen?

Keine Verletzung von Art. 3 EMRK erkannte der Gerichtshof etwa in Kurkowski/POL ${ }^{585}$ (4 Tage mit 2,1 $\mathrm{m}^{2}$ persönlichem Platz und dann nochmals 4 Tage mit 2,6 m² Platz), in Dmitriy Rozhin/RUS ${ }^{586}$ (11 Tage mit $2 \mathrm{~m}^{2}$ persönlichem Platz [in Disziplinarzelle]), in Fetisov u.a./RUS ${ }^{57}$ (19 Tage mit $2 \mathrm{~m}^{2}$ persönlichem Platz) oder in Vladimir Belyayev/RUS ${ }^{58}$ (10 Tage mit 2,95 $\mathrm{m}^{2}$ persönlichem Platz, dann zwei Tage mit 2,65 $\mathrm{m}^{2}$ und noch 26 Tage mit 2,97 $\mathrm{m}^{2}$ ).

In MuršićlCRO nahm die Grosse Kammer eine relativ kurze Dauer („relatively short duration“) bei Zeitspannen von einem Tag (bei 2,62 $\mathrm{m}^{2}$ ), bei zwei Tagen (bei 2,62 $\mathrm{m}^{2}$ ), drei Tagen (bei 2,62 $\mathrm{m}^{2}$ resp. 2,55 $\mathrm{m}^{2}$ ) und bei acht Tagen (bei 2,55 $\mathrm{m}^{2}$ ) an, so dass für diese Zeiträume keine Verlet-

584 Vgl. auch EGMR - Kalashnikov/RUS, Urt. v. 15.07.2002, 47095/99, Rn. 102: "Combined with the length of the period“; EGMR - Kehayov/BUL, Urt. v. 18.01.2005, 41035/98, Rn. 64: „When assessing conditions of detention, account has to be taken of the cumulative effects of those conditions and the duration of the detention“; EGMR - Novoselov/RUS, Urt. v. 02.06.2005, 66460/01, Rn.39; EGMR - Alver/EST, Urt. v. 08.11.2005, 64812/01, Rn. 50; EGMR (GK) - Idalov/RUS, Urt. v. 22.05.2012, Rn. 94; EGMR - Orchowski/POL, Urt. v. 22.10.2009, 17885/04, Rn. 121; EGMR - Torreggiani u.a./ITA, Urt. v. 08.01.2013, 43517/09 u.a., Rn. 66; EGMR - Ananyev u.a./RUS, Urt. v. 10.01.2012, 42525/07 u. 60800/08, Rn. 142.

585 EGMR - Kurkowski/POL, Urt. v. 09.04.2013, 36228/06, Rn. 66 f.

586 EGMR - Dmitriy Rozhin/RUS, Urt. v. 23.10.2012, 4265/06, Rn. 49 ff., 52 f.: Bezüglich des direkt vorangehenden Zeitraums von rund 4,5 Monaten, in welchem dem Inhaftierten zwischen $1,1 \mathrm{~m}^{2}$ und $2 \mathrm{~m}^{2}$ zur Verfügung standen, erkannte der Gerichtshof demgegenüber einen Verstoss gegen Art. 3 EMRK.

587 EGMR - Fetisov u.a./RUS, Urt. v. 17.01.2012, 43710/07 u.a., Rn. $134 \mathrm{ff}$.

588 EGMR - Vladimir Belyayev/RUS, Urt. v. 17.10.2013, 9967/06, Rn. 33 ff. 
zung von Art. 3 EMRK festgestellt wurde. ${ }^{589}$ Anders wurde dies bei einer Zeitspanne von 27 Tagen (bei 2,62 $\mathrm{m}^{2}$ ) beurteilt. Diese erfüllte für den Gerichtshof die Anforderungen einer kurzen, geringfügigen Unterschreitung (,short and minor reduction") nicht mehr, so dass eine Widerlegung der Vermutung durch kompensierende Faktoren nicht mehr in Frage kam und für diesen Zeitraum eine Verletzung von Art. 3 EMRK festgestellt wurde. 590

Sind die Kriterien der kurzen und geringfügigen Unterschreitung als kumulative Voraussetzungen zu verstehen („short and minor reduction“), macht der Gerichtshof dennoch ihre Definition vom jeweils anderen Kriterium abhängig, so dass "short" und „minor" immer im Lichte des jeweils anderen Kriteriums zu beurteilen sind. Je schwerwiegender die Unterschreitung der $3 \mathrm{~m}^{2}$, desto schneller ist nicht mehr von einer kurzen Dauer der Behandlung auszugehen. Mit Blick auf die Rechtsprechung des Gerichtshofs scheint die Grenze zwischen einer kurzen und einer langen Dauer, immer relativ zur Schwere der Unterschreitung, bei etwa einem Monat zu liegen (vgl. 27 Tage bei Muršić/CRO).

Die Analyse der Rechtsprechung des Gerichtshofs zeigt weiter, dass der persönliche Platz, welcher dem betroffenen Inhaftierten zusteht, häufigen Schwankungen unterliegt. ${ }^{591}$ Diese können auf Verlegungen in andere Zellen oder andere Haftanstalten oder auf wechselnde Zellenbelegung zurückzuführen sein (der Betroffene verbleibt in derselben Zelle, diese teilt er aber mit unterschiedlich vielen Inhaftierten). ${ }^{592}$ Letzteres dürfte in der Praxis wohl häufiger der Fall sein. Diese Schwankungen veranlassen den Gerichtshof, die einzelnen Zeitspannen zumindest in Bezug auf ihre Dauer separat voneinander zu prüfen. ${ }^{593}$

Zusammenfassend ist festzuhalten, dass das zeitliche Element der Widerlegbarkeit der Vermutung Grenzen setzt. Wird der persönliche Platz von $3 \mathrm{~m}^{2}$ (Auslösung für die Vermutung) für eine nicht bloss kurze Dauer unterschritten, kommen kompensierende Massnahmen zur Widerlegung der Vermutung von vorneherein nicht mehr in Betracht, so dass die Verletzung von Art. 3 EMRK zwingend direkt festzustellen ist. Die Beurteilung,

589 EGMR (GK) - Muršić/CRO, Urt. v. 20.10.2016, 7334/13, Rn. 149 f.

590 EGMR (GK) - Muršić/CRO, Urt. v. 20.10.2016, 7334/13, Rn. 151 ff.

591 Vgl. etwa EGMR (GK) - Muršić/CRO, Urt. v. 20.10.2016, 7334/13, Rn. 146; EGMR - Vladimir Belyayev/RUS, Urt. v. 17.10.2013, 9967/06, Rn. 33 ff.

592 Gerade Überbelegungssituationen können dazu führen, dass es zu organisationsbedingten Verlegungen kommt, um die vorhandenen Kapazitäten optimal auszunutzen. Vgl. dazu auch Devresse, Droit et Société 2013, 339 (351).

593 EGMR (GK) - Muršić/CRO, Urt. v. 20.10.2016, 7334/13, Rn. 149. 
ob eine kurze Dauer vorliegt, erfolgt mit Blick auf die Schwere der Unterschreitung der 3- $\mathrm{m}^{2}$-Grenze und ist insofern relativ. Die Rechtsprechungsanalyse lässt vermuten, dass ab ca. einem Monat nicht mehr von einer kurzen Behandlung auszugehen ist.

2.4 Prüfungsmethodisches Vorgehen des Gerichtshofs und Auswirkungen auf den Grundrechtsschutz

Der Gerichtshof betont in prüfungsmethodischer Hinsicht die Relativität des Massstabes und nimmt eine Gesamtbetrachtung der Umstände vor. ${ }^{594}$ Diese Gesamtbetrachtung manifestiert sich wie aufgezeigt entweder in der Kumulation aggravierender Umstände oder aber bei Eintritt der Vermutungswirkung in der Kompensationsmöglichkeit. In demselben Fall können auch gleichzeitig Kumulations- und Kompensationselemente vorliegen; komplexe Gewichtungsfragen sind die Folge. Dieses prüfungsmethodische Vorgehen der Gesamtbetrachtung wird im Folgenden mit Blick auf seine Menschenrechtswirkung und insbesondere betreffend Schutzniveau kritisch analysiert, wobei die Kumulationswirkung und die Kompensationswirkung separat voneinander zu beurteilen sind.

a Kumulation und Menschenrechtsschutz

Treffen mehrere ungenügende materielle Haftbedingungen aufeinander, kommt es zu einer Kumulation im Sinne einer Addition der negativen Umstände. 595 Wäre ein einzelner dieser Umstände für sich alleine so schwer, dass er als solcher schon als Verstoss gegen Art. 3 EMRK anzuse-

594 Der EGMR nimmt nicht nur bei Art. 3 EMRK und in Bezug auf Haftbedingungen eine Gesamtbetrachtung vor. Ein solches Vorgehen findet sich auch etwa bei der Art. 6 EMRK-Prüfung „overall fairness“. Vgl. dazu SK-StPO/MeYer Art. 6 EMRK Rn. 32; EGMR (GK) - Ibrahim u.a./GBR, Urt. v. 13.09.2016, 50541/08 u.a., Rn. 274: Faktoren welche bei der Gesamtbewertung der Fairness i.S.v. Art. 6 EMRK berücksichtigt werden können; EGMR (GK) - Schatschaschwili/GER, Urt. v. 15.12.2015, 9154/10, Rn. 113: Möglichkeit zur Abweichung von der Prüfungsreihenfolge aufgrund der ,overall fairness“.

595 Zur Belastungskumulation im völkerrechtlichen Menschenrechtsschutz vgl. auch Ruschemeier S. 230 ff.; zu kumulierenden Haftbedingungen auch Brade S. 43 ff. m.V.z. Rechtsprechung des deutschen Bundesverfassungsgerichts etwa betr. Wechselwirkungen von besonderen Sicherungsvorkehrungen; zur Dogmatik kumulierender Belastungen ausf.: Kromrey I. S. 105. 
hen ist, ändert die Kumulation nichts am Ausgang des Verfahrens. Würden hingegen die einzelnen berücksichtigten Umstände für sich alleine noch nicht so gravierend sein, dass sie eine Menschenrechtsverletzung bilden würden, ist die Möglichkeit der Kumulation entscheidend für den Ausgang des Verfahrens; erst durch diese ist die Verletzung von Art. 3 EMRK festzustellen. Argumentiert wird damit, dass erst durch die Gesamtbetrachtung, d.h. durch die Berücksichtigung sämtlicher Haftumstände, die Wirkungen des Vollzugs auf den Betroffenen angemessen beurteilt werden können. ${ }^{596}$

Statuieren Menschenrechte in unterschiedlichen Dimensionen Ansprüche des Individuums gegenüber dem Konventionsstaat (Freiheitsrechte, Schutzrechte, Leistungsrechte) und sind die Individuen ihrerseits demgegenüber nie Menschenrechtsverpflichtete ${ }^{597}$ (keine Ansprüche des Staates gegenüber dem Individuum aufgrund eines Menschenrechts), führt dies dazu, dass die Berücksichtigung der kumulierenden Wirkung einzelner Haftumstände den Konventionsstaat vermehrt in die Pflicht nimmt. Mit Blick auf die Schutzrichtung des Art. 3 EMRK ist die Berücksichtigung kumulierender Elemente geradezu geboten. Im Ergebnis führen sie zu einem erhöhten Schutzniveau.

\section{b Kompensation und Menschenrechtsschutz}

Ist der Strafvollzug aber einerseits durch ungenügende Bedingungen geprägt und liegen andererseits in anderen Bereichen der Haft genügende Bedingungen vor bzw. gehen einzelne Kriterien sogar über das erforderliche Mass hinaus, führt die Gesamtbetrachtung i.S.e. Neutralisierungswirkung zu Kompensationsmöglichkeiten. Diese manifestieren sich darin, dass die Unterschreitung der Platzanforderungen i.d.R. bloss die Vermutung einer Verletzung von Art. 3 EMRK auslöst, welche durch kompensierende Massnahmen widerlegt werden kann. Der Relativierungsaspekt der Gesamtbetrachtung führt mit anderen Worten dazu, dass beim Vorliegen von Kompensationsmöglichkeiten die Konformität mit Art. 3 EMRK festgestellt werden muss, während ohne diese für sich alleine betrachtet von

596 EGMR (GK) - Muršić/CRO, Urt. v. 20.10.2016, 7334/13, Rn. 123; vgl. auch Richter Pinto de Albuquerque partly diss. op., EGMR (GK) - MuršićlCRO, Urt. v. 20.10.2016, 7334/13, Rn. 49 f.

597 Zur Partei- und Prozessfähigkeit und zur Grundrechtsberechtigung vgl. auch SK-StPO/MeYER Verfahrensrecht (EMRK) Rn. $32 \mathrm{ff}$. 
einer Verletzung von Art. 3 EMRK auszugehen wäre. Bei der Anwendung der Kompensationskriterien als Teilaspekt der Gesamtbetrachtung wird wie schon bei den kumulierenden Wirkungen - argumentiert, dass nur die Berücksichtigung aller Aspekte die Beurteilung der Wirkungen auf den Einzelnen zulassen würde. ${ }^{598}$ Gegen die Beurteilung nur eines einzelnen Kriteriums ohne Berücksichtigung des Gesamtkontextes wird vorgetragen, dass ein starrer Standard zu kurz greifen würde und mithin zu einer zu formalistischen Herangehensweise führen würde, welche darüber hinaus auch den Grundprinzipien des Fallrechts des Gerichtshofs widerspräche. ${ }^{599}$

Die Bedeutung der einzelnen Haftumstände wird durch die Berücksichtigung derer Wechselwirkungen gleichwohl insgesamt abgeschwächt. Kompensationsmöglichkeiten können deshalb die Gefahr der Senkung des menschenrechtlichen Schutzniveaus beinhalten, weil sie den Standard der einzelnen Kriterien aufweichen. Dies würde der Schutzrichtung von Art. 3 EMRK zuwiderlaufen. Sieht man in der Kompensationsmöglichkeit aber einen Anreiz für den Konventionsstaat, ungenügende Bedingungen zumindest durch alternative Massnahmen auszugleichen, könnte die Kompensationsmöglichkeit auch dahingehend interpretiert werden, dass sie dem Inhaftierten zugute kommt. Dies gilt zumindest solange, als sich der Staat durch die Kompensationsmöglichkeit nicht davon entbunden sieht, auch der Nichterfüllung des Kriteriums selbst abzuhelfen.

Insbesondere mit Blick auf den absoluten Charakter von Art. 3 EMRK stellt sich die grundsätzliche Frage der Zulässigkeit von Kompensationsmöglichkeiten, wie sie vom Gerichtshof vorgesehen sind. Wie ausgeführt, ist aufgrund der absoluten Natur von Art. 3 EMRK bei einem Eingriff in den Schutzbereich die Grundrechtsverletzung festzustellen; eine Rechtfertigung kommt aufgrund des Anwendbarkeitskriteriums nicht in Betracht. Bei der Schutzbereichsbestimmung dürfen jedoch relative Kriterien berücksichtigt werden, sofern sie sich auf den Grad des erlittenen Leidens bzw. die Schwere der zugefügten Behandlung auswirken. Die Kompensationsprüfung, wie sie vom EGMR durchgeführt wird, erfolgt formell auf der Ebene der Schutzbereichsprüfung, weshalb dies hinsichtlich des Anwendbarkeitskriteriums von vorneherein unproblematisch scheint. Es

598 EGMR (GK) - Muršić/CRO, Urt. v. 20.10.2016, 7334/13, Rn. 123 ff.: „[...] only a comprehensive approach to the particular conditions of detention can provide an accuarate picture of the reality for detainees."

599 Im Kontext der Überbelegung und eines absoluten Anspruchs auf eine Mindestfläche an persönlichen Platz: EGMR (GK) - Muršić/CRO, Urt. v. 20.10.2016, 7334/13, Rn. 85 (Vorbringen der Regierung Kroatiens). 
stellt sich hier jedoch die Frage, ob unter dem Deckmantel der Ermittlung des notwendigen Mindestmasses an Schwere die Essenz des Anwendbarkeitskriteriums unterwandert wird, ob also das Zulassen von Kompensationsmöglichkeiten materiell einer Rechtfertigungsprüfung gleichkommt. Dies ist vorliegend zu verneinen, werden doch bei den kompensierenden Elementen keine Argumente der Regierung berücksichtigt, welche die ungenügende Bedingung rechtfertigen sollen, sondern vielmehr Umstände, welche diesen prekären Umstand ausgleichen sollen. Die berücksichtigten Kriterien wirken sich somit auf den Grad des erlittenen Leidens aus, weshalb sie auch bei absoluten Rechten berücksichtigt werden sollen.

Mit Blick auf das Spezifitätskriterium absoluter Rechte, welches auf die besondere Bedeutung eines konkreten Massstabes vorbehaltsloser Rechte hinweist, ist gleichwohl festzuhalten, dass die Berücksichtigung von nicht näher definierten Kompensationselementen als Variablen bei der Schutzbereichsbestimmung zu einer Variabilität des Ergebnisses führt, welche eine gewisse Rechtsunsicherheit zur Folge hat, da nicht mehr von vorneherein klar ist, was denn nun durch Art. 3 EMRK absolut gewährleistet ist. Das prüfungsmethodische Vorgehen ist deshalb nicht unproblematisch, weshalb Kompensationsmechanismen schon deshalb nur restriktiv angewandt werden sollten. Gleichwohl muss auch erkannt werden, dass durch das Vorsehen von Kompensationsmechanismen auch Raum für die Einzelfallprüfung und eine Weiterentwicklung des Standards geschaffen wird.

\section{Dogmatische Einordnung der Überbelegung durch den EGMR}

Während der Schwerpunkt der bisherigen Analyse darauf lag, den materiellen Kern des EGMR-Standards im Lichte von Art. 3 EMRK als solchem und das vom EGMR angewandte Prüfprogramm zu eruieren, wird in einem zweiten Schritt der Blick auf die dogmatische Einordnung der Überbelegung innerhalb von Art. 3 EMRK (zu den Begriffsmerkmalen der unmenschlichen und erniedrigenden Behandlung im Allgemeinen vgl. Teil 2 Kap. 3 II. 1.) sowie auf die Kriterien des Gerichtshofs bei der Standardbildung gerichtet.

\section{1 Überbelegung - unmenschlich oder erniedrigend?}

Im Piloturteil Ananyev u.a./RUS führte der Gerichtshof im Allgemeinen in Übereinstimmung mit den Begriffsmerkmalen der unmenschlichen resp. 
der erniedrigenden Behandlung zunächst aus, dass das notwendige Mindestmass an Schwere in der Regel mit einer tatsächlichen Körperverletzung oder mit schwerem körperlichem oder geistigem Leiden verbunden sei. ${ }^{600}$ Selbst wenn dies jedoch nicht der Fall sei, könne eine Behandlung, welche eine Person herabsetze oder entwürdige, einen Mangel an Respekt für ihre Menschenwürde zeige oder Gefühle der Angst oder Minderwertigkeit wecke, welche geeignet seien, den moralischen oder körperlichen Widerstand einer Person zu brechen, als erniedrigend i.S.v. Art. 3 EMRK bezeichnet werden. ${ }^{601}$ Der Staat habe aufgrund von Art. 3 EMRK sicherzustellen, dass die Haftbedingungen mit der Menschenwürde vereinbar sind, dass die Art und Weise und die Methode der Durchführung den Betroffenen nicht einer Belastung oder Härte aussetzen, die über das unvermeidbare Mass an Leiden, welches mit der Haft verbunden ist, hinausgehen und dass die Gesundheit und das Wohlergehen angesichts der praktischen Anforderungen an die Haft angemessen gesichert sind. ${ }^{602}$

Die Nichterfüllung einer der drei kumulativen Voraussetzungen des Ananyev-Tests habe die starke Vermutung einer erniedrigen Behandlung i.S.v. Art. 3 EMRK zur Folge. ${ }^{603}$ Die Haftumstände im konkreten Fall qualifizierte der EGMR schliesslich, ohne die betreffenden Begriffsmerkmale noch einmal im Einzelnen aufzugreifen, als unmenschlich und erniedrigend i.S.v. Art. 3 EMRK. ${ }^{604}$

Im Urteil Muršić/CRO wiederholte der Gerichtshof die bereits in Ananyev u.a./RUS ausgeführten allgemeinen theoretischen Grundsätze zum erforderlichen Mindestmass an Schwere. Das Verbot der Folter, der unmenschlichen und erniedrigenden Behandlung sei ein zivilisatorischer Wert, welcher eng mit der Achtung der Menschenwürde verbunden sei. ${ }^{605}$ Im Zusammenhang mit dem Freiheitsentzug müsse der Staat sicherstellen, dass die Haftbedingungen die Menschenwürde wahren, dass die Art und Weise und die Methode der Durchführung den Beschwerdeführer nicht einer Härte aussetzen, welche das unvermeidbare Mass übersteigt, und dass angesichts der praktischen Anforderungen der Inhaftierung die Gesundheit und das Wohlergehen angemessen gesichert seien. ${ }^{606}$ Es sei zwar zu

600 EGMR - Ananyev u.a./RUS, Urt. v. 10.01.2012, 42525/07 u. 60800/08, Rn. 140.

601 EGMR - Ananyev u.a./RUS, Urt. v. 10.01.2012, 42525/07 u. 60800/08, Rn. 140.

602 EGMR - Ananyev u.a./RUS, Urt. v. 10.01.2012, 42525/07 u. 60800/08, Rn. 141.

603 EGMR - Ananyev u.a./RUS, Urt. v. 10.01.2012, 42525/07 u. 60800/08, Rn. 147.

604 EGMR - Ananyev u.a./RUS, Urt. v. 10.01.2012, 42525/07 u. 60800/08, Rn. 166.

605 EGMR (GK) - Muršić/CRO, Urt. v. 20.10.2016, 7334/13, Rn.98; vgl. so auch schon EGMR (GK) - Bouyid/BEL, Urt. v. 28.09.2015, 23380/09, Rn. 81.

606 EGMR (GK) - Muršić/CRO, Urt. v. 20.10.2016, 7334/13, Rn. 99. 
berücksichtigen, ob der Konventionsstaat mit oder ohne Absicht gehandelt habe, einen Inhaftierten durch die schlechte Behandlung herabzusetzen oder zu entwürdigen, gleichwohl sei eine entsprechende Absicht für die Feststellung eines Verstosses gegen Art. 3 EMRK keine zwingende Voraussetzung. ${ }^{607}$

Bei der folgenden systematischen Aufarbeitung seiner bisherigen Rechtsprechung greift der Gerichtshof zwar seine bisherigen unterschiedlichen Rechtsprechungslinien auf, sein Fokus liegt dabei allerdings beim zu gewährleistenden Mindeststandard im Lichte des Art. 3 EMRK als solchem resp. beim konkret anzuwendenden Prüfprogramm. ${ }^{608}$ Eine eingehende Auseinandersetzung damit, ob in der Vergangenheit die Überbelegungssituation an den Kriterien einer unmenschlichen oder aber einer erniedrigenden Behandlung geprüft wurde, fand durch den Gerichtshof nicht statt. Vielmehr scheint der Gerichtshof, ausgehend vom Ananyev-Test, von vorneherein die Erniedrigung ins Zentrum seiner Menschenrechtsprüfung zu rücken. ${ }^{609}$ Gleichwohl nimmt er aber bei seinen Ausführungen bisweilen sowohl auf Begriffsmerkmale der unmenschlichen als auch der erniedrigenden Behandlung i.S.v. Art. 3 EMRK Bezug. ${ }^{610}$

Durchleuchtet man die bisherige Rechtsprechung des Gerichtshofs, so wird klar, dass die Einordnung durch den Gerichtshof zur unmenschlichen resp. zur erniedrigenden Behandlung weder vor noch nach dem Urteil Ananyev u.a./RUS immer eindeutig ist. ${ }^{611}$ Der Gerichtshof qualifizierte zwar in zahlreichen Fällen - mit mal mehr, mal weniger ausführlichen Subsumtionen - Überbelegungssituationen im Ergebnis eindeutig als eine erniedrigende Behandlung i.S.v. Art. 3 EMRK, ${ }^{612}$ in einer Reihe

607 EGMR (GK) - Muršić/CRO, Urt. v. 20.10.2016, 7334/13, Rn. 100; vgl. unter vielen auch schon: EGMR - Peers/GRE, Urt. v. 19.04.2001, 28524/95, Rn. 74.

608 EGMR (GK) - MuršićlCRO, Urt. v. 20.10.2016, 7334/13, Rn. 116 ff.

609 EGMR (GK) - Muršić/CRO, Urt. v. 20.10.2016, 7334/13, Rn. 119, 128: „Once a credible and reasonably detailed description of the allegedly degrading conditions of detention, constituting a prima facie case of ill-treatment, has been made, the burden of proof is shifted to the respondent Government [...]."

610 EGMR (GK) - Muršić/CRO, Urt. v. 20.10.2016, 7334/13, Rn. 131: „[...] the Court has already held that, while the length of a detention period may be a relevant factor in assessing the gravity of suffering or humiliation caused to a detainee by the inadequate conditions of his or her detention [...].“

611 Vgl. auch SK-StPO/MeYer Art. 3 EMRK Rn. 49; Morgenstern StV 2016, 395 (397, Fn. 34): Es komme dem Gerichtshof mehr auf die „Wesentlichkeitsschwelle" an.

612 Vgl. unter vielen EGMR - Varga u.a./HUN, Urt. v. 10.03.2015, 14097/12 u.a., Rn. 89 ff.; EGMR - Lin/GRE，Urt. v. 06.11.2012，58158/10，Rn.53 f., 56; 
von anderen Fällen stellte der Gerichtshof beim Unterschreiten des Mindestmasses an persönlichem Platz aber ohne nähere dahingehende Begründung auch immer wieder eine Verletzung von Art. 3 EMRK als solche ${ }^{613}$ oder eine unmenschliche und erniedrigende Behandlung ${ }^{614}$ fest. Die Feststellung sowohl einer unmenschlichen als auch einer erniedrigenden Behandlung kann zwar grundsätzlich Folge dessen sein, dass aufgrund der Gesamtbetrachtung bisweilen ganz unterschiedliche Eingriffe zusammen beurteilt werden. Es zeigt sich aber, dass die Gesamtbetrachtung nicht immer Ursache für die Feststellung sowohl einer unmenschlichen als auch einer erniedrigenden Behandlung ist. Vielmehr wird tatsächlich die Überbelegungssituation als solche als unmenschlich und erniedrigend i.S.v. Art. 3 EMRK qualifiziert. ${ }^{615}$

Im Fall Muršić/CRO stellte der Gerichtshof vor diesem Hintergrund fest, dass eine 27-tägige Unterbringung mit 2,62 $\mathrm{m}^{2}$ persönlichem Platz dem Beschwerdeführer eine Härte ausgesetzt hätte, welche über das unvermeidliche Mass an Leiden, das mit der Haft verbunden sei, hinausgehe und somit eine erniedrigende Behandlung i.S.v. Art. 3 EMRK vorliege. ${ }^{616}$ Für weitere kürzere Zeitspannen der Unterbringung mit weniger als $3 \mathrm{~m}^{2}$ Platz stellte der Gerichtshof fest, dass andere Haftumstände wie

EGMR - Nieciecki/GRE, Urt. v. 04.12.2012, 11677/11, Rn. 49 ff.; EGMR Shishkov/RUS, Urt. v. 20.02.2014, 26746/05, Rn. 93; EGMR - Karalevicius/LTU, Urt. v. 07.04.2005, 53254/99, Rn.36ff.; in diesem Sinne vgl. auch SK-StPO/ Meyer Art. 3 EMRK Rn. 49: Die massive Überbelegung gehöre eindeutig zu den erniedrigenden Behandlungen.

613 Vgl. bspw. EGMR - Torreggiani u.a./ITA, Urt. v. 08.01.2013, 43517/09, Rn. 79; EGMR - Orchowski/POL, Urt. v. 22.10.2009, 17885/04, Rn. 135.

614 Vgl. unter vielen EGMR - Neshkov u.a./BUL, Urt. v. 27.01.2015, 36925/10 u.a., Rn. 247, 253; EGMR - Trepashkin/RUS (Nr. 2), Urt. v. 16.12.2010, 14248/05, Rn. 103 ff.; EGMR - Ivakhnenko/RUS, Urt. v. 04.04.2013, 12622/04, Rn.36; EGMR - Asyanov/RUS, Urt. v. 09.10.2012, 25462/09, Rn. 43; EGMR - Suldin/ RUS, Urt. v. 16.10.2014, 20077/04, Rn. 46.

615 Vgl. etwa EGMR - Suldin/RUS, Urt. v. 16.10.2014, 20077/04, Rn. 45: Der Gerichtshof prüft die übrigen Haftbedingungen nämlich kumulierend zur Überbelegung (und nicht für sich alleine genommen) an den Kriterien der Erniedrigung. Die Überbelegung scheint für die Erniedrigung damit massgebend zu sein. Stellt der Gerichtshof dann im Ergebnis zusätzlich eine unmenschliche Behandlung fest - ohne auch diese Begriffsmerkmale bei den übrigen Haftbedingungen thematisiert zu haben - kann der Gerichtshof $e$ contrario nur aufgrund der Überbelegung auch zu diesem Schluss gelangt sein. Eine effektive Subsumtion unter die Begriffsmerkmale der unmenschlichen Behandlung nahm der Gerichtshof dazu allerdings nicht vor.

616 EGMR (GK) - MuršićlCRO, Urt. v. 20.10.2016, 7334/13, Rn. 153. 
genügende Bewegungsmöglichkeiten als erheblich erleichternde Faktoren zu berücksichtigen seien und der Beschwerdeführer in einer insgesamt angemessenen Haftanstalt untergebracht gewesen sei, weshalb die strenge Vermutung eines Verstosses gegen Art. 3 EMRK widerlegt worden sei. ${ }^{617}$ Das Mindestmass an Schwere für eine unmenschliche oder erniedrigende Behandlung i.S.v. Art. 3 EMRK sei damit trotz der nicht gänzlich adäquaten Platzverhältnisse nicht erreicht, weshalb der Gerichtshof zusammenfassend feststellte, dass in diesen Perioden keine erniedrigende Behandlung i.S.v. Art. 3 EMRK vorliege. 618

Zusammenfassend konzentriert sich der Gerichtshof vornehmlich auf die Frage, ob das Mindestmass an Schwere i.S.v. Art. 3 EMRK als solches erreicht sei. Die dogmatische Einordnung der Überbelegung zu einer unmenschlichen oder erniedrigenden Behandlung i.S.v. Art. 3 EMRK tritt in der Strassburger Rechtsprechung in den Hintergrund. Tendenziell scheint der Gerichtshof Überbelegungssituationen allerdings den erniedrigenden Behandlungen i.S.v. Art. 3 EMRK zuzuordnen.

Prima vista scheint die Frage, ob nun ein Konventionsverstoss vorliegt oder nicht, auch bedeutender zu sein als die Frage, ob es sich in concreto um eine unmenschliche oder erniedrigende Behandlung handelt. Diesbezügliche Unklarheiten sind aber nicht unproblematisch, setzt die Bestimmung des Mindestmasses an Schwere i.S.v. Art. 3 EMRK doch gerade Kenntnis über die Voraussetzungen voraus, anhand welcher man diese Mindestschwelle bemisst, selbst wenn dies sowohl für die unmenschliche als auch die erniedrigende Behandlung zu prüfen ist. Diese Voraussetzungen sind bei der unmenschlichen resp. erniedrigenden Behandlung aufgrund ihrer unterschiedlichen Art der Einwirkung auf den Betroffenen nämlich je andere. Mit anderen Worten muss erst klar sein, ob man die Voraussetzungen einer unmenschlichen oder erniedrigenden Behandlung i.S.v. Art. 3 EMRK prüft, um dann nach einer Subsumtion der konkreten Fakten im Einzelfall unter die entsprechenden Voraussetzungen zum Schluss zu gelangen, ob die je spezifischen Voraussetzungen der unmenschlichen oder erniedrigenden Behandlung im konkreten Fall vorliegen oder nicht. Nur auf diese Weise lässt sich die Frage beantworten, ob das erforderliche Mindestmass an Schwere i.S.d. konkret zu prüfenden Tatbestandes erreicht ist oder nicht. Darüber hinaus sollte die konkrete Qualifikation als unmenschliche resp. erniedrigende Behandlung auch dem

617 EGMR (GK) - Muršić/CRO, Urt. v. 20.10.2016, 7334/13, Rn. 163 ff.

618 EGMR (GK) - Muršić/CRO, Urt. v. 20.10.2016, 7334/13, Rn. 170 f. 
Konventionsstaat Klarheit darüber vermitteln, welcher Unrechtsgehalt in dem ihm vorgeworfenen Verhalten erkannt wird.

\subsection{Kriterien der Standardbildung}

Führt das Unterschreiten der $3 \mathrm{~m}^{2}$ zur Vermutung einer erniedrigenden Behandlung i.S.v. Art. 3 EMRK, nimmt der Gerichtshof mit Blick auf die allgemeinen Begriffsmerkmale einer Erniedrigung mit anderen Worten an, dass das Unterschreiten von $3 \mathrm{~m}^{2}$ persönlichem Platz i.d.R. beim Betroffenen in menschenwürderelevanter Hinsicht zu einem Demütigungsmoment führt und Gefühle der Angst, Furcht oder Unterlegenheit hervorgerufen werden bzw. der Betroffene in unzulässiger Weise herabgesetzt und sein physischer und psychischer Widerstand gebrochen wird. Wie auch bei anderen Fallgruppen verlangt der Gerichtshof aber gerade keinen Nachweis der konkreten Auswirkungen auf den Betroffenen. Vielmehr orientiert er sich bei der Definition dessen, was als eine Erniedrigung im Kontext von Überbelegungssituationen zu verstehen ist, an der Auslegungsdirektive des „no more harm principle“. Aufgrund von deren geringem Beschränkungspotential prüft der Gerichtshof allerdings nicht tatsächlich, ob die Haftbedingungen de facto verbessert werden könnten, sondern nimmt vielmehr eine eigens für Haftsituationen kreierte Zumutbarkeitsprüfung ${ }^{619}$ vor. Der Gerichtshof stellt sich folglich implizit die Frage, ob die konkreten Haftbedingungen unter Berücksichtigung sämtlicher relativer Kriterien gegenüber dem Beschwerdeführer im konkreten Fall zu verantworten waren oder nicht. ${ }^{620}$ Verneint der Gerichtshof dies, stellt er - wiederum das „no more harm principle" aufgreifend - fest, dass die Haftumstände über das Mass an Leiden hinaus gingen, welches dem Freiheitsentzug als solchem inhärent ist, womit eine erniedrigende Behandlung i.S.v. Art. 3 EMRK vorliegt. ${ }^{621}$

619 Vgl. auch Dörr/Grote/Marauhn/Bank Kap. 11 Rn. 22.

620 Es handelt sich dabei folglich nicht um eine Zumutbarkeitsprüfung, wie sie bei Abwehrrechten bei der Verhältnismässigkeitsprüfung i.e.S. durchgeführt wird. Es erfolgt hier nämlich keine Abwägung zwischen privaten und öffentlichen Interessen.

621 EGMR (GK) - Muršić/CRO, Urt. v. 20.10.2016, 7334/13, Rn. 153: „[...] the conditions of the applicant's detention subjected him to hardship going beyond the unavoidable level of suffering inherent in detention and thus amounting to degrading treatment prohibited by Article 3 of the Convention." 
Ausgangspunkt dieser Zumutbarkeitsprüfung sind die in $A n$ anyev u.a./RUS festgelegten Kriterien $\left(3 \mathrm{~m}^{2}\right.$ persönlicher Platz, individueller Schlafplatz, freie Bewegungsmöglichkeit zwischen den Einrichtungsgegenständen). Den Erwägungen des Gerichtshofs ist dabei nicht ausdrücklich zu entnehmen, ob diese Kriterien auf normativen oder faktischen Schlussfolgerungen beruhen. Eine eigene empirische, wissenschaftliche Untermauerung dieser Kriterien erfolgt jedenfalls nicht; sie scheinen vielmehr einer von der Menschenwürde her gedachten, normativ wertenden Beurteilung zu entspringen, welche ihrerseits aber soziomedizinische Überlegungen aufgreift. Ein ähnliches Bild zeigt sich auch bei den Voraussetzungen für die Widerlegung der Vermutung. Die kompensierenden Elemente basieren auf der Annahme soziomedizinischer Erleichterungs- resp. Erholungseffekte, ohne dass aber der Fokus auf wissenschaftlich belegte, kausale Effekte gelegt würde. Ob eine Kompensation, welche geeignet ist, die Vermutung zu widerlegen, vorliegt oder nicht, ist letztlich wiederum Ergebnis einer von der Menschenwürde her gedachten, normativ wertenden Betrachtung. Mit anderen Worten scheint der Gerichtshof soziomedizinischen Elementen mit Blick auf die Achtung der Menschenwürde einen gewissen normativen Wert in der Gesamtbetrachtung zuzuschreiben. Die soziomedizinische Annahme einer Belastung resp. der Erleichterungsresp. Erholungswirkung ist damit lediglich, aber immerhin, Fundament der normativen Gesamtbewertung.

Zusammenfassend hat der Gerichtshof einen für Überbelegungssituationen spezifischen Prüfungsmechanismus kreiert, welcher im Ergebnis zu einer an der Menschenwürde orientierten, normativ geprägten Zumutbarkeitsprüfung geführt hat, welche ihrerseits zumindest teilweise auf soziomedizinischen Schlussfolgerungen beruht. Die allgemeinen Begriffsmerkmale der erniedrigenden Behandlung rücken bei dieser Zumutbarkeitsprüfung in den Hintergrund, was auch zur dogmatischen Unschärfe des Gerichtshofs bei der Zuordnung von Überbelegungssituationen zur unmenschlichen oder erniedrigenden Behandlung geführt haben dürfte. Das hier ausgemachte spezifische Prüfprogramm für Überbelegungssituationen und die vom Gerichtshof angewandten Kriterien dürfen allerdings nicht darüber hinwegtäuschen, dass auf ihrer Basis letztlich eine normative Bewertung einer Situation an den je spezifischen Gehalten der erniedrigenden oder aber der unmenschlichen Behandlung i.S.v. Art. 3 EMRK erfolgt. Grundvoraussetzung auch dieses spezifischen Prüfprogramms ist folglich Kenntnis über die Voraussetzungen, anhand welcher man die normative Bewertung bei der „Zumutbarkeitsprüfung“ vornimmt. Eine Verselbstän- 
digung der Prüfung, losgelöst von den Kriterien einer unmenschlichen oder erniedrigenden Behandlung, ist ausgeschlossen.

\section{Notwendigkeit einer revidierten Prüfungsmethodik? - Ein Lösungsvorschlag}

Aufgrund der vorstehenden Ausführungen wird ein eigener Vorschlag gemacht, wie und anhand welcher Kriterien Überbelegungssituationen im Lichte des Art. 3 EMRK zu prüfen sind. Die Überbelegung ist aufgrund ihrer Einwirkungsweise, welche in der Regel den Demütigungsmoment in den Vordergrund rückt und einen besonders engen Konnex zur Menschenwürde aufweist, in der Regel anhand des Massstabs einer erniedrigenden Behandlung zu prüfen. Auch in diesem Vorschlag soll einem normativen Ansatz gefolgt werden, welcher soziomedizinische Faktoren bei seiner Bewertung aufgreift, ohne dass aber ein Nachweis der Auswirkungen auf den Einzelnen im konkreten Fall gefordert würde. In diesem Sinne wird grundsätzlich dem Gerichtshof gefolgt. Werden ausnahmsweise vom Beschwerdeführer konkrete und erhebliche, erlittene seelische und körperliche Qualen vorgebracht, ist die Feststellung (auch) einer unmenschlichen Behandlung keineswegs ausgeschlossen. Eine entsprechende Prüfung und Begründung der Voraussetzungen der unmenschlichen Behandlung hätten dann allerdings zwingend zu erfolgen.

Weil die Prüfungsmethodik der Gesamtbetrachtung des Gerichtshofs mit Blick auf den Menschenrechtsschutz nicht unproblematisch ist, wird hier eine leicht modifizierte bzw. ergänzte Prüfungsmethodik vorgeschlagen, welche sich aus den einzelnen Elementen der Vorgehensweise des Gerichtshofs zusammensetzt. Ziel dieser Modifikation ist, der Aufweichung der Standards bei den Haftbedingungen entgegenzutreten, ohne dabei einer Weiterentwicklung des Standards im Wege zu stehen. Mit anderen Worten soll der Standard in der Weise festgelegt werden, dass ganz im Sinn des Spezifitätskriteriums eine Konkretisierung des Standards stattfindet, womit es zu einer grösseren Rechtssicherheit kommt.

In Anerkennung der Wechselwirkungen zwischen den einzelnen Haftumständen wird in einem ersten Schritt an einer Gesamtbetrachtung festgehalten. Der Einbezug kumulierender und kompensierender Elemente führt zu einem umfassenden Bewertungsgegenstand, womit aus normativ wertender Sicht schliesslich Aussagen über eine Gesamtbelastung getroffen werden können. Bezüglich der kumulierenden Elemente soll weiter wie bisher verfahren werden, birgt dieses Vorgehen hinsichtlich des Schutzni- 
veaus des Art. 3 EMRK doch keinerlei Risiko. Auch die kompensierenden Elemente sollen nicht ausser Acht gelassen werden, so dass am Vermutungsmodell des Gerichtshofs festgehalten wird. Dieses ist aber insoweit zu modifizieren, dass die Kompensation von vorneherein nur für eine genau bestimmte Unterschreitung des Massstabes möglich sein soll, der Widerlegbarkeit der Vermutung also in einem zweiten Schritt eine Grenze gesetzt wird. Der relative Massstab, bei welchem Kompensationen möglich sind, ist also durch einen auch in numerischer Hinsicht absoluten Massstab zu ergänzen (im Sinne eines absoluten Kerns der Garantie). Wo diese Grenze liegt, ist mit Blick auf die Kriterien der erniedrigenden Behandlung festzulegen. Es stellt sich die Frage, welcher Platz aufgrund normativ wertender Überlegungen trotz hypothetisch maximal vorliegender Kompensation und bei minimaler Dauer niemals unterschritten werden darf, weil damit in jedem Fall zwingend eine Herabsetzung und Geringschätzung des Betroffenen in seiner Person erfolgen würde, welche das Mindestmass an Schwere automatisch erreicht. Abweichungen von diesem absoluten Kern sollen keinesfalls mehr möglich sein (im Unterschied zum EGMR „normally“622). Weitere absolute Untergrenzen sollen mit Blick auf die Menschenwürde darüber hinaus weiterhin das zweite und das dritte Kriterium des Ananyev-Tests bleiben, dass also dem Inhaftierten ein eigener Schlafplatz und freie Bewegungsmöglichkeit zwischen den Einrichtungsgegenständen zugestanden werden muss. Letzteres wäre etwa dadurch sicherzustellen, dass beim Mindeststandard des persönlichen Platzes jener Platz, der von Einrichtungsgegenständen belegt wird, abgezogen wird - wie dies jetzt schon für die sanitären Anlagen der Fall ist. ${ }^{623}$

Weiter soll nur dann von einer Kompensation ausgegangen werden, welche geeignet ist, die Vermutung zu widerlegen, wenn die kompensierenden Faktoren klar über dem adäquaten resp. genügenden Mindeststandard liegen. Die blosse Einhaltung der übrigen Kriterien soll noch nicht als Kompensation qualifiziert werden. ${ }^{624}$ Dies gestützt auf die Überlegung, dass Erleichterungswirkungen als soziomedizinische Grundlage der nor-

622 EGMR (GK) - Muršić/CRO, Urt. v. 20.10.2016, 7334/13, Rn. 130; vgl. auch EGMR - Fetisov u.a./RUS, Urt. v. 17.01.2012, 43710/07 u.a., Rn. 134 ff.; EGMR Dmitriy Rozhin/RUS, Urt. v. 23.10.2012, 4265/06, Rn. 52 f.; EGMR - Vladimir Belyayev/RUS, Urt. v. 17.10.2013, 9967/06, Rn. 33 ff.; EGMR - Kurkowski/POL, Urt. v. 09.04.2013, 36228/06, Rn. 66 f.

623 Vgl. auch schon Richter Pinto de Albuquerque partly diss. op., EGMR (GK) MuršićlCRO, Urt. v. 20.10.2016, 7334/13, Rn. 52.

624 In diese Richtung auch bereits Richter Sajó, López Guerra u. Wojtyczek, joint partly diss. op., EGMR (GK) - Muršić/CRO, Urt. v. 20.10.2016, 7334/13, Rn. 8. 
mativen Bewertung bei gerade genügenden Haftbedingungen noch nicht angenommen werden können. Mit anderen Worten soll die Vermutung eines Demütigungsmoments resp. der Geringschätzung des Betroffenen aufgrund inadäquater Platzverhältnisse nicht durch die Einhaltung gerade genügender anderer Haftbedingungen widerlegt werden können. Als Kompensationsfaktoren sollen aber weiterhin sämtliche erleichternden Faktoren in Betracht kommen, welche sich auf den Grad des erlittenen Leidens bzw. die Schwere der zugefügten Behandlung auswirken können. 625

Mit derselben Argumentation ist bei der Anwendung des Elementes der Dauer Abstand von der Praxis des Gerichtshofs zu nehmen. Eine separate Prüfung der Zeitspannen - wie sie vom Gerichtshof vorgenommen wird - überzeugt nämlich nur bedingt. ${ }^{626}$ Einerseits ist bei der Beurteilung der Dauer sicherzustellen, dass kumulierende Elemente und damit die Gesamtbelastung als soziomedizinische Basis der normativen Bewertung weiterhin berücksichtigt werden, und andererseits ist zu gewährleisten, dass keine kompensierende Wirkungen angenommen werden, wo gestützt auf soziomedizinischen Schlussfolgerungen gar keine positiven Effekte zu erwarten sind.

Unterbrechungen des bemängelten Umstandes sind also nur dann zu berücksichtigen, wenn sie geeignet sind, zu Erleichterungen zu führen. ${ }^{627}$ Dies setzt zum einen voraus, dass während dieser Zeit mindestens der garantierte Mindeststandard eingehalten wird, und zum anderen, dass der Unterbruch lange genug ist, um Erholungswirkungen überhaupt zu ermöglichen. In Situationen, bei welchen zwar ohne Unterbruch ungenügende Platzverhältnisse vorliegen und bloss das Mass der Unterschreitung variiert, wäre die Annahme, dass dies zu Erleichterungen führen würde, welche als soziomedizinische Basis dazu dienen könnten, die Vermutung einer Herabsetzung resp. Geringschätzung der Person zu widerlegen, ge-

625 A.A. Richter Lazarova Trajkovska, de Gaetano u. Grozev, joint partly diss. op., EGMR (GK) - Muršić/CRO, Urt. v. 20.10.2016, 7334/13, Rn. 10: Als kompensierender Faktor komme von vorneherein nur ein grösseres Platzangebot in den Gemeinschaftsräumen in Betracht. Nur durch diese Beschränkung könne sichergestellt werden, dass der zur Verfügung stehende Platz Schlüsselelement der Beurteilung von Art. 3 EMRK sei.

626 Kritisch zu dieser Vorgehensweise vgl. auch Richter Pinto de Albuquerque partly diss. op., EGMR (GK) - Muršić/CRO, Urt. v. 20.10.2016, 7334/13, Rn. 54.

627 Vgl. auch Richter Sajó, López Guerra u. Wojtyczek, joint partly diss. op., EGMR (GK) - Muršić/CRO, Urt. v. 20.10.2016, 7334/13, Rn.9. 
radezu befremdlich. ${ }^{628}$ Die für positive Effekte erforderliche Zeitspanne müsste auf jeden Fall in Relation zur Dauer der vorausgegangenen Zeitspanne mit ungenügenden Bedingungen und den effektiv erfahrenen Haftbedingungen stehen: Je länger die Zeitspanne mit ungenügenden Bedingungen und je gravierender die erfahrenen Haftbedingungen, desto länger hätte der Unterbruch zu sein, damit überhaupt von einer entlastenden Wirkung ausgegangen werden könnte. Mit Blick auf die soziomedizinische Belastung müsste weiter der Inkonstanz der Situation (ständige Wechsel, immer wieder neue Inhaftierte in der Zelle etc.) bei deren normativer Bewertung ein negativer Eigenwert zugeschrieben werden. ${ }^{629}$ Unterbrechungen sollen mit Blick auf die Missbrauchsgefahr (Umgehung des Mindeststandards durch Unterbrechungen der Unterschreitung des Standards) sodann im Allgemeinen nur restriktiv berücksichtigt werden.

Alternativ könnte dem Votum von Richter Pinto de Albuquerque gefolgt werden, und es könnten sämtliche Zeiträume unter ungenügenden Bedingungen zusammengerechnet und ins Verhältnis mit der gesamten Haftzeit gesetzt werden. ${ }^{630}$ Wird diesem prozentualen Ansatz gefolgt, müsste dieser allerdings zwingend mit einer absoluten zeitlichen Obergrenze kombiniert werden, da ansonsten insbesondere bei längeren Haftstrafen ein relativ geringer Prozentsatz die Gefahr birgt, über eine effektiv doch längere Zeitspanne mit ungenügenden Bedingungen hinwegzutäuschen, welche mit den Garantien von Art. 3 EMRK nicht mehr zu vereinbaren wäre.

Schliesslich hat der Eintritt der strengen Vermutung im Vergleich zur Erbringung eines Anscheinsbeweises durch den Beschwerdeführer auf beweisrechtlicher Ebene zu einer gesteigerten Begründungspflicht des Konventionsstaates zu führen, so dass zur Widerlegung der Vermutung auch hinsichtlich des Beweismasses generell höhere Anforderungen zu stellen wären als zur Erbringung eines Exkulpationsbeweises. Hinsichtlich dieser besonderen Begründungspflicht wird noch einmal deutlich, wie wichtig Klarheit darüber ist, ob dem Konventionsstaat eine unmenschliche oder erniedrigende Behandlung vorgeworfen wird und anhand welcher Kriterien deren Voraussetzungen beurteilt werden. Es ist dem Konventionsstaat

628 Vgl. auch bereits Richter Sajó, López Guerra u. Wojtyczek, joint partly diss. op., EGMR (GK) - Muršić/CRO, Urt. v. 20.10.2016, 7334/13, Rn.9.

629 Verlegungen sind für den Betroffenen insbesondere dann nicht in seinem Interesse, wenn ausgearbeitete Wiedereingliederungspläne oder andere Programme abrupt unterbrochen werden. Vgl. dazu auch Devresse, Droit et Société 2013, 339 (351).

630 Richter Pinto de Albuquerque partly diss. op., EGMR (GK) - Muršić/CRO, Urt. v. 20.10.2016, 7334/13, Rn. 54 ff. 
nämlich nur bei Kenntnis über den ihm vorgeworfenen Unrechtsgehalt möglich, zielgerichtete Argumente und Beweise vorzubringen, um die entsprechende Vermutung zu widerlegen.

Durch das Fortbestehen des zweiteiligen Prüfungsmodells mit grundsätzlicher Zulässigkeit der Widerlegung der Vermutung, aber ergänzt um den absoluten Kern jedes einzelnen Kriteriums, würde sowohl den Wechselwirkungen zwischen den einzelnen Kriterien, welche der tatsächlichen Wirklichkeit entsprechen, als auch dem Fundamentalcharakter von Art. 3 EMRK Rechnung getragen. Letzteres, indem die Aufweichungsmöglichkeit der einzelnen Kriterien eingedämmt und so auch der Konkretisierungsforderung des Spezifitätskriteriums absoluter Rechte nachgekommen würde, ohne dabei die Relativität der Schutzbereichsbestimmung zu beschneiden. Mit anderen Worten würde durch das hier vorgeschlagene Prüfungsmodell sowohl der Konzeption der Einzelfallbetrachtung als auch dem Umstand, dass gerade der absolute Charakter nach einer gesteigerten Rechtssicherheit verlangt, Rechnung getragen werden. Die vorgeschlagenen Modifikationen sollen mit Blick auf das zu gewährleistende Schutzniveau weiter dazu führen, dass die Widerlegung der Vermutung die Ausnahme bleibt und nicht zur Regel wird. ${ }^{631}$ Erleichterungs- und Erholungswirkungen als soziomedizinische Basis der normativen Bewertung dürfen bei kompensierenden Elemente nämlich keinesfalls leichthin angenommen werden. Insgesamt ist von diesem prüfungsmethodischen Vorgehen auch zu erwarten, dass es nicht zuletzt durch mehr Klarheit und eine flankierende numerische absolute Grenze zu einer Erhöhung des Schutzniveaus kommt, was mit Blick auf einen effektiven Menschenrechtsschutz zu begrüssen wäre.

5. EGMR im Verhältnis zu anderen Standards und zu anderen Institutionen

Sowohl bei der materiellen Genese des Mindeststandards als auch beim prüfungsmethodischen Vorgehen nahm der Gerichtshof immer wieder Bezug zu anderen Regelungsinstrumentarien und Institutionen. Hinsichtlich der Wechselwirkungen zwischen Rechtsprechung und Soft Law sind von vorneherein zwei Ebenen zu unterscheiden: die Bezugnahme auf der Sach-

631 Plädoyer zur nur ausnahmsweisen Widerlegung der Vermutung: Richter Sajó, López Guerra u. Wojtyczek, joint partly diss. op., EGMR (GK) - Muršić/CRO, Urt. v. 20.10.2016, 7334/13, Rn. 3. 
verhaltsebene („The Facts“) und auf der Rechtsebene („The Law“632). Letzteres vor dem Hintergrund, dass Regelungswerke anderer Institutionen und Gremien dem Gerichtshof als Rechtserkenntnisquelle dienen. ${ }^{633}$

\subsection{Einfluss anderer Institutionen auf die Rechtsprechung des EGMR bei der Überbelegung}

a Rechtsebene

Implementiert der Gerichtshof auf der Rechtsebene Soft Law und formt dieses unter dem Gesichtspunkt von Art. 3 EMRK zu einem harten, einklagbaren Standard, wird von einem Transformationsprozess gesprochen. Es stellt sich die Frage, ob ein solcher Prozess, mit den damit einhergehenden Befürchtungen, im Bereich der Überbelegung tatsächlich stattfindet bzw. wie sich das Soft Law in der Rechtsprechung des Gerichtshofs tatsächlich auswirkt. 634

Die Analyse der einschlägigen Regelungsinstrumentarien hat gezeigt, dass auf europäischer Ebene das Prinzip der Einzelunterbringung in den Europäischen Strafvollzugsgrundsätzen (EPR) prominent empfohlen wurde, während das CPT keine klare Präferenz zwischen Einzelunterbringung und einer Unterbringungsform mit zwei bis vier Inhaftierten äusserte. Zum zur Verfügung zu stellenden Platz nahmen die Europäischen Strafvollzugsgrundsätze insofern eine zurückhaltende Position ein, als sie die Mitgliedstaaten lediglich auffordern, einen konkreten Mindeststandard zu setzen; Vorschläge für einen solchen sind ihnen jedoch nicht zu entnehmen. Das CPT positionierte sich zu dieser Fragestellung mit der Festlegung seines Mindeststandards und seines desirable standard klar.

$632 \mathrm{Im}$ hier verwendeten Sinne wird auch dann von einer Implementation auf Rechtsebene gesprochen, wenn die Soft Law-Dokumente zunächst auf der Ebene "The Facts" aufgeführt werden, sie aber vom Gerichtshof bei seinen rechtlichen Ausführungen unter dem Kapitel „The Law" ausdrücklich wieder aufgegriffen werden. Vgl. so etwa in EGMR (GK) - Muršić/CRO, Urt. v. 20.10.2016, 7334/13, Rn. 46 ff., Rn. 77 u. 111. Von einer Bezugnahme auf Sachverhaltsebene ist im hier verstandenen Sinne demgegenüber dann die Rede, wenn Dokumente anderer Gremien bei der Sachverhaltserstellung aufgegriffen werden.

633 SK-StPO/Meyer Art. 3 EMRK Rn. 44; Schabas S. 41.

634 Zum „hardening of prison law" vgl. auch Richter Pinto de Albuquerque partly diss. op., EGMR (GK) - Muršić/CRO, Urt. v. 20.10.2016, 7334/13, Rn. 34 ff. 
Bei der Beurteilung der Konformität mit Art. 3 EMRK rezipiert der Gerichtshof diese Regelungsinstrumentarien, ${ }^{635}$ geht im Folgenden allerdings nicht mehr näher auf die Frage der konkreten Unterbringungsform (Einzelzelle vs. Mehrbettzelle) ein und beschränkt sich auf die Festlegung des konkret zur Verfügung zu stellenden Platzes, wobei er aber ausdrücklich vom CPT-Massstab abweicht. Im Urteil Ananyev u.a./RUS griff der EGMR die vom CPT vor allem in den Länderberichten statuierten $4 \mathrm{~m}^{2}$ persönlicher Platz zwar auf, interpretiert sie aber nicht als Mindeststandard, sondern als wünschenswerten Standard („desirable standard“) und grenzt seinen Massstab in der Folge mit Verweis auf seine bisherige Rechtsprechung explizit vom CPT-Standard ab. ${ }^{636}$ Im Urteil Muršić/CRO argumentiert der Gerichtshof dann anders. Die Argumentation aus Ananyev u.a./RUS hätte zum Urteilszeitpunkt von Muršić/CRO auch nicht mehr überzeugt. Spätestens mit der Herausgabe des konsolidierten Dokuments „Living Space per Prisoner in Prison Establishments" 637 des CPT, ergibt sich nämlich unmissverständlich, dass die vorgeschlagenen $4 \mathrm{~m}^{2}$ aus Sicht des CPT ein Mindeststandard und keinesfalls ein desirable standard sind. Begründet wird die Abweichung des Gerichtshofs vom Standard des CPT nun mit der unterschiedlichen Herangehensweise und der unterschiedlichen Rolle des Gerichtshofs und des CPT. ${ }^{638}$ Aus Sicht des Gerichtshofs sei es seine Aufgabe, sämtliche Umstände des Einzelfalls zu berücksichtigen, während das CPT aus Sicht des Gerichtshofs bestrebt sei, einen generellen Massstab festzulegen. ${ }^{639}$ Die Rolle des CPT und des Gerichtshofs würden sich damit konzeptionell unterscheiden. ${ }^{640}$ Während der EGMR für eine justizielle Kontrolle im Einzelfall zuständig ist, seien die Tätigkeiten des CPT im Verständnis des Gerichtshofs (rein) präventiver Natur und gerade nicht darauf gerichtet zu beurteilen, ob eine konkrete Situation unmenschlich oder erniedrigend sei. ${ }^{641}$ Daraus folgert die Grosse Kammer im Urteil Muršićl

635 Vgl. etwa EGMR (GK) - Muršić/CRO, Urt. v. 20.10.2016, 7334/13, Rn. 46 ff.

636 EGMR - Ananyev u.a./RUS, Urt. v. 10.01.2012, 42525/07 u. 60800/08, Rn. 144 f.: "Whereas the provision [from the CPT] of four square meters remains the desirable standard of multi-occupancy accommodation, the Court has found that where the applicants have at their disposal less than three square meters of floor surface, the overcrowding must be considered to be so severe as to justify of itself a finding of a violation of Article 3."

637 CPT - Living Space per Prisoner [CPT/Inf (2015) 44].

638 EGMR (GK) - Muršić/CRO, Urt. v. 20.10.2016, 7334/13, Rn. $112 \mathrm{f}$.

639 EGMR (GK) - Muršić/CRO, Urt. v. 20.10.2016, 7334/13, Rn. 112.

640 EGMR (GK) - Muršić/CRO, Urt. v. 20.10.2016, 7334/13, Rn. 112.

641 EGMR (GK) - MuršićlCRO, Urt. v. 20.10.2016, 7334/13, Rn. 113. 
CRO, dass der vom CPT geforderte Standard schon von seiner Konzeption her ein höherer Standard zu sein hat als derjenige des EGMR. ${ }^{642}$

Ist dem Ergebnis zuzustimmen, dass je nach Fragestellung und je nach Institution aufgrund je anderer normativer Wertungen soziomedizinischer Gegebenheiten unterschiedliche Standards möglich sind, so scheinen die vom Gerichtshof beschriebenen Rollen von EGMR und CPT doch allzu stark vereinfacht. Die Prävention ist sicher Hauptaufgabe des CPT ${ }^{643}$; gleichwohl beurteilt dieses aber auch konkrete Fälle bzw. zumindest konkrete Strafvollzugsanstalten. Gerade in den Länderberichten des CPT werden nämlich spezifische Situationen hinsichtlich ihrer Konformität mit Art. 3 EMRK beurteilt. ${ }^{644}$ Auch im Dokument „Living Space per Prisoner in Prison Establishments"645, in welchem klar zwei unterschiedliche Standards aufgestellt wurden (der Mindeststandard und der wünschenswerte Standard), wird die Zielsetzung des CPT noch einmal ausdifferenziert: Der wünschenswerte Standard ist klar auf die präventive Funktion des CPT ausgerichtet, ${ }^{646}$ während der Mindeststandard nicht nur auf diese Funktion beschränkt ist. ${ }^{647}$ So sieht auch das CPT selbst seine Aufgabe als integrierten Bestandteil des Europaratssystems zum Schutz der Menschen-

642 EGMR (GK) - Muršić/CRO, Urt. v. 20.10.2016, 7334/13, Rn. 113.

643 Vgl. bereits den Namen des Komitees: European Committee for the Prevention of Torture and Inhuman or Degrading Treatment or Punishment; vgl. auch CPT - 26th General Report [CPT/Inf (2017) 5], S. 45: „The CPT implements its essentially preventive function through two kinds of visits - periodic and ad hoc.“

644 Vgl. bspw. CPT - 27th General Report [CPT/Inf (2018) 4], Rn. 31 mit Verweis auf den Länderbericht zu seinem Besuch in Frankreich im Jahr 2015: „The CPT's report notes that the three remand prisons visited (Fresnes, Nîmes and Villepinte) were affected by severe overcrowding. In particular, in the CPT's view, the conditions of detention (e.g. lack of living space per prisoner in cells, insufficient number of beds and lack of out-of-cell activities) could be considered inhuman and degrading."

645 CPT - Living Space per Prisoner [CPT/Inf (2015) 44].

646 CPT - Living Space per Prisoner [CPT/Inf (2015) 44], Rn. 18: „The CPT encourages all Council of Europe member states to apply these higher standards, in particular when constructing new prisons."

647 Hier sei auf die 4. Hauptschlussfolgerung des CPT zu seinem präventiven Charakter im 1. General Report verwiesen, wo auch das CPT folgerte, dass bei der Prüfung einer Verletzung von Art. 3 EMRK sein Standard ein höherer zu sein habe als jener vom EGMR: CPT - 1st General Report [CPT/Inf (91) 3], Rn. 51. Dazumals hatte das CPT allerdings noch keine Differenzierung zwischen Mindeststandard und wünschenswertem Standard vorgenommen. Seitdem diese Differenzierung nun aber besteht, kann diese Schlussfolgerung für den Mindeststandard nicht mehr ohne Weiteres gezogen werden. 
rechte, welche es neben dem EGMR wahrnimmt. ${ }^{648}$ Die Beurteilung der Einzelfälle durch den Gerichtshof beschränkt sich sodann nicht immer auf rein individuell-konkrete Wirkungen; eine Präventionsfunktion für ähnlich gelagerte Fälle ist auch der Rechtsprechungsaktivität inhärent.

Es blieb denn auch in der Praxis nicht ohne Kritik, dass der Gerichtshof die massgebliche Grenze bei $3 \mathrm{~m}^{2}$ persönlichem Platz festlegt. Selbst in Anerkennung gewisser Unterschiede betreffend Funktion und Aufgabe des CPT und des Gerichtshofs wird von einzelnen Richtern des Gerichtshofs bisweilen gefordert, die Grenze dennoch in Übereinstimmung mit dem Mindeststandard des CPT bei $4 \mathrm{~m}^{2}$ zu setzen. ${ }^{649}$ Untermauert wird die Notwendigkeit zur Anlehnung des Gerichtshofs an das CPT mit der besonderen Expertise des CPT in diesem Bereich und dem Beizug von sozialwissenschaftlichen Erkenntnissen, welche die Auswirkungen von geringem Platz auf die Betroffenen aufzeigen würden. ${ }^{650}$ Werde leichthin vom CPT-Standard abgewichen, würde die Bedeutung seiner Arbeit herabgesetzt. ${ }^{651}$ Gerade im Bereich der Haftbedingungen sei aufgrund der Komplexität des Gebietes und auch mit Blick auf die Effektivität des Standards ein klarer Massstab notwendig, weshalb der Gerichtshof angehalten wird, seinen Mindeststandard mit demjenigen anderer Akteure zu synchronisieren. ${ }^{652}$ Hinzu komme, dass selbst die $4 \mathrm{~m}^{2}$, wenn auch die momentan beste Alternative, mit Blick auf den desirable standard des CPT noch nicht vollständig überzeugten. ${ }^{653}$

Auch meines Erachtens überzeugt die Argumentation des Gerichtshofs nicht vollständig. Weder Funktion noch Aufgabe des Gerichtshofs (im

648 CPT - 26th General Report [CPT/Inf (2017) 5], S. 45: „The work of the CPT is designed to be an integrated part of the Council of Europe system for the protection of human rights, placing a proactive non-judicial mechanism alongside the existing reactive judicial mechanism of the European Court of Human Rights."

649 Vgl. etwa Richter Sajó, López Guerra u. Wojtyczek, joint partly diss. op., EGMR (GK) - MuršićlCRO, Urt. v. 20.10.2016, 7334/13, Rn. 5.

650 Richter Sajó, López Guerra u. Wojtyczek, joint partly diss. op., EGMR (GK) Muršić/CRO, Urt. v. 20.10.2016, 7334/13, Rn. 4 f.; Richter Lazarova Trajkovska, de Gaetano u. Grozev, joint partly diss. op., EGMR (GK) - Muršić/CRO, Urt. v. 20.10.2016, 7334/13, Rn.9.

651 Richter Pinto de Albuquerque partly diss. op., EGMR (GK) - Muršić/CRO, Urt. v. 20.10.2016, 7334/13, Rn. 36.

652 Richter Lazarova Trajkovska, de Gaetano u. Grozev, joint partly diss. op., EGMR (GK) - Muršić/CRO, Urt. v. 20.10.2016, 7334/13, Rn. 3: „overall effectiveness" und "clarity of the standards".

653 Richter Sajó, López Guerra u. Wojtyczek, joint partly diss. op., EGMR (GK) Muršić/CRO, Urt. v. 20.10.2016, 7334/13, Rn. 5. 
Vergleich zum CPT) sind genügende Begründung für den vom EGMR festgesetzten Standard. Sowohl der vom CPT festgesetzte Massstab von $4 \mathrm{~m}^{2}$ als auch der 3- $\mathrm{m}^{2}$-Massstab des Gerichtshofs beruhen auf einer von der Menschenwürde her gedachten normativen Bewertung, welche auf soziomedizinischen Schlussfolgerungen beruht. Inwieweit sich bei einer solchen Beurteilung gewisse Unterschiede bei Funktion und Aufgabe von CPT und EGMR auswirken sollen, legt der Gerichtshof nicht schlüssig dar. Aus seiner Begründung lässt sich darüber hinaus schon gar nicht ableiten, wie viel tiefer der Standard des Gerichtshofs denn anzulegen sei.

Konzentriert man sich auf die Berechnungsmodalitäten sowie die Methodik der Beurteilung einer Verletzung von Art. 3 EMRK, wird deutlich, dass CPT und Gerichtshof sehr ähnlich vorgehen. Bei beiden wird der Platz für die sanitären Einrichtungen zum Mindeststandard hinzugezählt, während der Platz für Einrichtungsgegenstände in diesem bereits inkludiert ist. Sowohl CPT als auch Gerichtshof nehmen sodann eine Gesamtbetrachtung der Haftumstände vor und berücksichtigen Kompensationsmöglichkeiten, wobei sich auch die Kompensationsfaktoren weitgehend decken.

Bewertungskriterien und Betrachtungsgegenstand scheinen bei CPT und EGMR damit identisch zu sein, so dass die Festlegung des Mindeststandards durch den Gerichtshof bei $3 \mathrm{~m}^{2}$ persönlichen Platzes ohne weitergehende sozialwissenschaftliche Studien zu den Folgen dieses Platzangebotes auf den Betroffenen oder eingehendere normative Begründung schon fast willkürlich erscheint.

Zusammenfassend ist festzuhalten, dass der Gerichtshof bei der Frage der Unterbringungsform nicht der Tendenz des Soft Law (insbesondere der Europäischen Strafvollzugsgrundsätze) folgt und im Rahmen von Art. 3 EMRK keine klare Präferenz für die Einzelunterbringung fordert. Hinsichtlich des minimal zur Verfügung zu stellenden Platzes grenzt sich der Gerichtshof bewusst vom klaren CPT-Standard ab. Sowohl der von der Menschenwürde her gedachte wertende Begründungsansatz, welcher soziomedizinische Erkenntnisse aufgreift, als auch die Berechnungsmodalitäten und das methodische Vorgehen vom EGMR und CPT sind jedoch sehr ähnlich. Begründet wird die Abweichung vom Mindeststandard des CPT hinsichtlich des minimal zur Verfügung zu stellenden Platzes vom EGMR mit ihren je unterschiedlichen Aufgaben und Funktionen. Diese Argumentation greift allerdings zu kurz; insbesondere eine Reduktion um $1 \mathrm{~m}^{2}$ Platz (von $4 \mathrm{~m}^{2}$ beim CPT auf $3 \mathrm{~m}^{2}$ bei Art. 3 EMRK) lässt sich ohne weitergehende sozialwissenschaftliche Studien zu den Folgen dieses 
Platzangebotes auf den Betroffenen oder eine eingehendere normative Begründung jedenfalls nicht plausibel machen.

Insgesamt kann zum Schluss gelangt werden, dass im Bereich der Überbelegung die CPT-Dokumente für den Gerichtshof eine wichtige Rechtserkenntnisquelle sind. Ein Transformationsprozess von Soft Law zu Hard Law findet im Bereich der Festlegung des notwendigen persönlichen Platzes in der Rechtsprechung zu Art. 3 EMRK aber, zumindest was einen präzisen Mindeststandard des zur Verfügung zu stellenden Platzes anbelangt, nicht statt. Durch die Rezeption des CPT-Massstabes hinsichtlich Berechnungsmodalitäten und Prüfungsmethodik könnte gleichwohl argumentiert werden, dass die CPT-Empfehlungen einen gewissen Härtungsprozess durchlaufen und deren Normativität durch die EGMR-Rechtsprechung zunimmt.

\section{b Sachverhaltsebene}

Im Allgemeinen gilt, dass sich der EGMR bei divergierenden Vorbringen der Parteien bei der Sachverhaltserstellung mit den gleichen Schwierigkeiten konfrontiert sieht wie ein erstinstanzliches Gericht. ${ }^{654}$ Auch vor dem Gerichtshof gilt das Prinzip der freien Beweiswürdigung. ${ }^{655}$ Berichte anderer Institutionen sind damit, wie alle anderen Beweismittel auch, hinsichtlich des Beweiswertes zu beurteilen. Hinsichtlich des Beweismasses hat der Beschwerdeführer zunächst nur einen Anscheinsbeweis zu erbringen, dem Beschwerdegegner steht in der Folge der Exkulpationsbeweis offen. Lässt sich der Sachverhalt nicht erstellen, regelt die Beweislast die Folgen der Beweislosigkeit. ${ }^{656}$

Nachdem im Allgemeinen schon aufgezeigt wurde, dass Soft Law durch nachgelagerte Implementierungskontrollen praktische Wirksamkeit erlangen kann und dass gerade im Rahmen der stetigen Kontrolle des Umsetzungsstandes eine entsprechende (öffentliche) Berichterstattung erfolgt, stellt sich die Frage, ob diese Monitoringberichte hinsichtlich Überbelegungssituationen auch tatsächlich zur Sachverhaltserstellung genutzt werden.

Im Bereich der Feststellung von Haftbedingungen greift der EGMR tatsächlich Berichte anderer Institutionen auf, wobei vornehmlich den Be-

654 EGMR - Yunusova u. Yunusov/AZE, Urt. v. 02.06.2016, 59620/14, Rn. 139.

655 EGMR - Yunusova u. Yunusov/AZE, Urt. v. 02.06.2016, 59620/14, Rn. 139.

656 EGMR - Yunusova u. Yunusov/AZE, Urt. v. 02.06.2016, 59620/14, Rn. 139. 
richten des CPT besondere Bedeutung zukommt. ${ }^{657}$ So hält der Gerichtshof bisweilen etwa fest, dass er zwar selbst keinen Besuch vor Ort durchgeführt habe, die Behauptungen des Beschwerdeführers jedoch mit den Schlussfolgerungen des CPT-Berichts übereinstimmen würden, weshalb in der Folge von einer schweren Überbelegung und fehlenden Schlafmöglichkeiten ausgegangen werde. ${ }^{658}$ Der Anscheinsbeweis des Beschwerdeführers gelang damit, so dass es am Konventionsstaat gewesen wäre, den Exkulpationsbeweis zu erbringen.

CPT-Berichte werden im Einzelfall vom EGMR selbst dann zur Sachverhaltserstellung hinzugezogen, wenn die Inhaftierung des Beschwerdeführers nicht genau den Berichtszeitraum betraf, aber wenn gleichwohl davon ausgegangen werden muss, dass die ungenügenden Umstände unverändert sind. ${ }^{69}$ Auch werden Berichte zu anderen Haftanstalten des Konventionsstaates bei der Sachverhaltserstellung beigezogen, zumindest sofern unbestritten ist, dass in der besuchten und der verfahrensgegenständlichen Haftanstalt ähnliche Bedingungen herrschen. ${ }^{660}$

Es deutet alles darauf hin, dass der Beweiswert von CPT-Berichten vom EGMR als grundsätzlich gross eingestuft wird. Die Verlässlichkeit der Informationen von CPT-Berichten darf in aller Regel denn auch ange-

657 Vgl. bspw.: EGMR (GK) - Muršić/CRO, Urt. v. 20.10.2016, 7334/13, Rn. 54; EGMR - Dougoz/GRE, Urt. v. 06.03.2001, 40907/98, Rn. 46 f.: Die Ausführungen betreffen hier Sachverhaltselemente, selbst wenn sie formell unter der rechtlichen Würdigung „En Droit“ ausgeführt werden. EGMR - Peers/GRE, Urt. v. 19.04.2001, 28524/95, Rn. 61.

658 Vgl. etwa EGMR - Dougoz/GRE, Urt. v. 06.03.2001, 40907/98, Rn. 46.

659 EGMR - Neshkov u.a./BUL, Urt. v. 27.01.2015, 36925/10 u.a., Rn. 246: Der Besuch des CPT erfolgte fünf Jahre nach der Inhaftierung des Beschwerdeführers in der betreffenden Haftanstalt. Es gebe aber keine Hinweise darauf, dass sich in diesen Jahren etwas geändert hätte resp. dass die Belegungssituation eine andere gewesen sei. Vgl. ferner EGMR - Dougoz/GRE, Urt. v. 06.03.2001, 40907/98, Rn. 46 f.: Der Beschwerdeführer war von Juli 1997 bis Dezember 1998 inhaftiert. Herangezogen wurden CPT-Schlussfolgerungen von 1994. Aufgrund eines weiteren Besuchs von 1997 habe es das CPT weiter für notwendig gehalten, das betreffende Gefängnis 1999 erneut zu besuchen.

660 EGMR - Dougoz/GRE, Urt. v. 06.03.2001, 40907/98, Rn. 46 f.: Der EGMR zog einen Bericht über das Alexandras-Avenue-Polizeigefängnis von 1994 hinzu, wobei die Regierung Griechenlands die Bedingungen in diesem Gefängnis gleich beschrieb wie die Bedingungen in der verfahrensgegenständlichen Haftanstalt des Polizeigefängnisses in Drapetsona. Der EGMR fügte dann aber weiter an, dass auch die verfahrensgegenständliche Haftanstalt vom CPT bereits besucht und auch anlässlich dieses Besuchs die Notwendigkeit eines erneuten Besuchs festgestellt worden sei. 
nommen werden, handelt das CPT doch als unabhängiger Akteur mit im Wesentlichen gleicher Zielsetzung wie der Gerichtshof (Schutz der Inhaftierten vor Verletzungen von Art. 3 EMRK). Es hat sich aufgrund jahrelanger Erfahrung als Expertenkomitee auf dem Gebiet der Haftbedingungen etabliert.

Zusammenfassend wird deutlich, welch grosse Bedeutung der EGMR den CPT-Berichten hinsichtlich der Sachverhaltserstellung zumisst. Informationen des CPT zur allgemeinen Lage im Strafvollzug werden als wichtiges Indiz auch hinsichtlich der Lage des konkret Betroffenen gesehen, weshalb der Gerichtshof immer wieder von der Gesamtsituation auf die Situation im konkret zu beurteilenden Fall schliesst.

\subsection{Zukunftsperspektiven des Soft Law in Bezug auf prekäre Platzbedingungen}

Bezüglich der konkreten Unterbringungsform werden die Europäischen Strafvollzugsgrundsätze wohl auch in Zukunft an der Empfehlung der grundsätzlichen Einzelunterbringung festhalten und damit versuchen, die europäische Strafvollzugspolitik zu beeinflussen. Es ist allerdings in näherer Zukunft kaum zu erwarten, dass der Gerichtshof diese Empfehlung im Rahmen von Art. 3 EMRK aufgreifen wird, da eine Unterbringung in Mehrbettzellen wohl kaum a priori die Mindestschwelle einer Verletzung von Art. 3 EMRK erreichen wird, zumindest solange es sich bei der Unterbringungsform nicht um Grossraumschlafräume handelt.

Es ist zwar auch in Zukunft zu erwarten, dass der Gerichtshof die Entwicklung des Standards anderer europäischer Regelgeber beobachten und bisweilen auch in seiner Argumentation aufgreifen wird. Eine Anpassung des Standards des Gerichtshofs von $3 \mathrm{~m}^{2}$ auf $4 \mathrm{~m}^{2}$ im Sinne des CPT-Standards ist aber (noch) nicht absehbar, scheint sich der 3-m²-Standard doch mit dem Urteil Muršić/CRO (vorerst) gefestigt zu haben. Gleichwohl ist für die Zukunft eine erneute Auseinandersetzung des Gerichtshofs zum materiellen Kern des Mindeststandards anzuregen, wobei der gesetzte Standard von $3 \mathrm{~m}^{2}$ persönlichem Platz nicht zuletzt mit Blick auf den CPT-Standard noch einmal hinsichtlich seiner Auswirkungen auf den Einzelnen und seines normativen Gehalts - gemessen an den Kriterien einer erniedrigenden Behandlung i.S.v. Art. 3 EMRK - zu überdenken ist.

Hinsichtlich der Sachverhaltserstellung ist auch in Zukunft zu erwarten, dass Umsetzungsberichten anderer Institutionen - insbesondere des CPT weiterhin eine gewichtige Bedeutung zukommt. 


\section{Zwischenfazit}

Die Grosse Kammer hat sich, ausgehend vom Ananyev-Test und mit Blick auf die bislang mäandernde Rechtsprechung im Urteil Muršić/CRO, nicht nur in materieller Hinsicht, sondern auch in prüfungsmethodischer Hinsicht positioniert. Nicht immer eindeutig ist demgegenüber die dogmatische Einordnung von Überbelegungssituationen durch den Gerichtshof zur unmenschlichen oder erniedrigenden Behandlung i.S.v. Art. 3 EMRK. In der Tendenz scheint der Gerichtshof bei Überbelegungssituationen allerdings von einer erniedrigenden Behandlung i.S.v. Art. 3 EMRK auszugehen. In Zukunft wäre vom Gerichtshof mehr Bewusstsein für die Relevanz dieser Zuordnung für die zu prüfenden je spezifischen Voraussetzungen zu fordern, unterscheiden sich die unmenschliche und erniedrigende Behandlung doch nicht allein aufgrund der Intensität des Eingriffs, sondern eben auch aufgrund ihrer je anderen Einwirkungsweise auf den Einzelnen.

Der Mindeststandard wurde anhand einer von der Menschenwürde her gedachten, normativen Bewertung bei $3 \mathrm{~m}^{2}$ persönlichem Platz festgelegt. Dabei wurden auch soziomedizinische Faktoren aufgegriffen, ohne aber einen konkreten Nachweis tatsächlich erlittener Auswirkungen auf das Opfer zu fordern. Das Unterschreiten dieser Grenze führt für sich alleine jedoch noch nicht zu der Feststellung eines Verstosses gegen Art. 3 EMRK, sondern nur zur Geltung einer starken Vermutung für einen solchen. Für jene Fälle, bei welchen zwar der CPT-Standard von $4 \mathrm{~m}^{2}$ unterschritten wird, aber noch keine Unterschreitung der $3 \mathrm{~m}^{2}$ vorliegt, auferlegt sich der Gerichtshof bei der Beurteilung des konkreten Einzelfalls zumindest eine erhöhte Prüfungsdichte und -tiefe.

Beim Unterschreiten der 3- $\mathrm{m}^{2}$-Grenze hat der Vermutungseintritt eine Beweislastumkehr zur Folge. Vor dem Hintergrund der allgemeinen Regelungen zu Beweismass und Beweislast in Strafvollzugskonstellationen ist der Mehrwert der aufgestellten Vermutungsregelung in Überbelegungskonstellationen bei weniger als $3 \mathrm{~m}^{2}$ Platz für den Inhaftierten allerdings relativ gering.

Für die Widerlegbarkeit an sich setzt der Gerichtshof normalerweise voraus, dass die Unterschreitung der 3- $\mathrm{m}^{2}$-Grenze nur eine geringe, kurzzeitige und gelegentliche war. Durch die separate Berücksichtigung der einzelnen Zeitspannen setzt der Gerichtshof hier allerdings keinen hohen Standard, wobei die gesonderte Berücksichtigung einzelner Zeitspannen ohnehin nicht richtig in das Konzept der Gesamtbetrachtung passt, so dass dieses Vorgehen auch prüfungsmethodisch zu einer Inkonsistenz führt. Der Konventionsstaat kann die Vermutung sodann normalerweise nur dann 
widerlegen, wenn kompensierende Elemente aufgezeigt werden können und die Unterbringung gesamthaft angemessen war. An die kompensierenden Elemente setzt der Gerichtshof allerdings keine hohen Anforderungen, so dass zumindest in Frage steht, in wie weit diese überhaupt geeignet sind, für den Betroffenen Erleichterungswirkung herbeizuführen, welche als soziomedizinische Basis bei der normativen Bewertung zu berücksichtigen wären. In Zukunft wird mit Blick auf die Gewährung eines effektiven Menschenrechtsschutzes darauf zu achten sein, dass die Widerlegung der Vermutung von der Ausnahme nicht zur Regel wird. Alles andere würde auch dem vom Gerichtshof aufgestellten Bekenntnis, dass Platzmangel ein wichtiger Aspekt bei der Frage der Konformität mit Art. 3 EMRK ist, zuwiderlaufen.

Die prüfungsmethodische Herangehensweise der Gesamtbetrachtung der Haftumstände kann sich im Einzelfall sowohl zu Gunsten als auch zu Ungunsten des Betroffenen auswirken. Ersteres, indem kumulative Effekte mehrerer negativer Merkmale berücksichtigt werden - Letzteres durch die Möglichkeit der Berücksichtigung kompensierender Massnahmen. Während die Schutzrichtung von Art. 3 EMRK die Berücksichtigung kumulierender Elemente geradezu gebietet, ist die Berücksichtigung kompensierender Elemente nicht unproblematisch. Die faktische Wechselwirkung einzelner Kriterien im Strafvollzug (etwa Platzangebot in der Zelle und Aufschlusszeiten) ist zwar nicht in Abrede zu stellen; dennoch bleibt anzumerken, dass der Gerichtshof auf diese Weise Scheu zeigt, einen klaren anzuwendenden Mindeststandard festzusetzen. Dies ist insbesondere auch mit Blick auf den absoluten Charakter von Art. 3 EMRK zu bedauern. Der eigene Lösungsvorschlag, wonach flankierend zur Gesamtbetrachtung eine absolute numerische Untergrenze festzusetzen wäre, würde hier Abhilfe schaffen.

Soft Law-Regulatorien und die Arbeit der anderen internationalen Akteure werden vom Gerichtshof immer wieder aufgegriffen. Der Gerichtshof folgt im Rahmen seiner Rechtsprechung zu Art. 3 EMRK auf Rechtsebene allerdings weder der Tendenz des Soft Law hin zu einer Einzelunterbringung, noch folgt er - was den zur Verfügung zu stellenden Platz angeht - dem klaren CPT-Standard, wonach dem Inhaftierten mindestens $4 \mathrm{~m}^{2}$ Platz zu gewähren wären. Insbesondere vor dem Hintergrund, dass CPT und EGMR dieselben Bewertungskriterien anwenden und denselben Bewertungsgegenstand beurteilen, rechtfertigt sich ein Abweichen des EGMR vom CPT-Standard ohne weitergehende sozialwissenschaftliche Studien oder normative Begründung hinsichtlich des Mindestmasses an zu gewährendem persönlichem Platz allerdings nicht. Ein Verweis auf 
unterschiedliche Rollen und Aufgaben sind jedenfalls keine ausreichende Begründung für eine Senkung des vom CPT festgesetzten Standards um $1 \mathrm{~m}^{2}$. Ein Transformationsprozess von Soft Law zu Hard Law kann hinsichtlich dieser Fragen jedenfalls nicht festgestellt werden und ist in näherer Zukunft auch nicht zu erwarten. Anders ist die Situation hinsichtlich der Berechnungsmodalitäten sowie der Prüfungsmethodik zu beurteilen. Indem der EGMR diesbezüglich die CPT-Empfehlungen aufgreift, könnte geschlussfolgert werden, dass die CPT-Empfehlungen durch die Rechtsprechung des EGMR hier einen Härtungsprozess durchlaufen und die Normativität der CPT-Empfehlungen dadurch zunimmt.

Von grosser Bedeutung sind bei der Sachverhaltserstellung die Umsetzungsberichte des CPT, welchen in Anerkennung der Arbeit des CPT insgesamt ein grosser Beweiswert zugemessen wird.

\section{Situation in der Schweiz}

Vor dem Hintergrund der europäischen resp. internationalen Regelwerke sowie der detaillierten Rechtsprechung des EGMR stellt sich die Frage nach deren nationaler Umsetzung, sind es doch die Nationalstaaten, welche Haftstrafen vollziehen. Wie eine solche nationale Umsetzung sowohl auf legislativer als auch auf judikativer Ebene aussehen kann, wird im Folgenden anhand einer Analyse der Situation in der Schweiz aufgezeigt.

Aufgrund der europarechtlichen Empfehlungen, welche auch für die Schweiz gelten, ist im nationalen schweizerischen Recht eine klare Normierung des Mindeststandards zu erwarten, auch was den zur Verfügung zu stellenden Platz angeht (vgl. insb. Regel 18.3 EPR [2006]). Dass auf nationaler Ebene ein höherer Standard möglich ist als der Standard, welcher durch die EMRK garantiert wird, ergibt sich aus Art. 53 EMRK, in welchem das Günstigkeitsprinzip statuiert wird: Garantien der EMRK vermögen als festgelegte Mindestgarantien die in einzelnen Konventionsstaaten vorgesehenen höheren Standards in keiner Weise zu beschränken oder zu beeinträchtigen. ${ }^{661}$

661 Vgl. Karpenstein/Mayer/Thienel Art. 53 Rn. 2: Ein Anspruch auf Ausschliesslichkeit für die Gewährung von Grundrechten erhebe die EMRK nicht. Vgl. auch Meyer-Ladewig/Renger Art. 53 Rn. 1. 
1. Materieller Mindeststandard - Analyse der Gesetzeslage in der Schweiz

Aus den bundesrechtlichen Vollzugsgrundsätzen ergibt sich zwar ein Normalisierungsprinzip; konkrete Vorgaben bezüglich der Unterbringungsform und des zur Verfügung zu stellenden Platzes finden sich allerdings nicht. Auch in den interkantonalen Strafvollzugskonkordaten finden sich diesbezüglich keine spezifischen Normen, weshalb der Blick im Folgenden auf die kantonale Ebene gerichtet wird am Beispiel der Kantone Zürich und Genf. Diese Auswahl scheint deshalb besonders geeignet, weil mit dem Kanton Zürich ein Kanton herausgegriffen wird, in dem im Bereich des Strafvollzugsrechts grundsätzlich umfassend legiferiert wurde, und mit dem Kanton Genf einer, in welchem dies grundsätzlich nur rudimentär geschah. Die Strafvollzugsanstalten des Kantons Genf sind denn auch jene, welche in menschenrechtlicher Hinsicht am meisten kritisiert werden (vgl. sogleich).

Im Kanton Zürich liegt die Regelungskompetenz für den Vollzug freiheitsentziehender Sanktionen, insbesondere für die Rechte und Pflichten der Verurteilten im Anstaltsalltag, beim Regierungsrat ( $\$ 31$ lit. b StJVG $\mathrm{ZH}$ ). In der Justizvollzugsverordnung wird in $\$ 98$ Abs. $1 \mathrm{JVV} \mathrm{ZH}$ statuiert, dass verurteilte Personen i.d.R. in einer Einzelzelle resp. in einem Einzelzimmer untergebracht werden. ${ }^{662}$ Eine Unterbringung in Mehrbettzellen oder Mehrbettzimmern ist insbesondere für den Fall der Überbelegung vorgesehen. Spezifische Vorschriften zum zu gewährenden persönlichen Platz wurden in Zürich nicht erlassen.

Im Kanton Genf findet das Règlement sur le régime intérieur de la prison et le status de personnes incarcérées (RRIP) Anwendung. Auch dieses statuiert für den Normalvollzug das Prinzip der Einzelunterbringung, sofern es das Platzangebot zulässt (Art. 52 RRIP). Ausnahmen von diesem Prinzip aus logistischen Gründen sind folglich auch im Kanton Genf möglich. Spezifische Vorschriften zum zu gewährenden persönlichen Platz wurden auch im Kanton Genf nicht erlassen. ${ }^{663}$

Mit der Einzelunterbringung folgt die Schweiz dem in den Europäischen Strafvollzugsgrundsätzen von 2006 empfohlenen Grundsatz. Die im nationalen Recht vorgesehenen Abweichungsmöglichkeit von diesem

662 Dieser Grundsatz gilt v.a. für den geschlossenen Vollzug. Für die Halbgefangenschaft und den offenen Vollzug sieht $\$ 98$ Abs. 1 JVV die Möglichkeit einer Unterbringung in Mehrbettzellen/Mehrbettzimmern vor.

663 Zur genferischen Rechtslage vgl. auch die Ausführungen des Bundesgerichts in BGer 1B_335/2013, Urt. v. 26.02.2014, E. 3.1. 
Prinzip im Falle der Überbelegung entsprechen allerdings nicht mehr den Anforderungen der Europäischen Strafvollzugsgrundsätze von 2006, welche seit ihrer Revision eine Abweichung grundsätzlich nur dann vorsehen, wenn eine Unterbringung in einer Mehrbettzelle dem Strafgefangenen selbst Vorteile verschafft; logistische Gründe alleine genügen somit grundsätzlich nicht. Den Anforderungen des CPT entsprechen die Kantone Zürich und Genf mit ihren Regelungen zumindest so lange, als die Mehrbettzellen nicht als Grossraumschlafräume ausgestaltet werden. Der Forderung der Europäischen Strafvollzugsgrundsätze, dass im nationalen Recht auch hinsichtlich des zur Verfügung zu stellenden Platzes ein konkreter Massstab niedergelegt werden sollte, kommen diese beiden Kantone nicht nach.

Es bleibt damit auch auf nationaler Ebene der Konkretisierung durch die Rechtsprechung überlassen, den Mindeststandard der Platzverhältnisse aus schweizerischer Perspektive festzulegen. Im Sinne der Rechtssicherheit besteht Handlungsbedarf. Aus schweizerischer Sicht wäre damit eine gesetzliche Regel de lege ferenda wünschenswert.

\section{Rechtsprechung in der Schweiz - Analyse}

\subsection{Materieller Kern des Standards}

Die bundesgerichtliche Rechtsprechung verdeutlicht die menschenrechtliche Relevanz der Mindeststandards materieller Haftbedingungen auch für schweizerische Strafvollzugsanstalten. Insbesondere die Haftbedingungen in der grössten Genfer Haftanstalt Champ-Dollon sind vom Bundesgericht mehrfach gerügt und als Verletzung von Art. 3 EMRK befunden worden. ${ }^{664}$ Das Bundesgericht erkannte im Februar 2014 gleich in zwei Urteilen erstmals überhaupt aufgrund von Haftbedingungen in schweizerischen Vollzugsanstalten einen Verstoss gegen Art. 3 EMRK. ${ }^{665}$ Das Bun-

664 Vgl. etwa BGE 140 I 125; BGer 1B_335/2013, Urt. v. 26.02.2014; BGer 1B_239/2015, Urt. v. 29.09.2015; BGer 1B_152/2015, Urt. v. 29.09.2015; BGer 6B_456/2015, Urt. v. 21.03.2016; BGer 6B_71/2016, Urt. v. 05.04.2017. Rügen bezüglich materieller Haftbedingungen beschränkten sich jedoch nicht nur auf Genfer Haftanstalten: vgl. etwa BGer 1B_325/2017, Urt. v. 14.11.2017 (Kanton Waadt).

665 BGE 140 I 125: Der Beschwerdeführer war während 199 Tagen (davon 157 aufeinanderfolgende Tage) in einer Zelle von $23 \mathrm{~m}^{2}$ untergebracht worden. Die Zelle teilte er mit fünf weiteren Inhaftierten, so dass ihm für die entsprechende Zeitspanne ein persönlicher Platz von $3,83 \mathrm{~m}^{2}$ zur Verfügung stand. Während 
desgericht hält zur Situation der Genfer Strafvollzugsanstalt fest, dass die Überbelegung schon seit Jahren ein schweres und chronisches Ausmass (,un état grave et chronique") erreicht habe. ${ }^{666}$

Hinsichtlich der Platzverhältnisse hält das Bundesgericht konkret fest, dass eine Unterbringung mit $4 \mathrm{~m}^{2}$ persönlichem Platz (inkl. Platz für die Einrichtungsgegenstände) zwar eine beschwerliche Unterbringung sein kann, aber noch nicht automatisch eine Verletzung von Art. 3 EMRK bedeute. ${ }^{667}$ Anders sei die Situation, wenn dem Inhaftierten während längerer Zeit lediglich $3,83 \mathrm{~m}^{2}$ persönlicher Platz zur Verfügung stehen und weitere erschwerende Umstände hinzukommen würden. ${ }^{668}$ Eine beinahe dreimonatige Unterbringung (89 Tage) erachtete das Bundesgericht als lange Dauer und eine tägliche Einschlusszeit von 23 Stunden in der Zelle als erschwerenden Umstand. ${ }^{669}$ Auch eine Unterbringung von drei Personen in einer 10- $\mathrm{m}^{2}$ Zelle (persönlicher Platz damit: $3,3 \mathrm{~m}^{2}$ ) während 328 Tagen mit nur einer täglich einstündigen Möglichkeit für Bewegung an der frischen Luft wurde als Verstoss gegen Art. 3 EMRK befunden. ${ }^{670}$ Dies bisweilen unabhängig davon, ob der Betroffene eine Arbeitsmöglichkeit, welche ihm mehr Zeit ausserhalb der Zelle ermöglicht hätte, ablehnte. ${ }^{671}$

weiterer 27 Tage war er in einer 12- $\mathrm{m}^{2}$-Zelle untergebracht. Diese Zelle, welche der Beschwerdeführer mit zwei weiteren Inhaftierten teilte, verfügte dabei über zwei Betten sowie über eine am Boden liegende Matratze. Welcher der Inhaftierten die Matratze nutzte, wurde nicht dokumentiert. BGer 1B_335/2013, Urt. v. 26.02.2014: Der Beschwerdeführer verbrachte unter denselben Platzbedingungen wie im Urteil BGE 140 I 12589 Tage. Die Beschwerdeführer verbrachten dabei 23 Stunden pro Tag in der Zelle und hatten während einer Stunde täglich die Möglichkeit, sich im Freien zu bewegen.

666 BGE 140 I 125, E. 3.6.3; BGer 1B_335/2013, Urt. v. 26.02.2014, E. 3.6.3.

667 BGE 140 I 125, E. 3.6.3; BGer 1B_335/2013, Urt. v. 26.02.2014, E. 3.6.3; BGer 1B_152/2015, Urt. v. 29.09.2015, E. 2.4.

668 BGE 140 I 125, E. 3.6.3; BGer 1B_335/2013, Urt. v. 26.02.2014, E. 3.6.3: Im vorliegenden Fall ging es um präventive Haft, wobei das Bundesgericht hierzu ausdrücklich festhielt, dass präventive Haft aufgrund der Flucht- oder Kollisionsgefahr sowie aufgrund von Sicherheitsbedenken grundsätzlich restriktivere Haftbedingungen erlaube. Bei einer längeren Unterbringung in präventiver Haft (über drei Monate) sei von diesen an sich zulässigen Restriktionen aber abzusehen. Aufgrund der langen Zeitspanne der präventiven Haft im vorliegenden Fall wurde die Unterbringung demzufolge am Standard des Normalvollzugs gemessen.

669 BGer 1B_335/2013, Urt. v. 26.02.2014, E. 3.6.3; BGE 140 I 125, E. 3.6.3.

670 BGer 1B_239/2015, Urt. v. 29.09.2015, E. 2.5.2 f.

671 BGer 1B_239/2015, Urt. v. 29.09.2015, E. 2.5.2 f.: Der Beschuldigte hätte vorliegend jede zweite Woche die Möglichkeit gehabt, täglich eine bis 53/4 Stunden zu arbeiten. 
Eine Arbeitsmöglichkeit könne zwar zu einer Erleichterung der Bedingungen führen; im vorliegenden Fall hätte diese aber nicht ausgereicht, um wieder einen menschenrechtskonformen Zustand herzustellen. ${ }^{672}$ In einer am Boden liegenden Matratze als Schlafmöglichkeit erkennt das Bundesgericht nicht zwingend einen Verstoss gegen Art. 3 EMRK, insbesondere dann nicht, wenn seitens des Beschwerdeführers nicht auch vorgebracht wird, dass die Bettwäsche nicht regelmässig gewechselt würde. ${ }^{673}$ Hinsichtlich der Berechnungsmodalitäten hält das Bundesgericht konstant fest, dass der sanitäre Bereich nicht dem persönlich zur Verfügung stehenden Platz anzurechnen ist. ${ }^{674}$

Das Bundesgericht gewährt in Bezug auf den konkret zur Verfügung zu stellenden Platz mit seiner 4- $\mathrm{m}^{2}$-Marke in materieller Hinsicht einen höheren Standard als der EGMR, welcher zumindest seit dem Urteil Muršić/CRO die 3-m²-Grenze für massgebend erachtet. Dies ist vor dem Hintergrund zu sehen, dass sich die Rechtsprechungslinie des Bundesgerichts zu einer Zeit entwickelte, als der Europäische Gerichtshof sich noch nicht festgelegt hatte und mal $3 \mathrm{~m}^{2}$, mal $4 \mathrm{~m}^{2}$ als massgebend bezeichnete. Post-Muršić greift das Bundesgericht diesen Entscheid auf, folgt ihm hinsichtlich der 3- $\mathrm{m}^{2}$-Grenze bisher aber nicht. ${ }^{675} \mathrm{Im}$ Sinne

672 BGer 1B_239/2015, Urt. v. 29.09.2015, E. 2.5.3. Selbst wenn die angebotene Arbeitstätigkeit ausgereicht hätte, um wieder zu einer mit Art. 3 EMRK konformen Situation zu führen, dürfte diese nicht in hypothetischer Weise angerechnet werden, wenn der Inhaftierte sie zuvor abgelehnt und nicht ausgeführt hat. Dies würde im Ergebnis nämlich zu einem Verzicht auf die Garantien von Art. 3 EMRK führen, was unzulässig ist. Zur Frage des Menschenrechtsverzichts ausf.: SK-StPO/MeYER Methodik der Grundrechtsanwendung (EMRK) Rn. 59 ff. In Abwesenheit anderer Kompensationsmöglichkeiten könnte dies eine Arbeitspflicht des Beschuldigten zur Folge haben. Hinsichtlich einer Arbeitspflicht stellen sich dann Fragen im Zusammenhang mit Art. 4 EMRK, wobei der Gerichtshof festgehalten hat, dass eine Arbeitsverpflichtung im Strafvollzug selbst über das Rentenalter hinaus - zulässig ist: vgl. EGMR - Meier/SUI, Urt. v. 09.02.2016, 10109/14, Rn. 31.

673 BGer 6B_1244/2016, Urt. v. 31.10.2017, E. 2.3, 2.6; BGE 140 I 125, E. 3.6.4.

674 BGer 6B_456/2015, Urt. v. 21.03.2016, E. 2.4.2.

675 BGer 1B_325/2017, Urt. v. 14.11.2017, E. 3.3: „Depuis lors, le Tribunal fédéral - s'inspirant également de la jurisprudence de la Cour européenne des droits de l'homme $[\ldots]-$ s'en est tenu au critère de la surface individuelle inférieure à $4 \mathrm{~m}^{2}$ [...]. Dans un arrêt de principe Muršić contre Croatie du 20 octobre 2016 [...], la Grande Chambre de la Cour européenne des droits de l'homme s'est cependant écartée de cet ordre de grandeur de $4 \mathrm{~m}^{2}$, déduit des normes établies par le CPT: désormais, la Cour retient qu'une surface de $3 \mathrm{~m}^{2}$ au sol par détenu constitue la norme minimale pertinente." Dabei verweist das Bundesgericht auf 
eines effektiven Menschenrechtsschutzes und vor dem Hintergrund der am EGMR-Massstab angebrachten Kritik ist dies zu begrüssen. Es ist zu hoffen, dass das Bundesgericht auch in Zukunft seinen Schutzstandard nicht mit Blick auf die Strassburger Rechtsprechung von $4 \mathrm{~m}^{2}$ auf $3 \mathrm{~m}^{2}$ senken wird. Das Genügenlassen einer auf dem Boden liegenden Matratze als individueller Schlafplatz ist in Zukunft weiter zu beobachten. ${ }^{676} \mathrm{Nach}$ dem heutigen Stand würde der EGMR hier im Rahmen von Art. 3 EMRK wohl aber auch nicht weiter gehen und darin auch keinen Verstoss gegen Art. 3 EMRK erkennen, weshalb die Schweiz hier momentan wohl keine Verurteilungen durch den Gerichtshof zu befürchten hätte.

\subsection{Prüfungsmethodik des Schweizerischen Bundesgerichts und Vergleich mit dem EGMR}

Wie schon der EGMR nimmt das Bundesgericht die Quadratmeterzahl des zur Verfügung stehenden persönlichen Platzes zwar als Ausgangspunkt der Prüfung von Art. 3 EMRK, stellt diese dann aber mit der Berücksichtigung sowohl aggravierender ${ }^{677}$ als auch kompensierender ${ }^{678}$ Elemente in einen Gesamtkontext.

Hinsichtlich der kompensierenden Elemente führt das Bundesgericht aus, dass insbesondere längere Aufschlusszeiten einen Erleichterungseffekt für den Inhaftierten hätten. ${ }^{679}$ Eine genügende Kompensation, um trotz

den kritischen Beitrag zu diesem Entscheid: Tulkens RTDH 2017, 989 ff.; vgl. auch BGer 6B_1244/2016, Urt. v. 31.10.2017, E. 2.5.

676 Ein Bett, wie es von den Europäischen Strafvollzugssätzen vorgesehen ist, ist nicht nur aufgrund des subjektiven Gefühls, welche es einem Strafgefangenen vermittelt, zu bevorzugen. Es hat auch den Vorteil, dass der Platz unter dem Bett als Stauraum genutzt werden kann und das Bett gleichzeitig eine bequeme Sitzgelegenheit bietet. Dies ist umso wichtiger, als der Platz für Einrichtungsgegenstände vom zur Verfügung zu stellenden Platzes nicht abgezogen wird. Umgekehrt kann die Matratze leichter aufgestellt werden, womit auch wieder Platz geschaffen werden kann, um sich frei zu bewegen.

677 BGer 1B_335/2013, Urt. v. 26.02.2014, E. 3.6.3; BGer1B_325/2017, Urt. v. 14.11.2017, E. 3.5, BGer 6B_1244/2016, Urt. v. 31.10.2017.

678 BGer 1B_152/2015, Urt. v. 29.09.2015, E. 2.5.3: „La possibilité de sortir de la cellule, entre $1 \mathrm{~h} 00$ par jour et $5 \mathrm{~h} 45$ par jour une semaine sur deux, est certes susceptible d'alléger les conditions de détention." Im Folgenden wurde im konkreten Fall aber festgehalten, dass diese Erleichterung hier noch nicht ausreiche, um wieder einen mit Art. 3 EMRK konformen Zustand herzustellen.

679 BGer 1B_394/2016, Urt. v. 25.04.2017, E. 2.2.1. 
prekärer Platzverhältnisse von menschenrechtskonformen Haftbedingungen auszugehen, nimmt das Bundesgericht nicht leichthin an: Die Möglichkeit, sich 31/2 Stunden oder 51/2 Stunden ausserhalb der Zelle aufzuhalten, genügt nicht unbedingt. ${ }^{680}$ Kommen aber weitere erleichternde Faktoren hinzu, geht auch das Bundesgericht von einer genügenden Kompensation aus, wenn nicht gleichzeitig zu den prekären Platzbedingungen weitere erschwerende Bedingungen vorgebracht werden. ${ }^{681}$ Bei den Aufschlusszeiten dürften neben den Aufschlusszeiten des Inhaftierten auch jene der Mitinhaftierten berücksichtigt werden. ${ }^{682}$ Während der Zeit, welche die Mitinhaftierten ausserhalb der Zelle verbringen würden, stünde nämlich den in der Zelle verbleibenden Inhaftierten mehr persönlicher Platz zur Verfügung. Die kurze Dauer dieser Erleichterung führe allerdings dazu, dass Aufschlusszeiten der Mitinhaftierten in der Regel für eine Annahme einer kompensierenden Wirkung nicht ausreichen. ${ }^{63}$

Wie schon für den EGMR ist auch für das Bundesgericht die Dauer der Unterbringung eines der entscheidenden Kriterien. ${ }^{684}$ Bei einer Zeitdauer von rund drei Monaten sei von einer langen Dauer auszugehen. ${ }^{685}$ Die drei Monatsgrenze gelte aber nicht absolut, sie sei vielmehr als Indikation bei der Betrachtung der gesamten Haftumstände zu berücksichtigen. ${ }^{66}$ Dies bedeutet, dass vom Bundesgericht auch längere Zeitspannen mit weniger als $4 \mathrm{~m}^{2}$ als konform mit Art. 3 EMRK qualifiziert werden können, im Einzelfall ohne weitere erschwerende Elemente, aber auch ohne wirklich kompensierende Elemente. ${ }^{67}$ Bei der Berechnung der relevanten Dauer berücksichtigt das Bundesgericht grundsätzlich zusammenhängende Zeitspannen, in denen der Inhaftierte der fraglichen Situation ausgesetzt ist. Kleinere Unterbrüche, in welchen dem Inhaftierten vorübergehend bessere Haftbedingungen gewährt werden, führen allerdings nicht dazu, dass die Zeitspannen mit ungenügenden Bedingungen nicht zusammenzurech-

680 BGer 1B_84/2016, Urt. v. 27.07.2016, E. 2.4; vgl. aber auch BGer 6B_1085/2016, Urt. v. 28.08.2017, E. 3.2: Das Bundesgericht sah hier in einem durchschnittlich fünfstündigen täglichen Aufschluss der Zelle eine genügende Kompensation für die Unterschreitung der 4- $\mathrm{m}^{2}$-Grenze während 201 Tagen.

681 BGer 1B 394/2016, Urt. v. 25.04.2017, E. 2.4

682 BGer 1B_394/2016, Urt. v. 25.04.2017, E. 2.4.

683 Vgl. bspsw. BGer 1B_325/2017, Urt. v. 14.11.2017, E. 3.5.

684 Vgl. etwa BGer 1B_335/2013, Urt. v. 26.02.2014, E. 3.6.3.

685 BGE 140 I 125, E. 3.6.3.

686 BGE 140 I 125, E. 3.6.3.

687 BGer 6B_1314/2015, Urt. v. 10.10.2016, E. 4.2: 3,39 $\mathrm{m}^{2}$ persönlicher Platz während 98 Tagen wurden als mit Art. 3 EMRK konform befunden. 
nen sind ${ }^{688}$ Auch hinsichtlich der Unterbrechungsdauer setzt das Bundesgericht keine fixe Grenze, wann die Zeitspannen nicht mehr zusammenzurechnen, sondern separat zu betrachten wären. Im Einzelfall hatte das Bundesgericht auch bei einer Unterbrechung von 45 Tagen nicht von einer Addition der Zeitspannen abgesehen; in der Folge wurde auf eine „lange Dauer“ erkannt. ${ }^{689}$

Das Bundesgericht geht in prüfungsmethodischer Hinsicht ähnlich vor wie der EGMR, indem es von einer festgelegten Quadratmeterzahl ausgehend kumulierende und kompensierende Elemente würdigt. Die Grundrechtsprüfung des Bundesgerichts scheint allerdings insofern weniger formalisiert zu sein als jene des EGMR, als nicht mit dem Eintritt einer starken Vermutung, welche es zu widerlegen gilt, argumentiert, sondern einfach eine Gesamtbetrachtung vorgenommen wird. Die beweisrechtlichen Wirkungen des Vermutungseintritts sind ohnehin gering, weshalb die beiden Herangehensweisen im Kern dieselben sind. Auch die berücksichtigten Kompensationsfaktoren sind die gleichen, wenngleich die Anforderungen an eine kompensierende Wirkung vom Bundesgericht höher angesetzt werden. Auch das Element der Dauer wird sowohl vom EGMR als auch vom Bundesgericht als zentrales Kriterium betrachtet. Beiden gemein ist, dass eine Zeitspanne ab etwa drei Monaten als lange angesehen wird, wobei der EGMR bei Unterbrüchen die beiden Zeitspannen separat betrachtet, während das Bundesgericht bei kurzen Unterbrüchen die Zeitspannen addiert. Das Berechnungsmodell des Bundesgerichts fügt sich jedenfalls besser in das angewandte Gesamtbetrachtungsmodell ein.

Das nationale Schutzniveau ist damit nicht nur aufgrund eines höheren Massstabes hinsichtlich der für entscheidend befundenen Quadratmeterzahl, sondern auch aufgrund der höheren Anforderungen an kompensierende Wirkungen und der Addition von unterschiedlichen Zeitspannen bei kurzen Unterbrüchen höher als dasjenige des EGMR. Unter dem Gesichtspunkt eines effektiven Grundrechtsschutzes ist dies zu begrüssen. Dennoch ist auch an das Bundesgericht zu appellieren, klarere, absolut

688 BGer 1B 152/2015, Urt. v. 29.09.2015, E. 2.5.1 f.: Dem Beschwerdeführer wurde zwischen zwei Zeitspannen mit ungenügenden Haftbedingungen während 14 Tagen, 5-18 $\mathrm{m}^{2}$ persönlicher Platz gewährt. Die Zeitdauer der ungenügenden Haftbedingungen wurde gleichwohl addiert.

689 BGer 1B_325/2017, Urt. v. 14.11.2017, E. 3.5: Die einzelnen Zeitspannen von 98, 250 und 300 Tagen hätten im konkreten Fall aber auch bei einer separaten Würdigung jeweils für sich genommen die Voraussetzungen einer „langen Dauer" erfüllt. 
geltende Anforderungen zu stellen, um so mit Blick auf den absoluten Charakter von Art. 3 EMRK noch mehr Rechtssicherheit zu schaffen.

2.3 Dogmatische Einordnung der Überbelegung durch das Bundesgericht und Kriterien der Standardbildung

Bereits in den ersten beiden Urteilen des Bundesgerichts zu inadäquaten Platzverhältnissen in der Strafvollzugsanstalt Champ-Dollon qualifizierte es die Unterbringung der Beschwerdeführer ausdrücklich als eine erniedrigende Behandlung i.S.v. Art. 3 EMRK. ${ }^{690}$ Eine solche Inhaftierung führe nämlich dazu, dass der Inhaftierte eine Härte erleiden müsse, welche über das hinausgehe, was dem Freiheitsentzug von vorneherein inhärent sei. Damit würden die Haftbedingungen nicht den Anforderungen an die Achtung der Menschenwürde und der Privatsphäre entsprechen. ${ }^{691}$ Auch in den Folgeurteilen griff das Bundesgericht diese Ausführungen auf und prüfte die Haftbedingungen an den Voraussetzungen einer erniedrigenden Behandlung i.S.v. Art. 3 EMRK, ${ }^{692}$ woran auch der Umstand nichts zu ändern vermag, dass das Bundesgericht im Ergebnis zusammenfassend in der Regel nur noch festhielt, dass die Haftbedingungen (nicht) dem Mindeststandard entsprachen, ohne die Erniedrigung hier noch einmal zu nennen. ${ }^{693}$

Greift das Bundesgericht das „no more harm principle“ auf und führt dabei aus, dass ein Verstoss gegen Art. 3 EMRK nur dann vorliege, wenn die materiellen Haftbedingungen ein höheres Mass an Demütigung oder Erniedrigung verursachen würden als mit dem Freiheitsentzug üblicherweise verbunden sei, ist dies wiederum als blosse Auslegungsdirektive zu verstehen. ${ }^{694} \mathrm{Im}$ Kern prüft das Bundesgericht nämlich auch hier nicht etwa,

690 BGer 1B_335/2013, Urt. v. 26.02.2014, E. 3.6.3; BGE 140 I 125, E. 3.6.3.

691 BGer 1B_335/2013, Urt. v. 26.02.2014, E. 3.6.3; BGE 140 I 125, E. 3.6.3.

692 BGer 1B_239/2015, Urt. v. 29.09.2015, E. 2.4; BGer 1B_152/2015, Urt. v. 29.09.2015, E. 2.4; BGer 1B_394/2016, Urt. v. 25.04.2017, E. 2.2.1; BGer 6B_1244/2016, Urt. v. 31.10.2017, E. 2.1; BGer 1B_325/2017, Urt. v. 14.11.2017, E. 3.3 (Im Unterschied zum Bundesgericht hatte die Vorinstanz offenbar mit dem Begriff der unmenschlichen Behandlung operiert.).

693 BGer 1B_239/2015, Urt. v. 29.09.2015, E. 2.6; BGer 1B_152/2015, Urt. v. 29.09.2015, E. 2.6; BGer 1B_325/2017, Urt. v. 14.11.2017, E. 3.6; BGer 1B_394/2016, Urt. v. 25.04.2017, E. 2.5 (keine Verletzung von Art. 3 EMRK).

694 BGer 1B_335/2013, Urt. v. 26.02.2014, E. 3.5; BGE 140 I 125, E. 3.5; vgl. auch BGE 141 I 141, E. 6.3.4. 
ob bessere Haftbedingungen denkbar wären. Das Bundesgericht setzt seinen Massstab für das Vorliegen einer erniedrigenden Behandlung i.S.v. Art. 3 EMRK vielmehr von der Menschenwürde her gedacht anhand einer normativ wertenden Beurteilung fest, welche soziomedizinische Schlussfolgerungen ${ }^{695}$ aufgreift. Ein Nachweis tatsächlicher Auswirkungen auf den Beschwerdeführer wird der normativen Wertung entsprechend nicht gefordert.

Unklarer ist die Einordnung des Bundesgerichts hingegen, wenn es um die Beurteilung geht, ob eine am Boden liegende Matratze als individueller Schlafplatz genügen soll, wenn die Bettwäsche regelmässig gewechselt wird. ${ }^{696}$ So hielt das Bundesgericht in seinem Leitentscheid fest, dass dies nicht zwingend eine unmenschliche Behandlung i.S.v. Art. 3 EMRK sei, ohne dabei aber näher auf deren spezifische Begriffsmerkmale einzugehen. ${ }^{697}$ In der Folgerechtsprechung führte das Bundesgericht mit Verweis auf seinen Leitentscheid aus, dass das Schlafen auf einer am Boden liegenden Matratze nicht die ausreichende Schwere erreiche, um eine unmenschliche oder erniedrigende Behandlung im Sinne von Art. 3 EMRK zu sein. ${ }^{698}$

Insbesondere vor dem Hintergrund der ausgemachten dogmatischen Unschärfe des EGMR bei der Frage, ob er inadäquate Platzverhältnisse nun an den Kriterien einer unmenschlichen oder einer erniedrigenden Behandlung i.S.v. Art. 3 EMRK prüfte, sticht die diesbezügliche klare Positionierung des Bundesgerichts positiv heraus. Irritierend sind demgegenüber diesbezügliche Unklarheiten bei der Beurteilung des Schlafplatzes, zumal das Bundesgericht das Heranziehen (auch) der unmenschlichen Behandlung nicht weiter begründet und auch auf deren Voraussetzungen nicht eingeht. Auch vor dem Hintergrund, dass das Bundesgericht eine Gesamtbetrachtung vornimmt, erscheint es unstimmig, für diesen Einzelaspekt auf die Kriterien einer unmenschlichen Behandlung abstellen zu wollen. Im Ergebnis scheint die Schlafsituation nämlich vielmehr ein Aspekt der bundesgerichtlichen Prüfung einer erniedrigenden Behandlung im Gesamten zu sein.

695 Das Aufgreifen soziomedizinischer Schlussfolgerungen zeigt sich besonders deutlich, wenn das Bundesgericht beim Heranziehen kompensierender Elemente auf Erleichterungs- resp. Erholungseffekte abstellt.

696 BGer 6B_1244/2016, Urt. v. 31.10.2017, E. 2.3, 2.6; BGE 140 I 125, E. 3.6.4.

697 BGE 140 I 125, E. 3.6.4.

698 BGer 6B_1244/2016, Urt. v. 31.10.2017, E. 2.3, 2.6. 
3. Bundesgerichtliche Rechtsprechung und das Soft Law

\subsection{Rechtsebene}

Das Bundesgericht rezipiert in seiner Rechtsprechung, wenn es international rechtsverbindliche Normen oder national geltendes Vollzugsrecht anwendet, immer wieder Soft Law-Bestimmungen. ${ }^{699}$ In der bundesgerichtlichen Rechtsprechung finden die Europäischen Strafvollzugsgrundsätze (2006) und die Empfehlungen des CPT besondere Beachtung. ${ }^{700} \mathrm{Zu}$ deren Rechtsnatur führt das Bundesgericht aus, dass diese Spiegelbild der gemeinsamen Rechtstradition der Mitgliedstaaten des Europarates seien, weshalb sie bei der Verwirklichung der persönlichen Freiheit und anderer durch die Verfassung und die EMRK garantierter Grundrechte berücksichtigt würden, wenngleich sie den Charakter von blossen Leitlinien hätten. ${ }^{701}$ Die Bestimmungen des Soft Law, welche für die Behörden gleichwohl „relativ verbindlich“ seien („relativement contraignante pour les autorités"), hätten im Gegensatz zu den einschlägigen Bundes- und Kantonsgesetzen den Vorteil, Präzisierungen zu enthalten, etwa zu dem jedem Inhaftierten zur Verfügung zu stellenden Platz. ${ }^{702}$

Hinsichtlich des notwendigerweise zur Verfügung zu stellenden persönlichen Platzes setzt das Bundesgericht seinen Mindeststandard in Übereinstimmung mit den Empfehlungen des CPT bei $4 \mathrm{~m}^{2}$ fest. Das Bundesgericht greift dazu zunächst die Empfehlungen des CPT auf und führt die von diesem vorgeschlagenen Mindeststandards für Einzelzellen sowie für Mehrbettzellen im Einzelnen aus, wobei es auch auf die vom CPT vorgenommene Gesamtbetrachtung eingeht. ${ }^{703}$ Mögliche unterschiedliche Aufgaben und Funktion von Bundesgericht und CPT werden in der bundesgerichtlichen Rechtsprechung nicht thematisiert, so dass - im Un-

699 Vgl. etwa BGer 1B_152/2015, Urt. v. 29.09.2015, E. 2.2.; BGer 1B_335/2013, Urt. v. 26.02.2014, E. 3.2; BGer 6B_456/2015, Urt. v. 21.03.2016.

700 Vgl. etwa BGer 1B_152/2015, Urt. v. 29.09.2015, E. 2.2; BGer 1B_335/2013, Urt. v. 26.02.2014, E. 3.2; BGer 6B_456/2015, Urt. v. 21.03.2016.

701 BGE 140 I 125, E. 3.2; BGer 1B_335/2013, Urt. v. 26.02.2014, E. 3.2; BGer 1B 152/2015, Urt. v. 29.09.2015, E. 2.2; BGer 1B_239/2015, Urt. v. 29.09.2015, E. 2.2.

702 BGE 140 I 125, E. 3.2; BGer 1B_335/2013, Urt. v. 26.02.2014, E. 3.2; BGer 1B_152/2015, Urt. v. 29.09.2015, E. 2.2; BGer 1B_239/2015, Urt. v. 29.09.2015, E. 2.2.

703 BGE 140 I 125, E. 3.2; BGer 1B_335/2013, Urt. v. 26.02.2014, E. 3.2; BGer 1B_152/2015, Urt. v. 29.09.2015, E. 2.2; BGer 1B_239/2015, Urt. v. 29.09.2015, E. 2.2. 
terschied zum EGMR - auch kein Anlass gesehen wird, mit Blick auf eine erniedrigende Behandlung i.S.v. Art. 3 EMRK eine andere normative Bewertung der erforderlichen minimalen Platzverhältnisse vorzunehmen. Ein Transformationsprozess der CPT-Empfehlungen von Soft Law zu Hard Law setzt ein. Das Soft Law erlangt praktische Wirksamkeit.

Da der Auslegungskanon der EMRK die Berücksichtigung von Soft Law auch bei absoluten Rechten zulässt und das Bundesgericht für die einzelnen Soft Law-Normen sorgfältig zu prüfen scheint, ob nach seinem eigenen Verständnis die Soft Law-Forderung vom menschenrechtlichen Gehalt von Art. 3 EMRK tatsächlich umfasst wird, ${ }^{704}$ ist diesem Vorgehen nichts entgegenzuhalten. Vielmehr ist es positiv zu werten, dass sich das Bundesgericht hinsichtlich des minimal zu gewährleistenden Platzes an den Empfehlungen des CPT orientiert und diesen in der Praxis zum Durchbruch verhilft.

Selbst wenn das Bundesgericht auch die überragende Bedeutung der Europäischen Strafvollzugsgrundsätze bei der Konkretisierung der verfassungsmässigen Garantien anerkennt, ${ }^{705}$ weicht es bisweilen doch ausdrücklich von diesen ab. ${ }^{706}$ Begründet wird dies mit dem reinen Empfehlungscharakter des Soft Law und damit, dass ein Abweichen von den Empfehlungen nicht zwingend eine Verletzung von Art. 3 EMRK begründe. ${ }^{707}$ In Anbetracht des Erfordernisses der Mindestschwere der unmenschlichen bzw. erniedrigenden Behandlung unter dem Blickwinkel von Art. 3 EMRK ist dem wohl auch zuzustimmen, wenngleich eine Umsetzung der Empfehlungen im Allgemeinen wünschenswert bleibt.

704 Dies zeigt sich etwa darin, dass das Bundesgericht einzelne Forderungen der Europäischen Strafvollzugsgrundsätze nicht von Art. 3 EMRK umfasst sieht, bei der Quadratmeterzahl des CPT dann aber zu einem anderen Schluss gelangt.

705 Vgl. etwa BGE 140 I 125, E. 3.2; BGer 1B_152/2015, Urt. v. 29.09.2015, E. 2.2.

706 BGE 140 I 125, E. 3.6.2. Die Europäischen Strafvollzugsgrundsätze empfehlen einen Schlafplatz bestehend aus einem separaten Bett mit Bettwäsche, welche genügend oft gewechselt werden muss. Der erläuternde Bericht zu den Europäischen Strafvollzugsgrundsätzen hält sodann fest, dass unter einem Bett ein Bettrahmen mit Matratze verstanden wird. Das Bundesgericht hat im Rahmen von Art. 3 EMRK demgegenüber eine am Boden liegende Matratze als mit Art. 3 EMRK konform befunden.

707 In konstanter Rechtsprechung vgl. etwa BGE 140 I 125, E. 3.6.2. 


\subsection{Sachverhaltsebene}

Auch vor Bundesgericht können Umsetzungsberichte internationaler und nationaler Gremien Beachtung finden. ${ }^{708}$ Im Vergleich zu deren Bedeutung bei der Sachverhaltserstellung vor dem EGMR kommt ihnen vor dem Bundesgericht allerdings weit weniger Bedeutung zu. Die unterschiedliche Relevanz dieser Berichte dürfte darauf zurückzuführen sein, dass das Bundesgericht seinem Urteil den Sachverhalt zugrunde legt, den die Vorinstanz festgestellt hat (Art. 105 Abs. 1 BGG). Gemäss Art. 105 Abs. 2 BGG kann das Bundesgericht die Sachverhaltsfeststellung der Vorinstanz von Amtes wegen berichtigen oder ergänzen, wenn sie offensichtlich unrichtig ist oder auf einer Rechtsverletzung i.S.v. Art. 95 BGG beruht. Der Beschwerdeführer kann die Feststellung des Sachverhalts gemäss Art. 97 Abs. 1 BGG vor Bundesgericht sodann nur rügen, wenn sie offensichtlich unrichtig ist oder auf einer Rechtsverletzung im Sinne von Art. 95 BGG beruht und wenn die Behebung des Mangels für den Ausgang des Verfahrens entscheidend sein kann. Die Sachverhaltserstellung als solche ist damit vor dem Bundesgericht weit weniger oft relevant als vor dem EGMR.

\section{Zwischenfazit}

Hinsichtlich der Unterbringungsform ist auf kantonaler Ebene sowohl in Zürich als auch in Genf der Grundsatz der Einzelunterbringung festgehalten. Logistische Gründe dürfen allerdings zu Abweichungen von diesem Grundsatz führen. Hinsichtlich des konkret zur Verfügung zu stellenden Platzes finden sich weder auf Bundes- noch auf kantonaler Ebene generell-abstrakte Regelungen. Die Analyse der bundesgerichtlichen Rechtsprechung lässt aber erkennen, dass das Schutzniveau sowohl im materiellen Kern selbst als auch bedingt durch die Prüfungsmethodik höher ist als beim EGMR. Prüfungsmethodisch führen die strengeren Anforderungen an eine Kompensation einerseits und die Zusammenrechnung der einzelnen ungenügenden Zeitspannen beim Element der langen Dauer andererseits dazu, dass das Unterschreiten der Mindestgrenze an persönlichem Platz nicht leichthin kompensiert werden kann und dass deshalb früher von einer erniedrigenden Behandlung i.S.v. Art. 3 EMRK ausgegangen wird

708 Vgl. etwa BGer 1B_335/2013, Urt. v. 26.02.2014, E. 3.6.1: Das Bundesgericht setzt sich mit einem Monitoringbericht der Nationalen Kommission zur Verhütung von Folter auseinander. 
als vor dem EGMR. Dennoch sieht auch das Bundesgericht viele Relativierungsmöglichkeiten vor; hier ist ein klarer Standard für die Zukunft wünschenswert. Auch das Bundesgericht berücksichtigt Soft Law, wobei es dieses genau auf dessen menschenrechtlichen Gehalt prüft. Nur dort, wo das Bundesgericht eine Relevanz der Soft Law-Bestimmung für Art. 3 EMRK erkennt, greift es bei der Schutzbereichsbestimmung auf diese zurück. Dieses Vorgehen ist zu begrüssen, entspricht das Bundesgericht mit der Berücksichtigung des europäischen Konsenses doch dem Auslegungskanon der EMRK. Eine klare Ursache für den im Vergleich zum EGMR höheren Standard lässt sich nicht abschliessend bestimmen. Mitursächlich dürfte jedenfalls sein, dass das Bundesgericht dem Soft Law, insbesondere den Empfehlungen des CPT, eine grössere Bedeutung beimisst als der EGMR. Letztlich ist der höhere Standard beim Bundesgericht Ergebnis der normativen Bewertung des konkret erforderlichen Mindestmasses an Schwere. Dies mit Blick auf die Voraussetzungen einer erniedrigenden Behandlung i.S.v. Art. 3 EMRK.

\section{Kapitel 3: Gesundheitsversorgung im Strafvollzug}

Art. 3 EMRK geht hinsichtlich der Gesundheitsversorgung einerseits inhaltlich weiter als der reine Lebensschutz ${ }^{709}$, garantiert andererseits aber kein allgemeines Recht auf Gesundheit ${ }^{710}$. Es stellt sich deshalb die Frage, welchen Standard der Staat jedem einzelnen Strafgefangenen aus der positivrechtlichen Dimension und insbesondere aus dem leistungsrechtlichen Gehalt des Art. 3 EMRK aufgrund des besonderen Näheverhältnisses und der Fürsorgepflicht in medizinischen Belangen zu gewährleisten hat. Untersucht werden sowohl die Anforderungen an die Phase des Hafteintrittes hinsichtlich der Dokumentation des Gesundheitszustandes, die Anforderungen an die Gesundheitsversorgung während der ganzen Dauer des Strafvollzugs sowie allfällige bezüglich Art. 3 EMRK relevante Pflichten im Hinblick auf die Phase der Haftentlassung.

Ist das erforderliche Mindestmass an Schwere für eine Verletzung von Art. 3 EMRK nicht erreicht, bliebe jedenfalls eine Prüfung von Art. 8 EMRK denkbar, worauf im Folgenden aber nicht näher eingegangen

709 SCHÄFER S. 52.

710 Lines International Journal of Prisoner Health 2006, 269 (276); EGMR Rohde/DEN, Urt. v. 21.07.2005, 69332/01; EGMR (GK) - Kudta/POL, Urt. v. 26.10.2000, 30210/96; EGMR - Melnik/UKR, Urt. v. 28.03.2006, 72286/01. 
wird. ${ }^{711}$ Nicht behandelt wird sodann die Frage, wann besonders bedenkliche andere materielle Haftbedingungen, die zu einer gesundheitlichen Beeinträchtigung führen, eine Verletzung von Art. 3 EMRK bilden. Ferner wird hier nicht der Frage nachgegangen, ob und unter welchen Voraussetzungen der Gesundheitszustand eines Inhaftierten im Rechtshilfeverkehr unter Art. 3 EMRK-Gesichtspunkten von Relevanz ist.

Die Gesundheitsversorgung ist folglich als eigenständige Fallgruppe materieller Haftbedingungen zu betrachten und für sich alleine an Art. 3 EMRK zu messen. Wechselwirkung zwischen der Gesundheitsversorgung im Strafvollzug und weiteren Haftbedingungen werden aber nicht von der Hand gewiesen. ${ }^{712}$

\section{Gesundheitsversorgung im Strafvollzug - Einführung, Definition und Auswirkungen}

Die Begriffe Gesundheitsversorgung und medizinische Versorgung werden in diesem Kapitel als Synonyme verwendet und weit ausgelegt. Sie umfassen nicht nur die rein ärztliche Versorgung im Krankheitsfall, sondern sollen zum einen auch den Bereich der Prävention umfassen und zum anderen die Versorgung durch weitere medizinische Leistungsträger einschliessen. So sind auch weitere Massnahmen, welche auf den Gesundheitszustand des einzelnen Strafgefangenen gerichtet sind (geeignete Diäten, Physiotherapien, Rehabilitationsmassnahmen etc.), vom hier verwendeten Begriff umfasst.

711 SK-StPO/Meyer Art. 8 EMRK Rn. 11. Vgl. auch Pösl S. 53, welcher darauf hinweist, dass der gewährte Schutz unter Art. 8 EMRK auch aufgrund der Schrankenbestimmungen in Art. 8 Abs. 2 EMRK erheblich geringer sei.

712 So ist etwa der Zusammenhang zwischen der Problematik der Überbelegung und der medizinischen Versorgung bzw. der Behandlungsbedürftigkeit der Strafgefangenen evident. Überbelegte Zellen haben nachweislich Folgen, sowohl auf den mentalen als auch auf den physischen Zustand sowohl des gesunden als auch des bereits gesundheitlich angeschlagenen Inhaftierten. Weitere Haftbedingungen können darüber hinaus auch besondere Anforderungen an die Gesundheitsversorgungen im Strafvollzug stellen, zu denken ist etwa an die Anforderungen an die Behandlung ansteckender Krankheiten, bei welchen die Unterbringungsmodalitäten (Einzelzelle, Mehrbettzellen etc. oder mögliche Überbelegungen) eine wichtige Rolle spielen. Vgl. auch etwa EGMR - Raffray Tadei/FRA, Urt. v. 21.12.2010, 36435/07: Der EGMR hielt fest, dass mehrere Verlegungen des Beschwerdeführers aufgrund seines gesundheitlich labilen $\mathrm{Zu}$ stands nicht konform mit Art. 3 EMRK waren. 
Die Gesundheitsversorgung im Strafvollzug ist mit Blick auf Art. 3 EMRK auch deshalb von besonderer Relevanz, weil Inhaftierte häufig mehr gesundheitliche Probleme haben als Personen ausserhalb des Vollzugs. ${ }^{713}$ Gründe dafür können sowohl das Vorleben der Gefangenen - häufig können die Inhaftierten im Freiheitsentzug erstmals medizinisch erreicht werden - ${ }^{714}$ als auch das Umfeld des Strafvollzugs sein. ${ }^{715}$ Statistisch gesehen haben Strafgefangene folglich einen höheren Bedarf an Gesundheitsversorgung, wobei gewisse Krankheitsbilder im Strafvollzug besonders häufig vertreten sind. ${ }^{716}$ Ferner ist der Strafvollzug prima vista ein atypisches Umfeld für die Gesundheitsversorgung und zur Genesung. ${ }^{717}$

713 Lines International Journal of Prisoner Health 2006, 269 (271 f.); Charles/DraPer Journal of Medical Ethics 2012, 215 (216); JotTerand/Wangmo The American Journal of Bioethics 2014/7, 4 ff.; Vademekum: Übertragbare Krankheiten und Abhängigkeiten im Gefängnis, zuletzt abgerufen am 16.02.2020; WoLfF/SEbo/Haller/Eytan/Niveau/Bertrand/Gétaz/Ceruttin BMC Public Health 11, 245 (2011), 1 ff.; Hillenkamp in: Hillenkamp/Tag, S. 11 (12 f.); vgl. SAMW - Stellungnahme der Zentralen Ethikkommission (ZEK) der Schweizerischen Akademie der Medizinischen Wissenschaften (SAMW) betreffend Finanzierung medizinischer Leistungen im Gefängnis, S. 1 ff.; zur erhöhten Prävalenz an Infektionskrankheiten insb. HIV- und Hepatitisvirusinfektionen in der Gefängnispopulation vgl. KünZLi/ACHermann S. 7.

714 SAMW - Stellungnahme der Zentralen Ethikkommission (ZEK) der Schweizerischen Akademie der Medizinischen Wissenschaften (SAMW) betreffend Finanzierung medizinischer Leistungen im Gefängnis, S. 2; vgl. auch HillenKAMP in: Hillenkamp/Tag, S. 11 (14).

715 Jotterand/Wangmo The American Journal of Bioethics 2014/7, 4 (10); KünZLi/Achermann S.7, insb. Hafteinrichtungen in mittel- und osteuropäischen Staaten würden als „Herde der Tuberkuloseinfektion“ gelten, auch Schweizer Strafvollzugsanstalten seien Risikoorte für schwere Infektionskrankheiten. Vgl. auch Hillenkamp in: Hillenkamp/Tag, S. 11 (14f.); Ligthart/van Oploo/MeiJers/Meynen/Kooijmans NJECL 2019, 287 (292f.): Eine Studie in einem niederländischen Untersuchungsgefängnis hat gezeigt, dass das reizarme Umfeld des Vollzugs schon nach drei Monaten negative neurokognitive Auswirkungen hat. Hirnfunktionen, welche für die Selbstregulierung zuständig seien, würden dann abnehmen.

716 Niveau Journal of Medical Ethics 2007, 610 (611): Gemäss Niveau sind dies etwa: Hepatitis, HIV, Tuberkulose sowie Geschlechtskrankheiten, woraus schon eine andere Schwerpunktsetzung bei der Gesundheitsversorgung zu folgen habe. Gerade hier sei auch die Prävention im Gefängnis besonders bedeutend.

717 Charles/Draper Journal of Medical Ethics 2012, 215 (216); Niveau Journal of Medical Ethics 2007, 610 (611 f.): Eine Äquivalenz der psychiatrischen Behandlung könne nicht erreicht werden, denn der Strafvollzug biete für eine solche kein geeignetes Umfeld, und die wichtigsten Elemente wie stabile Familienbeziehungen, ein stabiles Umfeld, eine erfüllende Arbeit und Freiheit könnten 
Situationen, die für Art. 3 EMRK relevante sind, können sich etwa aus dem potentiellen Spannungsverhältnis zwischen den Aufgaben und Zielen des Vollzugs wie Kontrolle und Überwachung und den Zielen der Gesundheitsversorgung wie Pflege und Behandlung ergeben. ${ }^{718}$

Die Gesundheitsversorgung im Strafvollzug hat nicht nur für den betroffenen Inhaftierten Auswirkungen, sondern indirekt auch für deren Familien, Freunde, die Mitarbeiter der Strafvollzugsanstalt, Mitinhaftierte und sämtliche weiteren Personen, mit denen der Strafgefangene Kontakt aufnimmt. ${ }^{719}$ Betroffen sind - insbesondere bei ansteckenden Krankheiten - sämtliche Bereiche des Vollzugs (Schlaf- und Bewegungsmöglichkeiten, Möglichkeit von und Zugang zu Aktivitäten inner- und ausserhalb der Zelle, hygienische Bedingungen etc.). Es gilt, den Bedürfnissen des kranken Inhaftierten Rechnung zu tragen und insbesondere bei ansteckenden Krankheiten die Kontaktpersonen zu schützen sowie den Haftalltag für die weiteren Inhaftierten aufrechtzuerhalten. Schwerpunkt der folgenden Analyse von Art. 3 EMRK sind die Auswirkungen auf den Betroffenen selbst.

\section{Gesundheitsversorgung im Strafvollzug - Prinzipien}

Bevor die einzelnen Regelungsinstrumente und die Rechtsprechung analysiert werden, ist zunächst auf drei Prinzipien einzugehen, welche sich mit Blick auf Art. 3 EMRK als prägend erweisen werden; das Äquivalenzprinzip, das Freiwilligkeitsprinzip und das Vertrauensprinzip.

nicht gewährt werden. Vgl. ferner Jotterand/Wangmo The American Journal of Bioethics 2014/7, $4(5,8)$.

$718 \mathrm{Zu}$ diesem Spannungsverhältnis allgemein: JotTerand/Wangmo The American Journal of Bioethics 2014/7, 4 (8): Widerspruch zwischen den Zielen. Dies geht meines Erachtens aber zu weit. Zu bedenken ist, dass die Ziele des Strafvollzugs sich bei einer zeitgemässen Betrachtung keineswegs auf Kontrolle und Überwachung beschränken.

719 Melis International Journal of Prisoner Health 2009, 303 (304); vgl. auch Schweizerisches Kompetenzzentrum für den Justizvollzug - Gesundheit, zuletzt abgerufen am 23.12.2019; SAMW - Stellungnahme der Zentralen Ethikkommission (ZEK) der Schweizerischen Akademie der Medizinischen Wissenschaften (SAMW) betreffend Finanzierung medizinischer Leistungen im Gefängnis, S. 2 . 


\section{1. Äquivalenzprinzip}

Das Äquivalenzprinzip statuiert, dass das Niveau der Gesundheitsversorgung in der Strafvollzugsanstalt demjenigen in der freien Gesellschaft zu entsprechen hat. ${ }^{720}$ Gleichwertig haben sowohl ärztliches, pflegerisches und technisches Personal als auch die für die Gesundheitsversorgung notwendigen Räumlichkeiten, Installationen und Ausrüstungen $\mathrm{zu}$ sein. ${ }^{721}$ Sowohl die somatische als auch die psychiatrische Versorgung werden von diesem Prinzip umfasst.

Der Grundsatz der Gleichwertigkeit wurzelt in ethischen Gesichtspunkten. ${ }^{722}$ In der normativen Ethik wird das Prinzip der Äquivalenz als einer der Grundpfeiler der Gesundheitsversorgung im Strafvollzug aufgeführt. ${ }^{723}$ In der angewandten Ethik wird im Äquivalenzprinzip die Verwirklichung der Gerechtigkeit und der Solidarität gesehen. ${ }^{724}$ In der MedizinEthik ist das Äquivalenzprinzip Ausdruck des Postulats, dass der Arzt für das Wohl des Kranken besorgt zu sein hat. ${ }^{725}$ Dabei gebietet es die berufsethische Pflicht des Arztes, dass er nicht aufgrund des Status des Kranken differenziert. ${ }^{726}$

Auf rechtsphilosophischer Ebene wird das Äquivalenzprinzip durch die Grundprinzipien der Billigkeit „equity“ und der distributiven Gerechtigkeit (Verteilungsgerechtigkeit; „distributive justice“) gestützt. ${ }^{727}$

Das Äquivalenzprinzip bringt zum Ausdruck, dass auch der Inhaftierte Teil der Gesellschaft bleibt, ${ }^{728}$ womit es auch Ausdruck der Umsetzung des Normalisierungsgebotes ${ }^{729}$ resp. des „no more harm principles“ ist. Die Bestrafung erfolgt durch die Beschränkung der Freiheit und nicht durch weitere prekäre Haftbedingungen, weshalb auch im Strafvollzug eine ad-

720 Lines International Journal of Prisoner Health 2006, $269 \mathrm{ff}$.

721 Vgl. etwa MeIer in: Keppler/Stöver, S. 76 ff.; Pont International Journal of Prisoner Health 2006, 259 (264).

722 Niveau Journal of Medical Ethics 2007, $610 \mathrm{ff}$.

723 Niveau, Journal of Medical Ethics 2007, $610 \mathrm{ff}$.

724 Niveau, Journal of Medical Ethics 2007, $610 \mathrm{ff}$.

725 MeIER in: Keppler/Stöver, S. $76 \mathrm{ff}$.

726 MeIER in: Keppler/Stöver, S. 76 (77): Kommt es zu einer Differenzierung sei diese aus medizinethischer Sicht zu begründen. Im Unterschied dazu sei eine äquivalente Behandlung nicht begründungsbedürftig.

727 Charles/Draper Journal of Medical Ethics 2012, $215 \mathrm{ff}$.

728 Melis International Journal of Prisoner Health 2009, 303 (305).

729 NAGLER S. 36. 
äquate medizinische Versorgung zu gewährleisten ist. ${ }^{730}$ Ein äquivalenter Massstab der Gesundheitsversorgung sei auch ein „sichtbares Zeichen des Niveaus der Menschlichkeit und der Fürsorge in dem betroffenen Gefängnissystem".731

Die Anwendung des Äquivalenzprinzips leuchtet prima vista ein. Deshalb werden im Folgenden dessen Inhalt und dessen rechtliches Potential näher untersucht. Fragen drängen sich zum einen bei der Ermittlung des Referenzmassstabes und zum anderen bei den Beurteilungskriterien der Gleichwertigkeit auf.

Besteht ein einheitlicher medizinischer Standard in der freien Gesellschaft, wird dieser evidenterweise als Referenzmassstab beizuziehen sein. Besteht kein einheitlicher Standard in der freien Gesellschaft, könnte entweder der durchschnittlich angebotene Standard in der Gesellschaft, der höchste angebotene Standard in der Gesellschaft oder der hypothetische Standard, welcher dem konkret Betroffenen in der freien Gesellschaft zu Teil werden würde (individualisierter Massstab), als Referenzmassstab dienen.

Schon die Beantwortung der Frage, ob ein einheitlicher Massstab vorliegt oder nicht, setzt die Festlegung einer Vergleichsgruppe voraus. Hier ist meines Erachtens zu differenzieren: Wird das Äquivalenzprinzip auf europaratlicher Ebene statuiert, werden alle Europaratsstaaten zu dieser gehören. Wird das Äquivalenzprinzip demgegenüber auf nationaler Ebene gefordert, wird die Vergleichsgruppe die freie Gesellschaft dieses Staates sein. Je grösser die Vergleichsgruppe, desto weniger wahrscheinlich ist ein einheitlicher Massstab, und je heterogener die Gruppe, desto schwieriger wird es sein, einen sinnvollen Referenzmassstab zu eruieren.

Bei der Beurteilung müsste darauf geachtet werden, dass man die Personen im Strafvollzug immer mit einer Kontrollpopulation vergleicht, die auch versicherungstechnisch den Inhaftierten gleichgestellt ist. Geht es um einen menschenrechtlichen Mindeststandard, dürften jegliche Leistungen aus Zusatzversicherung von vorneherein nicht mitberücksichtigt werden.

Nimmt man den durchschnittlichen resp. den höchsten angebotenen Standard, kann es sein, dass das Äquivalenzprinzip dazu führt, dass der konkreten Person im Einzelfall im Strafvollzug eine bessere oder bisweilen wohl auch eine schlechtere medizinische Versorgung zukommt als in

730 Meier in: Keppler/Stöver, S. 76 ff.; Jotterand/Wangmo The American Journal of Bioethics 2014/7, $4 \mathrm{ff}$.; Charles/Draper Journal of Medical Ethics 2012, 215 ff.; STÖvER in: Keppler/Stöver, S. $301 \mathrm{ff}$.

731 Vókó S. 155 f. 
der freien Gesellschaft. ${ }^{732}$ Ein individualisierter Massstab würde dies zwar vermeiden, hätte allerdings auch zur Folge, dass eine benachteiligte gesellschaftliche Position im Strafvollzug perpetuiert würde. Solche individualisierten Massstäbe liessen sich jedenfalls aus einer menschenrechtlichen Perspektive nicht begründen.

Neben der Festlegung des Referenzmassstabes stellen sich auch bei der Beurteilung der Äquivalenz besondere Fragen. ${ }^{733}$ Von einer Gleichwertigkeit kann etwa dann ausgegangen werden, wenn die gewährten Leistungen innerhalb und ausserhalb des Strafvollzugs dieselben sind. Gleichwertigkeit könnte aber auch erst dann vorliegen, wenn nicht nur die gewährten Leistungen, sondern auch das erzielte Ergebnis das Gleiche ist. ${ }^{734}$ Die Gleichwertigkeit des Ergebnisses bedingt häufig eine höhere bzw. eine bessere medizinische Leistung, da im Strafvollzug im Vergleich zur freien Gesellschaft mit mehr und schwerwiegenderen Krankheitsfällen zu rechnen ist, welche komplexere Therapien erfordern. ${ }^{735}$ Würde ein gleiches Ergebnis gefordert, hätte dies zur Folge, dass auch bei Einhaltung einer äquivalenten Gesundheitsleistung die Rechte des Strafgefangenen verletzt sein könnten. ${ }^{736}$ Die Erzielung eines gleichwertigen Ergebnisses bedeutet aber nicht immer eine erhöhte Leistung; bisweilen erfordert ein gleichwertiges Ergebnis eher eine an die Umstände des Strafvollzugs angepasste Leistung. ${ }^{737}$ Beispielhaft kann hier die Verschreibung von Opiatanalgetika und Sedativa erwähnt werden. Dabei darf nicht ausser Acht gelassen werden, welches Missbrauchspotential solche Substanzen innerhalb einer Strafvoll-

732 Niveau Journal of Medical Ethics 2007, 610 (612): Gerade bei der Population der Strafgefangenen sei davon auszugehen, dass diese in der freien Gesellschaft eher eine unterdurchschnittliche Gesundheitsversorgung erhalten würden.

$733 \mathrm{Zu}$ methodischen Schwierigkeiten für einen empirischen Vergleich intra- und extramuraler Versorgung sowie zu validen Indikatoren für einen solchen Vergleich vgl. auch MeIER in: Hillenkamp/Tag, S. 35, $42 \mathrm{ff}$.

734 Charles/Draper Journal of Medical Ethics 2012, 215 ff.; Jotterand/Wangmo The American Journal of Bioethics 2014/7, 4 ff. In englischer Terminologie wird hier zwischen "equivalence of the process of care" und „equivalence of outcomes of care" unterschieden.

735 Lines International Journal of Prisoner Health 2006, 269 (271 f.): Als Beispiele somatischer Krankheiten werden etwa die Tuberkulose und HIV-Infektionen angeführt. Eine Häufung besteht aber auch bei psychischen Krankheiten. Vgl. auch Charles/Draper Journal of Medical Ethics 2012, 215 (216); Jotterand/ Wangmo The American Journal of Bioethics 2014/7, $4 \mathrm{ff}$.

736 Lines International Journal of Prisoner Health 2006, 269 (270).

737 Charles/Draper Journal of Medical Ethics 2012, 215 (216). 
zugsanstalt haben. ${ }^{738}$ Angepasste Leistungen können weiter auch aufgrund von Sicherheitsüberlegungen erforderlich sein, wobei Gefahrenanalysen mit entsprechenden Sicherheitskonzepten insbesondere bei Verlegungen in externe Behandlungseinrichtungen von besonderer Relevanz sein dürften.

Eine höhere Leistungsverpflichtung wird bisweilen aus der besonderen Schutzpflicht abgeleitet. Argumentiert wird, dass zwar kein allgemeines Recht auf Gesundheit in der freien Gesellschaft besteht, wohl aber während des Strafvollzugs, was aus der besonderen Fürsorgepflicht des Staates während der Dauer des Vollzugs abzuleiten ist. ${ }^{739}$

In vielen Situationen kann die Gleichwertigkeit des Ergebnisses aber einer ausgleichenden Gerechtigkeit entsprechen, weil auf diese Weise vorbestehende Nachteile ausgeglichen werden können. Eine Gleichwertigkeit ist aber keineswegs unter allen Bedingungen anzustreben, zum Beispiel bei der vertraulichen Handhabung einer Diagnose. ${ }^{740}$ Auch Befürworter des ergebnisorientierten Ansatzes räumen deshalb ein, dass nicht sämtliche Unterschiede zwischen Strafgefangenen und der freien Gesellschaft ausgeglichen werden müssen. ${ }^{741}$ Eine Äquivalenz im Ergebnis sei nur insofern anzustreben, als sie „unfair or avoidable difference[s]“ betreffe. ${ }^{742}$ Lässt sich noch relativ leicht eruieren, ob der Unterschied vermeidbar ist, wird näher zu konkretisieren sein, was ein unfairer und damit auszugleichender Unterschied ist.

738 Charles/Draper Journal of Medical Ethics 2012, 215 (217): Es sei bekannt, dass solche Substanzen im Gefängnis sowohl für den persönlichen Gebrauch als auch aus ökonomischer Sicht zum Tausch von anderen Produkten gebraucht würden.

739 Pont International Journal of Prisoner Health 2006, 259 (264): Dies werde insbesondere bei einem ungenügenden Standard in der freien Gesellschaft relevant. Vgl. auch Jotterand/Wangmo The American Journal of Bioethics 2014/7, $4 \mathrm{ff}$.; Lines International Journal of Prisoner Health 2006, 269 (276): „This higher standard of health care is owed by virtue of the custodial relationship between keepers and the kept."

740 Vgl. etwa das Beispiel von: Charles/Draper Journal of Medical Ethics 2012, 215 (217): Zu denken sei etwa an den Aspekt der Vertraulichkeit der Patientendaten. Der Entscheid über ihre Offenlegung erfolge nach einer Abwägung zwischen den Interessen des Patienten an der Vertraulichkeit der Daten und den öffentlichen Interessen, zu denen etwa auch Sicherheitsanliegen gehören würden. Diese Abwägung werde im Strafvollzug im Ergebnis häufiger zu Gunsten der öffentlichen Interessen ausfallen. Eine Gleichwertigkeit des Ergebnisses sei hier nicht anzustreben.

741 Charles/Draper Journal of Medical Ethics 2012, 215 (218).

742 Charles/Draper Journal of Medical Ethics 2012, 215 (218). 
Es lässt sich somit nicht allgemein festlegen, ob die Gleichwertigkeit der Leistung oder jene des Ergebnisses anzustreben sei.

Die Gleichwertigkeit der Leistung, oder auch des Ergebnisses, soll etwa durch institutionelle und personelle Vorkehrungen sichergestellt werden. Aus dem Äquivalenzprinzip wird nämlich abgeleitet, dass die Gesundheitsversorgung des Gefängnisses institutionell soweit wie möglich in die generelle öffentliche Gesundheitsversorgung eingebettet sein sollte. ${ }^{73}$ Von einer Zuständigkeitskonzentration sowohl für die Gesundheitsversorgung im Strafvollzug als auch für die Gesundheitsversorgung in der freien Gesellschaft wird eine inhaltliche Annäherung durch einheitliche medizinische Entscheide erhofft. ${ }^{744}$ Ferner wird aus dem Gleichwertigkeitsgebot abgeleitet, dass ein im Gefängnis praktizierender Arzt weiterhin auch in der freien Gesellschaft praktizieren sollte. ${ }^{745}$ Auf diese Weise sei die Unvoreingenommenheit und die Neutralität des Arztes sicherzustellen, was zu einer möglichst natürlichen Arzt-Patienten-Beziehung führen soll. ${ }^{746}$ Hinzu komme, dass auf diese Weise auch der aktuellste Standard der medizinischen Versorgung ausserhalb des Krankenhauses dem Arzt bekannt sei. ${ }^{777}$ Vom Äquivalenzprinzip jedoch anerkanntermassen nicht umfasst ist die freie Arztwahl. ${ }^{748}$

Unabhängig davon, welcher Methode man bei der Festsetzung des Referenzmassstabes folgt, und ebenso unabhängig davon, ob die Gleichwertigkeit der Leistung oder die Gleichwertigkeit des Ergebnisses massgebend ist, wird der Massstab, der aus dem Äquivalenzprinzip folgt, immer ein relativer sein. Kommt im konkreten Fall hinzu, dass in der freien Gesellschaft prekäre Bedingungen bei der Gesundheitsversorgung herrschen weil etwa grössere Teile der Gesellschaft nicht (pflicht-)versichert sind -, stellt sich zusätzlich die Frage des Genügens des Referenzmassstabes aus einer menschenrechtlichen Perspektive.

Es stellt sich deshalb die Frage, ob das Äquivalenzprinzip aus einem menschenrechtlichen Blickwinkel ein geeigneter und ein genügender Standard

743 Charles/Draper Journal of Medical Ethics 2012, 215 (216); eine „fachlich medizinische" Verknüpfung und keine Abkoppelung fordert auch: MeIER in: Keppler/Stöver, S. $76 \mathrm{ff}$.

744 Zur Situation in Grossbritannien etwa: Charles/Draper Journal of Medical Ethics 2012, 215 (216).

745 Jotterand/Wangmo The American Journal of Bioethics 2014/7, 4 (8).

746 Niveau Journal of Medical Ethics 2007, 610 (611).

747 Jotterand/Wangmo The American Journal of Bioethics 2014/7, 4 (8).

748 Niveau Journal of Medical Ethics 2007, 610 (611); Jotterand/Wangmo The American Journal of Bioethics 2014/7, 8. 
ist, um die Mindestbedingungen einer medizinischen Versorgung im Strafvollzug festzulegen (vgl. dazu näher Teil 3 Kap. 3 IV. 3.2.). ${ }^{74}$

2. Freiwilligkeitsprinzip im Strafvollzug - das Prinzip der Einwilligung (informed consent)

Selbstbestimmungsrecht und Autonomie des Patienten gebieten es, dass eine medizinische Untersuchung und Behandlung erst nach genügender Aufklärung und Einwilligung des Patienten erfolgt (vgl. auch die Garantien des Art. 8 EMRK). ${ }^{750}$ Beim informed consent handelt es sich um einen medizinethischen Grundsatz. Das Freiwilligkeitsprinzip wird in der Praxis allerdings immer wieder eingeschränkt; es erfolgt beispielsweise häufig schon bei Haftantritt eine obligatorische medizinische Untersuchung des Inhaftierten. Dabei wird versucht, vom Insassen eine Einverständniserklärung zu erlangen. Gelingt dies jedoch nicht, wird die Untersuchung bisweilen auch gegen den Willen des Strafgefangenen vorgenommen. ${ }^{751}$

Für das Obligatorium zur Eintrittsuntersuchung sind mehrere Gründe denkbar. Die Dokumentation zur Eintrittsuntersuchung kann etwa der Strafvollzugsanstalt in möglichen späteren Verfahren hinsichtlich vorbestehender gesundheitlicher Beeinträchtigungen als Beweis dienen, was insbesondere durch die aus dem Sonderstatusverhältnis des Inhaftierten resultierende Beweislastumkehr von besonderer Bedeutung ist. Insofern dient die Eintrittsuntersuchung mit entsprechender Dokumentation der Strafvollzugsanstalt als Schutz vor späteren ungerechtfertigten Vorwürfen. Sind die prozessualen Regelungen zu Gunsten des Strafgefangenen konzipiert worden (Beweiserleichterungen), stehen sie in diesem Fall gleichwohl in einem Spannungsverhältnis zur Autonomie des Strafgefangenen als Patient. Die Haftantrittsuntersuchung erfolgt aber auch aus dem Gesichtspunkt der besonderen Obhuts- und Fürsorgepflicht des Staates, können in der Untersuchung doch allfällige besondere Bedürfnisse des Inhaftierten festgehalten werden. ${ }^{752}$ Die Untersuchung ist folglich in der Regel

749 In diese Richtung auch bereits: Lines International Journal of Prisoner Health 2006, 269 (270).

750 Vgl. auch hinsichtlich des Selbstbestimmungsrechts und des gezielten Hungerstreiks: SK-StPO/MeYer Art. 3 EMRK Rn. 41.

751 Jotterand/Wangmo The American Journal of Bioethics 2014/7, 4 (8).

752 Immer vorausgesetzt ist dabei selbstverständlich, dass die Hafterstehungsfähigkeit sichergestellt ist. 
auch im Interesse des Betroffenen selbst. ${ }^{753}$ Darüber hinaus kommt die Strafvollzugsanstalt mit einer obligatorischen Eintrittsuntersuchung auch ihren Pflichten gegenüber den künftigen Mitinhaftierten und den Strafvollzugsbeamten nach, welche ihrerseits auch zum Staat in einem Sonderstatusverhältnis stehen, weshalb auch ihnen gegenüber besondere Obhutsund Fürsorgepflichten greifen.

Die Autonomie und der Wille des Strafgefangenen stehen hier folglich in einem besonderen Spannungsverhältnis zu prozessualen Vorschriften zum einen und Interessen von Dritten zum anderen. Hinsichtlich der entgegenstehenden Interessen des Staates aufgrund der Beweislastumkehr könnte sich in Abwägung mit dem Selbstbestimmungsrecht des Einzelnen eine Lösung abzeichnen, wenn die Antrittsuntersuchung nicht als Pflicht, sondern als Obliegenheit ausgestaltet werden könnte: Unterzieht sich der Strafgefangene der Untersuchung, kommen ihm die prozessualen Vorteile der Beweislastumkehr zugute; verweigert er indes eine solche, gelten die allgemeinen Beweislastregeln, die auch ausserhalb von Sonderstatusverhältnissen gelten (keine Beweislasterleichterungen für den Inhaftierten und damit Anwendung des allgemeinen Grundsatzes „affirmanti incumbit probatio"). Fehlt eine Einwilligung des Strafgefangenen, ist dies zu dokumentieren, und es ist sicherzustellen, dass der Inhaftierte die Folgen dessen versteht. Es ist explizit darauf hinzuweisen, dass er auf seine Entscheidung jederzeit zurückkommen kann, eine nachträgliche Untersuchung also möglich bleibt. ${ }^{754}$

Auf diese Weise würde dem Selbstbestimmungsrecht des Inhaftierten so weit als möglich Rechnung getragen; ob es sich bei der Einwilligung unter diesen Voraussetzungen allerdings tatsächlich um eine freiwillige (autonomer Wille) handelt, ist zumindest anzuzweifeln. Besteht das Spannungsverhältnis aber auch gegenüber Rechten von Dritten, scheint dieser Vorschlag ohnehin keine valable Lösung zu sein, so dass wohl auch in Zukunft an einer obligatorischen Eintrittsuntersuchung festzuhalten sein wird. Dabei bleibt weiterhin möglichst auf das Einverständnis des Inhaf-

753 Niveau Journal of Medical Ethics 2007, 610 (611).

754 Zur allgemeinen medizinischen Behandlung im Strafvollzug: Pont International Journal of Prisoner Health 2006, 259 (264). Im Zusammenhang mit der Eintrittsuntersuchung macht eine spätere Untersuchung in Bezug auf die Beweislast von im Vollzug entstandenen Verletzungen vor allem so lange Sinn, als sie gerade noch nicht entstanden sind. Wenn bereits Verletzungen entstanden sind, kann die Untersuchung zu Beweiszwecken immer noch sinnvoll sein, zum einen zur Dokumentation der entstandenen Verletzungen, zum anderen aber auch für den Fall, dass weitere Verletzungen dazukommen sollten. 
tierten hinzuwirken, indem ihm etwa die Gründe der Untersuchung und deren Vorteile für ihn selbst erläutert werden.

Die medizinische Versorgung während der Haft hat in aller Regel, wie in der freien Gesellschaft auch, erst nach einer Einverständniserklärung des urteilsfähigen Inhaftierten zu erfolgen. Ausnahmen können sich unter Umständen bei der Diagnostik und Behandlung ansteckender und lebensbedrohlicher Krankheiten ergeben (im Einzelnen Teil 3 Kap. 3 IV. 1.). Bei Ersterem ist zu berücksichtigen, dass die Umstände des Strafvollzugs viele Personen auf relativ engem geschlossenem Raum - ein erhöhtes Verbreitungsrisiko bergen, wobei wiederum der besonderen Fürsorgepflicht gegenüber den weiteren Inhaftierten Rechnung zu tragen ist.

Sind weder die Interessen des Inhaftierten noch unmittelbare Drittinteressen (insb. bzgl. Mitinhaftierte, Strafvollzugsbeamten etc.) berührt, sondern geht es vielmehr um allgemeine medizinische Versuche resp. Forschung, hat das Einwilligungsprinzip einschränkungslos zu gelten, wobei auch hier auf die besondere Fürsorgepflicht des Staates gegenüber dem konkret Betroffenen zu verweisen ist.

3. Vertrauensprinzip und Vertraulichkeit im Strafvollzug - ArztPatienten-Beziehung

Mit dem Prinzip der Einwilligung, d.h. der Freiwilligkeit der medizinischen Versorgung, eng verknüpft sind das Vertrauensprinzip und der Grundsatz der Vertraulichkeit. Strafvollzugsbedingte Ausnahmen bleiben auch hier möglich.

Die Arzt-Patienten-Beziehung sollte durch Vertrauen geprägt sein. Wie gesehen, umfasst das Äquivalenzprinzip gerade keine freie Arztwahl; der Arzt wird vielmehr durch die Vollzugsbehörde bestimmt - meist der Arzt, der für die konkrete Strafvollzugsanstalt zuständig ist. Sowohl das Fehlen der freien Arztwahl im Allgemeinen als auch das Verhältnis des Arztes zur Strafvollzugsanstalt können zu einem gewissen Spannungsverhältnis führen, weshalb der Aufbau des Vertrauensverhältnisses nicht immer einfach ist. $^{755}$ Führt ein fehlendes Vertrauensverhältnis dazu, dass der Inhaftierte

$755 \mathrm{Zu}$ diesem Spannungsverhältnis vgl. ausf. auch Hillenkamp in: Hillenkamp/ Tag, S. 11 (20 ff.), der für das „Primat der Medizin“ eintritt. Vgl. auch TAG in: Hillenkamp/Tag, S. 89, welche dieses Spannungsverhältnis mit einem Überschneiden der Pflichtenkreise aus vollzugsorientierter Tätigkeit und aus der 
eine Behandlung durch den zur Verfügung stehenden Arzt ablehnt ${ }^{756}$, kann dies weitreichende Konsequenzen für den Betroffenen haben, wobei sich insbesondere die Frage stellt, wann ein Beizug eines anderen externen Arztes erforderlich wird.

Der Aufbau eines Vertrauensverhältnisses hängt nicht zuletzt von der Gewährung der Vertraulichkeit der medizinischen Daten des Inhaftierten gerade auch gegenüber der Vollzugsanstalt ab, insbesondere wenn der Arzt von der Strafvollzugsanstalt bestimmt wird. Grundsätzlich gilt, wie in der freien Gesellschaft auch, dass der Arzt einem Berufsgeheimnis unterliegt, d.h., dass er ohne die Einwilligung des Inhaftierten grundsätzlich keine Informationen, welche er in Ausübung seines Berufes erfahren hat, an Dritte (auch nicht an die Leitung der Strafvollzugsanstalt) weitergeben darf. ${ }^{757}$ Auch das Prinzip der Vertraulichkeit dieser besonders sensiblen Informationen gilt jedoch nicht in jedem Fall. ${ }^{758}$ Der Grundsatz der Vertraulichkeit steht dabei wiederum in einem Spannungsverhältnis mit Drittinteressen. Zu denken ist beispielsweise an das Vorliegen ansteckender Krankheiten, aber auch etwa an Sicherheitsinteressen (für die Zeit während des Vollzugs aber auch für die Zeit nach dem Vollzug). Dieses Spannungsverhältnis gilt es im Einzelfall aufzulösen.

\section{Zwischenfazit}

Die Gesundheitsversorgung während des Strafvollzugs ist vom Prinzip der Äquivalenz geprägt, welches insbesondere auf ethischen, aber auch auf rechtsphilosophischen Grundsätzen beruht und welches aus dem Normalisierungsgebot und dem "no more harm principle“ abgeleitet werden kann. Im Strafvollzug soll die Gesundheitsversorgung damit gleichwertig mit jener in der freien Gesellschaft sein. Sowohl hinsichtlich der Festlegung des Referenzrahmens als auch bei der Frage der Beurteilungskriterien der Gleichwertigkeit besteht allerdings weiterer Konkretisierungsbedarf. Wei-

allgemeinen Gesundheitsfürsorge beschreibt. Vgl. ferner Pont International Journal of Prisoner Health 2006, 259 ff.

756 Niveau Journal of Medical Ethics 2007, 610 (611).

757 Entsprechende Vorschriften können sich aus dem jeweiligen nationalen Strafgesetzbuch ergeben oder auf berufsethischen Pflichten des Arztes beruhen. Vgl. auch TAG in: Hillenkamp/Tag, S. 89 (90 f.).

758 Charles/Draper Journal of Medical Ethics 2012, 215 (217); zur anstaltsärztlichen Schweigepflicht und deren Grenzen im deutschen Kontext vgl. ausf. TAG in: Hillenkamp/Tag, S. 89 (91 ff., 97 ff.). 
ter wurde deutlich, dass weder das Äquivalenzprinzip noch die weiteren Prinzipien der Freiwilligkeit und der Vertraulichkeit im Strafvollzug in jedem Fall Geltung beanspruchen. Gerade die Besonderheiten des Strafvollzugs führen zu Ausnahmen, insbesondere aufgrund der besonderen Fürsorgepflichten des Staates. Gleichwohl ist zu fordern, dass nie leichthin von den Grundprinzipien der Freiwilligkeit und der Vertraulichkeit abgewichen werden darf und Ausnahmen mithin nur bei klar umrissenen Konstellationen zur Anwendung kommen dürfen. Dies, um nicht Gefahr zu laufen, diese rechtlichen Grundprinzipien zu unterwandern.

III. Regelungsinstrumente zur Gesundheitsversorgung - vorgeschlagene bzw. gesetzte Standards

Die medizinische Versorgung ist nicht erst heute, sondern war auch schon in der Vergangenheit, Gegenstand von Regulierungsmechanismen und Empfehlungen auf verschiedenen Ebenen. Von ihnen werden einige zentrale in diesem Kapitel aufgegriffen, wobei der Fokus - wie schon bei der Überbelegungsproblematik - auf Entwicklungstendenzen, Gemeinsamkeiten und Unterschieden liegt. Besonderes Augenmerk wird weiter auf die Implementierung der vorgenannten Grundprinzipien gerichtet.

1. Internationaler Pakt über wirtschaftliche, soziale und kulturelle Rechte (UNO-Pakt I)

Art. 12 Abs. 1 UNO-Pakt I statuiert ein allgemeines Recht auf Gesundheit, welches das individuell erreichbare Höchstmass an körperlicher und geistiger Gesundheit umfasst. $\mathrm{Zu}$ beachten bleibt allerdings die Geltung von Art. 2 Abs. 1 UNO-Pakt I, wonach auch Art. 12 UNO-Pakt I einer progressiven Verwirklichung unterliegt, bei welcher die verfügbaren Ressourcen stets zu berücksichtigen bleiben. Verwirklicht werden soll dieses allgemeine Recht in den Konventionsstaaten etwa durch die Schaffung der Voraussetzungen, die für jedermann im Krankheitsfall den Genuss medizinischer Einrichtungen und ärztlicher Betreuung sicherstellen (Art. 12 Abs. 2 lit. d UNO-Pakt I).

Der Ausschuss für wirtschaftliche, soziale und kulturelle Rechte als Überwachungsorgan des UNO-Pakts I erkennt in dieser Garantie die Verpflichtung des Staates, qualitativ und quantitativ genügende öffentliche Gesundheitsinfrastruktur (Gesundheitseinrichtungen, Gesundheitsgüter, 
Gesundheitsdienstleistungen und Gesundheitsinformationen) verfügbar zu machen („availability“) und den Zugang zu diesen für alle Personen sicherzustellen („accessibility“).759 Aufgrund des gewährleisteten diskriminierungsfreien Zugangs zur Gesundheitsinfrastruktur gelten diese Garantien für die Gruppe der Strafgefangenen gleichermassen wie für den Rest der Gesellschaft. Das Verfügbarmachen von Gesundheitsinfrastruktur i.S.v. Art. 12 UNO-Pakt I umfasst insbesondere auch die Bereitstellung elementarer Arzneimittel sowie den Erlass und die Durchsetzung einer nationalen Gesundheitsstrategie und eines Aktionsplanes. ${ }^{760}$ Der gewährleistungsrechtliche Gehalt von Art. 12 UNO-Pakt I fordert von den Konventionsstaaten weiter, das Recht auf Gesundheit im eigenen Nationalstaat zu postulieren und diesem am besten durch gesetzgeberische Massnahmen Geltung zu verschaffen. ${ }^{761}$

Dieses allgemein gehaltene Postulat zeigt die grosse Bedeutung auf, welche die Vereinten Nationen der Gesundheitsversorgung im Allgemeinen zumessen, und ist Ankerpunkt für weitere, spezifischere Regelwerke.

2. UN-Mindestgrundsätze für die Behandlung von Gefangenen und Nelson-Mandela-Regeln

\subsection{UN-Mindestgrundsätze für die Behandlung von Gefangenen}

Schon in den UN-Mindestgrundsätzen für die Behandlung von Gefangenen aus dem Jahr 1955 finden sich umfassende Empfehlungen rund um die Gesundheitsversorgung Inhaftierter (insb. Regeln 22 ff., 53 Abs. 3, 62, 91) und zum Umgang mit kranken Inhaftierten (Regel $82 \mathrm{f}$.) sowie zum Recht des Inhaftierten auf Information seiner Angehörigen im Krankheitsfall (Regel 44).

In den UN-Mindestgrundsätzen von 1955 wird festgehalten, dass jeder Inhaftierte so rasch als möglich nach Haftantritt medizinisch zu unter-

759 Zum Ganzen vgl. WSK-Ausschuss, General Comment No. 14, insb. Ziff. 12: Das Erfordernis der Zugänglichkeit setze sich aus einer diskriminierungsfreien Zugänglichkeit, einer physischen Zugänglichkeit, einer ökonomischen Zugänglichkeit sowie einer Informationszugänglichkeit zusammen. Vgl. auch KüNZLI/ Eugster/Spring S. 45, S. 47; ausf. auch KäLIN/KünZli S. 353, 368 ff.

760 WSK-Ausschuss, General Comment No. 14, Ziff. 43; KünZli/Eugster/Spring S. 47.

761 WSK-Ausschuss, General Comment No. 14, Ziff. 60; Künzli/Eugster/Spring S. 45 . 
suchen ist (Regel 24). Bei der Eintrittsuntersuchung sind Gefangene mit Verdacht auf Infektionskrankheiten oder andere ansteckende Krankheiten zu separieren. Alle festgestellten physischen oder psychischen Krankheiten sind festzuhalten und die notwendigen Massnahmen zu treffen. Ferner sind Krankheiten, welche die Rehabilitation erschweren könnten, und die Arbeitsfähigkeit eines jeden Inhaftierten festzustellen (Regel 24). Damit gehen die UN-Mindestgrundsätze von einer obligatorischen Eintrittsuntersuchung aus, unabhängig von einer Einwilligung des Inhaftierten.

Für die Gesundheitsversorgung während der Dauer der Haft wird in Regel 22 gefordert, dass in jeder Strafvollzugsanstalt ein Arzt ${ }^{762}$ verfügbar sein muss. Im Weiteren wird die Ausgestaltung der Gesundheitsversorgung näher definiert: Die Gesundheitsdienste sind in enger Zusammenarbeit mit den Gesundheitsdiensten der freien Gesellschaft zu organisieren (Regel 22 Abs. 1). Ist eine spezialisierte Behandlung erforderlich, muss der Inhaftierte an eine spezialisierte Institution oder in ein ziviles Krankenhaus überwiesen werden (Regel 22 Abs. 2). Erfolgt die Behandlung in einer hafteigenen Krankenstation, haben die Einrichtung, die Ausstattung und die Medikation der medizinischen Behandlung angemessen zu sein (Regel 22 Abs. 2). Damit orientieren sich die UN-Mindestgrundsätze von 1955 an Einzelaspekten des Äquivalenzprinzips. Sowohl die Zusammenarbeit mit als auch die Überweisungsmöglichkeit zu den medizinischen Diensten der freien Gesellschaft zielen dabei auf eine gleichwertige Gesundheitsversorgung inner- und ausserhalb des Vollzugs ab. Erfolgt keine Überweisung in eine zivile Klinik, wird eine angemessene haftinterne medizinische Versorgung gefordert.

Zur Festsetzung, was die Angemessenheit der Gesundheitsversorgung beinhaltet, enthalten die UN-Mindestgrundsätze zahlreiche weitere konkretisierende inhaltliche Garantien. So wird etwa gefordert, dass der Arzt täglich bei allen kranken Inhaftierten und bei all jenen, die über eine Krankheit klagen, Visite abzuhalten hat (Regel 25 Abs. 1). Weiter hat für alle Strafvollzugsanstalten auch jederzeit ein Zahnarzt verfügbar zu sein (Regel 22 Abs. 3). Auch die besonderen Anforderungen an die Gesundheitsversorgung von schwangeren Frauen im Strafvollzug werden geregelt (Regel 23).

Mit Blick auf das Vertrauensprinzip und den Vertraulichkeitsgrundsatz ist insbesondere auf die Informationspflichten des Arztes gegenüber der Gefängnisdirektion hinzuweisen. Die UN-Mindestgrundsätze fordern et-

762 Dieser Arzt muss zumindest auch Kenntnisse über psychische Krankheiten haben (Regel 22 Abs. 1: „some knowledge of psychiatry“). 
wa, dass der Arzt Informationen, welche die Haftfähigkeit der Inhaftierten betreffen könnten, an die Direktion weiterzuleiten habe (Regel 25 Abs. 2). Der Arzt soll gemäss den UN-Mindestgrundsätzen zudem eine wichtige Hinweisfunktion bezüglich weiterer materieller Haftumstände innehaben (Regel 26 Abs. 1 lit. a-e). Der Arzt hat regelmässig die Nahrung, die Sauberkeit und Hygiene, die sanitären Umstände, die Lüftung und Heizung, die Eignung und Sauberkeit der Kleidung und Bettwäsche sowie bisweilen auch die Regeln zur körperlichen Betätigung und zur Ausübung von Sport zu betrachten und der Direktion Rückmeldung mit diesbezüglichen Ratschlägen zu erteilen. Stimmt die Direktion den Empfehlungen des Arztes zu und liegt deren Umsetzung innerhalb ihrer Kompetenz, setzt sie diese sofort um. Ist die Direktion jedoch mit den Empfehlungen des Arztes nicht einverstanden oder liegt deren Umsetzung ausserhalb ihrer Kompetenz, hat die Direktion selbst einen Bericht zu verfassen, welcher gemeinsam mit dem Bericht des Arztes an eine höhere Stelle (,higher authority") weiterzuleiten ist (Regel 26 Abs. 2).

Die ratio der Informationspflichten des Arztes gegenüber der Gefängnisdirektion ist im Schutz des Inhaftierten zu sehen; nichtsdestotrotz kann das bestehende (Austausch-)Verhältnis zwischen Arzt und Direktion im Einzelfall die Gefahr bergen, sich negativ auf die Bildung eines Vertrauensverhältnisses zwischen Arzt und inhaftiertem Patienten auszuwirken.

\subsection{Die Nelson-Mandela-Regeln}

Auch die Nelson-Mandela-Regeln sehen eine Eintrittsuntersuchung vor (Regel 30). Regel 30 entspricht dabei im Wesentlichen der Regelung in den UN-Mindestgrundsätzen von 1955. Als allgemeine Regel wird mit Blick auf das Äquivalenzprinzip und mit Blick auf die Arzt-PatientBeziehung nun aber ausdrücklich das Informed consent-Prinzip statuiert (Regel 32 lit. b). Nicht explizit geregelt sind Möglichkeiten zu Ausnahmen von diesem Prinzip. Was dies mit Bezug auf die Eintrittsuntersuchung zu bedeuten hat, scheint damit nicht abschliessend geklärt. Ein absolutes Verbot sehen die Nelson-Mandela-Regeln bezüglich jeglicher medizinischer oder wissenschaftlicher Versuche vor, die nicht im Einklang mit der Gesundheit des Inhaftierten stehen und welche Folter oder eine unmenschliche oder erniedrigende Behandlung zur Folge haben könnten, wobei hierbei ausdrücklich die Entnahme von Zellen, Gewebe oder Organen genannt werden (Regel 32 lit. d). 
Hinsichtlich der medizinischen Versorgung während des Strafvollzugs konsolidieren die Nelson-Mandela-Regeln das Äquivalenzprinzip, welches in Regel 24 wieder aufgenommen wird:

"Prisoners should enjoy the same standards of health care that are available in the community, and should have access to necessary health-care services free of charge without discrimination on the grounds of their legal status."

Die Gesundheitsversorgung in der Strafvollzugsanstalt ist weiterhin in enger Zusammenarbeit mit den zivilen Institutionen $\mathrm{zu}$ organisieren, wobei insbesondere der Grundsatz der Kontinuität der Behandlung zu beachten ist (Regel 24 Abs. 2). Sofern es der Gesundheitszustand resp. die Behandlung erfordern, ist der Inhaftierte an eine spezialisierte zivile Klinik zu überweisen (Regel 27 Abs. 1). Ausdrücklich festgehalten wird ferner, dass in der Arzt-Patienten-Beziehung die gleichen ethischen und beruflichen Massstäbe zu gelten haben wie in der freien Gesellschaft (Regel 32 Abs. 1).

Auch in den Nelson-Mandela-Regeln bleibt das Äquivalenzprinzip nicht das einzige massgebliche Kriterium. Ergänzt wird dieses vielmehr durch zahlreiche konkrete inhaltliche Einzelgarantien, welche im Vergleich zu den UN-Mindestgrundsätzen weiter ausgebaut wurden (vgl. Regel $25 \mathrm{ff}$.).

Wenngleich dieselben Informationspflichten des Arztes gegenüber der Gefängnisdirektion bestehen (Regel 33) und dem Arzt weiterhin dieselbe Hinweisfunktion zukommt (Regel 35), welche schon in den UN-Mindestgrundsätzen von 1955 statuiert wurden, wird in den Nelson-MandelaRegeln das Vertraulichkeitsprinzip nun ausdrücklich verankert (Regel 26). Medizinische Daten sollen in vertraulichen separaten medizinischen Akten aufbewahrt werden. Der inhaftierte Patient und weitere von ihm bezeichnete Personen müssen dabei jederzeit Zugriff auf diese haben. Wird der Inhaftierte in eine andere Strafvollzugsanstalt überführt, wird auch die medizinische Akte - unter Hinweis auf die medizinische Vertraulichkeit - der neuen Vollzugseinrichtung übergeben (Regel 26 Abs. 2). Auch unter dem Gesichtspunkt der Äquivalenz sollen bezüglich der Vertraulichkeit die gleichen Grundsätze wie in der freien Gesellschaft gelten. Ausdrücklich davon ausgenommen ist die Situation, dass der Arzt eine reale unmittelbar drohende Gefahr für den Inhaftierten oder für Dritte feststellt (Regel 32 lit. c). ${ }^{763}$

763 Meldepflichten können allerdings durchaus auch in der freien Gesellschaft bestehen. In der Schweiz sind solche für übertragbare Krankheiten etwa dem EpG i.V.m. der EPV und der EDI-VO über die Meldung von Beobachtungen übertragbarer Krankheiten des Menschen zu entnehmen. Bedarfslage und Dringlich- 
Die wichtige Stellung und Entscheidungsmacht des medizinischen Personals wird dadurch manifest, dass klinische Entscheidungen nur von diesem getroffen werden dürfen und Strafvollzugsbeamte (non-medical prison staff) nicht davon abweichen dürfen (Regel 27 Abs. 2). Auch dieser Grundsatz kann dem Vertrauensverhältnis zwischen Arzt und inhaftiertem Patient dienen.

Zusammengefasst finden in den Nelson-Mandela-Regeln im Vergleich zum UN-Mindeststandard von 1955 Ausdifferenzierungen einzelner Garantien statt. Ausgangspunkt bleibt dabei das weiterhin geltende Äquivalenzprinzip, welches durch die zahlreichen Garantien aber in materieller Sicht wesentlich ergänzt wird. Diese haben im Strafvollzug dann unabhängig davon zu gelten, ob sie auch in der freien Gesellschaft tatsächlich gewährt werden oder nicht. Zunehmend mehr Gewicht kommt in den Nelson-Mandela-Regeln dem Vertraulichkeitsprinzip zu.

3. Europäisches Komitee zur Verhütung von Folter und unmenschlicher oder erniedrigender Behandlung oder Strafe (CPT)

Auch das CPT erweist sich rund um die Thematik der Gesundheitsversorgung im Strafvollzug als wichtiger Akteur. Zur medizinischen Versorgung im Strafvollzug hat das CPT vorrangig zwei themenspezifische Dokumente veröffentlicht: (1) „Health care services in prisons"764 und (2) „Inspection of a prison medical service by CPT doctor - Checklist ${ }^{\text {"765 }}$.

Ersteres besteht aus Auszügen aus dem 3. General Report aus dem Jahr 1993. Thematisch ordnet das CPT dabei gesundheitsrelevante Fragen im Strafvollzug in sieben Teilbereiche ein: Zugang zu einem Arzt, Äquivalenzprinzip der Gesundheitsversorgung, Prinzip der Einwilligung des Patienten sowie das Vertraulichkeitsprinzip, präventive Gesundheitsversorgung, humanitärer Beistand, berufliche Unabhängigkeit und Fachkompetenz. ${ }^{766}$

An diese sieben Teilbereiche anknüpfend umfasst das zweite Dokument als Leitfaden für die CPT-Besuche einen nicht abschliessenden Fragenkatalog zur Gesundheitsversorgung in der Strafvollzugsanstalt, anhand dessen

keit einer Meldepflicht können sich im Übrigen aber selbstverständlich unterscheiden.

764 CPT - Health Care Services in Prisons [CPT/Inf (93) 12].

765 CPT - Inspection of a Prison Medical Service by a CPT Doctor [CPT/Inf (2017) 20].

766 CPT - Health Care Services in Prisons [CPT/Inf (93) 12]; resp. CPT - 3rd General Report CPT/Inf (93) 12. 
die Lage der medizinischen Versorgung von einem Arzt zu evaluieren ist. ${ }^{767}$ Die Checkliste sieht dabei ein dreistufiges Vorgehen vor. Zunächst sei ein Interview mit dem verantwortlichen Arzt zu führen, dann habe die Besichtigung der Räumlichkeiten der medizinischen Dienste zu erfolgen und schliesslich sei ein Abschlussgespräch mit dem behandelnden Arzt zu führen.

Hinsichtlich der sieben Themenbereiche wird unter dem Aspekt des $\mathrm{Zu}$ gangs zu einem Arzt zunächst festgehalten, dass jeder neu Inhaftierte sofort, d.h. in aller Regel am Tag der Inhaftierung, einem medizinischen Eintrittsgespräch und einer Eintrittsuntersuchung unterzogen werden soll. ${ }^{768}$ Durchzuführen ist dieses Eingangsscreening von einem Arzt und nur ausnahmsweise von voll ausgebildeten Krankenpflegern, welche dem Arzt Bericht erstatten. ${ }^{769}$ Hinsichtlich der Freiwilligkeit der Eintrittsuntersuchung selbst finden sich keine direkten Vorgaben, so dass auf die allgemeinen Prinzipien zur Einwilligung zurückzugreifen ist. ${ }^{770}$ So hält auch das CPT am Prinzip des informed consent fest und statuiert, dass eine medizinische Behandlung nur mit dem informierten Einverständnis des Patienten erfolgen könne. Ausnahmen davon seien nur in eng umschriebenen Fällen zulässig, wenn sie gesetzlich so vorgesehen sind und auf Prinzipien beruhen, wie sie auch ausserhalb des Strafvollzugs gelten. ${ }^{771}$

Konkret bedeutet dies, dass eine obligatorische Eintrittsuntersuchung gemäss dem CPT dann regelkonform wäre, wenn sie im konkreten Fall einerseits gesetzlich verankert ist und andererseits auf Prinzipien gründet, wie sie auch ausserhalb des Strafvollzugs gelten. Bei Letzterem wäre zur Begründung folglich etwa das Erkennen von ansteckenden Krankheiten und somit der Schutz von Dritten eine durchaus denkbare Argumentation.

Unter dem Aspekt des Äquivalenzprinzips führt das CPT aus, dass die zahlreichen Bereiche der Gesundheitsversorgung während des Strafvollzugs mit jenen der Gesellschaft ausserhalb des Vollzugs vergleichbar („com-

767 CPT - Inspection of a Prison Medical Service by a CPT Doctor [CPT/Inf (2017) 20]; zur Bedeutung der interdisziplinären Zusammensetzung des Besuchsgremiums vgl. auch KoEPpel S. 210.

768 CPT - Health Care Services in Prisons [CPT/Inf (93) 12], Rn. 33; resp. CPT 3rd General Report CPT/Inf (93) 12.

769 CPT - Health Care Services in Prisons [CPT/Inf (93) 12], Rn. 33; resp. CPT 3rd General Report CPT/Inf (93) 12.

770 CPT - Health Care Services in Prisons [CPT/Inf (93) 12], Rn. 45 ff.; resp. CPT 3rd General Report CPT/Inf (93) 12.

771 CPT - Health Care Services in Prisons [CPT/Inf (93) 12], Rn. 47; resp. CPT 3rd General Report CPT/Inf (93) 12. 
parable"; gemeint ist wohl gleichwertig) sein müssen. In der Folge wird auf dieses Gleichwertigkeitserfordernis nicht näher eingegangen; vielmehr werden unter dem Kapitel des Äquivalenzprinzips über eine relativ vergleichende Betrachtung hinaus konkrete inhaltliche Anforderungen gestellt; dies sowohl an die generelle medizinische Versorgung als auch an die psychiatrische Versorgung während des Strafvollzugs. ${ }^{772}$ Diese konkreten inhaltlichen Empfehlungen des CPT sind meines Erachtens - trotz ihrer systematischen Einordnung unter dem Gesichtspunkt der Äquivalenz dahingehend auszulegen, dass sie im Sinne eines Mindeststandards bei der Gesundheitsversorgung während des Strafvollzugs einzuhalten sind, und zwar grundsätzlich unabhängig davon, ob sie ausserhalb des Strafvollzugs gewährleistet sind oder nicht. Die Frage der Gleichwertigkeit der Gesundheitsversorgung inner- und ausserhalb des Strafvollzugs rückt damit auch beim CPT in den Hintergrund.

Auch das CPT greift das Vertraulichkeitsprinzip auf ${ }^{773}$ und setzt dabei schon beim Zugang zur Gesundheitsversorgung an. Dem Strafgefangenen muss es möglich sein, Mitteilungen oder Anfragen zu einem Termin vertraulich an den Arzt zu übermitteln (etwa schriftlich in einem geschlossenen Briefumschlag, ohne Vorprüfung durch nichtmedizinische Strafvollzugsbeamte). ${ }^{774}$ Weiter hat die Aufbewahrung von Patientenakten in der Verantwortung des Arztes zu liegen. ${ }^{775}$ Sodann haben Untersuchungen zum einen einzeln und nicht gruppenweise und zum anderen ausser Hörund, wenn vom Arzt nicht anders angeordnet, auch ausser Sichtweite von Strafvollzugsbeamten stattzufinden. ${ }^{776}$ Eng mit dem Vertrauens- und Vertraulichkeitsprinzip verbunden statuiert das CPT auch die berufliche Unabhängigkeit des Gesundheitspersonals in Anerkennung seiner besonders

772 CPT - Health Care Services in Prisons [CPT/Inf (93) 12], Rn. $38 \mathrm{ff}$.

773 Vgl. insb. CPT - Health Care Services in Prisons [CPT/Inf (93) 12], Rn. 34, 50 f.; resp. CPT - 3rd General Report CPT/Inf (93) 12.

774 CPT - Health Care Services in Prisons [CPT/Inf (93) 12], Rn. 34; resp. CPT 3rd General Report CPT/Inf (93) 12.

775 CPT - Health Care Services in Prisons [CPT/Inf (93) 12], Rn. 50; resp. CPT 3rd General Report CPT/Inf (93) 12.

776 CPT - Health Care Services in Prisons [CPT/Inf (93) 12], Rn. 51; resp. CPT 3rd General Report CPT/Inf (93) 12. Dem Arzt wird damit, insbesondere aufgrund eigener Sicherheitsaspekte, ein gewisses Ermessen eingeräumt. Gleichwohl hält das CPT auch fest, dass vor dem Hintergrund, dass dem inhaftierten Patienten keine freie Arztwahl zustehe, die Pflichten des Arztes grundsätzlich auch dann fortbestehen würden, wenn der Patient gegen medizinische Regeln verstosse oder wenn er auf Bedrohungen bzw. gar Gewalt zurückgreife (vgl. Rn. 74). 
diffizilen Situation. Dies insbesondere vor dem Hintergrund potentieller Interessenkonflikte zum Strafvollzugsmanagement. ${ }^{777}$ Zur Gewährleistung des Vertrauensverhältnisses zwischen Arzt und inhaftiertem Patienten hält das CPT in diesem Sinne auch fest, dass der Gefängnisarzt gewisse Funktionen gerade nicht übernehmen soll: Er soll weder für die Ausstellung eines Attestes zuständig sein, der bescheinigt, dass der Patient in der körperlichen Verfassung sei, eine besondere (Disziplinar-)Strafe über sich ergehen zu lassen, noch soll er die von den Behörden angeordneten Untersuchungen oder Durchsuchungen vornehmen. Letzteres habe allerdings zumindest in Notfallsituationen, wenn kein anderer Arzt verfügbar ist, möglich zu bleiben. ${ }^{778}$ Demgegenüber steht dem Vertrauensprinzip gemäss CPT die Zuständigkeit des Gefängnisarztes zur Feststellung der aus ärztlicher Sicht nicht mehr weiter vertretbaren Inhaftierung nicht entgegen (inkl. Erstellung eines entsprechenden Berichtes an die zuständige Behörde). ${ }^{779}$

Zusammenfassend kann festgehalten werden, dass die noch heute geltenden Empfehlungen des CPT zu einem grossen Teil in das Jahr 1993 zurückreichen. Auch die Empfehlungen des CPT sind prinzipiengetragen. So sind die Ausgangspunkte auch beim CPT der Äquivalenzgedanke, das Freiwilligkeitsprinzip und das Vertrauens- und Vertraulichkeitsprinzip. Einschränkungen von diesen Prinzipien sollen aber auch beim CPT möglich bleiben. Unbenommen von diesen Ausnahmen sind die einzelnen inhaltlichen Garantien des CPT schon von der Natur der Sache her kumulativ zu gewährleisten und sind damit schon von vorneherein nicht kompensabel. Weiter beinhalten die CPT-Dokumente über diese Fundamentalgrundsätze hinaus zahlreiche konkrete inhaltliche Anforderungen an die Gesundheitsversorgung im Strafvollzug. Diese beruhen zum Teil wiederum auf dem Äquivalenzgedanken, reichen aber weiter und gelten auch unabhängig von ihrer Gewährleistung ausserhalb des Strafvollzugs.

Die Herangehensweise des CPT trägt damit dem Umstand Rechnung, dass gewisse inhaltliche Garantien strafvollzugsspezifisch zu normieren sind und sich nicht immer auf die ausserhalb des Strafvollzugs geltenden Grundsätze der medizinischen Versorgung stützen lassen. Das CPT orien-

777 CPT - Health Care Services in Prisons [CPT/Inf (93) 12], Rn. 71 ff.; resp. CPT 3rd General Report CPT/Inf (93) 12.

778 CPT - Health Care Services in Prisons [CPT/Inf (93) 12], Rn. 73; resp. CPT 3rd General Report CPT/Inf (93) 12.

779 CPT - Health Care Services in Prisons [CPT/Inf (93) 12], Rn. 70; resp. CPT 3rd General Report CPT/Inf (93) 12. 
tiert sich damit an einem Mindeststandard, der von der Situation ausserhalb des Vollzugs abgekoppelt ist.

4. Europäische Mindestgrundsätze für die Behandlung der Gefangenen (ESMR) und Europäische Strafvollzugsgrundsätze (EPR)

Die ursprüngliche Version der Europäischen Mindestgrundsätze für die Behandlung der Gefangenen vom 19.01.1973 (ESMR) ${ }^{780}$ thematisiert die Gesundheitsversorgung im Strafvollzug in den Regeln $21 \mathrm{ff}$. Dabei halten auch diese in grundsätzlicher Weise fest, dass die medizinische Versorgung in enger Zusammenarbeit mit der allgemeinen Gesundheitsverwaltung der Gesellschaft bzw. des Staates organisiert werden soll (Äquivalenzprinzip; Regel 21 Abs.1). In der Folge werden einzelne inhaltliche Anforderungen an die Gesundheitsversorgung aufgestellt (dazu sogleich). Die Europäischen Strafvollzugsgrundsätze von 2006 widmen der Gesundheit im Strafvollzug einen eigenen Teil der Empfehlungen (Teil 3 der EPR). Auch in den EPR ist zunächst das Äquivalenzprinzip statuiert. Im Unterschied zu den ESMR wurde dieses allerdings weiter ausdifferenziert, wobei unterschiedliche Aspekte der Gleichwertigkeit ausdrücklich aufgeführt werden; beispielsweise die Gesundheitspolitik, der Zugang zur medizinischen Versorgung, Diagnosestellung und Behandlung (Regeln 40.1-40.5). Auch in den EPR folgen danach einzelne weitere konkrete inhaltliche Aspekte (Regeln 41 ff.; dazu sogleich).

In den ESMR von 1973 wird hinsichtlich einer Eintrittsuntersuchung festgehalten, dass jeder Strafgefangene direkt bei seiner Inhaftnahme untersucht werden soll (Regel 24). Besonderes Augenmerk soll dabei nicht nur auf die körperliche, sondern auch auf die psychische Verfassung des Strafgefangenen gerichtet werden. Ferner sind ansteckende Krankheiten oder Krankheiten, welche die Rehabilitation erschweren könnten, festzustellen und die Arbeitsfähigkeit der Strafgefangenen zu beurteilen (Regel 24). Auch in den EPR wird an einer Eintrittsuntersuchung, welche so rasch als möglich nach der Inhaftierung zu erfolgen hat, festgehalten, wobei die EPR als ausdrückliche Ausnahme vorsehen, dass eine solche dann unterbleiben kann, wenn sie offensichtlich unnötig („obviously unnecessary") sei (Regel 42. 1). Ausdrücklich verankert wird in den EPR anders als noch in den ESMR - sodann das Vertraulichkeitsprinzip (Regel 42.3).

780 Resolution (73) 5, Standard Minimum Rules for the Treatment of Prisoners. 
Damit ist sowohl in den ESMR als auch in den EPR (hier mit der oben genannten Ausnahme) eine obligatorische Eintrittsuntersuchung vorgesehen, wobei die diesbezügliche Einwilligung des Strafgefangenen in beiden Regelwerken nicht als erforderlich erachtet wird.

Die ESMR halten sodann fest, dass keine medizinischen oder wissenschaftlichen Versuche durchgeführt werden dürfen, welche zu physischen oder psychischen Beeinträchtigungen des Inhaftierten führen könnten (Regel 22). Die EPR gehen noch weiter und halten zunächst fest, dass Versuche nie ohne Einwilligung des Inhaftierten erfolgen dürfen (Regel 48.1). Versuche, welche zu physischen oder psychischen Beeinträchtigungen des Inhaftierten führen könnten, sind zu verbieten (Regel 48.2).

Für die Gesundheitsversorgung während der Dauer der Haft sehen die ESMR neben dem Äquivalenzprinzip einige spezifische Garantien vor, wie beispielsweise der Umgang mit schwangeren Inhaftierten. Insgesamt orientieren sich die ESMR allerdings hauptsächlich am Äquivalenzprinzip. Weiter sehen auch die ESMR wie bereits die UN-Mindestgrundsätze von 1955 Informationspflichten des Arztes gegenüber der Gefängnisdirektion vor. So hat der Arzt Informationen, die den Fortbestand der Haftfähigkeit betreffen, der Gefängnisdirektion weiterzuleiten (Regel 25 Abs.2). Weiter sehen auch die ESMR vor, dass dem Arzt eine wichtige Hinweisfunktion bezüglich weiterer materieller Haftumstände zukommt (Regel 26 Abs. 1 lit. a-e). Der Arzt hat regelmässig die Nahrung, die Sauberkeit und Hygiene, die sanitären Umstände, die Lüftung und Heizung, die Eignung und Sauberkeit der Kleidung und Bettwäsche sowie bisweilen auch die Regeln bei der körperlichen Betätigung und beim Sport zu betrachten und der Direktion Rückmeldung und diesbezügliche Ratschläge zu erteilen. Auch der Mechanismus für den Umgang mit diesen Empfehlungen des Arztes ist derselbe wie bei den UN-Mindestgrundsätzen.

Die Regeln der EPR sind gegenüber den ESMR wesentlich ausdifferenzierter und vertiefter. Einzelne Empfehlungen betreffen etwa den Umgang mit psychisch kranken oder suizidalen Inhaftierten (Regeln 47.1 und 47.2) und mit drogen-, medikamenten- und alkoholabhängigen Strafgefangenen (Regel 42.3 lit. d) oder die (medizinische) Überwachung von Inhaftierten in Einzelhaft (Regeln 43.2). Auch in den EPR wird an den Informationspflichten und der Hinweisfunktion des Arztes gegenüber der Gefängnisdirektion festgehalten (Regeln 43.3 und 44). Gleichwohl führen die EPR anders als die ESMR - den Grundsatz der Vertraulichkeit nun explizit auf (Regel 42.3).

Hinsichtlich des Fortbestands der Gesundheitsversorgung nach Beendigung des Strafvollzugs führen die EPR neu an, dass der Inhaftierte auf 
entsprechendes Verlangen hin auch bei seiner Entlassung medizinisch zu untersuchen sei (Regel 42.2). Mit dem Einverständnis des Inhaftierten sollen auch Arrangements mit Institutionen ausserhalb des Vollzugs getroffen werden können, um den Fortbestand einer Behandlung nach der Entlassung zu garantieren (Regel 42.3 lit. j).

Zusammenfassend kann damit festgehalten werden, dass die ESMR sich sehr stark an den UN-Mindestgrundsätzen von 1955 orientiert und diese teilweise fast wörtlich übernommen hat. Mit Blick auf die Entwicklung von den ESMR hin zu den EPR kann festgehalten werden, dass eine deutliche Ausdifferenzierung, einhergehend mit einem Ausbau der Garantien rund um die medizinische Versorgung, stattgefunden hat. Auffallend ist dabei, dass bei den ESMR das Äquivalenzprinzip noch der beherrschende Grundsatz der Empfehlungen betreffend die Gesundheitsversorgung im Strafvollzug insgesamt war, wobei dieser lediglich durch einzelne spezifische Garantien ergänzt wurde. Im Vergleich dazu rückte der Gleichwertigkeitsgrundsatz bei den EPR etwas in den Hintergrund - wenngleich er nach wie vor Geltung beansprucht -, während zahlreiche Einzelgarantien in den Vordergrund gelangten. Ursächlich dafür dürfte die Implementierung einzelner in der Zwischenzeit ergangener Empfehlungen auf Europaratsebene in die EPR sein, nicht zuletzt die Empfehlung R (98) 7 (vgl. zur Empfehlung R (98) 7 sogleich). Auch deren ethische Grundsätze, wie etwa der Vertraulichkeitsgrundsatz bei der medizinischen Behandlung, fanden nun Eingang in die EPR. Weiter konnte festgestellt werden, dass neu auch eine über den Haftvollzug hinaus dauernde medizinische Versorgung thematisiert wird, was insbesondere mit Blick auf die Langfristigkeit des Behandlungserfolgs zu begrüssen ist.

\section{Weitere Europaratsempfehlungen}

Der Europarat hat neben den Europäischen Mindestgrundsätzen für die Behandlung der Gefangenen eine Reihe weiterer Empfehlungen erlassen, welche auch bereits Eingang in die überarbeiteten Europäischen Strafvollzugsgrundsätze aus dem Jahr 2006 gefunden haben. Diese Empfehlungen setzen sich mit der Gesundheitsversorgung im Strafvollzug im Allgemeinen und konkret in Bezug auf einzelne Problembereiche der medizinischen Versorgung im Vollzug auseinander. $\mathrm{Zu}$ den umfassenden Empfehlungen zählt etwa die Empfehlung R (98) 7 über die ethischen und organi- 
satorischen Aspekte der Gesundheitsversorgung im Gefängnis. ${ }^{781}$ Bei den bereichsspezifischen Empfehlungen ist es etwa die Empfehlung R (93) 6 des Ministerkomitees betreffend strafvollzugsrechtliche und kriminologische Aspekte der Bekämpfung übertragbarer Krankheiten - einschliesslich AIDS - und die damit zusammenhängenden Gesundheitsprobleme im Strafvollzug.

Die Empfehlung R (98) 7 nimmt Bezug auf einige der grundsätzlichen Prinzipien. So ist etwa der Grundsatz der Äquivalenz ${ }^{782}$ verankert. In der Folge wird weiter festgehalten, dass verschiedene Erwartungen an den Gefängnisarzt seitens der Strafvollzugsadministration und der Strafgefangenen die strikte Einhaltung ethischer Richtlinien umso wichtiger machen. ${ }^{783}$ Schliesslich finden auch die Grundsätze der Freiwilligkeit bzw. des informed consent und der Vertraulichkeit Eingang in diese Empfehlung. ${ }^{784}$ Neben dem Postulat der Einhaltung dieser fundamentalen Prinzipien werden in ihr aber auch erstaunlich konkrete Empfehlungen statuiert. So wird beispielsweise festgehalten, dass eine Schutzimpfung gegen Hepatitis B sowohl für die Inhaftierten als auch für das Personal verfügbar sein soll (Regel 42). Weitere spezifische Themen, mit denen sich diese Empfehlung detailliert auseinandersetzt, sind etwa der Umgang mit drogen-, alkohol- und medikamentenabhängigen Inhaftierten (Regeln $43 \mathrm{ff}.)^{785}$ oder mit psychisch kranken oder suizidalen Strafgefangenen (Regeln $52 \mathrm{ff}$.). Weiter werden insbesondere auch Richtlinien aufge-

781 Recommendation R (98) 7 Concerning the Ethical and Organisational Aspect of Health Care in Prison.

782 Recommendation R (98) 7 Concerning the Ethical and Organisational Aspect of Health Care in Prison, Präambel: „Aware that the respect for the fundamental rights of prisoners entails the provision to prisoners of preventive treatment and health care equivalent to those provided to the community in general", vgl. auch weiter die Regeln $10 \mathrm{ff}$.

783 Recommendation R (98) 7 Concerning the Ethical and Organisational Aspect of Health Care in Prison, Präambel: „Recognising that the medical practitioner in prison often faces difficult problems which stem from conflicting expectations from the prison administration and prisoners, the consequences of which require that the practitioner should adhere to very strict ethical guidelines [...]."

784 Recommendation R (98) 7 Concerning the Ethical and Organisational Aspect of Health Care in Prison: Zum informed consent und der Vertraulichkeit vgl. insb. Regeln 13 ff.

785 Recommendation R (98) 7 Concerning the Ethical and Organisational Aspect of Health Care in Prison: Die Empfehlung nimmt hier mithin auch Bezug auf die Empfehlungen der „Cooperation Group to Combat Drug Abuse and Illicit Trafficking in Drugs; Pompidou Group“. 
stellt, wie mit Gefangenen umzugehen ist, die eine Behandlung ablehnen oder die in den Hungerstreik treten (Regeln 60 ff.)

\section{Zwischenfazit}

Es kann festgehalten werden, dass die betrachteten Regelwerke allesamt auf dem Grundsatz der Äquivalenz aufbauen, über diesen hinaus aber konkrete inhaltliche Vorgaben zur Gewährleistung einer angemessenen Gesundheitsversorgung machen, wobei die Ausdifferenziertheit dieser Garantien variiert. Insgesamt ist festzustellen, dass beim Thema der Gesundheitsversorgung keine Scheu vor konkreten inhaltlichen Empfehlungen besteht. Auch die medizinethischen Grundsätze der Freiwilligkeit und Vertraulichkeit haben mit der Zeit an Bedeutung gewonnen und wurden als Leitprinzipien aufgenommen, wenngleich Ausnahmen von diesen zulässig bleiben. Es kann sodann eine Tendenz erkannt werden, dass eine Einwilligung zwingend vorauszusetzen ist, wenn es um medizinische oder wissenschaftliche Versuche geht. Damit wird dem Strafgefangenen im besonders sensiblen Bereich der Gesundheitsversorgung jedenfalls Schutz vor einer Instrumentalisierung gewährt.

Was die Statuierung des Äquivalenzprinzips betrifft, beschäftigt sich keines der Regelwerke näher mit dem anzuwendenden Referenzmassstab. Aus den einzelnen Normen lässt sich jeweils lediglich (meist nur implizit) entnehmen, dass sich der Massstab meist am Standard einer wohl durchschnittlichen zivilen Institution (Krankenhaus) zu orientieren hat. Eine abschliessende Festlegung eines solchen Massstabes erscheint vor dem Hintergrund der einzelnen konkreten, themenspezifischen Garantien allerdings auch nur zweitrangig; die Prinzipien (insbesondere das Äquivalenzprinzip) sind denn auch mehr als Auslegungsdirektive der spezifischen Garantien, und nicht etwa für die Festsetzung des konkreten Standards, heranzuziehen.

IV. Rechtsprechung des EGMR - Analyse und Kritik

Die Auswirkungen ungenügender medizinischer Versorgung im Strafvollzug auf den Einzelnen wurden bereits aufgezeigt; damit liegt die Relevanz 
von Art. 3 EMRK auf der Hand. ${ }^{786}$ Analog zur Analyse der Regelungsinstrumente wird auch die Rechtsprechung des EGMR in drei Phasen gegliedert: den Zeitpunkt des Eintritts in den Strafvollzug, die Zeit während des Strafvollzugs und die Phase nach Beendigung des Strafvollzugs. Besondere Beachtung kommt - wie auch schon beim vorangehenden Regelinstrumentarium - in prüfungsmethodologischer Hinsicht dem Verhältnis zwischen einem prinzipiengetragenen Massstab (etwa Äquivalenzprinzip, Freiwilligkeitsprinzip, Vertrauensprinzip und Vertraulichkeit) und einem Massstab mit spezifischen Einzelgarantien zu.

\section{Genese der Rechtsprechung - materieller Kern des Standards}

Hinsichtlich einer Eintrittsuntersuchung sieht der EGMR in Bezug auf Art. 3 EMRK kein Obligatorium im eigentlichen Sinne vor, er knüpft aber prozedurale resp. beweisrechtliche Folgen für den Konventionsstaat an ein Unterlassen einer solchen Untersuchung bzw. an eine mangelhafte Dokumentation der erfolgten Untersuchung. ${ }^{787}$ Dem Inhaftierten kann in einem solchen Fall bei einer allfälligen Beschwerde in der Regel nicht entgegengehalten werden, er habe schon vor Haftantritt an einer bestimmten Erkrankung gelitten. ${ }^{788}$

Während des Strafvollzugs gilt, dass bei Krankheit des Inhaftierten aus Art. 3 EMRK grundsätzlich kein Anspruch auf Entlassung aus humanitären Gründen erwächst. Die Notwendigkeit einer Entlassung aus dem Vollzug aufgrund des Gesundheitszustandes des Inhaftierten ist nur in Ausnahmefällen von Art. 3 EMRK umfasst. ${ }^{789}$ Eine Entlassung erfordert, dass der Gesundheitszustand mit einer Inhaftierung überhaupt nicht (mehr) vereinbar ist. ${ }^{790}$ Die Vereinbarkeit der Inhaftierung mit dem Gesundheits-

786 Neben Art. 3 EMRK kann im Falle des Todes des Inhaftierten aufgrund der mangelhaften Gesundheitsversorgung im Strafvollzug auch eine Verletzung von Art. 2 EMRK vorliegen. Vgl. etwa EGMR - Tarariyeva/RUS, Urt. v. 14.12.2006, 4353/03, Rn. 76 ff., Rn. 89.

787 EGMR - Salmanoğlu u. Polattaş/TUR, Urt. v. 17.03.2009, 15828/03, Rn. 80 ff.

788 EGMR - Türkmen/TUR, Urt. v. 19.12.2006, 43124/98, Rn. 42 ff.; EGMR - Carabulea/ROM, Urt. v. 13.07.2010, 45661/99 Rn. 113.

789 EGMR - Khudobin/RUS, Urt. v. 26.10.2006, 59696/00, Rn. 92.

790 Vgl. etwa EGMR - Khudobin/RUS, Urt. v. 26.10.2006, 59696/00, Rn. 92. 
zustand prüft der EGMR anhand dreier Kriterien: ${ }^{791}$ Ausgangspunkt ist der Gesundheitszustand des Strafgefangenen ${ }^{792}$, weiter zu berücksichtigen ist die Adäquanz der Gesundheitsversorgung im Vollzug und schliesslich die Zweckdienlichkeit der Aufrechterhaltung der Inhaftierung mit Blick auf den Gesundheitszustand. ${ }^{793}$ Die Adäquanz der Gesundheitsversorgung im Vollzug ist damit mit Blick auf eine allfällige Entlassung eines der massgeblichen Entscheidungskriterien.

Die Adäquanz ist aber nicht nur für die Frage des Bestehens eines Anspruchs des Inhaftierten auf Entlassung von gewichtiger Relevanz; Art. 3 EMRK verbrieft darüber hinaus vielmehr allgemein einen Anspruch auf eine adäquate Gesundheitsversorgung während des Strafvollzugs. ${ }^{794}$ Die Festsetzung, was eine adäquate Gesundheitsversorgung beinhaltet, ist auch für den EGMR die grösste Herausforderung. ${ }^{795}$

Zur Frage, ob eine angemessene Gesundheitsversorgung nach dem vergleichenden Massstab der Äquivalenz zu beurteilen sei, hielt der Gerichtshof fest, dass das CPT zwar das Äquivalenzprinzip proklamiere, der Gerichtshof sich jedoch nicht immer nach diesem richte. ${ }^{796}$ So urteilte der

791 EGMR - Mouisel/FRA, Urt. v. 14.11.2002, 67263/01, Rn. 40 ff.; EGMR - Gelfmann/FRA, Urt. v. 14.12.2004, 25875/03; EGMR - Khudobin/RUS, Urt. v. 26.10.2006, 59696/00, Rn. 92.

792 EGMR - Mouisel/FRA, Urt. v. 14.11.2002, 67263/01, Rn. 42; EGMR - Khudobin/ RUS, Urt. v. 26.10.2006, 59696/00, Rn. 92.

793 EGMR - Khudobin/RUS, Urt. v. 26.10.2006, 59696/00, Rn. 92; EGMR - Hüseyin Yildirim/TUR, Urt. v. 03.05.2007, 2778/02, Rn. 74; EGMR - Dybeku/ALB, Urt. v. 18.12.2007, 41153/06, Rn. 41.

794 EGMR - Pakhomov/RUS, Urt. v. 30.09.2010, 44917/08, Rn. 61; EGMR - Yevgeniy Alekseyenko/RUS, Urt. v. 27.01.2011, 41833/04, Rn.99; EGMR (GK) - Kudtal RUS, Urt. v. 26.10.2000, 30210/96, Rn.93; EGMR - Khudobin/RUS, Urt. v. 26.10.2006, 59696/00, Rn.93; EGMR - Kalashnikov/RUS, Urt. v. 15.07.2002, 47095/99, Rn.95; EGMR - Mouisel/FRA, Urt. v. 14.11.2002, 67263/01, Rn. 40; EGMR - Kaprykowski/POL, Urt. v. 03.02.2009, 23052/05, Rn. 69.

795 EGMR - Pakhomov/RUS, Urt. v. 30.09.2010, 44917/08, Rn. 62; EGMR - Yevgeniy Alekseyenko/RUS, Urt. v. 27.01.2011, 41833/04, Rn. 100; EGMR - Wenner/ GER, Urt. v. 01.09.2016, 62303/13, Rn. 55; EGMR (GK) - Blokhin/RUS, Urt. v. 23.03.2016, 47152/06, Rn. 137: „The adequacy of medical assistance remains the most difficult element to determine."

796 Dies habe vor allem hinsichtlich verurteilter Straftäter (im Unterschied zu Untersuchungsgefangenen) zu gelten: EGMR - Pakhomov/RUS, Urt. v. 30.09.2010, 44917/08, Rn. 62: „The CPT proclaimed the principle of the equivalence of health care in prison with that in the outside community. However, the Court does not always adhere to this standard, at least when it comes to medical assistance for convicted prisoners (as opposed to those in pre-trial detention)."; vgl. auch EGMR - Aleksanyan/RUS, Urt. v. 22.12.2008, 46468/06. 
Gerichtshof im Einzelfall etwa, dass die Gesundheitsversorgung, wie sie dem Inhaftierten zuteil wurde, zwar nicht äquivalent zu jener ausserhalb des Strafvollzugs, aber dennoch im konkreten Fall angemessen und somit konform mit Art. 3 EMRK gewesen sei. ${ }^{797}$ Gelegentlich statuierte der EGMR gleichwohl, dass eine angemessene medizinische Behandlung etwa dann vorliegt, wenn sie das Niveau erreicht, das gleichwertig mit jenem ist, welches der Staat der Gesellschaft als Ganzes zur Verfügung stellt. ${ }^{798}$ Art. 3 EMRK garantiert sodann nicht jedem Strafgefangenen diejenige medizinische Versorgung, welche in der besten zivilen Institution verfügbar wäre. ${ }^{799}$ Der Gerichtshof akzeptiert prinzipiell, dass die Ressourcen medizinischer Einrichtungen innerhalb des Strafvollzugs gegenüber jenen ausserhalb des Vollzugs eingeschränkt sind. ${ }^{800}$ Ein grundsätzlicher Anspruch auf eine Verlegung in ein Krankenhaus besteht nicht. ${ }^{801}$

Jenseits dieses vergleichenden Massstabes hält der EGMR fest, dass eine medizinische Behandlung im Sinne von Art. 3 EMRK dann angemessen sei, wenn eine prompte Diagnose und eine akkurate Behandlung erfolgen. ${ }^{802}$ Ersteres fordert als zeitliche Komponente ein sofortiges bzw. schnellstmögliches Tätigwerden. Im Einzelfall urteilte der EGMR diesbezüglich etwa, dass dieses Erfordernis dann nicht erfüllt sei, wenn die Konsultation des

797 EGMR - Dankevich/UKR, Urt. v. 29.04.2003, 40679/98, Rn. 135: „[...] on the basis of the evidence given by the prison doctor it considers that the medical and dental care provided by the prison's medical unit was adequate, although certainly not of the same standard as the treatment provided outside the prison environment."

798 EGMR - Wenner/GER, Urt. v. 01.09.2016, 62303/13, Rn. 55: „[...] the adequacy of medical assistance remains the most difficult element to determine. Medical treatment provided within prison facilities must be appropriate, that is, at a level comparable to that which the State authorities have committed themselves to provide to the population as a whole." Zu berücksichtigen ist bei dieser Aussage, dass es bei bei diesem Entscheid gerade darum ging, eine ausserhalb des Strafvollzugs begonnene Therapie (Substitutionstherapie eines Heroinabhängigen) im Strafvollzug fortzusetzen.

799 EGMR - Pakhomov/RUS, Urt. v. 30.09.2010, 44917/08, Rn. 62.

800 EGMR - Grishin/RUS, Urt. v. 15.11.2007, 30983/02, Rn. 76. Dies ändert allerdings nichts an der Rechtsprechung des Gerichtshofs zur Ressourcenunabhängigkeit des Massstabes des EGMR im Allgemeinen.

801 EGMR (GK) - Kudta/RUS, Urt. v. 26.10.2000, 30210/96, Rn.93; EGMR Kalashnikov/RUS, Urt. v. 15.07.2002, 47095/99, Rn.95.

802 EGMR - Pakhomov/RUS, Urt. v. 30.09.2010, 44917/08, Rn. 62; EGMR - Hummatov/AZE, Urt. v. 29.11.2007, 9852/03 u. 13413/04, Rn. 115; EGMR - Melnik/ UKR, Urt. v. 28.03.2006, 72286/01, Rn. 104 ff.; EGMR - Yevgeniy Alekseyenko/ RUS, Urt. v. 27.01.2011, 41833/04, Rn. 100; EGMR - Lutsenko/UKR, Urt. v. 11.06.2015, 29334/11, Rn. 133. 
Gefängnisarztes erst 14 Tage resp. 9 Tage nach dem Ersuchen nach medizinischem Beistand eines an Tuberkulose erkranktem Strafgefangenen erfolge. ${ }^{803}$ Auch eine Diagnosestellung (wiederum Tuberkulose) erst zweieinhalb Monate nach den ersten Symptomen entspreche nicht einer rechtzeitigen und damit adäquaten Gesundheitsversorgung. ${ }^{804}$ Hinsichtlich der Diagnosestellung fliesst aus Art. 3 EMRK die Pflicht, dem Strafgefangenen seine Diagnose klar zu kommunizieren; der Inhaftierte muss dabei über seinen Gesundheitszustand klar ins Bild gesetzt werden. ${ }^{805}$

Akkurat ist die medizinische Versorgung gemäss EGMR weiter dann, wenn die Behandlung nach einer umfassenden Therapiestrategie erfolgt, welche auf Heilung bzw. auf die Verhinderung einer weiteren Verschlechterung des Gesundheitszustandes ausgerichtet ist, und wenn der Gesundheitszustand regelmässigen und systematischen Kontrollen unterliegt, jedenfalls wenn dies aufgrund der bestehenden Erkrankung notwendig ist. ${ }^{806}$ Beispielsweise kann bei drogenabhängigen Strafgefangenen eine Substitutionsbehandlung angemessen sein. ${ }^{807}$ Die medizinische Versorgung ist ferner nicht schon dann adäquat, wenn der Inhaftierte von einem Arzt untersucht und eine geeignete Behandlung verordnet wurde; vielmehr müssen auch im Strafvollzug die Voraussetzungen geschaffen werden, damit die verordnete Behandlung auch durchgeführt werden kann. ${ }^{808}$ Zudem hat die Behandlung zeitnah zu erfolgen, was selbst dann

803 EGMR - Hummatov/AZE, Urt. v. 29.11.2007, 9852/03 u. 13413/04, Rn. 115.

804 EGMR - Melnik/UKR, Urt. v. 28.03.2006, 72286/01, Rn. $104 \mathrm{ff}$.

805 EGMR - Testa/CRO, Urt. v. 12.07.2007, 20877/04, Rn. 52. Die Kenntnis über Diagnose mit all ihren Folgen ist sodann auch als Grundvoraussetzung für eine selbstbestimmte Einwilligung (informed consent) zu sehen.

806 EGMR - Pakhomov/RUS, Urt. v. 30.09.2010, 44917/08, Rn. 62; EGMR - Yevgeniy Alekseyenko/RUS, Urt. v. 27.01.2011, 41833/04, Rn. 100; EGMR - Hummaov/ AZE, Urt. v. 29.11.2007, 9852/03 u. 13413/04, Rn. 109, 114; EGMR - Lutsenko/ UKR, Urt. v. 11.06.2015, 29334/11, Rn. 133.

807 EGMR - Wenner/GER, Urt. v. 01.09.2016, 62303/13, Rn. 59: Der Inhaftierte war Jahrzehnte lang heroinabhängig und während 17 Jahren in Substitutionsbehandlung. Der EGMR stellte fest, dass der Abbruch dieser Behandlung zu abrupt erfolgt sei. Das Leid des Strafgefangenen durch die Entzugserscheinungen sei durch den Umstand verstärkt worden, dass er um die Möglichkeit einer Substitutionsbehandlung wusste. Damit wird deutlich, dass auch der Kontinuität einer bereits begonnenen Behandlung im Strafvollzug Rechnung zu tragen ist. Hinsichtlich des Erfordernisses der Kontinuität einer ausserhalb des Strafvollzugs begonnenen Therapie im Allgemeinen vgl. auch EGMR - Khayledinov/ RUS, Urt. v. 12.01.2016, 2763/13, Rn. 73.

808 EGMR - Hummatov/AZE, Urt. v. 29.11.2007, 9852/03 u. 13413/04, Rn. 116: Im konkreten Fall wurden eine Diät und warme Sitzbäder verordnet, ohne 
gilt, wenn es sich im konkreten Fall weder um einen Notfall noch um anhaltende Schmerzen handelt. ${ }^{809}$ Die medizinische Behandlung darf dem Inhaftierten grundsätzlich auch nicht mit dem Argument vorenthalten werden, dass von ihm eine Gefahr ausgehe. ${ }^{810}$ Werden dem Inhaftierten indes Möglichkeiten angeboten, hat er diese auch wahrzunehmen. ${ }^{811}$

Liegen hinsichtlich der notwendigen Behandlung zwei divergierende medizinische Ansichten vor, hält der EGMR fest, dass aus Art. 3 EMRK eine positive Verpflichtung fliessen kann, eine zusätzliche Meinung von einem Spezialisten einzuholen. ${ }^{812}$ Es besteht weiter - wie schon hinsichtlich der Eintrittsuntersuchung - eine Dokumentationspflicht über die medizinischen Belange. ${ }^{813}$

Hinsichtlich der Vertraulichkeit im Bereich der Gesundheitsversorgung im Strafvollzug hält der EGMR fest, dass eine medizinische Untersuchung ausser Hör- und vorzugsweise auch ausser Sichtweite von Vollzugsbeam-

dass diese Massnahmen näher umschrieben wurden (Art, Länge, Dauer). Die Befolgung der Therapie war zudem schon deshalb nicht gewährleistet, weil dem Inhaftierten zum einen seitens der Strafvollzugsbehörden keine speziellen Diätmahlzeiten zur Verfügung gestellt wurden, und zum anderen, da der Inhaftierte in seiner Zelle nicht über warmes Wasser verfügte und er nur ein Mal pro Woche die Möglichkeit hatte zu duschen.

809 EGMR - Ashot Harutyunyan/ARM, Urt. v. 15.06.2010, 34334/04, Rn. 114: Der Umstand, dass der Inhaftierte um eine medizinische Versorgung ersuchte und eine solche auch benötigte, diese für ihn (zu diesem Zeitpunkt) aber nicht verfügbar war, genügte, um eine Verletzung von Art. 3 EMRK festzustellen.

810 SK-StPO/Meyer Art. 3 EMRK Rn. 33. Der vom Inhaftierten ausgehenden Gefahr ist mit anderen Mitteln zu begegnen, wobei Zwangsmittel ihrerseits an Art. 3 EMRK zu messen sind. Vgl. etwa EGMR - Henaf/FRA, Urt. v. 27.11.2003, 65436/01; EGMR - Mouisel/FRA, Urt. v. 14.11.2002, 67263/01.

811 EGMR - Pandjikidze/GEO, Entsch. v. 20.06.2006, 30323/02; EGMR (GK) Kudta/POL, Urt. v. 26.10.2000, 30210/96, Rn. 96; EGMR - Tymoshenko/UKR, Urt. v. 30.04.2013, 49872/11, Rn. 217 ff.

812 EGMR - Wenner/GER, Urt. v. 01.09.2016, 62303/13, Rn. 57; vgl. auch EGMR Xiros/GRE, Urt. v. 09.09.2010, 1033/07, Rn. 87 und 89f.; EGMR - Budanov/ RUS, Urt. v. 09.01.2014, 66583/11, Rn. 73; EGMR - Khudobin/RUS, Urt. v. 26.10.2006, 59696/00: Das Fehlen einer qualifizierten und rechtzeitigen ärztlichen Versorgung sowie die Ablehnung einer unabhängigen medizinischen Untersuchung müssten beim Inhaftierten unweigerlich starke Unsicherheiten ausgelöst haben. Vgl. weiter EGMR - Xiros/GRE, Urt. v. 09.09.2010, 1033/07.

813 Vgl. etwa EGMR - Salmanoglu u. Polatta/TUR, Urt. v. 17.03.2009, 15828/03, Rn.76ff.; vgl. auch EGMR - Keenan/GBR, Urt. v. 03.04.2001, 27229/95, Rn. 114: Der EGMR hielt fest, dass die medizinische Dokumentation nicht ausreichen würde, so dass nicht genügend nachgewiesen sei, dass genügende Abklärungen getroffen worden seien, bevor man den suizidgefährdeten Inhaftierten in Einzelhaft versetzt habe. 
ten zu erfolgen hat. ${ }^{814}$ Ausnahmen von diesem Grundsatz sollen jedenfalls dann möglich sein, wenn der Arzt aus Sicherheitsgründen darum ersucht. ${ }^{815}$ Weiter hält der EGMR fest, dass medizinische Untersuchungen einzeln (und nicht etwa gruppenweise) zu erfolgen haben. ${ }^{816}$ Dem Vertrauensverhältnis zwischen Arzt und inhaftiertem Patienten trägt der EGMR insoweit Rechnung, dass der Wunsch des inhaftierten Patienten zum Beizug eines externen Arztes (Vertrauensarztes) grundsätzlich respektiert werden sollte. ${ }^{817}$ Sofern diesem Beizug jedoch kein genuin medizinischer Grund zukommt, ist es allerdings zulässig, dass der Inhaftierte die zusätzlichen Kosten zu tragen hat. ${ }^{818}$

Auch im Strafvollzug geht der EGMR im Grundsatz davon aus, dass eine medizinische Behandlung des Einverständnisses des urteilsfähigen Patienten bedarf. Gleichwohl können bzw. müssen ausnahmsweise medizinische Eingriffe auch gegen den Willen des Strafgefangenen durchgeführt werden. Hierbei sind vorab zwei Konstellationen zu unterscheiden: (1) Der medizinische Eingriff erfolgt als Zwangsmassnahme etwa zur Sicherung von Beweisen (wohl insbesondere bei Untersuchungsgefangenen) oder (2) dieser verfolgt einen wie auch immer gearteten therapeutischen Zweck. Aufgrund des vorliegenden Untersuchungsgegenstands steht hier die zweite Konstellation im Vordergrund.

Der EGMR hatte sich insbesondere mit der Frage der Zulässigkeit einer Zwangsernährung eines sich im Hungerstreik befindenden Inhaftierten auseinanderzusetzen, ${ }^{819}$ wobei er einerseits das Selbstbestimmungsrecht des Inhaftierten zu respektieren und andererseits der besonderen Fürsorgepflicht des Staates gegenüber dem Strafgefangenen ${ }^{820}$ Rechnung zu tragen hat. Zur Lösung dieses Interessenkonflikts stellte der EGMR ausdifferenzierte Kriterien auf: So ist eine Zwangsernährung etwa dann keine Verletzung von Art. 3 EMRK, wenn sie dokumentierterweise medizinisch notwendig ist, eine ständige medizinische Beobachtung und Versorgung garantiert sind, die Art der Durchführung als solche Art. 3 EMRK nicht ver-

814 EGMR - Salmanoglu u. Polatta/TUR, Urt. v. 17.03.2009, 15828/03, Rn. 80.

815 EGMR - Salmanoglu u. Polatta/TUR, Urt. v. 17.03.2009, 15828/03, Rn. 83.

816 EGMR - Salmanoglu u. Polatta/TUR, Urt. v. 17.03.2009, 15828/03, Rn. 80.

817 EGMR - Mathew/NED, Urt. v. 29.09.2005, 24919/03, Rn. 187.

818 EGMR - Mathew/NED, Urt. v. 29.09.2005, 24919/03, Rn. 187.

819 Vgl. etwa EGMR - Rappaz/SUI, Entsch. v. 26.03.2013, 73175/10, Rn. 60 ff.

820 EGMR - Rappaz/SUI, Entsch. v. 26.03.2013, 73175/10, Rn. 62; EGMR - Tekin Yildiz/TUR, Urt. v. 10.11.2005, 22913/04, Rn. 82; vgl. dazu auch SK-StPO/ Meyer Art. 3 EMRK Rn. 41. 
letzt ${ }^{821}$ und wenn verfahrensrechtliche Massnahmen getroffen wurden, um der Selbstbestimmung sowie der physischen Integrität des Strafgefangenen Beachtung zu schenken. ${ }^{822}$ Von besonderer Bedeutung ist bei Letzterem auch die eigentliche Motivation des Inhaftierten zum Hungerstreik; zielt dieser auf die Änderung seiner Haftsituation (sei es die Entlassung oder die „Erpressung besserer Haftbedingungen") ab, haben die positivrechtlichen Verpflichtungen von Art. 3 EMRK umso deutlicher zu greifen. ${ }^{823}$

Hinsichtlich des Freiwilligkeitsprinzips führt die Missachtung des Willens des Inhaftierten auch bei einer medizinischen Massnahme damit nicht zwingend zu einer Verletzung von Art. 3 EMRK. Eine zwangsweise Behandlung gegen den autonomen Willen des Inhaftierten kann sich unter Einhaltung der oben genannten Voraussetzungen - aufgrund der besonderen Fürsorgepflicht sogar aufdrängen.

Weiter ist an die Konstellation zu denken, in welcher der Inhaftierte beispielsweise aufgrund des Vorliegens einer psychischen Erkrankung nicht (mehr) in der Lage ist, eine Entscheidung zu treffen. ${ }^{824}$ Es ist dann an den Behörden, anhand der anerkannten Regeln der medizinischen Wissenschaft über die angemessene Behandlung zu entscheiden, welche, wenn erforderlich, auch mittels Zwang durchzuführen ist. ${ }^{825}$ Als allgemeine Regel hält der Gerichtshof fest, dass eine medizinische Behandlung, welche nach anerkannten medizinischen Grundsätzen notwendig ist, grundsätzlich Art. 3 EMRK nicht verletzen kann. ${ }^{826}$

Die Grundgedanken dieser mittlerweile konsolidierten Rechtsprechung können auch in Bezug auf anders geartete therapeutische Zwangsbehandlungen analog hinzugezogen werden. $\mathrm{Zu}$ denken ist insbesondere an die zwangsweise Behandlung ansteckender Krankheiten oder die Behandlung eines Inhaftierten mit erhöhtem Gefährdungspotential, wobei hier das

821 Dazu gehört etwa, dass die Zwangsernährung auf nicht demütigende Weise, d.h. ohne unnötigen und unangemessenen Zwang, zu erfolgen hat. Vgl. im Einzelnen dazu SK-StPO/Meyer Art. 3 EMRK Rn. 40.

822 Vgl. etwa EGMR - Nevmerzhitsky/UKR, Urt. v. 05.04.2005, 54825/00, Rn. 94; EGMR - Ciorap/MDA, Urt. v. 19.06.2007, 12066/02; im Einzelnen auch: SKStPO/Meyer Art. 3 EMRK Rn. 40.

823 Vgl. auch SK-StPO/MeYer Art. 3 EMRK Rn. 41.

824 Vgl. etwa EGMR - M.S./CRO, Urt. v. 19.02.2015, 75450/12, Rn. 98.

825 EGMR - M.S./CRO, Urt. v. 19.02.2015, 75450/12, Rn. 98.

826 EGMR - M.S./CRO, Urt. v. 19.02.2015, 75450/12, Rn. 98; EGMR (GK) - Jalloh/ GER, Urt. v. 19.06.2007, 12066/02, Rn. 69; EGMR - Herczegfalvy/AUT, Urt. v. 24.09.1992, 10533/83, Rn. 82; EGMR - Nevmerzhitsky/UKR, Urt. v. 05.04.2005, 54825/00, Rn. 94; EGMR - Özgül/TUR, Entsch. v. 06.03.2007, 7715/02; vgl. auch SK-StPO/MeYer Art. 3 EMRK Rn. 40. 
Drittinteresse - sei es der Mitinhaftierten oder der Vollzugsbeamten - als weitere zu beachtende Komponente hinzutritt.

Hinsichtlich der Zeit nach dem Vollzug hatte sich der EGMR weniger mit der Frage zu beschäftigen, ob eine fortwirkende Verpflichtung zur Gesundheitsversorgung nach der Entlassung besteht ${ }^{827}$, als vielmehr mit der oben bereits aufgeworfenen Frage, wann eine Entlassung aufgrund des Gesundheitszustands notwendig ist, wenn die angemessene Gesundheitsversorgung im Strafvollzug nicht mehr gewährleistet werden kann und die Weiterführung des Vollzugs folglich gegen Art. 3 EMRK verstösst. ${ }^{828}$ Bei der Beurteilung dieser Frage berücksichtigt der EGMR - neben den oben genannten drei Hauptkriterien - den Krankheitsverlauf, die Möglichkeit einer bedingten Entlassung sowie das Verhalten des Inhaftierten (insbesondere hinsichtlich seiner Kooperation mit der Ärzteschaft). 829

Zusammenfassend kann festgehalten werden, dass sich Art. 3 EMRK an den Grundprinzipien der Freiwilligkeit resp. des informed consent, der Vertraulichkeit der medizinischen Versorgung und der Äquivalenz der Versorgung inner- und ausserhalb des Strafvollzugs orientiert. Gleichwohl hat sich eindrücklich gezeigt, dass der EGMR unter dem Gesichtspunkt von Art. 3 EMRK Einschränkungen dieser Grundsätze zulässt und so den besonderen Gegebenheiten des Strafvollzugs Rechnung trägt. Gleichzeitig belässt es der EGMR nicht dabei, den Inhalt von Art. 3 EMRK auf diese Grundprinzipien zu stützen; vielmehr verlangt er unter Art. 3 EMRK eine angemessene Gesundheitsversorgung, wobei er diesen unbestimmten

827 Gemeint ist eine Entlassung aus dem Strafvollzug als solchen. Ganz anders ist die Konstellation zu beurteilen, wenn es sich um eine Verlegung in eine andere Vollzugsanstalt geht. Bei Letzterem gilt die besondere Fürsorgepflicht mit den damit einhergehenden positivrechtlichen Pflichten selbstverständlich weiter: Eine Kontinuität der medizinischen Versorgung muss damit grundsätzlich gewährleistet werden: vgl. etwa EGMR - Khayledinov/RUS, Urt. v. 12.01.2016, 2763/13, Rn. 73: „The Court reiterates that ensuring continuity of care between the community and prison, as well as within the prison system, is a fundamental component of successful treatment." Im konkreten Fall ging es um eine Therapie, welche durch den Haftantritt unterbrochen wurde.

828 Vgl. etwa EGMR - Mouisel/FRA, Urt. v. 14.11.2002, 67263/01: Der Gesundheitszustand des Strafgefangenen war immer weniger mit der fortdauernden Inhaftierung vereinbar. Frankreich entliess den Strafgefangenen schliesslich bedingt, mit der Auflage, sich in medizinische Behandlung oder Pflege zu begeben. Der EGMR stellte bis zum Zeitpunkt der Entlassung eine Verletzung von Art. 3 EMRK fest.

829 EGMR - Gelfmann/FRA, Urt. v. 14.12.2004, 25875/03; vgl. auch EGMR - Aleksanyan/RUS, Urt. v. 22.12.2008, 46468/06, Rn. 137. 
Rechtsbegriff durch weitere eigene Kriterien und Einzelgarantien gefüllt hat.

2. Dogmatische Einordnung der Gesundheitsversorgung durch den EGMR und Kriterien der Standardbildung

Der Vielgestaltigkeit der Fragestellungen und Fallkonstellationen entsprechend, stellt der Gerichtshof hinsichtlich inadäquater Gesundheitsversorgungen je nach Fall eine erniedrigende ${ }^{830}$ oder bisweilen auch eine erniedrigende und unmenschliche Behandlung ${ }^{831}$ i.S.v. Art. 3 EMRK fest. In aller Regel hält er eindeutig fest, welche spezifische Einzelgarantie von Art. 3 EMRK er prüft und welche Kriterien er zu dieser Prüfung hinzuzieht. Die Entscheidung trifft er dabei immer mit Blick auf die Menschenwürde einerseits und die praktischen Bedürfnisse des Strafvollzugs (,the practical demands of imprisonment ") andererseits. ${ }^{832}$

Bei seiner Prüfung greift der Gerichtshof vorab i.d.R. die allgemeinen Begriffsmerkmale der unmenschlichen oder erniedrigenden Behandlung auf und verweist darüber hinaus auf die für Strafvollzugssituationen aufgestellte Auslegungsdirektive des „no more harm principle“. ${ }^{833}$ Eine unmenschliche Behandlung erkennt der Gerichtshof in Übereinstimmung mit den allgemeinen Begriffsmerkmalen (vgl. Teil 2 Kap. 3 II. 1.) insbesondere in jenen Situationen, in denen der Beschwerdeführer nachweist ${ }^{834}$, dass die inadäquate Gesundheitsversorgung zu einem verstärkten Gefühl der Qual und des körperlichen Leidens geführt hat. ${ }^{835}$ Hinsichtlich der Frage

830 Vgl. etwa EGMR - Hummatov/AZE, Urt. v. 29.11.2007, 9852/03 u. 13413/04, Rn. 120 ff.; EGMR - Sarban/MDA, Urt. v. 04.10.2005, 3456/05, Rn. 90.

831 Vgl. etwa EGMR - Mouisel/FRA, Urt. v. 14. 11.2002, 67263/01, Rn. 4.

832 EGMR - Pakhomov/RUS, Urt. v. 30.09.2010, 44917/08, Rn. 63; EGMR (GK) Blokhin/RUS, Urt. v. 23.03.2016, 47152/06, Rn. 138; EGMR - Aleksanyan/RUS, Urt. v. 22.12.2008, 46468/06, Rn. 140.

833 Vgl. etwa EGMR - Nevmerzhitsky/UKR, Urt. v. 05.04.2005, 54825/00, Rn. 81.

834 Die Anforderungen an den Nachweis richten sich nach den Beweisregeln des EGMR. Dem Beschwerdeführer kommen hier beweisrechtliche Erleichterungen zugute.

835 Vgl. etwa EGMR - Kaprykowski/POL, Urt. v. 03.02.2009, 23052/05, Rn. 75: Der an Epilepsie leidende Beschwerdeführer hatte vorgetragen, dass es durch die Umstellung auf Generika zu einem Anstieg der Zahl seiner täglichen Anfälle gekommen sei und deren Auswirkungen sich verschlimmert hätten. Die Regierung hatte diese Behauptungen des Beschwerdeführers nicht bestritten, weshalb sich der Gerichtshof bei der Prüfung einer unmenschlichen Behandlung i.S.v. 
der erniedrigenden Behandlung stellt der Gerichtshof wiederum in Übereinstimmung mit den allgemeinen Begriffsmerkmalen hauptsächlich darauf $\mathrm{ab}$, ob der Beschwerdeführer durch die ungenügende Behandlung in seiner Persönlichkeit herabgesetzt und ob seine Menschenwürde gewahrt wurde. ${ }^{836}$ Mit Blick auf den Demütigungsmoment erkennt der EGMR etwa eine erniedrigende Behandlung, wenn eine einschneidende Behandlung durchgeführt wird, welche nicht medizinisch oder rechtlich indiziert war; und zwar unabhängig davon, ob im konkreten Fall eine Zustimmung durch den Betroffenen erteilt wurde oder nicht. ${ }^{837}$ Eine medizinische

Art. 3 EMRK auf diese stützte. Vgl. auch EGMR - Hummatov/AZE, Urt. v. 29.11.2007, 9852/03 u. 13413/04, Rn. 121: Der Gerichtshof stellte hier fest, dass aus den vorliegenden Beweisen nicht hervorgehe, dass der Beschwerdeführer während der verfahrensgegenständlichen Zeit einen Rückfall seiner Tuberkuloseerkrankung erlitten habe oder dass das Fehlen einer angemessenen medizinischen Behandlung im Gobustan-Gefängnis erhebliche psychische Leiden verursacht habe, weshalb keine unmenschliche Behandlung i.S.v. Art. 3 EMRK festzustellen sei. Vgl. ferner EGMR - Sarban/MDA, Urt. v. 04.10.2005, 3456/05, Rn. 86: Der Gerichtshof führte aus, dass der Beschwerdeführer nicht geltend gemacht habe, im relevanten Zeitraum einen medizinischen Notfall erlitten oder aufgrund inadäquater medizinischer Unterstützung anderweitig schweren oder langanhaltenden Schmerz erlitten zu haben. Eine unmenschliche Behandlung i.S.v. Art. 3 EMRK liege deshalb nicht vor.

836 EGMR - Hummatov/AZE, Urt. v. 29.11.2007, 9852/03 u. 13413/04, Rn. 121; EGMR - Kaprykowski/POL, Urt. v. 03.02.2009, 23052/05, Rn.76; vgl. auch EGMR - Sarban/MDA, Urt. v. 04.10.2005, 3456/05, Rn. 87 ff.: Der Gerichtshof hält zunächst fest, dass der Beschwerdeführer Kenntnis davon gehabt habe, dass im Falle eines medizinischen Notfalls, welcher aufgrund seiner Gesundheitssituation durchaus hätte auftreten können, keine sofortige medizinische Hilfe verfügbar gewesen wäre. Der Gerichtshof kam sodann zum Schluss, dass dies beim Beschwerdeführer erhebliche Ängste ausgelöst haben müsse. Das Versäumnis, dem Beschwerdeführer eine medizinische Grundversorgung zu gewähren, als er diese eindeutig benötigte und auch beantragt hatte, würden zusammen mit anderen Formen der erlittenen Demütigung (Transport in Handschellen ans Gericht, Gerichtsverhandlung in einem Käfig etc.) eine erniedrigende Behandlung i.S.v. Art. 3 EMRK sein.

837 EGMR - Salmanoglu u. Polatta/TUR, Urt. v. 17.03.2009, 15828/03, Rn. 88: Anlässlich der Eintrittsuntersuchung wurde die erst 16-jährige Beschwerdeführerin einer gynäkologischen Untersuchung unterzogen, bei welcher deren Jungfräulichkeit festgestellt werden sollte. Der Gerichtshof hielt fest, dass nicht nachgewiesen worden war, dass diese Untersuchung auf einer gesetzlichen Grundlage basierte und dass sich die Beschwerdeführerin zu diesem Zeitpunkt noch nicht über sexuelle Übergriffe beschwert hätte. Die Untersuchung an sich könne damit als diskriminierend und erniedrigend betrachtet werden. 
Behandlung gegen den Willen des Betroffenen ist demgegenüber weder unmenschlich noch erniedrigend, wenn sie therapeutisch notwendig war. ${ }^{838}$

Indem der Gerichtshof bei der Beurteilung einer unmenschlichen Behandlung i.S.v. Art. 3 EMRK einen konkreten Nachweis der tatsächlichen Auswirkungen fordert, stellt der Gerichtshof zunächst auf soziomedizinische resp. empirische Kriterien ab. Gleichwohl ist die Feststellung einer unmenschlichen Behandlung i.S.v. Art. 3 EMRK letztlich Ergebnis einer normativen Bewertung dieses Leidens. Nach der Feststellung des konkreten Leidens stellt der Gerichtshof sich nämlich die Frage, ob dieses das erforderliche Mindestmass an Leiden i.S.v. Art. 3 EMRK erreicht oder nicht. Bei der erniedrigenden Behandlung greift von Beginn an eine von der Menschenwürde her gedachte, normative Bewertung der Situation, wobei der Gerichtshof auch dieser soziomedizinische Schlussfolgerungen zugrunde legt, ohne hier aber einen konkreten Nachweis zu fordern. ${ }^{839}$

Zusammenfassend prüft der Gerichtshof bei der Beurteilung der Gesundheitsversorgung die vorgetragene Situation an den je spezifischen Voraussetzungen der erniedrigenden resp. der unmenschlichen Behandlung i.S.v. Art. 3 EMRK. Stellt der Gerichtshof im Ergebnis sowohl eine unmenschliche als auch eine erniedrigende Behandlung fest, ist dies nicht Folge dogmatischer Unschärfe, sondern eine klare und eindeutige Feststellung zu den konkreten Unrechtsgehalten, welche dem Konventionsstaat zur Last gelegt werden. Sowohl die Prüfung der unmenschlichen als auch der erniedrigenden Behandlung i.S.v. Art. 3 EMRK enthält sowohl soziomedizinische als auch normative Elemente. Dies hat aber nicht darüber hinwegzutäuschen, dass die normative Bewertung der soziomedizinischen Elemente auf je anderen Voraussetzungen beruht.

838 EGMR - Nevmerzhitsky/UKR, Urt. v. 05.04.2005, 54825/00, Rn.94; vgl. auch EGMR - Herczegfalvy/AUT, Urt. v. 24.09.1992, 10533/83, Rn. 82.

839 Vgl. etwa EGMR - Hummatov/AZE, Urt. v. 29.11.2007, 9852/03 u. 13413/04, Rn. 121: „[...] the Court considers that the lack of adequate medical treatment in Gobustan Prison must have caused the applicant considerable mental suffering diminishing his human dignity, which amounted to degrading treatment within the meaning of Article 3 of the Convention." 


\section{Prüfungsmethodik des EGMR}

\subsection{Prüfungstiefe und -dichte und Beweislast}

Bei der Beurteilung der Frage, ob eine dem Inhaftierten gewährte Gesundheitsversorgung adäquat und mit Art. 3 EMRK konform ist, nimmt der Gerichtshof sich insoweit zurück, als er unter Hinweis auf seine subsidiäre Rolle festhält, dass es gerade nicht seine Aufgabe sei, Fragen zu beurteilen, welche ausschliesslich im Kompetenzbereich eines medizinischen Spezialisten liegen. ${ }^{840}$ Der EGMR trägt damit insbesondere seiner fehlenden fachlichen Kompetenz bei der Beurteilung der Behandlungsbedürftigkeit oder der Wahl einer konkreten Therapie Rechnung. ${ }^{841}$ Insofern nimmt der EGMR denn auch keine umfassende Prüfung der medizinischen Therapiestrategie vor. Vielmehr begnügt er sich mit einer reduzierten Prüfungstiefe; er nimmt in inhaltlicher Sicht eine reine Willkürkontrolle ${ }^{842}$ vor.

In beweisrechtlicher Hinsicht anerkennt der EGMR die besondere Stellung des Strafgefangenen und insbesondere seine durch die Situation des Strafvollzugs gesteigerte Verletzlichkeit (im Einzelnen Teil 2 Kap. 2 II. 1.1.). ${ }^{843}$ Es liegt deshalb, nachdem der Inhaftierte den Anscheinsbeweis einer inadäquaten medizinischen Versorgung erbracht hat, am Staat, glaubhaft und überzeugend nachzuweisen, dass dem Inhaftierten eine umfassende und angemessene medizinische Versorgung zuteil wurde. ${ }^{844}$

Aus beweisrechtlicher Perspektive ist denn auch das Erfordernis der Einholung einer zusätzlichen unabhängigen Meinung eines Spezialisten zu werten. ${ }^{845}$ Fehlt diese, dürfte dem Konventionsstaat im Streitfall regelmässig der Nachweis misslingen, dass dem Inhaftierten eine umfassende und angemessene medizinische Versorgung gewährt wurde.

840 EGMR - Wenner/GER, Urt. v. 01.09.2016, 62303/13, Rn. 58; EGMR - Sergey Antonov/UKR, Urt. v. 22.10.2015, 40512/13, Rn. 86; EGMR - E.A./RUS, Urt. v. 23.05.2013, 44187/04, Rn. 57; EGMR - Ukhan/UKR, Urt. v. 18.12.2008, 30628/02, Rn. 76.

841 EGMR - Sergey Antonov/UKR, Urt. v. 22.10.2015, 40512/13, Rn. 86.

842 Vgl. auch Ronc S. 226.

843 EGMR - Wenner/GER, Urt. v. 01.09.2016, 62303/13, Rn. 58.

844 EGMR - Wenner/GER, Urt. v. 01.09.2016, 62303/13, Rn. 58; EGMR - Sergey Antonov/UKR, Urt. v. 22.10.2015, 40512/13, Rn. 86. Damit wird vom allgemeinen Grundsatz „affirmanti incumbit probatio“ abgewichen.

845 EGMR - Wenner/GER, Urt. v. 01.09.2016, 62303/13, Rn. 57; vgl. auch EGMR Xiros/GRE, Urt. v. 09.09.2010, 1033/07, Rn. 87 und 89 f.; EGMR - Budanov/RUS, Urt. v. 09.01.2014, 66583/11, Rn. 73. 
In die gleiche Richtung zielt das Erfordernis einer Eintrittsuntersuchung; das Unterlassen oder eine ungenügende Dokumentation ziehen wie bereits aufgezeigt - beweisrechtliche Folgen nach sich, und der EGMR lässt in solchen Konstellationen das Vorbringen des Konventionsstaates, dass eine spezifische Verletzung oder Erkrankung vorbestanden habe, in der Regel nicht genügen.

\section{2 Äquivalenzprinzip vs. Angemessenheitsprüfung}

a Bedeutung in der Rechtsprechung des Gerichtshofs

Es wurde bereits aufgezeigt, dass der EGMR bei der Prüfung von Art. 3 EMRK mit dem Prinzip der Gleichwertigkeit der Gesundheitsversorgung inner- und ausserhalb des Strafvollzugs operiert, dieses Prinzip prüfungsmethodologisch aber zu Gunsten einer „Angemessenheitsprüfung“ in den Hintergrund rückt. Die beiden Prinzipien gilt es deshalb auf ihre Eignung und Werthaltigkeit bei der Beurteilung einer Verletzung von Art. 3 EMRK zu prüfen.

b Äquivalenz oder Angemessenheit als geeigneter Mindeststandard?

Weder die Proklamation der Äquivalenz noch jene der Angemessenheit genügt mit Blick auf den absoluten Charakter von Art. 3 EMRK für sich allein, um einen im Sinne des Spezifitätskriteriums genügend konkreten Standard aufzustellen; beide Begriffe bedürfen zunächst weiterer Definition. Hinsichtlich der Äquivalenz sind die Fragen des massgeblichen Referenzmassstabes ausserhalb des Strafvollzugs und der Beurteilungskriterien der Äquivalenz zu klären. Auch die Angemessenheit der Gesundheitsversorgung ist als unbestimmter Rechtsbegriff mit konkreten inhaltlichen Garantien bzw. strukturellen Elementen zu füllen.

Gleichwohl unterscheiden sich die prüfungsmethodologischen Herangehensweisen wesentlich: Das Äquivalenzprinzip impliziert einen relativen, d.h. vergleichenden Massstab, während die Ermittlung der Angemessenheit auf einem wertebasierten Massstab beruht, wobei im Rahmen der normativen Bewertung einer Situation soziomedizinische Elemente aufgegriffen werden.

Beim vergleichenden Massstab ist bei der Wahl des Referenzmassstabes schon deshalb Vorsicht geboten, weil innerhalb des Geltungsbereichs 
der EMRK schon aufgrund ihrer Konzeption grundsätzlich ein auf dem europäischen Konsens beruhender einheitlicher Mindeststandard im Rahmen von Art. 3 EMRK statuiert wird, welcher von sämtlichen Konventionsstaaten einzuhalten ist. Ein nationaler Massstab würde dieser Konzeption in grundlegender Weise widersprechen. Hinzu kommt die Frage nach der Legitimität eines Massstabes, welcher anhand der Gesundheitsversorgung ausserhalb des Strafvollzugs in den Konventionsstaaten ermittelt wird: Das Äquivalenzprinzip setzt letztlich bei einem Umstand, wie er ausserhalb des Strafvollzugs existiert, an und fingiert, dass dies der Massstab sei, welcher auch im Vollzug zu gelten hat, ohne dabei den Massstab in der freien Gesellschaft in materieller Hinsicht in irgendeiner Weise zu überprüfen. Das Äquivalenzprinzip berücksichtigt damit in keiner Weise, ob der Massstab der freien Gesellschaft, wie er tatsächlich besteht, überhaupt erstrebenswert ist. Mit anderen Worten wird die „Sein-Ebene“ der freien Gesellschaft automatisch zur „Soll-Ebene“ des Strafvollzugs, ohne dabei die „Sein-Ebene“ zunächst zu prüfen.

Dieses Problem akzentuiert sich dadurch, dass sich schon die rechtlichen Vorgaben an die Gesundheitsversorgung im Strafvollzug von jenen in der freien Gesellschaft unterscheiden. In der freien Gesellschaft existiert schon von vorneherein kein allgemeines Recht auf Gesundheit ${ }^{846}$ und auf Gesundheitsversorgung, woran die tatsächlichen Gegebenheiten gemessen werden könnten. Im Strafvollzug kommt aufgrund des Sonderstatusverhältnisses - wie bereits aufgezeigt - aber eine besondere Fürsorgepflicht des Staates zum Tragen, welche insbesondere bezüglich der positiv- und leistungsrechtlichen Aspekte des Menschenrechtsschutzes besondere Implikationen hat.

Möchte man dennoch an einem vergleichenden Massstab festhalten, wäre in einem ersten Schritt immer sicherzustellen, dass der Referenzmassstab auch dem „Sollen“ entspricht. Dies zu prüfen, würde wiederum inhaltliche Kriterien erfordern. In einem zweiten Schritt müsste dann zusätzlich geprüft werden, ob im konkreten Fall den Besonderheiten des Strafvollzugs noch Rechnung zu tragen ist. Damit stellt sich dann allerdings die Frage, welchen Vorteil der Umweg über einen Referenzmassstab

846 Ein solches kann auch aus Art. 8 EMRK nicht abgeleitet werden. Dieser garantiert auch nicht die Einführung bestimmter Formen staatlicher Gesundheitssysteme. Vom Schutz von Informationen, die einen Aspekt des Privatlebens betreffen, werden aber selbstverständlich auch medizinische Daten umfasst. Bezüglich des Vertraulichkeitsgrundsatzes könnte deshalb auch ausserhalb des Strafvollzugs Art. 8 EMRK herangezogen werden: SK-StPO/MeYer Art. 8 EMRK Rn. 11, 38, 41. 
hat, könnten inhaltliche Kriterien doch ebenso gut direkt für die Situation des Strafvollzugs aufgestellt werden.

Auch vor dem Hintergrund, dass es sich bei Art. 3 EMRK um eine absolut geschützte Fundamentalgarantie handelt, scheint eine auf einem reinen Vergleich basierende Äquivalenzprüfung für sich alleine kein geeignetes prüfungsmethodologisches Vorgehen zu sein. Einerseits liesse sich die Konkretisierungsforderung des Spezifitätskriteriums mit einem variablen Referenzmassstab nicht vereinen, und andererseits würde die genuin menschenrechtlichen Wertigkeit des erlittenen Leids bzw. der Demütigung bei der Schutzbereichsbestimmung nicht genügend berücksichtigt. Eine im Vergleich zur freien Gesellschaft schlechtere medizinische Behandlung kann zwar ein bedeutender Faktor sein, dass sich der Beschwerdeführer in seinen Augen etwa in seiner Persönlichkeit herabgesetzt oder in seiner Menschenwürde getroffen fühlt, kann für sich allein genommen dafür aber in der Regel wohl nicht genügen. Für die Annahme eines Demütigungsmoments im Sinne einer erniedrigenden Behandlung i.S.v. Art. 3 EMRK wird in aller Regel nämlich entscheidender sein, wie die konkret gewährte Behandlung mit Blick auf das Krankheitsbild des Betroffenen zu beurteilen ist. Bei dieser normativen Bewertung können medizinische Möglichkeiten ausserhalb des Strafvollzugs aber selbstverständlich miteinfliessen. Gleiches hat bei der Prüfung einer unmenschlichen Behandlung i.S.v Art. 3 EMRK zu gelten. Auch hier hat die normative Bewertung der konkret gewährten Behandlung im Vordergrund zu stehen.

Anders wäre die vergleichende Herangehensweise zu beurteilen, wenn es um die Prüfung des Diskriminierungsverbots ginge. ${ }^{847}$ Das Diskriminierungsverbot statuiert gerade, dass hinsichtlich eines in der EMRK gewährten Rechts keine Differenzierung aufgrund der in Art. 14 EMRK genannten Gründe vorgenommen werden darf. ${ }^{848}$ Ein Konnex zwischen dem Diskriminierungsverbot und Art. 3 EMRK bestünde aber insofern, als eine Diskriminierung, die auf die Verletzung der Menschenwürde hinausläuft, eine erniedrigende Behandlung im Sinne von Art. 3 EMRK sein kann. ${ }^{849}$

847 An die Stelle des Eingriffs tritt beim Diskriminierungsverbot die Ungleichbehandlung - in dieser liegt bei Art. 14 EMRK gerade die Beeinträchtigung. Dies im Unterschied zu den positiven Verpflichtungen (etwa materielle Haftbedingungen), denn bei diesen ist die Beeinträchtigung in einem Unterlassen des Staates zur Vornahme der garantierten Handlung an und für sich zu sehen. Vgl. auch SK-StPO/MeYer Methodik der Grundrechtsanwendung (EMRK) Rn. 9, 22.

848 Vgl. etwa Grabenwarter/Pabel $\$ 26$ Rn. 1; Meyer-Ladewig/Lehner Art. 14 Rn. 5 ff., 9 ff., 16 ff.; SCHILling Rn. 738.

849 Dazu ausf. Mavronicola HRLR 2012, 723 (751). 
Da allerdings kein allgemeines Recht auf Gesundheitsversorgung in der EMRK verbrieft ist, scheint auch ein Heranziehen von Art. 14 EMRK diesbezüglich wenig zweckmässig. Der ratio des Äquivalenzprinzips, nämlich einem Normalisierungsgebot, sollte deshalb auf andere Weise Rechnung getragen werden.

Die Angemessenheitsprüfung, wie sie der Gerichtshof vornehmlich anwendet, nimmt die Frage der Äquivalenz denn auch auf, stellt aber fest, dass sich der Gerichtshof selbst nicht immer danach richtet. Im Einzelfall kann dies verschiedene Folgen haben: (1) Inner- und ausserhalb des Strafvollzugs besteht eine gleichwertige Gesundheitsversorgung, welche auch angemessen ist, womit keine Verletzung von Art. 3 EMRK vorliegt, (2) es liegt zwar eine gleichwertige Gesundheitsversorgung vor, diese ist aber im konkreten Fall für den Strafgefangenen gleichwohl nicht angemessen, weil sie entweder auch ausserhalb des Strafvollzugs nicht angemessen ist oder weil sie mit Blick auf die besondere Fürsorge- und Obhutspflicht nicht angemessen ist; es ist eine Verletzung von Art. 3 EMRK festzustellen, (3) es besteht zwar keine gleichwertige Gesundheitsversorgung, aber die dem Inhaftierten gewährte medizinische Versorgung ist gleichwohl angemessen, womit keine Verletzung von Art. 3 EMRK vorliegt.

Vor diesem Hintergrund stellt sich die Frage, welchen Stellenwert der EGMR dem Äquivalenzprinzip bei der Angemessenheitsprüfung konkret einräumt. Der Beizug des Äquivalenzprinzips geht jedenfalls nicht so weit, dass die Gewährung einer gleichwertigen Gesundheitsversorgung vermutungsweise eine angemessene Gesundheitsversorgung oder eine ungleiche Gesundheitsversorgung vermutungsweise eine unangemessene Gesundheitsversorgung implizieren würde. Die Äquivalenz bleibt bei der normativ geprägten Beurteilung der Angemessenheit vielmehr eine allgemeine Auslegungsdirektive bzw. ein zu berücksichtigender Faktor.

Wird die Angemessenheitsprüfung anhand vorgängig festgelegter Kriterien vorgenommen, erfüllt sie auch die Konkretisierungsforderung eines absoluten Rechts. Der besonderen Bedeutung der Grenze zwischen einer zu spezifischen und einer zu allgemeinen Rechtsprechung bei absoluten Rechten wird hier beispielsweise mit den Kriterien einer zeitnahen Diagnose und einer akkuraten Behandlung Rechnung getragen, welche zusammengefasst schnellstmögliches Tätigwerden mit einer umfassenden Therapiestrategie nach Massgabe der anerkannten Grundsätze der medizinischen Wissenschaften fordern. Mit diesen Kriterien wird ein Standard geschaffen, welcher ein ausreichendes Mass an Allgemeingültigkeit und Klarheit gewährleistet, aber auch Raum für die Einzelfallbetrachtung lässt. 
Letzteres ist umso wichtiger, als gerade medizinische Fragen individuelle Lösungen erfordern.

\section{c Zwischenfazit}

Zusammenfassend kann damit festgestellt werden, dass der EGMR bei der Festsetzung des Mindeststandards der Gesundheitsversorgung im Strafvollzug im Sinne von Art. 3 EMRK prüft, ob diese im Einzelfall angemessen ist. Das Äquivalenzprinzip tritt als ein bei der wertungsbasierten Angemessenheitsprüfung mitzuberücksichtigender Faktor in den Hintergrund. Soweit die Äquivalenz in der Angemessenheitsprüfung zu berücksichtigen ist, wird auch ihr ein normativer Wert zugeschrieben; Fragen rund um den anzuwendenden Referenzmassstab und die Beurteilungskriterien der Gleichwertigkeit verlieren damit an Bedeutung. Das methodische Vorgehen des EGMR, direkt die Angemessenheit der medizinischen Versorgung in das Zentrum der Menschenrechtsprüfung zu stellen, überzeugt. Ein auf einem blossen Vergleich basierender Massstab, wie ihn das Äquivalenzprinzip postuliert, würde für sich alleine dem Fundamentalcharakter von Art. 3 EMRK und der genuin menschenrechtlichen Wertigkeit des erlittenen Leids bzw. der Demütigung nicht genügend Rechnung tragen. Indem der EGMR die Angemessenheitsprüfung anhand klar formulierter Kriterien vornimmt, schafft er einen Massstab, welcher auch den Prüfungsanforderungen eines absoluten Rechts genügt.

\subsection{Wechselwirkungen - Kumulation und Kompensation?}

Der EGMR beurteilt das Vorliegen einer angemessenen medizinischen Versorgung grundsätzlich unabhängig von anderen Haftbedingungen; gleichwohl anerkennt der Gerichtshof, dass der Gesundheitszustand des Inhaftierten Folgen für dessen Unterbringung und damit auf andere Haftbedingungen haben kann. ${ }^{850}$ Inadäquate Haftbedingungen können die medizinische Versorgung des Inhaftierten unwirksam machen, womit diese nicht mehr als angemessen beurteilt werden kann. Mit anderen Worten

850 EGMR - Topekhin/RUS, Urt. v. 10.05.2016, 78774/13, Rn. 87: Der EGMR erkennt eine Pflicht des Mitgliedstaates zur Zurverfügungstellung einer druckvermindernden Matratze für einen kranken Inhaftierten. Vgl. ferner EGMR Raffray Tadei/FRA, Urt. v. 21.12.2010, 36435/07. 
trägt der Gerichtshof den kumulativen Effekten der Haftsituation Rechnung. Die medizinische Versorgung muss gerade vor deren Hintergrund auf ihre Angemessenheit geprüft werden. Ist eine medizinisch adäquate Unterbringung eines kranken Strafgefangenen nicht möglich, hat dies insoweit Konsequenzen, als eine Verlegung in eine zivile Krankeninstitution oder in schwerwiegenden Fällen gar eine Entlassung ins Auge gefasst werden muss. Kompensierende Effekte der Haft sind bei der Gesundheitsversorgung kaum denkbar: Eine menschenrechtskonforme Unterbringung dispensiert in seiner menschenrechtlichen Wertigkeit den Konventionsstaat in keiner Weise von einer angemessenen Gesundheitsversorgung. Auch die einzelnen Garantien der Gesundheitsversorgung lassen sich nicht gegenseitig aufwiegen. So kann die Freiwilligkeit der Behandlung an sich nicht etwa mangelnde Vertraulichkeit der Untersuchung kompensieren. Ebenso wenig wäre denkbar, dass eine an und für sich adäquate Behandlung eine verspätete Behandlung kompensiert oder dass eine zwar prompte Behandlung nicht mehr den qualitativen Anforderungen entsprechen müsste. Kompensationsmechanismen werden vom EGMR bei der Prüfung von Art. 3 EMRK hinsichtlich der medizinischen Versorgung denn auch nicht ins Feld geführt.

Anders gelagert sind Situationen konfligierender Interessen. Im Kontext der Gesundheitsversorgung im Strafvollzug ist allem voran an das Spannungsverhältnis zwischen Sicherheitsaspekten und dem menschenrechtlichen Anspruch einer angemessenen Behandlung i.S.v. Art. 3 EMRK auch gefährlicher Straftäter zu denken. Nachdem bei Art. 3 EMRK als absolutem Recht grundsätzlich nur relative Kriterien berücksichtigt werden dürfen, welche direkten Einfluss auf das Mass des erlittenen Leidens oder die Schwere der zugefügten Behandlung haben und sich die Gefährlichkeit des Täters gerade nicht auf das Mass des erlittenen Leidens auswirkt, stellt sich die Frage, welcher Wert der Gefährlichkeit des Täters bei der Prüfung von Art. 3 EMRK beigemessen werden darf.

Soweit irgend möglich ist der Gefährlichkeit des Täters mit angepassten Leistungen zu begegnen. In Frage kommen insbesondere bei Verlegungen in externe Behandlungseinrichtungen flankierende (Zwangs-) Massnahmen. Denkbar ist etwa, dass der Betroffene in Handfesseln gelegt wird oder dass er auch bei medizinischen Untersuchungen von Aufsehern resp. Polizeibeamten begleitet wird, obwohl Letzteres klar dem allgemeinen Vertraulichkeitsgrundsatz zuwiderläuft. ${ }^{851}$ Solche Massnahmen dürfen allerdings nur gerade so weit reichen, als sie durch ein objektiv nachvollziehbares

851 EGMR - Duval/FRA, Urt. v. 26.05.2011, 19868/08, Rn. 48 ff. 
Sicherheitsbedürfnis begründet werden können. ${ }^{852}$ Bei der Beurteilung ist insbesondere auch der Gesundheitszustand des Betroffenen zu berücksichtigen. ${ }^{853}$ Gehen die angewandten Massnahmen über das absolut notwendige Mass hinaus, hält der Gerichtshof fest, dass sie beim Betroffenen Gefühle der Willkür, der Minderwertigkeit und der Angst hervorrufen können, welche über das hinausgehen, was unweigerlich mit der medizinischen Untersuchung von Inhaftierten einhergehe, weshalb eine erniedrigende Behandlung i.S.v. Art. 3 EMRK zu erkennen ist. ${ }^{854}$ Das Überschreiten des absolut notwendigen Masses kann je nach Fallkonstellation aber auch zu einer unmenschlichen Behandlung i.S.v. Art. 3 EMRK führen. ${ }^{855}$

Schon bei den Kernmerkmalen absoluter Rechte wurde ausgeführt, dass bei absoluten Rechten aufgrund des Anwendbarkeitskriteriums keine Verhältnismässigkeitsprüfung im eigentlichen Sinn erfolgt und es damit auch zu keiner Interessenabwägung zwischen öffentlichen und Privatinteressen kommt. Gleichwohl schliesst der absolute Charakter auf der Schutzbereichsebene die Berücksichtigung konfligierender Interessen nicht aus. Aufgrund des Hierarchieverhältnisses zwischen absoluten und nicht absoluten Rechten hat das Drittinteresse denn auch ein absolutes zu sein, um überhaupt Berücksichtigung zu finden. In der vorliegenden Konstellation könnte sich der Arzt seinerseits auf Art. 3 EMRK berufen; auch er geniesst grundsätzlich besondere Schutzpflichten seitens der Strafvollzugsanstalt. Das weitere Hierarchieverhältnis besagt, dass keine absolute positive Pflicht bestehen kann, wenn diese eine negative absolute Pflicht verletzen würde. Während der Arzt sich vorliegend auf die abwehrrechtliche Dimension von Art. 3 EMRK beruft, beruft sich der Inhaftierte, was die Untersuchung als solche angeht, auf den positivrechtlichen Gehalt. Das konfligierende Interesse des Arztes führt deshalb dazu, dass in diesem Umfang keine positive Pflicht bestehen kann. Die Prüfung des Gerichtshofs, ob eine Massnahme absolut erforderlich war, legt damit mit anderen Worten fest, ob und in

852 EGMR - Henaf/FRA, Urt. v. 27.11.2003, 65436/01, Rn. 52 ff.; EGMR - Duval/FRA, Urt. v. 26.05.2011, 19868/08, Rn. 48 ff.; vgl. auch SK-StPO/MeYeR Art. 3 EMRK Rn. 88.

853 EGMR - Mouisel/FRA, Urt. v. 14.11.2002, 67263/01, Rn. 47.

854 EGMR - Duval/FRA, Urt. v. 26.05.2011, 19868/08, Rn. 52 f.

855 EGMR - Henaf/FRA, Urt. v. 27.11.2003, 65436/01, Rn. 52 ff.: Der Gerichtshof stellte fest, dass es in Anbetracht des Gesundheitszustandes und der vom Beschwerdeführer ausgehenden Gefahr nicht notwendig war, diesen in der Nacht vor seiner Operation an das Krankenbett zu fesseln, zumal zwei Polizeibeamte vor seinem Zimmer Wache hielten. In der Folge erkannte der Gerichtshof eine unmenschliche Behandlung i.S.v. Art. 3 EMRK. 
welchem Umfang eine negative absolute Pflicht gegenüber Drittpersonen besteht. Ist die Massnahme absolut erforderlich, kann in diesem Umfang folglich keine positive Pflicht des Betroffenen bestehen. Gehen sie aber über das erforderliche Mass hinaus, besteht eine positive absolute Pflicht und eine Verletzung von Art. 3 EMRK wird erkannt.

Das Vorgehen des Gerichtshofs entspricht folglich auch den besonderen Prüfungsanforderungen eines absoluten Rechts. Das Anwendbarkeitskriterium absoluter Rechte wird durch die Berücksichtigung konfligierender Interessen auf Schutzbereichsebene nicht untergraben.

\subsection{Zwischenfazit}

Der EGMR garantiert im Rahmen von Art. 3 EMRK eine „individuellgesundheitsadäquate" 856 Versorgung, wobei der Gerichtshof zur Konkretisierung des Angemessenheitsbegriffs keine Scheu zeigt, klare Kriterien und inhaltliche Aspekte seiner Beurteilung zu benennen. Die Beurteilung, ob im konkreten Fall das erforderliche Mindestmass an Schwere einer unmenschlichen oder erniedrigenden Behandlung i.S.v. Art. 3 EMRK vorliegt, bleibt eine normative, wobei auch der äquivalenten Gesundheitsversorgung als einem zu berücksichtigenden Faktor der Angemessenheit ein normativer Wert zugeschrieben wird. Starre Leistungskataloge liessen sich weder mit Blick auf die Vielgestaltigkeit potentieller Fallkonstellationen noch mit dem "Living Instrument-Charakter" der EMRK ohne Weiteres vereinbaren. Die vom Gerichtshof benannten strukturellen Elemente zur Konkretisierung des Angemessenheitsbegriffs sind in Ermangelung eines solchen Katalogs umso wichtiger. Der so aufgestellte Standard gilt grundsätzlich unabhängig von irgendwelchen anderen Haftbedingungen, wobei erschwerende Haftbedingungen bei der Angemessenheitsprüfung der Gesundheitsversorgung zu berücksichtigen sind. Konfligierenden Interessen ist bei der Bestimmung des Schutzbereichs zu begegnen: In dem Umfang, in dem eine negative absolute Pflicht gegenüber Drittpersonen besteht, kann keine positive absolute Pflicht erwachsen.

Die Einhaltung dieses menschenrechtlichen Standards sichert der Gerichtshof schliesslich auf beweisrechtlicher Ebene ab; der Inhaftierte hat nur einen Anscheinsbeweis zu erbringen, wonach es nicht mehr am Inhaftierten ist, nachzuweisen, dass ihm keine adäquate medizinische Versorgung gewährt wurde, sondern am Konventionsstaat liegt, zu belegen, dass

856 Vgl. auch SK-StPO/Meyer Art. 3 EMRK Rn. 33. 
er dem Strafgefangenen eine angemessene Gesundheitsversorgung zukommen liess.

4. EGMR im Verhältnis zu anderen Standards und zu anderen Institutionen

Auch hinsichtlich der medizinischen Versorgung nimmt der Gerichtshof sowohl bei der Statuierung einzelner materieller Garantien als auch beim prüfungsmethodischen Vorgehen - etwa in Bezug auf die Anwendung des Äquivalenzprinzips - Bezug auf andere Regelungsinstrumentarien. Im Folgenden wird auf einige Wechselwirkungen exemplarisch eingegangen. Unterschieden werden dabei wiederum die Sachverhalts- und die Rechtsebene. Schliesslich wird die Frage beantwortet, ob und inwieweit im Bereich der medizinischen Versorgung ein Transformationsprozess stattfindet (Soft Law zu Hard Law; rechtspolitische Zielbestimmungen zu rechtsverbindlichen subjektiven Rechten).

4.1 Einfluss anderer Institutionen auf die Rechtsprechung des EGMR bei der Gesundheitsversorgung im Strafvollzug

a Rechtsebene

Die Analyse der einschlägigen Regelungsinstrumentarien hat für die Phase des Beginns des Strafvollzugs gezeigt, dass sowohl auf internationaler als auch europäischer Ebene bei Haftantritt eine medizinische Untersuchung mit entsprechender Dokumentation empfohlen wird (vgl. NelsonMandela-Regeln, CPT und EPR). Ratio dieser Eintrittsuntersuchung sind sowohl auf UN-Ebene als auch auf europäischer Ebene die Separation der Gefangenen mit Verdacht auf Infektionskrankheiten oder andere ansteckende Krankheiten, das Treffen der indizierten Massnahmen sowie das Feststellen der Arbeitsfähigkeit. Während auf UN-Ebene diesbezüglich keine Ausnahmen vorgesehen sind, sehen die Europäischen Strafvollzugsgrundsätze eine Ausnahmemöglichkeit vor, wenn die Untersuchung offensichtlich unnötig („obviously unnecessary“) ist. Auch das CPT empfiehlt eine Eintrittsuntersuchung; dies systematisch unter der Garantie des $\mathrm{Zu}$ gangs zu einem Arzt.

Der EGMR nimmt die Forderung nach einer Eintrittsuntersuchung in seiner Rechtsprechung auf, bettet diese anders als die vorgenannten 
Soft Law-Regelungsinstrumente aber nicht primär in einen materiellrechtlichen, sondern in einen beweisrechtlichen Kontext. Ratio und telos der Eintrittsuntersuchung scheint beim EGMR nämlich vornehmlich die Frage der Nachweisbarkeit zu sein, ob eine Erkrankung bzw. Verletzung während des Strafvollzugs entstand oder ausbrach oder ob es sich um einen vorbestehenden Zustand handelt.

Mit anderen Worten bedeutet dies, dass die Soft Law-Regulatorien die Eintrittsuntersuchung unabhängig von irgendwelchen Folgen garantiert sehen wollen, während der EGMR erst dann eine menschenrechtliche Konsequenz an deren Unterlassen knüpft, wenn es zu einer Schädigung des Beschwerdeführers kam. Würde der Gerichtshof die Forderung der Soft Law-Regulatorien vollständig implementieren wollen, d.h. die medizinische Untersuchung bei Haftantritt als inhaltliche Garantie gewährleisten wollen, müsste er konsequenterweise in deren Nichtbeachtung unabhängig von einer Schädigung des Inhaftierten eine Verletzung von Art. 3 EMRK erkennen und diese folglich als eigenständiges Angemessenheitskriterium der medizinischen Versorgung betrachten. Das Unterlassen der Eintrittsuntersuchung müsste dann für sich alleine das Mindestmass an Schwere von Art. 3 EMRK überschreiten. Gestützt auf die allgemeinen Begriffsmerkmale der erniedrigenden Behandlung i.S.v. Art. 3 EMRK könnte diesbezüglich etwa dahingehend argumentiert werden, dass das Unterlassen von Eintrittsuntersuchungen bei Inhaftierten Gefühle der Angst und Hilfslosigkeit auslösen müsste, zumal sie nicht beurteilen können, ob sie im Strafvollzug übermässigen Gesundheitsgefahren ausgesetzt werden. ${ }^{857}$ Solange der Gerichtshof dies aber nicht annimmt, orientiert er sich zwar an den aufgeführten Regelwerken, misst der Eintrittsuntersuchung aber einen eigenen Gehalt zu. Von einem eigentlichen Transformationsprozess von Soft Law zu Hard Law kann deshalb hier nicht die Rede sein.

Für die Phase der Dauer des Vollzugs hat die Analyse der genannten Soft Law-Regelwerke in Bezug auf die Anwendung des Freiwilligkeitsprinzips - mithin des Prinzips des informed consent - gezeigt, dass diesem im Laufe der Zeit nicht nur in ethischer, sondern auch in rechtlicher Sicht zunehmende Bedeutung zugemessen wurde. Gleichwohl fordern die Soft Law-Regelwerke nicht deren absolute Anwendbarkeit. Ausnahmen bleiben möglich. So statuiert etwa das CPT, dass im konkreten Fall dann von die-

857 Ohne Eintrittsuntersuchungen besteht etwa die Gefahr, dass ansteckende Krankheiten von aussen in eine Vollzugseinrichtung eingeschleppt werden könnten, was die Inhaftierten insbesondere aufgrund der hohen sozialen Dichte im Vollzug besonders gefährdet (etwa: Coronavirus). 
sem Grundprinzip abgewichen werden darf, wenn dazu eine gesetzliche Grundlage besteht und wenn die Ausnahme auf Prinzipien beruht, die auch ausserhalb des Strafvollzugs gelten. Eine Einwilligung hat gemäss den Nelson-Mandela-Regeln und den Europäischen Strafvollzugsgrundsätzen jedenfalls zwingend vorzuliegen, wenn es sich nicht um eine therapeutische Massnahme, sondern um die Durchführung medizinischer oder wissenschaftlicher Versuche handelt. Wie aufgezeigt, geht auch der EGMR vom Grundsatz der Freiwilligkeit aus, anerkennt unter gewissen Voraussetzungen aber auch Ausnahmemöglichkeiten. Die ratio der Kriterien, welche zur zulässigen Abweichung vom Freiwilligkeitsprinzip vorliegen müssen, ist sowohl beim CPT als auch beim EGMR die bestmögliche Sicherstellung, dass Ausnahmen nicht leichthin bzw. gar willkürlich angenommen werden. Sowohl CPT als auch der EGMR stellen deshalb sowohl formelle als auch materielle Anforderungen an eine Behandlung gegen resp. bei Urteilsunfähigen ohne den Willen des inhaftierten Patienten. Durch seine ausdifferenzierten Kriterien entspricht die Rechtsprechung des EGMR folglich den Empfehlungen des CPT.

Die Analyse des Soft Law hat gezeigt, dass neben dem Freiwilligkeitsprinzip auch das Vertraulichkeitsprinzip zunehmend an Bedeutung gewonnen hat. Basierend auf dem Vertraulichkeitsprinzip werden von den Soft Law-Regulatorien diverse Empfehlungen aufgestellt. So fordert etwa das CPT, dass medizinische Untersuchungen ausser Hör- und, wenn vom Arzt nicht anders angeordnet, auch ausser Sichtweite von Strafvollzugsbeamten stattzufinden haben. Weiter fordert das CPT, dass die Untersuchungen auch in Abwesenheit anderer Strafgefangenen einzeln und nicht etwa gruppenweise zu erfolgen haben. Zur Sicherstellung der Vertraulichkeit fordert das CPT ferner die Unabhängigkeit des Gesundheitspersonals vom Strafvollzugsmanagement. Der EGMR verweist in seiner Rechtsprechung zum Grundsatz der Vertraulichkeit ausdrücklich auf die Forderungen des CPT. Er konsolidiert in seiner Rechtsprechung die Forderungen, die medizinische Versorgung ausserhalb von Hör- und, wenn nicht anders angeordnet, ausser Sichtweite zu gewährleisten, ${ }^{858}$ die Untersuchung einzeln

858 Vgl. etwa EGMR - Duval/FRA, Urt. v. 26.05.2011, 19868/08, Rn. 51: „[...] le CPT a recommandé d'effectuer les examens/consultations/soins médicaux de détenus hors de l'écoute et - sauf demande contraire du médecin concerné dans un cas particulier - hors de la vue du personnel d'escorte. Le Comité a ajouté que l'examen des détenus soumis à des moyens de contrainte était une pratique hautement contestable tant du point de l'éthique que du point de vue clinique." Im konkreten Fall nahm der EGMR mitunter aufgrund der Anwesenheit von Strafvollzugsbeamten während der medizinischen Untersuchung, ohne dass 
durchzuführen ${ }^{859}$ und die Unabhängigkeit des medizinischen Personals von der Strafvollzugsanstalt zu gewährleisten. ${ }^{860}$ Die Empfehlungen der Soft Law-Regelwerke werden folglich diesbezüglich vom EGMR vollständig rezipiert, und deren Gehalt wird auf diese Weise konsolidiert.

Die Auseinandersetzung des EGMR mit den Soft Law-Regelwerken betrifft auch weitere Bereiche der „Angemessenheitsprüfung“. Der EGMR weist nach der Rezeption des entsprechenden Regelwerks bisweilen ausdrücklich darauf hin, dass er diesem grosses Gewicht beimesse, räumt gleichzeitig aber auch ein, dass das Soft Law für die Mitgliedstaaten keinen bindenden Charakter habe. ${ }^{861}$ Basiert die Entscheidung des EGMR in der Folge dennoch auf diesen nicht bindenden Regelwerken, beginnt der Transformationsprozess von blosser Empfehlung hin zu einem harten, einklagbaren Standard. Die Normativität der entsprechenden Empfehlung nimmt durch die Rechtsprechung des EGMR folglich zu. Gleichwohl scheint der Gerichtshof nicht leichthin Soft Law-Bestimmungen in seiner Prüfung von Art. 3 EMRK zu implementieren, sondern die Bestimmungen stets sorgfältig mit Blick auf deren menschenrechtlichen Gehalt zu prüfen. Nur dort, wo er Art. 3 EMRK in seinem eigenen Verständnis bei Nichteinhaltung der Bestimmung verletzt sieht, zieht er die Soft Law-Bestimmung heran, was der Auslegungskanon der EMRK auch gerade gebietet.

dies Sicherheitsinteressen absolut notwendig gemacht hätten, eine Verletzung von Art. 3 EMRK an. Vgl. auch EGMR - Salmanoglu u. Polatta/TUR, Urt. v. 17.03.2009, 15828/03, Rn. 80.

859 EGMR - Salmanoglu u. Polatta/TUR, Urt. v. 17.03.2009, 15828/03, Rn. 80.

860 EGMR - Salmanoglu u. Polatta/TUR, Urt. v. 17.03.2009, 15828/03, Rn. 80: „The Court has already reaffirmed the European Committee for the Prevention of Torture's („CPT“) standards on the medical examination of persons in police custody $[\ldots]$. They [the health professionals] should not compromise their professional independence by contractual or other considerations but should provide impartial evidence, including making clear in their reports any evidence of ill-treatment [...].“

861 Vgl. etwa EGMR - Rivière/FRA, Urt. v. 11.07.2006, 33834/03, Rn. 72: „La Cour relève en outre que la Recommandation du Comité des Ministres du Conseil de l'Europe relative aux aspects éthiques et organisationnels des soins de santé en milieu pénitentiaire prévoit que les détenus souffrant de troubles mentaux graves devraient pouvoir être placés et soignés dans un service hospitalier doté de l'équipement adéquat et disposant d'un personnel qualifié. La Cour a déjà eu l'occasion de citer cette recommandation, et elle y attache un grand poids, même si elle admet qu'elle n'a pas en soi valeur contraignante à l'égard des Etats membres."; vgl. ferner EGMR - Gennadi Naoumenko/UKR, Urt. v. 10.02.2004, 42023/98, Rn. 94. 
In Bezug auf die Phase nach Beendigung des Strafvollzugs wurde aufgezeigt, dass die Europäischen Strafvollzugsgrundsätze auch diese Phase aufgreifen: Sie statuieren, dass Arrangements mit Institutionen ausserhalb des Vollzugs für die weitere Gewährleistung der Behandlung im Einverständnis mit dem Inhaftierten möglich sein sollen. Die Empfehlung geht damit nicht so weit, den Nationalstaaten zu empfehlen, in jedem Fall die Weiterführung der Behandlung zu gewährleisten. Auch der EGMR war soweit ersichtlich nicht damit konfrontiert, über das Bestehen einer solchen Garantie aus Art. 3 EMRK zu entscheiden. ${ }^{862}$

Rückt man die Methodik der Beurteilung einer Verletzung von Art. 3 EMRK in das Zentrum der Betrachtung, ist zunächst festzustellen, dass der Gerichtshof bezüglich der Frage der Geltung des Äquivalenzprinzips Soft Law rezipiert und dabei festhält, dass diese Instrumentarien das Äquivalenzprinzip als relevanten Prüfmassstab hinzuziehen. ${ }^{863}$ Bisweilen hält der EGMR jedoch fest, dass er sich selbst, entgegen der Proklamation von Soft Law-Regulatorien, nicht immer nach diesem Prinzip richte. ${ }^{864}$ Hier könnte man prima vista zum Schluss gelangen, dass der EGMR eine abweichende Prüfungsmethodik anwendet. Dieser Schluss erweist sich allerdings als unzutreffend: Es trifft zwar zu, dass das Äquivalenzprinzip mit seinen verschiedenen Ausprägungen in sämtlichen im Rahmen dieser Arbeit näher betrachteten Regelwerken verankert ist, so insbesondere in den Nelson-Mandela-Regeln (insb. Regel 24), beim CPT 865 , bei den Europäischen Strafvollzugsgrundsätzen von 2006 (Regel 40.1-40.5) und bei der Empfehlung R (98) 7 über die ethischen und organisatorischen Aspekte

862 Der Gedanke der Kontinuität beschäftigte den EGMR aber etwa bei der Frage, ob eine ausserhalb des Strafvollzugs begonne Behandlung in gewohnter Weise fortzuführen ist. Vgl. dazu EGMR - Wenner/GER, Urt. v. 01.09.2016, 62303/13.

863 Vgl. etwa EGMR - Wenner/GER, Urt. v. 01.09.2016, 62303/13, Rn. 66: „Both the CPT standards and the Committee of Ministers' Recommendation Rec (2006) 2 on the European Prison Rules (which do not specifically focus on drug therapy), as well as the Committee of Ministers' Recommendation no. $\mathrm{R}$ (98) 7 concerning the ethical and organisational aspects of health care in prison, lay down the principle of equivalence of care."; vgl. ferner EGMR Pakhomov/RUS, Urt. v. 30.09.2010, 44917/08, Rn. 62; EGMR - Aleksanyan/RUS, Urt. v. 22.12.2008, 46468/06, Rn. 139.

864 Insbesondere in Bezug auf das CPT vgl. etwa EGMR - Pakhomov/RUS, Urt. v. 30.09.2010, 44917/08, Rn. 62: „The CPT proclaimed the principle of the equivalence of health care in prison with that in the outside community. However, the Court does not always adhere to this standard.“; vgl. ferner EGMR - Aleksanyan/ RUS, Urt. v. 22.12.2008, 46468/06, Rn. 139.

865 CPT - Health Care Services in Prisons [CPT/Inf (93) 12]. 
der Gesundheitsversorgung im Gefängnis (Ziff. I. Teil B), doch fordern auch diese keineswegs, wie es die Aussage des EGMR impliziert, eine absolute Äquivalenz. So führt beispielsweise das CPT in seinen Empfehlungen resp. Berichten, wenn auch systematisch unter dem Titel der Äquivalenz, vielmehr inhaltliche Kriterien verschiedener Aspekte der Gesundheitsversorgung auf. Auch beim CPT ist damit im Endeffekt die Angemessenheit der medizinischen Versorgung im Strafvollzug der Leitgedanke. Dasselbe gilt auch für die anderen Soft Law-Regelwerke, wobei allgemein die Tendenz festzustellen ist, dass die Soft Law-Regulatorien im Laufe der Zeit zunehmend ausdifferenziertere Empfehlungen enthalten. Je ausdifferenzierter das Regelwerk ist, desto geringere Bedeutung kommt letztlich dem Äquivalenzprinzip zu. Das Äquivalenzprinzip rückt folglich nicht nur beim EGMR, sondern auch bei den Soft Law-Regelwerken zunehmend in den Hintergrund. Das Äquivalenzprinzip wird damit sowohl vom EGMR als auch im Soft Law durch konkrete, unabhängig von einem vergleichenden Massstab geltende inhaltliche Empfehlungen abgelöst, wobei dem Äquivalenzprinzip bei der Angemessenheitsprüfung immerhin noch ein gewisser normativer Wert zugeschrieben wird, welchen es weiterhin zu berücksichtigen gilt.

Anders als prima vista zu erwarten war, geht der EGMR bei seiner Konventionsrechtsprüfung in prüfungsmethodischer Hinsicht insgesamt ähnlich vor wie die Soft Law-Gremien. Es kann gleichwohl keineswegs davon ausgegangen werden, dass sich der EGMR in prüfungsmethodologischer Sicht bewusst an den Soft Law-Regulatorien orientiert. Im Gegenteil: Es hat sich gezeigt, dass, selbst wenn sich das Soft Law tatsächlich (nur) am Äquivalenzprinzip orientieren würde, der EGMR bereit wäre, sich nicht danach zu richten. Indem der Gerichtshof das Äquivalenzprinzip unter Verweis auf das Soft Law aber dennoch aufgreift und diesem in der Folge auch einen gewissen normativen Wert zuschreibt, könnte aber auch diesbezüglich argumentiert werden, dass ein gewisser Härtungsprozess der Soft Law-Empfehlung einsetzt.

b Sachverhaltsebene

Die Sachverhaltserstellung stützt sich aufgrund der zumeist komplexen Materie in aller Regel auf fachliche Berichte, d.h. vornehmlich auf Arztbe- 
richte, Expertenmeinungen und Gutachten. ${ }^{866}$ Diese Berichte haben sich insbesondere dazu zu äussern, welche Massnahmen, d.h. Untersuchungen, Behandlungen etc., im konkreten Fall ergriffen wurden, um eine adäquate Versorgung zu garantieren. ${ }^{867}$ Weiter sollte aus ihnen hervorgehen, ob ein Zusammenhang zwischen dem medizinischen Befund und einer unmenschlichen und erniedrigenden Behandlung besteht. ${ }^{868}$

Der Gerichtshof stellt auch Anforderungen formeller Natur an die ärztlichen Unterlagen, die als Beweismittel herangezogen werden. Er legt beispielsweise Wert auf die Vollständigkeit der Offenlegung sämtlicher medizinischer Daten. ${ }^{869}$ Weiter wird der zeitnahen Erstellung der Berichte sowie ihrer sauberen und datierten Dokumentation Beachtung geschenkt. ${ }^{870}$

Wurde im Rahmen einer vorsorglichen Massnahme ein unabhängiges medizinisches Gutachten zu spezifischen Fragen verlangt, wird diesem besonders grosse Bedeutung beigemessen. ${ }^{871}$ Kam der Konventionsstaat den vorsorglichen Massnahmen nicht nach und unterliess die Einholung eines Gutachtens, können andere Berichte, in denen der Konventionsstaat etwa selbst die vom EGMR gestellten Fragen beantwortete, dieses Versäumnis

866 Vgl. etwa EGMR - Salmanoglu u. Polatta/TUR, Urt. v. 17.03.2009, 15828/03, Rn. 80; EGMR - Mehmet Emin Yüksel/TUR, Urt. v. 20.07.2004, 40154/98, Rn. 29; EGMR - Arutyunyan/RUS, Urt. v. 10.01.2012, 48977/09, Rn. 74.

867 EGMR - Yunusova u. Yunusov/AZE, Urt. v. 02.06.2016, 59620/14, Rn. 143: „[...] However, it notes that although the domestic authorities were aware of the applicants' poor health from the very beginning of their detention, it does not appear from the case file that they were provided with adequate medical treatment in detention."

868 EGMR - Salmanoglu u. Polatta/TUR, Urt. v. 17.03.2009, 15828/03, Rn. 80: „Moreover, an opinion by medical experts on a possible relationship between physical findings and ill-treatment was found to be a requirement by the Court."

869 EGMR - Yunusova u. Yunusov/AZE, Urt. v. 02.06.2016, 59620/14, Rn. 143.

870 EGMR - Yunusova u. Yunusov/AZE, Urt. v. 02.06.2016, 59620/14, Rn. 145: „[...] Moreover, when communicating this case, the Court also asked the Government to provide full information on the medical treatment received by the applicants in respect of all their health problems. However, neither the Court nor the applicants were provided with the medical prescriptions or recommendations made by C.W. or the other doctors following these medical examinations and the Government contented themselves with submitting an undated note signed by [the doctor] [...]. No explanation was given by the Government for this failure."

871 EGMR - Kondrulin/RUS, Urt. v. 20.09.2016, 12987/15, Rn. 41 ff.; EGMR Khloyev/RUS, Urt. v. 05.02.2015, 46404/13, Rn. 35 ff., 84 ff.; EGMR - Amirov/ RUS, Urt. v. 27.11.2014, 51857/13, Rn. 31 ff., 91 ff. 
nicht kompensieren. ${ }^{872}$ Folgen hat ein solches Versäumnis damit nicht nur in Bezug auf die Feststellung einer eigenständigen Verletzung von Art. 34 Satz 2 EMRK (dazu vgl. Teil 4 Kap. 2), sondern auch in Bezug auf die Prüfung der Konformität der medizinischen Versorgung als solcher mit Art. 3 EMRK. In aller Regel geht der Gerichtshof in diesen Konstellationen nämlich davon aus, dass der Inhaftierte den Anscheinsbeweis mit der Folge der Beweislastumkehr erbracht hat und der Exkulpationsbeweis des Konventionsstaates misslungen ist. ${ }^{873}$

Neben den medizinischen Unterlagen, welche sich in erster Linie zum Gesundheitszustand des Inhaftierten äussern, zieht der EGMR auch allgemeine Berichte betreffend die Gesundheitsversorgung in der verfahrensgegenständlichen Strafvollzugsanstalt hinzu ${ }^{874}$ Länderberichte etwa des CPT, welche im Nachgang an dessen regelmässige Besuche erstellt werden, äussern sich sowohl zu infrastrukturellen, organisatorischen und personellen Mängeln als auch zur Frage, ob die medizinische Versorgung im Allgemeinen als adäquat erscheint oder nicht. ${ }^{875}$

Vor diesem Hintergrund stellt sich die Frage, welche Bedeutung der EGMR den internationalen bzw. europäischen Instituten bei der Sachverhaltserstellung insgesamt beimisst. Zu unterscheiden sind zunächst folgende zwei Fallgruppen: Von Relevanz sind (1) Fragen rund um strukturelle und organisatorische resp. personelle und infrastrukturelle Belange sowie

872 EGMR - Kondrulin/RUS, Urt. v. 20.09.2016, 12987/15, Rn. 47; EGMR - Kbloyev/ RUS, Urt. v. 05.02.2015, 46404/13, Rn. 35 ff., 84 ff.; EGMR - Amirov/RUS, Urt. v. 27.11.2014, 51857/13, Rn. $31 \mathrm{ff} ., 91 \mathrm{ff}$.

873 EGMR - Kondrulin/RUS, Urt. v. 20.09.2016, 12987/15, Rn. 57 ff.; EGMR Khloyev/RUS, Urt. v. 05.02.2015, 46404/13, Rn. 84 f.; EGMR - Amirov/RUS, Urt. v. 27.11.2014, 51857/13, Rn. $91 \mathrm{f}$.

874 Vgl. etwa EGMR - Xiros/GRE, Urt. v. 09.09.2010, 1033/07, Rn. 55.

875 Vgl. etwa EGMR - Xiros/GRE, Urt. v. 09.09.2010, 1033/07, Rn. 55 mit Auszug aus dem Bericht des CPT im Nachgang an seinen Besuch des Gefängnisses in Korydallos im Jahr 2007: „Le CPT visita le dispensaire de la prison de Korydallos et constata que la qualité du service médical était inacceptable et que des recommandations formulées antérieurement sur ce sujet n'avaient pas été suivies par les autorités internes. En particulier, le CPT souligna que les ressources médicales du dispensaire étaient complètement inadaptées pour une prison d'une telle capacité et qu'il n'y avait pas eu de progrès depuis sa dernière visite en 2005. Le service du dispensaire était principalement assuré par des détenus, qui tenaient le registre médical des détenus et secondaient le personnel médical. Pour le CPT, cette situation était inacceptable puisque, à titre d'exemple, l'administration de médicaments devait être effectuée par une personne qualifiée sous la direction d'un pharmacien." 
(2) Fragen rund um die Angemessenheit der medizinischen Untersuchung und Behandlung als solcher.

Rügt der Inhaftierte strukturelle, organisatorische oder personelle Mängel in der medizinischen Versorgung, können die Berichte von anerkannten Institutionen wie etwa des CPT grossen Beweiswert haben. ${ }^{876}$ Dies insbesondere dann, wenn die Berichte die konkrete Strafvollzugsanstalt betreffen, sich zur verfahrensgegenständlichen Zeitspanne äussern und der Inhaftierte nur in dieser medizinisch versorgt wurde (keine Verlegung in eine zivile Klinik) ${ }^{877}$ Der geforderte Anscheinsbeweis für eine Verletzung von Art. 3 EMRK lässt sich mitunter auf diese Weise regelmässig erbringen..$^{878}$

Komplexer erscheint die Situation bei der Frage, ob dem Inhaftierten eine angemessene medizinische Untersuchung oder Behandlung zuteil wurde. Die Vielgestaltigkeit der einzelnen relevanten Gegebenheiten, nicht zuletzt der konkrete Gesundheitszustand des Inhaftierten, verlangt hier mehr als anderswo eine individuelle Betrachtung. Berichte, welche sich lediglich insgesamt resp. allgemein zur Frage der Einhaltung einer angemessenen Gesundheitsversorgung äussern, vermögen den individuellen Umständen des Einzelfalls nicht gerecht zu werden. Gleichwohl können die Berichte des CPT auch bei solchen Fragen im Rahmen der freien Beweiswürdigung beweisrechtliche Wirkungen entfalten. Auch sie können die Vorbringen des Inhaftierten glaubhaft erscheinen lassen und so zum Gelingen des Anscheinsbeweises beitragen. ${ }^{879}$ Die Bedeutung von Umsetzungsberichten unabhängiger Institutionen bei der Sachverhaltserstellung im Bereich der medizinischen Versorgung ist damit insgesamt nicht $\mathrm{zu}$ unterschätzen. Implementierungskontrollen können damit über die Sachverhaltserstellung zu praktischer Wirksamkeit gelangen.

876 EGMR - Xiros/GRE, Urt. v. 09.09.2010, 1033/07, Rn. 55; EGMR - Duval/FRA, Urt. v. 26.05.2011, 19868/08, Rn. $22 \mathrm{ff} ., 51 \mathrm{f}$.

877 EGMR - Xiros/GRE, Urt. v. 09.09.2010, 1033/07, Rn. 55 ff.; vgl. insbesondere auch Richter Jebens, Malinverni u. Nicolaou, diss. op., EGMR - Xiros/GRE, Urt. v. 09.09.2010, 1033/07, Rn. 7: Es wird kritisiert, dass beim Beizug des CPT-Berichts nicht genügend berücksichtigt worden sei, dass dieser sich nur zur Versorgung innerhalb der Strafvollzugsanstalt, in welcher auch der Beschwerdeführer inhaftiert war, äussere, die medizinische Behandlung im konkreten Fall aber auch vorwiegend in einer zivilen Klinik durchgeführt worden sei.

878 Vgl. etwa EGMR - Xiros/GRE, Urt. v. 09.09.2010, 1033/07, Rn. 88.

879 Vgl. etwa EGMR - Xiros/GRE, Urt. v. 09.09.2010, 1033/07. 
4.2 Zukunftsperspektiven des Soft Law in Bezug auf eine angemessene Gesundheitsversorgung im Strafvollzug

Insbesondere bezüglich des Vertraulichkeitsprinzips hat sich gezeigt, dass der EGMR bereit ist, einzelne vom Soft Law empfohlene Aspekte in seiner Rechtsprechung zu konsolidieren, indem er ihnen aus menschenrechtlicher Sicht einen normativen Wert zuschreibt. Es hat sich mit Blick auf die Forderung einer gleichwertigen Gesundheitsversorgung inner- und ausserhalb des Strafvollzugs aber auch gezeigt, dass der Gerichtshof durchaus bereit ist, bewusst von Soft Law-Empfehlungen abzuweichen. Für die Phase nach der Haftentlassung ist in naher Zukunft nicht zu erwarten, dass der EGMR die Empfehlung der Europäischen Strafvollzugsgrundsätze im Rahmen von Art. 3 EMRK aufgreifen wird, da das erforderliche Mindestmass an Schwere kaum erreicht sein dürfte. Insgesamt kann aber davon ausgegangen werden, dass der Gerichtshof auch in Zukunft weitere einzelne materielle Empfehlungen in Bezug auf eine angemessene Gesundheitsversorgung während des Strafvollzugs herausgreifen und diese - nach einer sorgfältigen Prüfung auf ihre Art.-3-EMRK-Relevanz - in seiner Rechtsprechung rezipieren und konsolidieren wird, womit bei den betreffenden Bestimmungen ein Härtungsprozess einsetzen und deren Normativität zunehmen wird.

Den Empfehlungen ist damit mit Blick auf den „Living instrumentCharakter" der EMRK auch in Zukunft eine gewisse "Motorfunktion“ zu prognostizieren, wenngleich kein einheitlicher Standard des Soft Law und des EGMR zu erwarten ist. Es ist von Implementierungsberichten von Institutionen wie etwa dem CPT auch in Zukunft zu erwarten, dass ihnen bei der Sachverhaltserstellung gewichtige Bedeutung zukommt dies schwerpunktmässig wohl weiterhin rund um Fragen betreffend infrastrukturelle, organisatorische und personelle Gegebenheiten der medizinischen Versorgung in konkreten Strafvollzugsanstalten.

\section{Zwischenfazit}

Eine medizinische Untersuchung bei Haftantritt hat bei der Prüfung von Art. 3 EMRK beweisrechtliche Folgen. Wird die Eintrittsuntersuchung versäumt oder nur ungenügend dokumentiert, misst der Gerichtshof Ausführungen, wonach gesundheitliche Beschwerden schon vor Haftantritt bestanden hätten, keinen Beweiswert zu. In diesem Sinne ist der Konventi- 
onsstaat i.S.v. Art. 3 EMRK nicht verpflichtet, eine Eintrittsuntersuchung durchzuführen, es handelt sich vielmehr um eine Obliegenheit.

Für die Dauer der Inhaftierung gewährleistet der EGMR gestützt auf Art. 3 EMRK eine angemessene Gesundheitsversorgung. Angemessenheit setzt eine prompte Diagnose und eine akkurate Behandlung voraus. Weiter hat der Gerichtshof medizinethische Grundsätze, wie das Vertrauens- resp. Vertraulichkeitsprinzip und das Freiwilligkeitsprinzip resp. den Grundsatz des informed consent in seine Rechtsprechung implementiert und ihnen einen normativen Wert bei der Angemessenheitsprüfung zugeschrieben. Beim Vorliegen klar umschriebener Voraussetzungen ist der Gerichtshof allerdings bereit, von prinzipiengetragenen Einzelgarantien abzuweichen. In Abweichung des Freiwilligkeitsprinzips bleiben etwa Zwangsbehandlungen denkbar, wenn sie medizinisch notwendig sind, die Art und Durchführung als solche nicht gegen Art. 3 EMRK verstösst und flankierende verfahrensrechtliche Massnahmen getroffen wurden, um der Selbstbestimmung sowie der physischen Integrität des Inhaftierten Rechnung zu tragen.

Konfligierende Interessen werden bei der Bestimmung des Schutzbereichs berücksichtigt. So lässt der Gerichtshof beispielsweise trotz allgemeiner Gewährleistung des Vertraulichkeitsgrundsatzes die Anwesenheit eines Strafvollzugsbeamten bei der medizinischen Versorgung eines Inhaftierten zu, wenn der Arzt aus Sicherheitsgründen um eine solche ersucht hat. Soweit nämliche eine negative absolute Pflicht gegenüber einer Drittperson (etwa einem Gefängnisarzt) besteht, kann in diesem Umfang keine positive absolute Pflicht des Inhaftierten erwachsen.

Aus Art. 3 EMRK kann der Inhaftierte grundsätzlich keinen Anspruch auf Entlassung aus humanitären Gründen ableiten. In Ausnahmefällen, wenn der Gesundheitszustand des Inhaftierten mit einer Inhaftierung überhaupt nicht (mehr) vereinbar ist, kann der Gerichtshof, gestützt auf Art. 3 EMRK aber eine solche verlangen. Für die Phase nach der Haftentlassung, sei dies nun aufgrund einer ordentlichen Haftentlassung nach Verbüssung der schuldangemessenen Freiheitsstrafe oder aufgrund einer Haftentlassung aus humanitären Gründen, sieht der EGMR aber keine auf Art. 3 EMRK gestützten spezifischen Garantien hinsichtlich der Fortdauer resp. der Gewährung einer angemessenen medizinischen Versorgung des Entlassenen vor.

Die Analyse der prüfungsmethodischen Herangehensweise des Gerichtshofs hat gezeigt, dass der EGMR sich vom Äquivalenzprinzip löst und im Rahmen von Art. 3 EMRK vielmehr eine individuell gesundheitsadäquate Versorgung garantiert. Gleichwohl wird einer äquivalenten Ge- 
sundheitsversorgung bei der wertungsbasierten Angemessenheitsprüfung ein gewisser normativer Wert zugeschrieben. Bei dieser Bewertung verlieren der konkret anzuwendende Referenzmassstab und die denkbaren Beurteilungskriterien der Gleichwertigkeit allerdings an Bedeutung. Das Äquivalenzprinzip hat sich insgesamt zwar als ethischer, nicht aber als eigenständiger menschenrechtlicher Standard etabliert. Besondere Bedeutung kommt dem Äquivalenzgedanken aber nach wie vor dann zu, wenn der Gerichtshof garantiert, dass eine ausserhalb des Strafvollzugs begonnene Therapie fortzuführen ist. Hier bleibt allerdings anzumerken, dass es auch in dieser Konstellation nicht um eine strikte Äquivalenz, sondern vielmehr um Kontinuität geht, wobei diese niemals eine Obergrenze, sondern höchstens eine Untergrenze bildet. Zur Ausfüllung des Angemessenheitsbegriffs hat der Gerichtshof klare, strukturelle Elemente benannt.

Wenngleich erschwerende Haftbedingungen bei der Angemessenheit der medizinischen Versorgung zu berücksichtigen sind, gelten die aufgestellten Garantien in der medizinischen Versorgung als Untergrenze absolut. Kompensationsmöglichkeiten durch andere Haftbedingungen, oder die Gewährleistung anderer Einzelgarantien der medizinischen Versorgung, sind zu Recht nicht vorgesehen.

Der fehlenden Kompetenz in spezifisch medizinischen Fragen (etwa der Entscheidung über eine geeignete Therapieform) liegt zu Grunde, dass der EGMR in besonders ausgeprägtem Masse auf seine subsidiäre Rolle verweist und der Problematik auf beweisrechtlicher Ebene begegnet.

Insgesamt kann festgehalten werden, dass dem Soft Law eine wichtige „Motorfunktion“ zukommt. Einzelne Empfehlungen nimmt der EGMR auf und implementiert diese in seine Rechtsprechung, so dass sie zu in Art. 3 EMRK verankerten menschenrechtlichen Garantien werden und mithin ein Transformationsprozess einsetzt. Die Normativität der entsprechenden Soft Law-Bestimmungen nimmt damit zu. Dieser Härtungsprozess erfolgt allerdings immer erst nach einer sorgfältigen Prüfung der Bestimmungen auf ihre menschenrechtliche Wertigkeit. Auf Sachverhaltsebene haben sich Monitoringberichte rund um die Gesundheitsversorgung als zentrale Beweismittel sowohl hinsichtlich struktureller, organisatorischer und personeller Fragen als auch in Bezug auf die Angemessenheitsprüfung im engeren Sinne erwiesen. 


\section{Situation in der Schweiz}

1. Materieller Mindeststandard - Analyse der Regulierung in der Schweiz

Auf bundesrechtlicher Ebene finden sich mehrere Vorschriften, welche die Gesundheitsversorgung im Strafvollzug tangieren. Auf verfassungsrechtlicher Ebene ist zwar kein allgemeines Recht auf Gesundheit, wie etwa im UNO-Pakt I, vorgesehen, gleichwohl ist in Art. 41 Abs. 1 lit. b BV im Kapitel der Sozialziele ${ }^{880}$ festgehalten, dass sich Bund und Kantone in Ergänzung zur persönlichen Verantwortung und privaten Initiative dafür einsetzen, dass jede Person die für ihre Gesundheit notwendige Pflege erhält. Der in Art. 41 BV verankerte Subsidiaritätsgedanke kann indes nur dann greifen, wenn die betreffenden Personen diesen Bedürfnissen auch eigenständig nachkommen können, was gerade in Haftsituationen in aller Regel nicht der Fall sein dürfte. ${ }^{881}$

Darüber hinaus beinhalten einige der Grundrechtsgarantien der Bundesverfassung als Teilgehalte ein Recht auf Gesundheit. So wird etwa aus der Garantie der persönlichen Freiheit i.S.v. Art. 10 BV ein unmittelbarer Anspruch auf eine „einwandfreie ärztliche Betreuung“ erkannt, ${ }^{882}$ und auch Art. 12 BV beinhaltet Leistungsansprüche betreffend die Gesundheitsversorgung inner- und ausserhalb des Strafvollzugs. ${ }^{883}$

880 Zum Begriff des Sozialziels vgl. Kiener/KäLin/Wyttenbach $\ 3$ Rn. 8: Sozialziele begründen als Staatszielbesimmungen keine unmittelbar wirksamen und gerichtlich durchsetzbaren Ansprüche Privater. Sozialziele richten sich vielmehr an den Gesetzgeber. Vgl. ferner auch KüNZLI/Weber S. 14, welcher festhält, dass Sozialziele auch zur Interpretation von Normen herangezogen werden könnten, welche selbst Leistungsansprüche statuieren. Vgl. auch BSK BV/GäCHTER/WERDER Art. 41 Rn. 12, 26: Art. 41 Abs. 4 BV stehe der Ableitung von Leistungsansprüchen aus bestehenden Grundrechten nicht entgegen.

881 KünZli/Achermann S. 12.

882 KünZli/Weber S. 13 f., 23 ff., 30, 264 ff. m.w.H.

883 Hinsichtlich des im Art. 12 BV garantierten Leistungsumfang bestehen allerdings unterschiedlichste Auffassungen, welche von einer Minimalversorgung im Sinne einer Notfallversorgung bis hin zum vollen medizinischen Versorgungsumfang, welcher sich am Leistungskatalog des KVG zu orientieren hat, reichen. Zu dieser Debatte vgl. Künzli/Weber S. 14. Für einen über die medizinische Notfallversorgung hinausgehenden Anspruch vgl. insb. GächteR in: Zäch/Breining-Kaufmann/Ernst/Oberhammer/Portmann/Thier, S. 473 (482 f. und 485 f.), welcher die Einschränkung auf Leistungen der Notfallmedizin mit dem an der Menschenwürde orientierten Schutzgedanken von Art. 12 BV für unvereinbar hält. Vgl. auch bereits Coullery AJP 2001, 632 ff., welcher bei der 
Auf Gesetzesebene ist für die Gesundheitsversorgung im Strafvollzug das in Art. 75 Abs. 1 StGB statuierte Normalisierungsprinzip Ausgangspunkt. Dieses verkörpert in grundlegender Weise den Gleichwertigkeitsgedanken des Äquivalenzprinzips, ohne konkrete einzelne Garantien zu nennen. Weiter ist etwa Art. 80 Abs. 1 lit. a StGB einschlägig, welcher statuiert, dass von den für den Vollzug geltenden Regeln zu Gunsten des Strafgefangenen abgewichen werden darf, wenn sein Gesundheitszustand dies erfordert. Art. 234 Abs. 2 StPO hält sodann für den Vollzug der Untersuchungsund Sicherheitshaft fest, dass die zuständige kantonale Behörde die inhaftierte Person in ein Spital oder eine psychiatrische Klinik einweisen könne, wenn dies aus medizinischen Gründen angezeigt sei. Weiter sieht Art. 92 StGB vor, dass beim Vorliegen „wichtiger Gründe“ die Möglichkeit besteht, den Vollzug zu unterbrechen. Schliesslich wird die Vertraulichkeit der medizinischen Versorgung auch im Strafvollzug grundsätzlich durch Art. 321 StGB geschützt, welcher die Verletzung des Berufsgeheimnisses von Ärzten, Zahnärzten, Apothekern, Hebammen sowie ihrer Hilfspersonen unter Strafe stellt. Vorbehalten bleiben jedoch eidgenössische und kantonale Bestimmungen über die Zeugnispflicht und über die Auskunftspflicht gegenüber Behörden (Art. 321 Ziff. 3 StGB). Weitergehende Vorgaben finden sich auf der interkantonalen und kantonalen Ebene, weshalb der Blick im Folgenden auf diese gerichtet wird. Beispielhaft wird wieder der Kanton Zürich herausgegriffen; eine zusätzliche Analyse der Genfer Bestimmungen drängt sich hinsichtlich der medizinischen Versorgung im Strafvollzug nicht auf. Anders als bei der Überbelegung haben Genfer Strafvollzugsanstalten in diesem Bereich nicht zu besonderer Kritik geführt.

Die Konkordatsvereinbarung der ostschweizerischen Kantone über den Vollzug von Strafen und Massnahmen vom 29. Oktober 2004 selbst enthält keine expliziten Bestimmungen zur Gesundheitsversorgung im Strafvollzug. Sie hält jedoch fest, dass Sozialversicherungsbeiträge (also auch die KVG-Beiträge) von der eingewiesenen Person zu begleichen sind, soweit es ihr möglich und zumutbar ist (Art. 14 lit.c). ${ }^{884}$ Konkretisiert

Schutzbereichsbestimmung von Art. 12 BV dafür plädiert, am Krankheitsbegriff des KVG anzusetzen.

884 Vgl. dazu kritisch: SAMW - Stellungnahme der Zentralen Ethikkommission (ZEK) der Schweizerischen Akademie der Medizinischen Wissenschaften (SAMW) betreffend Finanzierung medizinischer Leistungen im Gefängnis, S. $1 \mathrm{ff}$. 
wird diese Bestimmung durch das Merkblatt ${ }^{885}$ "Gesundheitskosten im Straf- und Massnahmenvollzug “. ${ }^{886}$ Dieses hält fest, dass auch Kosten, welche von der Krankenversicherung nicht gedeckt sind, grundsätzlich von der inhaftierten Person zu begleichen sind. ${ }^{887}$ Personen ohne Versicherungspflicht ${ }^{888}$ hat die Vollzugseinrichtung der Einweisungsbehörde zu melden, welche dann darüber zu entscheiden hat, ob sie die betreffende Person selbst versichert, ob sie für medizinische Leistungen direkt aufkommt oder ob sie die medizinische Leistungen an einen anderen innerkantonalen Kostenträger (z.B. eine kantonale Fürsorgedirektion) zur Bezahlung weiterleitet. Vor dem Erbringen von Gesundheitsdienstleistungen für Personen ohne Versicherungspflicht hat die Vollzugseinrichtung - Notfallbehandlungen ausgenommen - vorgängig eine Kostengutsprache der Einweisungsbehörde einzuholen. ${ }^{889}$ Dem Merkblatt ist weiter zu entnehmen, dass die Gesundheitsversorgung im Rahmen der obligatorischen Grundversicherung gemäss dem KVG einen Teil des absoluten Existenzminimums bilde und in jedem Fall sicherzustellen sei, weshalb Vollzugseinrichtungen zur Sicherstellung dieser medizinischen Versorgung das nötige medizinische Personal und die nötige medizinische Infrastruktur bereitzustellen oder dafür besorgt zu sein hätten, dass die entsprechende medizi-

885 Die Zentralstelle kann gemäss Art. 6 lit. c des Organisationsreglements zur Vereinfachung und Verbesserung der interkantonalen Zusammenarbeit Merkblätter erlassen: OSK - Organisationsreglement v. 29.03.2019.

886 OSK - Merkblatt Gesundheitskosten im Straf- und Massnahmenvollzug v. 24.10.2008.

887 OSK - Merkblatt Gesundheitskosten im Straf- und Massnahmenvollzug v. 24.10.2008: Ist die Inhaftierte Person dazu nicht in der Lage, ist der zuständigen Sozialhilfebehörde ein Kostenübernahmegesuch einzureichen, S. 2 f.

888 Personen mit zivilrechtlichem Wohnsitz in der Schweiz unterstehen dem Obligatorium des KVG (Art. 3 Abs. 1 KVG; Art. 1 Abs. 1 KVV i.V.m. Art. 23 bis 26 ZGB). Der Wohnsitz i.S.v. Art. 23 ZGB wird vom Freiheitsentzug nicht berührt. Der Bundesrat hat weiter von der Möglichkeit i.S.v. Art. 3 Abs. 3 KVG Gebrauch gemacht und mit Art. 1 Abs. 2 KVV die Versicherungspflicht auf verschiedene weitere Personenkategorien ausgedehnt. Vgl. im Einzelnen auch Künzli/Weber S. 8: Ein „beträchtlicher Teil der inhaftierten Personen“ verfüge über keinen Versicherungsschutz im Sinne des KVG.

889 OSK - Merkblatt Gesundheitskosten im Straf- und Massnahmenvollzug v. 24.10.2008, S.3; kritisch dazu KüNZLI/Weber S. 6 ff.: Es werde damit nur der Leistungsumfang gewährt, dessen Kosten getragen würden. Personen ohne Versicherungsschutz seien damit schlechter gestellt, könnten sie im Einzelfall doch andere Leistungen erhalten als Personen mit Versicherungsschutz. Die Vorgaben im Merkblatt könnten dazu führen, dass eine Behandlungsverweigerung drohe, bis eine Notfallbehandlung notwendig sei. 
nische Unterstützung zeitgerecht von aussen beigezogen werden könne oder ausserhalb der Vollzugseinrichtung bereitstehe. ${ }^{890}$ Hinsichtlich der Frage der Vertraulichkeit medizinischer Angaben enthält die Richtlinie der Strafvollzugskommission nach Massgabe von Art. 2 Abs. 2 lit.c der Konkordatsvereinbarung über die Laufakte ${ }^{891}$ Bestimmungen zur Weitergabe von medizinischen Informationen. Zur Einweisung von Gefangenen aus Vollzugseinrichtungen, welche unter den Anwendungsbereich des Ostschweizer Strafvollzugskonkordats fallen, in ein Spital oder eine psychiatrische Klinik veröffentlichte die Zentralstelle ein Merkblatt, welches festhält, dass medizinische Abklärungen und Behandlungen soweit möglich in der Vollzugseinrichtung zu erfolgen haben (Ziff. 2 Abs. 1). ${ }^{892}$ In der Folge sieht es Modalitäten und Vorgehen vor für den Fall, dass diese ausserhalb der Vollzugsanstalt erfolgen müssen.

Das Straf- und Justizvollzugsgesetz des Kantons Zürich (StJVG ZH) hält in organisatorischer Hinsicht zur medizinischen Versorgung fest, dass die Direktion diese mit eigenem Personal zu erbringen hat ( $\$ 24$ Abs. 1 lit. a StJVG ZH). Kann die Leistung nicht mit eigenem Personal erbracht werden, beauftragt die Direktion Sachverständige ( $\$ 24$ Abs. 2 StJVG ZH). Ein Anspruch auf freie Wahl der Leistungserbringer besteht für Personen im Straf- und Massnahmenvollzug nicht ( $\$ 24$ Abs. 3 StJVG ZH). Weiterführende Einzelgarantien enthält das StJVG ZH hinsichtlich der Gesundheitsversorgung im Strafvollzug nicht.

Die Justizvollzugsverordnung des Kantons Zürich (JVV ZH) normiert systematisch unter dem Titel „Eintritt“, dass der Gesundheitszustand der verurteilten Personen durch medizinisches Fachpersonal abgeklärt wird ( $\$ 96$ Abs. 1 JVV ZH). Nicht anwendbar ist diese Bestimmung auf verurteilte Personen, die ihre Strafe im Regime der Halbgefangenschaft verbüssen oder in die Vollzugsstufe des Arbeitsexternats übertreten. Ihnen steht die Möglichkeit offen, selbst eine Ärztin oder einen Arzt aufzusuchen ( $\$ 96$ Abs. 2 JVV ZH). Beim Vorliegen begründeter Zweifel an der Richtigkeit des Arztzeugnisses kann die Leitung der Vollzugseinrichtung medizinische Abklärungen durch einen Vertrauensarzt anordnen ( $\$ 96$ Abs. 3 JVV ZH).

890 OSK - Merkblatt Gesundheitskosten im Straf- und Massnahmenvollzug, v. 24.10.2008, S. 1.

891 OSK - Richtlinie über die Laufakte v. 19.04.2012, Kap. 3.2.

892 OSK - Merkblatt betreffend Einweisungen von Gefangenen aus Vollzugseinrichtungen des OSK in ein Spital oder eine psychiatrische Klinik vom Juli 2019, S. $1 \mathrm{ff}$. 
Für die Dauer des Vollzugs sind Fragen zur Gesundheit und Betreuung des Inhaftierten in den $\$ \mathbb{S} 106 \mathrm{ff}$. JVV ZH geregelt. $\$ 108 \mathrm{JVV} \mathrm{ZH}$ statuiert dabei in grundlegender Weise, dass die Vollzugseinrichtung für die körperliche und geistige Gesundheit der verurteilten Person sorgt. Zur Vermeidung von gesundheitlichen Risiken können ärztliche oder psychiatrische Untersuchungen und Abklärungen veranlasst werden. $\$ 109$ JVV ZH normiert weiter, dass die ärztliche Betreuung im Normalvollzug durch den Gefängnisarzt erfolgt. Liegen erhebliche Gründe dafür vor, dass dieser vom Inhaftierten abgelehnt wird, sind Stellvertreter des Gefängnisarztes oder andere von der Vollzugseinrichtung zu bestimmende Ärzte beizuziehen. Die verurteilten Personen haben sich weiter den ärztlichen Anweisungen zu unterziehen, wobei die Leitung der Vollzugseinrichtung Abklärungen durch einen Vertrauensarzt anordnen kann ( $\$ 109$ Abs. 2 JVV ZH). Eine zahnärztliche Behandlung erfolgt nur in dringenden Fällen, eine weitergehende zahnärztliche Behandlung erfolgt auf Kosten der verurteilten Person, nach Vorliegen einer Kostengutsprache durch die Krankenversicherung oder nach Bewilligung durch die fürsorgerechtlich zuständige Behörde ( $\$ 109$ Abs. 3 JVV ZH). Gemäss $\$ 106$ Abs. 2 und 3 JVV ZH dürfen verurteilte Personen nur jene Medikamente besitzen und einnehmen, welche vom Gefängnisarzt zugelassen oder verschrieben wurden. Personen in Halbgefangenschaft haben für ihre Medikamente ein ärztliches Zeugnis vorzuweisen.

Die Überweisung in eine Klinik oder in ein Spital hat zu erfolgen, wenn der Gesundheitszustand der verurteilten Person dies erfordert. Vorausgesetzt wird grundsätzlich die vorgängige Zustimmung der einweisenden Behörde. In dringenden Fällen hat die Vollzugseinrichtung unter gleichzeitiger Information der einweisenden Stelle die Verlegung selbst zu veranlassen $(\$ 110$ Abs. $1 \mathrm{JVV} \mathrm{ZH})$. Die Bewachung des Inhaftierten ist sicherzustellen, wenn er flucht- oder gemeingefährlich ist $(\mathbb{\$} 110 \mathrm{Abs.} 2$ JVV ZH).

$\mathrm{Zu}$ den Behandlungskosten ist in $\mathbb{1 1 1}$ Abs. $1 \mathrm{JVV}$ ZH festgehalten, dass die notwendige hausärztliche Behandlung von der Vollzugseinrichtung getragen werde, sofern Krankenkasse oder Unfallversicherung nicht dafür aufkommen würden. Weitergehende Behandlungen oder die Einweisung in ein Spital oder eine Klinik würden nur erfolgen, wenn die Kosten von der verurteilten Person übernommen werden oder eine Kostengutsprache vorliegt. ${ }^{893}$ Bei medizinischer Indikation ist eine solche Kostengutsprache

893 Kritisch dazu Künzli/Weber S. 43 f.: Entscheide, ob und welche medizinische Massnahmen wann vorgenommen werden, sollten von der Frage der Kostentra- 
von der Vollzugseinrichtung bei der fürsorgerechtlichen zuständigen Behörde oder, wenn die verurteilte Person von einem anderen Kanton eingewiesen worden ist, bei der einweisenden Behörde einzuholen $(\mathbb{1} 111 \mathrm{Abs.} 2$ JVV ZH). Die Behandlung ist ohne Kostengutsprache anzuordnen, wenn es sich um dringende Fälle handelt. Diesfalls bleibt eine Kostengutsprache so rasch wie möglich einzuholen ( $\mathbb{1 1 1}$ Abs. $3 \mathrm{JVV} \mathrm{ZH})$.

$\$ 112$ JVV ZH sieht schliesslich vor, dass die verurteilte Person pro behandelten Krankheitsfall und pro zahnärztliche Behandlung eine Umtriebsentschädigung in der Höhe von CHF 5.- zu entrichten hat.

Neben den strafvollzugsspezifischen Regelungen haben auch die Gesundheitsgesetze Geltung. Auf bundesrechtlicher Ebene ist dies etwa das Epidemiengesetz $(\mathrm{EpG})^{894}$ mit der dazugehörenden Epidemienverordnung $(\mathrm{EpV})^{895}$ sowie den Verordnungen des Eidgenössischen Department des Innern ${ }^{896}$; auf kantonaler Ebene sind es die allgemeinen Gesundheitsgesetze. 897

Konkrete, spezifisch für den Strafvollzug ausschlaggebende Normen finden sich damit vornehmlich auf kantonaler Ebene. Ausgehend vom im Jahr 2008 vom Bundesamt für Gesundheit (BAG) und vom Bundesamt für Justiz (BJ) unter Zustimmung der Konferenz der kantonalen Justizund Polizeidirektorinnen und -direktoren $\left(\mathrm{KKJPD}^{898}\right)$ lancierten Projekt „Bekämpfung von Infektionskrankheiten im Gefängnis“ (BIG) wurde der Bedarf nach einer weitergehenden interkantonalen Harmonisierung der Gesundheitsversorgung im Strafvollzug festgestellt. ${ }^{899}$ Im Jahr 2013 erliess der Steuerungsausschuss des BIG die „Empfehlung zur Harmonisierung der Gesundheitsversorgung im schweizerischen Freiheitsentzug". Eine der Empfehlungen betraf die Schaffung eines „Fachrats für Gesundheitsfragen im Justizvollzug“ (später „Santé Prison Suisse“ [SPS]), welcher der Vereinheitlichung der über 100 Institutionen des Freiheitsentzugs dienen sollte.

gung entkoppelt werden. De lege ferenda sei die Finanzierung der Gesundheitsversorgung mit einer Fondslösung zu regeln.

894 Bundesgesetz über die Bekämpfung übertragbarer Krankheiten des Menschen (Epidemiengesetz) vom 28. September 2012.

895 Verordnung über die Bekämpfung übertragbarer Krankheiten des Menschen (Epidemienverordnung) vom 29. April 2015.

896 Vgl. etwa Verordnung des EDI über die Meldung von Beobachtungen übertragbarer Krankheiten des Menschen vom 1. Dezember 2015.

897 Etwa: Gesundheitsgesetz des Kantons Zürich vom 2. April 2007.

898 Zur Rolle des KKJPD bei der Umsetzung menschenrechtlicher Vorgaben: KIENER in: Fink/Arnold/Genillod-Villard/Oberholzer, S. 3 (21).

899 Schweizerisches Kompetenzzentrum für den Justizvollzug - Gesundheit im Justizvollzug, Chronologie, zuletzt abgerufen am 23.12.2019. 
In seiner Pilotphase in den Jahren 2014 und 2015 veröffentlichte die SPS die Informationspapiere „Gesundheit im Freiheitsentzug“ je für das Justizvollzugspersonal sowie für die Inhaftierten. Im Schlussbericht über die Pilotphase wurde ein grosser Handlungsbedarf bezüglich der Erstellung, Umsetzung und Überprüfung von medizinischen Minimalstandards und in der Klärung wichtiger Schnittstellenbereiche (z.B. Arztgeheimnis oder Medikamentenabgabe) festgehalten. ${ }^{900}$ Im Jahr 2018 wurde die SPS in die neuen Strukturen des Schweizerischen Kompetenzzentrums für den Justizvollzug integriert. Eine weitere Harmonisierung bei einzelnen Fragen der Gesundheitsversorgung im Strafvollzug ist damit in Zukunft zu erwarten.

Wirkungen auf gesamtschweizerischer Ebene entfalten auch die von der Zentralen Ethikkommission der Schweizerischen Akademie der medizinischen Wissenschaften (SAMW) von medizinethischer Seite her erlassene Richtlinie zur Ausübung der ärztlichen Tätigkeit bei inhaftierten Personen, mit denen Ärzten und anderen Gesundheitsfachpersonen konkrete Hilfestellung für die medizinische Praxis geboten wird. ${ }^{901}$ Verschiedene vollzugsspezifische Themen werden bisweilen sehr detailliert angesprochen: Die Verweigerung aus Gewissensgründen, Untersuchungsbedingungen, Gutachtertätigkeiten und -situationen, Disziplinarstrafen, Gleichwertigkeit der Behandlung, durch die Behörden beschlossene Zwangsmassnahmen im Polizeigewahrsam oder im Strafvollzug, Einwilligung in eine Zwangsbehandlung, ansteckende Krankheiten, Hungerstreik, Vertraulichkeit, Erstattung einer Anzeige über eventuelle Misshandlungen, ärztliche Unabhängigkeit und Ausbildung. Die im Nachgang an den Bundesgerichtsentscheid zum „Fall Rappaz“902 erfolgte Evaluation einer durch die Zentrale Ethikkommission der SAMW (ZEK) bestellten Arbeitsgruppe

900 Schweizerisches Kompetenzzentrum für den Justizvollzug - Gesundheit im Justizvollzug, Chronologie, zuletzt abgerufen am 23.12.2019; vgl. auch Informationen zum Straf- und Massnahmenvollzug, info bulletin 1/2015, Fokus: Gesundheitsversorgung, zuletzt abgerufen am 23.12.2019: Gemäss TroxLeR, dem Chef Fachbereich Straf- und Massnahmenvollzug, kenne die Schweiz in den einzelnen Kantonen verschiedene Modelle der Gesundheitsversorgung, diese reichten von höchst professionell bis verbesserungsbedürftig. Insbesondere wegen häufiger Verlegungen zwischen Vollzugseinrichtungen und zwischen Kantonen würden sich einheitliche Standards aufdrängen. Dies insbesondere bzgl. der Medikamentenabgabe, des Führens der Patientenakten, der Schulung des medizinischen Hilfspersonals oder der Mitgabe von Medikamenten und Patientenakten bei Verlegung.

901 SAMW - Medizin-ethische Richtlinien, Ausübung der ärztlichen Tätigkeit bei inhaftierten Personen.

902 BGE 136 IV 97; EGMR - Rappaz/SUI, Ensch. v. 26.03.2013, 73175/10, Rn. 60 ff. 
hat ergeben, dass die in den Richtlinien festgehaltenen Prinzipien noch unvollständig umgesetzt seien und in einzelnen Bereichen Konkretisierungsbedarf bestehe, weshalb ein Anhang zur vorgenannten Richtlinie verabschiedet wurde. ${ }^{903}$ Zur Umsetzung des Anspruchs auf gleichwertige Behandlung inner- und ausserhalb des Strafvollzugs wurde darin beispielsweise festgehalten, dass es problematisch sei, wenn aus Ressourcengründen Medikamente durch Strafvollzugspersonal abgegeben würden. Dieses Vorgehen tangiere sowohl das Berufsgeheimnis als auch das Heilmittelgesetz $(\mathrm{HMG})^{904}$. Die Realität in Schweizer Haftanstalten zwinge dazu, Lösungen zu finden, die sowohl ein angemessenes Funktionieren der medizinischen Betreuung ermöglichen als auch die rechtlichen Vorgaben respektieren. Hierbei sieht die SAMW eine Delegationspraxis vor, welche verschiedene Voraussetzungen einzuhalten hat. ${ }^{905}$ Hinsichtlich der Sicherstellung einer äquivalenten Gesundheitsversorgung inner- und ausserhalb des Strafvollzugs postuliert die ZEK, künftig alle inhaftierten Personen dem Krankenversicherungsobligatorium zu unterstellen. ${ }^{906}$

Zusammengefasst ist damit festzuhalten, dass aus dem bundesrechtlichen Normalisierungsgebot der Leitgedanke der äquivalenten Gesundheitsversorgung inner- und ausserhalb des Vollzugs fliesst. Einzelne konkrete Garantien finden sich insbesondere in den kantonalen Vorschriften.

903 SAMW - Medizin-ethische Richtlinien, Ausübung der ärztlichen Tätigkeit bei inhaftierten Personen, Anhang.

904 Bundesgesetz über Arzneimittel und Medizinprodukte (Heilmittelgesetz) vom 15. Dezember 2000.

905 Im Einzelnen vgl. SAMW - Medizin-ethische Richtlinien, Ausübung der ärztlichen Tätigkeit bei inhaftierten Personen, Anhang lit. b: Es sei vorauszusetzen, dass das Arzneimittel von einem Arzt verordnet wurde und es von einer öffentlichen Apotheke in die Anstalt geliefert wurde. Weiter hat das Arzneimittel solange es in der Anstalt aufbewahrt wird der Aufsicht und Kontrolle eines ermächtigten Apothekers zu unterstehen. Wenn immer möglich sollten die Arzneimittel sodann in einer neutralen Form (Dosierbehälter, Pillenbox) abgegeben werden, die es ermöglicht, die Vertraulichkeit zu wahren. Der Aufseher hat sich schliesslich darauf zu beschränken, zu prüfen, dass die Verteilung der Dosierbehälter korrekt vorgenommen wird. Im Zweifelsfall muss er den Apotheker oder den zuständigen Arzt kontaktieren und deren Anweisungen befolgen.

906 Vgl. dazu SAMW - Stellungnahme der Zentralen Ethikkommission (ZEK) der Schweizerischen Akademie der Medizinischen Wissenschaften (SAMW) betreffend Finanzierung medizinischer Leistungen im Gefängnis, S. 1 ff.; so auch der Vorschlag de lege ferenda von KüNZLI/WeBER S. 44 in ihrem Rechtsgutachten betreffend die Gesundheitsversorgung von inhaftierten Personen ohne Krankenversicherung. 
Auch das Vertrauens- bzw. das Vertraulichkeitsprinzip ist durch die Pönalisierung der Verletzung des Berufsgeheimnisses schon bundesrechtlich verankert. Einzelheiten mit Ausnahmemöglichkeiten bezüglich Informationspflichten gegenüber der Vollzugseinrichtung finden sich wiederum in den kantonalen Bestimmungen. Das Vorgehen bei einem nicht mehr bestehenden Vertrauensverhältnis zwischen inhaftiertem Patienten und Gefängnisarzt ist auf kantonaler Ebene verankert. Auch das Freiwilligkeitsprinzip, welches schon aufgrund des Äquivalenzprinzips grundsätzlich Wirkung entfaltet, erfährt durch kantonale Bestimmungen Einschränkungsmöglichkeiten (vgl. insb. $\$ 96$ Abs. 1 JVV ZH und $\$ 108$ Abs. 1 JVV ZH). Wie schon in den europarechtlichen Regelwerken wird auch in der Schweiz nur die Phase des Eintritts in den Vollzug und die Phase während des Vollzugs normiert. Vorschriften zur Gesundheitsversorgung, welche die Phase der Haftentlassung betreffen, finden sich keine.

Einzelne organisatorische Fragen, welche die Einhaltung dieser Prinzipien und Rechtsnormen betreffen, werden den einzelnen Institutionen überlassen. In diesen Ermessensspielraum greifen die Harmonisierungsbemühungen des Schweizerischen Kompetenzzentrums für den Justizvollzug. Die Harmonisierungsempfehlungen können sich darüber hinaus auf kantonale Rechtssetzungsvorhaben auswirken.

\section{Rechtsprechung in der Schweiz - Analyse}

\subsection{Materieller Kern des Standards}

Das Bundesgericht garantiert von Verfassungs wegen kranken Inhaftierten, die sich gesundheitlich beeinträchtigt fühlen, das Recht auf medizinische Hilfe bzw. eine ärztliche Untersuchung. ${ }^{907}$ Dies habe namentlich auch bei Haftantritt zu gelten. ${ }^{908}$ Es sei jedoch zulässig, diese Untersuchung von einem entsprechenden Wunsch des Inhaftierten abhängig zu machen. ${ }^{909}$

907 BGE 123 I 221, E. II.2.

908 BGE 123 I 221, E. II.2.

909 BGE 123 I 221, E. II.2: Im Entscheid handelte es sich um eine staatsrechtliche Beschwerde der Demokratischen JuristInnen der Schweiz, Regionalgruppe Basel, mit welcher sie den Erlass der Verordnung über das Gefängniswesen (VG/BS) des Regierungsrats des Kantons Basel-Stadt anfochten. Die Basler Rechtsnorm sah vor, dass jede neu eintretende Person u.a. zur Vorabklärung allfälliger Beeinträchtigungen des Gesundheitszustandes einer körperlichen Untersuchung unterzogen werden könne. Die Beschwerdeführer argumentierten 
Wenn die medizinische Betreuung durch einen Gefängnisarzt ausreichend sichergestellt ist, haben Gefangene grundsätzlich kein Recht auf freie Arztwahl. ${ }^{910}$ Der grundrechtliche Anspruch des Inhaftierten auf ausreichende (spezial-)ärztliche Versorgung kann im Einzelfall gleichwohl den Beizug eines weiteres Arztes oder die Verlegung in ein öffentliches Spital oder eine psychiatrische Klinik notwendig machen. ${ }^{911}$ Dieser Anspruch kann auch dann bestehen, wenn das Verhältnis zum Gefängnisarzt aus objektiven Gründen gestört ist. ${ }^{912}$

Mit Blick auf die zeitliche Komponente der medizinischen Versorgung hält das Bundesgericht fest, dass Überbelegungssituationen Einschränkungen mitunter hinsichtlich des Zugangs zur medizinischen Versorgung mit Ausnahme der Versorgung eines Notfalls - mit sich bringen können, ohne dass darin zwingend eine Verletzung von Art. 3 EMRK zu sehen sei. ${ }^{913}$

Zur Frage der medizinischen Versorgung während einer Disziplinarmassnahme würdigte das Bundesgericht, dass der Inhaftierte vor der Disziplinarmassnahme ärztlich untersucht worden sei und er während der fünf-

sinngemäss, dass diese Norm zu kurz greife und jedem neuen Inhaftierten vielmehr ein Anspruch zustehen müsse, bei Eintritt von einer medizinischen Fachperson auf mögliche Krankheiten untersucht zu werden. Gerügt wurde vorliegend eine Verletzung der persönlichen Freiheit.

910 BGE 123 I 221, E. II.2; BGE 102 Ia 302, E. 2c: Die Einschränkung des Rechts auf freie Arztwahl begründet das Bundesgericht mit dem „Interesse einer vernünftigen Ordnung und Organisation der Anstalt", wobei es insbesondere auf den erheblichen Mehraufwand verweist, welcher durch den Beizug externer Ärzte entstehen würde.

911 BGE 123 I 221, E. II.2; BGE 102 Ia 303, E. 2c; BGE 105 IA 379, E. 5; vgl. auch Art. 234 Abs. 2 StPO.

912 BGE 123 I 221, E. II.2; BGE 102 Ia 302, E. 2c: Das Vertrauensverhältnis sei als gestört anzusehen, wenn gewichtige Anhaltspunkte dafür bestünden, dass persönliche Gründe eine einwandfreie Betreuung des Gefangenen verunmöglichen oder erheblich erschweren würden. Eine förmliche Beschwerde gegen den Gefängnisarzt sei zur Annahme eines gestörten Vertrauensverhältnisses nicht erforderlich.

913 BGer 1B_404/2013, Urt. v. 26.02.2014, E. 2.6.3: Die Klinikdirektion hatte einen Bericht verfasst, in welchem sie festhielt, dass die Wartefristen für eine medizinische Behandlung von der Schwere der Erkrankung abhängen würden. Im Notfall sei eine sofortige Versorgung garantiert, während bei somatischen Erkrankungen eine Wartefrist von einem Monat und für psychische nicht notfallmässige Erkrankungen eine solche von mehreren Monaten möglich sei. Im konkreten Fall wurde vom Beschwerdeführer allerdings keine Verzögerung gerügt. Vgl. auch BGer 1B_152/2015, Urt. v. 29.09.2015, E. 2.5; BGer 1B_239/2015, E. 2.5; BGer 1B_336/2013, Urt. v. 26.02.2014, E. 4.6.1. 
tägigen Disziplinarmassnahme nicht um den Beizug eines Arztes ersucht habe. Eine Verletzung von Art. 3 EMRK sei damit nicht bewiesen. ${ }^{914}$

Das Bundesgericht hatte sich mit Blick auf die medizinische Versorgungssituation im Strafvollzug sodann mit Fragen rund um die Verschiebung des Strafantritts bzw. Vollzugsaufschubs erkrankter Personen ${ }^{915}$, eine Unterbrechung der Strafe ${ }^{916}$ oder eine Haftentlassung ${ }^{917}$ auseinanderzusetzen. Eine Verletzung von Art. 3 EMRK wurde, sofern überhaupt gerügt, hier aber nie erkannt. Hervorzuheben ist der „Fall Rappaz“, in welchem das Bundesgericht in Bezug auf das Freiwilligkeitsprinzip festhielt, dass die Strafvollzugsbehörde die Zwangsernährung anordnen könne. ${ }^{918}$ Eine Vollzugsunterbrechung käme aufgrund ihrer Subsidiarität erst in Frage, wenn der Gefahr für die Gesundheit des Inhaftierten nicht durch eine Zwangsernährung begegnet werden könne. ${ }^{919}$ Hinsichtlich der Voraussetzungen zur zulässigen Durchführung einer Zwangsernährung greift das Bundesgericht auf die Kriterien der Strassburger Rechtsprechung zurück. ${ }^{920}$ Weiter stehe der Strafantritt insbesondere auch aufgrund einer potentiell lebensgefährlichen Erkrankung nicht mit Art. 3 EMRK im Widerspruch, wenn die Lebensgefahr sowohl inner- als auch ausserhalb des Strafvollzugs vorliege, wobei das Bundesgericht insbesondere den Umstand würdigte, dass die Erkrankung innerhalb des Vollzugs gemäss vorliegenden Gutachten gar besser überwacht werden könne als ausserhalb des Vollzugs. Das Leben des Betroffenen sei nicht wegen des Vollzugs, sondern unabhängig davon gefährdet und ein weiterer Aufschub des Strafvollzugs deshalb ausgeschlossen..$^{921}$

914 BGE 124 I 231, E. 2c.

915 Vgl. etwa BGer 6B_1343/2016, Urt. v. 06.02.2017; BGer 6B_72/2018, Urt. v. 13.03.2018; BGer 6B_510/2008, Urt. v. 09.10.2008.

916 BGE 136 IV 97 „Fall Rappaz“. Zu Art.3 EMRK und der Auslegung von Art. $40 \mathrm{aStGB}$, in welchem festgehalten war, dass der Strafvollzug nur aus wichtigen Gründen unterbrochen werden dürfe vgl. BGE 106 IV 321.

917 Vgl. etwa BGer 1B_212/2008, Urt. v. 21.08.2008.

918 BGE 136 IV 97, E. 6.

919 BGE 136 IV 97, E. 6; vgl. auch EGMR - Rappaz/SUI, Entsch. v. 26.03.2013, 73175/10, Rn. 63.

920 Vgl. insb. BGE 136 IV 97, E. 6.1.1.

921 BGer 6B_510/2008, Urt. v. 09.10.2008, E. 3.5 f.: Das Bundesgericht nahm vorliegend keine konkrete Prüfung von Art. 3 EMRK vor, sondern wendete Bundesrecht an. Es kam zum Schluss, dass, soweit der Beschwerdeführer die Verletzung von Grundrechten und von kantonalem Recht rügte, die Beschwerde den Begründungsanforderungen von Art. 106 Abs. 2 BGG nicht genügte (qualifizierte Rügepflicht). 
2.2 Prüfungsmethodik des Schweizerischen Bundesgerichts und Vergleich mit dem EGMR

Die Prüfungsmethodik des Bundesgerichts unterscheidet sich bei der Beurteilung der medizinischen Versorgung im Strafvollzug wesentlich von jener des EGMR. Dies schon aufgrund dessen, dass Menschenrechtsverletzungen vor Bundesgericht tendenziell häufiger als vor dem EGMR hauptsächlich in Bezug auf eine Verletzung der persönlichen Freiheit und nicht zwingend auch mit Blick auf eine unmenschliche oder erniedrigende Behandlung gerügt werden. ${ }^{922}$ Weiter lässt sich die Tendenz feststellen, dass vor Bundesgericht die medizinische Versorgung häufiger als vor dem EGMR mit Blick auf eine Haftentlassung oder einen Unterbruch des Strafvollzugs gerügt wird. ${ }^{923}$ Das Bundesgericht prüft dann typischerweise die Voraussetzungen der nationalen Rechtsnorm, wobei oft deren menschenrechtskonforme Auslegung im Vordergrund steht. ${ }^{924}$

Prüft das Bundesgericht die materiellen Vollzugsbedingungen, fällt weiter auf, dass die Thematik einer mangelhaften medizinischen Versorgung vor Bundesgericht - anders als vor dem EGMR - oft nur Nebenpunkt ist. Im Vordergrund stehen die Frage der Überbelegung und die Frage nach dem jedem Einzelnen zur Verfügung stehenden Platz, wobei das Bundesgericht die medizinische Versorgung als einen der zu berücksichtigenden Faktoren in der Gesamtwürdigung wertet. ${ }^{925}$

Bei der Prüfung von Art. 3 EMRK orientiert sich das Bundesgericht bisweilen ausdrücklich an den Kriterien der Strassburger Rechtsprechung. ${ }^{926}$ Obwohl der Äquivalenzgedanke legislatorisch auch in der Schweiz fest verankert ist, nimmt dieser in der bundesgerichtlichen Rechtsprechung keine wegweisende Funktion ein.

Aufgrund der analysierten Rechtsprechung kann zusammengefasst festgehalten werden, dass der EGMR die medizinische Versorgung im Strafvollzug öfter als eigenständige Garantie prüft, während das Bundesgericht diese vermehrt im Rahmen einer Gesamtwürdigung miteinfliessen lässt. Weder das Äquivalenzprinzip noch der Adäquanzbegriff des EGMR haben

922 Vgl. etwa BGE 123 I 221, E. II.2; BGer 6B_580/2010, Urt. v. 26.07.2010.

923 BGE 136 IV 97; BGE 106 IV 321; BGer 1B_212/2008, Urt. v. 21.08.2008.

924 Vgl. etwa BGE 136 IV 97; BGE 106 IV 321.

925 Vgl. etwa BGer 1B_404/2013, Urt. v. 26.02.2014; BGer 1B_152/2015, Urt. v. 29.09.2015; BGer $1 \bar{B} \_336 / 2013$, Urt. v. 26.02.2014: In die Gesamtwürdigung fliessen daneben regelmässig die Arbeitsmöglichkeiten, Besuchszeiten und Einschlusszeiten ein.

926 Vgl. etwa BGE 136 IV 97. 
sich in der nationalen Rechtsprechung etabliert. Dennoch rezipiert das Bundesgericht in seiner Rechtsprechung einzelne Elemente des Strassburger Adäquanzbegriffs. ${ }^{927}$ Diese unterschiedliche Entwicklung hat mehrere potentielle Erklärungen: (1) Das Bundesgericht ist nicht mit der gleich grossen Fallzahl betraut wie der EGMR, weshalb der EGMR sich zu deutlich mehr Einzelfragen zu äussern hatte, (2) die medizinische Versorgung im schweizerischen Strafvollzug ist im europäischen Vergleich nicht gleich mangelhaft, 928 (3) die beanstandeten Umstände betrafen in der Schweiz mehr die Unterbringungssituation als Ganzes als ein konkretes Versäumnis betreffend die medizinische Versorgung, (4) einzelne Garantien, welche der EGMR aus dem Adäquanzbegriff herleitet, sind in der Schweiz (auf kantonaler Ebene) bereits genügend konkret auf legislativer Ebene normiert, und sie werden in der Praxis eingehalten. ${ }^{929}$ Den Beizug des Adäquanzbegriffes bedarf es deshalb nicht gleichermassen.

927 Vgl. etwa BGE 136 IV 97.

928 Es bleibt allerdings zu bemerken, dass gemäss diversen Berichten von NGOs, welche repräsentative Untersuchungen und Überprüfungen vorgenommen haben, auch im schweizerischen Strafvollzug bei der medizinischen Versorgung durchaus organisatorische, personelle und materielle Defizite zu bemängeln sind, welche in Zukunft möglicherweise auch vom Bundesgericht an Art. 3 EMRK zu messen sein werden: vgl. etwa NKVF - Gesamtbericht über die schweizweite Überprüfung der Gesundheitsversorgung im Freiheitsentzug durch die Nationale Kommission zur Verhütung von Folter (2018-2019), zuletzt abgerufen am 31.12.2019, insb. S. 28 ff., S. 44: Handlungsbedarf erkannte die Kommission etwa hinsichtlich Massnahmen zur Prävention von Infektionskrankheiten und anderen sexuell oder durch Blut übertragbaren Krankheiten, bei der medizinischen Eintrittsuntersuchung und der Medikamentenabgabe durch fachmedizinisches Personal, bei der psychiatrischen und zahnärztlichen Versorgung und bei der Gesundheitsversorgung von weiblichen Inhaftierten. Weiter kritisierte die Kommission die (kantonal) unterschiedliche Beteiligung der inhaftierten Personen an den Kosten für medizinische Behandlung. Vgl. ferner CPT - Rapport au Conseil fédéral suisse [CPT/Inf (2016) 18]: Das CPT empfiehlt unter anderem den Behörden des Kantons Genf eine personelle Verstärkung der medizinischen Teams in den Haftanstalten La Farera, La Stampa und Champ-Dollon (Rn. 53). Weiter empfiehlt das CPT die Sicherstellung, dass täglich mindestens eine Pflegefachperson anwesend ist (Rn. 54). Sodann empfiehlt das CPT ausdrücklich, eine umfassende Eintrittsuntersuchung innert 24 Stunden nach Haftantritt umzusetzen (Rn. 55). Auch hinsichtlich des Grundsatzes der Vertraulichkeit der Gesundheitsversorgung spricht das CPT Empfehlungen aus, insbesondere, dass der Zugang zu den Patientendossiers ausschliesslich auf Gesundheitsfachpersonen beschränkt sein soll (Rn. 57) etc.

$929 \mathrm{Zu}$ beachten ist hier allerdings, dass vor Bundesgericht ohnehin nicht kantonales Recht gerügt werden könnte (vgl. Art. 95 BGG). Zu rügen wäre eine Verlet- 
3. Bundesgerichtliche Rechtsprechung und das Soft Law

\subsection{Rechtsebene}

Die Rezeption des Soft Law durch das Bundesgericht erfolgt im Bereich der medizinischen Versorgung im Strafvollzug grundsätzlich einerseits durch die Thematisierung einer Soft Law-Bestimmung als solcher ${ }^{930}$ und andererseits durch die Auseinandersetzung mit einzelnen, durch den EGMR entwickelten materiellen Kriterien, welche ihrerseits wiederum auf Soft Law-Bestimmungen oder Grundsätzen beruhen.

Hinsichtlich der Phase des Beginns des Strafvollzugs nahm das Bundesgericht in seinem Leiturteil BGE 123 I 221 die Europäischen Mindestgrundsätze für die Behandlung der Gefangenen bei der Frage nach einer zwingenden Eintrittsuntersuchung auf und hielt zunächst fest, dass diese eine blosse Empfehlung und keine völkerrechtlich verbindlichen und gerichtlich durchsetzbaren subjektiven Rechte des Einzelnen enthielten. ${ }^{931}$ In der Folge erwägt das Bundesgericht, dass es mit Blick auf das Recht von Inhaftierten auf medizinische Versorgung zulässig sein müsse, auch die Eintrittsuntersuchung vom Wunsch des Inhaftierten abhängig zu machen. ${ }^{932}$ Eine vertiefte Auseinandersetzung auch mit anderen Empfehlungen zu dieser Thematik erfolgte nicht. ${ }^{933}$

Während das Soft Law für die Phase des Vollzugs für die Gesundheitsversorgung das Äquivalenzprinzip proklamiert und gleichzeitig auch inhaltliche Vorgaben zur Gewährleistung einer angemessenen Gesundheitsversorgung macht, hat sich in der bundesgerichtlichen Rechtsprechung der Äquivalenzgedanke hinsichtlich einzelner Fragen der Gesundheitsversorgung im Vollzug nicht als wegweisend erwiesen, wenngleich das Äquivalenzprinzip schon bundesrechtlich für den Strafvollzug verankert ist (vgl. Art. 75 Abs. 1 StGB). Diese unterschiedliche Herangehensweise lässt sich mit der angewandten Gesamtbetrachtung des Bundesgerichts und allenfalls mit anders gelagerten Sachverhalten erklären.

zung von Art. 3 EMRK oder aber die willkürliche Anwendung der kantonalen Bestimmung.

930 Vgl. etwa BGE 123 I 221, E. II.2.

931 BGE 123 I 221, E. II.2.; BGE 118 Ia 64, E. 2 a.

932 BGE 123 I 221, E. II.2.; BGE 118 Ia 64, E. 2a.

933 Hinsichtlich der Eintrittsuntersuchung empfiehlt das CPT der Schweiz in seinem Rapport au Conseil fédéral suisse [CPT/Inf (2016) 18] jetzt auch ausdrücklich, eine umfassende Eintrittsuntersuchung innert 24 Stunden nach Haftantritt umzusetzen (Rn. 55). 
Insgesamt erfolgt zwar auch in der bundesgerichtlichen Rechtsprechung eine gewisse Rezeption des Soft Law; diese ist aber keineswegs so ausgeprägt wie vor dem EGMR. Dies ist allerdings auch vor dem Hintergrund zu würdigen, dass viele der Soft Law-Prinzipien in der Schweiz bereits positivrechtlich verankert sind, so dass in diesen Fällen die verbindliche Rechtsnorm gerügt werden kann. ${ }^{934}$

\subsection{Sachverhaltsebene}

Nachdem auch vor Bundesgericht Implementierungsberichte internationaler und nationaler Gremien berücksichtigt werden können und sowohl Institutionen auf Europaratsebene als auch nationale Institutionen Schweizer Strafvollzugsanstalten besuchen und im Nachgang (Monitoring-)Berichte verfassen, welche auch die Gesundheitsversorgung Schweizer Haftanstalten betreffen, ${ }^{935}$ stellt sich die Frage nach deren tatsächlicher Bedeutung bei der Sachverhaltserstellung. Soweit ersichtlich nehmen diese Berichte wie schon bei Überbelegungsfragen - auch hinsichtlich der medizinischen Versorgung im Strafvollzug, anders als vor dem EGMR, (bislang) keine essentielle Rolle ein. Auch hier ist dies damit zu begründen, dass die Feststellung des Sachverhalts vor Bundesgericht nur gerügt werden kann, wenn sie offensichtlich unrichtig ist oder auf einer Rechtsverletzung von Art. 95 BGG beruht, d.h., die Sachverhaltserstellung damit vor Bundesgericht nicht gleich relevant ist wie vor dem EGMR.

\section{Zwischenfazit}

Das bundesrechtliche Normalisierungsgebot impliziert Äquivalenz der medizinischen Versorgung inner- und ausserhalb des Strafvollzugs. Viele einzelne Garantien der Gesundheitsversorgung im Strafvollzug sind auf kantonalzürcherischer Ebene festgehalten. Diese richten sich an denselben Grundprinzipien aus, die auch auf Europaratsebene zur Anwendung ge-

$934 \mathrm{Zu}$ beachten bleiben selbstverständlich die vor Bundesgericht zulässigen Beschwerdegründe i.S.v. Art. 95 BGG.

935 Vgl. etwa CPT - Rapport au Conseil fédéral suisse [CPT/Inf (2016) 18]; NKVF Gesamtbericht über die schweizweite Überprüfung der Gesundheitsversorgung im Freiheitsentzug durch die Nationale Kommission zur Verhütung von Folter (2018-2019), zuletzt abgerufen am 31.12.2019. 
langen (Freiwilligkeitsprinzip, Vertraulichkeitsgrundsatz etc.), wobei auch die kantonalen Bestimmungen Ausnahmemöglichkeiten ausdrücklich vorsehen. Besonders detaillierte und spezifische Handlungsanweisungen enthalten sodann die medizinethischen Richtlinien der SAMW. Es gibt verschiedene Harmonisierungsbemühungen, welche etwa durch das Schweizerische Kompetenzzentrum für den Justizvollzug vorangetrieben werden. Weitere Harmonisierungen sind damit in der Zukunft zu erwarten.

Die Analyse der bundesgerichtlichen Rechtsprechung hat gezeigt, dass die mangelhafte medizinische Versorgung, anders als vor dem EGMR, auf nationaler Ebene oft nur Nebenpunkt der Prüfung von Art. 3 EMRK war und vom Bundesgericht prüfungsmethodologisch im Rahmen einer Gesamtbetrachtung der materiellen Haftbedingungen beurteilt wurde. Weder dem Äquivalenzprinzip noch dem Adäquanzbegriff als solchen kommt damit in der bundesgerichtlichen Rechtsprechung eine essentielle Bedeutung zu; ausschlaggebend bleibt gleichwohl die Angemessenheit der medizinischen Versorgung.

Die Rolle des Soft Law betreffend die Gesundheitsversorgung im Strafvollzug ist in der bundesgerichtlichen Rechtsprechung zu Art. 3 EMRK nicht gleich bedeutend wie in der Strassburger Rechtsprechung, was allerdings auch vor dem Hintergrund zu würdigen ist, dass viele der Soft Law-Prinzipien in der Schweiz bereits positivrechtlich verankert sind, so dass in diesen Fällen die verbindliche Rechtsnorm gerügt werden kann. 


\section{Teil 4: Durchsetzungsmechanismen bei unzureichenden Haftbedingungen}

\section{Kapitel 1: Ausgangspunkt}

In erster Linie statuieren die Konvention und die dazugehörige Rechtsprechung den aus menschenrechtlicher Sicht erforderlichen Mindeststandard, welchen es von den Konventionsstaaten einzuhalten gilt. Dies darf aber nicht darüber hinwegtäuschen, dass ein wirksamer Menschenrechtsschutz weder mit dem Gerichtsurteil selbst beginnt noch bei diesem endet. Die Durchsetzung des Mindeststandards ist vielmehr in drei Phasen sicherzustellen. ${ }^{936} \mathrm{Zu}$ unterscheiden sind die Phase vor dem Urteilszeitpunkt, die Phase des Urteils selbst sowie die Phase nach dem Urteilszeitpunkt. ${ }^{937}$ In allen drei Phasen sind denn auch im Konventionssystem Massnahmen vorgesehen, welche die Wirksamkeit des eigenen Mindeststandards sicherstellen sollen. In Anbetracht der verheerenden Folgen unzureichender Haftbedingungen für den Einzelnen ist von diesen Möglichkeiten in der Praxis auch Gebrauch zu machen, denn nur so kann dem statuierten Mindeststandard auch auf tatsächlicher Ebene zum Durchbruch verholfen werden.

Während des laufenden Verfahrens können, durch die fehlende aufschiebende Wirkung der Beschwerdeeinlegung, ${ }^{938}$ im Sinne der Sicherung der praktischen Wirksamkeit und der Entscheidungsoffenheit Massnahmen des vorsorglichen Rechtsschutzes notwendig werden. ${ }^{939}$ Ihr Stellenwert und ihr Potential bei unmenschlichen bzw. erniedrigenden Haftbedingungen wird deshalb im Folgenden analysiert, wobei sowohl die Anordnungspraxis des Gerichtshofes als auch die Befolgungspraxis der Konventionsstaaten massgebend sind. Im Urteilszeitpunkt sind für die praktische Wirksamkeit zunächst die konkreten Urteilswirkungen selbst massgebend. Die klassischen Urteilswirkungen haben durch die weiterentwickelte Verfahrenstechnik - des Piloturteilsverfahrens - Modifikationen

936 Vgl. auch Keller/Marti EJIL 2015, 829 (831), auch Keller und Marti unterteilen die Implementierung des Standards in drei Phasen.

937 Keller/Marti EJIL 2015, 829 (831).

938 SK-StPO/MeYER Verfahrensrecht (EMRK) Rn. 261.

939 SK-StPO/MeYER Verfahrensrecht (EMRK) Rn. 278. 
erfahren. Neben den allgemeinen Urteilswirkungen und deren Bedeutung für die Umsetzung des Mindeststandards ist deshalb insbesondere die Praxis der Piloturteilsverfahren bei unmenschlichen bzw. erniedrigenden Haftbedingungen von Interesse. Es werden dabei sowohl individuelle als auch generelle Massnahmemöglichkeiten und deren Potential in Bezug auf die Durchsetzung des Mindeststandards bei unzureichenden Haftbedingungen austariert.

Für die Effektivität des festgelegten Mindeststandards nicht minder bedeutend, aber in der Literatur häufig weniger Beachtung findend, ${ }^{940}$ ist die Phase nach dem Urteilsspruch, wobei insbesondere Art. 46 EMRK zentral ist, wonach die Konventionsstaaten verpflichtet sind, das endgültige Urteil des Gerichtshofs zu befolgen. Es wird aufgezeigt, dass in dieser Phase das Ministerkomitee zu einem zentralen Akteur wird. Ministerkomitee, Gerichtshof und weitere Gremien wirken jedoch zusammen, so dass hier Wechselwirkungen auszumachen sind. Sodann werden mögliche Umsetzungsschwächen im Bereich inadäquater Haftbedingungen und deren Ursachen eruiert, so dass Optimierungsvorschläge und Zukunftsperspektiven ausgearbeitet werden können.

Kapitel 2: Gewährleistung eines Mindeststandards vor dem Urteilszeitpunkt? Vorsorgliche Massnabmen

I. Bedeutung der vorsorglichen Massnahmen für die Durchsetzung von EGMR-Urteilen

Aufgrund der fehlenden aufschiebenden Wirkung der Beschwerdeeinlegung ${ }^{941}$ verändert diese allein die Situation des Inhaftierten noch nicht; der Inhaftierte hat bis auf weiteres seine Haft unter den gerügten Bedingungen zu verbüssen. ${ }^{942}$ Regel 39 VerfO sieht deshalb, subsidiär zum innerstaatlichen vorsorglichen Rechtsschutz, die Möglichkeit von vorsorglichen Massnahmen vor; dies von Amtes wegen oder auf Antrag ${ }^{943}$ der betroffenen Person. ${ }^{944}$ Im Rahmen einer vorsorglichen Massnahme können

940 So auch Pabel in: Zimmermann, S. $81 \mathrm{ff}$.

941 SK-StPO/Meyer Verfahrensrecht (EMRK) Rn. 261.

942 Dies wiegt umso schwerer, je gravierender die vorgebrachten Mängel und je länger die Verfahrensdauer vor dem Gerichtshof sind.

$943 \mathrm{Zu}$ den formellen Voraussetzungen des Antrags vgl. Practice Directives „Requests for Interim Measures“.

944 SK-StPO/MeYer Verfahrensrecht (EMRK) Rn. 262. 
die Strassburger Organe ${ }^{945}$ die Konventionsstaaten anweisen, was während der Verfahrensdauer bzw. bis zu einer anderslautenden Anordnung des Gerichtshofs zu geschehen oder zu unterbleiben hat (Anordnung der Ergreifung einer einstweiligen aktiven Massnahme oder einer einstweiligen Unterlassung). ${ }^{946}$

Vorsorgliche Massnahmen sollen verhindern, dass während der Verfahrensdauer Tatsachen geschaffen werden, die den Sinn und Zweck der Beschwerde vereiteln. ${ }^{947}$ Vorausgesetzt ist deshalb, dass der Beschwerdeführer glaubhaft macht, dass ein ernsthaftes Risiko (real risk) eines irreversiblen, insbesondere physischen ${ }^{948}$ Schadens unmittelbar droht (imminent risk of irreversible harm). ${ }^{949}$ Diese relativ hohen Hürden widerspiegeln den Ausnahmecharakter ${ }^{950}$ der vorsorglichen Massnahmen. Vor- resp. Bindungswirkungen für die Hauptsacheentscheidung hat der Erlass vorsorglicher Massnahmen nicht, dient er doch gerade auch der praktischen Entscheidungsoffenheit. ${ }^{951}$

Die vom Gerichtshof entschiedene vorsorgliche Massnahme kann nur vom betroffenen Konventionsstaat selbst umgesetzt werden;952 dem EGMR kommt nämlich keine unmittelbar wirkende Anordnungsbefugnis zu. ${ }^{953}$ Vorsorgliche Massnahmen sind für den Konventionsstaat aber verbindlich. ${ }^{954}$ Der Entscheid des Gerichtshofs über die vorsorglichen

945 Die Zuständigkeit für vorsorgliche Massnahmen richtet sich nach Regel 39 VerfO. Zuständig ist die Kammer oder gegebenenfalls der Sektionspräsident oder ein nach Regel 39 Abs. 4 VerfO benannter Dienst habender Richter. Gemäss Regel 39 Abs. 4 VerfO kann der Präsident des Gerichtshofs Vizepräsidenten der Sektion als Dienst habende Richter für die Entscheidung über Anträge auf vorläufige Massnahmen bestimmen.

946 Vgl. EGMR - Aleksanyan/RUS, Urt. v. 22.12.2008, 46468/06, Rn. 228; vgl. SKStPO/Meyer Verfahrensrecht (EMRK) Rn. 264.

947 Keller/Marti EJIL 2015, 829 (833f.); SK-StPO/Meyer Verfahrensrecht (EMRK) Rn. 261.

948 SK-StPO/MeYer Verfahrensrecht EMRK Rn. 269.

949 Karpenstein/Mayer/Schäfer Art. 34 Rn. 101; SK-StPO/MeYER Verfahrensrecht (EMRK) Rn. 262.

950 Factsheet Interim Measures, S. 1, zuletzt abgerufen am 10.11.2019.

951 Karpenstein/Mayer/Schäfer Art. 34 Rn. 105.

952 SK-StPO/MEYER Verfahrensrecht (EMRK) Rn. 264.

953 SK-StPO/Meyer Verfahrensrecht (EMRK) Rn. 264.

954 Der Wortlaut von Regel 39 VerfO „indicate“ hätte auch eine Auslegung hin zu einer blossen Vermutung zugelassen. Die Practice Directions „Request for Interim Measures" sprechen sich in Übereinstimmung mit den Leitentscheiden des EGMR aber für die Verbindlichkeit aus, was nun auch die Konventionsstaaten bestätigt haben. Vgl. dazu auch SK-StPO/Meyer Verfahrensrecht 
Massnahmen kann deshalb an das Ministerkomitee weitergeleitet werden (Regel 39 Abs. 2 VerfO), wodurch der Befolgungsdruck auf den Konventionsstaat erhöht wird.955 Setzt der Konventionsstaat die vorsorgliche Massnahme nicht oder nicht rechtzeitig um, droht ihm eine Verurteilung nach Art. 34 Satz 2 EMRK.

Hauptanwendungsfeld der vorsorglichen Massnahme sind Fälle betreffend Art. 2 EMRK und Art. 3 EMRK, insbesondere Abschiebungs- und Auslieferungsfälle. ${ }^{956}$ Zur Anwendung kommt sie aber auch im Gebiet der unmenschlichen und erniedrigenden Haftbedingungen.

II. Vorsorgliche Massnahmen bei unmenschlichen und erniedrigenden Haftbedingungen i.S.v. Art. 3 EMRK

\section{Anordnung vorsorglicher Massnahmen nach Regel 39 VerfO}

Gegen Art. 3 EMRK verstossende Haftbedingungen können grundsätzlich das Risiko bergen, einen schweren, irreversiblen Schaden herbeizuführen. Die Effektivität der Mindeststandards des Gerichtshofs im Bereich der Haftbedingungen hängt folglich entscheidend von der Anordnungspraxis vorsorglicher Massnahmen einerseits und von der Befolgungspraxis der Konventionsstaaten andererseits ab.

Bei Haftbedingungen, die gegen Art. 3 EMRK verstossen, sind verschiedene Konstellationen denkbar, bei denen vorsorgliche Massnahmen praxis-

(EMRK) Rn. 277 f.; vgl. zur Rechtsprechung EGMR - Mamatkulov u. Askarov/ TUR, Urt. v. 04.02.2005, 46827/99 u. 4951/99, Rn. 104; EGMR - Savriddin Dzhurayev/RUS, Urt. v. 25.04.2013, 71386/10, Rn. 213; vgl. ferner Izmir Declaration v. 27.04.2011, Follow-up Plan A., Rn.3. Vor den Leitentscheiden des EGMR wurde allgemein von einem reinen Empfehlungscharakter der vorsorglichen Massnahmen ausgegangen: vgl. dazu etwa noch deutlich Alleweldt EJIL 1993, 360 (374). Dieser Empfehlungscharakter wurde aber schon früh immer wieder kritisiert: Vgl. etwa Addo/Grief European Law Review - Human Rights Survey 1998, 17 (20 ff.), welche vor dem Hintergrund der Wesensmerkmale eines absoluten Rechts argumentierten, dass der Empfehlungscharaker vorsorglicher Massnahmen die Absolutheit von Art. 3 EMRK unterwandere.

955 SK-StPO/Meyer Verfahrensrecht (EMRK) Rn. 285.

956 Karpenstein/Mayer/Schäfer Art. 34 Rn. 101 f.; vgl. etwa EGMR (Pl) - Soering/ GBR, Urt. v. 07.07.1989, 14038/88, Rn. 4; EGMR - Jabari/TUR, Urt. v. 11.07.2000, 40035/98, Rn. 6; EGMR - Babar Abmad u.a./GBR, Urt. v. 10.04.2012, 24027/07, Rn.5; EGMR (GK) - Saadi/ITA, Urt. v. 28.02.2008, $37201 / 06$. 
relevant werden. In erster Linie ist an schwer erkrankte Inhaftierte zu denken, insbesondere wenn ihr Gesundheitszustand kein längeres Abwarten mehr erlaubt. Bringt der Beschwerdeführer in der Hauptsache eine inadäquate medizinische Versorgung in der Haftanstalt vor, wird bisweilen denn auch eine unabhängige ärztliche Begutachtung angeordnet, welche mit einer konkreten Fragestellung an den Arzt verbunden sein kann. ${ }^{957}$

Im Sinne einer vorsorglichen Massnahme ordnet der EGMR regelmässig direkt einen Transfer in eine Spezialklinik oder in ein (ziviles) Krankenhaus bzw. in eine andere geeignete medizinische Institution an, in denen alle notwendigen Untersuchungen sowie Behandlungen durchzuführen sind. ${ }^{958}$ Die Strassburger Organe können sogar spezifische Vorgaben zum weiteren Vorgehen in der Klinik machen. ${ }^{959}$ So empfahl der EGMR, als zusätzliche vorsorgliche Massnahme etwa ein parteiübergreifendes medizinisches Konsil zu gründen, welches sowohl über die Diagnose als auch über die Behandlung zu beraten habe. ${ }^{960}$ Angeordnete vorsorgliche Massnahmen betreffen keineswegs nur die Behandlung somatischer Krankheiten sondern können auch eine Behandlung psychischer Krankheiten umfassen. ${ }^{961}$ Mit einem Transfer in eine auswärtige Institution resp. eine zivile Klinik können auch Vorgaben bezüglich der Rücküberstellung verknüpft werden, ${ }^{962}$ etwa die Notwendigkeit eines ärztlichen Attests, welches die

957 Vgl. etwa EGMR - Kondrulin/RUS, Urt. v. 20.09.2016, 12987/15, Rn. 21: Konkret wurden an den medizinischen Experten drei Fragen gestellt: erstens, ob die Behandlung, welche dem Beschwerdeführer in der Haft zuteil wurde, mit Blick auf seinen Gesundheitszustand adäquat sei, zweitens, ob der Gesundheitszustand des Beschwerdeführers eine Haft in einem Gefängniskrankenhaus zulasse, und drittens, ob aufgrund des Gesundheitszustandes ein Transfer in eine spezialisierte (zivile) Klinik erforderlich sei. Sofern Letzteres bejaht würde, sei der Transfer in eine solche Klinik als weitere vorsorgliche Massnahme sicherzustellen.

958 EGMR - Kotsaftis/GRE, Urt. v. 12.06.2008, 39780/06, Rn.36; EGMR (GK) Paladi/MDA, Urt. v. 10.03.2009, 39806/05, Rn. 4; EGMR - Salakhov u. Islyamova/UKR, Urt. v. 14.03.2013, 28005/08, Rn. 29; EGMR - Yakovenko/UKR, Urt. v. 25.10.2007, 15825/06, Rn. 3; EGMR - Ghvaladze/GEO, Entsch. v. 11.09.2007, 42047/06; EGMR - Grori/ALB, Urt. v. 07.07.2009, 25336/04; EGMR - Bamouhammad/BEL, Urt. v. 17.11.2015, 47687/13.

959 EGMR - Aleksanyan/RUS, Urt. v. 22.12.2008, 46468/06, Rn. 80.

960 EGMR - Aleksanyan/RUS, Urt. v. 22.12.2008, 46468/06, Rn. 80.

961 Vgl. etwa EGMR - Bamouhammad/BEL, Urt. v. 17.11.2015, 47687/13; EGMR Prezec/CRO, Entsch. v. 28.08.2008, 7508/05.

962 EGMR - Kotsaftis/GRE, Urt. v. 12.06.2008, 39780/06, Rn.36; EGMR - Groril ALB, Urt. v. 07.07.2009, 25336/04, Rn. 64. 
Rückverlegung gutheisst ${ }^{963}$ oder zumindest bescheinigt, dass eine Rücküberstellung keine Lebensgefahr für den Betroffenen bildet. ${ }^{964}$

Bisweilen werden vorsorgliche Massnahmen zu einem späteren Zeitpunkt ergänzt, konkretisiert oder ${ }^{965}$ auch erneuert ${ }^{966}$. Die Strassburger Organe können den Konventionsstaat im Rahmen vorsorglicher Massnahmen auch anweisen, dem Gerichtshof in regelmässigen Abständen Informationen über den weiteren Gesundheitszustand des Inhaftierten zukommen zu lassen. ${ }^{967}$

Adressat der vorsorglichen Massnahme kann nicht nur der Konventionsstaat, sondern ausnahmsweise auch der Beschwerdeführer sein. ${ }^{968}$ Bei medizinischen Fragestellungen im Bereich unmenschlicher oder erniedrigender Haftbedingungen i.S.v. Art. 3 EMRK haben insbesondere Anweisungen des Gerichtshofs zur Einstellung eines Hungerstreiks in der Praxis Relevanz erlangt. ${ }^{969}$ An Beschwerdeführer adressierte Massnahmen dürften allerdings auch in Zukunft eine absolute Ausnahme bleiben.

Der Gerichtshof verwendet bei vorsorglichen Massnahmen in der Regel eine weiche Formulierung wie, that it was desirable and in the interest of the parties and the proper conduct of the proceedings before the Court ${ }^{\text {(9970 }}$ gefolgt von der entsprechenden Anweisung. Die Formulierung ändert aber nichts an der Verbindlichkeit der vorsorglichen Massnahme. Eine Formulierung, welche schon nach ihrem Wortlaut die Verbindlichkeit erkennen lässt, wäre für die Zukunft allerdings gleichwohl wünschenswert.

Während vorsorgliche Massnahmen im Bereich der medizinischen Versorgung in Strafvollzugsanstalten in der Praxis keine Seltenheit sind, spielen sie bei vielen anderen Aspekten der unmenschlichen und erniedrigen-

963 EGMR - Grori/ALB, Urt. v. 07.07.2009, 25336/04, Rn. 64.

964 EGMR - Kotsaftis/GRE, Urt. v. 12.06.2008, 39780/06, Rn. 36.

965 EGMR - Aleksanyan/RUS, Urt. v. 22.12.2008, 46468/06, Rn. 80.

966 EGMR - Grori/ALB, Urt. v. 07.07.2009, 25336/04: Im konkreten Fall lehnte der Gerichtshof die Erneuerung der Massnahme jedoch ab.

967 EGMR - Grori/ALB, Urt. v. 07.07.2009, 25336/04: Albanien wurde zusätzlich noch einmal darauf hingewiesen, dass der Beschwerdeführer aufgrund seines Gesundheitszustandes während der Inhaftierung möglicherweise weitere spezialisierte Begleitung benötige. Die Regierung Albaniens informierte den Gerichtshof daraufhin über die regelmässige Medikamentenabgabe.

968 Karpenstein/Mayer/Schäfer Art. 34 Rn. 99; SK-StPO/Meyer Verfahrensrecht (EMRK) Rn. 263; vgl. etwa EGMR (GK) - Ilascu u.a./MDA u. RUS, Urt. v. 08.07.2004, 48787/99, Rn. 11; EGMR - Rodic/BIH, Urt. v. 27.05.2008, 22893/05, Rn. 4 (Anordnung, den Hungerstreik zu beenden).

969 Vgl. etwa EGMR - Bamouhammad/BEL, Urt. v. 17.11.2015, 47687/13.

970 Vgl. etwa EGMR - Yakovenko/UKR, Urt. v. 25.10.2007, 15825/06, Rn. 3. 
den Haftbedingungen - wie etwa der Überbelegung - gar keine bzw. nur eine marginale Rolle. Bei Überbelegungskonstellationen, welche für Art. 3 EMRK relevant sind, wäre aber durchaus denkbar, dass das Vorliegen der hohen Voraussetzungen von Regel 39 VerfO $^{971}$ regelmässig angenommen werden könnte. Die Belastungssituation bei Überbelegung einer Haftanstalt kann nämlich durchaus unmittelbares und irreversibles Schädigungspotential für die Gesundheit des Beschwerdeführers haben (zu den Auswirkungen von Überbelegungssituationen Teil 3 Kap. 2 II.). Mehrere Gründe sind denkbar, weshalb der Gerichtshof Regel 39 VerfO in Überbelegungssituationen nicht bzw. kaum heranzieht.

Bei der Überbelegung dürfte in erster Linie die psychische Gesundheit und erst zu einem späteren Zeitpunkt auch die physische Gesundheit beeinträchtigt werden. Psychische Gesundheitsschäden werden von der Regel 39 VerfO zwar nicht ausgeschlossen, aber der Schwerpunkt der bisherigen Anordnung vorsorglicher Massnahmen liegt klar auf physischen Gesundheitsschäden. ${ }^{972}$ Angesichts der Schwere und der Irreparabilität der Auswirkungen, welche auch psychische Gesundheitsschäden zeitigen können, überzeugt dies jedoch nicht. Denkbar wäre allerdings, dass ernsthafte Gefährdungen im Sinne eines real risk durch psychische Faktoren weniger "sichtbar" und damit schwerer zu beweisen sind als drohende physische Gesundheitsschäden. Auch dieser Erklärungsansatz greift allerdings zu kurz, erfordert eine Anordnung vorsorglicher Massnahmen doch gerade keinen Nachweis, sondern nur eine substantiierte Glaubhaftmachung des unmittelbar drohenden irreversiblen Schadens, ${ }^{973}$ was auch bei psychischen Schäden grundsätzlich sollte gelingen können. Zumindest mitursächlich für die restriktive Praxis des Gerichtshofs bei Überbelegungssituationen könnte vor diesem Hintergrund auch sein, dass der Gerichtshof den Unrechtsgehalt von Überbelegungssituationen in seiner Rechtsprechung nicht durchgehend spezifiziert. Während dies im Hauptsacheentscheid zur dogmatischen Unschärfe bei der Einordnung zur unmenschlichen oder erniedrigenden Behandlung i.S.v. Art. 3 EMRK führt, kann dies betreffend die Notwendigkeit der Anordnung vorsorglicher Massnahmen Verschleierungen tatsächlicher physischer und psychischer Folgen mit sich bringen. Möchte ein Beschwerdeführer aufgrund einer Unterbringung in

971 Rules of Court, zuletzt abgerufen am 06.08.2020.

972 SK-StPO/MeYER Verfahrensrecht (EMRK) Rn. 269.

973 SK-StPO/Meyer Verfahrensrecht (EMRK) Rn. 268: Die substantiierte Glaubhaftmachung habe mit Unterlagen, die in den Practice Directives genannt werden, und anhand der eigenen plausiblen Ausführungen zu erfolgen. 
einer überbelegten Haftzelle die Anordnung vorsorglicher Massnahmen i.S.v. Regel 39 VerfO beantragen, ist es deshalb umso wichtiger, dass er die unmittelbaren tatsächlichen Auswirkungen der Überbelegungssituation für seine Gesundheit konkret benennt und diese glaubhaft macht. Da vorsorgliche Massnahmen aber auch von Amtes wegen anzuordnen sind, hat beim Gerichtshof insbesondere auch in diesem Kontext zwingend eine Sensibilisierung hinsichtlich des tatsächlichen Schädigungspotentials von Überbelegungssituationen für die körperliche und psychische Gesundheit einzusetzen. ${ }^{974}$ Dabei hat sich der Gerichtshof jeweils am aktuellen Stand der medizinischen Wissenschaft zu orientieren.

Vergleicht man allerdings Überbelegungssituationen mit den Situationen der Anordnung vorsorglicher Massnahmen bei der medizinischen Versorgung, wird deutlich, dass bei Letzteren die Situation in aller Regel akuter, d.h. aus einer zeitlichen Sicht dringlicher ist als in den Überbelegungsfällen, weshalb bei diesen besonderes Augenmerk auf das „Unmittelbarkeitserfordernis“ zu legen sein wird. Dies wird dabei mit Blick auf die potentielle Verfahrensdauer auszulegen sein.

Es stellt sich weiter die Frage, ob die aus dem Sonderstatus fliessende besondere Fürsorgepflicht nicht dazu führen müsste, die Voraussetzungen des unmittelbar drohenden irreparablen Schadens grosszügiger auszulegen. Dies ist wohl aber nicht der Fall; das besondere Näheverhältnis zwischen Inhaftiertem und Staat führt zwar in materieller Hinsicht zu weitergehenden positiven Verpflichtungen, die Voraussetzungen von Regel 39 VerfO sind aber nicht mit der Hauptsacheentscheidung verknüpft, weshalb eine Lockerung der hohen Anforderungen wohl nicht der ratio und dem telos von Regel 39 VerfO entsprechen würde. Auswirkungen auf den vorsorglichen Rechtsschutz hat das Sonderstatusverhältnis insofern also nicht.

Rückt man die Rechtsfolgenseite einer hypothetischen Anordnung vorsorglicher Massnahmen aufgrund massiver Überbelegung ins Blickfeld, gäbe es durchaus geeignete und schnell realisierbare Abhilfemassnahmen. Diese könnten weitestgehend den individuellen Massnahmen, welche im Anschluss an einen Verstoss gegen Art. 3 EMRK angeordnet werden (vgl. Teil 4 Kap. 3 III. 2.1.), entsprechen. Sie wären bei einer vorsorglichen Massnahme aber selbstverständlich nur vorübergehender Natur und hätten hinsichtlich der Hauptsacheentscheidungen keine Vor- resp. Bindungs-

974 Hinsichtlich des Hauptsacheentscheides ist daraus aber keineswegs der Schluss zu ziehen, dass eine Verletzung von Art. 3 EMRK nur beim Vorliegen eines Nachweises physischer oder psychischer Folgen anzunehmen wäre. 
wirkungen. Es ist dementsprechend nicht davon auszugehen, dass die $\mathrm{Zu}$ rückhaltung bei der Anordnung vorsorglicher Massnahmen auf einem fehlenden Massnahmenrepertoire beruht. Vor diesem Hintergrund ist das Potential vorsorglicher Massnahmen durch den Gerichtshof in Zukunft noch besser auszuschöpfen. Für Überbelegungssituationen bedeutet dies, dass der Gerichtshof in all jenen Fällen, bei welchen die Überbelegungssituation zu einem unmittelbar irreversiblen physischen oder psychischen Schaden zu führen droht, vorsorgliche Massnahmen anzuordnen hat.

Der Anordnung vorsorglicher Massnahmen kommt insgesamt eine grosse Bedeutung bei der Sicherstellung der praktischen Wirksamkeit des Mindeststandards des Gerichtshofs zu; sie gewährleisten, dass das Urteil seine „menschenrechtserhaltenden resp. menschenrechtsermöglichenden Wirkungen"975 überhaupt noch entwickeln kann. Vor dem Hintergrund, dass der Gerichtshof dem Bestreben folgt, den Beschwerdeführer wann immer möglich in die Situation zu versetzen, in der er sich ohne Konventionsverletzung befinden würde, ${ }^{976}$ ist es umso bedenklicher, dass der Gerichtshof dieses Potential im Bereich von Überbelegungsfällen noch nicht erkannt hat und folglich auch nicht ausnutzt. Für die Zukunft ist der Gerichtshof deshalb dringlich anzuhalten, dem unmittelbaren und irreversiblen Schädigungspotential physischer und psychischer Art von Überbelegungssituationen konsequent mit der Anordnung vorsorglicher Massnahmen zu begegnen. Erfreulich ist demgegenüber die Praxis der Anordnung vorsorglicher Massnahmen, wenn es um die Gesundheitsversorgung des Beschwerdeführers im Strafvollzug geht.

2. Befolgungspraxis der Konventionsstaaten bei vorsorglichen Massnahmen und die Rolle von Art. 34 EMRK

Um dem Mindeststandard in der Praxis bestmöglich zum Durchbruch zu verhelfen, ist entscheidend, dass die vom Gerichtshof angeordneten vorsorglichen Massnahmen von den Mitgliedstaaten umgesetzt werden. Tun sie dies nicht oder nicht rechtzeitig, ist die Reaktion des Gerichtshofs von Bedeutung - nicht zuletzt, um Prognosen über die zukünftige Befolgungspraxis machen zu können.

Die rechtliche Verpflichtung zur Umsetzung einer vorsorglichen Massnahme ist heute unbestritten. Die Missachtung einer vorsorglichen Mass-

975 SK-StPO/MeYER Verfahrensrecht (EMRK) Rn. 278.

976 Keller/Marti EJIL 2015, 829 (834). 
nahme führt selbst schon zu einer Verletzung von Art. 34 Satz 2 EMRK, ${ }^{977}$ welche im Urteil als eigenständige Verletzung festgestellt wird. Ob eine Verletzung von Art.34 EMRK vorliegt, prüft der Gerichtshof in zwei Schritten. In einem ersten Schritt stellt er fest, ob der Konventionsstaat die vorsorgliche Massnahme (rechtzeitig) umgesetzt hat. ${ }^{978}$ Ist dies nicht der Fall, prüft der Gerichtshof in einem zweiten Schritt, ob die Nichtbefolgung bzw. die verspätete Umsetzung der vorsorglichen Massnahme gerechtfertigt war. ${ }^{979}$

Trotz der unbestrittenen Verpflichtungswirkung ist im Allgemeinen die Tendenz zu beobachten, dass vor allem einige osteuropäische Staaten die Anordnungen nicht beachten oder umgehen. ${ }^{980}$ Diese allgemeine Tendenz der Nichtbefolgung vorsorglicher Massnahmen entspricht auch der Situation im Bereich inadäquater Haftbedingungen, so dass der Gerichtshof auch in diesem Kontext regelmässig eine Verletzung von Art. 34 EMRK feststellt. ${ }^{981}$

Bei Anordnungen vorsorglicher Massnahmen im Kontext der medizinischen Versorgung im Strafvollzug kommt es auf der ganzen Bandbreite der angeordneten Massnahmen zu Verstössen: Es wurden etwa verlangte unabhängige medizinische Experten nicht beigezogen, ${ }^{982}$ sondern die

977 Karpenstein/Mayer/Schäfer Art. 34 Rn. 106.

978 Vgl. etwa EGMR - Grori/ALB, Urt. v. 07.07.2009, 25336/04, Rn. 185 ff.

979 Vgl. etwa EGMR - Grori/ALB, Urt. v. 07.07.2009, 25336/04, Rn. 188 ff.

980 SK-StPO/Meyer Verfahrensrecht (EMRK) Rn. 265 m.w.N.

981 Vgl. etwa EGMR (GK) - Paladi/MDA, Urt. v. 10.03.2009, 39806/05; EGMR Kondrulin/RUS, Urt. v. 20.09.2016, 12987/15; EGMR - Aleksanyan/RUS, Urt. v. 22.12.2008, 46468/06; EGMR - Grori/ALB, Urt. v. 07.07.2009, 25336/04; EGMR - Makharadze u. Sikharulidze/GEO, Urt. v. 22.11.2011, 35254/07; EGMR - Salakhov u. Islyamova/UKR, Urt. v. 14.03.2013, 28005/08; EGMR Amirov/RUS, Urt. v. 27.11.2014, 51857/13; EGMR - Andrey Lavrov/RUS, Urt. v. 01.03.2016, 66252/14; EGMR - Klimov/RUS, Urt. v. 04.10.2016, 54436/14; EGMR - Maylenskiy/RUS, Urt. v. 04.10.2016, 12646/15; EGMR - Pivovarnik/UKR, Urt. v. 06.10.2016, 29070/15; EGMR - Konovalchuk/UKR, Urt. v. 13.10.2016, 31928/15.

982 EGMR - Kondrulin/RUS, Urt. v. 20.09.2016, 12987/15, Rn. 41 ff.; EGMR Aleksanyan/RUS, Urt. v. 22.12.2008, 46468/06, Rn. 231: Es wurde trotz entsprechender Anordnung keine gemischte Expertenkommission einberufen. Der Beschwerdeführer wäre, wie von ihm gefordert, bereit gewesen, betreffend die Zusammensetzung der Kommission mit den Behörden zusammenzuarbeiten. Vgl. weiter EGMR - Amirov/RUS, Urt. v. 27.11.2014, 51857/13, Rn. 72; EGMR Andrey Lavrov/RUS, Urt. v. 01.03.2016, 66252/14, Rn. 38; EGMR - Klimov/RUS, Urt. v. 04.10.2016, 54436/14; EGMR - Maylenskiy/RUS, Urt. v. 04.10.2016, 12646/15, Rn. 34 ff.: Es wurden zwar Experten beigezogen, diese beantworteten allerdings nicht die an sie gestellten Fragen. 
an die unabhängigen Experten zu stellenden Fragen vom Konventionsstaat selbst beantwortet. ${ }^{983}$ Auch angeordnete Überweisungen in Kliniken wurden nicht ausgeführt, ${ }^{984}$ angeordnete Untersuchungen nicht durchgeführt ${ }^{985}$ oder Rücküberstellungen von solchen Kliniken in den normalen Vollzug oder in das Gefängniskrankenhaus trotz anderslautenden Anweisungen vorgenommen. ${ }^{986}$ Auch verspätete Umsetzungen führten regelmässig zu einem Verstoss gegen Art. 34 EMRK.987

Für die Frage der Umsetzung entscheidend ist zunächst die Formulierung der angeordneten vorsorglichen Massnahme. ${ }^{988}$ Massgebend ist aber auch ihr telos ${ }^{989}$ Bei angeordneter Überweisung in eine andere Institution prüft der Gerichtshof deshalb etwa, ob die neue Einrichtung grundsätzlich den in der Anordnung gestellten Anforderungen entspricht. 990 Dabei ist entscheidend, ob etwa eine Überweisung in ein ziviles Krankenhaus oder in eine Spezialklinik gefordert wurde. ${ }^{991}$ Wurde ein Transfer in eine spezialisierte Institution angeordnet, prüft der Gerichtshof, ob die Klinik über die spezifisch notwendige Laboreinrichtung sowie über die notwendigen Medikamente, und das Personal der Klinik über die spezialisierten Fähig-

983 In diesem Bereich sticht insbesondere die negative Befolgungspraxis Russlands hervor: EGMR - Kondrulin/RUS, Urt. v. 20.09.2016, 12987/15, Rn. 47; vgl. auch EGMR - Amirov/RUS, Urt. v. 27.11.2014, 51857/13, Rn.69; EGMR - Andrey Lavrov/RUS, Urt. v. 01.03.2016, 66252/14, Rn. 35; EGMR - Klimov/RUS, Urt. v. 04.10.2016, 54436/14, Rn. 45; EGMR - Maylenskiy/RUS, Urt. v. 04.10.2016, 12646/15, Rn. 39.

984 EGMR - Aleksanyan/RUS, Urt. v. 22.12.2008, 46468/06, Rn. 230.

985 EGMR - Pivovarnik/UKR, Urt. v. 06.10.2016, 29070/15, Rn. 55 ff.

986 EGMR (GK) - Paladi/MDA, Urt. v. 10.03.2009, 39806/05, Rn. 94.

987 EGMR - Aleksanyan/RUS, Urt. v. 22.12.2008, 46468/06, Rn. 230: Der Transfer in die Spezialklinik erfolgte erst zwei Monate nach deren vorsorglicher Anordnung. Vgl. weiter EGMR - Grori/ALB, Urt. v. 07.07.2009, 25336/04, Rn. 187: Der Transfer zur weiteren Diagnosestellung und zur Behandlung in ein Krankenhaus erfolgte erst nach 17 Tagen. Vgl. auch EGMR - Salakhov u. Islyamoval UKR, Urt. v. 14.03.2013, 28005/08, Rn. 216 ff.: Der Transfer in ein Krankenhaus erfolgte erst nach drei Tagen.

988 EGMR (GK) - Paladi/MDA, Urt. v. 10.03.2009, 39806/05, Rn. 91.

989 EGMR (GK) - Paladi/MDA, Urt. v. 10.03.2009, 39806/05, Rn.91; EGMR Kondrulin/RUS, Urt. v. 20.09.2016, 12987/15, Rn.44; EGMR - Amirov/RUS, Urt. v. 27.11.2014, 51857/13, Rn. 71.

990 EGMR - Makharadze u. Sikharulidze/GEO, Urt. v. 22.11.2011, 35254/07, Rn. $100 \mathrm{ff}$.

991 EGMR - Makharadze u. Sikharulidze/GEO, Urt. v. 22.11.2011, 35254/07, Rn. $100 \mathrm{ff}$. 
keiten verfügen. ${ }^{992}$ In zeitlicher Hinsicht verlangen die dringliche Natur ${ }^{993}$ und das immanente Risiko, aufgrund derer die vorsorgliche Massnahme überhaupt erst angeordnet wurde und die keinen Aufschub dulden, eine unverzügliche Umsetzung der angeordneten Massnahme - vorbehaltlich einer anderslautenden Anordnung (Festsetzung einer expliziten Frist). ${ }^{994}$ Unter einer unverzüglichen Umsetzung versteht der Gerichtshof eine Umsetzung am selben Tag. ${ }^{995}$ Bereits eine Verzögerung von drei Tagen kann zu einer Verletzung von Art. 34 EMRK führen. ${ }^{996}$ Für dringliche Angelegenheiten, wie es insbesondere vorsorgliche Massnahmen sind, hat im Konventionsstaat nicht nur an Arbeitstagen ${ }^{997}$, sondern auch an Wochenenden und Feiertagen ein zuständiger Richter verfügbar zu sein. ${ }^{998}$

Vom Konventionsstaat erwartet der Gerichtshof i.S.d. Art.34 Satz 2 EMRK, dass dieser alle Schritte unternommen hat, welche vernünftigerweise von ihm verlangt werden konnten, um der vorsorglichen Anordnung des Gerichtshofs zu entsprechen. ${ }^{999}$ Bei der Rechtfertigungsprüfung kann der Konventionsstaat deshalb nur geltend machen, dass objektive Gründe vorlagen, welche die Befolgung verhinderten. ${ }^{1000}$ Ist der Konventionsstaat der Meinung, Gründe für eine anderweitige Beurteilung und insbesondere für eine Aufhebung der vorsorglichen Massnahme zu haben, hat er den Gerichtshof über diese zu informieren. Es liegt dann am Gerichtshof, über diese zu befinden. ${ }^{1001}$ Wurde die vorsorgliche Mass-

992 EGMR - Makharadze u. Sikharulidze/GEO, Urt. v. 22.11.2011, 35254/07, Rn. 101.

993 EGMR (GK) - Paladi/MDA, Urt. v. 10.03.2009, 39806/05, Rn. 98.

994 Vgl. etwa EGMR - Salakhov u. Islyamova/UKR, Urt. v. 14.03.2013, 28005/08, Rn. 217; EGMR - Grori/ALB, Urt. v. 07.07.2009, 25336/04, Rn. 190.

995 EGMR - Salakhov u. Islyamova/UKR, Urt. v. 14.03.2013, 28005/08, Rn. 220.

996 EGMR - Salakhov u. Islyamova/UKR, Urt. v. 14.03.2013, 28005/08, Rn. 216.

997 EGMR (GK) - Paladi/MDA, Urt. v. 10.03.2009, 39806/05, Rn. 97: Der Gerichtshof bemängelte im vorliegenden Fall, dass an einem Arbeitstag keine für die Umsetzung der vorsorglichen Massnahme besorgte Person erreichbar war.

998 Als obiter dictum etwa: EGMR (GK) - Paladi/MDA, Urt. v. 10.03.2009, 39806/05, Rn. 100.

999 EGMR (GK) - Paladi/MDA, Urt. v. 10.03.2009, 39806/05, Rn. 88.

1000 EGMR (GK) - Paladi/MDA, Urt. v. 10.03.2009, 39806/05, Rn. 92.

1001 EGMR (GK) - Paladi/MDA, Urt. v. 10.03.2009, 39806/05, Rn.90. An der dringlichen und schwerwiegenden Natur der Sache, die häufig keinen Aufschub zulässt, liegt es, dass der Gerichtshof bei der Entscheidung, ob er eine vorsorgliche Massnahme erlässt oder nicht, zunächst auf die bereits vorhandenen Fakten abstellt: vgl. etwa EGMR - Makharadze u. Sikharulidze/GEO, Urt. v. 22.11.2011, 35254/07, Rn. 98. Der Gerichtshof ist deshalb meines Erachtens anzuhalten, lieber einmal zu viel als einmal zu wenig von der Möglichkeit 
nahme vom Konventionsstaat nicht umgesetzt, ohne zuvor dem Gerichtshof seine Gründe vorgebracht zu haben, berücksichtigt der Gerichtshof bei einer Prüfung von Art. 34 EMRK diese potentiellen Gründe nicht mehr. ${ }^{1002}$ Selbst wenn objektive Hindernisse vorliegen, bleibt der Konventionsstaat aufgrund Art. 34 EMRK dazu verpflichtet, zum einen wiederum alle möglichen Schritte zur Beseitigung dieser Hindernisse vorzunehmen und zum anderen den Gerichtshof regelmässig über die aktuelle Situation $\mathrm{zu}$ informieren. ${ }^{1003}$

$\mathrm{Zu}$ den Rechtfertigungsgründen im Einzelnen kommt der Gerichtshof zum Schluss, dass im Umstand, dass sich die vom Gerichtshof angenommene ernsthafte Gefahr einer irreparablen Gesundheitsschädigung i.S.d. Regel 39 VerfO nicht realisiert hat, keine Rechtfertigung gesehen werden kann. ${ }^{1004}$ Eine Verletzung von Art. 34 EMRK setzt dementsprechend nicht voraus, dass ein irreversibler Gesundheitsschaden eingetreten ist. Der Vorwurf, der dem Mitgliedstaat gemacht wird, ist vielmehr, dass dieser einen irreparablen Gesundheitsschaden in Kauf genommen hat. Auch die eigene Beurteilung des Konventionsstaats, dass keine ernsthafte Gefahr eines irreversiblen Gesundheitsschadens vorgelegen habe, ist kein tauglicher Rechtfertigungsgrund. ${ }^{1005}$ Unterliess der Konventionsstaat den Beizug eines unabhängigen Experten zur Beantwortung der Fragen des Gerichtshofs, spielt es auch keine Rolle, dass der Gerichtshof im Hauptsacheverfahren schliesslich eine genügende Informationsgrundlage zur Beurteilung des Falles hatte. ${ }^{1006}$

Als Rechtfertigungsgrund bei einem angeordneten Transfer in eine Spezialklinik scheint der Gerichtshof aber zu akzeptieren, dass ein Transfer in eine Spezialklinik deshalb unterbleibt, weil im Konventionsstaat überhaupt keine spezialisierte Einrichtung existiert. ${ }^{1007}$ Gibt es allerdings spezialisier-

der Anordnung einer vorsorglichen Massnahme Gebrauch zu machen. Dies soll dem Konventionsstaat aber gerade nicht dazu dienen, einen Grund zu haben, die vorsorgliche Massnahme nicht umzusetzen.

1002 EGMR (GK) - Paladi/MDA, Urt. v. 10.03.2009, 39806/05, Rn. 92.

1003 EGMR (GK) - Paladi/MDA, Urt. v. 10.03.2009, 39806/05, Rn. 92.

1004 EGMR (GK) - Paladi/MDA, Urt. v. 10.03.2009, 39806/05, Rn. 89; EGMR Grori/ALB, Urt. v. 07.07.2009, 25336/04, Rn. 194; EGMR - Salakhov u. Islyamova/UKR, Urt. v. 14.03.2013, 28005/08, Rn. 223.

1005 EGMR (GK) - Paladi/MDA, Urt. v. 10.03.2009, 39806/05, Rn.90; EGMR Makharadze u. Sikharulidze/GEO, Urt. v. 22.11.2011, 35254/07, Rn. 98 ff.

1006 EGMR - Aleksanyan/RUS, Urt. v. 22.12.2008, 46468/06, Rn. 231.

1007 EGMR - Makharadze u. Sikharulidze/GEO, Urt. v. 22.11.2011, 35254/07, Rn. 102: Im konkreten Fall hält der Gerichtshof allerdings fest, dass es in Georgien eine spezialisierte Klinik gab, in die der Beschwerdeführer hätte ver- 
te zivile Einrichtungen, kann das Fehlen eines spezialisierten Gefängniskrankenhauses ein Unterlassen der Umsetzung der vorsorglichen Massnahme nicht rechtfertigen. Der Inhaftierte ist in einem solchen Fall in die zivile Klinik zu überstellen. ${ }^{1008}$

Hinsichtlich der vom Gerichtshof festgelegten Frist zur Umsetzung ist es nicht am Mitgliedstaat, die Dringlichkeit anderweitig zu beurteilen und die Frist auf diese Weise eigenmächtig zu verlängern; solche Vorbringen sind keine tauglichen Rechtfertigungsgründe. ${ }^{1009}$ Eine zu späte Umsetzung der vorsorglichen Massnahme kann vom Konventionsstaat auch nicht mit Organisationsschwierigkeiten gerechtfertigt werden. ${ }^{1010}$ Bringt der Konventionsstaat vor, dass notwendige Sicherheitsvorkehrungen zu einer verspäteten Umsetzung geführt hätten, prüft dies der Gerichtshof eingehend und kommt regelmässig zum Schluss, dass letztlich nicht rechtfertigende Organisationsprobleme für die Verspätung ausschlaggebend waren. ${ }^{1011}$ Der Gerichtshof anerkennt aber, dass auch das Verhalten des Beschwerdeführers für eine verspätete Umsetzung verantwortlich sein kann. ${ }^{1012}$ Weigert sich der Beschwerdeführer etwa, bei einem Transfer in eine (Spezial-) Klinik zu kooperieren und tritt stattdessen etwa in einen Hungerstreik, ist dies für die entsprechende Zeitperiode ein Rechtfertigungsgrund für die nicht rechtzeitig erfolgte Umsetzung der vorsorglichen Massnahme. ${ }^{1013}$ Ein solches Verhalten des Beschwerdeführers hebt die vorsorgliche Massnahme aber keineswegs auf. Der Konventionsstaat muss weiterhin alle

legt werden können. Objektive Rechtfertigungsgründe lagen damit keine vor. Selbst wenn der Gerichtshof in diesem Entscheid ausdrücklich darauf abstellte, ob im Inland eine solche Klinik vorhanden ist, wären meines Erachtens durchaus auch Situationen denkbar, in denen in Ermangelung einer spezialisierten Klinik im Inland aufgrund der positiven Gewährleistungspflicht des Staates eine Verpflichtung zur Verlegung auch in eine ausländische Klinik erkannt werden müsste. Dies insbesondere dann, wenn der Staat im eigenen Land nicht einmal eine Minimalversorgung gewährleisten kann. Es stellt sich dann allerdings auch die Frage, inwieweit ausländische (Konventions-)Staaten ihre Gesundheitsinfrastruktur zur Verfügung stellen müssen.

1008 EGMR - Makharadze u. Sikharulidze/GEO, Urt. v. 22.11.2011, 35254/07, Rn. 102.

1009 EGMR (GK) - Paladi/MDA, Urt. v. 10.03.2009, 39806/05, Rn. 90.

1010 EGMR - Grori/ALB, Urt. v. 07.07.2009, 25336/04, Rn. $189 \mathrm{ff}$.

1011 EGMR - Grori/ALB, Urt. v. 07.07.2009, 25336/04, Rn. 189.

1012 EGMR - Grori/ALB, Urt. v. 07.07.2009, 25336/04, Rn. 192.

1013 EGMR - Grori/ALB, Urt. v. 07.07.2009, 25336/04, Rn. 192; vgl. auch EGMR Konovalchuk/UKR, Urt. v. 13.10.2016, 31928/15, Rn. 84, 87. 
Schritte vornehmen, um die angeordnete Massnahme so rasch wie möglich umzusetzen. ${ }^{1014}$

Die relativ grosse Zahl an Nichtbefolgungen vorsorglicher Massnahmen im Kontext inadäquater medizinischer Versorgung im Strafvollzug darf nicht über die praktische Bedeutung der vorsorglichen Massnahmen hinwegtäuschen. In jedem Fall, in welchem sie befolgt wird, tragen sie entscheidend dazu bei, die effektive Durchsetzung des vom Gerichtshof garantierten Mindeststandards sicherzustellen. Der Gerichtshof scheint auf jeden Fall sehr bemüht, die Befolgungspraxis der Konventionsstaaten zu verbessern, indem er bei der Prüfung, ob die angeordneten vorsorglichen Massnahmen vom Konventionsstaat (rechtzeitig) umgesetzt wurden oder nicht, klare und stringente Grundsätze anwendet, Rechtfertigungsgründe nur restriktive zulässt und Nichtbefolgungen resp. zu späte Befolgungen mit der Feststellung eigenständiger Verletzungen von Art. 34 EMRK quittiert. ${ }^{1015}$

\section{Zwischenfazit}

Für die Verfahrensdauer haben sich besonders im Bereich der inadäquaten medizinischen Versorgung im Strafvollzug die vorsorglichen Massnahmen als wichtiger Garant des Mindeststandards herausgestellt. Die Anordnungspraxis des Gerichtshofs verdeutlicht, dass er das Potential vorsorglicher Massnahmen vor allem bei inadäquater medizinischer Versorgung erkannt hat und dort auch ausschöpft. Andere Teilaspekte inadäquater Haftbedingungen werden hingegen sehr selten zum Anlass der Anordnung einer vorsorglichen Massnahme genommen. Der Gerichtshof ist mit Blick auf wissenschaftliche Erkenntnisse der Medizin anzuhalten, insbe-

1014 Vgl. implizit EGMR - Grori/ALB, Urt. v. 07.07.2009, 25336/04, Rn. 192; vgl. auch EGMR - Konovalchuk/UKR, Urt. v. 13.10.2016, 31928/15, Rn. 89.

1015 Vgl. etwa EGMR (GK) - Paladi/MDA, Urt. v. 10.03.2009, 39806/05, Rn. 100; EGMR - Kondrulin/RUS, Urt. v. 20.09.2016, 12987/15, Rn. 48; EGMR Aleksanyan/RUS, Urt. v. 22.12.2008, 46468/06, Rn. 232; EGMR - Grori/ALB, Urt. v. 07.07.2009, 25336/04, Rn. 195; EGMR - Makharadze u. Sikharulidzel GEO, Urt. v. 22.11.2011, 35254/07, Rn. 104 f.; EGMR - Salakhov u. Islyamoval UKR, Urt. v. 14.03.2013, 28005/08, Rn. 224; EGMR - Amirov/RUS, Urt. v. 27.11.2014, 51857/13, Rn. 75; EGMR - Andrey Lavrov/RUS, Urt. v. 01.03.2016, 66252/14, Rn. 40; EGMR - Klimov/RUS, Urt. v. 04.10.2016, 54436/14, Rn. 50; EGMR - Maylenskiy/RUS, Urt. v. 04.10.2016, 12646/15, Rn. 40; EGMR - Pivovarnik/UKR, Urt. v. 06.10.2016, 29070/15, Rn. 61 f.; EGMR - Konovalchuk/ UKR, Urt. v. 13.10.2016, 31928/15, Rn. 90 f. 
sondere auch dem unmittelbaren und irreversiblen Schädigungspotential physischer und psychischer Art von Überbelegungssituationen Beachtung zu schenken und auch in solchen Konstellationen nicht davor zurückzuschrecken, vorsorgliche Massnahmen anzuordnen.

Die Befolgungspraxis einzelner Staaten entspricht nicht dem wichtigen und dringenden Anliegen der vorsorglichen Massnahmen. So verstösst etwa Russland wiederholt gegen die Anordnung der Einberufung unabhängiger Experten zur Beantwortung der vom Gerichtshof vorgelegten Fragen. Die säumigen Konventionsstaaten sind für die Zukunft eindringlich anzuhalten, die angeordneten und verbindlichen Massnahmen unverzüglich und mit grösserer Zuverlässigkeit umzusetzen. Schon jetzt weist der Gerichtshof durch Verurteilungen aufgrund von Art. 34 EMRK die Mitgliedstaaten auf ihre diesbezüglichen Pflichten hin. Auch die stringente Rechtsprechung hinsichtlich der Befolgungs- und Rechtfertigungskriterien erleichtert es den Konventionsstaaten, sich ihren Pflichten zu stellen. Weitere Möglichkeiten, auf die Befolgungspraxis einzuwirken, hat der Gerichtshof nicht. Er schöpft sein diesbezügliches Potential folglich aus. Entscheidend bleibt hier also letztlich die Kooperationsbereitschaft der einzelnen Konventionsstaaten.

Kapitel 3: Mindeststandard im Urteil - Wirkung eines EGMR-Urteils

Für die Frage, wie der vom Gerichtshof festgesetzte Mindeststandard in der Praxis Wirksamkeit erlangt, sind die Urteilswirkungen als solche relevant.

\section{Feststellungsurteil}

Der Gerichtshof erlässt Feststellungsurteile, d.h., im Urteil wird festgestellt, ob das gerügte Verhalten des Mitgliedstaates ein garantiertes Konventionsrecht verletzt oder ob es als konventionskonform zu qualifizieren ist und somit keine Verletzung vorliegt und die Beschwerde damit abzuweisen ist. ${ }^{1016}$ Diese Feststellungsurteile unterscheiden sich insbesondere von Gestaltungsurteilen; eine rechtsgestaltende Wirkung kommt den Urteilen des Gerichtshofs nämlich gerade nicht zu. ${ }^{1017} \mathrm{Im}$ Zusammenhang

1016 Baumann S. 75.

1017 Meyer-Ladewig/Brunozzi Art. 46 Rn. 21. 
mit der Möglichkeit des Gerichtshofs, dem Beschwerdeführer eine gerechte Entschädigung i.S.v. Art. 41 EMRK zuzusprechen, wird seinen Urteilen auch ein leistungsrechtlicher Gehalt attestiert. ${ }^{1018}$ Passend erscheint auch die Bezeichnung des „Verpflichtungsurteils“, ${ }^{1019}$ enthält das Urteil bei Feststellung eines Konventionsverstosses doch gegenüber dem betroffenen Mitgliedstaat eine Verpflichtung, die Verletzung abzustellen und soweit nötig und möglich Ersatz für die Folge des Konventionsverstosses zu leisten. ${ }^{1020}$

Nachdem schon die Beschwerdeeinlegung keine unmittelbaren Folgen hinsichtlich der Situation des Inhaftierten hat, führt auch das rechtskräftige Urteil für sich alleine noch zu keiner automatischen Änderung der Unterbringungsumstände, selbst wenn die Situation im konkreten Fall vom EGMR für konventionswidrig befunden wird. Die Natur des Feststellungsurteils und seine fehlende Gestaltungskraft erfordern vielmehr die Umsetzung des Urteils durch die nationalen Behörden. Nur diese sind in der Lage, einen EMRK-konformen Zustand herzustellen. Wie weitreichend eine solche Umsetzung zu sein hat, ergibt sich aus der Bindungsund Orientierungswirkung.

\section{Bindungswirkung und Orientierungswirkung - ein Überblick}

Die Urteile des Gerichtshofs sind rechtsverbindlich. ${ }^{1021}$ Bindungswirkung haben die Urteile in erster Linie für die Verfahrensparteien in Bezug auf den konkret abgeurteilten Sachverhalt (Inter partes-Wirkung). ${ }^{1022}$ Darüber hinaus entfalten sie eine Orientierungswirkung, worunter eine faktische, nicht aber rechtliche Wirkung zu verstehen ist, welche den konkreten Staat dazu veranlassen soll, seine Verhaltensweise für die Zukunft zu überdenken und EMRK-konform zu gestalten, um weitere gleichartige Verletzungen und damit drohende Verurteilungen zu vermeiden. ${ }^{1023}$ Verschiede-

1018 Baumann S. 75; Meyer-Ladewig/Brunozzi Art. 46 Rn. 21.

1019 Meyer-Ladewig/Brunozzi Art. 46 Rn. 21.

1020 Meyer-Ladewig/Brunozzi Art. 46 Rn. 22; vgl. auch Matscher FS Bernhardt, S. 503 (508): Die Urteilswirkungen gingen weit über diejenigen von Feststellungsurteilen im Sinne der allgemeinen Verfahrenslehre hinaus. Vgl. ferner Aemisegger in: Jaag/Kaufmann, S. 201 (225 f.).

1021 Karpenstein/Mayer/Breuer Art. 46 Rn. 1.

1022 Karpenstein/Mayer/Breuer Art. 46 Rn. 43 ff.; Grabenwarter/Pabel $\$ 16$ Rn. 2.

1023 Karpenstein/Mayer/Breuer Art. 46 Rn. 45; Grabenwarter/Pabel $\mathbb{1} 16$ Rn. 8: In diesem Kontext werde auch von „indirekter Wirkung“ oder „Präjudiz- 
ne Ansätze begründen diese Orientierungsfunktion: (1) die Anwendung von Art. 32 EMRK, welcher die Auslegung und Anwendung der Konvention in die Zuständigkeit des EGMR legt, ${ }^{1024}$ (2) die Erwartungshaltung der Konventionsstaaten, dass der EGMR nicht ohne Grund von seiner bisherigen Rechtsprechung abweicht, auch wenn er im formellen Sinne nicht an seine eigenen Rechtsprechungslinien gebunden ist; dies im Sinne der Rechtssicherheit, der Vorhersehbarkeit und der Rechtsgleichheit ${ }^{1025}$, (3) die Anwendung von Art. 1 EMRK, der die Mitgliedstaaten zum Schutz der in der Konvention garantierten Rechte verpflichtet ${ }^{1026}$.

Wie weit die Orientierungswirkung reicht resp. wie stark diese ist, hängt von verschiedenen Faktoren ab. Entscheidend ist einerseits, ob es sich um denselben bereits verurteilten Staat oder um einen anderen Konventionsstaat handelt, und andererseits, wie ähnlich gelagert die Sachverhalte sind. ${ }^{1027}$ Besonders ausgeprägt ist die Orientierungswirkung bei Parallelfäl-

wirkung“ gesprochen. Vgl. auch MiLej S.614ff., welcher im Einzelnen auf die Hintergründe und Intensität dieser Wirkung eingeht. Vgl. auch Glas EYHR 2018, 287 (295 ff.): Der Geltungsanspruch durchlaufe folglich eine Transformation. Der „Inter-partes-Charakter" werde mit „Erga-omnesElementen“ angereichert. Der Begriff der Erga-omnes-Wirkung beschlage aber nicht Fragen der Drittwirkung von Menschenrechten oder der Rechtskraftwirkung. Zu den Fragen des Bestehens, der Bedeutung und der Rechtfertigung eines „Erga-omnes-Effekts“ der EGMR-Urteile vgl. im Einzelnen Besson in: Besson, S. $125 \mathrm{ff}$., welche sowohl hinsichtlich der Inter-partes- als auch der Erga-omnes-Wirkung zwischen der res judicata und der res interpretata unterscheidet.

1024 So Karpenstein/Mayer/Breuer Art. 46 Rn. 45: Autorität des EGMR bei der Auslegung der Konvention; vgl. auch Grabenwarter/Pabel $\mathbb{1} 16$ Rn. 8.

1025 Vgl. auch EGMR (Pl) - Cossey/GBR, Urt. v. 27.09.1990, 10843/84, Rn. 35: „[...] the Court is not bound by its previous judgments; indeed, this is borne out by Rule 51 para. 1 of the Rules of Court. However, it usually follows and applies its own precedents, such a course being in the interests of legal certainty and the orderly development of the Convention case-law. Nevertheless, this would not prevent the Court from departing from an earlier decision if it was persuaded that there were cogent reasons for doing so. Such a departure might, for example, be warranted in order to ensure that the interpretation of the Convention reflects societal changes and remains in line with present-day conditions."; vgl. auch Karpenstein/Mayer/Breuer Art. 46 Rn. 45 m.w.N.: Der EGMR weiche nur aus „triftigen Gründen“ von seiner bisherigen Rechtsprechung ab. So auch Stone Sweet/Keller in: Keller/Stone Sweet, S. 3 (14); vgl. auch Keller/Marti Justice - Justiz - Giustizia 2015/1, 1 (5f.): Eine klare und kohärente Rechtsprechung sei auch Grundvoraussetzung dafür, dass die innerstaatliche Berücksichtigungspflicht überhaupt umsetzbar ist.

1026 Grabenwarter/Pabel $\mathbb{1} 16$ Rn. 8.

1027 Eingehend Karpenstein/Mayer/Breuer Art. 46 Rn. 43 ff. 
len. ${ }^{1028}$ Dies sind Fälle, in welchen die Konventionsverletzung des konkret abgeurteilten Falles auf einem Rechtszustand beruht, der weitere Personen desselben Mitgliedstaates in derselben Weise betrifft, wie es den Beschwerdeführer im abgeurteilten Fall betroffen hat. ${ }^{1029}$ Es bedarf dann keiner weiteren Übertragung der EGMR-Rechtsprechung, ist doch die Konventionsverletzung durch diesen Rechtszustand bereits festgestellt worden. ${ }^{1030}$

Ist der Feststellungscharakter des EGMR-Urteils und die damit einhergehende fehlende Gestaltungswirkung dafür verantwortlich, dass es einer Umsetzung des Urteils bedarf, geben die Bindungswirkung sowie die Orientierungswirkung vor, wie weitreichend eine solche Umsetzung zu sein hat.

III. Befolgungspflicht

\section{Beendigungspflicht}

Art. 46 Abs. 1 EMRK enthält eine Befolgungspflicht, welche ein Beendigungsgebot umfasst. ${ }^{1031}$ Die Beendigung einer noch andauernden Konventionsverletzung ${ }^{1032}$ kann dabei entweder als Sekundärpflicht oder sogar als „fortdauernde Primärverpflichtung“ angesehen werden. ${ }^{1033}$ Auf welche Art und Weise und mit welchen Mitteln der Staat dieser Pflicht nachkommt, liegt i.d.R. in seinem Ermessen. ${ }^{1034}$ Abweichungen von diesem Grundsatz

1028 Vgl. etwa Grabenwarter JZ 2010, 857 (857 ff., 869 f.) am Beispiel der Sicherungsverwahrung in Deutschland; vgl. auch Frowein FS Wildhaber, S. 261 (263 f.).

1029 Grabenwarter/Pabel $\$ 16$ Rn. 9; vgl. auch Stone Sweet/Keller in: Keller/Stone Sweet, S. 3 (13f.): Anders als bei nationalen Verfassungsgerichten könne der Gerichtshof aufgrund des Feststellungscharakters seiner Urteile nämlich gerade keine nationalen Rechtsbestimmungen ausser Kraft setzen, welche er für konventionswidrig befunden hat.

1030 Grabenwarter/Pabel $\$ 16$ Rn. 9.

1031 SK-StPO/MeYer Verfahrensrecht (EMRK) Rn.327; Glas EYHR 2018, 287 (292); zum hierfür massgeblichen Zeitpunkt der Rechtskraft vgl. FroweIn FS Wildhaber, S. 261 (264 ff.).

1032 Zur Diskussion über fortgesetzte Verstösse und Dauerdelikte im Einzelnen vgl. LeEb S. $29 \mathrm{ff}$.

1033 Karpenstein/Mayer/Breuer Art. 46 Rn. 8; Breuer EuGRZ 2004, 257 ff.; vgl. auch SK-StPO/MeYer Verfahrensrecht (EMRK) Rn. 289: Beendigung als unmittelbare Folge der Primärverpflichtung.

1034 SK-StPO/MeYer Verfahrensrecht (EMRK) Rn. 327; Karpenstein/Mayer/Breuer Art. 46 Rn. 5; Meyer-Ladewig/Brunozzi Art. 46 Rn. 22. Diesen Grundsatz hält 
können sich unter Umständen ergeben, wenn der EGMR bestimmte Massnahmen anordnet.

\section{Anordnung bestimmter Abhilfemassnahmen}

Aus dem feststellenden Charakter des EGMR-Urteils leitet der Gerichtshof $\mathrm{ab}$, dass es grundsätzlich Aufgabe des Konventionsstaates ist, unter Aufsicht des Ministerkomitees das geeignete Mittel zu bestimmen, um seinen Primär- und Sekundärverpflichtungen nachzukommen. ${ }^{1035}$ Diese vom EGMR standardmässig verwendete Formel wird auch als Ausdruck des Subsidiaritätsprinzips gesehen. ${ }^{1036}$ Dem liegt die Annahme zugrunde, dass die nationalen Behörden grundsätzlich besser in der Lage sind als der EGMR, die geeignete Massnahme zu bestimmen. ${ }^{1037}$ Gleichwohl kann der Gerichtshof neben der Feststellung der Konventionsverletzung im Einzelfall auch bestimmte individuelle oder generelle Massnahmen anordnen, ${ }^{1038}$ wobei auch eine Kombination von individuellen und generellen Massnahmen möglich ist. ${ }^{1039}$

der Gerichtshof sogar bei der Anwendung der Piloturteilstechnik fest. Vgl. etwa EGMR - Orchowski/POL, Urt. v. 22.10.2009, 17885/04, Rn. 148.

1035 Vgl. auch Glas EYHR 2018, 287 (293); EGMR - Tagayeva u.a./RUS, Urt. v. 13.04.2017, 26562/07, Rn. 638.

1036 Keller/Marti EJIL 2015, 829 (835). Vgl. indes auch Mowbray HRLR 2015, 313 ( 325 ff.), welche vorbringt, dass die Anordnung bestimmter genereller Abhilfemassnahmen - bei gleichzeitigem Aufzeigen des strukturellen Problems dem Konventionsstaat im Sinne des Subsidiaritätsprinzips erst ermögliche, das festgestellte Problem so rasch als möglich zu beheben und damit weiteren Fällen auf nationaler Ebene zu begegnen. Zur Frage der Subsidiarität bei der Anordnung individueller Abhilfemassnahmen ausf. JaHn ZaörV 2014, 1 (22 ff.).

1037 Keller/Marti EJIL 2015, 829 (835): Damit werde das, was für die Primärverpflichtung nach Art. 1 EMRK gelte, auch auf die Sekundärverpflichtung, welche aus einer EMRK-Verletzung fliesst, übertragen.

1038 Glas EYHR 2018, 287 (293); Karpenstein/Mayer/Breuer Art. 46 Rn. 6: Die deklaratorische Wirkung der Urteile gebe der Gerichtshof aber nicht auf. Vgl. kritisch Nicolaou FS Jaeger, S. 163 (165): „When, however, a judgment is qualified as "essentially“ declaratory, the question arises as to the extent to which that attenuates its purely declaratory nature and thus allows for a more active form, by indicating or requiring something to be done, especially when that is included in the operative part. There is inevitably some difficulty in drawing the line."

1039 Meyer-Ladewig/Brunozzi Art. 46 Rn. 5. 
Seit einiger Zeit ist in der Rechtsprechung des Gerichtshofs die Tendenz eines Bedeutungszuwachses solcher Massnahmen zu beobachten, geht doch der Gerichtshof dazu über, mehr und mehr direkt bestimmte Abhilfemassnahmen in seinen Urteilen vorzusehen. ${ }^{1040}$ Der Gerichtshof legt damit nicht nur fest, welchen Konventionsverpflichtungen der Mitgliedstaat nicht nachgekommen ist, sondern macht auf diese Weise gleich noch genaue Vorgaben, mit welchen Mitteln der Staat diesen Verpflichtungen jetzt nachzukommen hat. ${ }^{1041}$ Mit der Anordnung individueller oder genereller Massnahmen bezweckt der Gerichtshof eine Vereinfachung des Implementierungsverfahrens. Eine effizientere Umsetzung der Urteile durch den Konventionsstaat soll letztlich die Arbeitsbelastung des gesamten Konventionssystems senken. ${ }^{1042}$ Vor diesem Hintergrund können drei zusammenhängende Faktoren ausgemacht werden, welche den Gerichtshof dazu veranlasst haben dürften, auch mit der Anordnung konkreter individueller oder genereller Massnahmen auf den Implementierungsprozess einzuwirken: (1) die schlechte Befolgungspraxis der Konventionsstaaten bei der Umsetzung der Urteile des Gerichtshofs, (2) die grosse Anzahl pendenter Fälle am Gerichtshof und (3) die grosse Anzahl repetitiver Fälle in gewissen Konventionsstaaten. ${ }^{1043}$ Insbesondere bei Staaten mit schwachen Durchsetzungsstrukturen soll die Anordnung konkreter Abhilfemassnahmen das Umsetzungsverfahren auf nationaler Ebene beschleunigen können. ${ }^{1044}$ Allgemein könne mit klaren Vorgaben zu den zu ergreifenden Implementierungsmassnahmen auch der politische Druck zur effizienten Umsetzung eines Urteils verschärft werden. ${ }^{1045}$ Fügt sich die spezifische Massnahme nur schlecht in das nationale System ein, ist in Einzelfällen allerdings auch der gegenteilige Effekt zu befürchten, bleibt doch gera-

1040 Vgl. Jahn ZaöRV 2014, 1 ff.; Keller/Marti EJIL 2015, 829 (836); Sicilianos in: Seibert-Fohr/Villiger, S. 285 (286); Cremer in: Seibert-Fohr/Villiger, S. 39 (40).

1041 Keller/Marti EJIL 2015, 829 (836); Glas EYHR 2018, 287 (293).

1042 Vgl. etwa EGMR - Varga u.a./HUN, Urt. v. 10.03.2015, 14097/12 u.a., Rn. 95.

1043 Vgl. so Glas HRLR 2014, 671 (680 ff., 684 ff.)

1044 Keller/Marti EJIL 2015, 829 (840); vgl. auch Anagnostou/Mungiu-Pippidi EJIL 2014, 205 (222 ff.): Insbesondere Griechenland und Rumänien hätten gar nicht die notwendigen Strukturen, die effektivsten Abhilfemassnahmen überhaupt erst zu evaluieren. Institutionell werde die Umsetzung schon dadurch erschwert, dass dieselbe Behörde (Ministry of Foreign Affairs) für die Verteidigung des Konventionsstaats vor dem EGMR sowie (im Falle des Unterliegens) für die Implementierung des Urteils zuständig ist. Das Menschenrechtsbewusstsein sei zudem erst schwach ausgeprägt.

1045 Keller/Marti EJIL 2015, 829 (840) m.w.H. 
de nur noch wenig politischer Verhandlungsspielraum. Durch die dem Ministerkomitee in Art. 46 Abs. 2 EMRK zugeschriebene Rolle, welche sich auf die Überwachung der Umsetzung der EGMR-Urteile beschränkt (im Einzelnen vgl. Teil 4 Kap. 4 I.), beschneidet die Anordnung spezifischer Massnahmen durch den Gerichtshof nicht nur den Ermessensspielraum des betroffenen Staates, sondern auch jenen des Ministerkomitees bei seiner Beurteilung, ob der Konventionsstaat seinen Umsetzungspflichten genügend nachgekommen ist. ${ }^{1046}$ Die Aufgabenverteilung zwischen Gerichtshof und Ministerkomitee erfordert folglich immer ein sorgfältiges Abwägen, ob es im konkreten Fall angezeigt sei, spezifische Massnahmen anzuordnen, oder ob die Wahl der infolge des Urteils zu treffenden Abhilfemassnahmen vollständig dem Konventionsstaat in Absprache mit dem Ministerkomitee überlassen bleiben soll.

Die Kompetenzgrundlage für die Anordnung solcher bestimmter Abhilfemassnahmen ist noch nicht abschliessend geklärt. ${ }^{1047}$ In Frage kommen grundsätzlich Art. 41 EMRK, Art. 46 EMRK oder eine Annexkompetenz, ${ }^{1048}$ wobei in der Rechtsprechung des Gerichtshofs eine Tendenz hin zu einer Abstützung auf Art. 46 EMRK als Rechtsgrundlage festgestellt werden kann. ${ }^{1049}$ Meines Erachtens sollte die konkrete Rechtsgrundlage vom Einzelfall abhängig gemacht werden. Hängt die bestimmt angeordnete Massnahme nämlich eng mit der Entschädigung des Beschwerdeführers zusammen, scheint Art. 41 EMRK die passendere Rechtsgrundlage. Geht es im Kern der angeordneten Abhilfemassnahme aber eher um die Frage, auf welche Art und Weise die Konventionsverletzung zu beenden ist, wird Art. 46 EMRK die treffendere Rechtsgrundlage sein. ${ }^{1050}$

1046 EGMR - Orchowski/POL, Urt. v. 22.10.2009, 17885/04, Rn. 148: „The respondent State remains free, subject to monitoring by the Committee of Ministers, to choose the means by which it will discharge its legal obligation under Article 46 of the Convention, provided that such means are compatible with the conclusions set out in the Court's judgment."; vgl. ferner EGMR (GK) - Broniowski/POL, Urt. v. 22.06.2004, 31443/96, Rn. 192.

1047 Karpenstein/Mayer/Breuer Art. 46 Rn. 6.

1048 Vgl. etwa vgl. auch Cremer in: Seibert-Fohr/Villiger, S. 39 (47 ff.); zur Diskussion hinsichtlich einer Annexkompetenz: Breuer EuGRZ 2004, 257 (261); Rohleder S. 70 ff.; Hass S. $193 \mathrm{ff}$.

1049 Karpenstein/Mayer/Breuer Art. 46 Rn. 6; vgl. hierzu auch Keller/Marti EJIL 2015 (829) 836 f.; vgl. auch Sicilianos in: Seibert-Fohr/Villiger, S. 285 (301 ff.).

1050 In diese Richtung auch schon Karpenstein/Mayer/Breuer Art. 46 Rn. 7 f.; vgl. auch SK-StPO/MeYer Verfahrensrecht (EMRK) Rn. 298: In der Sache gehe es um die Feststellung von Primärverpflichtungen von Art. 46 EMRK. 
Für die Frage, inwieweit die Vorgaben des Gerichtshofs bezüglich der bestimmten Abhilfemassnahmen für Konventionsstaat und Ministerkomitee verbindlich sind, ist weniger die Frage ihrer konkreten Rechtsgrundlage entscheidend als vielmehr, ob der Gerichtshof die bestimmten Abhilfemassnahmen im Urteilstenor oder in den Urteilsgründen nennt. ${ }^{1051}$ Hinweise auf den Verpflichtungsgrad lassen sich darüber hinaus auch den konkreten Formulierungen des Gerichtshofs entnehmen. ${ }^{1052}$ Weist der Gerichtshof in den Urteilsgründen auf die vom Konventionsstaat zu ergreifenden Abhilfemassnahmen hin, sind diese unbenommen ihrer Formulierung nie rechtlich bindend, ${ }^{1053}$ in Rechtskraft erwächst nämlich nur der Urteilstenor. Trotz dieser fehlenden Rechtsbindung entfaltet die gewählte Formulierung dennoch auch hier Wirkungen, indem sie im Implementierungsverfahren massgeblicher Ausgangspunkt für Konventionsstaat und Ministerkomitee ist. ${ }^{1054}$ Bei Fällen mit „weicher Formulierung“ in den Urteilsgründen können die Abhilfemassnahmen damit auch einen rein empfehlenden Charakter haben. ${ }^{1055}$ Absolut formulierte Anweisung im Urteilstenor sind für den Konventionsstaat und Ministerkomitee jedoch grundsätzlich bindend. ${ }^{1056}$

Für die konkrete Bindungswirkung entscheidend ist weiter, ob der Gerichtshof eine Auswahl an verschiedenen möglichen Massnahmen vorschlägt oder aber eine spezifische Massnahme benennt. ${ }^{1057}$ Kommt bei Ersterem dem Konventionsstaat noch ein - wenn auch sehr begrenzter Ermessensspielraum zu, besteht dieser bei Letzterem nicht. Das Vorsehen nur einer bestimmten Massnahme begründet der Gerichtshof bisweilen damit, dass die Natur der vom Konventionsstaat begangenen Verletzung dem Konventionsstaat ohnehin keine echte Wahl bezüglich der zu ergreifenden Massnahme lasse. ${ }^{1058}$

1051 Meyer-Ladewig/Brunozzi Art. 46 Rn. 23; vgl. auch Glas EYHR 2018, 287 (293).

1052 Vgl. auch Meyer-Ladewig/Brunozzi Art. 46 Rn. 5.

1053 Meyer-Ladewig/Brunozzi Art. 46 Rn. 5; Glas EYHR 2018, 287 (293).

1054 Meyer-Ladewig/Brunozzi Art. 46 Rn. 5; EGMR - Tagayeva u.a./RUS, Urt. v. 13.04.2017, 26562/07, Rn. 638.

1055 Vgl. Sicilianos in: Seibert-Fohr/Villiger, S. 285 (293 ff. m.w.H.); vgl. auch EGMR - Tagayeva u.a./RUS, Urt. v. 13.04.2017, 26562/07, Rn. 638.

1056 Vgl. etwa auch Meyer-Ladewig/Brunozzi Art. 46 Rn. 23; Glas EYHR 2018, 287 (293).

1057 Keller/Marti EJIL 2015, 829 (835), m.w.H.; Glas EYHR 2018, 287 (293).

1058 Anstatt vieler: EGMR - Aleksanyan/RUS, Urt. v. 22.12.2008, 46468/06, Rn. 239; EGMR - Ghavtadze/GEO, Urt. v. 03.03.2009, 23204/07, Rn. 106; vgl. auch EGMR (GK) - Assanidze/GEO, Urt. v. 08.04.2004, 71503/01, Rn. 202. 
In Anbetracht der grundsätzlich an Bedeutung zunehmenden Abhilfemassnahmen durch den Gerichtshof, ${ }^{1059}$ den zahlreichen pendenten Fällen und der grossen Anzahl repetitiver Fälle in gewissen Konventionsstaaten betreffend inadäquate Haftbedingungen erstaunt es nicht, dass der Gerichtshof auch in diesem Bereich sowohl bereits individuelle als auch generelle Abhilfemassnahmen anordnete.

\subsection{Anordnung individueller Massnahmen bei unmenschlichen und erniedrigenden Haftbedingungen}

Der Gerichtshof ordnete individuelle Abhilfemassnahmen beispielsweise etwa im Urteilstenor an, nachdem er aufgrund einer inadäquaten medizinischen Versorgung im Strafvollzug eine Verletzung von Art. 3 EMRK festgestellt hatte. ${ }^{1060}$ Dabei sah er die Verpflichtung zur schnellstmöglichen Sicherstellung adäquater Haftbedingungen in einer spezialisierten Institution vor, verknüpft mit der Verpflichtung der Gewährleistung, dass diese Institution dem Inhaftierten die notwendige psychiatrische Betreuung zukommen lassen kann und dass eine stetige medizinische Überwachung stattfindet. ${ }^{1061}$ Während dem Konventionsstaat betreffend die reine Verlegungshandlung hier kein Ermessensspielraum zukommt, kommt ihm dieser - in Zusammenarbeit mit dem Ministerkomitee (zur Rolle des Ministerkomitees vgl. Teil 4 Kap. 4 I.) - wohl aber bei der Beurteilung zu, welche innerstaatliche Einrichtung diese Kriterien erfüllt. Erfüllen aus Sicht des Konventionsstaates (in Übereinstimmung mit dem Ministerkomitee) mehrere mitgliedstaatliche Einrichtungen diese Voraussetzungen, ist es am Mitgliedstaat zu entscheiden, in welche dieser Einrichtungen der Inhaftierte zu verlegen ist. Eine Ermessensreduzierung auf Null - indem der Gerichtshof etwa festhalten würde, in welche konkrete Einrichtung der Beschwerdeführer zu verlegen ist - wäre in Anbetracht der subsidiären Rolle des Gerichtshofs auch nicht zu rechtfertigen; sind mehrere Optionen gleich geeignet, den Mindeststandard zu erfüllen, ist es nicht an ihm, hier die Wahl zu treffen.

1059 Keller/Marti EJIL 2015, 829 (836); vgl. auch EGMR - Tagayeva u.a./RUS, Urt. v. 13.04.2017, 26562/07, Rn. 638.

1060 Vgl. etwa EGMR - Slawomir Musial/POL, Urt. v. 20.01.2009, 28300/06, Rn. 108, Ziff. 4 Urteilstenor; EGMR - Ghavtadze/GEO, Urt. v. 03.03.2009, 23204/07, Ziff. 3 lit. a Urteilstenor.

1061 EGMR - Slawomir Musial/POL, Urt. v. 20.01.2009, 28300/06, Rn. 108, Ziff. 4 lit. a Urteilstenor. 
In den Urteilsgründen sah sich der Gerichtshof beispielsweise mitunter aufgrund der Schwere der Krankheit des Beschwerdeführers veranlasst, unter Art. 46 EMRK zu befinden, dass die Unterbringung in der Untersuchungshaft durch eine oder mehrere weniger einschneidende Massnahme(n), die im mitgliedstaatlichen Recht vorgesehen sind, zu ersetzen ist. ${ }^{1062}$ Im Urteilstenor hielt der Gerichtshof in der Folge fest, dass die Unterbringung in der Untersuchungshaft aufzuheben ist („should be discontinued“"). ${ }^{1063}$ Was den Ermessensspielraum betrifft, sind hier die Frage der Entlassung aus der Untersuchungshaft und die Frage nach einem geeigneten Follow-up zu unterscheiden. Ersteres belässt dem Konventionsstaat keinen Ermessensspielraum, auch wenn die „weiche Formulierung“ (,should be discontinued“) prima vista anderes vermuten liesse. ${ }^{1064}$ Bezüglich des weiteren Vorgehens hat der Mitgliedstaat aber die Wahl - nicht nur weil der Gerichtshof das weitere Vorgehen nicht in den Urteilstenor aufgenommen hat, sondern auch aufgrund der gewählten Formulierung des Gerichtshofs in der Urteilsbegründung. ${ }^{1065}$ Einzige Vorgabe des Gerichtshofs ist hier, dass die Massnahme eine weniger einschneidende zu sein hat; im Übrigen ist es am Konventionsstaat, wiederum in Übereinstimmung mit dem Ministerkomitee die geeignete Follow-up-Massnahme zu eruieren.

Es ist jedoch nicht immer der Fall, dass der Gerichtshof zumindest einen Teil der angeordneten Massnahmen auch im Urteilstenor aufgreift. Bisweilen belässt es der Gerichtshof nach der Feststellung einer inadäquaten medizinischen Versorgung im Strafvollzug im Sinne von Art. 3 EMRK auch dabei, in der Urteilsbegründung die Notwendigkeit einer Verlegung in eine spezialisierte medizinische Institution auszuführen, ohne nähere

1062 EGMR - Aleksanyan/RUS, Urt. v. 22.12.2008, 46468/06, Rn. 240; zur Pflicht des Haftrichters, einen unter Art.-3-EMRK-widrigen Umständen untergebrachten Beschwerdeführer aus der Untersuchungshaft zu entlassen oder präzise menschenrechtskonforme Haftbedingungen anzuordnen, vgl. eingehend Hohl-Chirazi forumpoenale 2018, $187 \mathrm{ff}$.: Der Haftrichter dürfe sich aufgrund des Erfordernisses eines effektiven präventiven Rechtsbehelfs i.S.v. Art. 13 EMRK nicht damit begnügen, die EMRK-Widrigkeit der Haftumstände festzustellen.

1063 EGMR - Aleksanyan/RUS, Urt. v. 22.12.2008, 46468/06, Ziff. 9 Urteilstenor.

1064 Eine solche klare Anordnung im Urteilstenor mit einer weichen Formulierung zu versehen wie "should be discontinued", ist deshalb auch wenig überzeugend, birgt sie die Gefahr, über den rechtsverbindlichen Charakter der im Urteilstenor vorgesehenen Anordnung hinwegzutäuschen.

1065 EGMR - Aleksanyan/RUS, Urt. v. 22.12.2008, 46468/06, Rn. 240. 
Vorgaben an diese zu machen oder zumindest die Verlegungshandlung als solche im Urteilstenor anzuordnen. ${ }^{1066}$

Der Gerichtshof ordnete individuelle Massnahmen auch nicht nur bei einer festgestellten inadäquaten medizinischen Versorgung im Strafvollzug an. Er sah sich in Einzelfällen auch bei Art. 3 EMRK relevanten Überbelegungsfällen dazu veranlasst, im Urteilstenor konkrete individuelle Massnahmen anzuordnen. ${ }^{1067}$ So hielt er im konkreten Fall etwa fest, dass einer der Beschwerdeführer, wenn er dies wünscht, mit Rechtskraft des Urteils unverzüglich in eine andere Strafvollzugsanstalt zu verlegen ist. ${ }^{1068}$ Auch hier hat der Konventionsstaat hinsichtlich der Verlegungshandlung als solcher keinen Spielraum, welcher ihm bei der Wahl der Vollzugsanstalt, in die er den Beschwerdeführer verlegt, aber zukommt. Weiter darf das Anknüpfen an einen entsprechenden Willen des Beschwerdeführers nicht dahingehend ausgelegt werden, dass der Konventionsstaat von seiner Pflicht zur Beendigung der unmenschlichen und erniedrigenden Behandlung befreit würde, sollte der Beschwerdeführer keine Verlegung wünschen. Es wäre dann vielmehr am Konventionsstaat, eine menschenrechtskonforme Unterbringungssituation in der betreffenden Strafvollzugsanstalt herzustellen.

Insgesamt ist die Anordnung individueller Massnahmen bei unmenschlichen bzw. erniedrigenden Haftbedingungen zwar keine Seltenheit, aber auch keineswegs die Regel. Es ist durchaus denkbar, dass sich dieses Vorgehen in der Zukunft auch im Bereich inadäquater Haftbedingungen weiter etablieren wird.

\subsection{Anordnung genereller Massnahmen bei unmenschlichen und erniedrigenden Haftbedingungen}

Obwohl der Gerichtshof auch ausserhalb von Piloturteilsverfahren generelle Massnahmen anordnen kann, ${ }^{1069}$ sind Piloturteilsverfahren Hauptanwendungsbereich der Anordnung genereller Massnahmen durch den Gerichtshof, weshalb auf diese Verfahrenstechnik im Folgenden eingegangen

1066 Vgl. bspw. EGMR - Dybeku/ALB, Urt. v. 18.12.2007, 41153/06, Rn. 64.

1067 EGMR - Neshkov u.a./BUL, Urt. v. 27.01.2015, 36925/10, Ziff. 7 lit. b Urteilstenor.

1068 EGMR - Neshkov u.a./BUL, Urt. v. 27.01.2015, 36925/10, Ziff. 7 lit. b Urteilstenor.

1069 Keller/Marti EJIL 2015, 829 (838). 
wird, wobei auf die Anordnung genereller Massnahmen zurückgekommen wird.

\section{Piloturteilstechnik}

Die Piloturteilstechnik als besondere Verfahrenstechnik hat bei der Durchsetzung von Mindeststandards neue Möglichkeiten geschaffen. ${ }^{1070}$ Piloturteilsverfahren gehen strukturelle und systemische Probleme in den einzelnen Konventionsstaaten an, indem sie diese Probleme bezeichnen und konkrete Abhilfemassnahmen vorsehen. Im Folgenden wird deshalb zunächst allgemein auf diese innovative Verfahrenstechnik eingegangen, wobei mit einem kurzen Blick auf die Entstehungsgeschichte begonnen wird, gefolgt von einer knappen Erklärung der Funktionsweise des Piloturteilsverfahrens anhand der relevanten gesetzlichen Grundlage in der Verfahrensordnung. Abzugrenzen ist das Piloturteil sodann von „Quasi-Piloturteilen“ oder "Semi-Piloturteilen“. Es folgt eine Analyse der bisher ergangenen Piloturteile im Bereich der unmenschlichen bzw. erniedrigenden Haftumstände. Besondere Beachtung finden hier die Anwendungsvoraussetzung des Vorliegens eines strukturellen Problems zum einen und die Anordnung bestimmter Abhilfemassnahmen zum anderen.

1. Möglichkeit von Piloturteilsverfahren - im Allgemeinen

\subsection{Entstehungsgeschichte und Überblick}

Das Piloturteilsverfahren wurde durch den Gerichtshof erstmals im Jahr 2004 im Fall Broniowski/POL ${ }^{1071}$ angewandt. Der Gerichtshof verwies mangels ausdrücklicher konventionsrechtlicher Kompetenzgrundlage damals auf die Entschliessung Res (2004) 3 des Ministerkomitees v. 12. Mai

1070 Vgl. auch Nicolau FS Jaeger, S. 163 (171): Piloturteilstechnik als „most impressive and far-reaching of all the Court's procedural innovations".

1071 EGMR (GK) - Broniowski/POL, Urt. v. 22.06.2004, 31443/96 (eigentumsrechtlicher Fall); zur Entstehungsgeschichte inkl. einer Auseinandersetzung mit Vorläuferentscheiden des Gerichtshofs, in denen er sich schon früher mit strukturellen Problemen auseinanderzusetzen hatte vgl. Sснмaнl EuGRZ 2008, 369 (371 ff.). 
2004, ${ }^{1072}$ welche zum Massnahmenpaket zur Gewährleistung der Effektivität des Gerichtshofs gehört. Ausgangspunkt dieser Entwicklung ist die Konfrontation des Gerichtshofs mit immer grösseren Fallzahlen, wozu auch repetitive Fälle gehören, die alle auf derselben strukturellen oder systembedingten Ursache basieren. ${ }^{1073}$ Der Gerichtshof sieht es deshalb als seine Aufgabe, den Staat zum einen bei der Benennung des für die Konventionsverletzung ursächlichen strukturellen Problems und zum anderen auch bei der Suche nach angemessenen Massnahmen zu unterstützen. ${ }^{1074}$ Insofern ist das Piloturteilsverfahren nicht nur Ausgangspunkt der Thematisierung struktureller und systemischer Probleme, sondern dient auch als Anknüpfungspunkt für die Anordnung genereller Abhilfemassnahmen. Zusammenfassend erlaubt das Piloturteilsverfahren dem Gerichtshof, in einem einzelnen Verfahren das festzustellen, auf was er ansonsten in einer Vielzahl weiterer ähnlich gelagerter Fälle zurückkommen müsste. ${ }^{1075}$ Uneinigkeit besteht darüber, ob die Einführung des Piloturteilsverfahrens auch einen qualitativen Sprung beabsichtigte. ${ }^{1076}$

Die Entschliessung Res (2004) 3 des Ministerkomitees weist auch für Piloturteilsverfahren auf die Rolle des Ministerkomitees bei der Überwa-

1072 EGMR (GK) - Broniowski/POL, Urt. v. 22.06.2004, 31443/96, Rn. 190; Resolution Res (2004) 3 on Judgments Revealing an Underlying Systemic Problem. Die Eignung der Entschliessung des Ministerkomitees für die Einführung des Piloturteilsverfahrens ist indes nicht unumstritten: vgl. auch GARLICKI FS Wildhaber, S. 177 (183 f.); kritisch Zagrebelsky FS Wildhaber, S. 521 (530 ff.).

1073 EGMR (GK) - Broniowski/POL, Urt. v. 22.06.2004, 31443/96, Rn. 190; vgl. auch Breuer in: Zimmermann, S. 51 (64 f.); Frowein FS Wildhaber, S. 261 (262); Glas HRLR 2020, 121 (125, 132 ff.); Glas HR\&ILD 2019, 73 (73 f.); zur Entwicklung der Arbeitsbelastung des Gerichtshofs ausf. Bates S. 3 f.; vgl. auch GiLch S. 68, 70.

1074 Resolution Res (2004) 3 on Judgments Revealing an Underlying Systemic Problem, Paragraph I; vgl. auch Schmahl EuGRZ 2008, 369 (370); Тісну ZaöRV 2016, 415 (417f.).

1075 JAEGER in: Zimmermann, S. 125 (126).

1076 Dies verneinend MiLEj S. 516 ff.: Die Einzelfallfokussierung des Gerichtshofs sollte auch mit Blick auf diese Rechtsentwicklung nicht aufgegeben werden, was bisher auch nicht geschehen sei. A.A. Glas HRLR 2014, 671 (689f.): GLAS erkennt eine Entwicklung des Gerichtshofs weg von „einer Gerechtigkeit in individualisierter Form“ und hin zu einer „allgemeinen Gerechtigkeit“. Zur Frage, ob die „verfassungsgerichtliche Rolle" mittels der Piloturteilstechnik gestärkt werde vgl. Breuer in: Zimmermann, S. 51 (62 ff.); diese Frage bejahend Garlicki FS Wildhaber, S. 177 (186). 
chung der Durchsetzung der Urteile des Gerichtshofs hin. ${ }^{1077}$ Das Ministerkomitee selbst befürwortete in der Empfehlung Rec (2010) 3 v. 24. Februar $2010^{1078}$ die Einführung des Piloturteilsverfahrens und attribuierte diesem eine wichtige Anleitungs- und Instruktionsfunktion. ${ }^{1079}$ Seit dem 1. April 2011 ist der prozessrechtliche Rahmen der Piloturteile in Regel 61 VerfO des EGMR kodifiziert. Eine explizite konventionsrechtliche Regelung existiert bislang hingegen weiterhin nicht. Als implizite konventionsrechtliche Kompetenzgrundlage wird Art. 46 EMRK herangezogen.

\subsection{Funktionsweise und Begriff des Piloturteilsverfahrens}

Die Durchführung eines Piloturteilsverfahrens setzt das Vorliegen eines strukturellen oder systemischen Problems im betroffenen Mitgliedstaat voraus, welches Ausgangspunkt weiterer (potentieller) Beschwerden ist oder sein kann (Regel 61 Abs. 1 VerfO). Den Parteien wird das rechtliche Gehör sowohl zur Frage des Vorliegens eines solchen strukturellen oder systembedingten Mangels als auch zur Frage der Eignung der konkreten Beschwerde für die Durchführung eines Piloturteilsverfahrens gegeben (Regel 61 Abs. 2 lit. a VerfO). Der Beschluss zur Durchführung eines Piloturteilsverfahrens kann im Anschluss entweder von Amtes wegen erfolgen oder auf Antrag einer oder beider Parteien (Regel 61 Abs. 2 lit.b VerfO). Es liegt im Ermessen des Gerichtshofs zu beschliessen, wann er ein Piloturteilsverfahren durchführt. ${ }^{1080}$ Die Beurteilung eines Piloturteils wird i.S.d. Regel 41 VerfO vorrangig behandelt.

Ergeht ein Piloturteil, hält der EGMR im Urteilstenor den strukturellen oder systembedingten Mangel fest und benennt in diesem konkret auch die durch den Mitgliedstaat zu ergreifenden Abhilfemassnahmen (Regel 61 Abs. 3 VerfO). Letztere können mit einer Frist versehen werden, welche sich an der Art der geforderten Massnahmen und der Geschwindigkeit, mit welcher diese ergriffen werden können, zu orientieren hat (Regel 61 Abs. 4 VerfO). Die Frage einer gerechten Entschädigung kann sodann im konkreten Fall solange offenbleiben, bis der Mitgliedstaat

1077 Resolution Res (2004) 3 on Judgments Revealing an Underlying Systemic Problem, Paragraph I.

1078 Recommendation $\mathrm{CM} / \operatorname{Rec}$ (2010) 3 on Effective Remedies for Excessive Length of Proceedings.

1079 Recommendation $\mathrm{CM} / \operatorname{Rec}$ (2010) 3 on Effective Remedies for Excessive Length of Proceedings, E. 6 der Präambel.

1080 Art. 61 Abs. 1 VerfO: Der Gerichtshof „kann“ beschliessen. 
sowohl die individuell geforderten als auch die generellen Massnahmen umgesetzt hat (Regel 61 Abs. 5 VerfO).

Wendet der Gerichtshof die Piloturteilstechnik an, ist es sodann in seinem Ermessen, die Parallelfälle bis zur erfolgten Umsetzung der im Urteilstenor des Piloturteils angeordneten Massnahmen zurückzustellen (Regel 61 Abs. 6 lit. a VerfO). Gegenüber den Beschwerdeführern der Parallelfälle hat der Gerichtshof lediglich Informationspflichten. ${ }^{1081}$ Dem EGMR bleibt es aber unbenommen, zunächst zurückgestellte Beschwerden im Interesse einer geordneten Rechtspflege vorzeitig doch wieder zu prüfen (Regel 61 Abs. 6 lit. c VerfO). Auch in jenen Fällen, in denen der Mitgliedstaat die im Urteilstenor des Piloturteils angeordneten Massnahmen nicht umsetzt, erfolgt i.d.R. eine Wiederaufnahme der Parallelfälle (Regel 61 Abs. 8 VerfO).

Legt die Regel 61 VerfO vor allem den prozessualen Rahmen der Anwendung eines Piloturteilsverfahrens fest, fehlt es weiterhin an einer Legaldefinition, welche die konstitutiven Begriffselemente eines Piloturteils festlegen würde - neben dem bereits genannten Kriterium des Vorliegens eines strukturellen oder systembedingten Problems. Durch ein interdisziplinäres Forschungsteam ${ }^{1082}$ wurden drei Kernelemente als zwingende Merkmale ausgemacht: (1) Der Gerichtshof habe explizit von einem Piloturteil oder einem Piloturteilsverfahren zu sprechen, (2) inhaltlich habe in der mitgliedstaatlichen Rechtsordnung ein strukturelles Defizit vorzuliegen, welches kausal für die Konventionsverletzung zu sein hat und welches zu einer Belastung des Gerichtshofs geführt haben muss oder potentiell führen kann, und (3) im Urteilstenor müsse der Gerichtshof generelle Abhilfemassnahmen angeordnet haben. ${ }^{1083}$ Während die letzteren beiden Argumente als Definitionsmerkmale überzeugen, scheint ersteres einer zu formalistischen Betrachtungsweise zu entspringen. Die reine Bezeichnung des Urteils als Piloturteil kann an dessen Charakter meines Erachtens nichts ändern. Als prozessrechtliches Kriterium könnte stattdessen seit Inkrafttreten der Regel 61 VerfO am 1. April 2011 vielmehr die Einhaltung der Verfahrensvorschriften treten, z.B. die Anhörung der Parteien zur Durchführung dieser besonderen Verfahrensart. ${ }^{1084}$ Wobei hier einzu-

1081 Die Informationspflichten umfassen etwa die Bekanntgabe der Zurückstellung sowie allenfalls Auskünfte über neue Entwicklungen (Regel 61 Abs. 6 lit. b VerfO).

1082 Team des Forschungsinstituts für Menschenrechte und Soziale Gerechtigkeit der London Metropolitan University.

1083 Vgl. Leach/Hardman/Stephenson/Blitz S. 14 ff., 22.

1084 In diese Richtung auch Meyer-Ladewig/Brunozzi Art. 46 Rn. 11. 
räumen bleibt, dass die Anwendung dieser Vorschriften ein Urteil nicht zu einem Piloturteil macht, sondern dass ihre Anwendung vielmehr Folge dessen ist, dass es sich um ein Piloturteil handeln soll.

Hält der Gerichtshof in einem Urteil zwar fest, dass ein strukturelles Defizit besteht, ohne aber gleichzeitig generelle Abhilfemassnahmen im Urteilstenor anzuordnen oder in prozessualer Hinsicht nach Regel 61 VerfO vorzugehen, wird in der Lehre für diese Urteile bisweilen der Ausdruck „Semi-Piloturteil“1085 bzw. „Quasi-Piloturteil“1086 verwendet.

Für die effektive Durchsetzung des Mindeststandards ist eine klare Kategorisierung der ergangenen Urteile in „echte Piloturteile“ oder in die ähnlichen „Semi-“ resp. „Quasi-Piloturteile“ zweitrangig. Entscheidender sind das Bestehen und das Nutzen der Möglichkeit der vorrangigen Behandlung von Fällen, die strukturelle bzw. systemische Defizite angehen. Entscheidend ist dabei zum einen, dass der EGMR die strukturellen Probleme bezeichnet und diese mit generellen Abhilfemassnahmen angeht. Dieses Vorgehen ist denn auch kennzeichnend für die aktive Rolle des Gerichtshofs.

2. Piloturteile bei unmenschlichen und erniedrigenden Haftbedingungen i.S.v. Art. 3 EMRK

\subsection{Vorbemerkungen}

Gut fünf Jahre nach dem ersten Piloturteil ${ }^{1087}$ wird das Piloturteilsverfahren seit Orchowski/POL ${ }^{1088}$ und Norbert Sikorski/POL ${ }^{1089}$ auch bei Verfahren wegen unmenschlicher und erniedrigender Haftbedingungen i.S.v.

1085 Vgl. etwa Meyer-Ladewig/Brunozzi Art. 46 Rn. 11.

1086 Baumann S. 96 ff.; vgl. auch Leach/Hardman/Stephenson/Blitz S. 13 ff., welche gar drei Kategorien unterscheiden. Von einem Piloturteil sei auszugehen, wenn die oben genannten drei Voraussetzungen vorliegen würden. Von Quasi-Piloturteilen sei auszugehen, wenn der Gerichtshof strukturellen Problemen mit generellen Massnahmen begegne, ohne das Verfahren aber als Piloturteil zu bezeichnen. In die dritte Kategorie würden jene Fälle fallen, in denen der Gerichtshof zwar strukturelle Probleme feststelle, dabei aber keine generellen Massnahmen benenne.

1087 EGMR (GK) - Broniowski/POL, Urt. v. 22.06.2004, 31443/96.

1088 EGMR - Orchowski/POL, Urt. v. 22.10.2009, 17885/04.

1089 EGMR - Norbert Sikorski/POL, Urt. v. 22.10.2009, 17599/05. 
Art. 3 EMRK angewandt. ${ }^{1090}$ Da es sich bei Fragen rund um unmenschliche und erniedrigende Behandlungen nicht nur um potentiell schwerwiegende Konventionsverletzungen handelt, sondern der Strafvollzug zudem auch einen politisch sensiblen Bereich betrifft, wird die Anwendung des Piloturteilsverfahrens auch in diesem Themengebiet als Zeichen dafür erkannt, dass der Gerichtshof seine anfängliche Zurückhaltung gegenüber Piloturteilsverfahren abgelegt hat. ${ }^{1091}$

Es bleibt aber festzuhalten, dass bei unmenschlichen und erniedrigenden Haftbedingungen i.S.v. Art. 3 EMRK bislang „nur“ gegen einige wenige Konventionsstaaten Piloturteilsverfahren resp. „Quasi-Piloturteile“ geführt wurden bzw. ergangen sind. ${ }^{1092}$ Betroffen waren Belgien, Bulgarien, Italien, Polen, Russland, Ungarn, die Ukraine und Rumänien. ${ }^{1093}$ In Anbetracht des in Europa flächendeckenden Problems der Überbelegung (vgl. Teil 2 Kap. 1; Teil 3 Kap. 2 I.) erstaunt es, dass nicht bereits gegen mehr Konventionsstaaten das Piloturteilsverfahren zur effektiveren Durchsetzung des Mindeststandards angewendet wurde. ${ }^{1094}$

\subsection{Vorliegen eines strukturellen oder systemischen Problems}

Gedenkt der Gerichtshof das Piloturteilsverfahren anzuwenden, prüft er zunächst, ob im konkreten Land von einem strukturellen bzw. systemi-

1090 Je nach Definition des Piloturteils wären die Urteile Orchowski/POL und Norbert Sikorski/POL als "Quasi-Piloturteile“ resp. als "Semi-Piloturteile“ zu qualifizieren. Generelle Massnahmen werden nur in den Urteilsgründen, nicht jedoch im Urteilstenor selbst angeordnet. Da der Gerichtshof die Urteile Orchowski/POL und Norbert Sikorski/POL allerdings selbst als Piloturteile bezeichnet, wenn er diese in späteren Urteilen aufgreift (vgl. etwa EGMR (GK) Muršić/CRO, Urt. v. 20.10.2016, 7334/13, Rn.94), werden diese hier auch bei den Piloturteilen aufgenommen.

1091 Baumann S. 130.

1092 Strecker ZeuS 2016, 235 (245): Strecker bezeichnet die Piloturteile zu Art. 3 EMRK allesamt als Quasi-Piloturteile: Dafür spricht, dass die Anordnung genereller Massnahmen im Urteilstenor Art. 13 EMRK i.V.m. Art. 3 EMRK betrifft und nicht die Verletzung von Art. 3 EMRK.

1093 Je nach Definition der "Quasi-Piloturteile“ wäre der Kreis der betroffenen Länder allerdings weiter zu ziehen.

1094 Vgl. auch Kromrey/Morgenstern ZIS 2017, 106 (118), welche v.a. auf die CPT-Berichte hinweisen, welche bezüglich Litauen und Griechenland über eine "flächendeckende Überfüllung“ berichten. Vor allem bei diesen beiden Ländern sei deshalb „in absehbarer Zeit“ eine Verurteilung wegen systemischer Verletzungen von Art. 3 EMRK zu erwarten. 
schen Problem auszugehen ist oder nicht. Die ergangenen Piloturteile werden deshalb im Folgenden hinsichtlich dieser Frage chronologisch analysiert, um mögliche Entwicklungstendenzen und Perspektiven sichtbar zu machen.

a Die Piloturteile im Einzelnen

In den beiden zeitgleich ergangenen Urteilen Orchowski/POL ${ }^{1095}$ und Norbert Sikorski/POL ${ }^{1096}$ stellt der Gerichtshof mit beinahe identischer Begründung das Vorliegen eines strukturellen Problems in Polen fest. Zum Urteilszeitpunkt waren gegen Polen ca. 160 weitere Beschwerden hängig. Sie alle hatten die Überbelegung polnischer Haftanstalten und die daraus resultierenden mangelhaften Unterbringungs- und Hygienebedingungen zum Gegenstand. ${ }^{1097}$ Die Schwere und der strukturelle Charakter des Problems waren im Mai 2008 auch bereits in einem polnischen Verfassungsgerichtsurteil festgestellt worden, und sie wurden auch im Verfahren vor dem EGMR von den weiteren staatlichen Behörden nicht bestritten. Polen weist gleichwohl auf die seither bereits ergriffenen Massnahmen zum Kampf gegen die Überbelegung hin. ${ }^{1098}$ Der Gerichtshof hält in der Folge fest, dass die Überbelegung in polnischen Haftanstalten schon über eine längere Zeitspanne andauere, ${ }^{1099}$ dass die gerügten Umstände eine Vielzahl von Personen betreffe und dass sie dies auch in Zukunft tun würden. ${ }^{1100}$ Die auf diesen Umständen gründende Verletzung von Art. 3 EMRK sei daher nicht Folge einzelner isolierter Vorfälle, sondern vielmehr Ausdruck eines strukturellen Problems, resultierend aus dem Versagen des polnischen Strafvollzugsystems. ${ }^{1101}$

1095 EGMR - Orchowski/POL, Urt. v. 22.10.2009, 17885/04.

1096 EGMR - Norbert Sikorski/POL, Urt. v. 22.10.2009, 17599/05.

1097 EGMR - Orchowski/POL, Urt. v. 22.10.2009, 17885/04, Rn. 147; EGMR - Norbert Sikorski/POL, Urt. v. 22.10.2009, 17599/05, Rn. 149.

1098 EGMR - Norbert Sikorski/POL, Urt. v. 22.10.2009, 17599/05, Rn. 148.

1099 EGMR - Norbert Sikorski/POL, Urt. v. 22.10.2009, 17599/05, Rn. 152; EGMR Orchowski/POL, Urt. v. 22.10.2009, 17885/04, Rn. 147 (seit mindestens dem Jahr 2000 und zum Urteilszeitpunkt mindestens bis in die erste Jahreshälfte 2008).

1100 EGMR - Norbert Sikorski/POL, Urt. v. 22.10.2009, 17599/05, Rn. 151; EGMR Orchowski/POL, Urt. v. 22.10.2009, 17885/04, Rn. 147.

1101 EGMR - Norbert Sikorski/POL, Urt. v. 22.10.2009, 17599/05, Rn. 151. 
In Ananyev u.a./RUS ${ }^{1102}$ knüpft der Gerichtshof in einem ersten Schritt an ein früheres, ähnliches EGMR-Urteil (Kalashnikov/RUS ${ }^{1103}$ ) an, welches er vor fast 10 Jahren gefällt hatte. In einem zweiten Schritt stellt der Gerichtshof fest, dass auf das Urteil Kalashnikov weitere 80 Urteile gegen Russland gefolgt waren, in welchen er wegen ähnlicher Umstände eine Verletzung von Art. 3 EMRK festgestellt hatte. ${ }^{1104}$ Beanstandet wurden der akute Platzmangel, zu wenige Schlafmöglichkeiten, unrechtmässige Restriktionen beim Zugang zu natürlichem Licht und zu frischer Luft sowie die mangelnde Privatsphäre während der Nutzung der sanitären Anlagen. ${ }^{1105}$ Bisweilen war zusätzlich zum Verstoss gegen Art. 3 EMRK eine Verletzung von Art.13 EMRK (Recht auf wirksame Beschwerde) festgestellt worden. Unter Rückgriff auf sein Fallbearbeitungssystem stellt der Gerichtshof weiter fest, dass zum Urteilszeitpunkt gegen Russland weitere rund 250 Beschwerden zum gleichen Gegenstand hängig seien. ${ }^{1106}$ Die Beschwerden zur Haftsituation hätten ihren Ursprung in unterschiedlichsten Untersuchungsgefängnissen, die geographisch in verschiedenen Regionen Russlands liegen, würden aber dennoch auf denselben Mängeln basieren. ${ }^{1107}$ Vor diesem Hintergrund geht der Gerichtshof davon aus, dass die Situation nicht auf besonderen Umständen der Einzelfälle beruhe. Der Gerichtshof stellt auch hier fest, dass die ungenügende Haftsituation eine grosse Anzahl Inhaftierter in Untersuchungsgefängnissen betreffe und die Gefahr bestehe, dass dies weiterhin der Fall bleibe. ${ }^{1108}$ Die grosse Zahl einschlägiger Beschwerden sei für sich alleine genügendes Zeichen für das Vorliegen eines strukturellen Problems. ${ }^{1109}$ Auch im konkreten Fall

1102 EGMR - Ananyev u.a./RUS, Urt. v. 10.01.2012, 42525/07 u. 60800/08.

1103 EGMR - Kalashnikov/RUS, Urt. v. 15.07.2002, 47095/99: Auch das Urteil Kalashnikov/RUS hatte die Haftsituation in einem russischen Untersuchungsgefängnis zum Gegenstand.

1104 EGMR - Ananyev u.a./RUS, Urt. v. 10.01.2012, 42525/07 u. 60800/08, Rn. 184: Zum Teil wurde in diesen wegen Fehlens eines effektiven nationalen Rechtsbehelfs zusätzlich eine Verletzung von Art. 13 EMRK festgestellt.

1105 EGMR - Ananyev u.a./RUS, Urt. v. 10.01.2012, 42525/07 u. 60800/08, Rn. 185.

1106 EGMR - Ananyev u.a./RUS, Urt. v. 10.01.2012, 42525/07 u. 60800/08, Rn. 184.

1107 Akuter Mangel an persönlichem Platz, zu wenige verfügbare Schlafmöglichkeiten, nicht gerechtfertigte Einschränkungen bezüglich Zugangs zu Tageslicht und frischer Luft und fehlende Privatsphäre bei der Nutzung der sanitären Anlagen: EGMR - Ananyev u.a./RUS, Urt. v. 10.01.2012, 42525/07 u. 60800/08, Rn. 185.

1108 EGMR - Ananyev u.a./RUS, Urt. v. 10.01.2012, 42525/07 u. 60800/08, Rn. 185.

1109 EGMR - Ananyev u.a./RUS, Urt. v. 10.01.2012, 42525/07 u. 60800/08, Rn. 184. 
sei das strukturelle Problem für die Konventionsverletzung ursächlich. ${ }^{1110}$ Weiter verweist der EGMR auf die beiden Zwischenresolutionen, welche vom Ministerkomitee während der Implementierung des Urteils Kalashnikov/RUS resp. während der Implementierung der Urteile der Kalashnikov Group verabschiedet wurden und in welchen auch das Ministerkomitee auf die Existenz struktureller Probleme hingewiesen hatte. ${ }^{1111}$

In Torreggiani u.a./ITA ${ }^{1112}$ stellt der Gerichtshof fest, dass die Überbelegungssituation in Italien die Haftanstalten de Busto Arsizio und Piacenza sowie eine grosse Anzahl weiterer italienischer Gefängnisse betreffe. ${ }^{1113}$ Vom konventionsrechtlich relevanten Sachverhalt seien nicht nur die Beschwerdeführer, sondern viele weitere Inhaftierte betroffen. ${ }^{1114}$ Zum Urteilszeitpunkt von Torreggiani u.a./ITA seien mehrere hundert Beschwerden bezüglich der Überbelegung vor dem Gerichtshof hängig. ${ }^{1115}$ Auch mit Blick auf die künftigen Entwicklungen sei nicht mit einer Eindämmung des Problems zu rechnen; es sei im Gegenteil sogar zu erwarten, dass die Anzahl Beschwerden zu dieser Thematik gegen Italien weiter steigen würden. ${ }^{1116}$ Der Gerichtshof kommt deshalb zum Schluss, dass die Überbelegung in Italien ein strukturelles bzw. systemisches Problem sei, welches auf ein chronisches Versagen des italienischen Strafvollzugssystems zurückzuführen sei. ${ }^{1117}$

Im Urteil Neshkov u.a./BUL ${ }^{1118}$ knüpft der Gerichtshof an ein früheres, gegen Bulgarien ergangenes Urteil (Iorgov/BUL ${ }^{1119}$ ) an, in welchem er vor beinahe elf Jahren erstmals unmenschliche und erniedrigende Haftbe-

1110 EGMR - Ananyev u.a./RUS, Urt. v. 10.01.2012, 42525/07 u. 60800/08, Rn. $184 \mathrm{f}$.: „widespread problem resulting from a malfunctioning of the Russian penitentiary system and insufficient legal and administrative safeguards against the proscribed kind of treatment".

1111 EGMR - Ananyev u.a./RUS, Urt. v. 10.01.2012, 42525/07 u. 60800/08, Rn. 187; Interim Resolution CM/ResDH (2010) 35, Execution of the Judgments of the European Court of Human Rights in 31 Cases/RUS Mainly Concerning Conditions of Detention in Remand Prisons (Kalashnikov Group); Interim Resolution CM/ResDH (2003) 123, Execution of the Judgment of the European Court of Human Rights in the Case of Kalashnikov/RUS.

1112 EGMR - Torreggiani u.a./ITA, Urt. v. 08.01.2013, 43517/09 u.a.

1113 EGMR - Torreggiani u.a./ITA, Urt. v. 08.01.2013, 43517/09 u.a., Rn. 54.

1114 EGMR - Torreggiani u.a./ITA, Urt. v. 08.01.2013, 43517/09 u.a., Rn. 54, 87: Im Urteil wurde dies mit einschlägigen Statistiken belegt.

1115 EGMR - Torreggiani u.a./ITA, Urt. v. 08.01.2013, 43517/09 u.a., Rn. 89.

1116 EGMR - Torreggiani u.a./ITA, Urt. v. 08.01.2013, 43517/09 u.a., Rn. 88 f.

1117 EGMR - Torreggiani u.a./ITA, Urt. v. 08.01.2013, 43517/09 u.a., Rn. 88.

1118 EGMR - Neshkov u.a./BUL, Urt. v. 27.01.2015, 36925/10 u.a.

1119 EGMR - Iorgov/BUL, Urt. v. 11.03.2004, 40653/98. 
dingungen i.S.v. Art. 3 EMRK in bulgarischen Haftanstalten festgestellt hatte. Seit dem Urteil Iorgov/BUL seien gegen Bulgarien wegen ähnlicher Haftumstände 25 weitere Verurteilungen erfolgt. Inadäquate Haftbedingungen gebe es in diversen Strafvollzugsanstalten. ${ }^{1120}$ Beanstandet wurden regelmässig zu enge Platzverhältnisse, unrechtmässige Einschränkungen hinsichtlich des Zugangs zu natürlichem Licht und frischer Luft, ungenügende Hygiene, ungenügende Privatsphäre und unzureichende Gewährung der persönlichen Würde während der Nutzung der sanitären Anlagen. Zum Urteilszeitpunkt von Neshkov u.a./BUL seien beinahe 40 weitere ähnliche Beschwerden vor dem EGMR hängiggewesen, ${ }^{1121}$ wobei der Gerichtshof festhält, dass es keinesfalls nur auf die Zahl der bereits hängigen Beschwerden ankomme. ${ }^{1122}$ Für die Beurteilung, ob ein strukturelles bzw. systemisches Problem vorliege, seien vielmehr die noch zu erwartenden Beschwerden massgebend. ${ }^{1123}$ Der EGMR stützt sich dabei direkt auf den Wortlaut der Regel 61 Abs. 1 VerfO: Ein strukturelles oder systemisches Problem liege dann vor, wenn es zu ähnlichen Beschwerden geführt habe oder führen könne (,has given or may give rise“). Das Vorliegen eines strukturellen bzw. systemischen Problems untermauert der EGMR in der Folge anhand nationaler Statistiken ${ }^{1124}$ sowie Berichten internationaler Organisationen. ${ }^{1125}$ Ca. 9'000 in Bulgarien Inhaftierte, welche überwiegend in überfüllten Haftanstalten untergebracht seien, würden für das Vorliegen eines strukturellen bzw. systemischen Problems sprechen. Der Gerichtshof deutete auch den Beschluss der bulgarischen Behörden, die gesetzliche Einführung der 4-m²-pro-Inhaftiertem Regel auf das Jahr 2019 zu verschieben, als Hinweis für die momentane praktische Unmöglichkeit, diese Regel zu befolgen, und für die gegenwärtige Unmöglichkeit Bulgari-

1120 EGMR - Neshkov u.a./BUL, Urt. v. 27.01.2015, 36925/10 u.a., Rn. 268.

1121 EGMR - Neshkov u.a./BUL, Urt. v. 27.01.2015, 36925/10 u.a., Rn. 270.

1122 EGMR - Neshkov u.a./BUL, Urt. v. 27.01.2015, 36925/10 u.a., Rn. 270.

1123 EGMR - Neshkov u.a./BUL, Urt. v. 27.01.2015, 36925/10 u.a., Rn. 270 mit Verweis auf EGMR - Hutten-Czapska/POL, Urt. v. 19.06.2006, 35014/97, Rn. 235.

1124 Es handelte sich dabei um Statistiken zur Überbelegung. Die Kapazität berechnet auf der Grundlage von $4 \mathrm{~m}^{2}$ Platz pro Strafgefangenem - wurde mit der effektiven Belegung ins Verhältnis gesetzt. Neun der elf Haftanstalten für männliche Inhaftierte waren aufgrund dieser Rechnung überbelegt. Die höchste Belegungsrate wies Ende 2013 die Haftanstalt Burgas auf (239\% der Kapazität).

1125 Im konkreten Fall nahm der EGMR auf CPT-Berichte, auf den jährlichen Bericht des nationalen Ombudsmanns, der die allermeisten Haftanstalten selbst besucht hatte, sowie auf einen Bericht des Bulgarian Helsinki Committee' Bezug. 
ens, der Situation Herr zu werden ${ }^{1126}$. Finanzielle und logistische Schwierigkeiten seien aber keine tauglichen Argumente, um den strukturellen oder systemischen Charakter einer Situation zu bestreiten. ${ }^{1127}$ Vor diesem Hintergrund geht der Gerichtshof davon aus, dass die Verstösse gegen Art. 3 EMRK nicht auf einzelnen Vorfällen beruhten, sondern einem weit verbreiteten Problem geschuldet seien, basierend auf einem Versagen des bulgarischen Strafvollzugssystems und einem ungenügenden Schutz vor unmenschlichen resp. erniedrigenden Behandlungen. ${ }^{1128}$ Der Gerichtshof stellt deshalb in Bulgarien ein strukturelles Problem fest.

In Varga u.a./HUN ${ }^{1129}$ hält der Gerichtshof zunächst fest, dass in bisher vier Urteilen ein Verstoss Ungarns gegen Art. 3 EMRK aufgrund mangelhafter Haftbedingungen festgestellt worden sei. ${ }^{1130} \mathrm{Im}$ Fallbearbeitungssystem des Gerichtshofs seien zum Urteilszeitpunkt aber ca. 450 weitere ähnliche Beschwerden hängig gewesen. Die grosse Zahl weiterer einschlägiger hängiger Beschwerden seien ein genügendes Zeichen für das Bestehen eines strukturellen Problems. ${ }^{1131}$ Die Verstösse gegen Art. 3 EMRK seien in unterschiedlichen ungarischen Haftanstalten in verschiedenen geografischen Regionen festgestellt worden, würden aber alle auf ähnlichen Fakten basieren. Die Verletzungen von Art. 3 EMRK hatten zu knappe Platzverhältnisse, einen zu eingeschränkten Zugang zu den Duschmöglichkeiten und zu Outdoor-Aktivitäten sowie mangelnde Privatsphäre bei der Nutzung der sanitären Anlagen als Ursache. Der Gerichtshof kommt vor diesem Hintergrund zum Schluss, dass die Situation im vorliegenden Fall als Versagen des ungarischen Strafvollzugssystems zu würdigen sei und

1126 EGMR - Neshkov u.a./BUL, Urt. v. 27.01.2015, 36925/10 u.a., Rn. 269 f.: Die fehlende Inkraftsetzung des entsprechenden Rechtsaktes hat zwar zur Folge, dass die bulgarischen Behörden bei einer Unterschreitung der 4- $\mathrm{m}^{2}$-Grenze keinen nationalen Regelverstoss begehen, an ihrer internationalen bzw. europaratsrechtlichen Verantwortung ändert sich hingegen nichts. Eine Verletzung von Art. 3 EMRK könnte die Folge sein. Dies zumindest dann, wenn auch die konventionsrechtlich massgebliche Grenze von $3 \mathrm{~m}^{2}$ unterschritten wird.

1127 EGMR - Neshkov u.a./BUL, Urt. v. 27.01.2015, 36925/10 u.a., Rn. 270.

1128 EGMR - Neshkov u.a./BUL, Urt. v. 27.01.2015, 36925/10 u.a., Rn. 268.

1129 EGMR - Varga u.a./HUN, Urt. v. 10.03.2015, 14097/12 u.a.

1130 Vgl. EGMR - Szél/HUN, Urt. v. 07.06.2011, 30221/06; EGMR - István Gábor Kovács/HUN, Urt. v. 17.01.2012, 15707/10; EGMR - Hagyó/HUN, Urt. v. 23.04.2013, 52624/10; EGMR - Fehér/HUN, Urt. v. 02.07.2013, 69095/10. In den Urteilen Szél/HUN und Hagyó/HUN wurden zudem Verletzungen von Art. 13 EMRK festgestellt. Im Urteil Hagyó/HUN bezog sich die Verletzung von Art. 13 EMRK allerdings auf Beschwerden in Zusammenhang mit Art. 8 EMRK.

1131 EGMR - Varga u.a./HUN, Urt. v. 10.03.2015, 14097/12 u.a., Rn. 98 m.w.H. 
dass von einem in Ungarn weit verbreiteten Missstand auszugehen sei, von welchem viele Inhaftierte betroffen seien und ohne Tätigwerden der Behörden auch in Zukunft sein würden.

In W.D./BEL ${ }^{1132}$ hält der Gerichtshof fest, dass er schon zuvor in diversen Grundsatzurteilen aufgrund fehlender therapeutischer Betreuung von psychisch kranken Strafgefangenen Verletzungen von Art. 3 EMRK und von Art. 5 Abs. 1 und Abs. 4 EMRK ${ }^{1133}$ erkannt und strukturelle Probleme festgestellt habe. ${ }^{1134}$ In Belgien bestünde ein Mangel an geeigneten Plätzen in psychiatrischen Kliniken ausserhalb von Strafvollzugsanstalten und ein Mangel an qualifiziertem Personal in den psychiatrischen Abteilungen der Haftanstalten. ${ }^{135}$ Ein Rechtsbehelf, bei welchem von einem psychisch kranken Inhaftierten ein Nachweis verlangt werde, dass seine derzeitige Unterbringung nicht geeignet sei, und welches fordere, dass der Beschwerdeführer selbst einen geeigneten therapeutischen Ansatz vorzubringen habe, genüge weiter den Anforderungen an einen wirksamen Rechtsbehelf i.S.v. Art. 13 EMRK nicht. Auch der Umstand, dass die Verlegung in eine psychiatrische Einrichtung trotz Anordnung nicht erfolgte und der Beschwerdeführer gezwungen war, ein Vollstreckungsverfahren einzuleiten, lasse sich nicht mit der angeblichen Wirksamkeit der bestehenden Rechtsbehelfe in Einklang bringen. ${ }^{1136}$ Die Zahl der Verstösse betreffend fehlende therapeutische Behandlung psychisch kranker Inhaftierter habe stetig zugenommen. Belgien räumte diesbezüglich selbst ein, dass es an freien Plätzen in externen psychiatrischen Einrichtungen mangle, und führte dazu aus, dass die Behörden auch nicht über Zwangsmittel verfügen würden, um externe psychiatrische Kliniken zu verpflichten, die betroffenen Inhaftierten aufzunehmen. ${ }^{1137}$ Der Gerichtshof erwog vor diesem Hintergrund, dass die Existenz eines dahingehenden strukturellen Problems

1132 EGMR - W.D./BEL, Urt. v. 06.09.2016, 73548/13.

1133 EGMR - W.D./BEL, Urt. v. 06.09.2016, 73548/13, Rn. 144 f.: Das belgische Rechtsmittelsystem sehe vor, dass Art. 3 EMRK und Art. 5 Abs. 1 EMRK sowohl vor den Sozialschutzbehörden als auch vor den Gerichten gemeinsam geltend gemacht werden können, weshalb es hier gerechtfertigt sei, diese Fragen insgesamt vom Standpunkt der Art. 5 Abs. 4 EMRK und Art. 13 EMRK i.V.m. Art. 3 EMRK zu beurteilen.

1134 EGMR - W.D./BEL, Urt. v. 06.09.2016, 73548/13, Rn. 161.

1135 EGMR - W.D./BEL, Urt. v. 06.09.2016, 73548/13, Rn. 151.

1136 EGMR - W.D./BEL, Urt. v. 06.09.2016, 73548/13, Rn. 149.

1137 EGMR - W.D./BEL, Urt. v. 06.09.2016, 73548/13, Rn. 139: Im konkreten Fall hatten sich externe psychiatrische Einrichtungen geweigert, den Beschwerdeführer bei sich aufzunehmen. 
in belgischen Strafvollzugsanstalten von Belgien nicht bestritten sei. ${ }^{1138}$ Der strukturelle Charakter werde weiter durch die Tatsache bestätigt, dass zum Urteilszeitpunkt rund 50 Klagen gegen Belgien anhängig waren, welche die Inhaftierung psychisch kranker Inhaftierter in verschiedenen belgischen Strafvollzugsanstalten ohne angemessene therapeutische Betreuung und ohne jeden Rechtsbehelf betreffen würden. ${ }^{1139}$ Auch die Konventionsverletzung im konkreten Fall beruhe gerade auf diesen strukturellen Problemen und könne deshalb nicht isoliert betrachtet werden. ${ }^{1140}$ Vor diesem Hintergrund und aufgrund der potentiellen Zahl weiterer Betroffener wendet der Gerichtshof in der Folge das Piloturteilsverfahren an.

In Rezmiveș u.a./ROM ${ }^{1141}$ hält der Gerichtshof fest, dass schon im Jahr 2012 festgestellt worden sei, dass die Überbelegung in Rumänien ein strukturelles Ausmass angenommen habe. ${ }^{1142}$ Bis zum Urteilszeitpunkt seien 150 Urteile ergangen, in denen Verletzungen von Art. 3 EMRK aufgrund der Überbelegung und weiterer inadäquater Haftbedingungen (etwa: schlechte Hygieneverhältnisse, defekte sanitäre Anlagen, ungenügende Duschmöglichkeiten, ungenügende Lüftung und Beleuchtung, ungenügendes Angebot an Nahrung, Ungeziefer in den Räumlichkeiten der rumänischen Haftanstalten) festgestellt worden seien. Im Fallbearbeitungssystem des Gerichtshofs seien im Urteilszeitpunkt sodann weitere 3'200 ähnliche Beschwerden hängig. Das Überbelegungsproblem beruhe auf dem Versagen des rumänischen Strafvollzugssystems, betreffe eine Vielzahl von Inhaftierten und werde dies auch in Zukunft tun. Vor diesem Hintergrund stellt der Gerichtshof auch hier einen strukturellen bzw. systemischen Mangel fest und wendet das Piloturteilsverfahren an.

In Sukachov/UKR ${ }^{1143}$ stellt der Gerichtshof fest, dass er seit seinem ersten Urteil zu Haftbedingungen in der Ukraine (Nevmerzhitsky/UKR ${ }^{1144}$ ) in 55 Urteilen aufgrund inadäquater Haftbedingungen in ukrainischen Untersuchungshaftanstalten Verstösse gegen Art. 3 EMRK festgestellt ha-

1138 EGMR - W.D./BEL, Urt. v. 06.09.2016, 73548/13, Rn. 163.

1139 EGMR - W.D./BEL, Urt. v. 06.09.2016, 73548/13, Rn. 165.

1140 EGMR - W.D./BEL, Urt. v. 06.09.2016, 73548/13, Rn. 164.

1141 EGMR - Rezmiveș u.a./ROM, Urt. v. 25.04.2017, 61467/12 u.a.

1142 EGMR - Rezmiveș u.a./ROM, Urt. v. 25.04.2017, 61467/12 u.a., Rn. 106, 110: Zwischen 2007 und 2012 seien 93 Urteile ergangen, in welchen aufgrund der Überbelegung und weiterer inadäquater materieller Haftbedingungen eine Verletzung von Art. 3 EMRK festgestellt worden sei.

1143 EGMR - Sukachov/UKR, Urt. v. 30.01.2020, 14057/17.

1144 EGMR - Nevmerzhitsky/UKR, Urt. v. 05.04.2005, 54825/00. 
be. ${ }^{1145}$ Bisweilen habe er in diesen Urteilen zudem aufgrund des Fehlens wirksamer innerstaatlicher Rechtsbehelfe für die Beschwerden der Antragssteller eine Verletzung von Art. 13 EMRK festgestellt. Er habe schon in vielen dieser Urteile festgestellt, dass die Probleme der Haftbedingungen struktureller Natur seien. Die Verletzungen von Art. 3 EMRK hätten Probleme der Überbelegung und verschiedene immer wiederkehrende Fragen im Zusammenhang mit den materiellen Haftbedingungen betroffen: unangemessene Hygiene- und Sanitärbedingungen, unzureichende Beleuchtung und Belüftung, Insekten und Schimmel in den Zellen etc. ${ }^{1146}$ Die unzureichenden Haftbedingungen würden damit nicht isolierten Ereignissen entspringen, sondern hätten ihren Ursprung in einem weit verbreiteten strukturellen Problem, welches auf einem Versagen des ukrainischen Strafsystems und unzureichenden Schutzmassnahmen gegen unmenschliche resp. erniedrigende Behandlungen beruhe. Obwohl der Gerichtshof diese Schlüsse seit dem Jahr 2005 fast jährlich gezogen habe, scheine das Problem auf innerstaatlicher Ebene nach wie vor ungelöst zu sein, was sich darin zeige, dass im Fallbearbeitungssystem des Gerichtshofs zum Urteilszeitpunkt rund 120 Beschwerden verzeichnet seien, welche prima facie die gleichen Umstände betreffen würden. Diese Zahl alleine deute auf ein wiederkehrendes, strukturelles Problem hin. ${ }^{1147}$ Die Anwendung des Piloturteilsverfahren hänge aber nicht notwendigerweise nur von der Zahl der hängigen Verfahren ab; potentielle weitere Anträge seien ebenso zu berücksichtigen. ${ }^{1148}$ In der Ukraine gebe es zum Urteilszeitpunkt 20'346 Untersuchungsgefangene, wovon gemäss internationalen und nationalen Berichten viele in überfüllten und unzulänglichen Haftanstalten festgehalten würden. Auch das Ministerkomitee habe in einer Zwischenresolution im Dezember 2018 den strukturellen Charakter des Problems hervorgehoben und darauf hingewiesen, dass bei der Umsetzung früherer Urteile keine konkreten Fortschritte bezüglich der allgemeinen Situation hätten erzielt werden können, was zu einer zusätzlichen Belastung des Konventionssystems führe. ${ }^{1149}$ Weiter habe unter anderen auch das CPT wiederholt

1145 EGMR - Sukachov/UKR, Urt. v. 30.01.2020, 14057/17, Rn. 135.

1146 EGMR - Sukachov/UKR, Urt. v. 30.01.2020, 14057/17, Rn. 137.

1147 EGMR - Sukachov/UKR, Urt. v. 30.01.2020, 14057/17, Rn. 138.

1148 EGMR - Sukachov/UKR, Urt. v. 30.01.2020, 14057/17, Rn. 138.

1149 EGMR - Sukachov/UKR, Urt. v. 30.01.2020, 14057/17, Rn. 139; Interim Resolution CM/ResDH (2018) 472, Execution of the Judgments of the European Court of Human Rights Nevmerzhitsky/UKR, Yakovenko/UKR, Logninenko/ UKR, Isayev/UKR u. Melnik Groups/UKR. 
die anhaltenden Probleme in ukrainischen Haftanstalten bemängelt. ${ }^{1150}$ Vor diesem Hintergrund wendet der Gerichtshof in der Folge das Piloturteilsverfahren an.

\section{b Analyse der Piloturteile}

\section{aa Numerische und prognostizierende Betrachtung}

Prüft der Gerichtshof, ob strukturelle oder systemische Mängel vorliegen, nimmt der Gerichtshof in allen vorgenannten Piloturteilen zunächst eine numerische Betrachtung vor, indem er auf die gegen den konkreten Mitgliedstaat bereits ergangenen Urteile verweist oder die weiteren zum Urteilszeitpunkt noch vor dem Gerichtshof hängigen Verfahren gegen den betreffenden Konventionsstaat quantifiziert. ${ }^{1151}$

Während der Gerichtshof beispielsweise bei Ananyev u.a./RUS (bisher ca. 80 ergangene Urteile und ca. 250 weitere hängige Beschwerden) und bei Varga u.a./HUN (zwar bisher erst vier einschlägige Verurteilungen, aber ca. 450 weitere hängige Beschwerden) argumentiert, dass die grosse Zahl an Verfahren genügendes Kriterium für das Vorliegen eines strukturellen und systemischen Problems sei ${ }^{1152}$, damit auch automatisch die Gefahr zahlreicher künftiger Fälle als gegeben betrachtet und in der Folge die weitere Argumentation hinsichtlich des angenommenen strukturellen bzw. systemischen Defizits schlank hält ${ }^{1153}$, verfolgt der Gerichtshof im Urteil Neshkov u.a./BUL eine leicht andere Argumentationslinie. Vor dem Hintergrund der vergleichsweise geringen Zahl der ergangenen Urteile (25 Urteile) und der zum Urteilszeitpunkt hängigen Verfahren (knapp 40 weitere Beschwerden) hält der Gerichtshof hier ausdrücklich fest, dass es bei der Frage des strukturellen bzw. systemischen Problems nicht allein auf die Anzahl der ergangenen oder hängigen Verfahren ankomme, vielmehr sei auch auf die in Zukunft noch zu erwartenden Beschwerden abzustellen. Die darauffolgende Feststellung des Vorliegens eines strukturellen bzw. systemischen Problems untermauert der Gerichtshof in der Folge

1150 EGMR - Sukachov/UKR, Urt. v. 30.01.2020, 14057/17, Rn. 140.

1151 Berücksichtigt werden dabei sämtliche Urteile resp. hängigen Klagen, welchen materiell ähnliche Sachverhalte zugrunde liegen, welche auf derselben Ursache basieren.

1152 EGMR - Ananyev u.a./RUS, Urt. v. 10.01.2012, 42525/07 u. 60800/08, Rn. 184; EGMR - Varga u.a./HUN, Urt. v. 10.03.2015, 14097/12 u.a., Rn. 98 m.w.H.

1153 Besonders knapp ist seine Argumentation im Fall Varga u.a./HUN. 
mit einer aufwändigen Argumentation, wobei er insbesondere die Anzahl Inhaftierter in überfüllten Strafvollzugsanstalten insgesamt sowie Berichte internationaler Organisationen, unter anderem des CPT, heranzieht.

In Sukachov/UKR (50 Urteile und rund 120 hängige Verfahren) kombiniert der Gerichtshof diese beiden Argumentationslinien, indem er einerseits festhält, dass diese Zahl alleine schon auf ein strukturelles Problem hindeute, die Anwendung des Piloturteilsverfahren aber nicht zwingend nur von der Zahl hängiger Verfahren abhänge, sondern potentielle weitere Fälle ein ebenso bedeutender Faktor sei. Es folgt wiederum eine aufwändige Argumentation, wobei der Gerichtshof die Anzahl insgesamt in Untersuchungshaftanstalten Gefangener heranzieht und darauf verweist, dass gemäss internationalen und nationalen Berichten viele von ihnen in überfüllten und unzulänglichen Haftanstalten inhaftiert seien. Internationale Berichte, welche auch bereits anhaltende unzulängliche Zustände festgestellt hatten, werden vom Gerichtshof hinzugezogen. Auch die Zwischenresolution des Ministerkomitees, in welcher schon früher der strukturelle Charakter betont und darauf hingewiesen wurde, dass das Konventionssystem durch die mangelhafte Implementierung früherer ähnlicher Fälle belastet werde, wurde in der Argumentation aufgegriffen.

Zusammenfassend lässt sich festhalten, dass weder die Zahl abgeurteilter Fälle noch die weiteren bereits hängigen Fälle genügendes Kriterium für die Feststellung des Vorliegens eines strukturellen Mangels sind. Es wurde deutlich, dass der Gerichtshof bereits bei relativ geringen Fallzahlen durchaus bereit ist, dem prospektiven Kriterium potentiell weiterer Betroffener grosse Bedeutung zuzumessen. Bei dieser prognostizierenden Betrachtung stützt er sich immer wieder auch auf Berichte internationaler Organisationen, wobei unter anderem dem CPT grosse Bedeutung zukommt. Der Gerichtshof berücksichtigt auch Zwischenresolutionen des Ministerkomitees. Mit Blick auf die Gewährleistung einer effektiven menschenrechtsermöglichenden Umsetzung des Mindeststandards ist die prospektive Herangehensweise des Gerichtshofs jedenfalls zu begrüssen. In Anbetracht dessen, dass bereits eine geringere Anzahl an Urteilen resp. hängiger Verfahren die Anwendung des Piloturteilsverfahren auslösen kann, wenn für die $\mathrm{Zu}-$ kunft ohne Intervention keine positive Prognose möglich ist, erstaunt es umso mehr, dass der Gerichtshof das Piloturteilsverfahren bislang erst bei acht Konventionsstaaten angewendet hat. Auch vor diesem Hintergrund ist allerdings zu erwarten, dass Piloturteilsverfahren in naher Zukunft bei weiteren Konventionsstaaten zur Anwendung gelangen werden. 
bb Bedeutung der Kooperationsbereitschaft der Konventionsstaaten

Die ersten Piloturteile im Bereich der unmenschlichen bzw. erniedrigenden Haftbedingungen i.S.v. Art. 3 EMRK betrafen Polen. In der Literatur ist gar davon die Rede, dass Polen, was die Anwendung des Piloturteilsverfahrens angeht, eine Pionierrolle übernehme. ${ }^{154}$ Als Grund wird angeführt, dass Polen im Allgemeinen dem „neuen“ Verfahren der Piloturteilstechnik aufgeschlossen und positiv begegnete. ${ }^{1155}$ Diese grundsätzliche Kooperationsbereitschaft Polens manifestiert sich ein Stück weit auch in den Fällen Orchowski und Norbert Sikorski, in welchen Polen die strukturelle Natur des Überbelegungsproblems grundsätzlich anerkannte. ${ }^{1156}$ Gleichwohl erfolgte die Anwendung von Art. 46 EMRK letztlich aber doch gegen den Willen Polens. Polen war nämlich der Ansicht, dass das Problem auf nationaler Ebene erkannt wurde und man bereits Abhilfemassnahmen ergriffen habe, so dass ein Piloturteilsverfahren nicht mehr notwendig sei. Der Widerstand Polens gegen die Anwendung des Piloturteilsverfahrens ist im Vergleich zu anderen Staaten wohl dennoch relativ bescheiden ausgefallen.

Die darauffolgenden Piloturteile im Bereich der unmenschlichen bzw. erniedrigenden Haftbedingungen zeigen aber deutlich, dass das Einverständnis oder nur ein geringer Widerstand des Konventionsstaates für die Anwendung des Piloturteilsverfahrens keineswegs Voraussetzung ist. Der Gerichtshof wendet das Piloturteilsverfahren vielmehr auch dann an, wenn der strukturelle bzw. systemische Charakter vom Konventionsstaat entschieden bestritten wird. ${ }^{1157}$

Ist eine mangelnde Kooperationsbereitschaft kein Grund, von der Piloturteilstechnik abzusehen, spricht auch dies dafür, dass weitere Piloturteilsverfahren folgen werden.

1154 Nicht nur das erste Piloturteil überhaupt (EGMR [GK] - Broniowski/POL, Urt. v. 22.06.2004, 31443/96) erging gegen Polen. Auch in mehreren weiteren Sachbereichen wurden Piloturteilsverfahren als Erstes gegen Polen eingeleitet. Vgl. auch Eschment S. 162: Polen übernehme in dieser Hinsicht eine Pionierrolle. Vgl. ausf. auch Leach/Hardman/Stephenson/Blitz S. $41 \mathrm{ff}$.

1155 Vgl. auch Eschment S. 162; Zagrebelsky FS Wildhaber, S. 521 (531 f.).

1156 EGMR - Orchowski/POL, Urt. v. 22.10.2009, 17885/04, Rn. 146.

1157 EGMR - Neshkov u.a./BUL, Urt. v. 27.01.2015, 36925/10 u.a., Rn. 269. 
cc Geographische Verteilung

Mit Blick auf die Konventionsstaaten, bei welchen der Gerichtshof hinsichtlich unmenschlicher und erniedrigender Haftbedingungen das Piloturteilsverfahren bisher angewendet hat (Polen, Russland, Italien, Bulgarien, Ungarn, Belgien, Rumänien und die Ukraine), fällt auf, dass die meisten von ihnen zu den „neueren“, östlichen Europaratsmitgliedstaaten gehören ${ }^{1158}$. Mit den Urteilen Torreggiani u.a./ITA ${ }^{1159}$ sowie W.D./BEL ${ }^{1160}$ wird aber deutlich, dass durchaus auch Gründungsmitglieder des Europarats resp. süd- bzw. westeuropäische Länder bei Vorliegen struktureller Probleme mit der Anwendung eines Piloturteilsverfahrens zu rechnen haben. Die geographische Verteilung der Piloturteilsverfahren deutet damit eher nicht auf politische Gründe auf Europaratsebene hin, sondern widerspiegelt mehr die noch besonders mangelhafte Umsetzung des Mindeststandards in den ehemaligen Ostblockstaaten, sodass dort besonders frappante strukturelle und systemische Mängel festgestellt werden können.

dd Themenbereiche unmenschlicher und erniedrigender Haftumstände

Das strukturelle bzw. systemische Defizit wird in fast allen Piloturteilen rund um unmenschliche und erniedrigende Haftumstände in der Überbelegungssituation in den mitgliedstaatlichen Strafvollzugsanstalten erkannt. Bisweilen zieht der Gerichtshof dann in einem zweiten Schritt auch weitere für Art. 3 EMRK relevante, mit der Überbelegung eng verknüpfte Aspekte hinzu, wie etwa ungenügende Anzahl Schlafmöglichkeiten, unrechtmässige Restriktionen hinsichtlich des Zugangs zu natürlichem Licht und zu frischer Luft, ungenügende Hygiene, eingeschränkte Duschmöglichkeiten, mangelnde Möglichkeit zu Outdoor-Aktivitäten und die ungenügende Privatsphäre bei der Nutzung der sanitären Einrichtungen. Auch bei der Beurteilung, ob ein strukturelles resp. systemisches Problem vorliegt, nimmt der Gerichtshof damit eine gewisse Gesamtbetrachtung der Haftsituation vor. Die Gesamtbetrachtung widerspiegelt bei dieser Frage gewissermassen auch die "Grosswetterlage“ des Strafvollzugssystems im konkreten Mitgliedstaat.

1158 Diese Tendenz im Allgemeinen auch in anderen Sachgebieten aufzeigend: Eschment S. $170 \mathrm{ff}$.

1159 EGMR - Torreggiani u.a./ITA, Urt. v. 08.01.2013, 43517/09 u.a.

1160 EGMR - W.D./BEL, Urt. v. 06.09.2016, 73548/13. 
Bislang grosse Ausnahme ist der Entscheid W.D./BEL, in welchem das strukturelle Problem im Bereich einer inadäquaten medizinischen Versorgung im Strafvollzug bei psychisch kranken Inhaftierten lokalisiert wurde. Die Anwendung des Piloturteilsverfahrens auch in Themenbereichen ausserhalb von Überbelegungssituationen zeigt, dass in Zukunft auch in inhaltlicher Sicht durchaus mit einer Ausdehnung des Anwendungsgebiets der Piloturteile zu rechnen ist. Insbesondere vor dem Hintergrund der desolaten Situation hinsichtlich adäquater medizinischer Versorgung in Strafvollzugsanstalten gewisser Konventionsstaaten ${ }^{1161}$ erstaunt es vielmehr, dass hier nicht schon längst mehr Piloturteilsverfahren angewendet wurden.

Die Piloturteile ergingen zeitlich in ziemlich regelmässigen Abständen. ${ }^{1162}$ Die Piloturteilstechnik ist damit nicht als eine kurzzeitige Bemühung des Gerichtshofs im Kampf gegen unmenschliche und erniedrigende Haftbedingungen aufgrund struktureller und systemischer Mängel einzustufen, vielmehr widerspiegelt sie ein kontinuierliches Bestreben nach menschenrechtswahrenden Haftbedingungen. Das erst kürzlich ergangene Piloturteil Sukachov/UKR verdeutlicht, dass der Gerichtshof auch weiterhin an dieser Verfahrenstechnik festhalten wird, weshalb auch unter diesem Gesichtspunkt in naher Zukunft weitere Piloturteile zu erwarten sind.

\section{ff Zwischenfazit}

Das Potential des Piloturteilsverfahrens als Durchsetzungsinstrument der Konventionsrechte mit Blick auf Verletzungen von Art. 3 EMRK durch unmenschliche und erniedrigende Haftumstände scheint noch lange nicht ausgeschöpft zu sein. Alle Zeichen deuten darauf hin, dass sich die Pilotur-

1161 Vgl. etwa CPT - Report to the Greek Government [CPT/Inf (2020) 15], S. 5; CPT - 26th General Report [CPT/Inf (2017) 5], Rn.46; CPT - Report to the Government of „The Former Yugoslav Republic of Macedonia“ [CPT/Inf (2017) 30], Rn.34; CPT - Report to the Georgian Government [CPT/Inf (2019) 16], Rn. $83 \mathrm{f}$.

1162 Orchowski u. Norbert Sikorski: 22.10.2009, Ananyev: 10.01.2012, Torreggiani: 08.01.2013, Neshkov: 27.01.2015, Varga: 10.03.2015, W.D.: 06.09.2016, Rezmiveș: 25.04.2017, Sukachov: 30.01.2020. 
teilstechnik in diesem Bereich weiter etablieren wird, wobei weitere Piloturteile nicht nur in den ehemaligen Ostblockstaaten oder aufgrund von Überbelegungssituationen zu erwarten sind. Vielmehr ist von einer sowohl geografischen als auch inhaltlichen Ausdehnung dieser Verfahrenstechnik auszugehen, lassen sich aufgrund statistischer Daten und zahlreicher Berichte verschiedenster Gremien strukturelle Mängel doch nicht nur in den Konventionsstaaten vermuten, gegen die bereits Piloturteilsverfahren geführt wurden. Hinsichtlich der Überbelegungssituation in europäischen Staaten hat sich etwa gezeigt, dass neben Italien, Ungarn und Rumänien v.a. auch Haftanstalten in Nordmazedonien, Frankreich, Moldawien, Serbien, Portugal, Tschechien, Griechenland, Österreich, der Slowakei und Dänemark am stärksten mit der Überbelegung zu kämpfen haben (Teil 2 Kap. 1). ${ }^{1163}$ Im Sinne einer effektiven Durchsetzung des Mindeststandards wäre eine solche Expansion denn auch zu begrüssen.

\subsection{Anordnung konkreter individueller und genereller Massnahmen in den Piloturteilen}

Ist ein strukturelles bzw. systemisches Problem festgestellt, besteht umfassende Reformnotwendigkeit, ${ }^{1164}$ so dass der Gerichtshof bei Piloturteilen konkrete Abhilfemassnahmen anordnen kann, welche dem festgelegten Mindeststandard effektiv zum Durchbruch verhelfen sollen. Die konkreten angeordneten Massnahmen müssen deshalb geeignet und erforderlich sein, um im entsprechenden Mitgliedstaat wieder eine konventionsrechtskonforme Rechtslage oder Verwaltungspraxis zu schaffen. Welche Massnahmen der Gerichtshof in den einzelnen Konventionsstaaten als geeignet betrachtet hat, wird anhand der ergangenen Piloturteile aufgezeigt - wieder in chronologischer Reihenfolge; dies, um in der Folge verallgemeinerungsfähige Aussagen treffen zu können.

1163 Der Gerichtshof hat einige Konventionsstaaten schon in frühen Urteilen in Anwendung von Art. 46 EMRK darauf hingewiesen, dass die Haftbedingungen zu verbessern seien. So insbesondere auch Griechenland und Moldawien: vgl. EGMR - Samaras u.a./GRE, Urt. v. 28.02.2012, 11463/09; EGMR - Tzamalis u.a./GRE, Urt. v. 04.12.2012, 15894/09; EGMR - Al. K./GRE, Urt. v. 11.12.2014, 63542/11; EGMR - Shishanov/MDA, Urt. v. 15.09.2015, 11353/06. Auch dies deutet darauf hin, dass der Gerichtshof gegen weitere Konventionsstaaten die Piloturteilstechnik anwenden könnte.

1164 Vgl. etwa auch: EGMR - Ananyev u.a./RUS, Urt. v. 10.01.2012, 42525/07 u. 60800/08, Rn. 194. 
a Die Piloturteile im Einzelnen

In den Urteilen Orchowski/POL und Norbert Sikorski/POL ist eine Analyse der von Polen im Kampf gegen die Überbelegung zuvor bereits ergriffenen Massnahmen Ausgangspunkt der Frage, welche konkreten Massnahmen vom EGMR anzuordnen sind. ${ }^{1165}$ Im Kampf gegen die Überbelegung hatte Polen in erster Linie verschiedene legislative Massnahmen getroffen. Am 01.09.2009 trat etwa ein Gesetz über die elektronische Überwachung von Verurteilten ausserhalb von Strafvollzugsanstalten in Kraft mit dem Zweck, die Zahl der Inhaftierten zu senken. ${ }^{1166}$ Weitere Gesetzesentwürfe mit gleicher Zielsetzung lagen zum Urteilszeitpunkt vor. So sollte beispielsweise Gefängnisdirektoren die Möglichkeit eingeräumt werden, Haftantritte auf einen späteren Zeitpunkt zu verschieben, wenn sich abzeichnen sollte, dass zum Zeitpunkt des geplanten Haftantrittes in der entsprechenden Haftanstalt keine Kapazität besteht. Polnische Gesetzesartikel, welche vorsahen, dass es dem Gefängnisdirektor in „gerechtfertigten Fällen“ erlaubt ist, für eine gewisse Zeit die Unterbringung in einer Zelle mit weniger als $3 \mathrm{~m}^{2}$ persönlichem Platz pro Person zu beschliessen, wurden für verfassungswidrig erklärt. Bemängelt worden war der offene Wortlaut dieser Norm, weil er die Gefahr berge, Inhaftierte willkürlich in inadäquaten Haftbedingungen unterzubringen. ${ }^{1167}$ Neben den legislativen Massnahmen hatte Polen zudem bereits für die Jahre 2006-2009 ein

1165 EGMR - Orchowski/POL, Urt. v. 22.10.2009, 17885/04, Rn. 89 ff.; EGMR Norbert Sikorski/POL, Urt. v. 22.10.2009, 17599/05, Rn. 90 ff.

1166 Gemäss der polnischen Regierung soll diese Massnahme (nach einer sukzessiven Steigerung) ca. 15'000 Verurteilte pro Jahr betreffen, was zu einer entsprechenden Entlastung polnischer Haftanstalten führen soll: vgl. EGMR Orchowski/POL, Urt. v. 22.10.2009, 17885/04, Rn. 90; EGMR - Norbert Sikorskil POL, Urt. v. 22.10.2009, 17599/05, Rn. 92.

1167 Art. 248 Code of Execution of Criminal Sentences: „1. In particularly justified cases a governor of a prison or remand center may decide to place detainees, for a specified period of time, in conditions where the area of the cell is less than 3 square meters per person. Any such decision shall be promptly communicated to a penitentiary judge. 2. The Minister of Justice shall determine, by means of an ordinance, the rules which are to be followed by the relevant authorities in a situation where the number of persons detained in prisons and remand centers exceeds on a nationwide scale the overall capacity of such establishments $[\ldots]$. . 
Sonderprogramm ausgearbeitet, anhand welchem $17^{\prime} 000$ neue Haftplätze geschaffen werden sollten. ${ }^{1168}$

In der Folge führt der Gerichtshof aus, dass der Konventionsstaat nach einer festgestellten Konventionsverletzung gestützt auf Art. 46 EMRK grundsätzlich verpflichtet sei, allgemeine oder individuelle Massnahmen zu ergreifen, um die vom Gerichtshof festgestellte Verletzung zu beenden. ${ }^{1169}$ Die Wahl der Mittel zur Beseitigung struktureller bzw. systemischen Defizite stehe dem Mitgliedstaat zu - vorbehaltlich der Aufsicht des Ministerkomitees -, vorausgesetzt, diese Mittel seien mit den im Urteil des Gerichtshofs dargelegten Schlussfolgerungen vereinbar. ${ }^{1170}$ Der Gerichtshof hält weiter fest, dass die von Polen bereits ergriffenen allgemeinen Massnahmen zu begrüssen seien, das Ausmass des systemischen Problems aber konsequente, langfristige Bemühungen und weitere Massnahmen erfordere, um die Konformität des polnischen Strafvollzugs mit Art. 3 EMRK für die Zukunft wieder sicherzustellen. ${ }^{1171}$ Im Bewusstsein, dass die Lösung systembedingter Probleme der Überbelegung die Mobilisierung erheblicher finanzieller Mittel erfordere, hält der Gerichtshof sodann fest, dass der Mangel an Ressourcen keine Haftbedingungen rechtfertigen könne, die Art. 3 EMRK verletzen. Ist der Staat nicht in der Lage sicherzustellen, dass die Haftbedingungen den Anforderungen von Art. 3 EMRK entsprechen, müsse er seine strenge Strafpolitik aufgeben oder weitere alternative Massnahmen einführen, um die Zahl der inhaftierten Personen zu verringern. ${ }^{1172}$ Die Möglichkeit, auf zivilrechtlichem Weg Schadensersatz bei ungenügenden Bedingungen einzufordern, genüge für sich alleine jedenfalls nicht, um dem Überbelegungsproblem zu begegnen, da Schadensersatzzahlungen allein die Ursache des Problems nicht bekämpfen würden. Mit einer zivilrechtlichen Klage müsse vielmehr immer auch eine zeitnahe Reaktion der Behörden auf die konkrete Situation einhergehen; deshalb müsse ein System geschaffen werden, in welchem effiziente Beschwerden des Inhaftierten bei Behörden möglich seien, so dass schneller als auf dem Weg eines Gerichtsprozesses reagiert und, wenn

1168 Etwa die Hälfte der Plätze waren in den ersten beiden Jahren auch bereits effektiv realisiert worden: vgl. EGMR - Orchowski/POL, Urt. v. 22.10.2009, 17885/04, Rn. 91.

1169 EGMR - Orchowski/POL, Urt. v. 22.10.2009, 17885/04, Rn. 148.

1170 EGMR - Orchowski/POL, Urt. v. 22.10.2009, 17885/04, Rn. 148.

1171 EGMR - Orchowski/POL, Urt. v. 22.10.2009, 17885/04, Rn. 152.

1172 EGMR - Orchowski/POL, Urt. v. 22.10.2009, 17885/04, Rn. 153. 
angezeigt, ein zeitnaher Transfer in eine andere Strafvollzugsanstalt mit EMRK-konformen Haftbedingungen veranlasst werden könne. ${ }^{1173}$

In Ananyev u.a./RUS hält der Gerichtshof in den Urteilsgründen fest, dass das strukturelle Problem in Russland auf multiple Ursachen zurückzuführen sei. Grundlage für die Anordnung konkreter Massnahmen durch den Gerichtshof waren auch hier die innerstaatlich bereits ergriffenen Massnahmen: Russland hatte für die Jahre 2002-2006 einen Fahrplan zur Renovation und zum Wiederaufbau gewisser Untersuchungshaftanstalten entwickelt, mit dem Ziel, über eine grössere Zahl an Haftplätzen zu verfügen und den Inhaftierten mehr persönlichen Platz garantieren zu können. Im daran anschliessenden Fahrplan von 2007-2016 war der Bau von mehr als 20 neuen Untersuchungshaftanstalten vorgesehen, so dass im Jahr 2016 in allen russischen Untersuchungshaftanstalten der Mindeststandard von $4 \mathrm{~m}^{2}$ persönlichem Platz hätte gewährleistet sein sollen. ${ }^{1174}$

Der Gerichtshof anerkennt unter anderem mit Verweis auf die Zwischenresolutionen des Ministerkomitees die von den russischen Behörden ergriffenen Massnahmen, bemängelt gleichzeitig aber auch, dass wirksame, kurzfristig zu realisierende und kostengünstige Massnahmen wie etwa die Abschirmung der Toiletten durch Vorhänge, die Entfernung der dicken Netze vor den Fenstern, welche den Zugang zu natürlichem Licht verhindern, und die Gewährung von häufigeren Duschmöglichkeiten bisher nicht ergriffen worden waren. ${ }^{1175}$ Die Komplexität des Problems der Überbelegung erfordere - wie es schon das Ministerkomitee in seiner Zwischenresolution festgehalten habe - einen umfassenden Ansatz mit Änderungen sowohl in rechtlicher als auch in praktischer Hinsicht. ${ }^{1176}$ Auf gesetzlicher Ebene sei etwa sicherzustellen, dass die Anordnung der Untersuchungshaft die Ausnahme und nicht die Regel sei; eine Freilassung

1173 EGMR - Orchowski/POL, Urt. v. 22.10.2009, 17885/04, Rn. 154.

1174 EGMR - Ananyev u.a./RUS, Urt. v. 10.01.2012, 42525/07 u. 60800/08, Rn. 192.

1175 EGMR - Ananyev u.a./RUS, Urt. v. 10.01.2012, 42525/07 u. 60800/08, Rn. 193; Interim Resolution CM/ResDH (2010) 35, Execution of the Judgments of the European Court of Human Rights in 31 Cases/RUS Mainly Concerning Conditions of Detention in Remand Prisons (Kalashnikov Group); Interim Resolution CM/ResDH (2003) 123, Execution of the Judgment of the European Court of Human Rights in the Case of Kalashnikov/RUS.

1176 EGMR - Ananyev u.a./RUS, Urt. v. 10.01.2012, 42525/07 u. 60800/08, Rn. 194; Interim Resolution CM/ResDH (2010) 35, Execution of the Judgments of the European Court of Human Rights in 31 Cases/RUS Mainly Concerning Conditions of Detention in Remand Prisons (Kalashnikov Group). 
gelte es immer zu vermuten. ${ }^{1177} \mathrm{Um}$ dies in der Praxis sicherzustellen, hätten in anderen Konventionsstaaten Schulungen und der Einsatz spezialisierter Haftrichter Erfolg gebracht. ${ }^{1178}$ Der Gerichtshof räumt bei seinen Ausführungen aber ein, dass es über die Aufgabe des Gerichtshofs hinausgehe, eine Lösung für ein solch komplexes System zu finden. ${ }^{1179}$ Der Gerichtshof betont dennoch den Zusammenhang zwischen der Überbelegung und den überlangen Haftzeiten in der Untersuchungshaft, die es zu bekämpfen gelte. ${ }^{1180}$

Mit Verweis auf die Europäischen Strafvollzugsgrundsätze hält es der Gerichtshof weiter für angebracht, für jede Haftanstalt eine konventionskonforme Kapazitätsgrenze festzulegen. ${ }^{1181}$ Weiter seien die Befugnisse und Zuständigkeiten der Gefängnisdirektoren zu überprüfen. Die Situation könne nämlich verbessert werden, wenn ihnen die Möglichkeit eingeräumt würde, bei Erreichen der Kapazitätsgrenze die Aufnahme weiterer Personen abzulehnen. ${ }^{1182}$ Auch besondere Übergangsregelungen, welche in der Untersuchungshaft bis zu einer allgemeinen Verbesserung der Haftbedingungen gelten können, seien möglich. Während einer solchen Transitionsperiode könnten etwa Ausnahmen hinsichtlich des mindestens zur Verfügung zu stellenden Platzes möglich sein, ${ }^{1183}$ unter den Voraussetzungen, dass die Dauer der Unterbringung unter inadäquaten Platzverhältnissen nur kurz sei, sie unter rechtlicher Aufsicht erfolge und Kom-

1177 EGMR - Ananyev u.a./RUS, Urt. v. 10.01.2012, 42525/07 u. 60800/08, Rn. $197 \mathrm{ff}$.

1178 EGMR - Ananyev u.a./RUS, Urt. v. 10.01.2012, 42525/07 u. 60800/08, Rn. 203.

1179 EGMR - Ananyev u.a./RUS, Urt. v. 10.01.2012, 42525/07 u. 60800/08, Rn. 194: Gerade das Ministerkomitee sei besser geeignet, die ergriffenen Massnahmen zu überwachen.

1180 EGMR - Ananyev u.a./RUS, Urt. v. 10.01.2012, 42525/07 u. 60800/08, Rn. 196. Dies ist vor allem auch ein Problem von Art. 5 EMRK und wurde in diesem Zusammenhang vom Gerichtshof im Urteil Kalashnikov/RUS, Urt. v. 15.07.2002, 47095/99 dargelegt.

1181 EGMR - Ananyev u.a./RUS, Urt. v. 10.01.2012, 42525/07 u. 60800/08, Rn. 205: Die Kapazitätsgrenze sei anhand des zur Verfügung stehenden Platzes zu berechnen. Daneben könne aber auch eine operative Kapazitätsgrenze festgelegt werden, welche auf den Kriterien der Kontrolle, der Sicherheit und dem ordnungsgemässen Funktionieren des Regimes beruhe.

1182 EGMR - Ananyev u.a./RUS, Urt. v. 10.01.2012, 42525/07 u. 60800/08, Rn. 206: Auf diese Weise solle sichergestellt werden, dass die operative Kapazitätsgrenze der Untersuchungsgefängnisse nur unter streng definierten und aussergewöhnlichen Umständen überschritten werde.

1183 EGMR - Ananyev u.a./RUS, Urt. v. 10.01.2012, 42525/07 u. 60800/08, Rn. 207. 
pensationsmöglichkeiten zur Verfügung stünden. ${ }^{1184}$ Auf diese Weise solle sichergestellt werden, dass es trotz unbestrittenermassen inadäquaten Haftbedingungen zu keiner Verletzung von Art. 3 EMRK komme. ${ }^{1185}$ Die Kompensationsmöglichkeiten können gemäss dem Gerichtshof entweder monetärer Art sein oder aber in Form eines ausgedehnteren Zugangs zu Outdoor-Aktivitäten oder vermehrter Möglichkeit zu Aktivitäten ausserhalb der Zelle gewährt werden. ${ }^{1186}$ Wie lange eine solche Transitionsperiode andauern könne, richte sich nach den konkreten Umständen und sei jeweils vom Gericht zu bestimmen. Zusätzlich sei dafür aber auch eine gesetzliche Grundlage zu schaffen, welche eine absolut geltende Höchstdauer festlege. ${ }^{1187} \mathrm{Im}$ Gesetz seien sodann abschliessend auch die Situationen zu definieren, in welchen überhaupt von dieser Ausnahme Gebrauch gemacht werden dürfe.

Im Urteil Ananyev u.a./RUS werden die aus Art. 46 EMRK folgenden Pflichten zumindest partiell auch im Urteilstenor aufgenommen. Im Urteilstenor verpflichtet der Gerichtshof Russland, innert sechs Monaten nach Rechtskraft des Urteils gemeinsam mit dem Ministerkomitee einen verbindlichen Zeitrahmen auszuarbeiten. In inhaltlicher Sicht solle dieser die Einführung von Rechtsbehelfen sowohl präventiver als auch kompensatorischer Natur enthalten, welche zur Sicherstellung EMRK-konformer Haftumstände in russischen Haftanstalten führen sollen. ${ }^{1188}$ Weiter verpflichtet der Gerichtshof die russischen Behörden im Urteilstenor, innert zwölf Monaten seit Rechtskraft allen Beschwerdeführern der zum Urteilszeitpunkt hängigen Verfahren Wiedergutmachungen zu leisten. ${ }^{1189}$ Die fundamentale Natur des Art. 3 EMRK veranlasst den EGMR ferner, von der in Regel 61 Abs. 6 VerfO vorgesehenen Möglichkeit des Zurückstellens der Parallelfälle keinen Gebrauch zu machen. ${ }^{1190}$

In Torreggiani u.a./ITA führt der Gerichtshof zunächst die Situation in Italien aus. Italien habe auf nationaler Ebene wegen der Überbelegungssituation bereits im Jahr 2010 (Belegungsrate von 151\%) einen Ausnahmezustand angenommen und einen Interventionsplan zur Bekämpfung

1184 EGMR - Ananyev u.a./RUS, Urt. v. 10.01.2012, 42525/07 u. 60800/08, Rn. 207.

1185 EGMR - Ananyev u.a./RUS, Urt. v. 10.01.2012, 42525/07 u. 60800/08, Rn. 208.

1186 EGMR - Ananyev u.a./RUS, Urt. v. 10.01.2012, 42525/07 u. 60800/08, Rn. 208.

1187 EGMR - Ananyev u.a./RUS, Urt. v. 10.01.2012, 42525/07 u. 60800/08, Rn. 208.

1188 EGMR - Ananyev u.a./RUS, Urt. v. 10.01.2012, 42525/07 u. 60800/08, Ziff. 7 Urteilstenor.

1189 EGMR - Ananyev u.a./RUS, Urt. v. 10.01.2012, 42525/07 u. 60800/08, Ziff. 8 Urteilstenor.

1190 EGMR - Ananyev u.a./RUS, Urt. v. 10.01.2012, 42525/07 u. 60800/08, Rn. 236. 
der Überbelegung ausgearbeitet. ${ }^{1191}$ Dieser beinhaltete vor allem zweierlei Massnahmen: zum einen die Schaffung zusätzlicher Haftplätze (Neubau von 11 Haftanstalten sowie Ausbau von 20 bereits bestehenden Haftanstalten $)^{1192}$ und zum anderen das Ergreifen befristeter legislativer Massnahmen. Ausgesprochene Haftstrafen sollten unter gewissen Voraussetzungen ausserhalb von Strafvollzugsanstalten verbüsst werden können - etwa zu Hause oder an anderen öffentlichen oder privat betreuten Plätzen. ${ }^{1193}$ Diese Massnahmen hätten jedoch nicht zum gewünschten Erfolg geführt. Auch zwei Jahre später, im Jahr 2012, habe die Belegungsrate in Italien noch $148 \%$ betragen. ${ }^{1194}$ Der EGMR anerkennt die Anstrengungen Italiens im Kampf gegen die Überbelegung, bemängelt aber die Befristung des italienischen Interventionsplans und weist auf die Notwendigkeit langfristiger Massnahmen im Kampf gegen die Überbelegung hin. ${ }^{1195}$ Der Gerichtshof ermutigt Italien in der Folge, Massnahmen zur Reduktion der Zahl der Inhaftierten zu ergreifen. ${ }^{1196}$

Auch hier nimmt sich der EGMR zurück und stellt aufgrund der Komplexität einer stringenten Kriminalpolitik fest, dass dies über seinen Aufgabenbereich hinausgehen würde. Er verweist folglich auch auf die Empfehlungen des Ministerkomitees. ${ }^{1197}$ Den Hinweis auf die Notwendigkeit des Ergreifens sowohl präventiver als auch kompensatorischer Massnahmen lässt sich der Gerichtshof aber nicht nehmen. ${ }^{1198}$ Im Vordergrund müsse bei einer Konventionsverletzung immer ihre schnellstmögliche Behebung stehen; im Nachgang soll sodann eine Entschädigung für die bereits erlittene Konventionsverletzung erfolgen. ${ }^{1199}$

Im Urteilstenor verpflichtet der Gerichtshof Italien, innert eines Jahres nach Rechtskraft des Urteils einen Rechtsbehelf zu schaffen, welcher es

1191 EGMR - Torreggiani u.a./ITA, Urt. v. 08.01.2013, 43517/09 u.a., Rn. 25.

1192 Auf diesem Wege sollten insgesamt 9150 zusätzliche Haftplätze geschaffen werden.

1193 EGMR - Torreggiani u.a./ITA, Urt. v. 08.01.2013, 43517/09 u.a., Rn. 26 f.

1194 EGMR - Torreggiani u.a./ITA, Urt. v. 08.01.2013, 43517/09 u.a., Rn. 92.

1195 EGMR - Torreggiani u.a./ITA, Urt. v. 08.01.2013, 43517/09 u.a., Rn. 92 f.

1196 EGMR - Torreggiani u.a./ITA, Urt. v. 08.01.2013, 43517/09 u.a., Rn.91, 94: Der Gerichtshof verweist dabei vor allem auch auf die äusserst hohe Rate von Personen, welche sich in Untersuchungshaft befinden (40\% aller Inhaftierter befinden sich in Untersuchungshaft).

1197 EGMR - Torreggiani u.a./ITA, Urt. v. 08.01.2013, 43517/09 u.a., Rn. 95: Die Richter sollen insbesondere von der bestehenden Möglichkeit zum Erlass auch zur Freiheitsstrafe alternativer Strafen Gebrauch machen.

1198 EGMR - Torreggiani u.a./ITA, Urt. v. 08.01.2013, 43517/09 u.a., Rn. 96.

1199 EGMR - Torreggiani u.a./ITA, Urt. v. 08.01.2013, 43517/09 u.a., Rn. 96. 
den Inhaftierten ermöglicht, gegen Verletzungen von Art. 3 EMRK durch Überbelegungssituationen vorzugehen. ${ }^{1200}$ Ähnlich gelagerte Fälle gegen Italien, welche ausschliesslich die Überbelegung betreffen und noch nicht an die Regierung weitergeleitet wurden, werden für ein Jahr ab Rechtskraft des Urteils aufgeschoben. Dies, um Italien die Möglichkeit zu geben, die angesprochenen Massnahmen zu ergreifen. Die bereits an die Regierung weitergeleiteten Fälle werden jedoch ungehindert fortgeführt. ${ }^{1201}$

In Neshkov u.a./BUL hält der Gerichtshof zunächst fest, dass multiple Gründe für die Überbelegungssituation in Bulgarien verantwortlich seien. ${ }^{1202}$ Fehlende Investitionen seien für den schlechten Zustand der Haftanstalten verantwortlich. Personalmangel führe zusätzlich zur kritisierten Praxis, dass die Inhaftierten nachts keinen Zugang zu Toiletten hätten. ${ }^{1203}$ Der EGMR hält in der Folge wiederum fest, dass es nicht seine eigentliche Aufgabe sei, konkrete Vorgaben zur Lösung eines solch komplexen Problems vorzutragen, und weist dabei wiederum auf die Rolle des Ministerkomitees hin. ${ }^{1204}$ Bulgarien müsse aber bei der Implementierung des vorliegenden Urteils die Bekämpfung der Überbelegung und der weiteren inadäquaten materiellen Haftbedingungen, wie etwa der mangelnden Hygiene in den Haftanstalten, in Angriff nehmen. ${ }^{1205}$ Hinsichtlich der Überbelegung hält der EGMR fest, dass der Mitgliedstaat bei Unfähigkeit zur Art.-3-EMRK-konformen Unterbringung entweder seine strenge Kriminalpolitik aufgeben müsse oder die Einführung bzw. den Ausbau alternativer Strafmöglichkeiten vorantreiben müsse. ${ }^{1206}$ Unter Verweis darauf, dass es eben nicht seine Aufgabe sei, dem Mitgliedstaat vorzuschreiben, wie es sein Strafvollzugssystem auszugestalten habe, bezieht sich der Gerichtshof auf Empfehlungen des CPT und des Ministerkomitees sowie auf den Bulgarien-spezifischen MacManus Report. Diese würden allesamt potentielle Massnahmen zum Kampf gegen die Überbelegung vorschlagen, wobei die Massnahmen vom Bau neuer Haftanstalten über die bessere Verteilung der

1200 EGMR - Torreggiani u.a./ITA, Urt. v. 08.01.2013, 43517/09 u.a., Rn.96ff., Ziff. 4 Urteilstenor. Es liegt dabei im Ermessensspielraum von Italien, ob bestehende Rechtsbehelfe entsprechend geändert werden oder ob neue Rechtsbehelfe eingeführt werden.

1201 EGMR - Torreggiani u.a./ITA, Urt. v. 08.01.2013, 43517/09 u.a., Rn. 101, Ziff. 5 Urteilstenor.

1202 EGMR - Neshkov u.a./BUL, Urt. v. 27.01.2015, 36925/10 u.a., Rn. 272.

1203 EGMR - Neshkov u.a./BUL, Urt. v. 27.01.2015, 36925/10 u.a., Rn. 272.

1204 EGMR - Neshkov u.a./BUL, Urt. v. 27.01.2015, 36925/10 u.a., Rn. 274.

1205 EGMR - Neshkov u.a./BUL, Urt. v. 27.01.2015, 36925/10 u.a., Rn. 275 f.

1206 EGMR - Neshkov u.a./BUL, Urt. v. 27.01.2015, 36925/10 u.a., Rn. 276. 
Inhaftierten auf die bestehenden Haftanstalten bis hin zur Reduktion der Zahl der Inhaftierten reichen würden. ${ }^{1207}$ Der Gerichtshof bemängelt in der Folge, dass Bulgarien in den letzten Jahren trotz vieler Berichte zu den ungenügenden Umständen nicht mehr zur Behebung des Problems getan habe. Die bestehenden Haftanstalten seien umgehend entweder einer umfassenden Renovation zu unterziehen oder ganz zu ersetzen. ${ }^{1208}$ Ein Mangel an Ressourcen oder logistische Probleme dürften dabei keine Rolle spielen. ${ }^{1209}$ Hinsichtlich Art. 13 EMRK ordnet der EGMR sowohl den Erlass präventiver als auch kompensatorischer Rechtsbehelfe an.

Im Urteilstenor verpflichtet der Gerichtshof Bulgarien als generelle Massnahme, zur Einhaltung der in diesem Urteil aufgestellten Anforderungen an die Haftbedingungen innert 18 Monaten seit Rechtskraft des Urteils effektive Rechtsbehelfe sowohl präventiver als auch kompensatorischer Natur einzuführen. ${ }^{1210}$ Als individuelle Massnahme ordnet der EGMR an, den Beschwerdeführer Zlatev in eine andere Haftanstalt zu verlegen, wenn er dies wolle.

In Varga u.a./HUN hält der EGMR an seiner bisherigen Rechtsprechung fest und verweist zunächst auf die Wahlmöglichkeiten des Konventionsstaates bei der Ergreifung der Massnahmen zur Behebung der Konventionsverletzung, bevor der Gerichtshof mögliche Massnahmen zur Behebung des Problems ausführt. Der Gerichtshof betont die Notwendigkeit substantieller und stetiger Schritte seitens des Mitgliedstaates zur Behebung des systemischen Problems. ${ }^{1211}$ Die Überbelegung sei vor allem durch eine Reduktion der Zahl der Inhaftierten zu bekämpfen, wobei dies vor allem durch eine vermehrte Nutzung alternativer Massnahmen zu erfolgen habe. ${ }^{1212}$ Zur Untermauerung seiner Forderung verweist der Gerichtshof auf die Recommendation Rec (2006) 13 des Ministerkomitees $^{1213}$.

Im Urteilstenor verpflichtet der Gerichtshof Ungarn, innerhalb von sechs Monaten seit Rechtskraft des Urteils unter der Aufsicht des Ministerkomitees einen Zeitplan auszuarbeiten für die Ergreifung geeigneter Massnah-

1207 EGMR - Neshkov u.a./BUL, Urt. v. 27.01.2015, 36925/10 u.a., Rn. 276.

1208 EGMR - Neshkov u.a./BUL, Urt. v. 27.01.2015, 36925/10 u.a., Rn. 277.

1209 EGMR - Neshkov u.a./BUL, Urt. v. 27.01.2015, 36925/10 u.a., Rn. 278.

1210 EGMR - Neshkov u.a./BUL, Urt. v. 27.01.2015, 36925/10 u.a., Ziff. 7 Urteilstenor.

1211 EGMR - Varga u.a./HUN, Urt. v. 10.03.2015, 14097/12 u.a., Rn. $101 \mathrm{ff}$.

1212 EGMR - Varga u.a./HUN, Urt. v. 10.03.2015, 14097/12 u.a., Rn. 104 f.

1213 Recommendation Rec (2006) 13 on the Use of Remand in Custody, the Conditions in which it Takes Place and the Provision of Safeguards against Abuse. 
men und für die Einführung sowohl präventiver als auch kompensatorischer Rechtsbehelfe, mit denen effektive Beschwerden zu gewährleisten sind, die für Art. 3 EMRK relevante, inadäquate Haftbedingungen betreffen. ${ }^{1214}$ Von der Möglichkeit der Zurückstellung der Parallelfälle i.S.d. Art. 61 Abs. 6 VerfO macht der Gerichtshof keinen Gebrauch. ${ }^{1215}$

In W.D./BEL hält der Gerichtshof fest, dass Belgien im Rahmen einer weitreichenden Reform der psychiatrischen Versorgung während der Inhaftierung bereits wichtige Schritte unternommen habe, um das festgestellte strukturelle bzw. systemische Problem anzugehen. Der Gerichtshof begrüsst diese Schritte und ermutigt Belgien, seine Bemühungen fortzusetzen. Es sei nicht Aufgabe des Gerichtshofs, den Konventionsstaaten vorzuschreiben, wie sie mit psychisch kranken Inhaftierten umzugehen hätten; dieser Prozess werfe komplexe rechtliche und praktische Fragen auf, die über die richterliche Funktion des Gerichtshofs hinausgehen würden. ${ }^{1216}$ In der Folge ermutigt der Gerichtshof Belgien, Massnahmen zu ergreifen, um die Zahl psychisch kranker Inhaftierter ohne angemessene therapeutische Versorgung zu verringern. In Frage käme hier etwa eine Neudefinition der Kriterien, welche eine Massnahme rechtfertigen würden, wie es in der laufenden Gesetzesreform auch vorgesehen sei. Der Gerichtshof begrüsst auch das in Belgien nun gesetzlich verankerte Ziel, der inhaftierten Person im Hinblick auf ihre Wiedereingliederung in die Gesellschaft eine angemessene therapeutische Unterstützung zu gewähren. ${ }^{1217}$ Der Gerichtshof hält es für angemessen, Belgien eine Frist von zwei Jahren einzuräumen, um die individuellen und generellen Massnahmen umzusetzen. Hinsichtlich gegenwärtiger und zukünftiger Beschwerdeführer könne eine Wiedergutmachung durch Ad-hoc-Massnahmen erreicht werden, die Gegenstand gütlicher Regelungen oder einseitiger Erklärung sein können. ${ }^{1218}$

Im Urteilstenor verpflichtet der Gerichtshof Belgien, im Kontext der ähnlich gelagerten Fälle geeignete Massnahmen zu ergreifen, um die Konventionskonformität sicherzustellen, wobei diese Massnahmen innerhalb von zwei Jahren nach Rechtskraft dieses Urteils umgesetzt sein müssten.

1214 EGMR - Varga u.a./HUN, Urt. v. 10.03.2015, 14097/12 u.a., Ziff. 9 Urteilstenor.

1215 EGMR - Varga u.a./HUN, Urt. v. 10.03.2015, 14097/12 u.a., Rn. 114 ff.

1216 EGMR - W.D./BEL, Urt. v. 06.09.2016, 73548/13, Rn. $168 \mathrm{f}$.

1217 EGMR - W.D./BEL, Urt. v. 06.09.2016, 73548/13, Rn. 169.

1218 EGMR - W.D./BEL, Urt. v. 06.09.2016, 73548/13, Rn. 173. 
Alle anderen ähnlichen hängigen Verfahren gegen Belgien werden für die Dauer von zwei Jahren zurückgestellt. ${ }^{1219}$

In den Urteilsgründen von Rezmiveş u.a./ROM hält der Gerichtshof aufgrund des Feststellungscharakters seines Urteils fest, dass die von ihm angeordneten generellen Massnahmen als Vorschläge aufzufassen seien. ${ }^{1220}$ Der Gerichtshof würdigt die bereits ergriffenen Massnahmen Rumäniens beim Kampf gegen die Überbelegung, verweist aber auch auf Berichte des CPT, des Ministerkomitees und des Ombudsmanns, welche die weiterhin andauernde Überbelegungssituation in Rumänien rügen. ${ }^{1221}$ Rumänien habe deshalb weitere Massnahmen zur Reduktion der Anzahl Inhaftierter zu ergreifen. ${ }^{1222}$ Mögliche Haftverschiebungen, bedingte Entlassungen und Bewährungshilfen seien zu evaluieren. ${ }^{1223}$ Die Schaffung weiterer Haftplätze würdigt der Gerichtshof mit einem Verweis auf die Empfehlung R (99) 22 des Ministerkomitees kritisch. Der Ausbau der Infrastruktur alleine sei nämlich nicht geeignet, die Ursache des Problems der Überbelegung zu beheben. Erforderlich vor allem mit Blick auf die schlechten materiellen Haftbedingungen seien insbesondere Renovationsarbeiten an den bestehenden Strafvollzugsanstalten. ${ }^{1224}$ Weiter seien die präventiven und kompensatorischen Rechtsbehelfe auszubauen. ${ }^{1225}$

Im Urteilstenor verpflichtet der Gerichtshof Rumänien, innert sechs Monaten seit Rechtskraft einen Zeitplan auszuarbeiten, in welchem generelle Massnahmen zur Behebung des Überbelegungsproblems und der inadäquaten Haftbedingungen darzulegen sind. ${ }^{1226}$ Ähnlich gelagerte Fälle gegen Rumänien, welche die Überbelegung und damit zusammenhängende inadäquate Haftbedingungen betreffen und noch nicht an die Regierung weitergeleitet wurden, werden vorerst aufgeschoben, um Rumänien die Möglichkeit der Ergreifung der angesprochenen Massnahmen zu geben.

1219 EGMR - W.D./BEL, Urt. v. 06.09.2016, 73548/13, Ziff. 6 und Ziff. 7 Urteilstenor.

1220 EGMR - Rezmiveş u.a./ROM, Urt. v. 25.04.2017, 61467/12 u.a., Rn. 112.

1221 EGMR - Rezmiveş u.a./ROM, Urt. v. 25.04.2017, 61467/12 u.a., Rn. 113.

1222 EGMR - Rezmiveş u.a./ROM, Urt. v. 25.04.2017, 61467/12 u.a., Rn. 115 ff.

1223 EGMR - Rezmiveș u.a./ROM, Urt. v. 25.04.2017, 61467/12 u.a., Rn. 118.

1224 EGMR - Rezmiveș u.a./ROM, Urt. v. 25.04.2017, 61467/12 u.a., Rn. 119.

1225 EGMR - Rezmiveș u.a./ROM, Urt. v. 25.04.2017, 61467/12 u.a., Rn. 121.

1226 EGMR - Rezmiveș u.a./ROM, Urt. v. 25.04.2017, 61467/12 u.a., Ziff. 4 Urteilstenor. 
Die bereits an die Regierung weitergeleiteten Fälle werden jedoch ungehindert fortgeführt. ${ }^{1227}$

In Sukachov/UKR hält der Gerichtshof fest, dass, wenn der Konventionsstaat nicht in der Lage sei, jedem Strafgefangenen mit Art. 3 EMRK konforme Haftbedingungen zu gewähren, die Überbelegung am besten durch eine Minimierung der Zahl der Inhaftierten angegangen werden könne, indem häufiger alternative Massnahmen zum Freiheitsentzug zu ergreifen seien. ${ }^{1228}$ Der Gerichtshof hält in der Folge den Zusammenhang zwischen Art.-3-EMRK-relevanten Überbelegungssituationen und Art.-5EMRK-relevanten Fällen der übermässigen Nutzung und Dauer der Untersuchungshaft fest. ${ }^{1229}$ Der Gerichtshof begrüsst in der Folge die von der Ukraine bereits ergriffenen Massnahmen bezüglich der Eindämmung von Anordnungen der Untersuchungshaft, weist Staatsanwaltschaften und Gerichte aber erneut darauf hin, ihre Anträge auf resp. Anordnungen von Untersuchungshaft auf die schwerwiegendsten Fälle zu beschränken und Alternativen zur Untersuchungshaft zu nutzen. ${ }^{1230}$ Weiter begrüsst der Gerichtshof die von der Ukraine bereits ergriffenen Massnahmen, hält aber fest, dass sich die materiellen Bedingungen trotz dieser Bemühungen gar verschlechtert hätten, was durch internationale und nationale Berichte bestätigt worden sei. ${ }^{1231}$ Es seien konsequente und langfristige Bemühungen und die Verabschiedung weiterer Massnahmen notwendig. Grössere Renovierungsarbeiten in den bestehenden Haftanstalten oder der Ersatz veralteter Einrichtungen seien deshalb unverzüglich fortzusetzen, wozu auch angemessene Mittel bereitgestellt werden sollten. ${ }^{1232}$ Es sei aber den ukrainischen Behörden unter der Aufsicht des Ministerkomitees überlassen, angemessene Massnahmen zu ergreifen, wozu auch die genannten Massnahmen gehören könnten. ${ }^{1233}$ Das Ministerkomitee habe in seiner Zwischenresolution zwar bereits die dringende Notwendigkeit hervorgehoben, dass die ukrainischen Behörden weiterhin an der Annahme einer umfassenden langfristigen Strategie mit klaren und verbindlichen Zeitvorgaben arbeiten, die zur Lösung der strukturellen Probleme führen könne.

1227 EGMR - Rezmiveș u.a./ROM, Urt. v. 25.04.2017, 61467/12 u.a., Rn. 127 f., Ziff. 5 Urteilstenor.

1228 EGMR - Sukachov/UKR, Urt. v. 30.01.2020, 14057/17, Rn. 146.

1229 EGMR - Sukachov/UKR, Urt. v. 30.01.2020, 14057/17, Rn. 147 f.: Der Gerichtshof hatte in mehr als 90 Fällen Verstösse gegen Art. 5 Abs. 3 EMRK festgestellt.

1230 EGMR - Sukachov/UKR, Urt. v. 30.01.2020, 14057/17, Rn. 149.

1231 EGMR - Sukachov/UKR, Urt. v. 30.01.2020, 14057/17, Rn. 151.

1232 EGMR - Sukachov/UKR, Urt. v. 30.01.2020, 14057/17, Rn. 151.

1233 EGMR - Sukachov/UKR, Urt. v. 30.01.2020, 14057/17, Rn. 152. 
Das Ministerkomitee habe die Zeitvorgaben allerdings nicht weiter präzisiert. In Anbetracht des langwierigen Charakters des im vorliegenden Fall festgestellten strukturellen Problems und des offensichtlichen Fehlens einer konkreten Lösung, welche innerstaatlich geprüft werde, müsse nun eine spezifische Frist gesetzt werden. Spätestens innert 18 Monaten nach Rechtskraft habe eine Kombination wirksamer innerstaatlicher Rechtsbehelfe in Bezug auf die Haftbedingungen zur Verfügung zu stehen, die sowohl vorbeugende als auch ausgleichende Wirkung haben. ${ }^{1234}$

Im Urteilstenor verpflichtet der Gerichtshof die Ukraine (aufgrund der Verletzung von Art. 13 i.V.m. Art. 3 EMRK), innert 18 Monaten nach Rechtskraft eine Kombination wirksamer innerstaatlicher Rechtsbehelfe in Bezug auf die Haftbedingungen zur Verfügung zu stellen, die sowohl vorbeugende als auch ausgleichende Wirkung haben. ${ }^{1235}$

b Weiterführende Analyse

aa Grundsätze des Gerichtshofs und Vorgehensweise

Der Gerichtshof hat im Rahmen von Art. 46 EMRK verschiedene Grundsätze entwickelt. Dazu gehört, dass der Gerichtshof in Übereinstimmung mit seiner Funktion und dem Subsidiaritätsgedanken den Konventionsstaaten auch hinsichtlich des Ergreifens geeigneter und erforderlicher Massnahmen einen weiten Ermessensspielraum zugesteht. ${ }^{1236}$ Dies nicht zuletzt deshalb, weil der Gerichtshof erkennt, dass die Wahl der zu ergreifenden Massnahmen Resultat einer stringenten und komplexen Kriminalpolitik zu sein hat und die Ausarbeitung einer solchen über seinen Aufgabenbereich hinausgeht. ${ }^{1237}$ Nichtsdestotrotz lässt es sich der Gerichtshof auch nach einer Analyse der vom Konventionsstaat bereits ergriffenen

1234 EGMR - Sukachov/UKR, Urt. v. 30.01.2020, 14057/17, Rn. 160; vgl. auch Interim Resolution CM/ResDH (2018) 472, Execution of the Judgments of the European Court of Human Rights Nevmerzhitsky/UKR, Yakovenko/UKR, Logninenko/UKR, Isayev/UKR u. Melnik Groups/UKR.

1235 EGMR - Sukachov/UKR, Urt. v. 30.01.2020, 14057/17, Ziff. 7 Urteilstenor.

1236 Vgl. etwa EGMR - Neshkov u.a./BUL, Urt. v. 27.01.2015, 36925/10 u.a., Rn. 279.

1237 Vgl. etwa EGMR - Torreggiani u.a./ITA, Urt. v. 08.01.2013, 43517/09 u.a., Rn. 95. 
Massnahmen ${ }^{1238}$ in den Urteilsgründen der Piloturteile typischerweise nicht nehmen, eine Auswahl an Massnahmen zu nennen, die aus seiner Perspektive zur Lösung des strukturellen Problems geeignet und erforderlich sind. Bisweilen nimmt der Gerichtshof dabei auch auf die Arbeit des Ministerkomitees in Implementierungsverfahren früherer, ähnlich gelagerter Urteile Bezug. Bei den Formulierungen zu potentiellen Abhilfemassnahmen bleibt der Gerichtshof wiederum zurückhaltend.

bb Themenbereiche unmenschlicher und erniedrigender Haftumstände

Die Anordnung konkreter Massnahmen zielt darauf ab, das zuvor festgestellte strukturelle Problem zu lösen. Wird in den meisten Piloturteilen im Bereich unmenschlicher und erniedrigender Haftumstände aufgrund der Überbelegungssituation ein strukturelles oder systemisches Defizit angenommen, hat die anzuordnende Massnahme die Lösung des Überbelegungsproblems zu bezwecken. Es erstaunt deshalb nicht, dass der Gerichtshof im Rahmen des Art. 46 EMRK bei seinen Ausführungen zu den zu ergreifenden Massnahmen die Bekämpfung der Überbelegungssituation im jeweiligen Konventionsstaat ins Zentrum rückt und Abhilfemassnahmen zu weiteren prekären resp. kumulierenden Haftbedingungen nur am Rande thematisiert. ${ }^{1239}$ Einzig im Urteil W.D./BEL ging es bei den generellen Massnahmen nicht um die Bekämpfung der Überbelegungssituation, sondern um die Einführung der erforderlichen therapeutischen Massnahmen in Strafvollzugsanstalten. Dies, da zuvor auch diesbezüglich ein struktureller bzw. systemischer Mangel festgestellt worden war.

1238 Urteile Orchowski/POL, Norbert Sikorski/POL, Ananyev u.a./RUS, Torreggiani u.a./ITA, Rezmiveș u.a./ROM und Sukachov/UKR, nicht hingegen in Nesh$k o v$ u.a./BUL und in Varga u.a./HUN.

1239 So schlägt der Gerichtshof etwa in Ananyev u.a./RUS zwar konkrete weitere Massnahmen auch bezüglich der übrigen prekären materiellen Umstände vor, vertieft diese in der Folge jedoch nicht. Ähnlich im Urteil Rezmiveş u.a./ROM, in welchem der Gerichtshof zwar hinsichtlich der zu ergreifenden generellen Massnahmen auf die übrigen inadäquaten Haftbedingungen verweist, aber ausser der Renovation der Haftanstalten keine weiteren generellen Massnahmen vorschlägt. Einzig im Urteil Neshkov u.a./BUL behandelt der EGMR Massnahmen zur Bekämpfung der Überbelegung und Massnahmen zur Bekämpfung der übrigen prekären materiellen Haftumstände getrennt voneinander, was gleichwohl nicht zur Folge hat, dass er bezüglich der übrigen inadäquaten Umstände hinsichtlich allfälliger Abhilfemassnahmen konkreter werden würde. 
Sollen generelle Massnahmen im Rahmen von Piloturteilen über die Bekämpfung der Überbelegungssituation hinaus angeordnet werden, würde dies zunächst bedingen, den strukturellen oder systemischen Charakter des dafür ursächlichen Problems festzustellen.

\section{cc Art und Zeithorizont der vorgeschlagenen Massnahmen}

Es wird deutlich, dass der Gerichtshof das Ergreifen nur oberflächlicher Massnahmen nicht genügen lässt. Seine Forderungen sind vielmehr dahingehend zu verstehen, dass das festgestellte Problem bei seiner Ursache anzugehen ist. ${ }^{1240}$ Auch die Möglichkeit eines Private Enforcement genügt dem Gerichtshof nicht; vielmehr wird eine Reaktionen der Behörde auf die konkrete Situation gefordert. ${ }^{1241}$ Insgesamt verdeutlichen die in den Urteilsgründen genannten generellen Massnahmen den umfassenden Ansatz und die langfristige Perspektive des Gerichtshofs. ${ }^{1242}$ Dieser vom EGMR gewählte Ansatz entspricht denn auch der Empfehlung R (99) 22, welche der Gerichtshof in seinen Erwägungen aufgreift. ${ }^{1243}$

Die vorgeschlagenen Massnahmen betreffen sowohl die Legislative ${ }^{1244}$ als auch die Exekutive ${ }^{1245}$ und die Judikative ${ }^{1246}$. Verlangt werden insbesondere auch Investitionen in die Infrastruktur der Haftanstalten ${ }^{1247}$. Fast schon gebetmühlenartig hält der Gerichtshof immer wieder fest, dass

1240 Vgl. etwa EGMR - Rezmiveș u.a./ROM, Urt. v. 25.04.2017, 61467/12 u.a., Rn. 119: Der Ausbau der Strafvollzugsanstalten alleine genügt nicht, um dem Problem der Überbelegung zu begegnen.

1241 Vgl. etwa EGMR - Orchowski/POL, Urt. v. 22.10.2009, 17885/04, Rn. 154.

1242 EGMR - Varga u.a./HUN, Urt. v. 10.03.2015, 14097/12 u.a., Rn. 101 ff.: Der Gerichtshof betont die Notwendigkeit substantieller und stetiger Schritte des Mitgliedstaats.

1243 EGMR - Rezmiveş u.a./ROM, Urt. v. 25.04.2017, 61467/12 u.a., Rn. 119: „[...] the Court would draw attention to Recommendation Rec (99) 22 of the Committee of Ministers, according to which such a measure is generally unlikely to offer a lasting solution to this problem."

1244 EGMR - Ananyev u.a./RUS, Urt. v. 10.01.2012, 42525/07 u. 60800/08, Rn. 197 ff.: Es sei gesetzlich zu verankern, dass die Anordnung der Untersuchungshaft die Ausnahme und nicht die Regel ist.

1245 EGMR - Ananyev u.a./RUS, Urt. v. 10.01.2012, 42525/07 u. 60800/08, Rn. 193, 206.

1246 EGMR - Torreggiani u.a./ITA, Urt. v. 08.01.2013, 43517/09 u.a., Rn. 95.

1247 EGMR - Neshkov u.a./BUL, Urt. v. 27.01.2015, 36925/10 u.a., Rn. 277: umfassende Renovationen bestehender Haftanstalten oder vollständige Ersetzung; EGMR - Rezmiveș u.a./ROM, Urt. v. 25.04.2017, 61467/12 u.a., Rn. 119. 
durch ein entsprechendes Straf- resp. Sanktionenrecht die Anzahl Inhaftierter zu reduzieren ist, wenn der Konventionsstaat nicht in der Lage ist, allen Inhaftierten eine mit Art. 3 EMRK konforme Unterbringung zu gewährleisten. ${ }^{1248}$ Wie genau eine solche Reduktion erreicht werden soll, überlässt der Gerichtshof dann aber den Konventionsstaaten, ${ }^{1249}$ womit er die resolute Forderung des Ergebnisses wiederum in Einklang mit seiner Zurückhaltung bezüglich der innerstaatlichen Kriminalpolitik bringt.

Weit weniger zurückhaltend zeigt sich der Gerichtshof, wenn es um Fragen rund um Art. 13 EMRK i.V.m. Art. 3 EMRK geht. ${ }^{1250}$ Hier insistiert der Gerichtshof auf der Einführung wirkungsvoller, sowohl präventiver als auch kompensatorischer Rechtsmittel, welche den Inhaftierten die Möglichkeit effektiver Beschwerden über ihre Haftumstände ermöglichen müssen. ${ }^{1251}$ Dies ist vor dem Hintergrund zu sehen, dass ein niederschwelliger Zugang eines jeden Inhaftierten zu solchen Beschwerdemöglichkeiten wichtiger Bestandteil des Kampfes gegen inadäquate Haftbedingungen ist. Der präventive Rechtsbehelf stellt die rasche Kenntnis über (drohende) ungenügende Bedingungen sicher, wobei nur auf diese Weise auf nationaler Ebene überhaupt die Möglichkeit besteht, zeitnahe Abhilfemassnahmen zu ergreifen. Der kompensatorische Rechtsbehelf stellt flankierend sicher, dass ungenügende Haftbedingungen prompt geahndet werden. Die Einführung wirkungsvoller innerstaatlicher Rechtsbehelfe dient letztlich der Sicherstellung des Subsidiaritätsprinzips.

Der Ermessensspielraum des Konventionsstaates wird hier zwar dadurch eingeschränkt, dass die Abhilfemassnahme bereits durch den Gerichtshof definiert ist. Gleichwohl ist dem Konventionsstaat immerhin die genaue Ausgestaltung des Rechtsbehelfs überlassen und ebenso die Frage, ob die Konventionskonformität durch Anpassung bereits bestehender Rechtsbehelfe oder durch die Einführung neuer Rechtsbehelfe erfolgt. ${ }^{1252}$ Die Anordnung der Einführung präventiver resp. kompensatorischer Rechtsmittel ist auch vor dem Hintergrund zu sehen, dass die effektive Behebung

1248 EGMR - Torreggiani u.a./ITA, Urt. v. 08.01.2013, 43517/09 u.a., Rn.94; EGMR - Neshkov u.a./BUL, Urt. v. 27.01.2015, 36925/10 u.a., Rn. 276; EGMR - Varga u.a./HUN, Urt. v. 10.03.2015, 14097/12 u.a., Rn. $104 \mathrm{f}$.

1249 Vgl. etwa EGMR - Orchowski/POL, Urt. v. 22.10.2009, 17885/04, Rn. 153; EGMR - Neshkov u.a./BUL, Urt. v. 27.01.2015, 36925/10 u.a., Rn.276; EGMR - Torreggiani u.a./ITA, Urt. v. 08.01.2013, 43517/09 u.a., Rn. 95.

1250 EGMR - Neshkov u.a./BUL, Urt. v. 27.01.2015, 36925/10 u.a., Rn. 279.

1251 Vgl. etwa EGMR - Neshkov u.a./BUL, Urt. v. 27.01.2015, 36925/10 u.a., Rn. 279.

1252 Vgl. etwa EGMR - Varga u.a./HUN, Urt. v. 10.03.2015, 14097/12 u.a., Rn. 107. 
von Verletzungen von Art. 13 EMRK i.V.m. Art. 3 EMRK gar keine echten Alternativen liesse. Mit anderen Worten ist die Einschränkung des Ermessensspielraums von Konventionsstaat und Ministerkomitee in der Durchsetzungsphase durch diese Anordnung des Gerichtshofs sehr gering.

\section{dd Möglichkeit der Übergangslösungen}

Die langfristige Perspektive des Gerichtshofs führt zur Frage, was in der Zeit bis zur Wirkung dieser langfristigen Massnahmen zu geschehen hat. Der Gerichtshof sieht diesbezüglich zwar vor, dass langfristige Massnahmen mit schnell wirksamen Massnahmen zu kombinieren sind, scheint den Konventionsstaaten aber auch genügend Zeit zur Umsetzung der Massnahmen einräumen zu wollen. Letzteres, indem er die Möglichkeit von Transitionsperioden vorsieht, in welchen an und für sich inadäquaten Umständen mit Kompensationsmechanismen zu begegnen ist. ${ }^{1253}$ Der Gerichtshof scheint sich hier aber in gewisse Widersprüchlichkeiten zu verstricken: Die unter Art. 46 EMRK vorgeschlagenen Massnahmen zielen gerade darauf $\mathrm{ab}$, einen konventionskonformen Zustand herzustellen. Führt der Gerichtshof dann allerdings aus, in einer Transitionsperiode könnten etwa Ausnahmen hinsichtlich des mindestens zur Verfügung zu stellenden Platzes möglich sein, wenn die Dauer der Unterbringung unter inadäquaten Platzverhältnissen nur kurz sei, sie unter rechtlicher Aufsicht erfolge und Kompensationsmöglichkeiten zur Verfügung stünden, womit sichergestellt werde, dass es zu keiner Verletzung von Art. 3 EMRK komme, ${ }^{1254}$ suggeriert er einerseits, dass solche Kompensationsmechanismen nach einer Transitionsperiode den Anforderungen von Art. 3 EMRK nicht mehr genügen würden. Andererseits hält er aber auch fest, dass während der Transitionsperiode Art. 3 EMRK eingehalten würde. Somit scheint er entweder hinsichtlich Art. 3 EMRK von zwei unterschiedlichen Mindeststandards auszugehen, was wohl nicht mit dem absoluten Charakter und der daraus folgenden unbedingten Anwendbarkeitsforderung vereinbar wäre und was auch im Wissen, dass der Gerichtshof allgemein Kompensationsmöglichkeiten unter Art. 3 EMRK genügen lässt, kaum gemeint sein kann, oder er sieht unter Art. 46 EMRK Massnahmen vor, welche nach einer Transitionsperiode einen Zustand herstellen sollen, welcher über

1253 EGMR - Ananyev u.a./RUS, Urt. v. 10.01.2012, 42525/07 u. 60800/08, Rn. 207. 1254 EGMR - Ananyev u.a./RUS, Urt. v. 10.01.2012, 42525/07 u. 60800/08, Rn. 208. 
den konventionsrechtlich geforderten Mindeststandard hinausgehen. Letzteres ginge dann allerdings wohl über die ratio von Art. 46 EMRK hinaus.

\section{ee Anordnungen im Urteilstenor}

Der Gerichtshof hat in keinem der Piloturteile im Urteilstenor die Ergreifung einer spezifisch umschriebenen generellen Massnahme zur Behebung der für die Verletzung von Art. 3 EMRK ursächlichen Überbelegungssituation angeordnet. ${ }^{1255}$ Bisweilen ordnet er aber allgemein gehalten an, geeignete Massnahmen zu ergreifen. Anders verhält sich dies bei Massnahmen zu Art. 13 i.V.m. Art. 3 EMRK. Hier ordnet der Gerichtshof regelmässig auch im Urteilstenor die Einführung effektiver, sowohl präventiver als auch kompensatorischer Rechtsbehelfe an. ${ }^{1256}$ Der Gerichtshof setzt dem Konventionsstaat entweder direkt eine Frist zu deren Umsetzung resp. Einführung ${ }^{1257}$, oder er setzt dem Mitgliedstaat eine Frist zur Ausarbeitung eines Zeitplan für deren Umsetzung resp. für die Einführung des Rechtsbehelfs (zur Fristsetzung vgl. sogleich). ${ }^{1258}$

Grund für die Zurückhaltung des Gerichtshofs auch bei der gewählten Tenorierung dürfte wiederum die subsidiäre Rolle des Gerichtshofs gegenüber dem Konventionsstaat sein. Hinzu kommt sicherlich auch die vorgesehene Aufgabenverteilung zwischen Gerichtshof und Ministerkomitee. ${ }^{1259}$ Je konkreter nämlich die Anordnungen genereller Massnahmen seitens des Gerichtshofs sind, desto weniger Ermessensspielraum kommt dem Ministerkomitee zu, mit der Folge, dass es zu einer Aufgabenverschie-

$1255 \mathrm{Ob}$ es sich in Bezug auf Art. 3 EMRK um echte Piloturteile oder doch nur um "Quasi-Piloturteile“ handelt, ist deshalb zumindest fraglich. Da es letztlich nur eine terminologische Frage ist, kann dies hier aber offengelassen werden.

1256 Vgl. etwa EGMR - Ananyev u.a./RUS, Urt. v. 10.01.2012, 42525/07 u. 60800/08, Ziff. 7 Urteilstenor; EGMR - Neshkov u.a./BUL, Urt. v. 27.01.2015, 36925/10 u.a., Ziff. 7 lit. a Urteilstenor.

1257 Etwa: EGMR - Neshkov u.a./BUL, Urt. v. 27.01.2015, 36925/10 u.a., Ziff. 7 lit. a Urteilstenor.

1258 Etwa: EGMR - Ananyev u.a./RUS, Urt. v. 10.01.2012, 42525/07 u. 60800/08, Ziff. 7 Urteilstenor.

1259 Dies zeigt sich auch darin, dass der Gerichtshof immer wieder auf die Arbeit des Ministerkomitees hinweist: vgl. etwa EGMR - Orchowski/POL, Urt. v. 22.10.2009, 17885/04, Rn. 148; EGMR - Ananyev u.a./RUS, Urt. v. 10.01.2012, 42525/07 u. 60800/08, Rn. 194; EGMR - Neshkov u.a./BUL, Urt. v. 27.01.2015, 36925/10 u.a., Rn.274; EGMR - Rezmiveș u.a./ROM, Urt. v. 25.04.2017, 61467/12 u.a., Rn. 112. 
bung kommen könnte, was der Gerichtshof wohl gerade nicht intendiert. Gleichwohl erstaunt diese Zurückhaltung insofern, als Piloturteile dem Gerichtshof gerade ermöglichen, nicht nur den strukturellen bzw. systemischen Mangel aufzuzeigen, sondern eben auch selbst Abhilfemassnahmen für das festgestellte Problem anzuordnen.

Neben diesen rollenbedingten Gründen dürften aber auch rein pragmatische Gründe zu der Zurückhaltung des Gerichtshofs führen. Die Eruierung von effektiven Massnahmen im Kampf gegen Überbelegungssituationen ist nämlich - wie es der Gerichtshof auch selbst ausführte - ein komplexes Unterfangen. Dies nicht nur aufgrund der multiplen Ursachen von Überbelegungssituationen, sondern auch aufgrund stark politisierender Fragen, wenn es um kostenintensive Massnahmen und um straf- und strafprozessrechtliche Fragen geht. Konkrete Anweisungen im Urteilstenor würden die Verhandlungsmöglichkeiten im politisch geprägten Umfeld denn auch stark einschränken. ${ }^{1260}$ Ungleich einfacher gestaltet sich die Tenorierung hinsichtlich Abhilfemassnahmen rund um Art. 13 EMRK i.V.m. Art. 3 EMRK, da hier die Einführung eines effektiven Rechtsbehelfs für Inhaftierte ohnehin die einzige valable Abhilfemassnahme ist.

Die manifeste Zurückhaltung des Gerichtshofs hinsichtlich der Anordnung genereller Massnahmen bei Überbelegungssituationen hat für die effektive Durchsetzung des Mindeststandards, des obersten Ziels überhaupt, aber nicht zwingend nachteilige Folgen. Auch ohne die Anordnung spezifischer genereller Massnahmen hat das Ministerkomitee nämlich bemüht zu sein, eine umfassende Umsetzung des Urteils im jeweiligen Konventionsstaat zu erzielen. ${ }^{1261}$ Eine abschliessende Würdigung der Zurückhaltung des Gerichtshofs in der Tenorierung kann folglich erst nach der Analyse der Durchsetzungsmassnahmen des Ministerkomitees, und der Reaktion der Konventionsstaaten darauf, vorgenommen werden.

ff Umsetzungsfrist

Das Vorsehen einer Umsetzungsfrist für die im Urteilstenor angeordneten Massnahmen i.S.v. Regel 61 Abs. 4 VerfO soll der Umsetzungsverpflich-

1260 Eschment S. 217: Benennt der Gerichtshof nur in den Urteilsgründen die generellen Massnahmen, entspreche dies einer „weicheren Steuerung“.

1261 A.A. Eschment S. 218: Mit Blick auf die Durchsetzungskraft sei eine verstärkte Aufnahme genereller Abhilfemassnahmen im Urteilstenor von Piloturteilen zu begrüssen. 
tung der Konventionsstaaten Nachdruck verschaffen und garantieren, dass der betroffene Staat unverzüglich mit Implementierungsarbeiten beginnt. ${ }^{1262}$ Bei der Bemessung der Frist hat der Gerichtshof zu berücksichtigen, dass diese einerseits lange genug ist, damit der Konventionsstaat auch eine realistische Möglichkeit zur Umsetzung innert Frist erhält, ${ }^{1263}$ sie andererseits nicht zu lange ist, damit sie dem Ziel der Fristansetzung, nämlich der Dringlichkeit der Implementierung Nachdruck zu verschaffen, nicht diametral entgegenläuft.

Daraus ergibt sich folgendes Leitprinzip: Je schwerwiegender die Konventionsverletzung, desto dringlicher die Implementierung d.h., desto kürzer ist die Frist anzusetzen. Grenze muss stets die faktische Umsetzungsmöglichkeit bleiben. Besondere Dringlichkeit der Umsetzung genereller Massnahmen besteht insbesondere bei jenen Piloturteilen, in denen die Parallelfälle bis zur Umsetzung der Massnahmen zurückgestellt werden. ${ }^{264}$

Führten die festgestellten strukturellen bzw. systemischen Probleme zu Verletzungen von Art. 3 EMRK und zu Verletzungen von Art. 13 EMRK i.V.m. Art. 3 EMRK, machte der Gerichtshof regelmässig von der Möglichkeit der Fristsetzung Gebrauch. Die Dauer der angesetzten Fristen variierte von sechs Monaten ${ }^{1265}$ bis zu 24 Monaten ${ }^{1266}$, wobei sich diese nicht ohne Weiteres miteinander vergleichen lassen, da innert der angesetzten Fristen je unterschiedliche Massnahmen gefordert wurden (etwa: Erstellung

1262 Eschment S. 220: Eine Fristansetzung könne von den Konventionsstaaten aber auch als allzu interventionalistisch angesehen werden, was für die Kooperationsbereitschaft negative Folgen haben könne. Vgl. auch Leach/Hardman/STEPHENSON HRLR 2010, 346 (358): Fristansetzung auch als wichtiger Faktor für die Beurteilung der Angemessenheit der Reaktion der Staaten.

1263 Vgl. auch Eschment S. 221: Bei zu kurzen Fristen drohe ein Wirkungsverlust der Piloturteile, da die Glaubwürdigkeit der autoritären Fristfestsetzung erodiere, wenn diese öfter nicht eingehalten werde. Im Allgemeinen kritisch gegenüber zu kurzen Umsetzungsfristen vgl. auch: Richter Sajó, López Guerra u. Wojtyczek, joint partly diss. op., EGMR (GK) - Muršić/CRO, Urt. v. 20.10.2016, 7334/13, Rn. 7: Bei der Fristsetzung müsse insbesondere auch die Kostenintensität berücksichtigt werden, insbesondere in jenen Fällen, in denen von bisherigen Urteilen abgewichen werde (etwa bei einer Erhöhung des Standards).

1264 Vgl. auch Sicilianos in: Seibert-Fohr/Villiger, S. 285 (314f.).

1265 EGMR - Ananyev u.a./RUS, Urt. v. 10.01.2012, 42525/07 u. 60800/08, Ziff. 7 Urteilstenor; EGMR - Varga u.a./HUN, Urt. v. 10.03.2015, 14097/12 u.a., Ziff. 9 Urteilstenor.

1266 EGMR - W.D./BEL, Urt. v. 06.09.2016, 73548/13, Ziff. 6 Urteilstenor. 
eines Zeitplans zur Einführung des entsprechenden Rechtsbehelfs ${ }^{1267}$ vs. Einführung des Rechtsbehelfs selbst ${ }^{1268}$ ). So kann die Sechsmonatsfrist zur Ausarbeitung eines Zeitplans in relativer Hinsicht grosszügiger bemessen sein als die 24-monatige Frist zur definitiven Einführung des Rechtsbehelfs resp. zur Umsetzung der geeigneten Massnahmen. Da die Einführung des Rechtsbehelfs je nach Ausgangssituation ein legislatives Tätigwerden erfordert, müssen innert dieser 24 Monate bereits sämtliche nationalen (politischen) Hürden überwunden werden, während die Ausarbeitung eines Zeitplans den Konventionsstaaten weitaus mehr Spielraum lässt.

In der Variante der Fristsetzung zur Ausarbeitung eines Zeitplans manifestiert sich auch wieder die Zurückhaltung des Gerichtshofs. Indem dem Konventionsstaat (und dem Ministerkomitee bei der Überwachung und bei der Beurteilung des Zeitplans) hier ein grösserer Beurteilungs- und Ermessensspielraum belassen wird, können, etwa beim Rechtssetzungsprozess, nationale Besonderheiten besser berücksichtigt werden. ${ }^{1269}$

Ordnet der Gerichtshof die Ausarbeitung eines verbindlichen Zeitplans innert sechs Monaten seit Rechtskraft des Urteils an, ist dies auch mit Blick auf die allgemeinen Vorschriften des Ministerkomitees zu würdigen (im Einzelnen Teil 4 Kap. 4 I. 2.2. b). Im Implementierungsverfahren des Ministerkomitees ist nämlich vorgesehen, dass jeder Konventionsstaat dem Ministerkomitee spätestens nach sechs Monaten seit Rechtskraft einen Aktionsplan für die zu ergreifenden Massnahmen einzureichen hat. Der Gerichtshof formalisiert mit seiner Anordnung das Implementierungsverfahren, verlangt er vom Konventionsstaat doch einen verbindlichen Zeitplan. In inhaltlicher Sicht konkretisiert der Gerichtshof mit seiner Anordnung, was genau der erste Aktionsplan zu enthalten hat, der innert eben dieser sechs Monate einzureichen ist. Die Evaluation dieses Zeitplans, sowohl was die vom Konventionsstaat vorgesehenen Massnahmen als auch was die vom Konventionsstaat bezeichneten einzelnen Zeitspannen betrifft, bleibt aber beim Ministerkomitee. Der Mehrwert dessen, dass nun der Gerichtshof im Urteilstenor dem Konventionsstaat eine Sechsmonatsfrist zur Ausarbeitung eines Zeitplans setzt, liegt im Wesentlichen darin, der

1267 EGMR - Ananyev u.a./RUS, Urt. v. 10.01.2012, 42525/07 u. 60800/08, Ziff. 7 Urteilstenor; EGMR - Varga u.a./HUN, Urt. v. 10.03.2015, 14097/12 u.a., Ziff. 9 Urteilstenor.

1268 EGMR - W.D./BEL, Urt. v. 06.09.2016, 73548/13, Ziff. 6 Urteilstenor.

1269 Nationale Besonderheiten etwa beim Rechtssetzungsprozess können besser berücksichtigt werden. Vgl. auch Leach/Hardman/Stephenson HRLR 2010, 346 (358): In der Praxis bestünden Zweifel, ob Fristen zur Einführung von Rechtsbehelfen überhaupt realistisch sind. 
Verpflichtung des Konventionsstaates, zügig mit der Implementierung zu beginnen, noch einmal Nachdruck zu verschaffen, ${ }^{1270}$ ohne dass es aber zu einer wirklichen Verschiebung bei der Rollenverteilung zwischen Ministerkomitee und Gerichtshof kommen würde. Ein Mittelmass an Zurückhaltung und Gewährung eines Ermessensspielraums bei gleichzeitiger Druckausübung zur effizienten Durchsetzung des Mindeststandards ist auf diese Weise gewährleistet.

\section{gg Parallelfälle}

Der Gerichtshof hat gemäss Regel 61 Abs. 6 VerfO die Möglichkeit, die Parallelfälle zurückzustellen („Kann-Vorschrift“). In den Piloturteilen zu unmenschlichen und erniedrigenden Haftbedingungen i.S.d. Art. 3 EMRK macht der Gerichtshof allerdings nur zurückhaltend von dieser Möglichkeit Gebrauch.

Während der Gerichtshof in den Urteilen Torreggiani u.a./ITA, W.D./BEL und Rezmiveş u.a./ROM mindestens die noch nicht an die Regierung weitergeleiteten Fälle zurückstellte, ${ }^{1271}$ entschied er sich in allen anderen Piloturteilen aufgrund des fundamentalen Charakters von Art. 3 EMRK und der damit einhergehenden Dringlichkeit der Verfahren gegen eine Zurückstellung der Parallelfälle. ${ }^{1272}$ Laufenden Verfahren vor dem Gerichtshof komme die Funktion zu, den Konventionsstaat in regelmässigen Abständen an seine menschenrechtlichen Verpflichtungen zu erinnern. ${ }^{1273}$ Die Anzahl weiterer, bereits hängiger Parallelfälle scheint mit

1270 Es bliebe später auch eine separate Feststellung einer Verletzung von Art. 34 EMRK möglich, wenn der Konventionsstaat der Anordnung des Gerichtshofs nicht nachkommt.

1271 EGMR - Torreggiani u.a./ITA, Urt. v. 08.01.2013, 43517/09 u.a.: Der Gerichtshof wollte abwarten und schauen, ob die italienischen Behörden die notwendigen Massnahmen nach dem nationalen Plan ergreifen. Ausschlaggebend dürfte hier damit gewesen sein, dass Italien schon im nationalen Plan weiterführende Massnahmen geplant hatte. Vgl. auch EGMR - Rezmiveș u.a./ ROM, Urt. v. 25.04.2017, 61467/12 u.a., Rn. 127; EGMR - W.D./BEL, Urt. v. 06.09.2016, 73548/13, Rn.173 f.: Einzig in W.D./BEL stellte der Gerichtshof sämtliche ähnlichen Fälle zurück.

1272 Etwa: EGMR - Ananyev u.a./RUS, Urt. v. 10.01.2012, 42525/07 u. 60800/08, Rn. 236; EGMR - Varga u.a./HUN, Urt. v. 10.03.2015, 14097/12 u.a., Rn. 116.

1273 Etwa: EGMR - Ananyev u.a./RUS, Urt. v. 10.01.2012, 42525/07 u. 60800/08, Rn. 236; EGMR - Varga u.a./HUN, Urt. v. 10.03.2015, 14097/12 u.a., Rn. 116. 
Blick auf die Zurückstellungspraxis des Gerichtshofs aber nicht ausschlaggebendes Kriterium zu sein.

Im Sinne einer umfassenden, effizienten und vor allem prompten Durchsetzung des Mindeststandards ist die restriktive Zurückstellungspraxis des Gerichtshofs in diesem besonders menschenrechtssensiblen Themenbereich zu begrüssen. Die Weiterführung des Verfahrens vor dem Gerichtshof bietet den Beschwerdeführern in den Parallelfällen bessere Möglichkeiten zur Durchsetzung ihres eigenen individuellen Anspruchs. Dies insbesondere aufgrund der Langfristigkeit der zu ergreifenden generellen Massnahmen, welche wenig geeignet scheinen, schnelle Abhilfe für Einzelpersonen zu schaffen. ${ }^{1274}$ Macht der Gerichtshof von der Möglichkeit der Zurückstellung dennoch Gebrauch, so ist er zumindest anzuhalten, regelmässig zu prüfen, ob Notwendigkeit besteht, von der Möglichkeit Gebrauch zu machen, zunächst zurückgestellte Verfahren wieder vorzeitig zu behandeln (Regel 61 Abs. 6 VerfO und Regel 61 Abs. 8 VerfO).

\subsection{Zwischenfazit}

Das Piloturteilsverfahren hat sich im Bereich der unmenschlichen und erniedrigenden Haftbedingungen etabliert. Strukturelle bzw. systemische Probleme wurden insbesondere hinsichtlich der Überbelegungssituationen in den einzelnen Konventionsstaaten erkannt. Aufgrund statistischer Daten und zahlreicher Berichte verschiedenster Gremien, welche eindrücklich die Überbelegungssituation in weiteren Konventionsstaaten aufzeigen, sind denn auch in Zukunft weitere diesbezügliche Piloturteilsverfahren zu erwarten. Dass im Kontext materieller Haftbedingungen nicht nur hinsichtlich Überbelegungssituationen strukturelle bzw. systemische Probleme festzustellen sind, hat das Urteil W.D./BEL verdeutlicht. Insbesondere der Umstand, dass das CPT bei seinen Besuchen immer wieder weit verbreitete Unzulänglichkeiten der Gesundheitsversorgung im Strafvollzug festgestellt hat und auch der Gerichtshof bereits Grundsatzurteile gefällt hat, in welchen er hinsichtlich der therapeutischen Versorgung im

1274 EGMR - W.D./BEL, Urt. v. 06.09.2016, 73548/13, Rn. 173 f.: Der Gerichtshof wies in den Urteilsgründen aber immerhin auf die Möglichkeit von Ad-hocMassnahmen im Rahmen einer gütlichen Einigung oder einer einseitigen Erklärung hin. 
Strafvollzug strukturelle Probleme erkannte, macht es wahrscheinlich, dass auch in diesem Themengebiet Piloturteilsverfahren folgen werden. ${ }^{1275}$

Bei den Abhilfemassnahmen zur Bekämpfung der Überbelegung ist der umfassende und langfristige Ansatz des Gerichtshofs in den Urteilsgründen zu begrüssen. Gleichwohl hat sich gezeigt, dass die Formulierungen des Gerichtshofs hinsichtlich der vorgeschlagenen Abhilfemassnahmen sehr zurückhaltend sind, was einerseits seiner subsidiären Rolle gegenüber den Konventionsstaaten und andererseits der Aufgabenverteilung zwischen Gerichtshof und Ministerkomitee geschuldet sein dürfte. Resoluter ist der Gerichtshof, wenn er die Notwendigkeit der Reduktion der Anzahl Inhaftierter festhält oder aufgrund von Art. 13 EMRK i.V.m. Art. 3 EMRK die Einführung eines Rechtsbehelfs fordert.

Im Urteilstenor macht der Gerichtshof in Bezug auf Art. 3 EMRK und inadäquate Haftbedingungen keinen Gebrauch von der Möglichkeit der Anordnung bestimmter genereller Massnahmen. Anders sieht dies aus, wenn es um Verletzungen von Art. 13 EMRK i.V.m. Art. 3 EMRK geht. Hier ordnet der Gerichtshof im Sinne einer Abhilfemassnahme regelmässig verbindlich die Einführung sowohl präventiver als auch kompensatorischer

1275 Vgl. etwa CPT - Report to the Greek Government [CPT/Inf (2020) 15], S. 5: „[...] widespread deficiencies regarding the state of health care services in prisons persist"; CPT - 26th General Report [CPT/Inf (2017) 5], Rn. 46: Hinsichtlich der Situation in Nordmazedonien: „The provision of health care was deficient in all the prisons visited. "Auch bei seinem nächsten Besuch in Nordmazedonien stellte das CPT ernüchternde Bedingungen fest: „The material conditions of the medical facilities remained inadequate and in a state of neglect and dilapidation": CPT - Report to the Government of former Yugoslav Republic of Macedonia [CPT/Inf (2017) 30], Rn. 34; vgl. auch das „QuasiPiloturteil“ gegen Georgien EGMR - Poghossian/GEO, Urt. v. 24.02.2009, 9870/07, Rn. 63 ff.: In Georgien hatte der Gerichtshof strukturelle Missstände bei der medizinischen Betreuung kranker Inhaftierter festgestellt (strukturelles Ausmass würden insb. Hepatitis-C-Erkrankungen annehmen). In der Folge hielt der Gerichtshof in den Urteilsgründen, nicht aber im Urteilstenor fest, dass generelle Massnahmen ergriffen werden müssten. Es müssten unverzüglich die notwendigen legislativen und administrativen Massnahmen ergriffen werden, um die Übertragung der viralen Hepatitis $\mathrm{C}$ zu verhindern. Es müsse etwa ein Früherkennungssystem eingeführt und sichergestellt werden, dass diese Krankheit rasch wirksam behandelt werde. Anlässlich seines Besuchs in Georgien im Jahr 2018 stellte das CPT zwar fest, dass sich die Situation in Bezug auf Hepatitis B und Hepatitis-C-Erkrankungen verbessert habe und den Inhaftierten ein systematisches Screening angeboten werde. Hinsichtlich der Versorgung psychisch kranker Inhaftierter stellte das CPT allerdings anhaltende, schwerwiegende Mängel fest: CPT - Report to the Georgian Government [CPT/Inf (2019) 16], Rn. 83 f. 
Rechtsbehelfe innert einer bestimmten Frist an, oder er fordert innert einer solchen zumindest die Ausarbeitung eines Zeitplans zur Einführung des Rechtsbehelfs. Ein Ermessensspielraum bezüglich der konkreten Ausgestaltung des Rechtsbehelfs bleibt dem Konventionsstaat unter Aufsicht des Ministerkomitees aber in beiden Konstellationen erhalten.

Eine Ausdehnung der Anordnung genereller Massnahmen in andere Bereiche inadäquater Haftbedingungen kann erst erwartet werden, wenn der Gerichtshof auch bei diesen einen strukturellen bzw. systemischen Charakter feststellt und aufgrund dessen überhaupt erst das Piloturteilsverfahren anwendet.

\section{Gerechte finanzielle Entschädigung i.S.v. Art. 41 EMRK}

Der Gerichtshof kann dem Beschwerdeführer auf seinen Antrag hin, falls aufgrund der Billigkeit notwendig ${ }^{1276}$, eine gerechte finanzielle Entschädigung zusprechen (Art. 41 EMRK). Entschädigt werden können grundsätzlich sowohl materielle als auch immaterielle Schäden sowie Kosten und Auslagen. ${ }^{1277}$ Vorausgesetzt ist, dass innerstaatlich nur eine unvollkommene Wiedergutmachung für die erlittene Konventionsverletzung möglich ist. ${ }^{1278}$ Letzteres ist Ausdruck des Subsidiaritätsgedanken: Naturalrestitution (restitutio in integrum) und Schadensausgleich auf nationaler Ebene haben vorzugehen. ${ }^{1279}$ Für die Bestimmung der Höhe der Entschädigungszahlung bei immateriellen Schäden sind im Allgemeinen die Schwere der Konventionsverletzung, das Vorliegen einer concurring bzw. einer dissenting opinion, der beklagte Staat sowie die Frage, ob es sich beim Beschwerdeführer um eine natürliche oder juristische Person handelt, massgebliche Kriterien. ${ }^{1280}$

1276 Peters/Altwicker $₫ 37$ Rn. 4.

1277 Meyer-Ladewig/Brunozzi Art. 41 Rn. 1.

1278 SK-StPO/MeYer Verfahrensrecht (EMRK) Rn. 299; Karpenstein/Mayer/WeNzEL Art. 41 Rn. 5 f.: Von einer vollständigen Wiedergutmachung sei im Allgemeinen nur ausnahmsweise auszugehen, nämlich dann, wenn der Zustand vor der Konventionsverletzung wiederhergestellt werden kann.

1279 SK-StPO/MeYer Verfahrensrecht (EMRK) Rn. 302.

1280 Im Einzelnen ausf. dazu Altwicker-Hámori/Altwicker/Peters ZaöRV 2016, $1 \mathrm{ff} .:$ Altwicker-Hámori et al. führten eine empirische Analyse der EGMRRechtsprechung durch und identifizierten drei Elemente des „Billigkeitsprinzips" (Schwere der Verletzung, Faktoren im Zusammenhang mit den Antragsstellenden und allgemeine kontextbezogene Faktoren). Vgl. ferner КветsсHMER NJW 2009, 2406 (2411), der auf den reinen Symbolcharakter der Entschä- 
Zwischen den Massnahmen von Art. 46 EMRK und Art. 41 EMRK bestehen damit Wechselwirkungen. ${ }^{1281}$ Da die Wiedergutmachung der Entschädigungszahlung i.S.v. Art. 41 EMRK vorgeht, kann eine Zahlung nach Art. 41 EMRK den Konventionsstaat auch keinesfalls von seinen Verpflichtungen nach Art. 46 Abs. 1 und Abs. 2 EMRK i.V.m. Art. 1 EMRK befreien, und die Verpflichtung zur Behebung des konventionswidrigen Zustandes bleibt folglich bestehen - insbesondere auch zur Behebung eines strukturellen Problems. ${ }^{1282}$

Am Beispiel der im Bereich inadäquater Haftbedingungen ergangenen Piloturteile wird die Bedeutung der Entschädigungszahlung deutlich. In allen Piloturteilen wurden, neben der Anordnung der Massnahmen i.S.v. Art. 46 EMRK, auch finanzielle Entschädigungen zugesprochen. ${ }^{1283}$ Grund dafür dürfte sein, dass eine vollständige Naturalrestitution für die bereits ergangenen Konventionsverletzungen (Unterbringung unter inadäquaten Haftbedingungen) nicht erzielt werden kann, erfolgte doch gerade die Feststellung einer Konventionsverletzung trotz Ausschöpfung aller nationalen Rechtsbehelfe. ${ }^{1284}$ Die Art. 46 EMRK-Massnahmen können sich nur noch auf die zukünftige Unterbringung des konkreten Beschwerdeführers beziehen bzw. im Sinne der Orientierungswirkung auch auf die Unterbringung weiterer Inhaftierter in derselben Situation. Sie richten sich dagegen nicht gegen die vergangene inadäquate Unterbringung. Die Natur der Verletzung ermöglicht damit keine vollständige Naturalrestitution. ${ }^{1285}$

Auch wenn die Auferlegung einer Entschädigungspflicht nicht direkt auf die Umsetzung des Mindeststandards zielt, kann diese zusätzliche

digung hinweist und den Gedanken eines finanziellen Gewinns zurückweist und als Ausdruck falscher Empörung bezeichnet.

1281 SK-StPO/MeYer Verfahrensrecht (EMRK) Rn. 304.

1282 Vgl. etwa EGMR (GK) - Assanidze/GEO, Urt. v. 08.04.2004, 71503/01, Rn. 198; SK-StPO/Meyer Verfahrensrecht (EMRK) Rn. 304, Rn. 289: Art. 41 EMRK enthält eine sekundäre Kompensationsform.

1283 Vgl. zuletzt EGMR - Sukachov/UKR, Urt. v. 30.01.2020, 14057/17, Rn. 165, Ziff. 5 Urteilstenor; vgl. auch EGMR - Orchowski/POL, Urt. v. 22.10.2009, 17885/04, Ziff. 4 Urteilstenor; EGMR - Norbert Sikorski/POL, Urt. v. 22.10.2009, 17599/05, Ziff. 4 Urteilstenor; EGMR - Ananyev u.a./RUS, Urt. v. 10.01.2012, 42525/07 u. 60800/08, Ziff. 5 Urteilstenor; EGMR - Torreggiani u.a./ITA, Urt. v. 08.01.2013, 43517/09 u.a., Ziff. 6 Urteilstenor; EGMR Neshkov u.a./BUL, Urt. v. 27.01.2015, 36925/10 u.a., Ziff. 8 Urteilstenor; EGMR - Varga u.a./HUN, Urt. v. 10.03.2015, 14097/12 u.a., Ziff. 7 Urteilstenor; EGMR - W.D./BEL, Urt. v. 06.09.2016, 73548/13, Ziff. 8 Urteilstenor; EGMR - Sukachov/UKR, Urt. v. 30.01.2020, 14057/17, Ziff. 5.

1284 Vgl. SK-StPO/MeYer Verfahrensrecht (EMRK) Rn. 303.

1285 Vgl. hierzu auch LeEB S. 14. 
Leistungsverpflichtung doch dazu führen, der Beendigungs- bzw. der Befolgungspflicht weiteren Nachdruck zu verschaffen. Die Nichteinhaltung des Mindeststandards wirkt sich dadurch nämlich insbesondere bei einer grossen Anzahl „repetitve cases“ auch in monetärer Hinsicht negativ auf den Konventionsstaat aus. Gleichwohl ist festzuhalten, dass der Gerichtshof in Anbetracht des gravierenden Vorwurfs der Verletzung von Art. 3 EMRK nur relativ moderate Entschädigungszahlungen ausrichtet. ${ }^{1286}$

\section{Kapitel 4: Die Durchsetzung des Mindeststandards im Anschluss an das Urteil}

Die Phase im Anschluss an das Urteil ist von der fehlenden Gestaltungsmacht der EGMR-Urteile geprägt. Der Konventionsstaat hat das Feststellungsurteil des Gerichtshofs umzusetzen, d.h., sowohl die vom Gerichtshof angeordneten individuellen als auch die generellen Massnahmen zu ergreifen. Somit ist der effektive Menschenrechtsschutz mit dem Erlass eines Feststellungsurteils allein noch nicht garantiert. Umso wichtiger ist deshalb die Überwachung der Implementierungsphase im Nachgang an das Urteil. ${ }^{1287}$

Wichtigster Akteur in der Implementierungsphase ist das Ministerkomitee, ${ }^{1288}$ weshalb zunächst seine Arbeitsweise und seine Rolle betrachtet

1286 Vgl. bspw.: EGMR - Orchowski/POL, Urt. v. 22.10.2009, 17885/04, Ziff. 4 Urteilstenor: EUR 3'000; EGMR - Norbert Sikorski/POL, Urt. v. 22.10.2009, 17599/05, Ziff. 4 Urteilstenor: EUR 3'500; EGMR - Ananyev u.a./RUS, Urt. v. 10.01.2012, 42525/07 u. 60800/08, Ziff. 5 Urteilstenor: EUR 2'000 für den Beschwerdeführer Ananyev und EUR 13'000 für den Beschwerdeführer $B a$ shirov; EGMR - Torreggiani u.a./ITA, Urt. v. 08.01.2013, 43517/09 u.a., Ziff. 6 Urteilstenor: EUR 10'600 für den Beschwerdeführer Torreggiani und für die weiteren Beschwerdeführer Beträge zwischen EUR 11'000 und EUR 23'500; EGMR - Neshkov u.a./BUL, Urt. v. 27.01.2015, 36925/10 u.a., Ziff. 8 Urteilstenor: EUR 6'750 für den Beschwerdeführer Neshkov und EUR 10'000 und EUR 11'625 für die weiteren beiden Beschwerdeführer; EGMR - Varga u.a./ HUN, Urt. v. 10.03.2015, 14097/12 u.a., Ziff. 7 Urteilstenor: EUR 5'000 für den Beschwerdeführer Varga und zwischen EUR 150 und EUR 26 '000 für die übrigen Beschwerdeführer; EGMR - W.D./BEL, Urt. v. 06.09.2016, 73548/13, Ziff. 8 Urteilstenor: EUR 16'000; EGMR - Sukachov/UKR, Urt. v. 30.01.2020, 14057/17, Ziff. 5: EUR 9'500. Die Beträge sind tlw. zzgl. Kosten und Auslagen sowie weiterer Beträge zu verstehen.

1287 Vgl. auch Tichy ZaöRV 2016, 415 (418).

1288 Meyer-Ladewig/Brunozzi Art. 46 Rn. 43. Im Ministerkomitee sind die Regierungsvertreter der Mitgliedstaaten vertreten (Art. 14 Satzung des Europarats), womit es sich beim Ministerkomitee um ein politisches Organ handelt. 
werden. In einem nächsten Schritt werden der konkrete Umsetzungsstatus und das Verfahren zur Umsetzung der Piloturteile einerseits und der übrigen Urteile andererseits im Bereich inadäquater Haftbedingungen analysiert, gefolgt von einer Beurteilung der Effektivität der Durchsetzung des Mindeststandards.

I. Arbeitsweise und Rolle des Ministerkomitees bei der Durchsetzung von EGMR-Urteilen

1. Ausgangspunkt, Überwachungsgegenstand und Grundsätze

Gemäss Art. 46 Abs. 2 EMRK ist das endgültige Urteil dem Ministerkomitee zuzuleiten, welches dessen Umsetzung zu überwachen hat. Die Aufgabe des Ministerkomitees ist folglich auf das Monitoring beschränkt; ${ }^{1289}$ die Umsetzung hat durch den Konventionsstaat selbst zu erfolgen. ${ }^{1290}$ Weder der Gerichtshof noch das Ministerkomitee verfügen über direkte Gestaltungsmacht, so dass das Urteil im Konventionsstaat niemals mit Zwang umgesetzt werden kann. Einzige Möglichkeit ist die Erhöhung resp. die Aufrechterhaltung des politischen Drucks. ${ }^{1291}$ Das Ministerkomitee nimmt dabei aber keinesfalls eine passive Rolle ein; vielmehr begleitet es den Implementierungsprozess aktiv. ${ }^{1292}$

Überwachungsgegenstand des Ministerkomitees sind nicht nur die durch den Gerichtshof angeordneten individuellen, sondern auch die generellen Massnahmen. ${ }^{1293}$ Im Kontext inadäquater Haftbedingungen

1289 Die reine Überwachungsfunktion korrespondiert mit den Urteilswirkungen. Das Ministerkomitee handelt folglich zur Durchsetzung der Bindungswirkung des Urteils und zur Anmahnung der Befolgungspflicht der Konventionsstaaten.

1290 Vgl. auch Pabel in: Zimmermann, S. 81 (83): Das Ministerkomitee ist dementsprechend kein Vollzugsorgan. JAEger in: Zimmermann, S. 125: Vollstreckung über das Ministerkomitee als diplomatisches, d.h. völkerrechtliches Instrument.

1291 Vgl. auch Pabel in: Zimmermann, S. 81 (83, 86).

1292 Zum Dialog zwischen Ministerkomitee und Mitgliedstaat vgl. GLAS EYHR 2018, 287 (293).

1293 Annual Report 2011 Concerning Supervision of the Execution of Judgments and Decisions, S. 16; Pabel in: Zimmermann, S. 81 (86); Pabel in: Zimmermann, S. 81 (86f.): Dies widerspiegle sich auch in Regel 6 Abs. 2 der Verfahrensregeln des Ministerkomitees. Die Überwachungsbefugnis hänge auch von der Reichweite der Bindungswirkung des Urteils ab. Auch die Orientierungs- 
könnte eine Überwachung beispielhaft wie folgt aussehen: Das Ministerkomitee überwacht bei der Umsetzung einer individuellen Massnahme etwa, ob der Inhaftierte in eine andere Zelle, welche den Mindeststandard erfüllt, transferiert wurde, oder überprüft, ob dem Inhaftierten fortan eine adäquate Gesundheitsversorgung zuteil wird. Für die Umsetzung einer generellen Massnahme wäre das Ministerkomitee etwa dafür zuständig, zu überwachen, ob ein effektiver Rechtsbehelf i.S.v. Art. 13 EMRK i.V.m. Art. 3 EMRK eingeführt wurde, mit welchem fortan inadäquate Haftbedingungen gerügt werden können.

Der Implementierungsprozess ist vom Grundsatz geprägt, dass der Konventionsstaat i.d.R. bei der Wahl der Mittel zur Durchsetzung des EGMRUrteils frei ist, ${ }^{1294}$ so dass das Ministerkomitee i.d.R. keine Möglichkeit hat, vom Konventionsstaat eine spezifische Massnahme zu fordern. Die Arbeit des Ministerkomitees besteht damit in erster Linie darin, die vom Mitgliedstaat ergriffenen Massnahmen zu evaluieren und auf ihre Eignung, die Konventionsverletzung zu beseitigen, zu überprüfen. ${ }^{1295}$ Gleichwohl stehen das Ministerkomitee resp. das Department for Execution of Judgments and Decisions (nachfolgend: Execution Department) und der Konventionsstaat betreffend die zu ergreifenden Massnahmen in einem ständigen Dialog, in welchem auch seitens der Strassburger Organe Vorschläge betreffend die zu ergreifenden Massnahmen gemacht werden können (im Einzelnen vgl. sogleich; twin-track supervision).

\section{Entwicklung und Funktionsweise des Monitoringprozesses}

In der Ministerkonferenz in Rom im November $2000^{1296}$ wurde die Verbesserung des Umsetzungsprozesses zu einem der drei Hauptpfeiler bei

wirkung werde in der Überwachung durch das Ministerkomitee berücksichtigt. Vgl. auch: Annual Report 2011 Concerning Supervision of the Execution of Judgments and Decisions, S. 27: Das Ministerkomitee wird aufgefordert, sich strickt am Subsidiaritätsprinzip zu orientieren. Die Überwachung darf ausschliesslich auf der Grundlage der rechtlichen Analyse des Urteils des Gerichtshofs erfolgen.

1294 Vgl. etwa Karpenstein/Mayer/Breuer Art. 46 Rn. 5; Meyer-Ladewig/Brunozzi Art. 46 EMRK Rn. 5; Pabel in: Zimmermann, S. 81 (88).

1295 PABEL in: Zimmermann, S. 81 (88): Dies könne den Überwachungsprozess gerade mit Blick auf generelle Massnahmen langwierig machen.

1296 Anlässlich der Ministerkonferenz wurde der 50. Jahrestag des Bestehens der Konvention gefeiert. 
der Sicherstellung der langfristigen Wirksamkeit des Konventionsrechtssystems erklärt. ${ }^{1297}$ Die Erkenntnis, dass eine prompte und effektive Umsetzung der Urteile des Gerichtshofs essentiell für die Glaubwürdigkeit und Effektivität des ganzen Konventionssystems ist, ${ }^{1298}$ führt zu stetigen Bemühungen, den Implementierungsprozess weiterzuentwickeln und transparenter zu gestalten. ${ }^{1299}$ Es folgten diverse Massnahmen zur Steigerung der Effizienz des Implementierungsprozesses: Es wurden etwa Empfehlungen (recommendations) erlassen, ${ }^{1300}$ das 14 . Zusatzprotokoll in Kraft gesetzt und die Arbeitsmethoden des Ministerkomitees überarbeitet (new working methods).

\section{1 Änderungen infolge des 14. Zusatzprotokolls}

Das 14. Zusatzprotokoll, welches am 1. Juni 2010 in Kraft trat, sollte das Funktionieren des Konventionssystems langfristig gewährleisten. $\mathrm{Zu}$ diesem Zweck wurde eine Anzahl Änderungen eingeführt, welche sowohl den Gerichtshof selbst als auch das Ministerkomitee betreffen. Hinsichtlich des Monitoringprozesses wurden zentrale Änderungen vor allem in den Art. 46 EMRK und Art. 39 EMRK eingeführt:

Der neue Art. 46 Abs. 3 EMRK gibt dem Ministerkomitee die Möglichkeit, den Gerichtshof zum Entscheid von Auslegungsfragen eines Urteils anzurufen, falls die Überwachung der Umsetzung eines Urteils durch die Auslegungsfrage behindert wird. ${ }^{1301}$ Der Beschluss des Ministerkomitees, den Gerichtshof anzurufen, ist dabei allerdings an die relativ hohe Hür-

1297 Annual Report 2011 Concerning Supervision of the Execution of Judgments and Decisions, S. 23: Neben der Verbesserung des Umsetzungsprozesses wurde auch auf die Bedeutung der Effektuierung des Verfahrens vor dem Gerichtshof selbst sowie auf die Notwendigkeit zur Verbesserung der nationalen Implementierung der Konvention insgesamt hingewiesen.

1298 Committee of Ministers at its 120th session in May 2010: „[...] that prompt and effective execution of the judgments and decisions delivered by the Court is essential for the credibility and effectiveness of the Convention system and a determining factor in reducing the pressure on the Court."

1299 Annual Report 2011 Concerning Supervision of the Execution of Judgments and Decisions, S. 23.

1300 Insbesondere Recommendation CM/Rec (2008) 2 on Efficient Domestic Capacity for Rapid Execution of Judgments of the European Court of Human Rights.

1301 Vgl. dazu auch Pabel in: Zimmermann, S. 81 (90 f.). 
de $^{1302}$ einer Zweidrittelmehrheit der Stimmen der zur Teilnahme an den Sitzungen des Komitees berechtigten Mitglieder geknüpft.

Mit Art. 46 Abs. 4 und Abs. 5 EMRK wurde das sogenannte infringement proceeding (ein Versäumnisverfahren) eingeführt. ${ }^{1303}$ Ist das Ministerkomitee der Auffassung, dass ein Konventionsstaat ein Urteil nicht oder nur ungenügend befolgt, und weigert sich der Mitgliedstaat, weitere Umsetzungsmassnahmen zu ergreifen, hat das Ministerkomitee die Möglichkeit, an den Gerichtshof zu gelangen. Dazu sind die Zweidrittelmehrheit der Stimmen der zur Teilnahme an den Sitzungen des Komitees berechtigten Mitglieder und die vorgängige Mahnung des Mitgliedstaates erforderlich. Es ist dann am Gerichtshof darüber zu entscheiden, ob der Mitgliedstaat seiner Pflicht nach Art. 46 Abs. 1 EMRK zur Urteilsumsetzung Genüge getan hat. Kommt der EGMR zum Schluss, dass nur eine ungenügende Umsetzung erfolgte, stellt er eine diesbezügliche Pflichtverletzung ${ }^{1304}$ fest; Sanktionsmöglichkeiten wie etwa die Verhängung einer Geldbusse hat der Gerichtshof aber nicht. ${ }^{1305}$ Kommt der Gerichtshof zum Schluss, dass der Konventionsstaat seinen Pflichten nach Art. 46 Abs. 1 EMRK genügend nachgekommen ist, hält er dies fest und weist die Sache an das Ministerkomitee zurück, welches dann die Einstellung seiner Prüfung beschliesst (Art. 46 Abs. 5 EMRK). Ziel dieses Verfahrens ist die Erhöhung des politischen Drucks auf den Konventionsstaat, um diesen doch noch zu einer Umsetzung des Urteils zu veranlassen. ${ }^{1306}$

1302 Karpenstein/Mayer/Breuer Art. 46 Rn. 86; Pabel in: Zimmermann, S. 81 (90 f.): Ein exzessiver Gebrauch dieser Möglichkeit solle mit Blick auf die Arbeitsbelastung des Gerichtshofs verhindert werden. So auch GILCH S. $171 \mathrm{f}$.

1303 Zum Einführungsprozess des infringement proceeding ausf. GILCH S. 110 ff., 122, $135,173 \mathrm{f}$.

1304 Es geht nun nicht mehr um die ursprüngliche Konventionsverletzung. Die Pflichtverletzung bezieht sich jetzt nur auf die mangelnde Umsetzung des Urteils.

1305 Vgl. etwa SK-StPO/MeYer Verfahrensrecht (EMRK) Rn. 363.

1306 Vgl. auch PABEL in: Zimmermann, S. 81 (92 f.): Das infringement proceeding berge aber die Gefahr, das ohnehin schon langwierige Verfahren durch die Beteiligung des Gerichtshofs noch weiter in die Länge zu ziehen. Bisher wurde das infringement proceeding überhaupt erst ein Mal eingeleitet, wobei es um die Umsetzung des Urteils EGMR - Ilgar Mammadov/AZE, Urt. v. 22.05.2014, 15172/13 ging, in welchem Verstösse gegen Art. 5 Abs. 1 und 4, Art. 6 EMRK und Art. 18 i.V.m. Art. 5 EMRK festgestellt wurden. Mit Urteil vom 29. Mai 2019 stellte die Grosse Kammer eine Verletzung von Art. 46 Abs. 1 EMRK fest: EGMR (GK) - Ilgar Mammadov/AZE, Urt. v. 29.05.2019, 15172/13: Der Gerichtshof erwog, dass Aserbaidschan bei der Umsetzung des ersten Urteils nicht „in gutem Glauben“ und auch nicht in der Weise gehandelt habe, die 
Art. 39 Abs. 4 EMRK sieht zudem neu vor, dass das Ministerkomitee nun auch generell mit der Überwachung der Umsetzung von Vergleichen betraut ist. ${ }^{1307}$

\subsection{Priorisierungsverfahren des Ministerkomitees}

Der Verbesserung des Umsetzungsprozesses widmete sich im Februar 2010 auch die High Level Conference in Interlaken, anlässlich welcher ein Aktionsplan beschlossen wurde. ${ }^{1308}$ Das Ministerkomitee wurde eingeladen, sein Monitoring unter anderem durch ein Priorisierungsverfahren zu verstärken und seine Arbeitsmethoden zu überdenken. ${ }^{1309}$ Am 1. Januar 2011 erliess das Ministerkomitee in Umsetzung des Aktionsplans von Interlaken seine neuen Arbeitsmethoden (new working methods). Das Ministerkomitee führte ein System der doppelspurigen Überwachung (twin-track supervision) ein. Ein dem Ministerkomitee zugeleitetes Urteil wird von diesem dabei entweder der enhanced supervision oder der standard supervision zugeteilt, welche sich in der Intensität des Überwachungsverfahrens unterscheiden. ${ }^{1310}$ Das zweigleisige System bezweckt die an der Interlaken-Konferenz geforderte Priorisierung gewisser Kategorien von Fällen in der Umsetzungsphase. Bei diesem Priorisierungsverfahren handelt es sich aber nicht um ein offizielles Verfahren, welches in der Konvention festgelegt wäre, sondern um eine Strategie, welche verschiedene Massnahmen umfasst. 1311

den Schutz der Konventionsrechte, die der Gerichtshof im ersten Urteil für verletzt gehalten habe, praktisch und wirksam machen würde.

1307 Die Vorschrift von Art. 39 Abs. 4 EMRK ist als Sonderregel zu Art. 46 Abs. 2 EMRK zu sehen, welche vorsieht, dass nur Urteile vom Ministerkomitee überwacht werden: vgl. auch Karpenstein/Mayer/Wenzel Art. 39 Rn. 11 f.

1308 Interlaken Declaration v. 19.02.2010.

1309 Annual Report 2011 Concerning Supervision of the Execution of Judgments and Decisions, S. 25.

$1310 \mathrm{Zu}$ bedenken ist dabei, dass Überwachungsgrad und der durch das Monitoring ausgeübte politische Druck korrelieren.

1311 Vgl. auch GLas S. 210. 
a Twin-track supervision - Zuteilung der Fälle

Bei der Zuteilung der Fälle richtet sich das Ministerkomitee nach folgenden Kriterien:

Der enhanced supervision sind Fälle zuzuteilen, in denen individuelle Massnahmen dringend umgesetzt werden müssen, und zwar auch dann, wenn diese nicht auf strukturellen oder systemischen Problemen beruhen: Fälle, bei denen das Piloturteilsverfahren angewandt wurde, Urteile, welche erkennen lassen, dass strukturelle oder komplexe Probleme für die Konventionsverletzung verantwortlich sind, sowie Staatenbeschwerden (Regel 41312). ${ }^{1313}$ Darüber hinaus kann das Ministerkomitee auf Initiative eines Mitgliedstaates oder des Sekretariats auch jeden weiteren Fall der enhanced supervision zuteilen. ${ }^{1314}$

Sämtliche Urteile, welche nicht der enhanced supervision zugeleitet werden, unterliegen der standard supervision. Eine spätere Umteilung von der enhanced supervision zur standard supervision, oder umgekehrt von der standard supervision zur enhanced supervision, ist möglich, falls der Implementierungsprozess dafür Gründe liefert. ${ }^{1315}$ Die Relevanz des Priorisierungsverfahrens auch für das vorliegende Themengebiet zeigt sich darin, dass inadäquate Haftbedingungen und eine ungenügende medizinische Versorgung im Jahr 2019 8\% der Enhanced supervision-Urteile ausmachten und damit die viertgrösste Fallgruppe bei der enhanced supervision bildeten. ${ }^{1316}$

Die besondere Dringlichkeit der Umsetzung und die notwendige Priorisierung durch das Ministerkomitee ergeben sich damit typischerweise entweder aus der Schwere der Konventionsverletzung oder aus dem strukturellen bzw. systemischen Problem, welches der Konventionsverletzung zugrunde liegt. Die Priorisierung von Urteilen betreffend strukturelle Proble-

1312 Rules of the Committee of Ministers for the Supervision of the Execution of Judgments and of the Terms of Friendly Settlements; vgl. ausführlicher auch: iGuide, Committee of Ministers Procedures and Working Methods, Rn. 19, zuletzt abgerufen am 01.02.2020.

1313 Annual Report 2011 Concerning Supervision of the Execution of Judgments and Decisions, S. 19.

1314 Annual Report 2011 Concerning Supervision of the Execution of Judgments and Decisions, S. 19.

1315 Annual Report 2011 Concerning Supervision of the Execution of Judgments and Decisions, S. 19.

1316 Annual Report 2019 Concerning Supervision of the Execution of Judgments and Decisions, S. 50: Die grösste Fallgruppe bilden Handlungen von Sicherheitskräften. 
me dient insbesondere auch der Präventionsfunktion des Gerichtshofs. ${ }^{1317}$ Denn solange das für die Konventionsverletzung ursächliche strukturelle Problem, welches eine Vielzahl weiterer Personen betrifft, nicht beseitigt ist, drohen weitere ähnliche Beschwerden (repetitive cases). Eine effektivere Implementierungsphase kommt somit sowohl dem Konventionsstaat als auch dem Gerichtshof zugute, insbesondere bei strukturellen Problemen. Der Konventionsstaat muss nach Beseitigung des strukturellen Problems mit keinen weiteren Verurteilungen rechnen, ${ }^{1318}$ und die Arbeitsbelastung des Gerichtshofs wird fortan nicht weiter durch repetitive cases erhöht.

b Twin-track supervision - das Monitoringverfahren im Einzelnen

Unabhängig davon, ob ein Urteil der enhanced supervision oder der standard supervision zugeteilt wurde, verlangt das Ministerkomitee von den Konventionsstaaten so zeitnah als möglich, aber spätestens innert sechs Monaten nach Rechtskraft des Urteils, die Einreichung eines Aktionsplans (action plan) bzw. eines Aktionsberichts (action report). ${ }^{1319}$ Der Mitgliedstaat hat darin darzulegen, welche Massnahmen er in Reaktion auf die vom Gerichtshof festgestellte Konventionsverletzung zu ergreifen plant resp. bereits ergriffen hat. ${ }^{1320}$ Das Ministerkomitee resp. das Execution Department bleiben bis zur Einreichung der Aktionspläne aber nicht untätig. Vielmehr nehmen sie bereits zu diesem Zeitpunkt eine erste Evaluation der Situation vor und halten diese in einem internen Dokument fest, welches ihnen später dazu dienen wird, die von den Konventionsstaaten eingereichten Pläne zu beurteilen. ${ }^{1321}$

Ist ein Verfahren der enhanced supervision zugeleitet, wird dessen Umsetzung eng und aktiv ${ }^{1322}$ vom Ministerkomitee und dem Execution De-

1317 Vgl. implizit Annual Report 2011 Concerning Supervision of the Execution of Judgments and Decisions, S. 15.

1318 Dies gilt allerdings nur für neue Sachverhalte. Die Feststellung von Konventionsverletzungen bezüglich Sachverhalten, welche sich vor der Abhilfeschaffung bezüglich des strukturellen Problems ereignet haben, bleibt denkbar.

1319 Annual Report 2011 Concerning Supervision of the Execution of Judgments and Decisions, S. 19.

1320 Annual Report 2011 Concerning Supervision of the Execution of Judgments and Decisions, S. 19.

1321 Glas S. 379: Die Daten von Glas basieren auf Interviews mit Personen, welche mit dem Durchsetzungsverfahren betraut sind; etwa mit Mitarbeitern des Execution Department.

1322 Pabel in: Zimmermann, S. 81 (94); vgl. auch GLas S. 210. 
partment betreut. Der Implementierungsfortschritt wird anhand der vom Konventionsstaat eingereichten Dokumente (action plans / action reports) in regelmässigen Abständen beurteilt. Dazu werden sie vom Execution Department evaluiert, ${ }^{1323}$ bevor sie an das Ministerkomitee weitergeleitet werden. ${ }^{1324}$ Wenn es das Ministerkomitee für notwendig hält, greift es diese Fälle dann an seinen Menschenrechtssitzungen auf. ${ }^{1325}$ Das Ministerkomitee tagt zur Erfüllung seiner Monitoringfunktion nämlich vier Mal jährlich für je drei Tage. Jedes dieser Treffen hat eine „Agenda“, wobei alle noch nicht umgesetzten Urteile in die Agenda jedes Treffens aufgenommen werden (Regel 7). Das Ministerkomitee ist somit theoretisch jederzeit in der Lage, zu Entwicklungen im Umsetzungsprozess jedes Urteils Stellung zu nehmen. Die grosse Anzahl pendenter Fälle macht allerdings eine Selektion der Fälle notwendig, welche an den Menschenrechtssitzungen aufgegriffen werden sollen. ${ }^{1326}$

Ein Aufgreifen des Umsetzungsverfahrens in den Menschenrechtssitzungen ist insbesondere in jenen Fällen angezeigt, in denen zwischen Executive Department und Konventionsstaat ein Dissens besteht, oder wenn es beim Implementierungsprozess zu signifikanten Verzögerungen kommt. ${ }^{1327}$ Ein Aufgreifen des Implementierungsprozesses an den Menschenrechtssitzungen kann aber etwa auch dann geboten sein, wenn es die Situation des Beschwerdeführers erfordert oder wenn strukturelle Probleme, welche eine Vielzahl weiterer Fälle betreffen, die Ursache der Konventionsverletzung sind. ${ }^{1328}$ Letztere Selektionskriterien korrelieren weitgehend mit den Zuteilungskriterien zur enhanced supervision, weshalb Fälle, welche der enhanced supervision unterliegen, an den Menschenrechtssitzungen regelmässig wieder aufgenommen werden dürften. ${ }^{1329}$

1323 Ist das Execution Department mit den eingereichten Plänen bzw. Berichten noch nicht einverstanden bzw. genügen diese seines Erachtens noch nicht, kann es den Konventionsstaat anhalten, diese zunächst zu verbessern. Ist der Konventionsstaat mit den Änderungen einverstanden, ergänzt er diese entsprechend. Ist er nicht einverstanden, bleibt die Möglichkeit, den Dissens anlässlich bilateraler Treffen auszuräumen, was in der Praxis aber selten notwendig ist. Vgl. zum Ganzen GLas S. 379.

1324 Glas S. 378; Glas HR\&ILD 2019, 73 (79): Das Ministerkomitee habe damit wichtige Auslegungs- und Überwachungsaufgaben an das Department delegiert.

1325 Glas S. 400.

1326 Vgl. auch GLas S. 399.

1327 GLas S. $400 \mathrm{f}$.

1328 Glas S. 400 f. m.w.N.

1329 GLAS S. 401. 
Im Verfahren der standard supervision übernimmt das Ministerkomitee nur die formalen Schritte, ${ }^{1330}$ etwa wenn es um die Aufnahme der Fälle in seine Agenda geht, oder um formell Kenntnis von den eingereichten Aktionsplänen bzw. Aktionsberichten zu nehmen. Für die enge Begleitung der Umsetzung ist hier hauptsächlich das Execution Department zuständig. ${ }^{1331}$ Obwohl auch die Fälle der standard supervision in die Agenda eines jeden Treffens aufgenommen werden (Regel $7^{1332}$ ), führen die Selektionskriterien dazu, dass Fälle, welche der standard supervision unterliegen, nur sehr selten tatsächlich an den Menschenrechtssitzungen aufgegriffen werden. ${ }^{1333}$

Das Ministerkomitee kann weiter sowohl in Verfahren der enhanced supervision als auch in Verfahren der standard supervision in Form von Zwischenresolutionen (interim resolutions) zum Implementierungsprozess Stellung nehmen, Besorgnis ausdrücken oder weitere angemessene Umsetzungsmassnahmen vorschlagen (Regel 16) und auf diese Weise indirekt auf den Konventionsstaat einwirken; ein Dialog zwischen Mitgliedstaat und Ministerkomitee kann auf diese Weise intensiviert werden. Wenngleich Zwischenresolutionen auch im Verfahren der standard supervision möglich sind, wird von diesem Instrument in der Praxis fast ausschliesslich in Verfahren der enhanced supervision Gebrauch gemacht. ${ }^{1334}$

Unabhängig davon, ob das Verfahren der enhanced supervision oder der standard supervision unterliegt, werden Aktionspläne und Aktionsberichte i.d.R. gemeinsam mit allen weiteren relevanten Informationen unverzüglich im Internet öffentlich gemacht (Regel 8). Veröffentlicht werden insbesondere auch die an den Menschenrechtssitzungen gefassten Beschlüsse (Regel 8 Abs.4). Ausnahmsweise kann von einer Publikation abgesehen werden, wenn die Eingabe mit einer Vertraulichkeitsanfrage versehen wurde, über welche das Komitee jeweils in der nächsten Men-

1330 PABEL in: Zimmermann, S. 81 (94). Das Verfahren der standard supervision steht ganz im Zeichen des Grundprinzips der Subsidiarität, ist es doch Sache der Vertragsstaaten, die wirksame Durchführung der Urteile des Gerichtshofs zu gewährleisten. Vgl. dazu GLas HR\&ILD 2019, 73 (79).

1331 Annual Report 2011 Concerning Supervision of the Execution of Judgments and Decisions, S. 27.

1332 Regel 7 der Rules of the Committee of Ministers for the Supervision of the Execution of Judgments and of the Terms of Friendly Settlements wurde am 1100. Treffen des Ministerkomitees in diesem Sinne ausgeführt und präzisiert. Vgl. auch Pabel in: Zimmermann, S. 81 (88), welche in diesem Zusammenhang von einer „Prangerwirkung“ spricht.

1333 Glas S. 401.

1334 GLas S. 210. 
schenrechtssitzung entscheidet. Durch die Publikation sämtlicher Informationen kommt das Ministerkomitee der Forderung nach einer gesteigerten Transparenz nach, welche letztlich wieder der Effektivierung des Implementierungsprozesses dienen soll. ${ }^{1335}$ Die Veröffentlichung der Informationen ermöglicht, dass auch NGOs, nationale Menschenrechtsinstitutionen sowie Beschwerdeführer die Möglichkeit haben, dem Ministerkomitee Stellungnahmen zum Implementierungsprozess einzureichen (Regel 91336). Die Stellungnahmen von Beschwerdeführern haben sich i.d.R. allerdings auf die Umsetzung individueller Massnahmen und auf die Zahlung der finanziellen Entschädigung zu beschränken.

Ist der Konventionsstaat überzeugt, alle notwendigen Massnahmen zur Implementierung des Urteils ergriffen zu haben, reicht er dem Ministerkomitee einen Schlussbericht (final action report) ein. Es folgt eine sechsmonatige Frist, innert welcher andere Staaten oder das Sekretariat die Möglichkeit haben, Stellungnahmen hinsichtlich der Schliessung des Überwachungsprozesses einzureichen. ${ }^{1337}$ Das Ministerkomitee entscheidet nach Ablauf der Frist über die Beendigung des Monitoringprozesses. Ist das Ministerkomitee von der Umsetzung des Urteils - sowohl der individuellen als auch der generellen Massnahmen - überzeugt, beendet es das Überwachungsverfahren mit einer Schlussresolution (final resolution) (Regel 17). Dem Ministerkomitee ist es ferner möglich, die Überwachung auch der repetitiven Fälle abzuschliessen, sofern alle individuellen Massnahmen getroffen wurden. Ist es aber der Überzeugung, dass der Konventionsstaat seinen Umsetzungsverpflichtungen noch nicht genügend nachgekommen ist, kann es eine Zwischenresolution erlassen (Regel 16) und so den politischen Druck auf den Konventionsstaat zur Ergreifung weiterer Abhilfemassnahmen aufrechterhalten bzw. gar verstärken.

Mehr theoretischer Natur ${ }^{1338}$, da in aller Regel klar unangemessen ${ }^{1339}$, bleiben demgegenüber die Möglichkeiten des Ministerkomitees, einem Konventionsstaat das Stimmrecht zu suspendieren (Art. 8 Satzung des Eu-

$1335 \mathrm{Zu}$ Beginn war der Überwachungsprozess des Ministerkomitees noch vertraulich: Bis im Jahr 2000 waren nur die „Final Resolutions“ öffentlich.

1336 Regel 9 der Rules of the Committee of Ministers for the Supervision of the Execution of Judgments and of the Terms of Friendly Settlements wurde beim 1275. Treffen des Ministerkomitees entsprechend ergänzt.

1337 Vgl. Annual Report 2011 Concerning Supervision of the Execution of Judgments and Decisions, S. $20 \mathrm{f}$.

1338 PABEL in: Zimmermann, S. 81 (88).

1339 SK-StPO/Meyer Verfahrensrecht (EMRK) Rn. 364. 
roparats) oder als ultima ratio sogar den renitenten Staat aus dem Europarat auszuschliessen (Art. 3 Satzung des Europarats).

Zusammenfassend unterliegt der Konventionsstaat einem gewissen politischen Druck zur Umsetzung der Urteile, unabhängig davon, ob die Umsetzung des Urteils der enhanced supervision oder der standard supervision zugeteilt wurde, da alle noch nicht umgesetzten Urteile in der "Agenda“ einer jeden Menschenrechtssitzung aufgeführt und sämtliche Aktionspläne und Aktionsberichte im Internet öffentlich gemacht werden. Wird ein Fall in den Menschenrechtssitzungen im Einzelnen aufgegriffen, oder wird gar eine Zwischenresolution erlassen, steigt der politische Druck zur Umsetzung der Massnahmen noch einmal. Dies dürfte vornehmlich Urteile betreffen, welche der enbanced supervision zugeteilt wurden.

Aufgrund dessen, dass nach der Einführung der twin-track supervision jährlich deutlich mehr Fälle mit einer final resolution beendet werden können, wird bisweilen zum Schluss gelangt, dass die mit dem Twin-trackVerfahren angestrebte Effizienzsteigerung erreicht werden konnte. ${ }^{1340}$ Die Bedeutung und die Auswirkungen des Twin-track-Verfahrens für die Fälle inadäquater Haftbedingungen allgemein, sowie für die Piloturteilsverfahren dieses Themenbereiches im Speziellen, werden im Folgenden evaluiert. Ziel ist es, Aussagen darüber machen zu können, wie sich die neuen Arbeitsweisen im Implementierungsprozess bei Urteilen zu inadäquaten Haftbedingungen tatsächlich auswirken.

\section{3 Überwachung der Zahlungsverpflichtung}

Die Überwachung der Zahlungsverpflichtung i.S.v. Art. 41 EMRK unterscheidet sich etwas von der Überwachung der übrigen Massnahmen. Bei

1340 Wittling-Vogel in: Zimmermann, S. 103 (105): Im Jahr 2013 wurden die Überwachung von insgesamt 1398 Fällen mit einer final resolution beendet, im Jahr 2004 konnten indes nur gerade 163 Fälle beendet werden. Allerdings dürfen meines Erachtens auch weitere Faktoren, welche zu dieser Entwicklung möglicherweise beigetragen haben, nicht ausgeblendet werden. So bleibt meines Erachtens gerade auch zu berücksichtigen, dass die Fallzahlen vor dem Gerichtshof allgemein zugenommen haben und dass repetitive Fälle immer mehr Bedeutung erlangt haben. Indem es dem Ministerkomitee möglich ist, repetitive Fälle abzuschliessen, wenn alle individuellen Massnahmen getroffen wurden, konnte es nämlich bspw. die Überwachung von 100 polnischen und 250 ungarischen Urteilen zu inadäquaten Haftbedingungen abschliessen: Annual Report 2017 Concerning Supervision of the Execution of Judgments and Decisions, S. 14. 
der Überwachung der Zahlungsverpflichtung wird der Beschwerdeführer seit dem Jahr 2011 im Durchsetzungsverfahren vermehrt in die Pflicht genommen und auf diese Weise der Überwachungsprozess vereinfacht. ${ }^{1341}$ Mit Zustellung des Urteils des Gerichtshofs wird der Beschwerdeführer darauf hingewiesen, dass es in seiner Verantwortung liegt, auf Defizite bei der Umsetzung der Leistung der Entschädigungspflicht des Konventionsstaats durch Beschwerde beim Execution Department hinzuweisen. Informiert der Konventionsstaat das Execution Department, dass er die Zahlung geleistet habe, wird dies auf der Webseite publiziert. Geht innert zwei Monaten seit Publikation der geleisteten Zahlung seitens des Beschwerdeführers keine Beschwerde ein, so gilt die Angelegenheit der Entschädigungszahlung als geschlossen.

\subsection{Zusammenarbeit des Ministerkomitees mit weiteren Akteuren}

Die in Interlaken angestossenen Veränderungen des Implementierungsprozesses sind Gegenstand fortwährender Evaluierung und Weiterentwicklung. Die Interlaken-Deklaration und der Interlaken-Aktionsplan wurden in den folgenden Konferenzen in Izmir (2011), Brighton (2012), Brüssel (2015) und Kopenhagen (2018) wieder aufgenommen und Follow-upPläne beschlossen. ${ }^{1342}$ Insbesondere in der Brüssel-Deklaration ${ }^{1343}$ wurde noch einmal die Notwendigkeit der Zusammenarbeit mit anderen (Europarats-)Akteuren auch in der Durchsetzungsphase betont. ${ }^{1344}$ Die Zusammenarbeit betrifft in ihrem jeweiligen Aufgabengebiet etwa den Gerichts-

1341 Annual Report 2011 Concerning Supervision of the Execution of Judgments and Decisions, S. 20.

1342 Interlaken Declaration v. 19.02.2010; Izmir Declaration v. 27.04.2011; Brighton Declaration v. 20.04.2012; Brussels Declaration v. 27.03.2015; Copenhagen Declaration v. 13.04.2018; vgl. auch Annual Report 2017 Concerning Supervision of the Execution of Judgments and Decisions, S. $17 \mathrm{ff}$. Auch in der Konferenz in Oslo war die Effizienz des Durchsetzungsverfahrens Thema.

1343 Brussels Declaration v. 27.03.2015.

1344 Vgl. etwa Annual Report 2017 Concerning Supervision of the Execution of Judgments and Decisions, S. 22; vgl. aber auch bereits zuvor: Annual Report 2011 Concerning Supervision of the Execution of Judgments and Decisions, S. 13. 
hof selbst, die parlamentarische Versammlung oder die Arbeit des Menschenrechtskommissars. ${ }^{1345}$

Synergien sind darüber hinaus über Kooperationsprogramme mit Expertengremien denkbar, wie etwa der Venedig-Kommission, dem Europäischen Ausschuss für soziale Rechte, dem CPT oder der CEPEJ (Commission européenne pour l'efficacité de la justice). ${ }^{1346}$ Die Idee der Zusammenarbeit ist, dass sich die mit der Umsetzung betrauten nationalen Behörden auf die Empfehlungen und Informationen dieser Institutionen stützen können, insbesondere wenn es um die Ergreifung genereller Massnahmen geht. ${ }^{1347}$

Ferner wird eine Kooperation des Ministerkomitees mit dem Human Rights Trust Fund (HRTF) im Sinne eines effizienten Durchsetzungsverfahrens angestrebt. ${ }^{1348}$ Der HRTF, welcher 2008 von Norwegen, dem Europarat und der Entwicklungsbank des Europarates ins Leben gerufen wurde und an welchem sich später auch Deutschland, die Niederlande, Finnland, die Schweiz, Grossbritannien und Luxemburg beteiligten, hat zum Ziel, finanzielle Mittel zur Verfügung zu stellen, damit andere Staaten ihren Verpflichtungen aus der Europäischen Menschenrechtskonvention und anderen europaratsrechtlichen Menschenrechtsstandards nachkommen können. ${ }^{1349}$

Das Projekt 18 des HRTF hat die Implementierung von Urteilen in Bezug auf Haftbedingungen zum Gegenstand. ${ }^{1350}$ An diesem Projekt sind sechs Partnerstaaten beteiligt (Bulgarien, Polen, Moldawien, Rumänien, Russland und die Ukraine). Kernanliegen des Projekts ist die Unterstüt-

1345 Annual Report 2017 Concerning Supervision of the Execution of Judgments and Decisions, S. 22; vgl. aber auch bereits zuvor: Annual Report 2011 Concerning Supervision of the Execution of Judgments and Decisions, S. 22.

1346 Annual Report 2011 Concerning Supervision of the Execution of Judgments and Decisions, S. 13; Annual Report 2017 Concerning Supervision of the Execution of Judgments and Decisions, S. 24.

1347 Annual Report 2011 Concerning Supervision of the Execution of Judgments and Decisions, S. 13.

1348 Annual Report 2017 Concerning Supervision of the Execution of Judgments and Decisions, S. 24: „Targeted Convention-related cooperation projects“; vgl. auch bereits: Annual Report 2011 Concerning Supervision of the Execution of Judgments and Decisions, S. 27.

1349 Office of the Directorate General of Programmes, Human Rights Trust Fund, zuletzt abgerufen am 24.04.2020; vgl. auch WitTLING-VogeL in: Zimmermann, S. 103 (114): Finanzielle Hilfe könne den entscheidenden Anschub zur Umsetzung des Urteils geben.

1350 Department for the Execution of Judgments of the European Court of Human Rights, Human Rights Trust Fund, zuletzt abgerufen am 24.04.2020. 
zung der Einführung effektiver Rechtsbehelfe für Beschwerden über inadäquate Haftbedingungen. ${ }^{1351}$ Der HRTF analysiert nationale Gesetzesentwürfe und prüft deren Kompatibilität mit den Mindestvoraussetzungen der Konvention. Darüber hinaus beteiligt sich der HRTF auch bei der Suche nach notwendig zu ergreifenden generellen Massnahmen. ${ }^{1352} \mathrm{Im}$ Zeitraum vom 01.01.2012 bis 31.07.2015 unterstützte der HRTF die Implementierung von Piloturteilen resp. „Quasi-Piloturteilen“ im Bereich inadäquater Untersuchungshaftbedingungen mit insgesamt 800'000 Euro. ${ }^{1353}$ Ferner trug resp. trägt der HRTF zur Finanzierung verschiedener weiterer Vorhaben bei, so insbesondere vom 01.01.2012 bis 31.12.2014 mit 1'250'000 Euro zur Förderung einer wirksamen öffentlichen Überwachung von Orten des Freiheitsentzuges in Russland (PMC Project ${ }^{1354}$ ), vom 01.11.2016 bis 26.02.2018 mit 400'000 Euro zur Umsetzung der Urteile des EGMR und der Standards und Empfehlungen des CPT im Rahmen der Polizei- und Gefängnisreform in Bulgarien, und vom 01.06.2019 bis 31.05.2021 mit 600'000 Euro zur Verbesserung der Gesundheitsversorgung in griechischen Haftanstalten. ${ }^{1355}$ Der HRTF hat sich damit insgesamt als wichtiger Akteur bei der Implementierung eines Mindeststandards bei Haftbedingungen etabliert.

\section{Umsetzung der Piloturteile}

\section{Status der Umsetzung der angeordneten Massnahmen}

Die Implementierung der Urteile Orchowski/POL ${ }^{1356}$ und Norbert Sikorskil POL $^{1357}$ wurde mit der Resolution CM/ResDH (2016) 254 im Jahr 2016 formell abgeschlossen und damit auch das Monitoring des Ministerkomi-

1351 Department for the Execution of Judgments of the European Court of Human Rights, Human Rights Trust Fund, zuletzt abgerufen am 24.04.2020.

1352 Department for the Execution of Judgments of the European Court of Human Rights, Human Rights Trust Fund, zuletzt abgerufen am 24.04.2020.

1353 Office of the Directorate General of Programmes, Projects Funded, zuletzt abgerufen am 24.04.2020.

1354 Dies auch im Zusammenhang mit der Überwachung der Kalashnikov/Ananyev Group.

1355 Office of the Directorate General of Programmes, Projects Funded, zuletzt abgerufen am 24.04.2020.

1356 EGMR - Orchowski/POL, Urt. v. 22.10.2009, 17885/04.

1357 EGMR - Norbert Sikorski/POL, Urt. v. 22.10.2009, 17599/05. 
tees beendet. ${ }^{1358}$ Auf individueller Ebene hält das Ministerkomitee fest, dass die Beschwerdeführer nicht mehr den beklagten Haftumständen ausgesetzt sind; sie wurden entweder aus der Haft entlassen oder in andere Haftanstalten verlegt.

Polen hat eine Reihe genereller Massnahmen ergriffen, um dem strukturellen Problem der Überbelegung zu begegnen. Allem voran wurde Polen auf legislativer Ebene tätig: Der Code of Execution of Criminal Sentences wurde dahingehend geändert, dass eine Unterbringung mit weniger als $3 \mathrm{~m}^{2}$ nur noch in klar definierten Ausnahmesituationen sowie für eine beschränkte Zeit möglich sein soll. Gleichzeitig wurde zusätzlich noch ein Rechtsbehelf eingeführt, mit welchem sich die Betroffenen gegen eine solche Einschränkung bzw. gegen die Unterbringung in einer überbelegten Zelle zur Wehr setzen können. Weiter wurde ein Gesetz zur elektronischen Überwachung sowie zur Verbüssung kurzer Freiheitsstrafen auch ausserhalb von Haftanstalten erlassen, es wurden die Möglichkeiten des Zugangs zur bedingten Entlassung verbessert und auf materiell-strafrechtlicher Ebene gewisse Delikte (teilweise) entkriminalisiert. ${ }^{1359}$ Neben diesen legislativen Neuerungen wurden weitere Massnahmen getroffen: Durch Renovationsarbeiten und weitere Investitionen wurden mehr Haftplätze geschaffen, es wurde ein Monitoring zur Überwachung der Gefängnispopulation durch das Justizministerium eingeführt und die beiden Piloturteile wurden übersetzt sowie zur Sensibilisierung unter dem Gefängnispersonal verbreitet. Schliesslich wurde ein Leitfaden mit Standards zum Umgang mit Inhaftierten publiziert.

Die Implementierung des Piloturteils Ananyev u.a./RUS ${ }^{1360}$ dauert noch an. Die Durchsetzung des Urteils erfolgt im Verfahren der enhanced supervision und wird gemeinsam mit weiteren einschlägigen Urteilen über-

1358 Resolution CM/ResDH (2016) 254, Execution of the Judgments Orchowski u.a./ POL; vgl. auch Summary of Final Resolutions 2016, S.91, zuletzt abgerufen am 10.04.2018.

1359 Entkriminalisiert wurde etwa das Fahren von Nicht-Kraftfahrzeugen im angetrunkenen Zustand.

1360 EGMR - Ananyev u.a./RUS, Urt. v. 10.01.2012, 42525/07 u. 60800/08. 
wacht (Kalashnikov Group ${ }^{1361}$ ). ${ }^{1362}$ Die Umsetzung der Massnahmen der Kalashnikov Group wurde zunächst durch den HRTF und später durch einen Beitrag Grossbritanniens finanziert. ${ }^{1363}$

Das Ministerkomitee stellt fest, dass individuelle Abhilfemassnahmen bei den meisten Beschwerdeführern der Kalashnikov Group getroffen wurden und dass diese fortan nicht mehr unter inadäquaten Haftbedingungen untergebracht sind. Mit Resolution CM/ResDH (2018) 455 schloss der Gerichtshof die Überwachung individueller Massnahmen in 136 Fällen dieser Gruppe ab. ${ }^{1364}$ In anderen Fällen sind die von Russland zur Verfügung gestellten Informationen allerdings noch ungenügend; weitere Informationen müssen nachgereicht werden. Ein Beschwerdeführer reichte nach dem Transfer in eine andere Haftanstalt (was Folge des ersten Urteils ${ }^{1365}$ war) erneut Beschwerde ein, und der Gerichtshof stellte erneut eine Verletzung von Art. 3 EMRK fest. ${ }^{1366}$

Auf der Ebene der generellen Massnahmen stellte das Ministerkomitee im Juni 2017 fest, dass hinsichtlich der Überbelegung und der Haftbedingungen im Allgemeinen (Art. 3 EMRK betreffend) in den letzten Jahren deutliche Fortschritte erzielt wurden. Die Voraussetzungen für die Anordnung der Untersuchungshaft wurden durch eine Änderung der russischen Strafprozessordnung verschärft, und es wurden Alternativen

1361 Bei der Frage, ob ein strukturelles Problem vorliegt, hatte der EGMR im Urteil Ananyev u.a./RUS selbst bereits an das Urteil Kalashnikov/RUS angeknüpft: EGMR - Kalashnikov/RUS, Urt. v. 15.07.2002, 47095/99 u. 60800/08. Die geforderten Massnahmen beziehen sich deshalb auch auf weitere Urteile und beschränken sich nicht nur auf die in Ananyev u.a./RUS selbst angeordneten Massnahmen.

1362 Vgl. zum Ganzen: Annual Report 2017 Concerning Supervision of the Execution of Judgments and Decisions, S. $160 \mathrm{f}$.

1363 Annual Report 2017 Concerning Supervision of the Execution of Judgments and Decisions, S. 25.

1364 Resolution CM/ResDH (2018) 455, Execution of the Judgments of the European Court of Human Rights, 136 Cases/RUS (from the Kalashnikov Group).

1365 EGMR - Amirov/RUS, Urt. v. 27.11.2014, 51857/13.

1366 EGMR - Amirov/RUS, Urt. v. 17.10.2017, 56220/15: In diesem Entscheid wurde keine Verletzung von Art. 3 EMRK wegen mangelhafter medizinischer Versorgung festgestellt. Eine Verletzung von Art. 3 EMRK wurde aber hinsichtlich der weiteren Bedingungen festgestellt: Der Beschwerdeführer war auf die tägliche Hilfe der Mitinsassen angewiesen, Rampen und Aufzüge fehlten, auf die der Beschwerdeführer angewiesen gewesen wäre, und es war keine genügende Privatsphäre in den Waschräumen gewährleistet. 
zur Untersuchungshaft eingeführt. ${ }^{1367}$ Die materiellen Haftbedingungen in den föderalen Strafvollzugsanstalten sind durch Programme, welche den Wiederaufbau und die Renovation russischer Strafvollzugsanstalten (inkl. der Krankenstationen) und die Überwachung der Haftbedingungen zum Ziel haben, verbessert worden. ${ }^{1368}$ Das Ministerkomitee forderte Russland im Juni 2017 weiter auf, über weitere Verbesserungen und über CPT-Berichte zu russischen Haftanstalten zu informieren. ${ }^{1369}$ Im April 2018 verabschiedete Russland in der Folge das Programm „Penal System Development 2018-2026“, wobei 763 Mio. Euro investiert werden sollten. Das Programm umfasst unter anderem die Renovation und den Bau von 366 Gebäuden. Im Jahr 2018 wurden bereits 135 (wieder)aufgebaut und 183 Untersuchungshaftanstalten renoviert. ${ }^{1370}$ Weiter wurden Massnahmen zum Schutz besonders gefährdeter Gruppen Inhaftierter getroffen (Minderjährige, Schwangere, Behinderte oder Kranke), indem etwa Ernährungsvorschriften erlassen oder besondere Pflegeprodukte und Hilfsmittel bereitgestellt wurden. ${ }^{1371} \mathrm{Im}$ Jahr 2018 wurden sodann 2'400 Inhaftierte aus medizinischen Gründen aus der Haft entlassen; gleichzeitig wurde die medizinische Versorgung insbesondere bei Tuberkulose und AIDS verbessert und eine psychodiagnostische Untersuchung eines jeden neuen Inhaftierten eingeführt. ${ }^{1372}$ Im Juni 2019 wurden die ergriffenen Massnahmen begrüsst, gleichzeitig aber auch festgestellt, dass die Überbelegungssituation trotz einem Rückgang der Zahl der Untersuchungsinhaftierten in gewissen russischen Haftanstalten fortbesteht. Informationen darüber,

1367 Zum Ganzen vgl. Annual Report 2017 Concerning Supervision of the Execution of Judgments and Decisions, S. 161.

1368 Federal Target Programme „Development of the Correctional System“; vgl. auch Annual Report 2017 Concerning Supervision of the Execution of Judgments and Decisions, S. 161.

1369 Zum Ganzen: Annual Report 2017 Concerning Supervision of the Execution of Judgments and Decisions, S. 161.

1370 HUDOC, Status of Execution: EGMR - Ananyev u.a./RUS, Urt. v. 10.01.2012, 42525/07 u. 60800/08, zuletzt abgerufen am 25.04.2020.

1371 HUDOC, Status of Execution: EGMR - Ananyev u.a./RUS, Urt. v. 10.01.2012, 42525/07 u. 60800/08, zuletzt abgerufen am 25.04.2020.

1372 HUDOC, Status of Execution: EGMR - Ananyev u.a./RUS, Urt. v. 10.01.2012, 42525/07 u. 60800/08, zuletzt abgerufen am 25.04.2020: Dies hat sich auch in der Statistik niedergeschlagen: Im Jahr 2018 ist im Vergleich zum Jahr 2017 die Zahl der insgesamt kranken Inhaftierten um 5,3\%, die Zahl an Tuberkulose erkrankter Gefangener um 13\% und diejenige an HIV erkrankter Inhaftierter um 3,6\% zurückgegangen. 
welche Einrichtungen überbelegt sind, weshalb dies so ist und was die geplanten Abhilfemassnahmen sind, stehen aus. ${ }^{1373}$

Infolge der Verletzung von Art. 13 EMRK i.V.m. Art. 3 EMRK führte Russland einen präventiven Rechtsbehelf ein. Das Ministerkomitee forderte Russland in der Folge auf, weitere Informationen über das Funktionieren des Rechtsbehelfs bereitzustellen, insbesondere hinsichtlich der Zahl der bei den Gerichten eingereichten Beschwerden und der Massnahmen zur Durchsetzung von Gerichtsentscheiden. ${ }^{1374}$ Die Einführung auch eines effektiven kompensatorischen Rechtsbehelfs steht allerdings noch aus. Russland arbeitete allerdings bereits einen diesbezüglichen Gesetzesentwurf aus. ${ }^{1375}$ Zuvor hatte das Ministerkomitee Russland auf die Möglichkeiten eines „Reduktionssystems“ nach dem italienischen Modell (siehe sogleich) hingewiesen. ${ }^{1376}$ Finanzielle Entschädigungen für inadäquate Haftbedingungen werden in Russland aber immerhin schon heute ausgerichtet. ${ }^{1377}$

Der Implementierungsprozess des Urteils Torreggiani u.a./ITA ${ }^{1378}$ ist vom Ministerkomitee mit der Resolution CM/ResDH (2016) 28 beendet worden. ${ }^{1379}$ In individueller Hinsicht wurde der Konventionsverstoss durch den Transfer oder die Entlassung der Beschwerdeführer behoben. Als generelle Massnahme zur Behebung der Verletzung von Art. 13 EMRK führte Italien einen präventiven und einen kompensatorischen Rechtsbehelf ein. Inhaftierte können sich nun über Rechtsverletzungen in allgemeiner Weise beklagen und mittels des kompensatorischen Rechtsbehelfs bei einer Unterbringung in überbelegten Zellen eine Haftreduktion verlan-

1373 HUDOC, Status of Execution: EGMR - Ananyev u.a./RUS, Urt. v. 10.01.2012, 42525/07 u. 60800/08, zuletzt abgerufen am 25.04.2020.

1374 HUDOC, Status of Execution: EGMR - Ananyev u.a./RUS, Urt. v. 10.01.2012, 42525/07 u. 60800/08, zuletzt abgerufen am 25.04.2020.

1375 HUDOC, Status of Execution: EGMR - Ananyev u.a./RUS, Urt. v. 10.01.2012, 42525/07 u. 60800/08, zuletzt abgerufen am 25.04.2020.

1376 Zum Ganzen: Annual Report 2017 Concerning Supervision of the Execution of Judgments and Decisions, S. 161.

1377 HUDOC, Status of Execution: EGMR - Ananyev u.a./RUS, Urt. v. 10.01.2012, 42525/07 u. 60800/08, zuletzt abgerufen am 25.04.2020.

1378 EGMR - Torreggiani u.a./ITA, Urt. v. 08.01.2013, 43517/09 u.a.

1379 Resolution CM/ResDH (2016) 28, Execution of the Judgments Torregiani u.a./ITA u. Sulejmanovic/ITA; vgl. auch Summary of Final Resolutions 2016, S.51, zuletzt abgerufen am 10.04.2018; ausf. Evaluierung der ergriffenen Implementierungsmassnahmen auch in Favuzza HRLR 2017, $153 \mathrm{ff}$. 
gen. ${ }^{1380}$ Kommt eine Haftreduktion nicht mehr in Betracht (z.B. nach der Entlassung) oder betrug die Unterbringung in der überfüllten Zelle weniger als 15 Tage, ist die Möglichkeit einer zivilrechtlichen Schadenersatzklage vorgesehen. ${ }^{1381}$ Diese Umsetzungsmassnahmen hatten auch Einfluss auf die Folgerechtsprechung des Gerichtshofs. Die von Italien neu eingefügten präventiven und kompensatorischen Rechtsbehelfe i.S.v. Art. 13 EMRK i.V.m. Art. 3 EMRK hatten die Unzulässigkeit mehrerer Beschwerden zur Folge, weil der innerstaatliche Rechtsweg nicht ausgeschöpft worden war (vgl. Art. 35 Abs. 1 EMRK). ${ }^{1382}$ Der Gerichtshof führte aus, dass es keine Hinweise gebe, dass die innerstaatlich vorhandenen (neu eingeführten) Rechtsbehelfe nicht effektiv seien, weshalb von diesen Gebrauch zu machen sei. ${ }^{1383}$

In Bezug auf die Verletzung von Art. 3 EMRK hat Italien legislative, organisatorische und Instandsetzungsmassnahmen getroffen. Auf legislativer Ebene wurden für eine Reihe von geringeren Delikten neu alternative Strafen eingeführt (elektronische Überwachung, Hausarrest) und die präventive Haft eingeschränkt. Weiter wurden Möglichkeiten zur vorzeitigen Entlassung bei gleichzeitiger Bewachung ausgebaut. In organisatorischer Hinsicht wurden vermehrte Möglichkeiten für die Bewegung ausserhalb der eigenen Haftzelle geschaffen, was zur allgemeinen Verbesserung der Haftbedingungen beiträgt (zum Standard von Art. 3 EMRK und Kompensationsmechanismen vgl. Teil 3 Kap. 2 IV.). ${ }^{1384}$ Ferner wurden bestehende Haftanstalten einer Renovation unterzogen. Auch statistische Daten belegten vor Abschluss des Implementierungsprozesses den Rückgang der Gefängnispopulation in Italien und damit einhergehend die geringere

1380 Das Gesetz sieht vor, dass für alle 10 Tage in einer überfüllten Haftanstalt die zu verbüssende Haft um jeweils einen Tag zu reduzieren ist.

1381 Für jeden Tag in einer überfüllten Zelle soll eine Entschädigung in der Höhe von 8 Euro bezahlt werden.

1382 EGMR - Stella u.a./ITA, Entsch. v. 16.09.2014, 49169/09 u.a., Rn. 35 ff.; EGMR - Rexhepi u.a./ITA, Entsch. v. 16.09.2014, 47180/10, Rn. 35 ff.: Dies, obwohl die Beschwerden zwischen 2009 und 2010 anhängig gemacht wurden, d.h. vor Inkrafttreten der neuen gesetzlichen Bestimmung. Der EGMR wich vorliegend vom allgemeinen Grundsatz ab, dass das Erfordernis der Erschöpfung im Hinblick auf den Zeitpunkt der Beschwerdeeinlegung zu beurteilen ist. Diese Ausnahme wendete er sodann auf alle ähnlichen anhängigen Rechtssachen an.

1383 EGMR - Stella u.a./ITA, Entsch. v. 16.09.2014, 49169/09 u.a., Rn. 35 ff.; EGMR - Rexhepi u.a./ITA, Entsch. v. 16.09.2014, 47180/10, Rn. 35 ff.

1384 Der Mehrheit der Inhaftierten ist es nun möglich, mindestens 8 Stunden pro Tag ausserhalb der Zelle zu verbringen. 
Überbelegung italienischer Haftanstalten. ${ }^{1385}$ Zur Überwachung des Platzangebotes und der Gefängnispopulation wurde ein Computermonitoring entwickelt, welches auch Transfermöglichkeiten bei einzelnen überbelegten Haftanstalten und Vakanzen in anderen Haftanstalten anzeigt. Seit Mai 2014 soll dadurch sichergestellt sein, dass jedem Inhaftierten mindestens $3 \mathrm{~m}^{2}$ persönlicher Platz zustehen.

Der Implementierungsprozess des Piloturteils Neshkov u.a./BUL ${ }^{1386}$ dauert noch an. Die Überwachung auch dieses Urteils findet im Verfahren der enhanced supervision statt und wird gemeinsam mit einer Reihe weiterer Urteile durchgeführt, welche allesamt inadäquate Haftbedingungen zum Gegenstand haben (Kehayov Group ${ }^{1387}$ ). ${ }^{1388}$

Im Anschluss an das Piloturteil Neshkov u.a./BUL (und im Anschluss an die öffentliche Stellungnahme des CPT vom 26. März 2015 ${ }^{1389}$ ) ersuchte das Justizministerium Bulgariens den Europarat, Bulgarien bei der Durchführung der notwendigen Gefängnisreform zu unterstützen. ${ }^{1390} \mathrm{Im}$ Juli 2015 wurde in Sofia sodann eine Diskussionsrunde einberufen. Resultat der Gesprächsrunde war die Entwicklung eines Projekts mit dem Ziel der Umsetzung des Piloturteils sowie des CPT-Standards. ${ }^{1391}$ Das Projekt war zunächst auf zwölf Monate befristet und wurde schliesslich bis zum 26.02.2018 verlängert. Finanziert wurde das Projekt vom HRTF, der ein Gesamtbudget von 400 '000 Euro zur Verfügung stellte. ${ }^{1392}$

1385 Belegungsrate April 2012: 148\%; April 2014: 124\%; September 2014: 110\%: Summary of Final Resolutions 2016, S. 52, zuletzt abgerufen am 10.04.2018.

1386 EGMR - Neshkov u.a./BUL, Urt. v. 27.01.2015, 36925/10 u.a.

1387 EGMR - Kehayov/BUL, Urt. v. 18.01.2005, 41035/98.

1388 Zum Ganzen: Annual Report 2017 Concerning Supervision of the Execution of Judgments and Decisions, S. $155 \mathrm{f}$. Aus diesem Grund beschränken sich die Forderungen des Ministerkomitees auch nicht auf die in Neshkov u.a./BUL angeordneten generellen Massnahmen, sondern beziehen sich auch auf weitere Urteile.

1389 CPT - Public Statement Concerning Bulgaria [CPT/Inf (2015) 17].

1390 Support for the Implementation of the European Court of Human Rights Judgments and European Committee for the Prevention of Torture and Inhuman or Degrading Treatment of Punishment Standards and Recommendations in Bulgaria (Prison Reform), zuletzt abgerufen am 25.04.2020.

1391 Support for the Implementation of the European Court of Human Rights Judgments and European Committee for the Prevention of Torture and Inhuman or Degrading Treatment of Punishment Standards and Recommendations in Bulgaria (Prison Reform), zuletzt abgerufen am 25.04.2020.

1392 Support for the Implementation of the European Court of Human Rights Judgments and European Committee for the Prevention of Torture and In- 
Die angeordneten individuellen Massnahmen des Piloturteils Neshkov u.a./BUL wurden von Bulgarien umgesetzt. Weitere Informationen zu diversen Beschwerdeführern der Kehayov-Implementierungsgruppe sind jedoch noch ausstehend. ${ }^{1393}$ Hinsichtlich der generell zu ergreifenden Massnahmen wurden zur Abhilfe der Verletzung von Art. 13 EMRK i.V.m. Art. 3 EMRK im Januar 2017 innerstaatliche Rechtsmittel eingeführt, die im Mai 2017 in Kraft getreten sind. Diese entsprechen den Anforderungen des Gerichtshofs. ${ }^{1394}$ Weitere Informationen bezüglich des Funktionierens der Rechtsbehelfe in der Praxis seien gemäss Ministerkomitee aber noch ausstehend. ${ }^{1395}$ Die Effektivität des Rechtsbehelfs hänge vor allem von weiteren Verbesserungen bezüglich der Überbelegung und der allgemeinen Haftbedingungen ab. ${ }^{1396}$

Hinsichtlich der genuinen Verletzung von Art. 3 EMRK fordert das Ministerkomitee Bulgarien auf, ihm Informationen zu den Auswirkungen der getroffenen Massnahmen zur Verbesserung des Zugangs zu Aktivitäten ausserhalb der Zelle zukommen zu lassen. ${ }^{1397}$ Das Ministerkomitee anerkennt die Renovierungsarbeiten an den Strafvollzugsanstalten im Jahr 2016 und fordert weitere Renovationsarbeiten, wobei insbesondere deren Finanzierung sicherzustellen sei. ${ }^{1398}$ Die Projekte zur Verbesserung der Haftbedingungen seien so rasch als möglich abzuschliessen und die erzielten Ergebnisse zu bewerten und dem Ministerkomitee vorzulegen. ${ }^{1399}$ Ferner sind die mit Unterstützung des Europarats ausgearbeitete nationale

human or Degrading Treatment of Punishment Standards and Recommendations in Bulgaria (Prison Reform), zuletzt abgerufen am 25.04.2020.

1393 HUDOC, Status of Execution (Kehayov Group of Cases/BUL): EGMR Neshkov u.a./BUL, Urt. v. 27.01.2015, 36925/10 u.a., zuletzt abgerufen am 25.04.2020.

1394 Zum Ganzen: Annual Report 2017 Concerning Supervision of the Execution of Judgments and Decisions, S. 155.

1395 HUDOC, Status of Execution (Kehayov Group of Cases/BUL): EGMR Neshkov u.a./BUL, Urt. v. 27.01.2015, 36925/10 u.a., zuletzt abgerufen am 25.04.2020.

1396 Annual Report 2017 Concerning Supervision of the Execution of Judgments and Decisions, S. 155.

1397 Annual Report 2017 Concerning Supervision of the Execution of Judgments and Decisions, S. 155.

1398 Annual Report 2017 Concerning Supervision of the Execution of Judgments and Decisions, S. 155.

1399 HUDOC, Status of Execution (Kehayov Group of Cases/BUL): EGMR Neshkov u.a./BUL, Urt. v. 27.01.2015, 36925/10 u.a., zuletzt abgerufen am 25.04.2020. 
Strategie und der Aktionsplan für die Verbesserung der medizinischen Versorgung in den Gefängnissen umzusetzen. ${ }^{1400}$

Auch der Implementierungsprozess des Piloturteils Varga u.a./HUN1401 ist noch nicht abgeschlossen (István Gábor Kovács Group). Die Überwachung des Piloturteils Varga u.a./HUN findet im Verfahren der enhanced supervision statt. $^{1402}$

Nachdem das Ministerkomitee zuvor festgestellt hatte, dass einige der Beschwerdeführer der Implementierungsgruppe noch immer unter inadäquaten Haftbedingungen untergebracht waren und Ungarn bezüglich dieser Beschwerdeführer noch weitere Abhilfemassnahmen zu treffen hatte, ${ }^{1403}$ stellte es fest, dass sich nun die Situation einiger dieser Beschwerdeführer verbessert hat; Informationen zu weiteren Beschwerdeführern stehen aber nach wie vor aus. ${ }^{1404}$ Auf genereller Ebene wurde zur Abhilfe der Verletzung von Art. 13 EMRK i.V.m. Art. 3 EMRK sowohl ein präventiver als auch ein kompensatorischer (finanzielle Kompensation) Rechtsbehelf geschaffen. ${ }^{1405}$ Zur Überprüfung der Funktionsfähigkeit dieser Rechtsbehelfe in der Praxis forderte das Ministerkomitee aktualisierte Informationen einschliesslich umfassender statistischer Daten über den Ausgang der präventiven und kompensatorischen Verfahren. ${ }^{1406}$ Die Einführung dieser Rechtsbehelfe haben es dem Gerichtshof ermöglicht, 6’000 Fälle an die nationalen Behörden zurückzuweisen. ${ }^{1407}$

Hinsichtlich der Verletzung von Art. 3 EMRK selbst hat Ungarn bereits einige generelle Abhilfemassnahmen ergriffen, um der Überbelegung zu begegnen. Ungarn führte im Sinne einer Wiedereingliederungsmassnahme

1400 HUDOC, Status of Execution (Kehayov Group of Cases/BUL): EGMR Neshkov u.a./BUL, Urt. v. 27.01.2015, 36925/10 u.a., zuletzt abgerufen am 25.04.2020.

1401 EGMR - Varga u.a./HUN, Urt. v. 10.03.2015, 14097/12 u.a.

1402 Annual Report 2017 Concerning Supervision of the Execution of Judgments and Decisions, S. $157 \mathrm{f}$.

1403 Annual Report 2017 Concerning Supervision of the Execution of Judgments and Decisions, S. 158.

1404 HUDOC, Status of Execution (Istvan Gabor Kovacs Group of Cases/HUN): EGMR - Varga u.a./HUN, Urt. v. 10.03.2015, 14097/12 u.a., zuletzt abgerufen am 25.04.2020.

1405 Annual Report 2017 Concerning Supervision of the Execution of Judgments and Decisions, S. 158.

1406 HUDOC, Status of Execution (Istvan Gabor Kovacs Group of Cases/HUN): EGMR - Varga u.a./HUN, Urt. v. 10.03.2015, 14097/12 u.a., zuletzt abgerufen am 25.04.2020.

1407 Annual Report 2017 Concerning Supervision of the Execution of Judgments and Decisions, S. 13. 
die Möglichkeit ein, die letzten zehn bis zwölf Monate der Freiheitsstrafe zu Hause zu verbüssen. Ferner wurde die Anwendung des Hausarrestes gefördert und die Anforderungen hierzu gesenkt; damit zusammenhängend wurden die Möglichkeiten der elektronischen Überwachung ausgebaut. ${ }^{1408}$ Diese Massnahmen führten zu einem gewissen Rückgang der Zahl in Untersuchungshaft befindlicher Personen. ${ }^{1409}$ Das Ministerkomitee fordert Ungarn auf, seine diesbezüglichen Bemühungen fortzusetzen; ungarische Staatsanwälte und Richter hätten so oft als möglich auf alternative Strafen auszuweichen. ${ }^{1410}$

Die Umsetzung des Piloturteils W.D./BEL ${ }^{1411}$ ist noch pendent und wird im Verfahren der enhanced supervision durchgeführt (Group of Cases L.B./ BEL). In einzelnen Verfahren, in denen die individuellen Massnahmen bereits ergriffen wurden, schloss das Ministerkomitee die Überwachung ab. Hinsichtlich einiger Beschwerdeführer, welche auf Bewährung entlassen wurden, fordert das Ministerkomitee die Behörden auf, das Ministerkomitee über den weiteren Verlauf zu informieren. Hinsichtlich des festgestellten strukturellen Problems einer inadäquaten psychiatrischen Versorgung psychisch kranker Inhaftierter hat Belgien bereits gewisse Massnahmen ergriffen. Insbesondere wurden neue forensisch-psychiatrische Zentren eingeführt, die eine adäquate Betreuung der Inhaftierten bieten. Weiter wurde eine Verringerung der Anzahl Internierter in den Gefängnissen festgestellt. Auch eine Effizienzsteigerung des präventiven Rechtsbehelfs vor der Sozialschutzkammer (social protection chamber) konnte festgestellt werden. ${ }^{1412}$ Das Ministerkomitee begrüsste die getroffenen generellen Massnahmen und ermutigte Belgien - in Anlehnung an die Empfehlung des nationalen Ombudsmanns und die Empfehlungen des CPT -, einen nationalen Mechanismus zur Verhütung von Folter einzurichten, um alle

1408 Annual Report 2017 Concerning Supervision of the Execution of Judgments and Decisions, S. $157 \mathrm{f}$.

1409 HUDOC, Status of Execution (Istvan Gabor Kovacs Group of Cases/HUN): EGMR - Varga u.a./HUN, Urt. v. 10.03.2015, 14097/12 u.a., zuletzt abgerufen am 25.04.2020.

1410 HUDOC, Status of Execution (Istvan Gabor Kovacs Group of Cases/HUN): EGMR - Varga u.a./HUN, Urt. v. 10.03.2015, 14097/12 u.a., zuletzt abgerufen am 25.04.2020; vgl. auch bereits Annual Report 2017 Concerning Supervision of the Execution of Judgments and Decisions, S. 158.

1411 EGMR - W.D./BEL, Urt. v. 06.09.2016, 73548/13.

1412 HUDOC, Status of Execution (L.B. Group of Cases/BEL): EGMR - W.D./BEL, Urt. v. 06.09.2016, 73548/13, zuletzt abgerufen am 25.04.2020. 
Haftanstalten und forensisch-psychiatrischen Zentren zu überwachen. ${ }^{1413}$ Weiter sollen die Bemühungen zur Verbesserung der Gesundheitsversorgung in den Gefängnissen fortgesetzt und die diesbezüglich angekündigte Reform umgesetzt werden. ${ }^{1414}$

Auch das Piloturteil Rezmiveș u.a./ROM ${ }^{1415}$ wird vom Ministerkomitee im Verfahren der enhanced supervision überwacht (Bragadireanu Group). Rumänien reichte in einem ersten Schritt fristgerecht im Januar 2018 einen Aktionsplan mit einem Fahrplan für die Ergreifung genereller Massnahmen in den Jahren 2018-2024 ein. ${ }^{1416}$ Das Ministerkomitee stellte in der Folge fest, dass bedeutende Fortschritte insbesondere durch eine Verringerung der Überbelegung erzielt werden konnten. Rumänien ergriff etwa Massnahmen zur Stärkung des Bewährungsdienstes, zur Erreichung einer besseren Verteilung der Inhaftierten innerhalb des Strafvollzugs, zur Einstellung zusätzlicher Mitarbeiter in der Gefängnisverwaltung und zum Ausbau der zur Verfügung stehenden Aktivitäten ausserhalb der Zelle. Das Ministerkomitee weist aber auch darauf hin, dass es wichtig ist, diese Massnahmen fortzusetzen und weitere Massnahmen auf hoher politischer Ebene zu ergreifen, um zu einer raschen, umfassenden und nachhaltigen Lösung dieses Problems zu gelangen. ${ }^{1417}$ In formeller Hinsicht fordert das Ministerkomitee die Ausarbeitung eines neuen Aktionsplans, der von der neuen Regierung nach ihrem Amtsantritt rasch gebilligt werden kann; dies aufgrund der innenpolitischen Entwicklung, d.h. der Beendigung der Amtszeit der Regierung nach einem Misstrauensvotum. ${ }^{1418}$ Hinsichtlich der Verpflichtung nach Art. 13 EMRK i.V.m. Art. 3 EMRK drückt das Ministerkomitee sodann tiefstes Bedauern aus, dass ein Ausgleichsmechanismus in Form einer Strafminderung ohne Bereitstellung von alternativen, der Konvention entsprechenden Massnahmen erfolgte. Das Ministerkomitee befürchtet, dass es deshalb, zusätzlich zu den bereits 6'000

1413 HUDOC, Status of Execution (L.B. Group of Cases/BEL): EGMR - W.D./BEL, Urt. v. 06.09.2016, 73548/13, zuletzt abgerufen am 25.04.2020.

1414 HUDOC, Status of Execution (L.B. Group of Cases/BEL): EGMR - W.D./BEL, Urt. v. 06.09.2016, 73548/13, zuletzt abgerufen am 25.04.2020.

1415 EGMR - Rezmiveş u.a./ROM, Urt. v. 25.04.2017, 61467/12 u.a.

1416 Vgl. zum Ganzen: Annual Report 2017 Concerning Supervision of the Execution of Judgments and Decisions, S. 160.

1417 HUDOC, Status of Execution (Bragadireanu Group of Cases/ROM): EGMR Rezmiveş u.a./ROM, Urt. v. 25.04.2017, 61467/12 u.a., zuletzt abgerufen am 25.04.2020.

1418 HUDOC, Status of Execution (Bragadireanu Group of Cases/ROM): EGMR Rezmiveș u.a./ROM, Urt. v. 25.04.2017, 61467/12 u.a., zuletzt abgerufen am 25.04.2020. 
Anträgen, zu einem neuen, massiven Zustrom an Beschwerden vor dem Gerichtshof kommen wird, was eine Bedrohung für die Wirksamkeit des Konventionssystems sei. ${ }^{1419}$

Das Umsetzungsverfahren des Piloturteils Sukachov/UKR ${ }^{1420}$ steht noch ganz am Anfang; in einem ersten Schritt hat die Ukraine einen Aktionsplan eingereicht. ${ }^{1421}$

\section{Umsetzungsverfahren der Piloturteile}

Nachdem der Implementierungsstatus der einzelnen Urteile dargelegt wurde, wird der Blick auf den Ablauf des Implementierungsverfahrens gerichtet. Der Fokus wird hier nicht auf den Inhalt der einzelnen Implementierungsschritte gelegt, sondern auf einzelne Faktoren, welche Aufschluss insbesondere über das Bestehen eines Dialogs zwischen Ministerkomitee und Konventionsstaat geben können. Dies soll in der folgenden Analyse Aussagen darüber zulassen, ob die Implementierungsverfahren der Urteile tatsächlich eng und aktiv vom Ministerkomitee betreut wurden, wie es deren Zuteilung zur enhanced supervision vermuten lässt. Von Relevanz scheint diesbezüglich insbesondere, ob der Konventionsstaat Aktionspläne resp. Aktionsberichte eingereicht hat, ob die Implementierungsfortschritte an den Menschenrechtssitzungen des Ministerkomitees aufgegriffen wurden und ob die Abgeordneten Beschlüsse gefasst haben bzw. ob sogar mittels Zwischenresolutionen der politische Druck auf die Mitgliedstaaten verschärft wurde. Hinsichtlich der Aussenwirkung des Implementierungsprozesses wird weiter auch berücksichtigt, ob NGOs bzw. nationale Menschenrechtsinstitutionen sich mittels Stellungnahmen in den Umsetzungsprozess eingebracht haben und ob die Konventionsstaaten auf diese mit eigenen Stellungnahmen reagiert haben.

Es wurden hier nur jene Dokumente berücksichtigt, welche in der Datenbank des Execution Department (Hudoc-Exec) unter der jeweiligen Verfahrensnummer abrufbar sind. Besondere Vorsicht bei der Interpretation ist deshalb insbesondere bei jenen Urteilen geboten, welche in Imple-

1419 HUDOC, Status of Execution (Bragadireanu Group of Cases/ROM): EGMR Rezmiveş u.a./ROM, Urt. v. 25.04.2017, 61467/12 u.a., zuletzt abgerufen am 25.04.2020: Im Urteil Rezmiveș u.a./ROM selbst wurde allerdings keine Verletzung von Art. 13 EMRK i.V.m. Art. 3 EMRK festgestellt. Generelle Massnahmen diesbezüglich waren folglich nicht gefordert.

1420 EGMR - Sukachov/UKR, Urt. v. 30.01.2020, 14057/17.

1421 Action Plan v. 06.07.2020, DH-DD (2020) 625. 
mentierungsgruppen, d.h. gemeinsam mit weiteren Urteilen, überwacht werden.

Im Implementierungsverfahren Orchowski/POL und Norbert Sikorski/POL hat Polen insgesamt fünf Aktionspläne resp. Aktionsberichte eingereicht. ${ }^{1422} \mathrm{Zu}$ den Aktionsplänen resp. Aktionsberichten wurden vom Human Rights Defender sowie von der Helsinki Foundation for Human Rights und der Association for Legal Intervention Stellungnahmen eingereicht, auf welche die polnische Regierung jeweils ihrerseits wieder Stellung genommen hat. ${ }^{1423}$ Der Implementierungsprozess wurde sodann an verschiedenen Menschenrechtssitzungen aufgegriffen. In zwei Beschlüssen forderten die Abgeordneten ergänzende Informationen zum Implementierungsprozess. ${ }^{1424}$

Im Implementierungsverfahren rund um Ananyev u.a./RUS hat Russland bislang sieben Aktionspläne resp. Aktionsberichte eingereicht. ${ }^{1425} \mathrm{Zu}$ den Aktionsplänen resp. Aktionsberichten wurden fünf Stellungnahmen von NGOs eingereicht (Penal Reform International PRI/Association of Russian Lawyers for Human Rights/drei von der Public Verdict Foundation). ${ }^{1426}$ Auch in diesem Umsetzungsverfahren wurden anlässlich mehrerer Menschenrechtssitzungen die bisher ergriffenen Implementierungs-

1422 Action Plan / Action Report v. 17.03.2010, DH-DD (2011) 627; Action Plan / Action Report v. 12.09.2011, DH-DD (2011) 709; Consolidated Action Report v. 08.07.2014, DH-DD (2014) 950; Updated Action Report v. 28.04.2016, DHDD (2016) 560; Consolidated Action Report v. 21.06.2016, DH-DD (2016) 791.

1423 Communication from the Office of the Human Rights Defender and Reply of the Government v. 16.11.2011, DH-DD (2011) 1108; Communication from the Helsinki Foundation for Human Rights and the Association for Legal Intervention v. 21.06.2013, DH-DD (2013) 798; Communication from Poland v. 16.07.2013 in Reply to the Communication v. 21.06.2013, DHDD (2013) 86.

1424 Decision v. 14.09.2011, CM/Del/Dec (2011) 1120/7; Decision v. 07.03.2013, CM/Del/Dec (2013) 1164/17.

1425 Action Plan v. 10.10.2012, DH-DD (2012) 1009; Action Plan v. 14.08.2013, DH-DD (2013) 936; Action Plan v. 30.04.2014, DH-DD (2014) 580; Action Plan v. 10.08.2015, DH-DD (2015) 862; Action Plan v. 19.04.2017, DH-DD (2017) 467; Action Plan v. 26.04.2017, DH-DD (2017) 560; Action Plan v. 29.04.2019, DH-DD (2019) 473.

1426 Communication from the Penal Reform International PRI v. 27.09.12, DHDD (2012) 1026; Communication from the Association of Russian Lawyers for Human Rights v. 29.11.2012, DH-DD (2013) 92; Communication from the Public Verdict Foundation v. 07.10.2013, DH-DD (2014) 44; Communication from the Public Verdict Foundation v. 26.05.2017, DH-DD (2017) 668; Communication from the Public Verdict Foundation v. 30.04.2019, DH-DD (2019) 517. 
massnahmen thematisiert. Es erfolgten bislang sieben Beschlüsse der Abgeordneten, in welchen Russland unter anderem bisweilen aufgefordert wurde, weitere Informationen zum Umsetzungsstand bereit zu stellen. ${ }^{1427}$

Während des Umsetzungsprozesses von Torreggiani u.a./ITA reichte Italien insgesamt drei Aktionspläne resp. Aktionsberichte ein. ${ }^{1428}$ Es erfolgten sechs Stellungnahmen von NGOs (L'Altro Diritto/vier von Radicali Italiani/Nonviolent Radical Party), auf welche Italien bisweilen selber wieder Stellung nahm. ${ }^{1429}$ Auch die Umsetzung dieses Piloturteils wurde in mehreren Menschenrechtssitzungen aufgenommen. Es ergingen von den Abgeordneten drei Beschlüsse, in denen etwa weitere Informationen oder ein konsolidierter Aktionsplan gefordert wurde. ${ }^{1430}$ Mit Beschluss vom 4. Dezember 2014 wurden die Fälle in Anbetracht der bereits erzielten Fortschritte in die standard supervision übergeleitet. ${ }^{1431}$

Im Implementierungsprozess rund um Neshkov u.a./BUL reichte Bulgarien bisher neun Aktionspläne resp. Aktionsberichte ein. ${ }^{1432}$ Es erfolgten

1427 Decision v. 06.06.2012, CM/Del/Dec (2012) 1144/17; Decision v. 26.09.2012, CM/Del/Dec (2012) 1150/17; Decision v. 06.12.2012, CM/Del/Dec (2012) 1157/20; Decision v. 07.03.2013, CM/Del/Dec (2013) 1164/24; Decision v. 05.06.2014， CM/Del/Dec (2014) 1201/15; Decision v. 07.06.2017, CM/Del/Dec (2017) 1288/H46-24; Decision v. 06.06.2019, CM/Del/Dec (2019) 1348/H46-23.

1428 Action Report v. 29.11.2013, DH-DD (2013) 1368; Updated Action Plan v. 15.09.2014, DH-DD (2014) 1143; Action Report v. 20.11.2015, DH-DD (2015) 1251.

1429 Communication from the Nonviolent Radical Party Transnational and Transparty v. 10.04.2014, DH-DD (2014) 585; Communication from the Radicali Italiani v. 23.05.2014, DH-DD (2014) 740; Communication from the Radicali Italiani v. 22.09.2014, DH-DD (2014) 1131; Communication from the Radicali Italiani v. 24.10.2014, DH-DD (2014) 1325; Communication from the Radicali Italiani v. 05.05.2015, DH-DD (2015) 562; Communication from L'Altro Diritto v. 02.12.2015, DH-DD (2015) 1371; Communication de l'Italie v. 07.10.2013, DH-DD (2013) 1119; Communication from Italy v. 03.04.2014, DH-DD (2014) 471; Communication des autorités v. 25.01.2016, DH-DD (2016) 106.

1430 Decision v. 06.03.2014, CM/Del/Dec (2014) 1193/11; Decision v. 05.06.2014, CM/Del/Dec (2014) 1201/10; Decision v. 04.12.2014, CM/Del/Dec (2014) 1214/11.

1431 Decision v. 04.12.2014, CM/Del/Dec (2014) 1214/11.

1432 Bei den Aktionsberichten handelte es sich teilweise auch um Ergänzungen bisheriger Aktionsberichte: Action Plan v. 06.07.2015, DH-DD (2015) 755; Revised Action Plan v. 31.08.2015, DH-DD (2015) 755-rev; Revised Action Plan v. 04.01.2016, DH-DD (2016) 25; Addendum to the Revised Action Plan v. 16.02.2016, DH-DD (2016) 25-add; Addendum to the Revised Ac- 
zwei Stellungnahmen von NGOs (Bulgarian Helsinki Committee und Bulgarian Prisoners' Association) und eine Stellungnahme des Beschwerdeführers Neshkov selbst; auf eine der Stellungnahmen antwortete Bulgarien. ${ }^{1433}$ Der Implementierungsfortschritt wurde auch in diesem Verfahren in Menschenrechtssitzungen thematisiert. Es ergingen von den Abgeordneten vier Beschlüsse, in denen Bulgarien bisweilen aufgefordert wurde, weitere Informationen einzureichen. ${ }^{1434}$

Im Umsetzungsverfahren rund um Varga u.a./HUN reichte Ungarn bislang sieben Aktionspläne resp. Aktionsberichte ein. ${ }^{1435}$ Es erfolgten fünf Stellungnahmen von NGOs, allesamt vom Hungarian Helsinki Committee, auf welche hin Ungarn selbst eine Stellungnahme verfasste. ${ }^{1436}$ Der Implementierungsprozess wurde wiederum in mehreren Menschenrechtssitzungen thematisiert. Die Abgeordneten verabschiedeten drei Beschlüsse, in denen sie von Ungarn bisweilen weitere bzw. aktuellere Informationen

tion Plan (DH-DD (2016) 25, DH-DD (2016) 25-add) v. 29.04.2016, DH-DD (2016) 587; Updated Action Plan v. 16.12.2016, DH-DD (2017) 5; Addendum v. 26.01.2017 to Updated Action Plan v. 16.12.2016, DH-DD (2017) 5-add; Action Plan v. 21.12.2017, DH-DD (2018) 13; Addendum to the Action Plan v. 19.01.2018, DH-DD (2018) 13-add.

1433 Communication from the Bulgarian Helsinki Committee v. 16.09.2016, DHDD (2016) 1098; Communication from the Bulgarian Prisoners' Association v. 16.03.2018 and Reply from the Bulgarian Authorities, DH-DD (2018) 301; Communication from the Applicant v. 17.05.2017, DH-DD (2017) 593.

1434 Decision v. 24.09.2015, CM/Del/Dec (2016) 1250/H46-6; Decision v. 08.-10.03.2016, CM/Del/Dec (2016) 1250/H46-6; Decision v. 10.03.2017, CM/Del/Dec (2017) 1280/H46-9; Decision v. 15.03.2018, CM/Del/Dec (2018) 1310/H46-4.

1435 Updated Action Plan v. 14.12.2015, DH-DD (2015) 1373; Revised Action Plan v. 01.07.2016, DH-DD (2016) 855; Updated Action Plan v. 14.10.2016, DHDD (2016) 1223; Action Plan v. 03.03.2017, DH-DD (2017) 313; Action Plan v. 15.09.2017, DH-DD (2017) 1012; Action Plan v. 15.06.2018, DH-DD (2018) 690; Action Plan v. 08.03.2019, DH-DD (2019) 311.

1436 Communication from the Hungarian Helsinki Committee v. 28.08.2017, DHDD (2017) 1003; Communication from the Hungarian Helsinki Committee v. 20.01.2020, DH-DD (2020) 76; Communication from the Hungarian Helsinki Committee v. 21.01.2020, DH-DD (2020) 96; Reply from the Authorities v. 04.02.2020 to Communications from NGOs (DH-DD (2020) 76, DH-DD (2020) 96), DH-DD (2020) 107; Communication from the Hungarian Helsinki Committee v. 21.04.2020, DH-DD (2020) 396; Communication from the Hungarian Helsinki Committee v. 30.06.2020, DH-DD (2020) 624. 
forderten oder Ungarn dazu anhielten, ihre Bemühungen weiter vordringlich zu behandeln und die Implementierung nicht zu verlangsamen. ${ }^{1437}$

Im Umsetzungsverfahren von W.D./BEL reichte Belgien drei Aktionspläne ein. ${ }^{1438}$ Auch in diesem Verfahren wurden die Implementierungsfortschritte an mehreren Menschenrechtssitzungen thematisiert. Es erfolgten drei Beschlüsse der Abgeordneten des Ministerkomitees, in denen Belgien unter anderem aufgefordert wurde, aktualisierte Informationen einzureichen. ${ }^{1439}$

Im Implementierungsprozess Rezmiveş u.a./ROM reichte Rumänien einen Aktionsplan sowie mehrere Aktualisierungen zu diesem ein. ${ }^{1440}$ Die NGO Association for the Defence of Human Rights in Romania reichte eine Stellungnahme ein. ${ }^{1441}$ Auf Nachfrage des Execution Department an das Sekretariat des Ministerkomitees erklärte dieses, dass aktuell 6'625 Beschwerden vor dem Gerichtshof hängig seien, welche inadäquate Haftbedingungen in Rumänien betreffen würden. ${ }^{1442}$ Der Umsetzungsprozess wurde in mehreren Menschenrechtssitzungen aufgegriffen. Es erfolgten fünf Beschlüsse, in denen die Abgeordneten unter anderem festhielten, dass sie zutiefst bedauern, dass die Regierung durch ein Misstrauensvotum daran gehindert worden sei, einen neuen Aktionsplan vorzulegen, welcher den Zusicherungen anlässlich der letzten Menschenrechtssitzung entsprechen würde. ${ }^{1443}$ Weiter wurde Rumänien aufgefordert, das Ministerkomi-

1437 Decision v. 08.-10.03.2016, CM/Del/Dec (2016) 1250/H46-11; Decision v. 07.06.2017, CM/Del/Dec (2017) 1288/H46-16; Decision v. 15.03.2018, CM/Del/Dec (2018) 1310/H46-8.

1438 Plan d'action v. 15.02.2017, DH-DD (2017) 186; plan d'action révisé v. 07.11.2017, DH-DD (2017) 186-rev; plan d'action révisé v. 21.06.2018, DHDD (2018) 649.

1439 Décision H46-6 v. 10.03.2017, Groupe L.B. u. W.D/BEL, CM/Del/Dec (2017) 1280/H46-6; décision H46-5 v. 07.12.2017, L.B. group and W.D./BEL, CM/Del/Dec (2017) 1302/H46-5; décision H46-3 v. 20.09.2018, Groupe L.B. et W.D./BEL, CM/Del/Dec (2018) 1324/H46-3.

1440 Action plan v. 25.01.2018, DH-DD (2018) 90; Update v. 22.03.2018 to the Action Plan v. 25.01.2018, DH-DD (2018) 330; Update v. 31.10.2018 to the Action Plan v. 25.01.2018, DH-DD (2018) 1069; Update v. 23.04.2019 to the Action Plan v. 25.01.2018, DH-DD (2019) 440; Update v. 04.11.2019 to the Action Plan v. 25.01.2018, DH-DD (2019) 1273; Communication from the Authorities v. 14.02.2020, DH-DD (2020) 143.

1441 Communication from the Association for the Defence of Human Rights in Romania - Helsinki Committee v. 21.10.2019, DH-DD (2019) 1263.

1442 Letter from the Registrar v. 12.02.2020, DH-DD (2020) 141.

1443 Decision v. 15.03.2018, CM/Del/Dec (2018) 1310/H46-13; Decision v. 06.12.2018, CM/Del/Dec (2018) 1331/H46-23, Decision v. 06.06.2019, 
tee über die relevanten Entwicklungen auf dem Laufenden zu halten. Rumänien wurde auch darüber in Kenntnis gesetzt, dass eine erneute Prüfung der erzielten Fortschritte spätestens anlässlich seiner Tagung vom Dezember 2020 erfolgen wird und dass anlässlich dieser auch der Erlass einer Zwischenresolution in Betracht gezogen werden wird. ${ }^{1444}$

Im Implementierungsprozess des Piloturteils Sukachov/UKR hat die Ukraine bislang einen Aktionsplan eingereicht. ${ }^{1445}$

\section{Analyse der Umsetzung der Piloturteile}

\subsection{Priorisierungsverfahren und Stand der Umsetzung}

Die einzelnen Piloturteile befinden sich in unterschiedlichen Implementierungsstadien. Während die Überwachung der Urteile Orchowski/POL, Norbert Sikorski/POL und Torreggiani u.a./ITA abgeschlossen ist, wurden die anderen Piloturteile erst unvollständig umgesetzt.

Zunächst ist festzustellen, dass sämtliche noch nicht vollständig umgesetzten Piloturteile dem Enhanced supervision-Verfahren zugeteilt sind. Diese Zuteilung entspricht auch den Zuteilungskriterien; gerade Piloturteile sollen im Implementierungsverfahren priorisiert überwacht und begleitet werden. Das Implementierungsverfahren wird in sämtlichen Piloturteilsverfahren tatsächlich auch eng und aktiv vom Ministerkomitee überwacht. ${ }^{1446}$ Zwischen Konventionsstaat und Ministerkomitee besteht ein ständiger Dialog insbesondere in Form von Aktionsplänen resp. Aktionsberichten der Konventionsstaaten und Beschlüssen der Abgeordneten des Ministerkomitees. Die Implementierungsfortschritte wurden denn auch in allen Verfahren bereits mehrmals an den Menschenrechtssitzungen aufgegriffen. Von der Möglichkeit, den politischen Druck mit Zwischenresolutionen zu erhöhen, hat das Ministerkomitee in diesen Fällen (noch) keinen Gebrauch gemacht. Dies könnte sich in naher Zukunft aber ändern, haben die Abgeordneten des Ministerkomitees im Implementierungsverfahren des Piloturteils Rezmiveș u.a./ROM Rumänien doch bereits ausdrücklich

CM/Del/Dec (2019) 1348/H46-21; Decision v. 05.12.2019, CM/Del/Dec (2019) 1362/H46-19; Decision v. 05.03.2020, CM/Del/Dec (2020) 1369/H46-23.

1444 Decision v. 05.03.2020, CM/Del/Dec (2020) 1369/H46-23.

1445 Action Plan v. 06.07.2020, DH-DD (2020) 625.

1446 Nicht berücksichtigt werden kann hier das Umsetzungsverfahren von Sukachov/UKR, da dieses noch ganz am Anfang steht. 
darauf hingewiesen, dies in Betracht zu ziehen. Umgekehrt ist das Ministerkomitee auch durchaus bereit, die Überwachungsintensität zu verringern, wenn der Implementierungsfortschritt dies zulässt. So waren etwa die Fälle im Implementierungsverfahren Torreggiani u.a./ITA in das Verfahren der standard supervision überführt worden, als schon beträchtliche Umsetzungsfortschritte erzielt worden waren. Das Implementierungsverfahren dieses Piloturteils konnte später auch abgeschlossen werden, was zeigt, dass die Umteilung in die standard supervision berechtigt war. Die Korrelation von Überwachungsintensität und Implementierungsstand ist auch Ausdruck des Subsidiaritätsgedankens.

Da die Piloturteile nicht alle gleichzeitig gefällt wurden ${ }^{1447}$, können deren Implementierungsstände nicht direkt miteinander verglichen werden. Dennoch können aus der Gesamtschau der Urteile gewisse generelle Aussagen getroffen werden.

Hinsichtlich des Implementierungsstands der noch nicht vollständig umgesetzten Piloturteile sind im Folgenden drei Bereiche zu unterscheiden: (1) der Implementierungsstand der aufgrund der Art. 3 EMRK zu ergreifenden individuellen Massnahme, (2) der Implementierungsstand der generellen Massnahme aufgrund der Verletzung von Art. 13 EMRK i.V.m. Art. 3 EMRK und (3) der Implementierungsstand der generellen Massnahme aufgrund der Verletzung von Art. 3 EMRK selbst.

1. Der Implementierungsstand der zu ergreifenden individuellen Abhilfemassnahmen der Verletzung von Art. 3 EMRK weist insgesamt noch Defizite auf. Die Konventionsstaaten haben zwar in der Regel individuelle Massnahmen für einzelne Beschwerdeführer getroffen, andere Beschwerdeführer der jeweiligen Implementierungsgruppen sind aber entweder nachweislich noch immer unter inadäquaten Haftbedingungen untergebracht, oder dem Ministerkomitee liegen keine genaueren Informationen hinsichtlich ihrer aktuellen Unterbringung vor, so dass davon auszugehen ist, dass deren Haftsituation noch nicht den Anforderungen des Gerichtshofs entsprechen. Das Ministerkomitee fordert folglich die betroffenen Konventionsstaaten auf, ihren diesbezüglichen Verpflichtungen nachzukommen und dem Ministerkomitee für dessen Beurteilung genügende Informationen zur Verfügung zu stellen.

1447 Orchowski/POL, Norbert Sikorski/POL (22.10.2009); Ananyev u.a./RUS (10.01.2012); Torreggiani u.a./ITA (08.01.2013); Neshkov u.a./BUL (27.01.2015); Varga u.a./HUN (10.03.2015); W.D./BEL (06.09.2016); Rezmiveş u.a./ROM (25.04.2017); Sukachov/UKR (30.01.2020). 
2. Der Implementierungsstand hinsichtlich der generellen Abhilfe der Verletzungen von Art. 13 EMRK i.V.m. Art. 3 EMRK ist insgesamt weit fortgeschritten. In Bulgarien und Ungarn wurden die erforderlichen Rechtsbehelfe bereits eingeführt. Russland ist den Forderungen des Gerichtshofs allerdings erst teilweise nachgekommen, indem ein präventiver Rechtsbehelf eingeführt wurde. Die Einführung eines kompensatorischen Rechtsbehelfs steht hier indes noch aus. Auch von Belgien erwartet das Ministerkomitee hinsichtlich des kompensatorischen Rechtsbehelfs weitere Informationen über die Rechtsprechung zu Entschädigungsmitteln. Im Urteil Rezmiveş u.a./ROM wurde keine Verletzung von Art. 13 EMRK i.V.m. Art. 3 EMRK festgestellt, so dass auch keine dahingehenden generellen Massnahmen zu erwarten sind. Gleichwohl merkt das Ministerkomitee im Rahmen des Überwachungsprozesses hier kritisch an, dass ein bestehender kompensatorischer Rechtsbehelf in Rumänien abgeschafft wurde.

3. Der Implementierungsstand der generellen Abhilfemassnahmen zur festgestellten Verletzung von Art. 3 EMRK weist in allen offenen Piloturteilen noch Defizite auf. Keiner der Konventionsstaaten ist aber vollständig untätig geblieben. Die bereits getroffenen Massnahmen sind weiterzuführen und weitere Massnahmen zu ergreifen. ${ }^{1448}$

\subsection{Implementierungsdauer}

Mit Blick auf die Urteilsdaten der noch nicht vollständig umgesetzten Piloturteile (Ananyev u.a./RUS: 10.01.2012; Neshkov u.a./BUL: 27.01.2015; Varga u.a./HUN: 10.03.2015; W.D./BEL: 06.09.2016; Rezmiveş u.a./ROM: 25.04.2017; Sukachov/UKR: 30.01.2020) bleibt zu konstatieren, dass der Implementierungsprozess Jahre in Anspruch nehmen kann. Auch die beiden bereits abgeschlossenen Implementierungsverfahren haben bis zu ihrem Abschluss einiges an Zeit benötigt. In Polen dauerte der Durchsetzungsprozess rund sieben Jahre, während er in Italien rund drei Jahre erforderte. ${ }^{1449}$ Die Umsetzung des Piloturteils Orchowski/POL verdeutlicht aber immerhin, dass auch nach mehreren Jahren konstanter Implementierungsbemühungen noch mit einer genügenden Umsetzung gerechnet wer-

1448 Vgl. etwa Annual Report 2017 Concerning Supervision of the Execution of Judgments and Decisions, S. 155, 158.

1449 Orchowski/POL (Urt. v. 22.10.2009 und Implementierungsprozess förmlich abgeschlossen im Jahr 2016) und Torreggiani u.a./ITA (Urt. v. 08.01.2013 und Implementierungsprozess förmlich abgeschlossen im Jahr 2016). 
den kann, was auch auf die Implementierung der noch nicht vollständig umgesetzten Piloturteile hoffen lässt.

Der lang andauernde und teilweise nur schleppend vorangehende Implementierungsprozess ist insbesondere mit Blick auf die Schwere der fortdauernden Menschenrechtsverletzungen gleichwohl bedenklich. Die Umsetzung der Massnahmen ist auch mit Blick auf die grosse Anzahl Betroffener äusserst dringlich. ${ }^{1450}$ Eine unverzügliche Implementierung setzt in jedem Fall zunächst unabdingbar den Kooperationswillen des betroffenen Konventionsstaates voraus, sind die Massnahmen doch gerade von ihm zu treffen und können sie nicht mit Zwang gegen seinen Willen durchgeführt werden. Die Kooperationsbereitschaft setzt in aller Regel auch die Bereitschaft zur Finanzierung der Massnahmen voraus. Die lange Dauer der Umsetzung ist indes nicht zwingend (nur) auf eine fehlende Kooperationsbereitschaft zurückzuführen. Vielmehr beruhen gerade bei Piloturteilen die Konventionsverletzungen auf strukturellen bzw. systemischen Problemen, welchen gerade immanent ist, dass eine Abhilfeschaffung bezüglich der Situation nicht innert kürzester Zeit möglich ist. ${ }^{1451}$ So sind langfristige Massnahmen gefordert, die entsprechend viel Zeit bei der Umsetzung in Anspruch nehmen. Grund für die vergleichsweise relativ zügige Implementierung Italiens dürfte vor diesem Hintergrund auf der einen Seite der vorhandene Kooperationswille Italiens und auf der anderen Seite die schon vor Urteilsfällung laufenden generellen Massnahmen zur Behebung des Überbelegungsproblems gewesen sein.

Die Priorisierungsbemühungen des Ministerkomitees mit der Anwendung des Verfahrens der enhanced supervision und der tatsächlich engen und aktiven Betreuung des Umsetzungsverfahrens können auf die Kooperationsbereitschaft der Konventionsstaaten hinwirken und den Implementierungsprozess mit konstruktiven Beiträgen zur Eignung der zu ergreifenden Massnahmen effizienter machen. Gleichwohl kann die Implementierung eine lange Zeit in Anspruch nehmen, welche auf umfassende Reformmassnahmen zurückzuführen ist und damit nicht $a$ priori als Zeichen gesehen

1450 Vgl. auch Glas HR\&ILD 2019, 73 (81).

1451 Vgl. zum Kooperationswillen auch Bосcн S. 1; vgl. weiter auch die Studie von Anagnostou/Mungiu-Pippidi EJIL 2014, 205 (227): Der politische Umsetzungswille sei ein zentraler Faktor bei der Umsetzung von EGMR-Urteilen. Dieser alleine sei aber noch kein Garant für eine effektive Implementierung. Es komme vielmehr auch der innerstaatlichen „rechtlichen Infrastruktur“ und der Effektivität der Regierung eines Staates eine wichtige Bedeutung zu. Im Ergebnis übereinstimmend, aber zur gewählten Methode von Anagnostou/ Mungiu-Pippidi kritisch vgl. auch Voeten EJIL 2014, 229 ff. 
werden kann, dass die Effektivierungsbemühungen des Ministerkomitees nicht greifen würden.

Für die Implementierungsdauer von geringerer Bedeutung dürfte der Verbindlichkeitsgrad der Formulierungen in den Urteilsgründen und im Urteilstenor sein. Den Mitgliedstaaten dürfte die Dringlichkeit der Angelegenheit und der Nachdruck sowohl des Gerichtshofs als auch des Ministerkomitees schon allein durch die Anwendung des Piloturteilsverfahrens und der Zuteilung in das Enhanced supervision-Verfahren klar sein.

\subsection{Strukturelles Problem und Art der ergriffenen Massnahmen}

Der Gerichtshof fordert bei Vorliegen struktureller resp. systemischer Probleme das Ergreifen nicht nur oberflächlicher Massnahmen, sondern auch solcher, die das Problem von Grund auf bekämpfen und dessen Ursache beheben. Die Konventionsstaaten nehmen in ihren Aktionsplänen i.d.R. die Massnahmen auf, welche der Gerichtshof in den Urteilsgründen vorgeschlagen hat. Auch das Ministerkomitee nimmt bei der Würdigung der Aktionspläne die Urteilsgründe des konkreten Urteils zum Ausgangspunkt, verweist aber auch auf erfolgreiche Abhilfemassnahmen, welche andere Länder bei derselben Konventionsverletzung getroffen haben. ${ }^{1452}$ Insgesamt folgt das Ministerkomitee dem umfassenden Ansatz des Gerichtshofs, wenn es die von den Konventionsstaaten eingereichten Aktionspläne resp. Aktionsberichte evaluiert und wenn es mittels Beschlüssen auf tiefgreifende Reformmassnahmen hinwirkt.

Die Urteile Orchowski/POL, Norbert Sikorski/POL und Torreggiani u.a./ITA zeigen, dass auch strukturelle Probleme im Bereich der Überbelegung und weiterer inadäquater Haftbedingungen so weit behoben werden können, dass das Ministerkomitee den Implementierungsprozess als abgeschlossen betrachtet und seine Überwachung einstellt.

Das bessere Fazit des Implementierungsstandes der Verletzungen von Art. 13 EMRK i.V.m. Art. 3 EMRK könnte darauf beruhen, dass hier die Ergreifung legislativer Massnahmen genügt und die Anforderungen an die Abhilfeschaffung sehr klar sind. Gerade der Umstand, dass zur Einführung selbst keine zu hohen finanziellen Hürden überwunden werden müssen,

1452 Annual Report 2017 Concerning Supervision of the Execution of Judgments and Decisions, S. 161: Bei der Einführung eines kompensatorischen Rechtsbehelfs in Russland verweist das Ministerkomitee etwa auf das italienische Modell eines „Reduktionssystems“. 
kommt der Implementierung wohl zusätzlich zugute. Kompensatorische Rechtsbehelfe können zwar zur Folge haben, dass der Konventionsstaat finanziellen Ausgleich für erlittene inadäquate Haftbedingungen schaffen muss und so doch finanziell belangt wird. Die monetäre Entschädigung ist allerdings nur eine Art der kompensatorischen Möglichkeiten. Kostenneutrale Folgen, wie eine Strafminderung, sofern eine Strafe noch nicht vollständig verbüsst wurde, werden vom Ministerkomitee sogar bevorzugt gesehen. Fehlt ein solcher Rechtsbehelf auf nationaler Ebene befreit ihn dies sodann nicht von seiner Verpflichtung, eine gerechte Entschädigung zu bezahlen; diese kann dann vor dem Gerichtshof gemäss Art. 41 EMRK fällig werden. Effektive Rechtsbehelfe sind weiter grundsätzlich sowohl im Interesse des EGMR als auch im Interesse des Mitgliedstaates, weil Beschwerden dann innerstaatlich geklärt werden können. Nur so kann die grundsätzliche Subsidiarität des Strassburger Systems zum Tragen kommen. Die Vorteile der Umsetzung dieser Abhilfemassnahmen liegen damit auf der Hand und dürften sich auch auf den Kooperationswillen der betroffenen Konventionsstaaten auswirken, was wiederum den meist fortgeschrittenen Implementierungsstand dieser Massnahmen erklärt.

\subsection{Generelle Implementierungsmassnahmen und Folgerechtsprechung}

Der Implementierungsstand der Piloturteile hat auch Auswirkungen auf die Folgerechtsprechung. So hat die Einführung eines effektiven Rechtsbehelfs i.S.v. Art. 13 i.V.m. Art. 3 EMRK etwa die Unzulässigkeit weiterer Beschwerden aufgrund von Art. 35 Abs. 1 EMRK zur Folge. ${ }^{1453}$ Auch in materieller Hinsicht schlägt sich der Implementierungsstand in der Folgerechtsprechung nieder. Die bisher nur unvollständige Implementierung eines Piloturteils und die Notwendigkeit der Ergreifung weiterer genereller Massnahmen ziehen weitere Verurteilungen des betroffenen Konventionsstaates nach sich. ${ }^{1454}$ Erneute Verurteilungen erinnern den Konven-

1453 EGMR - Stella u.a./ITA, Entsch. v. 16.09.2014, 49169/09 u.a., Rn. 35 ff.; EGMR - Rexhepi u.a./ITA, Entsch. v. 16.09.2014, 47180/10, Rn. 35 ff.

1454 Vgl. bspw. EGMR (GK) - Simeonovi/BUL, Urt. v. 12.05.2017, 21980/04, Rn. 85 ff.: An der Verletzung von Art. 3 EMRK änderte auch der Hinweis der Regierung auf die laufenden Gefängnisreform nichts. Vgl. auch EGMR - Stanculeanu/ROM, Urt. v. 09.01.2018, 26990/15, Rn. 34 ff.: Der Gerichtshof nimmt hier direkt Bezug auf das Piloturteil Rezmiveş u.a./ROM. Vgl. ferner EGMR Dudchenko/RUS, Urt. v. 07.11.2017, 37717/05, Rn. 116; EGMR - Bandur/HUN, Urt. v. 05.07.2016, 50130/12, Rn. 34 ff. Es ist aber zu bedenken, dass auch 
tionsstaat wiederkehrend an seine Implementierungspflichten. Auch die nach einem Piloturteil ergangenen Urteile werden dem Ministerkomitee zugeleitet, welches die Umsetzung auch dieser Urteile überwacht. Umgekehrt schützt das abgeschlossene Implementierungsverfahren eines Piloturteils selbstverständlich nicht vor weiteren Verfahren gegen den Konventionsstaat, wenn trotz genereller Massnahmen vereinzelt wieder inadäquate Haftbedingungen herrschen. Durch die Implementierung genereller Massnahmen sollten im betreffenden Mitgliedstaat aber immerhin nicht mehr Verhältnisse herrschen, die auf strukturelle bzw. systemische Defizite schliessen lassen.

\subsection{Bedeutung weiterer Akteure im Implementierungsverfahren}

Korrelierend zur Rechtsprechung des Gerichtshofs, in welcher er auf Sachverhaltsebene Bezug auf Berichte internationaler resp. europäischer Gremien nimmt, zeigt sich eine solche Verbindung auch in der Durchsetzungsphase der EGMR-Urteile. Die Berichte internationaler Organisationen sind nämlich auch Informationsquelle des Ministerkomitees etwa bei der Beurteilung des Implementierungsstands. ${ }^{1455}$

Insbesondere der Implementierungsprozess des Piloturteils Neshkov u.a./BUL zeigt auch, dass die Umsetzung des Urteils des Gerichtshofs gleichzeitig an die Umsetzung eines Soft Law-Standards (im konkreten Fall CPT-Standard) geknüpft werden kann. ${ }^{1456}$ Die Verpflichtung zur Umsetzung auch des höheren CPT-Standards erwächst zwar nicht aus Art. 46 EMRK i.V.m. Art. 1 EMRK, kann aber bei einer Finanzierungsunterstützung etwa durch den HRTF ausbedungen werden. So zielte das durch den HRTF unterstützte Implementierungsprojekt in Bulgarien ausdrücklich nicht nur auf die Umsetzung der Urteile des Gerichtshofs, sondern auch auf eine künftige Einhaltung des CPT-Standards ab.

bei einer Abhilfeschaffung zunächst weitere Verurteilungen erfolgen können, wenn sie sich auf vergangene Sachverhalte - etwa vor der Abhilfeschaffung der Konventionsverletzung - beziehen.

1455 Annual Report 2017 Concerning Supervision of the Execution of Judgments and Decisions, S. 161: Russland wird aufgefordert, dem Ministerkomitee weitere CPT-Berichte zu russischen Haftanstalten zukommen zu lassen.

1456 Support for the Implementation of the European Court of Human Rights Judgments and European Committee for the Prevention of Torture and Inhuman or Degrading Treatment of Punishment Standards and Recommendations in Bulgaria (Prison Reform), zuletzt abgerufen am 25.04.2020. 
Ausschlaggebend für diese Bezugnahme auf den CPT-Standard auch in der Durchsetzungsphase dürfte hier das Urteil Neshkov u.a./BUL selbst gewesen sein, scheint doch gerade in diesem Urteil die Arbeit des CPT massgebend zu sein. Der Gerichtshof bezog sich nicht nur bei der Frage des Vorliegens eines strukturellen Problems ${ }^{1457}$, sondern gerade auch bei der Thematisierung der Notwendigkeit genereller Massnahmen ${ }^{1458}$ auf die Arbeit des CPT. Gleichwohl hat sich das Ministerkomitee aufgrund seiner Rolle (Art. 46 Abs. 2 EMRK) auf die Überwachung des Urteils des Gerichtshofs zu beschränken. Geht der CPT-Standard über den Standard des Gerichtshofs hinaus, hat das Ministerkomitee die Überwachung einzustellen, sobald das Urteil des Gerichtshofs umgesetzt ist, und zwar auch dann, wenn der CPT-Standard noch nicht erreicht sein sollte. Es wird sich in Zukunft zeigen müssen, wie das Ministerkomitee die Situation in diesem Implementierungsverfahren handhaben wird.

Auch im Implementierungsverfahren des Urteils W.D./BEL hat sich die Bedeutung von Empfehlungen anderer Akteure für das Ministerkomitee gezeigt. Nachdem der nationale Ombudsmann und das CPT die Bedeutung der Unterzeichnung des OPCAT hervorgehoben haben, ermutigte auch das Ministerkomitee Belgien zur Einführung eines nationalen Präventionsgremiums zur Verhütung von Folter. Mittlerweile informierte Belgien das Ministerkomitee, dass es das OPCAT unterzeichnet habe und die Einsetzung eines nationalen Präventionsgremiums zur Verhütung von Folter plane. Auch in diesem Implementierungsverfahren wird besonderes Augenmerk auf die Rolle des Ministerkomitees i.S.v. Art. 46 Abs. 2 EMRK zu legen sein, wenn es um den Abschluss des Durchsetzungsverfahrens geht. Das Ministerkomitee kann Belgien wohl ermutigen, ein nationales Präventionsgremium i.S.d. OPCAT einzuführen, und hat eine allfällige Einführung sicherlich auch positiv in seine Gesamtwürdigung einzubeziehen, kann den Abschluss des Implementierungsverfahrens wohl aber nicht von der Einführung eines solchen Gremiums abhängig machen, würde dies doch über die Verpflichtung des Konventionsstaats i.S.v. Art. 46 Abs. 1 EMRK i.V.m. Art. 1 EMRK hinausgehen. Wäre Belgien nicht dazu bereit gewesen, das OPCAT zu unterzeichnen und sich damit völkerrechtlich zu verpflichten, einen solchen Präventionsmechanismus einzuführen (Art. 3 OPCAT), hätte es diesbezüglich mit dem Ministerkomitee den Dialog suchen und seinen Standpunkt darlegen können.

1457 EGMR - Neshkov u.a./BUL, Urt. v. 27.01.2015, 36925/10, Rn. 269.

1458 EGMR - Neshkov u.a./BUL, Urt. v. 27.01.2015, 36925/10, Rn. 276. 
3.6 Stellungnahmen von NGOs, nationalen Menschenrechtsinstitutionen und Beschwerdeführern

Die gesteigerte Transparenz des Implementierungsverfahrens ermöglicht durch die Publikation sämtlicher Informationen, dass NGOs, nationale Menschenrechtsorganisationen und auch die Beschwerdeführer zum Implementierungsprozess Stellung nehmen können (Regel 91459). In vielen der Implementierungsverfahren wurde diese Möglichkeit insbesondere von regionalen NGOs ergriffen. Die Praxis der Konventionsstaaten, ob sie nun ihrerseits auf diese Stellungnahmen mit eigenen Stellungnahmen reagieren oder nicht, variiert von Konventionsstaat zu Konventionsstaat. Während Polen etwa auch zu beiden eingereichten Stellungnahmen selbst Stellung nahm, blieb Russland diesbezüglich passiv. Andere Konventionsstaaten wie Italien, Bulgarien oder Ungarn nahmen zu einem Teil der Stellungnahmen selbst direkt Stellung. Die Beteiligung mehrerer NGOs in den meisten Fällen lässt jedenfalls den Schluss zu, dass nicht nur die Verfahren vor dem Gerichtshof, sondern auch die Umsetzungsverfahren im Nachgang an die Urteile eine gewisse Öffentlichkeitswirkung entfalten und auch die Implementierungsfortschritte regional mit Interesse beobachtet und einer kritischen Würdigung unterzogen werden. Der politische Druck zur zügigen Implementierung dürfte durch die Stellungnahmen regelmässig zunehmen.

Einzig im Fall Neshkov u.a./BUL hat auch der Beschwerdeführer selbst von der Möglichkeit Gebrauch gemacht, eine eigene Stellungnahme einzureichen. Die Stellungnahmen von Beschwerdeführern haben sich i.d.R. auf die Umsetzung individueller Massnahmen und auf die Zahlung der finanziellen Entschädigung zu beschränken. Es erstaunt, dass die Beschwerdeführer so selten von dieser Möglichkeit Gebrauch gemacht haben, zumal der Implementierungsstand der zu ergreifenden individuellen Massnahmen zur Abhilfe gegen die Verletzung von Art. 3 EMRK insgesamt noch Defizite aufweist. Selbstverständlich wirkt das Ministerkomitee aber auch ohne Stellungnahmen der Beschwerdeführer darauf hin, dass alle individuellen Massnahmen ergriffen werden, was sich auch darin manifestiert, dass das Ministerkomitee in zahlreichen Beschlüssen die Konventionsstaaten aufforderte, weitere Informationen betreffend einzelne Beschwerdeführer einzureichen. Gleichwohl könnte der politische Druck zur Umsetzung der

1459 Rules of the Committee of Ministers for the Supervision of the Execution of Judgments and of the Term of Friendly Settlements. 
individuellen Massnahme mit einer Stellungnahme nochmals verschärft werden.

\subsection{Bedeutung des infringement proceeding}

Der noch ungenügende Implementierungsstand der Piloturteile könnte Grund für die Einleitung eines infringement proceeding durch das Ministerkomitee sein. Grundsätzlich kann das Verfahren nach Art. 46 Abs. 4 EMRK sowohl bei prinzipieller Nichtumsetzung eines Urteils als auch bei nur schleppender Umsetzung angewendet werden. ${ }^{1460} \mathrm{Im}$ Bereich der inadäquaten Haftbedingungen kam es seit seiner Einführung allerdings noch nie zur Anwendung. Keiner der Konventionsstaaten hat sich prinzipiell geweigert, das Piloturteil umzusetzen, und in allen Konventionsstaaten wurden bereits gewisse Abhilfemassnahmen ergriffen. Die Anwendung des Verfahrens nach Art. 46 Abs. 4 EMRK könnte den politischen Druck auf die entsprechenden Konventionsstaaten erhöhen. Da der Druck auf den betroffenen Konventionsstaat zur weiteren Implementierung insbesondere durch die Anwendung des Piloturteilsverfahrens, die Überwachung durch die enhanced supervision und durch die grosse Zahl weiterer hängiger Fälle ohnehin bereits gross sein dürfte, wären meines Erachtens auch bei der Anwendung des infringement proceeding keine schnelleren Umsetzungsfortschritte zu erwarten. ${ }^{1461}$

\subsection{Zwischenfazit}

Insgesamt kann festgehalten werden, dass bis anhin erst zwei Piloturteile vollständig umgesetzt sind. Die restlichen Piloturteile sind alle dem Überwachungsverfahren der enhanced supervision zugeteilt; deren Umsetzung wird eng und aktiv vom Ministerkomitee begleitet. Die vollständige Implementierung dieser Urteile setzt insbesondere die Ergreifung weiterer genereller Massnahmen zur Beseitigung der strukturellen Gründe der Überbelegung voraus. Eine vollständige Umsetzung erfordert wegen der fehlenden Gestaltungskraft des Gerichtshofs und des Ministerkomitees

1460 De Londras/Dzehtsiarou International \& Comparative Law Quarterly 2017, 467 (474 ff.).

1461 Insgesamt kritisch zum infringement proceeding De Londras/DzeHTSIarou International \& Comparative Law Quarterly 2017, 467 ff. 
dabei immer den Kooperationswillen des Konventionsstaates. Es ist an ihm, die konkreten Massnahmen unter der Aufsicht des Ministerkomitees vorzuschlagen und in die Praxis umzusetzen. Es wurde festgestellt, dass die Implementierung von Piloturteilen zu inadäquaten Haftbedingungen in aller Regel eine längere Zeit in Anspruch nimmt. Dies ist allerdings vor allem mit Blick auf das Bestehen eines strukturellen Problems, d.h. der Notwendigkeit langfristiger und kostenintensiver Massnahmen, zu würdigen. ${ }^{1462}$ Die Beispiele Orchowski/POL und Norbert Sikorski/POL haben aber verdeutlicht, dass auch nach mehreren Jahren durchaus noch mit dem Abschluss des Implementierungsprozesses zu rechnen ist. Weiter ist auch im Implementierungsprozess eine Wechselwirkung zwischen der Arbeit des Gerichtshofs und jener des Ministerkomitees sowie internationaler und europäischer Gremien festzustellen, wobei in Zukunft ein Augenmerk darauf zu richten sein wird, dass vom Ministerkomitee zum Abschluss des Implementierungsverfahren nicht mehr verlangt wird, als es seine Rolle i.S.v. Art. 46 Abs. 2 EMRK zulässt. Es hat sich schliesslich gezeigt, dass nicht nur das Verfahren vor dem Gerichtshof, sondern auch das Implementierungsverfahren eine gewisse Öffentlichkeitswirkung erzielt, wobei insbesondere regionale NGOs immer wieder von der Möglichkeit Gebrauch gemacht haben, eigene Stellungnahmen einzureichen, in denen sie den Implementierungsfortschritt kritisch würdigen.

\section{Umsetzung übriger Urteile}

Aufgrund der immens grossen Zahl von Urteilen im Bereich inadäquater Haftbedingungen kann es in diesem Kapitel nicht zielführend sein, den Implementierungsstand aller Urteile zu untersuchen. Vielmehr geht es darum, überblicksartig generelle Aussagen über bestehende Tendenzen zu machen.

1462 Den Erfolg der Anwendung des Piloturteilsverfahrens nur an der Anzahl abgeschlossener Implementierungsverfahren zu messen, würde deshalb zu kurz greifen. Vgl. auch GLAS HR\&ILD 2019, 73 (81 f.). 
1. Parallelfälle vs. Fälle ohne Konnex zu Piloturteilen und die Bedeutung des Priorisierungsverfahrens

Neben den Piloturteilen lassen sich die übrigen Urteile in zwei Fallgruppen unterteilen. Zum einen sind es Parallelfälle zu Piloturteilen. Zum anderen können es Urteile gegen Konventionsstaaten sein, gegen die noch kein Piloturteil ergangen ist, resp. Urteile in Themengebieten, in denen überhaupt noch keine Piloturteile gefällt wurden.

Die Umsetzung Ersterer ist eng mit der Umsetzung des dazugehörigen Piloturteils verknüpft. Nicht selten erfolgt die Umsetzung sodann im Rahmen einer „Implementierungsgruppe“. Die für das Piloturteilsverfahren charakteristische Ergreifung genereller Massnahmen kann zugleich Abhilfemassnahme in parallelen Urteilen sein. Darüber hinausgehende individuelle Massnahmen können aber weiter erforderlich bleiben. Demgegenüber erfolgt die Implementierung ausserhalb eines Piloturteilsverfahrens in der Regel nicht im Kontext der Anordnung genereller Massnahmen. Auch in diesen Fällen kann die Überwachung der Durchsetzung aber in Implementierungsgruppen erfolgen. ${ }^{1463}$

Die Jahresberichte des Ministerkomitees verdeutlichen, dass im Bereich inadäquater Haftbedingungen eine grosse Zahl von Urteilen auch ausserhalb des Piloturteilsverfahrens im Verfahren der enhanced supervision überwacht werden. ${ }^{1464}$ Diese Zuteilungen zum priorisierten Überwachungsverfahren entspricht der dem Art. 3 EMRK inhärenten Schwere der Konventionsverletzung, welche eine enge Überwachung zur schnellstmöglichen und vollständigen Behebung unabhängig vom Vorhandensein eines strukturellen bzw. systemischen Problems erfordert. Durch die konsequente Anwendung des Verfahrens der enhanced supervision stehen die weiteren Verletzungen von Art. 3 EMRK bei inadäquaten Haftbedingungen den einschlägigen Piloturteilsverfahren resp. ihren Parallelfällen hinsichtlich der Art des Implementierungsverfahrens also in nichts nach.

1463 Vgl. etwa Ashot Harutyunyan Group, Nisiotis Group, Siasios u.a. Group, Ciorap Group etc.: Annual Report 2017 Concerning Supervision of the Execution of Judgments and Decisions, S. $154 \mathrm{ff}$.

1464 Vgl. bspw.: EGMR - Ashot Harutyunyan/ARM, Urt. v. 15.06.2010, 34334/04; EGMR - Insanov/AZE, Urt. v. 14.03.2013, 16133/08; EGMR - Martzaklis u.a./ GRE, Urt. v. 09.07.2015, 20378/13; vgl. auch Annual Report 2019 Concerning Supervision of the Execution of Judgments and Decisions, S. 50: 8\% aller Verfahren im Verfahren der enhanced supervision betreffen Haftbedingungen oder die medizinische Versorgung. 


\section{Status der Umsetzung und die Art ergriffener Massnahmen}

Die Fülle an Urteilen bringt es mit sich, dass sich die Urteile zu inadäquaten Haftbedingungen in den verschiedensten Implementierungsstadien befinden. Bei einer Reihe von Urteilen hat das Ministerkomitee bereits eine vollständige Umsetzung festgestellt und die Überwachung abgeschlossen, ${ }^{1465}$ während viele andere Urteile sich noch mitten im Implementierungsprozess befinden. Ist das Monitoringverfahren noch pendent, heisst dies, dass das Ministerkomitee vom Konventionsstaat die Ergreifung weiterer Abhilfemassnahmen fordert. Für eine Analyse, welche Massnahmen durch den Konventionsstaat getroffen und vom Ministerkomitee als genügend eingestuft wurden, eignen sich abgeschlossene resp. zumindest weit fortgeschrittene Implementierungsverfahren besser, weshalb in der Folge nur auf solche eingegangen wird.

\subsection{Umsetzungsverfahren und Notwendigkeit genereller Massnahmen}

Die bereits abgeschlossenen Implementierungsverfahren verdeutlichen, dass die Umsetzung eines Urteils auch ausserhalb des Piloturteilsverfahrens neben den zu ergreifenden individuellen Massnahmen oft mit der Ergreifung genereller Abhilfemassnahmen einhergeht. ${ }^{1466}$ Dies selbst dann,

1465 Vgl. etwa EGMR - Tunis/EST, Urt. v. 19.12.2013, 429/12: Resolution CM/ ResDH (2016) 22, Execution of the Judgment Tunis/EST, vgl. auch Annual Report 2016 Concerning Supervision of the Execution of Judgments and Decisions, S. 162; EGMR - Dybeku/ALB, Urt. v. 18.12.2007, 41153/06; EGMR - Grori/ALB, Urt. v. 07.07.2009, 25336/04: Resolution CM/ResDH (2016) 273, Execution of the Judgments Dybeku/ALB u. Grori/ALB, vgl. Annual Report 2016 Concerning Supervision of the Execution of Judgments and Decisions, S. 157; EGMR - Ghavtadze/GEO, Urt. v. 03.03.2009, 23204/07: Resolution CM/ResDH (2014) 209, Execution of the Judgments Ghavtadze u.a./ GEO; EGMR - Jashi/GEO, Urt. v. 08.01.2013, 10799/06: Resolution CM/ResDH (2014) 162, Execution of the Judgment Jashi/GEO; EGMR - Kaprykowski u.a./POL, Urt. v. 21.09.2016, 23052/05: Resolution CM/ResDH (2016) 278, Execution of the Judgments Kaprykowski u.a./POL; EGMR - Kasperovičius/ LTU, Urt. v. 20.11.2012, 54872/08: Résolution CM/ResDH (2017) 34, Exécution de l'arrêt Kasperovičius/LTU; EGMR - Bulatović/MNE, Urt. v. 22.07.2014, 67320/10: Resolution CM/ResDH (2017) 35, Execution of the Judgment Bulatović/MNE.

1466 Vgl. etwa die Umsetzung folgender Urteile: EGMR - Dybeku/ALB, Urt. v. 18.12.2007, 41153/06; EGMR - Grori/ALB, Urt. v. 07.07.2009, 25336/04; EGMR - Tunis/EST, Urt. v. 19.12.2013, 429/12; EGMR - Kasperovičius/LTU, 
wenn die generellen Abhilfemassnahmen im Urteil durch den Gerichtshof nicht angeordnet wurden, was im Folgenden anhand zweier ausgewählter Beispiele aufgezeigt wird:

Estland hat etwa im Rahmen der Implementierung eines Urteils des Gerichtshofs zu ungenügenden Platzverhältnissen in der Haft ${ }^{1467}$ seine nationalen Strafvollzugsvorschriften dahingehend geändert, dass jedem Inhaftierten mindestens $3 \mathrm{~m}^{2}$ Platz anstatt der bisher national vorgesehenen 2,5 $\mathrm{m}^{2}$ zur Verfügung stehen müssen, was so auch in der Praxis umgesetzt wurde. ${ }^{1468}$ Auf legislativer Ebene wurde weiter festgelegt, dass die Zahl der Inhaftierten die für die entsprechende Haftanstalt vorgesehene Quote nie überschreiten darf. ${ }^{1469}$

Die Ergreifung der generellen Massnahme - hier die nationale Gesetzesänderung ${ }^{1470}$ - ist im Urteil selbst nicht ausdrücklich genannt, ist aber dennoch logische Konsequenz. Im vorliegenden Fall waren die Platzverhältnisse auf dem innerstaatlichen Rechtsweg nämlich anhand der bisherigen nationalen Bestimmung geprüft und für gesetzeskonform befunden worden (dem Inhaftierten standen mehr als die national gesetzlich vorgesehenen 2,5 $\mathrm{m}^{2}$ zur Verfügung). Der Gerichtshof stellte in der Folge eine Verletzung von Art. 3 EMRK fest (Unterschreiten der $3 \mathrm{~m}^{2}$ als starke Vermutung für einen Konventionsverstoss: vgl. Teil 3 Kap. 2 IV.), ohne dass er weitere Ausführungen zum nationalen Recht oder Ausführungen im Rahmen von Art. 46 EMRK machte. In den Urteilsgründen wurden sodann weder konkrete individuelle noch generelle Massnahmen genannt. Im Urteilstenor blieb es bei der Feststellung einer Verletzung von Art. 3 EMRK und der Festlegung einer Schadenersatzzahlung. Auch ohne diese Anordnung blieb Estland als einzige Möglichkeit nur die innerstaatliche Gesetzesänderung resp. die Nichtmehranwendung der besagten Norm, um weitere einschlägige Verurteilungen in Zukunft zu verhindern. Die von Estland ergriffene generelle Massnahme zur Durchsetzung des Urteils erstaunt deshalb nicht. Im Implementierungsverfahren von Tunis/EST

Urt. v. 20.11.2012, 54872/08; EGMR - Bulatović/MNE, Urt. v. 22.07.2014, 67320/10.

1467 EGMR - Tunis/EST, Urt. v. 19.12.2013, 429/12.

1468 Zum Ganzen vgl. Annual Report 2016 Concerning Supervision of the Execution of Judgments and Decisions, S. 162.

1469 Vgl. Annual Report 2016 Concerning Supervision of the Execution of Judgments and Decisions, S. 162.

1470 Regulation no. 72 of the Minister of Justice on the Internal Prison Rules (Vangla sisekorraeeskiri). 
hat Estland zwei Aktionspläne bzw. Aktionsberichte eingereicht. ${ }^{1471}$ Stellungnahmen von NGOs oder dem Beschwerdeführer erfolgten nicht. Seitens des Ministerkomitees wurden keine Beschlüsse gefällt. Eine besonders intensive Überwachung oder eine Verschärfung des politischen Drucks schien hier folglich auch für die Ergreifung der generellen Massnahmen nicht nötig gewesen zu sein.

Die Urteile Dybeku/ALB ${ }^{1472}$ und Grori/ALB ${ }^{1473}$ machen deutlich, dass in der Praxis generelle Massnahmen in Umsetzung eines EGMR-Urteils ausserhalb von Piloturteilsverfahren auch hinsichtlich festgestellter ungenügender medizinischer Versorgungssituationen Inhaftierter ergriffen werden. Albanien hat nämlich im Rahmen der Implementierung dieser beiden Urteile des Gerichtshofs zur künftigen Sicherstellung der adäquaten medizinischen Versorgung während der Haft legislative Anpassungen vorgenommen. ${ }^{1474}$ Die Gesetzesänderungen zielten darauf ab, die Vorschriften zur Diagnose, zur medizinischen Behandlung, zur Medikation und zum medizinischen Equipment auszubauen. ${ }^{1475}$ Darüber hinaus wurde die obligatorische Krankenversicherung für Inhaftierte eingeführt. ${ }^{1476}$ Auch diese Massnahmen ergriff Albanien, ohne dass der Gerichtshof in den beiden Urteilen bestimmte generelle Massnahmen in den Urteilsgründen nannte oder vertiefte Ausführungen zu den entsprechenden nationalen Rechtsakten machte. Auch im Urteilstenor hatte sich der Gerichtshof auf die Feststellung der Verletzung von Art. 3 EMRK und auf die Festlegung eines Schadensersatzes beschränkt. Konkrete individuelle oder generelle Massnahmen hatte der Gerichtshof nicht angeordnet. Die Umsetzung der Urteile Dybeku/ALB und Grori/ALB erfolgte im Verfahren der enhanced supervision. Albanien hat sechs Aktionspläne bzw. Aktionsberichte eingereicht. ${ }^{1477}$ Stellungnahmen von NGOs oder den Beschwerdeführern erfolgten nicht. Die Implementierungsfortschritte wurden an mehreren Men-

1471 Action Report v. 12.09.2014, DH-DD (2014) 1146; Revised Action Report v. 29.09.2015, DH-DD (2015) 1002.

1472 EGMR - Dybeku/ALB, Urt. v. 18.12.2007, 41153/06.

1473 EGMR - Grori/ALB, Urt. v. 07.07.2009, 25336/04.

1474 Law on the Rights and Treatment of Prisoners and Detainees v. 17.04.2014 sowie Mental Health Law v. April 2012.

1475 Zum Ganzen Annual Report 2016 Concerning Supervision of the Execution of Judgments and Decisions, 2016, S. 157.

1476 Annual Report 2016 Concerning Supervision of the Execution of Judgments and Decisions, S. 157.

1477 Action Plan / Action Report v. 09.11.2011, DH-DD (2011) 1041; Action Plan v. 10.04.2013, DH-DD (2013) 428; Action Report v. 03.07.2014, DH-DD (2014) 893; Revised Action Report v. 30.07.2014, DH-DD (2014) 974; Revised 
schenrechtssitzungen aufgegriffen, und die Abgeordneten haben zwei Beschlüsse erlassen, in denen sie Albanien anhielten, weitere Informationen einzureichen. ${ }^{1478}$ Dies, indem sie Albanien etwa aufforderten, dem Ministerkomitee ohne weitere Verzögerung einen aktualisierten Aktionsplan vorzulegen, der sämtliche fehlenden Informationen insbesondere hinsichtlich der Ergreifung der generellen Massnahmen zu enthalten habe. Das Ministerkomitee stand hier folglich in einem engen Dialog mit dem Konventionsstaat und wirkte auf diese Weise aktiv auf die Ergreifung auch der generellen Massnahmen hin.

Im Allgemeinen beschränkten sich auch ausserhalb von Piloturteilen die generellen Massnahmen nicht auf legislative Anpassungen. Vielmehr wurden im Rahmen der Durchsetzung einzelner EGMR-Urteile aufgrund festgestellter inadäquater Haftbedingungen i.S.v. Art. 3 EMRK ganze Vollzugseinrichtungen geschlossen ${ }^{1479}$, Renovationsarbeiten durchgeführt, ${ }^{1480}$ generell der Zugang zu den sanitären Einrichtungen und Aktivitäten ausserhalb der Zellen verbessert ${ }^{1481}$ und die Möglichkeit alternativer Strafen für leichtere Straftaten eingeführt. ${ }^{1482}$ Die Feststellung von Verletzungen von Art. 13 EMRK i.V.m. Art. 3 EMRK hatten auch ausserhalb von Piloturteilen die Einführung effektiver Beschwerdemöglichkeiten zur Folge. ${ }^{1483}$

Es bleibt folglich festzuhalten, dass auch die Implementierung der Urteile, welche ausserhalb eines Piloturteilsverfahrens ergangen sind, umfassende Reformen nach sich ziehen kann - dies selbst dann, wenn vom Gerichtshof weder in den Urteilsgründen konkrete Massnahmen thematisiert noch solche im Urteilstenor angeordnet worden sind. Die Überwa-

Action Report v. 09.07.2015, DH-DD (2015) 768; Revised Action Report v. 03.06.2016, DH-DD (2016) 739.

1478 Decision v. 07.03.2013, CM/Del/Dec (2013) 1164/3; Decision v. 25.09.2014, CM/Del/Dec (2014) 1208/1.

1479 EGMR - Kasperovičius/LTU, Urt. v. 20.11.2012, 54872/08: Résolution CM/ResDH (2017) 34, Exécution de l'arrêt Kasperovičius/LTU; EGMR - Kadikis/LAT, Urt. v. 04.05.2006, 62393/00: Resolution CM/ResDH (2016) 122, Execution of the Judgments Kadikis u.a./LAT.

1480 EGMR - Kadikis/LAT, Urt. v. 04.05.2006, 62393/00: Resolution CM/ResDH (2016) 122, Execution of the Judgments Kadikis u.a./LAT; EGMR - Mathew/ NED, Urt. v. 29.09.2005, 24919/03: Resolution CM/ResDH (2016) 12, Execution of the Judgment Mathew/NED.

1481 EGMR - Kasperovičius/LTU, Urt. v. 20.11.2012, 54872/08: Résolution CM/ResDH (2017) 34 Exécution de l'arrêt Kasperovičius/LTU.

1482 EGMR - Bulatović/MNE, Urt. v. 22.07.2014, 67320/10: Resolution CM/ResDH (2017) 35, Execution of the Judgment Bulatović/MNE.

1483 EGMR - Kadikis/LAT, Urt. v. 04.05.2006, 62393/00: Resolution CM/ResDH (2016) 122, Execution of the Judgments Kadikis u.a./LAT. 
chungsintensität des Ministerkomitees variiert dabei stark; ist ein Fall dem Enhanced supervision-Verfahren zugeteilt, erfolgt auch hier eine enge und aktive Begleitung durch das Ministerkomitee.

\subsection{Konsequenz der Gesamtbetrachtung bei der Durchsetzung des Urteils}

Der Gerichtshof hatte in seinem Urteil Insanov/AZE ${ }^{1484}$ unter anderem die Haftbedingungen der aserbaidschanischen Strafvollzugsanstalt Baku (Gebäude 1 und Gebäude 13) zu beurteilen. Die Haftbedingungen in beiden Gebäuden wurden vom Gerichtshof als Verletzung von Art. 3 EMRK qualifiziert. ${ }^{1485}$ Die Implementierung findet, wie zu erwarten, im Verfahren der enhanced supervision statt. ${ }^{1486} \mathrm{Im}$ Rahmen der Durchsetzung des ergangenen Urteils wurde das Gebäude 1 abgerissen und ersetzt. In Gebäude 13 wurden die sanitären Einrichtungen renoviert und ein Heizungssystem installiert. ${ }^{1487}$ Hinsichtlich der Überbelegung hatte das Ministerkomitee Aserbaidschan zunächst aufgefordert, nähere Informationen zur aktuellen Überbelegungssituation zur Verfügung zu stellen, und verwies im weiteren Dialog mit dem Konventionsstaat auch auf die Bedeutung der Gesamtumstände der Inhaftierung, wobei es ausführte, dass die Verletzung von Art. 3 EMRK auf den kumulativen Effekten der Überbelegung, der inadäquaten Sanitäreinrichtungen und des fehlenden Heizsystems beruhe. ${ }^{1488}$ Die Beseitigung der letzten beiden kumulativen Umstände hätte zur Folge, dass die Überbelegung für sich alleine wohl nicht mehr als Verstoss gegen Art. 3 EMRK anzusehen sei. Der Fokus weiterer Implementierungsarbei-

1484 EGMR - Insanov/AZE, Urt. v. 14.03.2013, 16133/08: Im konkreten Fall wurde neben der Verletzung von Art. 3 EMRK auch in verschiedener Hinsicht Verletzungen von Art. 6 EMRK festgestellt. Das vorliegende Implementierungsverfahren ist noch pendent, was allerdings an den festgestellten Verletzungen von Art. 6 EMRK liegt, hinsichtlich welcher noch keine genügenden Abhilfemassnahmen getroffen wurden. Die folgenden Ausführungen beschränken sich aber auf die Abhilfemassnahmen der Verletzung von Art. 3 EMRK.

1485 EGMR - Insanov/AZE, Urt. v. 14.03.2013, 16133/08, Rn. 110.

1486 Zum Ganzen vgl. Annual Report 2016 Concerning Supervision of the Execution of Judgments and Decisions, S. $158 \mathrm{f}$.

1487 Annual Report 2016 Concerning Supervision of the Execution of Judgments and Decisions, S. 159.

1488 Annual Report 2016 Concerning Supervision of the Execution of Judgments and Decisions, S. 159. 
ten sei deshalb nicht mehr auf die Überbelegungssituation zu legen. ${ }^{1489}$ Diese Ausführungen des Ministerkomitees sind vor dem Hintergrund zu würdigen, dass der Gerichtshof im Urteil zuvor festgehalten hatte, dass die Überbelegung und die mangelhafte Platzsituation durch ungehinderte Bewegungsfreiheit tagsüber kompensiert würden. ${ }^{1490}$

Die Argumentation des Ministerkomitees bei seiner Beurteilung des Implementierungsstandes zeigt deutlich, wie es sich an den Ausführungen des Gerichtshofs in den Urteilsgründen orientiert. Dies ist auch vor dem Hintergrund einleuchtend, dass die Verpflichtung der Konventionsstaaten bei der Implementierung des Urteils i.S.v. Art. 46 Abs. 1 EMRK i.V.m. Art. 1 EMRK nur so weit geht, den EMRK-widrigen Zustand zu beseitigen. Hat der Gerichtshof festgestellt, dass die Überbelegungssituation für sich alleine keine solche Verletzung bildet, kann das Ministerkomitee letztlich in dieser Hinsicht auch kein Tätigwerden des Konventionsstaates fordern. Das Ministerkomitee ist gerade nur mit Überwachung der Durchsetzung des Urteils betraut. Gleichwohl zeigt das vorliegende Beispiel deutlich die Auswirkungen der Zulässigkeit von Kompensationsmechanismen in der Durchsetzungsphase. Überspitzt formuliert vereiteln Kompensationsmechanismen hier Anreiz und Pflicht zur Ergreifung von Massnahmen gegen die bestehende (und unbestrittene) aber noch nicht Art.-3-EMRKwidrige Überbelegungssituation. Mit der Gewährung einer kompensierenden Bewegungsfreiheit und der Beseitigung kumulativer Elemente im Sinne der Instandsetzung sanitärer Einrichtungen und des Heizsystems ist der Umsetzung von Art. 3 EMRK vielmehr bereits Genüge getan. Das Problem der Überbelegung besteht auf diese Weise fort, gebilligt durch den Gerichtshof. Die präventive Funktion wird durch die Kompensationsrechtsprechung auch in der Durchsetzungsphase beschränkt.

\section{Implementierungsdauer}

Die Umsetzungsdauer und damit verbunden die Dauer des Überwachungsverfahrens durch das Ministerkomitee sind sehr unterschiedlich. Wurde das Überwachungsverfahren bisweilen nach wenigen Jahren ${ }^{1491}$

1489 Annual Report 2016 Concerning Supervision of the Execution of Judgments and Decisions, S. 159.

1490 EGMR - Insanov/AZE, Urt. v. 14.03.2013, 16133/08, Rn. 120.

1491 Vgl. etwa EGMR - Tunis/EST, Urt. v. 19.12.2013, 429/12: Resolution CM/ResDH (2016) 22, Execution of the Judgment Tunis/EST; EGMR - Bulatović/MNE, 
beendet, erfolgte die Feststellung einer vollständigen Durchsetzung bisweilen auch erst nach deutlich längerer Zeit. ${ }^{1492}$ Daran können auch in diesen Urteilen die Priorisierungsbemühungen des Ministerkomitees mit der Anwendung des Verfahrens der enbanced supervision und der bisweilen tatsächlich engen und aktiven Betreuung des Umsetzungsverfahrens nichts ändern. Die lange Dauer der Umsetzung auch der übrigen Urteile dürfte auf die teilweise umfassenden Reformen, welche auch hier ergriffen werden, zurückzuführen sein. Vor dem Hintergrund dieser Massnahmen erstaunt die lange Implementierungsdauer jedenfalls nicht, sind doch viele von ihnen langfristiger Natur und brauchen Zeit (vgl. auch bereits bei den Piloturteilen). Die Priorisierungsbemühungen sind jedenfalls auch hier geeignet, auf die Kooperationsbereitschaft der Konventionsstaaten hinzuwirken und das Implementierungsverfahren mit konstruktiven Beiträgen zu den zu ergreifenden Massnahmen möglichst effizient zu gestalten.

Eine über längere Zeit andauernde, dafür umfassende und tiefgreifende Implementierung ist einer schnellen, oberflächlichen und auf den Einzelfall begrenzten Implementierung jedenfalls vorzuziehen. Ist eine lange Implementierungsdauer jedoch mangelndem Kooperationswillen eines Konventionsstaates geschuldet, ist dies selbstredend aus einer menschenrechtlichen Sicht kritisch, ist doch aufgrund des Feststellungsurteils gerade eine effektive Umsetzung durch den Konventionsstaat ausschlaggebend. Wichtiger als die abstrakte Zeitspanne ist damit die Ernsthaftigkeit und Kontinuität der Implementierungsarbeiten.

\section{Zwischenfazit}

Insgesamt hat die Analyse der Implementierung der übrigen Urteile gezeigt, dass diese, wie schon die einschlägigen Piloturteile, in der Regel dem Verfahren der enhanced supervision zugeteilt sind und ein enger Dialog zwischen Konventionsstaat und Ministerkomitee entstehen kann. Ferner wurde deutlich, dass die Implementierung auch ausserhalb des Piloturteilsverfahrens und folglich ohne zwingende Feststellung eines strukturellen bzw.

Urt. v. 22.07.2014, 67320/10: Resolution CM/ResDH (2017) 35, Execution of the Judgment Bulatović/MNE.

1492 Vgl. etwa EGMR - Dybeku/ALB, Urt. v. 18.12.2007, 41153/06: Resolution CM/ ResDH (2016) 273, Execution of the Judgments Dybeku/ALB u. Grori/ALB; EGMR - Grori/ALB, Urt. v. 07.07.2009, 25336/04: Resolution CM/ResDH (2016) 273, Execution of the Judgments Dybeku u. Grori/ALB. 
systemischen Problems und ohne die explizite Nennung genereller Massnahmen in den Urteilsgründen resp. deren Anordnung im Urteilstenor langfristige Massnahmen und umfassende Reformen erfordern kann. In diesem Sinne ergreifen die Konventionsstaaten unter der Aufsicht des Ministerkomitees bisweilen zur Schaffung von Abhilfe gegen die bestehenden Konventionsverletzungen generelle Massnahmen, was letztlich auch der Prävention von künftigen Konventionsverletzungen dient. Die präventive Funktion wird allerdings durch die Auswirkungen der Gesamtbetrachtung in der Rechtsprechung des Gerichtshofs auch in der Durchsetzungsphase beschränkt. Die Dauer des Implementierungsprozesses und der Implementierungserfolg sind ähnlich wie in Piloturteilsverfahren. Wesentliche Unterschiede beim Implementierungsverfahren zwischen Piloturteilen und den übrigen Urteilen lassen sich insgesamt auch nicht ausmachen.

\section{Zwischenfazit - Phase nach dem Urteil}

Die Phase im Anschluss an das Urteil ist geprägt von der Zusammenarbeit des Gerichtshofs, des Ministerkomitees und der Konventionsstaaten. Das Ministerkomitee als Hauptmonitoringakteur übt dabei eine zentrale und vermittelnde Rolle aus. Es wurde aufgezeigt, dass das Ministerkomitee seine Arbeitsweise insbesondere durch die Einführung des Twin-trackVerfahrens optimiert hat. Aufgrund der Zuteilungskriterien werden Urteile zu inadäquaten Haftbedingungen in der Regel dem Verfahren der enhanced supervision zugeführt, unabhängig davon, ob es sich um Piloturteile handelt oder nicht. Dies hat eine Priorisierung der Umsetzungsverfahren und eine enge und aktive Begleitung des Verfahrens durch das Ministerkomitee zur Folge. Das Implementierungsverfahren wird entsprechend durch den Dialog zwischen Konventionsstaat und Ministerkomitee mittels Aktionsplänen resp. Aktionsberichten der Konventionsstaaten und Beschlüssen des Ministerkomitees geprägt, wobei insbesondere die Menschenrechtssitzungen des Ministerkomitees wesentlicher Bestandteil der Überwachung der Umsetzung der Urteile sind.

Insbesondere durch die Anwendung der Piloturteilsverfahren im Bereich der Überbelegung, in denen der Gerichtshof in den Urteilsgründen die zu ergreifenden generellen Massnahmen nennt und im Urteilstenor vor allem hinsichtlich der Verletzung von Art. 13 EMRK i.V.m. Art. 3 EMRK die Einführung effektiver sowohl präventiver als auch kompensatorischer Rechtsbehelfe verbindlich anordnet, greift der Gerichtshof in die Implementierungsphase vor. Sowohl Ministerkomitee als auch 
Konventionsstaat richten sich in der Praxis im Durchsetzungsverfahren bezüglich der zu ergreifenden generellen Massnahmen denn auch tatsächlich nach den Vorgaben des Gerichtshofs. Das Ministerkomitee fordert im Bereich unzureichender Haftbedingungen vom Konventionsstaat aber keineswegs nur bei der Implementierung der Piloturteile neben den individuellen Massnahmen auch generelle Massnahmen. Deshalb nehmen die Konventionsstaaten unter der Aufsicht des Ministerkomitees auch zur Umsetzung anderer Urteile, in denen unmenschliche oder erniedrigende Haftbedingungen festgestellt wurden, tiefgreifende Massnahmen vor. Die Bedeutung der Anordnung genereller Massnahmen durch den Gerichtshof scheint vor diesem Hintergrund nicht so gross, wie dies a priori zu erwarten wäre.

Auch hinsichtlich des Implementierungsstands und der Implementierungsdauer lassen sich keine wesentlichen Unterschiede zwischen Piloturteilen und weiteren Urteilen feststellen; auch ohne die Anwendung eines Piloturteilsverfahrens setzten die Konventionsstaaten den EMRK-Standard um. Gleichwohl erhöht ein Piloturteilsverfahren durch eine gewisse Prangerwirkung den politischen Umsetzungsdruck auf den Konventionsstaat. Die Öffentlichkeitswirkung, welche Piloturteile auch in der Umsetzungsphase entfalten, zeigt sich etwa darin, dass regionale NGOs regelmässig Stellungnahmen zum Implementierungsfortschritt an das Ministerkomitee einreichen.

Eine lange Implementierungsdauer ist aus einer menschenrechtlichen Perspektive kritisch, vor allem mit Blick auf die einer Verletzung von Art. 3 EMRK innewohnenden Schwere. Kontinuierliche, tiefgreifende, langfristige generelle Abhilfemassnahmen sind zwar zu begrüssen, gleichzeitig sind aber im Sinne eines effektiven Individualrechtsschutzes schnell wirksame individuelle Massnahmen zu ergreifen. Ein unbedingter Kooperationswille des Konventionsstaates ist vorauszusetzen, und Scheu vor grösseren finanziellen Investitionen in Strafvollzugsanstalten ist nicht angebracht. Es ist gerade auch Pflicht des Konventionsstaates, finanzielle Mittel zur Abhilfe gegen Konventionsverletzungen entweder selbst zur Verfügung zu stellen oder eine anderweitige Finanzierungshilfe zu organisieren (etwa Unterstützung durch den HRTF). Die bisherige Implementierungspraxis zeigt jedenfalls, dass keiner der Konventionsstaaten vollständig untätig bleibt; eine grundsätzliche Kooperationsbereitschaft scheint damit vorzuliegen. Verbesserungsbedürftig scheint in einigen Konventionsstaaten allerdings der Informationsfluss - auch was die Ergreifung individueller Massnahmen anbelangt -, musste das Ministerkomitee doch immer wieder um zusätzliche Informationen ersuchen. 
Ein infringement proceeding wurde im Bereich inadäquater Haftbedingungen noch nicht durchgeführt. Sein Nutzen bei Piloturteilen wäre durch den ohnehin bereits bestehenden hohen politischen Druck wohl begrenzt. Bei den übrigen Verfahren müsste sich sein Nutzen erst in der Praxis erweisen. Die Anwendung dieses Verfahrens ist in naher Zukunft im Bereich inadäquater Haftbedingungen nicht zu erwarten. Vor allem bei frappierenden Haftbedingungen und bei einem völlig untätigen Konventionsstaat wäre eine Anwendung jedoch denkbar.

Kapitel 5: Der Einfluss des EGMR in der Durchsetzungsphase - eine Judizialisierung des Implementierungsvorgangs?

Die ständige Weiterentwicklung des Implementierungsprozesses und das ausdrückliche Bekenntnis zur Zusammenarbeit des Ministerkomitees mit weiteren Akteuren und besonders mit dem Gerichtshof ${ }^{1493}$ haben in mehrerer Hinsicht zu einer zunehmenden Verflechtung des Ministerkomitees und des EGMR in der Implementierungsphase geführt, ${ }^{1494}$ weshalb die verschiedenen Durchsetzungsphasen im Folgenden noch einmal aufgegriffen werden. Dabei wird der Blick nun auf das Zusammenspiel zwischen Gerichtshof, Ministerkomitee und Konventionsstaat gerichtet und der Frage nachgegangen, ob eine zunehmende Judizialisierung des Implementierungsvorgangs festzustellen sei.

Indem der Gerichtshof in der Phase vor dem Urteil mit dem Erlass vorsorglicher Massnahmen die Schaffung vollendeter Tatsachen durch den Konventionsstaat verhindert, sind die angeordneten vorsorglichen Massnahmen auch Voraussetzung dafür, dass das Urteil durch das Ministerkomitee überhaupt noch umgesetzt werden kann. Der Gerichtshof schafft folglich schon in dieser frühen Phase die Voraussetzungen für ein Tätigwerden des Ministerkomitees. Eine weitergehende Einwirkung auf

1493 Insbesondere die Brüssel-Deklaration betonte die geteilte Verantwortung („shared responsibility“) aller Akteure bei der Durchsetzung der Urteile des Gerichtshofs: vgl. Brussels Declaration v. 27.03.2015. Vgl. auch etwa Annual Report 2017 Concerning Supervision of the Execution of Judgments and Decisions, S. 22.

1494 Annual Report 2011 Concerning Supervision of the Execution of Judgments and Decisions, S. 21; Karpenstein/Mayer/Breuer Art. 46 Rn. 75: Der Gerichtshof habe sich von seinem ehemals sehr strikten Rollenverständnis gelöst. Vgl. auch Sicilianos in: Seibert-Fohr/Villiger, S. 285 ff.; auch Glas HRLR 2014, 671 (680 ff.); vgl. ausf. auch Haider S. 225 ff. 
den Implementierungsprozess des späteren Urteils besteht durch die Anordnung vorsorglicher Massnahmen indes nicht. Der Spielraum des Konventionsstaats bei der Erfüllung seiner Durchsetzungspflicht i.S.v. Art. 46 EMRK, bzw. des Ministerkomitees bei der Überwachung dieses Prozesses, wird keineswegs beschnitten. Die spezifisch angeordnete vorsorgliche Massnahme gilt höchstens bis zum Urteilszeitpunkt und wird dann bei Feststellung eines Konventionsverstosses durch die Verpflichtung zur Urteilsumsetzung ersetzt. Eine Judizialisierung des Implementierungsprozesses kann diesbezüglich folglich nicht festgestellt werden. Aus rein praktischer Sicht liegt allerdings die Vermutung nahe, dass sich sowohl Konventionsstaat als auch Ministerkomitee bei der konkreten Umsetzung der definitiven Massnahme an der vorsorglichen Massnahme orientieren, zumindest wenn sich diese während der Dauer des Verfahrens bereits bewährt hat.

Ungleich stärker kann der Gerichtshof in die Implementierungsphase vorgreifen, indem er im Urteil selbst konkrete, sowohl individuelle als auch generelle Massnahmen anordnet. ${ }^{1495}$ Durch die dem Ministerkomitee in Art. 46 Abs. 2 EMRK zugeschriebene Rolle, welche sich auf die Überwachung der Umsetzung der EGMR Urteile beschränkt, beschneidet die Anordnung spezifischer Massnahmen durch den Gerichtshof - wie bereits dargelegt - nicht nur den Ermessensspielraum des betroffenen Staates, sondern auch jenen des Ministerkomitees bei seiner Beurteilung, ob der Konventionsstaat seinen Umsetzungspflichten genügend nachgekommen ist. Die vom Konventionsstaat eingereichten Aktionsberichte sind vom Ministerkomitee nämlich im Lichte des Urteils und der konkret angeordneten Massnahmen zu evaluieren. ${ }^{1496}$ In anderen Themengebieten hat sich in der Vergangenheit allerdings gezeigt, dass das Ministerkomitee zum Abschluss des Implementierungsverfahrens bisweilen mehr vom Konventionsstaat forderte als der Gerichtshof effektiv angeordnet hatte. ${ }^{1497}$ Umgekehrt

1495 Vgl. auch Glas HRLR 2014, 671 (682 ff.); Sicilianos in: Seibert-Fohr/Villiger, S. $285 \mathrm{ff}$.

1496 EGMR - Orchowski/POL, Urt. v. 22.10.2009, 17885/04, Rn. 148: „The respondent State remains free, subject to monitoring by the Committee of Ministers, to choose the means by which it will discharge its legal obligation under Article 46 of the Convention, provided that such means are compatible with the conclusions set out in the Court's judgment"; vgl. auch EGMR (GK) - Broniowskil POL, Urt. v. 22.06.2004, 31443/96, Rn. 192.

1497 Vgl. dazu ausf. Glas HR\&ILD 2019, 73 (86 ff. m.w.N.): Dies, wenn der Gerichtshof nur kompensierende Massnahmen anordne, das Ministerkomitee aber auch präventive Massnahmen von den Konventionsstaaten fordere sowie 
wurde in Einzelfällen das Implementierungsverfahren vom Ministerkomitee auch nach Ergreifen einer von der Anordnung des Gerichtshofs abweichenden Massnahme geschlossen. ${ }^{1498}$ Dies erweckt den Anschein, dass das Ministerkomitee nicht immer bereit scheint, sich durch die Anordnung spezifischer Massnahmen einschränken zu lassen, und vielmehr bereit ist, seine eigene Würdigung der Situation an die Stelle derjenigen des Gerichtshofs zu setzen. Mit Blick auf Art. 46 Abs. 2 EMRK ist ein solches Vorgehen des Ministerkomitees allerdings klar abzulehnen.

Bei den vorliegend untersuchten Fällen zu inadäquaten Haftbedingungen hat sich gezeigt, dass sich der Gerichtshof seiner subsidiären Rolle im Implementierungsverfahren durchaus bewusst ist und diese in den Urteilsgründen auch immer wieder betont. Wenn es um Überbelegungssituationen geht, nimmt sich der Gerichtshof bei der Anordnung der Abhilfemassnahmen denn auch stark zurück und benennt in den Urteilsgründen meist nur eine Auswahl möglicher Massnahmen. ${ }^{1499}$ Mit anderen Worten konzentriert sich der Gerichtshof mehr auf eine globale Betrachtung und überlässt es weiterhin dem Ministerkomitee, eine detailliertere Bewertung der Situation vorzunehmen. ${ }^{1500}$ Auch die Formulierungen des Gerichtshofs sind hier von Zurückhaltung geprägt. Eine Aufgabenverschiebung scheint der Gerichtshof durch die Anordnung spezifischer Massnahmen diesbezüglich nicht anzustreben. Eine echte Judizialisierung des Implementierungsprozesses erfolgt hier folglich nicht.

wenn das Ministerkomitee das effektive praktische Funktionieren der eingeführten Massnahme prüfe und sich damit nicht nur auf die Prüfung des Ergreifens der Massnahme alleine beschränke. Vgl. etwa EGMR - Dimitrov u. Hamanov/ BUL, Urt. v. 10.05.2011, 48059/06 u. 2708/09, Ziff. 5 Urteilstenor (Verletzung von Art. 6 EMRK); EGMR - Finger/BUL, Urt. v. 10.05.2011, 37346/05, Ziff. 5 Urteilstenor.

1498 Vgl. dazu ausf. Glas HR\&ILD 2019, 73 (88 ff. m.w.N.): etwa EGMR - Greens u. M.T./GBR, Urt. v. 23.11.2010, 60041/08 u. 60054/08, Ziff. 6 Urteilstenor: Der Gerichtshof hatte legislative Vorschläge gefordert, das Ministerkomitee begnügte sich mit den von Grossbritannien ergriffenen administrativen Massnahmen.

1499 Vgl. etwa EGMR - Neshkov u.a./BUL, Urt. v. 27.01.2015, 36925/10 u.a., Rn. 279: „The Court has abstained from giving specific indications on the general measures that need to be taken by Bulgaria with a view to bringing conditions of detention in its correctional facilities into line with Article 3 of the Convention in execution of this judgment. While voicing some concerns and pointing out possible ways of dealing with deficiencies, the Court has found that, given the nature of the issues involved, specific instructions on these points would exceed its judicial function."

1500 Vgl. im Allgemeinen auch schon GLas HRLR 2014, 671 (693 f.). 
Ordnet der Gerichtshof infolge der Feststellung einer Verletzung von Art. 13 EMRK i.V.m. Art. 3 EMRK die Einführung wirksamer präventiver resp. kompensatorischer Rechtsbehelfe im Urteilstenor an und setzt gar eine Frist zu deren Einführung, greift er stärker in den Implementierungsvorgang ein. Dies relativiert sich - wie aufgezeigt - allerdings im Lichte der ohnehin sehr eingeschränkten Auswahl der Abhilfemassnahmen und des weiter bestehenden Ermessensspielraums hinsichtlich der konkreten Ausgestaltung der Rechtsbehelfe. Auch hier überlässt der Gerichtshof folglich die detaillierte Bewertung der Situation dem Konventionsstaat und dem Ministerkomitee. Einzig die Anordnung einer konkreten Frist zur Einführung des Rechtsbehelfs schränkt sowohl Konventionsstaat als auch Ministerkomitee erheblich ein. Eine gewisse Judizialisierung des Implementierungsverfahrens ist hier folglich zu erkennen.

Anzeichen dafür, dass das Ministerkomitee sich in den vorliegend untersuchten Fällen spezifischer Anordnungen des Gerichtshofs widersetzt und seine Beurteilung anstelle derjenigen des Gerichtshofs gesetzt hätte, konnten keine festgestellt werden; anderes würde auch die Glaubwürdigkeit des Konventionsrechtssystems gefährden.

Auch das eingeführte infringement proceeding führt zu einer weiteren Verflechtung des Ministerkomitees mit dem Gerichtshof (Art. 46 Abs. 4 und Abs. 5 EMRK). Anders als bei der Anordnung konkreter Massnahmen im Urteil geht diese hier allerdings vom Ministerkomitee aus. Es ist an ihm zu entscheiden, ob es den Gerichtshof anrufen soll, um über eine mögliche Pflichtverletzung i.S.v. Art. 46 Abs. 1 EMRK zu befinden. Der Gerichtshof selbst hat keine Möglichkeit, dies von sich aus zu tun. Die Feststellung der Verletzung von Art. 46 Abs. 1 EMRK hat denn auch einzig die Erhöhung des politischen Drucks zur Folge; für die Überwachung der weiteren Umsetzung des Urteils durch den Konventionsstaat ist weiterhin das Ministerkomitee zuständig, wobei der Gerichtshof auch keine weiteren Vorgaben macht. Anders sieht es aus, wenn der Gerichtshof festhält, dass keine Verletzung von Art. 46 Abs. 1 EMRK vorliegt. In diesem Fall hat das Ministerkomitee die Einstellung des Implementierungsprozesses zu beschliessen, wobei der Wortlaut von Art. 46 Abs. 5 Satz 2 EMRK einen Ermessensspielraum des Ministerkomitees ausschliesst. Das infringement proceeding weist dem Gerichtshof damit eine bedeutende Aufgabe in der Implementierungsphase zu. Ausserhalb eines infringement proceedings ist es nämlich am Ministerkomitee zu entscheiden, wann es den Überwachungsprozess beendet, weshalb das Ministerkomitee diese Kompetenzabgabe bei einer Weiterleitung i.S.v. Art. 46 Abs. 4 EMRK zu berücksichtigen hat. 
Praxisrelevanz hat das infringement proceeding zumindest im Bereich inadäquater Haftbedingungen aber noch nicht erlangt.

Zusammenfassend kommt es insbesondere bei Piloturteilen zu einem Zusammenspiel von Ministerkomitee und Gerichtshof, sind sie doch das Hauptanwendungsfeld für die Anordnung genereller Massnahmen. Die Judizialisierung des Durchsetzungsverfahrens scheint in der Praxis zumindest im Bereich inadäquater Haftbedingungen aber geringer zu sein, als man es prima vista vielleicht erwarten würde. Ermessensspielräume des Konventionsstaates und des Ministerkomitees bleiben relativ gross und Hauptakteur im Implementierungsverfahren bleibt klarerweise das Ministerkomitee. Der Mehrwert der Anwendung des Piloturteilsverfahrens scheint in Bezug auf die Überbelegungssituation damit auch nicht darin zu liegen, dass bereits im Urteil eine eingehende Evaluation der Situation vorgenommen würde, um etwa die Suche nach geeigneten Abhilfemassnahmen zu erleichtern. Bezweckt wird vielmehr, dem Konventionsstaat noch einmal zu verdeutlichen, dass das Ergreifen von generellen Massnahmen zwingend notwendig ist. Auf diese Weise soll der politische Druck auf den Konventionsstaat, auch tiefgreifende Massnahmen umzusetzen, verschärft werden. Die detaillierte Evaluation der innerstaatlichen Situation mit der Suche nach geeigneten Abhilfemassnahmen bleibt dabei weiterhin Aufgabe des Konventionsstaats in Zusammenarbeit mit dem Ministerkomitee. Mit anderen Worten erfolgt die durch das Piloturteilsverfahren angestrebte Effizienzsteigerung des Implementierungsprozesses nicht durch eine echte Aufgabenverschiebung zwischen Gerichtshof und Ministerkomitee, sondern mehr durch eine Bekräftigung der Bemühungen des Ministerkomitees durch den Gerichtshof. Dies auch vor dem Hintergrund, dass das Ministerkomitee keineswegs nur bei der Implementierung von Piloturteilen generelle Massnahmen im Durchsetzungsverfahren verlangt.

Kapitel 6: Schlussevaluation des Durchsetzungsverfahrens Optimierungsmöglichkeiten zur Gewährleistung einer effektiven Durchsetzung?

Die einleitende These, dass ein effektiver Menschenrechtsschutz im Strafvollzug nicht nur eines klaren Mindestmassstabs im Sinne einer normativen Erwartung bedarf, sondern dass die umfassende Umsetzung dieses Standards in der Praxis mindestens genauso bedeutend ist, und die Tatsache, dass in vielen der Konventionsstaaten der Strafvollzug nach wie vor durch unzureichende Haftbedingungen geprägt bleibt, werfen die 
Frage nach Optimierungsmöglichkeiten im Durchsetzungsverfahren auf. Deshalb werden im Folgenden die drei Phasen der Durchsetzung des Standards einer Schlussevaluation unterzogen und, wo nötig, Verbesserungen vorgeschlagen.

Bei der Anordnung vorsorglicher Massnahmen betreffend unmenschliche oder erniedrigende Haftbedingungen verwendet der Gerichtshof bislang in der Regel weiche Formulierungen. Aufgrund der unbestrittenen Verbindlichkeit der vorsorglichen Massnahmen i.S.v. Regel 39 VerfO ist diese Praxis des Gerichtshofs zu überdenken. Auch wenn nicht anzunehmen ist, dass sich die Konventionsstaaten nicht auch heute grundsätzlich im Klaren über die bindende Natur der Massnahmen wären, so könnte mit deutlicheren Formulierungen, welche die Verbindlichkeit schon ihrem Wortlaut nach erkennen liessen, der Bedeutung und der Dringlichkeit der Umsetzung der angeordneten vorsorglichen Massnahmen gleichwohl nochmals Nachdruck verschafft und so auf eine bessere Befolgungspraxis der Konventionsstaaten hingewirkt werden.

Vorsorgliche Massnahmen i.S.v. Regel 39 VerfO werden bislang vorwiegend im Bereich inadäquater medizinischer Versorgung in Strafvollzugsanstalten ergriffen. Der aktuelle Stand der medizinischen Wissenschaft gebietet aber auch eine Sensibilisierung des Gerichtshofs für das unmittelbare und irreversible Schädigungspotential physischer und psychischer Art, welches von Überbelegungssituationen ausgeht. In all jenen Fällen, in denen die Überbelegungssituation zu einem unmittelbar irreversiblen physischen oder psychischen Schaden zu führen droht, sind vom Gerichtshof konsequent vorsorgliche Massnahmen anzuordnen. Geeignete und schnell realisierbare Massnahmen stehen auch hier durchaus zur Verfügung. Diese können sich weitestgehend an den individuellen Massnahmen, welche im Anschluss an Verletzungen von Art. 3 EMRK angeordnet werden, orientieren. Das Potential vorsorglicher Massnahmen sollte in Zukunft folglich durch den Gerichtshof noch besser ausgeschöpft werden.

Die Piloturteilsverfahrenstechnik wurde durch den Gerichtshof bislang bei verhältnismässig wenigen Konventionsstaaten infolge unmenschlicher und erniedrigender Haftbedingungen i.S.v. Art. 3 EMRK angewandt. Die statistischen Daten zu Überbelegungssituationen zeigen allerdings, dass zahlreiche weitere Konventionsstaaten mit Überbelegungssituationen zu kämpfen haben, weshalb vom Gerichtshof auch in Zukunft immer sorgfältig geprüft werden muss, ob die Anwendung eines Piloturteilsverfahrens angezeigt ist. Für die Frage, ob ein strukturelles bzw. systemisches Problem im Konventionsstaat vorliegt, ist auch in Zukunft nicht allein auf die Zahl abgeurteilter Fälle abzustellen, sondern dem prospektiven 
Kriterium potentiell weiterer Betroffener grosse Bedeutung zuzumessen. Dies hat umso mehr zu gelten, als mit Verletzungen von Art. 3 EMRK schwere Menschenrechtsverletzungen drohen, welche ein rechtzeitiges Eingreifen erfordern. Nachdem insbesondere das CPT anlässlich seiner Besuchstätigkeit in mehreren Konventionsstaaten auch weit verbreitete Unzulänglichkeiten der Gesundheitsversorgung und strukturelle Probleme bei der therapeutischen Versorgung im Strafvollzug erkannt hat, ist in Zukunft auch bei solchen Beschwerden vom Gerichtshof immer sorgfältig zu prüfen, ob die Konventionsverletzungen auf einem strukturellen bzw. systemischen Problem beruhen, welches die Anwendung der Piloturteilsverfahrenstechnik gebieten würde. Das Potential der Piloturteilsverfahrenstechnik scheint hier noch nicht vollständig ausgenutzt zu werden.

Auch dem Ministerkomitee scheint eine gewisse Rolle zuzukommen, wenn es darum geht, das Potential der Piloturteilsverfahrenstechnik vollständig auszunutzen. Es hat sich nämlich in der Vergangenheit gezeigt, dass der Gerichtshof bei der Prüfung der Anwendung der Piloturteilstechnik hinsichtlich des Vorliegens eines strukturellen bzw. systemischen Problems auch Zwischenresolutionen des Ministerkomitees aufgegriffen hat, in welchen dieses festgehalten hatte, dass ein Konventionsstaat gewisse Urteile bislang nur mangelhaft umgesetzt hätte, dass dies das Konventionssystem weiter belastet hätte und dass eine langfristige Strategie zur Lösung des strukturellen Problems erarbeitet werden müsste. Ob das Ministerkomitee Zwischenresolutionen bereits bewusst auch in dieser Absicht genutzt hat, lässt sich nicht abschliessend beurteilen. Das Hervorheben des Vorliegens eines strukturellen bzw. systemischen Problems deutet aber darauf hin. ${ }^{1501}$ Vor diesem Hintergrund bleibt das Ministerkomitee jedenfalls anzuhalten, den Entscheid, eine Zwischenresolution zu erlassen, auch im Bewusstsein darüber zu treffen, welch wichtige Hinweisfunktion die

1501 Vgl. Interim Resolution CM/ResDH (2018) 472, Execution of the Judgments of the European Court of Human Rights Nevmerzhitsky/UKR, Yakovenko/UKR, Logninenko/UKR, Isayev/UKR u. Melnik Groups/UKR: „structural nature of the problems" „[...] noted that, in view of the increasing number of applications brought before the European Court, this lack of progress also puts an additional undue burden on the Convention system [...]"; Resolution CM/ResDH (2018) 455, Execution of the Judgments of the European Court of Human Rights, 136 Cases/RUS (from the Kalashnikov Group): „[...] the existence of structural problems and the pressing need for comprehensive general measures were stressed by the Committee and acknowledged by the Russian authorities since the adoption by the Court of the judgment in the case of Kalashnikov against Russia in 2002.“ 
Zwischenresolution auch für den Gerichtshof haben kann, wenn dieser in einem künftigen ähnlich gelagerten Fall über die Anwendung der Piloturteilstechnik zu befinden hat. Die Entscheidung, ob die Piloturteilsverfahrenstechnik tatsächlich angewandt und generelle Massnahmen angeordnet werden, liegt letztlich allerdings allein beim Gerichtshof. Es ist gleichwohl durchaus denkbar, dass sich dieser Interaktionsmechanismus zwischen Gerichtshof und Ministerkomitee in Zukunft etablieren und noch an Bedeutung gewinnen wird.

Von der Möglichkeit des Gerichtshofs, bei Anwendung der Piloturteilsverfahrenstechnik Parallelfälle für die Dauer des Verfahrens zurückzustellen, ist weiterhin nur restriktive Gebrauch zu machen; dies aufgrund des fundamentalen Charakters von Art. 3 EMRK und der damit einhergehenden Dringlichkeit der Verfahren. Laufende Verfahren vor dem Gerichtshof, welche den Konventionsstaat in regelmässigen Abständen an seine menschenrechtlichen Verpflichtungen erinnern, sind als subtiler Enforcementmechanismus weiter zu nutzen. Gleichwohl ist mit Blick auf den Subsidiaritätsgedanken immer zu prüfen, ob eine Zurückstellung der Fälle doch indiziert ist. Dies ist in Anlehnung an das Urteil Torreggiani u.a./ ITA ${ }^{1502}$ etwa in jenen Fällen denkbar, in welchen der Konventionsstaat zuvor bereits ein Konzept zur Ergreifung genereller Massnahmen entwickelt hat und es angezeigt scheint, abzuwarten, ob die notwendigen Massnahmen von den Behörden ergriffen werden. Macht der Gerichtshof von der Zurückstellung Gebrauch, so hat er aber i.S.v. Regel 61 Abs. 6 lit. c und Abs. 8 VerfO jedenfalls regelmässig zu prüfen, ob es notwendig ist, zunächst zurückgestellte Verfahren doch vorzeitig zu behandeln.

Die Anordnungspraxis des Gerichtshofs bezüglich genereller Abhilfemassnahmen ist von Zurückhaltung geprägt. Beim Entscheid darüber, ob die Anordnung bestimmter individueller oder genereller Abhilfemassnahmen im konkreten Fall indiziert ist oder nicht, bleibt vom Gerichtshof - wie es bisher auch der Fall war - stets eine sorgfältige Abwägung zu fordern, bei welcher er sowohl der Effektivität des Menschenrechtsschutzes als auch der Subsidiarität des Strassburger Systems Rechnung zu tragen hat. Dabei hat er weiter stets auf die Rollenverteilung zwischen Gerichtshof und Ministerkomitee zu achten. Konkrete Abhilfemassnahmen sind folglich auch in Zukunft so oft wie nötig, aber so selten wie möglich anzuordnen, und die angeordneten Abhilfemassnahmen haben so flexibel wie

1502 EGMR - Torreggiani u.a./ITA, Urt. v. 08.01.2013, 43517/09 u.a. 
möglich, aber so bestimmt wie nötig zu sein. ${ }^{1503}$ Die Praxis des Gerichtshofs, seine Ausführungen zu generellen Abhilfemassnahmen betreffend Überbelegungssituationen auf die Urteilsgründe zu beschränken und in den Urteilstenor nur generelle Abhilfemassnahmen zu Verletzungen von Art. 13 EMRK i.V.m. Art. 3 EMRK aufzunehmen, scheint diesen Grundsätzen zu entsprechen und ist auch in Zukunft beizubehalten. Beizubehalten ist weiter auch, sowohl Konventionsstaat als auch Ministerkomitee ein weites Auswahlermessen hinsichtlich der zu ergreifenden Massnahmen zu belassen und sich auf eine Globalbetrachtung der Situation zu beschränken. Die detaillierte Evaluation bleibt Sache von Konventionsstaat und Ministerkomitee. Betreffend die Anordnung der Einführung von präventiven und kompensatorischen Rechtsbehelfen ist das Modell des Gerichtshofs vorzuziehen, hierzu keine Frist zur Einführung, sondern eine Frist zur Einreichung eines verbindlichen Zeitplans zu setzen. Es bleibt dann am Ministerkomitee zu evaluieren, ob der Zeitplan realistisch ist und ob auch der Dringlichkeit der Angelegenheit genügend nachgekommen wird. Dies auch vor dem Hintergrund, dass die Anordnung genereller Massnahmen nicht bezweckt, die Arbeit des Ministerkomitees vorwegzunehmen, sondern nur, dessen Bemühungen zu bekräftigen. Eine Aufgabenverschiebung zwischen Gerichtshof und Ministerkomitee ist nämlich weder beabsichtigt, noch wäre von einer solchen eine Effizienzsteigerung des Implementierungsprozesses zu erwarten.

Hält der Gerichtshof es allerdings für nötig, individuelle Massnahmen im Urteilstenor anzuordnen, gebietet es die Klarheit, dass er diesbezüglich keine weichen Formulierungen wählt, wenn dem Konventionsstaat kein Auswahlermessen belassen wird, wie dies etwa der Fall ist, wenn der Gerichtshof feststellt, dass der Beschwerdeführer aus der Haft zu entlassen sei. Eine weiche Formulierung täuscht hier einzig über den rechtsverbindlichen Charakter der Anordnung hinweg.

Die Zuteilungskriterien der twin-track supervision führen dazu, dass Urteile betreffend inadäquate Haftbedingungen unabhängig davon, ob es sich um Piloturteilsverfahren handelt oder nicht, regelmässig dem Verfahren der enhanced supervision zugeführt werden. Diese Zuteilung ist auch in Zukunft beizubehalten, ergibt sich die Dringlichkeit der Umsetzung

1503 Vgl. auch Sснмaнl EuGRZ 2008, 369 (379f.), welche hinsichtlich der Margin of appreciation-Doktrin keine Bedenken äussert, solange der EGMR bestimmte Abhilfemassnahmen nur mit einer gewissen Flexibilität ausspreche und den Konventionsstaaten bei der Umsetzung einen grösstmöglichen Beurteilungsspielraum zugestehe. 
bei inadäquaten Haftbedingungen doch bereits aus der Schwere der Konventionsverletzung und nicht erst bei Vorliegen eines strukturellen bzw. systemischen Problems.

Insbesondere mit Blick auf die zu ergreifenden individuellen Massnahmen stellt sich die Frage, ob eine Sechsmonatsfrist bis zur Einreichung eines ersten Aktionsplans - wie sie vom Ministerkomitee vorgesehen ist - der Schwere der fortdauernden Menschenverletzungen tatsächlich gerecht werden kann. Denkbar wäre etwa, hinsichtlich der zu setzenden Frist je nach Schwere der Menschenrechtsverletzung oder zwischen individuellen und generellen Massnahmen zu differenzieren. Anders als bei den generellen Massnahmen erfordert die Evaluierung möglicher individueller Abhilfemassnahmen nämlich keine komplexe kriminalpolitische Abwägung, so dass ein rascheres Handeln durchaus möglich sein sollte. Die daraus resultierende Doppelspurigkeit der Überwachung individueller und genereller Implementierungsmassnahmen würde aber wohl das ohnehin schon strapazierte Konventionsrechtssystem weiter belasten, weshalb dies aus pragmatischen Überlegungen als keine valable Option erscheint. Eine allgemein kürzere Frist bei schweren Menschenrechtsverletzungen wie sie Verletzungen von Art. 3 EMRK sind - könnte dieser Problematik zwar auf den ersten Blick abhelfen, scheint der Komplexität der abzuklärenden Möglichkeiten für generelle Abhilfemassnahmen zu strukturellen und systemischen Problemen aber nicht gerecht zu werden. Resultiert aus einer kürzeren Frist ein Aktionsplan, welcher nicht auf einer fundierten Basis bzw. fundierten innerstaatlichen Abklärungen beruht, wäre letztlich nichts gewonnen. Änderungsbedarf hinsichtlich dieser Frist besteht folglich nicht. Die Konventionsstaaten bleiben aber eindringlich daran zu erinnern, den Aktionsplan so schnell als möglich einzureichen und die Sechsmonatsfrist nur auszuschöpfen, wenn ein rascheres Tätigwerden nicht möglich ist. Weiter sind individuelle Massnahmen wann immer möglich schon vor der der Einreichung des ersten Aktionsplans zu treffen. Im Aktionsplan bleibt dann über die bereits getroffenen Massnahmen Bericht zu erstatten, woraufhin das Ministerkomitee diesbezüglich nur noch festzustellen hat, ob den Verpflichtungen betreffend das Ergreifen individueller Massnahmen mit den getroffenen Massnahmen schon Genüge getan wurde.

Die Anwendung des Verfahrens der enhanced supervision führt in der Praxis erwartungsgemäss auch dazu, dass diese Verfahren eng und aktiv vom Ministerkomitee begleitet werden, so dass Konventionsstaat und Ministerkomitee in einem ständigen Dialog stehen. Die Implementierungsfortschritte werden auch regelmässig an den Menschenrechtssitzun- 
gen des Ministerkomitees thematisiert. Die Überwachungsintensität ist aber keineswegs starr. Es wird in der Praxis vielmehr auch von der Möglichkeit der Umteilung des Umsetzungsverfahrens von der enhanced supervision zur standard supervision Gebrauch gemacht, wenn der Implementierungsstand dies zulässt. Dem Subsidiaritätsprinzip wird folglich entsprochen, ohne dass die effiziente Durchsetzung der Urteile gefährdet würde. Insoweit besteht hier kein Änderungsbedarf.

Von der Möglichkeit, den politischen Druck mit Zwischenresolutionen i.S.v. Regel $16^{1504} \mathrm{zu}$ erhöhen, hat das Ministerkomitee in Implementierungsverfahren von Piloturteilen bislang allerdings noch keinen Gebrauch gemacht. Nachdem der Gerichtshof in der Vergangenheit Zwischenresolutionen des Ministerkomitees in seiner Rechtsprechung aufgegriffen hat, wenn es um die Frage ging, ob die Anwendung der Piloturteilsverfahrenstechnik angezeigt erscheint, könnten Zwischenresolutionen auch nach der Anwendung der Piloturteilsverfahrenstechnik für den Gerichtshof von gewisser Relevanz sein. So scheint es etwa denkbar, dass Zwischenresolutionen auch hier einen gewissen Einfluss auf die Folgerechtsprechung haben können. Hat der Gerichtshof während der Implementierung des Piloturteils etwa über einen Parallelfall zu entscheiden, ist anzunehmen, dass der Gerichtshof bei Ausführungen zu Art. 46 EMRK den Implementierungsstand des Piloturteils berücksichtigen wird. Hat das Ministerkomitee in einer Zwischenresolution festgehalten, dass keine oder nur geringe Implementierungsfortschritte erzielt werden konnten, könnte dies den Gerichtshof etwa dazu veranlassen, im Parallelfall erneut generelle Massnahmen anzuordnen und so den politischen Druck auf den Konventionsstaat nochmals zu erhöhen bzw. aufrechtzuerhalten. Auch hinsichtlich der Frage, ob es i.S.v. Regel 61 Abs. 8 VerfO angezeigt ist, zunächst zurückgestellte Parallelfälle wieder aufzunehmen, könnten Zwischenresolutionen in Zukunft eine wichtige Hinweisfunktion zukommen.

Nachdem das Ministerkomitee im Prozess zur Implementierung des Piloturteils Rezmiveș u.a./ROM ${ }^{1505}$ Rumänien zumindest in Aussicht gestellt hat, dass auch der Erlass einer Zwischenresolution in Betracht gezogen würde, sollten keine konkreten Fortschritte erzielt werden, ${ }^{1506}$ wird sich diese These vielleicht schon bald überprüfen lassen. Das Ministerkomitee ist vor diesem Hintergrund jedenfalls anzuhalten, in Zukunft auch

1504 Rules of the Committee of Ministers for the Supervision of the Execution of Judgments and of the Terms of Friendly Settlements.

1505 EGMR - Rezmiveș u.a./ROM, Urt. v. 25.04.2017, 61467/12 u.a.

1506 Decision v. 05.03.2020, CM/Del/Dec (2020) 1369/H46-23. 
im Implementierungsverfahren von Piloturteilen keine Scheu zu zeigen, wann immer nötig das Instrument des Erlasses von Zwischenresolutionen i.S.v. Regel $16^{1507} \mathrm{zu}$ nutzen, insbesondere auch, um Besorgnis auszudrücken oder weitere angemessene Umsetzungsmassnahmen vorzuschlagen.

Ist der Konventionsstaat der Ansicht, alle notwendigen Massnahmen zur Implementierung ergriffen zu haben, und reicht er dem Ministerkomitee einen Schlussbericht ein, folgt eine sechsmonatige Frist, innert welcher andere Staaten oder das Sekretariat die Möglichkeit haben, Stellungnahmen hinsichtlich der Schliessung des Überwachungsverfahrens einzureichen, bevor das Ministerkomitee darüber entscheidet, ob das Urteil umgesetzt wurde. Diese Frist erscheint relativ lang, was sich insbesondere in jenen Fällen negativ auswirken kann, in denen das Ministerkomitee zum Schluss gelangt, dass die ergriffenen Massnahmen noch nicht genügen. Die Notwendigkeit einer solch langen Frist sollte in Zukunft vom Ministerkomitee einer Prüfung unterzogen und die Frist falls nötig entsprechend gekürzt werden.

Hinsichtlich des Abschlusses der Umsetzungsverfahren wurde bei anderen Themengebieten Divergenzen festgestellt zwischen dem, was der Gerichtshof anordnete und dem, was das Ministerkomitee effektiv vom Konventionsstaat verlangte. Auch wenn dies im vorliegenden Themengebiet bislang nicht festgestellt werden musste, ist in Zukunft besonderes Augenmerk darauf zu legen, ob sich das Ministerkomitee an seine Rolle i.S.v. Art. 46 Abs. 2 EMRK hält. Besondere Vorsicht scheint in jenen Fällen geboten zu sein, in denen sich ein Konventionsstaat etwa Finanzierungsunterstützung organisiert hat und sich in diesem Kontext nicht nur zur Umsetzung der Urteile des Gerichtshofs, sondern zu einem höheren Soft Law-Standard (etwa dem CPT-Standard) verpflichtet hat. Die Pflichten nach Art. 46 EMRK i.V.m. Art. 1 EMRK und anderen vertraglichen Verpflichtungen sind im Kontext der Implementierung klar zu trennen. Sobald das Urteil umgesetzt ist, ist der Implementierungsprozess durch das Ministerkomitee abzuschliessen. Alles andere würde Zweifel an der Glaubwürdigkeit des Konventionsrechtssystems zur Folge haben.

1507 Rules of the Committee of Ministers for the Supervision of the Execution of Judgments and of the Terms of Friendly Settlements. 


\section{Teil 5: Schlussbetrachtung}

Nicht nur die Länge der Freiheitsstrafe bestimmt über deren Eingriffsintensität. Massgeblichen Einfluss haben auch die Modalitäten des Strafvollzugs, weshalb die menschenrechtlichen Mindestanforderungen an diesen ausschlaggebend sind. Dies gilt umso mehr, als der Inhaftierte in einem besonderen Näheverhältnis zum Staat steht und sich in einer besonders verletzlichen Position befindet. Besondere Obhuts- und Fürsorgepflichten des Staates gegenüber dem Inhaftierten sind die Folge. Da der Inhaftierte aufgrund des Freiheitsentzugs nicht mehr selbst in der Lage ist, für seine Grundbedürfnisse zu sorgen, werden dem Staat in materieller Sicht aus der leistungs-, schutz- und gewährleistungsrechtlichen Dimension der Menschenrechte besondere positive Pflichten auferlegt. Gleichzeitig wird aus prozessrechtlicher Sicht mit beweisrechtlichen Erleichterungen für den Inhaftierten dem Umstand Rechnung getragen, dass der Staat in der Regel über die Informationsherrschaft verfügt.

Vorgaben an den Strafvollzug finden sich auf sämtlichen Stufen des Mehrebenensystems. Aus menschenrechtlicher Sicht kommt den fundamentalen, absolut geltenden Schutzgehalten des Art. 3 EMRK besondere Bedeutung zu. Es wurden die zwei strafvollzugsspezifischen, menschenrechtlich relevanten Themen der Überbelegung resp. des persönlich zur Verfügung stehenden Platzes sowie der medizinischen Versorgung im Strafvollzug herausgegriffen und die menschenrechtlichen Anforderungen an diese im Lichte des Art. 3 EMRK untersucht. Den Spezifika eines absoluten Rechts, nämlich der unbedingten Anwendbarkeitsforderung (Anwendbarkeitskriterium) und den Besonderheiten des Spezifitätskriteriums bei der Schutzbereichsbestimmung, wurden besondere Bedeutung zugemessen.

Bei der Beurteilung einer Verletzung von Art. 3 EMRK aufgrund einer Überbelegungssituation scheut sich der EGMR bislang davor, einen verbindlichen konkreten Mindeststandard zu setzen. Vielmehr nimmt er eine Gesamtbetrachtung der Haftumstände vor, wobei er sowohl erschwerende (kumulierende) als auch erleichternde (kompensierende) Faktoren berücksichtigt. Konkret bedeutet dies, dass er beim Unterschreiten von $3 \mathrm{~m}^{2}$ persönlichem Platz von einer starken Vermutungswirkung eines Verstosses gegen Art. 3 EMRK ausgeht. Der Vermutungseintritt hat die Beweislastumkehr zur Folge, was vor dem Hintergrund der allgemeinen Regelun- 
gen zu Beweismass und Beweislast in Strafvollzugskonstellationen für den Inhaftierten allerdings nur zu einem geringen Mehrwert führt. Kompensierende Elemente können die Vermutung eines Konventionsverstosses umstossen, wobei der EGMR an die Kompensation keine hohen Anforderungen stellt.

Nicht immer eindeutig ist, ob der Gerichtshof bei Anwendung dieses eigens für Überbelegungssituationen kreierten Prüfprogramms die Voraussetzungen der unmenschlichen oder aber der erniedrigenden Behandlung prüft. Der Schwerpunkt der Prüfung des Gerichtshofs liegt bei Überbelegungssituationen i.d.R. bei der Frage, ob das erforderliche Mindestmass an Schwere i.S.v. Art. 3 EMRK erreicht ist. Auch zur Beantwortung dieser Frage ist allerdings entscheidend, welche konkrete Garantie von Art. 3 EMRK geprüft wird, da sich diese Garantien nicht allein aufgrund ihrer Eingriffsintensität, sondern auch aufgrund ihrer je anderen Einwirkungsweise auf den Einzelnen unterscheiden. Das erforderliche Mindestmass an Schwere bestimmt sich folglich nach anderen Kriterien.

In der Tendenz scheint der Gerichtshof bei Überbelegungssituationen allerdings zu prüfen, ob eine erniedrigende Behandlung i.S.v. Art. 3 EMRK vorliegt. Die 3- $\mathrm{m}^{2}$-Grenze scheint dabei einer von der Menschenwürde her gedachten, normativ wertenden Beurteilung zu entspringen, welche ihrerseits soziomedizinische Schlussfolgerungen aufgreift. Dasselbe gilt auch für die Voraussetzungen der Widerlegung der Vermutung. Die Berücksichtigung kompensierender Elemente basiert auf der Annahme soziomedizinischer Erleichterungs- resp. Erholungseffekte, welchen mit Blick auf die Achtung der Menschenwürde ein normativer Wert bei der Gesamtbetrachtung zugeschrieben wird. Der wissenschaftliche Nachweis kausaler Effekte auf den Einzelnen ist demnach gerade nicht gefordert. Auch wenn durch das vom Gerichtshof aufgestellte spezifische Prüfprogramm die Begriffsmerkmale der unmenschlichen und erniedrigenden Behandlung in den Hintergrund rücken, ist sich stets zu vergegenwärtigen, welche Einzelgarantie geprüft wird. Nur im Bewusstsein dessen kann die normative Bewertung der Situation überhaupt erfolgen. Eine verselbständigte Prüfung, losgelöst von den Voraussetzungen der unmenschlichen oder der erniedrigenden Behandlung i.S.v. Art. 3 EMRK, ist ausgeschlossen.

Die prüfungsmethodische Herangehensweise mit der Kompensationsmöglichkeit ist auch mit Blick auf die Schutzrichtung und den Fundamentalcharakter des absolut geltenden Art. 3 EMRK grundsätzlich nicht unproblematisch. Die Berücksichtigung der kompensierenden Elemente erfolgt zwar formell auf der Ebene der Schutzbereichsbestimmung, wo relative Kriterien berücksichtigt werden dürfen, sofern sie sich auf den 
Grad des erlittenen Leidens bzw. die Schwere der zugefügten Behandlung auswirken, und kommt auch materiell keiner Rechtfertigungsprüfung gleich. Nicht näher definierte Kompensationselemente führen bei der Schutzbereichsbestimmung aber zu einer Variabilität des Ergebnisses. Dies ist mit Blick auf das Spezifitätskriterium absoluter Rechte bedenklich. Der Kompensationsmechanismus sollte deshalb nur restriktiv zur Anwendung gelangen.

Vor diesem Hintergrund wurde in dieser Arbeit ein eigener Lösungsvorschlag zu einer revidierten Prüfungsmethodik in Überbelegungsfällen ausgearbeitet. Aufgrund dessen, dass die Überbelegung in der Regel den Demütigungsmoment in den Vordergrund rückt und einen besonders engen Konnex zur Menschenwürde aufweist, ist die Überbelegung in diesem Vorschlag in der Regel bewusst anhand der Voraussetzungen einer erniedrigenden Behandlung i.S.v. Art. 3 EMRK zu prüfen. Dabei wurde auch in diesem Vorschlag einem normativen Ansatz gefolgt, bei welchem soziomedizinische Faktoren aufgegriffen werden, ohne dass ein Nachweis der Auswirkungen auf den Einzelnen im konkreten Fall gefordert würde. Modifiziert wurde die Möglichkeit, mit Kompensationsmassnahmen die Vermutung zu widerlegen. Zum einen wurde der Kompensationsmöglichkeit eine klare Grenze gesetzt und zum anderen generell höhere Anforderungen an die kompensierenden Faktoren verlangt. Dies soll zu mehr Rechtssicherheit der Inhaftierten und zu einer Anhebung des Schutzniveaus führen. An der Berücksichtigung kumulierender Elemente wird aufgrund der Schutzrichtung von Art. 3 EMRK festgehalten.

Anders geht der EGMR vor, wenn er die Konformität der Gesundheitsversorgung im Strafvollzug mit Art. 3 EMRK zu prüfen hat. Für die Phase während der Haft garantiert der EGMR eine individuell gesundheitsadäquate medizinische Versorgung, wobei er zu deren Konkretisierung bereit ist, inhaltliche Einzelgarantien aufzustellen. Der EGMR zeigt damit keine Scheu, an die Gesundheitsversorgung konkrete Anforderungen zu stellen, welche über die Statuierung von auslegungsbedürftigen Grundprinzipien hinausgehen. In aller Regel hält der Gerichtshof dabei auch ausdrücklich fest, welche spezifische Einzelgarantie von Art. 3 EMRK er prüft und welche Kriterien er zu dieser Prüfung heranzieht. Eine unmenschliche Behandlung i.S.v. Art. 3 EMRK erkennt der EGMR in Übereinstimmung mit den allgemeinen Begriffsmerkmalen insbesondere in jenen Situationen, in denen der Beschwerdeführer nachweist, dass die inadäquate Gesundheitsversorgung zu einem verstärkten Gefühl der Qual und des körperlichen Leidens geführt hat. Bei der Prüfung der erniedrigenden Behandlung i.S.v. Art. 3 EMRK stellt der Gerichtshof demgegenüber 
hauptsächlich darauf $a b$, ob der Beschwerdeführer durch die ungenügende Behandlung in seiner Persönlichkeit herabgesetzt bzw. ob seine Menschenwürde gewahrt wurde. Der EGMR berücksichtigt bei seiner Prüfung sowohl bei der unmenschlichen als auch bei der erniedrigenden Behandlung die kumulativen Effekte der Haftsituation; einen Kompensationsmechanismus wendet er nicht an. Kompensierende Effekte, die tatsächlich eine Ausgleichsfunktion hätten, wären auch praktisch kaum denkbar, und zwar weder durch andere Haftbedingungen noch durch die Einhaltung anderer Garantien der Gesundheitsversorgung, lassen sich diese doch gerade nicht gegenseitig aufwiegen.

Das Vorgehen des Gerichtshofs bei der Beurteilung der Gesundheitsversorgung im Strafvollzug hat gezeigt, dass ein klarer Standard, welcher der absoluten Natur von Art. 3 EMRK gerecht wird, trotz der grossen Varietät potentieller Fallkonstellationen möglich ist. Ein solches Vorgehen wäre denn auch auf die Prüfung der Menschenrechtskonformität von Überbelegungssituationen übertragbar, etwa in Anwendung des vorgeschlagenen eigenen Lösungsvorschlags. Durch den Umstand, dass nach wie vor kumulative Effekte und ein Stück weit auch kompensierende Effekte zu berücksichtigen blieben, würde den Umständen des Einzelfalls genügend Rechnung getragen, ohne dass die Gefahr bestünde, dass der menschenrechtliche Mindeststandard durch (scheinbare) Kompensationen unterlaufen würde. Rechtssicherheit und ein effektiver Menschenrechtsschutz könnten auf diese Weise vorangetrieben werden.

Soft Law als rechtlich unverbindliche Bestimmungen, welche aber gleichwohl eine normative, verhaltensbeeinflussende Wirkung entfalten, kann - unter anderem über seine Rezeption in der Rechtsprechung zu praktischer Wirksamkeit gelangen. Von einem Transformationsprozess von Soft Law zu „hardened Soft Law" zu Hard Law wird dann ausgegangen, wenn die Soft Law-Bestimmung regelmässig gerichtlich durchgesetzt wird und dadurch immer mehr an Normativität gewinnt, bis die Bestimmung unter Umständen ganz ihre ursprüngliche rechtliche Unverbindlichkeit verliert, oder anders gesagt, wenn sich rechtspolitische Zielbestimmungen $\mathrm{zu}$ rechtsverbindlichen subjektiven Rechten formen. $\mathrm{Zu}$ wahren bleibt bei der Rezeption von Soft Law in der Rechtsprechung die Grenze richterlicher Rechtsfortbildung, welche ihrerseits für jede auszulegende Norm nach Massgabe des für sie anwendbaren Auslegungskanons zu bestimmen ist.

In Bezug auf die Implementierung von Soft Law in die Rechtsprechung des Gerichtshofs zu Art. 3 EMRK haben sich Unterschiede zwischen der Unterbringungsfrage und der Gesundheitsversorgung gezeigt. Hinsichtlich 
der Unterbringungsform folgt der Gerichtshof nicht der Tendenz des Soft Law, welche eine Einzelunterbringung fordert. Auch bei der Frage des zur Verfügung zu stellenden Platzes grenzt sich der Gerichtshof bewusst vom CPT-Standard ab und argumentiert, dass sich die Rollen des CPT und des Gerichtshofs konzeptionell unterscheiden; schon von seiner Konzeption her habe der Standard des CPT ein höherer zu sein als derjenige des EGMR. Während dem Ergebnis zuzustimmen ist, dass je nach Fragestellung und je nach Institution andere Standards möglich sind, überzeugt die vom EGMR beschriebene Rollenverteilung nicht; diese reduziert einerseits die umfassenderen Aufgaben des CPT auf eine rein präventive Tätigkeit und vernachlässigt andererseits die Präventionsfunktion des Gerichtshofs. Aus den unterschiedlichen Rollen von EGMR und CPT lässt sich weiter schon gar nicht ableiten, wie viel tiefer der Standard des Gerichtshofs denn anzusetzen ist. Sowohl der vom CPT festgesetzte Massstab von $4 \mathrm{~m}^{2}$ als auch der 3-m²-Massstab des EGMR beruhen, wie aufgezeigt, auf einer von der Menschenwürde her gedachten normativen Bewertung, welche sich auf soziomedizinische Schlussfolgerungen stützt. Weitergehende wissenschaftliche Studien zu den Folgen des Platzangebotes auf den Betroffenen oder eine eingehendere normative Begründung wären hier erforderlich, um schlüssig darzulegen, weshalb und inwieweit im Rahmen von Art. 3 EMRK vom CPT-Standard abgewichen werden kann. Prüfungsmethodologisch geht der EGMR insbesondere mit der Befürwortung einer Gesamtbetrachtung, welche die Berücksichtigung sowohl kumulativer als auch kompensatorischer Effekte zulässt, gleich vor wie das CPT. Insgesamt sind die Soft Law-Regulatorien (insbesondere die Dokumente des CPT) für den Gerichtshof eine wichtige Rechtserkenntnisquelle; ein Transformationsprozess von Soft Law zu Hard Law findet, zumindest was einen präzisen Mindeststandard des zur Verfügung zu stellenden Platzes anbelangt, aber nicht statt. Gleichwohl könnte argumentiert werden, dass die CPT-Empfehlungen durch die Rezeption in der EGMR-Rechtsprechung etwa hinsichtlich Berechnungsmodalitäten und Prüfungsmethodik an Normativität gewinnen und folglich einen gewissen Härtungsprozess durchlaufen.

Im Bereich der Gesundheitsversorgung im Strafvollzug hat sich gezeigt, dass der Gerichtshof ausdrücklich bereit ist, Soft Law in seine Rechtsprechung zu implementieren (etwa hinsichtlich Garantien aus dem Vertraulichkeitsgrundsatz). Mit anderen Worten setzt hier ein Transformationsprozess ein. Dieser erfolgt allerdings erst nach einer sorgfältigen Prüfung der konkreten Soft Law-Bestimmung auf ihren genuin menschenrechtlichen Gehalt. Dies zeigt sich mitunter darin, dass der Gerichtshof auch bei der Gesundheitsversorgung nicht sämtliche Empfehlungen über- 
nimmt, sondern zum Teil ausdrücklich von diesen abweicht (vgl. etwa unterschiedliche Folgen einer fehlenden Eintrittsuntersuchung). Auch prüfungsmethodologisch geht der Gerichtshof bei der Festsetzung seines Mindeststandards sehr ähnlich vor wie die Soft Law-Gremien. Insgesamt scheint die „Motorfunktion“ des Soft Law bei Fragen rund um eine adäquate, Art.-3-EMRK-konforme Gesundheitsversorgung tendenziell stärker zu sein als bei Fragen rund um die Unterbringungsmodalitäten.

Bei der Sachverhaltserstellung des EGMR spielen Monitoringberichte eine nicht unbedeutende Rolle, sowohl bei Unterbringungsfragen als auch bei Fragen rund um die Gesundheitsversorgung im Strafvollzug. Bei der Überbelegungssituation werden Informationen des CPT zur allgemeinen Lage im Strafvollzug als wichtiges Indiz hinsichtlich der Lage des konkret Betroffenen gesehen. Der Gerichtshof schliesst immer wieder von der Gesamtsituation auf die Situation im konkreten Fall. Der Anscheinsbeweis gelingt damit in der Regel. Bei der Gesundheitsversorgung ist die Bedeutung der Berichte dann am grössten, wenn es um infrastrukturelle, organisatorische und personelle Mängel geht, welche zu einer inadäquaten medizinischen Versorgung führen. Aber auch bei der Angemessenheitsprüfung der Gesundheitsversorgung, welche massgeblich von den Umständen des Einzelfalls abhängt, werden die Monitoringberichte im Rahmen der freien Beweiswürdigung berücksichtigt.

In der Schweiz erkannte das Bundesgericht bei Überbelegungssituationen mehrmals Verletzungen von Art. 3 EMRK. Der menschenrechtliche Standard des Bundesgerichts ist höher als jener der Strassburger Rechtsprechung; es geht von einem Minimum von $4 \mathrm{~m}^{2}$ zur Verfügung zu stellenden Platzes aus. Für die Beurteilung der Konfomität mit Art. 3 EMRK wendet das Bundesgericht aus prüfungsmethodischer Sicht die gleichen Elemente mit leicht anderen Modalitäten an wie der EGMR, d.h., auch das Bundesgericht nimmt eine Gesamtbetrachtung vor, wenngleich dies nicht in einem gleich formalisierten Rahmen erfolgt. Aus der bundesgerichtlichen Rechtsprechung geht weiter klar hervor, dass die Prüfung der Überbelegungssituation an den Voraussetzungen der erniedrigenden Behandlung i.S.v. Art. 3 EMRK erfolgt.

Betreffend die Beurteilung der Gesundheitsversorgung im Strafvollzug hatte das Bundesgericht eher Rügen der Verletzung der persönlichen Freiheit, und nicht etwa der unmenschlichen und erniedrigenden Behandlung i.S.v. Art. 3 EMRK, zu prüfen. Hatte das Bundesgericht inadäquate Haftbedingungen als solche zu beurteilen, betraf dies in den konkreten Fallkonstellationen die Gesundheitsversorgung nur als Nebenpunkt. Prüfungsmethodologisch liess das Bundesgericht dann die Gesundheitsversorgung im 
Strafvollzug in seine Gesamtbetrachtung der Haftumstände einfliessen. Abschliessende Aussagen, wie das Bundesgericht verfahren würde, wenn es bei einer Beschwerde im Kern um ein konkretes Versäumnis bei der medizinischen Versorgung ginge, können deshalb noch nicht gemacht werden. Mit Blick auf das durch den EGMR garantierte Schutzniveau ist zu erwarten, dass in solchen Fällen auch das Bundesgericht keine Scheu haben wird, im Rahmen von Art. 3 EMRK gewisse Einzelgarantien in seine Rechtsprechung aufzunehmen und diese unabhängig von einer Gesamtbetrachtung sämtlicher Haftumstände zu garantieren. Dies schon mit Blick darauf, dass bei einer Unterschreitung des Schutzniveaus des EGMR eine Beschwerde des Inhaftierten an den EGMR drohen würde, welche mit einer Verurteilung der Schweiz enden könnte.

Während das Bundesgericht bei der Überbelegungssituation mit der Festsetzung des 4- $\mathrm{m}^{2}$-Standards den CPT-Standard konsolidiert, ist die Rezeption des Soft Law in der bundesgerichtlichen Rechtsprechung betreffend die Gesundheitsversorgung noch nicht gleich ausgeprägt.

Einleitend wurde bereits darauf hingewiesen, dass ein mit Art.-3-EMRKkonformer Strafvollzug weitaus mehr Anforderungen zu erfüllen hat als die beiden hier speziell beleuchteten Themengebiete der Überbelegung und der Gesundheitsversorgung. Beispielhaft erwähnt wurden die Möglichkeit von Outdoor-Aktivitäten, Beschäftigungs- und Arbeitsmöglichkeiten, Schutz vor Witterung, Anforderungen an die hygienischen Verhältnisse im Strafvollzug, Zugang zu sanitären Einrichtungen und genügend Privatsphäre bei deren Nutzung, Anforderungen an die Licht-, Luft- und Temperaturverhältnisse in der Zelle sowie ausreichende und angemessene Ernährung während des Vollzugs. Die Analyse der Überbelegungsproblematik hat deutlich gemacht, dass all diese Faktoren in der Gesamtbetrachtung der Haftsituation mitberücksichtigt werden. In Anlehnung an das prüfungsmethodische Vorgehen des EGMR bei der Gesundheitsversorgung, und an den erarbeiteten eigenen Lösungsvorschlag bei der Überbelegung, ist insbesondere mit Blick auf das Spezifitätskriterium eines absoluten Rechts zu fordern, dass auch bei der Überprüfung dieser Umstände analogerweise vorgegangen würde. Kumulative Effekte bleiben dabei jeweils zu berücksichtigen, während kompensierende Kriterien nur zurückhaltend und unter Verweis auf eine zusätzliche, strikte Grenze zu würdigen sind. Was den materiellen Massstab betrifft, ist auch bei den weiteren Themengebieten zu erwarten, dass der Gerichtshof die Standards des Soft Law aufgreifen wird und dieses sorgfältig auf seine menschenrechtliche Wertigkeit prüft. Erkennt er eine Relevanz für Art. 3 EMRK, ist 
zu erwarten, dass er das Soft Law aufgreift und in seine Rechtsprechung implementiert.

Im Sinne eines effektiven Menschenrechtsschutzes wäre es schliesslich wünschenswert, dass der EGMR Einzelfragen ungenügender Haftbedingungen auch konkret der unmenschlichen resp. der erniedrigenden Behandlung zuordnen und auf diese Weise den Mitgliedstaaten den ihnen gemachten Vorwurf verdeutlichen würde. Wenn es durch die materiellen Haftbedingungen zu einer Verletzung der psychischen oder physischen Integrität kommt, ist von einer unmenschlichen Behandlung auszugehen. Typischerweise führen etwa prekäre Verhältnisse in den Bereichen der Belüftung, Belichtung, der Nahrung, der hygienischen Verhältnisse, der sanitären Anlagen sowie der medizinischen Versorgung zu einer für Art. 3 EMRK relevanten Gefährdung der körperlichen und psychischen Integrität. Auch ungenügende Platzverhältnisse können im Einzelfall dazu führen. Steht aber klar das Demütigungsmoment im Vordergrund, ist eine erniedrigende Behandlung i.S.v. Art. 3 EMRK anzunehmen.

Ein effektiver, auch in der Praxis gelebter Menschenrechts-Mindeststandard setzt nicht nur eine stringente Rechtsprechung voraus; vielmehr bedarf es in verschiedenen Phasen je unterschiedlicher Durchsetzungsmechanismen. Nur wenn in allen Phasen von sämtlichen zur Verfügung stehenden und im konkreten Fall angezeigten Mitteln Gebrauch gemacht wird, kann dem statuierten und vom Gerichtshof ausgearbeiteten Konventionsstandard in der Praxis zum Durchbruch verholfen werden.

Für die Dauer des Gerichtsverfahrens hat der EGMR mit dem Instrument der vorsorglichen Massnabmen (Regel 39 VerfO) eine Möglichkeit, die Schaffung vollendeter Tatsachen durch den Konventionsstaat, und damit die Vereitelung der Konventionsrechte, zu verhindern. Die Anordnungspraxis des Gerichtshofs im Bereich inadäquater medizinischer Versorgungssituationen bezeugt, dass der Gerichtshof die Bedeutung der vorsorglichen Massnahmen als Garanten für einen effektiven Menschenrechtsschutz in diesem Bereich erkannt hat. Eine akute Krankheit macht denn auch den besonderen Schwächezustand des Inhaftierten deutlich, so dass die Annahme eines irreparablen Gesundheitsschadens bei einem Untätigbleiben naheliegt und die hohen Anordnungsvoraussetzungen von Regel-39-Massnahmen häufig als erfüllt betrachtet werden. Umso besorgniserregender ist die schlechte Befolgungspraxis und Ignoranz einzelner Staaten. Es bleibt deshalb, an sämtliche Konventionsstaaten zu appellieren, verbindlich angeordnete vorsorgliche Massnahmen unverzüglich und vollständig umzusetzen. Der Gerichtshof ist in diesem Sinne anzuhalten, seine konsistente Rechtsprechungslinie und die konsequente Ahndung 
säumiger Konventionsstaaten nach Art. 34 EMRK fortzusetzen. Ferner ist für die Zukunft eine Erstreckung des Anwendungsgebietes von vorsorglichen Massnahmen auch auf andere Unterbringungsdefizite zu fordern, insbesondere auf Überbelegungssituationen, bei welchen im konkreten Fall unmittelbare und irreversible Schädigungen physischer oder psychischer Art drohen.

Weiter steht dem Gerichtshof bei Vorliegen der Voraussetzungen die Anwendung der Piloturteilsverfahrenstechnik offen. In einem Piloturteil stellt er anhand einer numerischen und prognostizierenden Betrachtung fest, dass die vorliegende Konventionsverletzung auf einem strukturellen bzw. systemischen Problem basiert, und nennt in der Folge nicht nur individuelle, sondern auch generelle Abhilfemassnahmen in den Urteilsgründen resp. ordnet solche im Urteilstenor an. Piloturteile haben für Parallelfälle eine gesteigerte Orientierungswirkung, und der politische Druck zur Behebung nicht nur der festgestellten Individualrechtsverletzung, sondern des strukturellen Problems als solchem, wird aufgrund der diesem Verfahren innewohnenden Prangerwirkung erhöht.

Im Bereich inadäquater Haftbedingungen wurden erst gegen einige wenige Konventionsstaaten Piloturteilsverfahren geführt. Die Konventionsverletzung wurde, ausser im Urteil W.D./BEL (ungenügende psychiatrische Versorgung psychisch kranker Internierter), auf die strukturell bzw. systemisch bedingte Überbelegungssituation im jeweiligen Mitgliedstaat zurückgeführt. Weitere kumulierende Haftbedingungen wurden bisweilen gleichzeitig auch bemängelt; diese waren aber niemals für sich alleine Anknüpfungspunkte für die Anwendung des Piloturteilsverfahrens. Statistischen Erhebungen zufolge nimmt die Überbelegung in vielen weiteren europäischen Ländern ein Ausmass an, das auf strukturelle Probleme schliessen lässt. Piloturteilsverfahren gegen weitere Konventionsstaaten erscheinen deshalb in naher Zukunft wahrscheinlich. Darüber hinaus bleibt eine Ausweitung des praktischen Anwendungsbereichs in Betracht zu ziehen; strukturelle bzw. systemische Probleme gibt es, wie zahlreiche CPTBerichte zeigen, insbesondere auch bei der allgemeinen medizinischen Versorgung während des Strafvollzugs. Solche Situationen könnten in $\mathrm{Zu}-$ kunft für sich alleine - ohne Konnex zur Überbelegung - Grund genug für die Anwendung der Piloturteilstechnik bilden.

Der Feststellungscharakter des Urteils des Gerichtshofs macht eine Umsetzung des rechtskräftigen Urteils durch den Konventionsstaat unter der Aufsicht des Ministerkomitees erforderlich. Aufgrund der in Art. 3 EMRK vorausgesetzten Schwere der Konventionsverletzung unterstehen Urteile im Bereich inadäquater Haftbedingungen im Implementierungsverfahren 
grundsätzlich der enhanced supervision des Ministerkomitees; ihre Umsetzung wird damit priorisiert überwacht. Zwischen Konventionsstaat und Ministerkomitee entsteht ein enger Dialog, insbesondere durch Aktionspläne bzw. Aktionsberichte der Konventionsstaaten, Beschlüsse des Ministerkomitees und den Umstand, dass die Implementierungsfortschritte der Piloturteile regelmässig an den Menschenrechtssitzungen aufgegriffen werden. Das Ministerkomitee fordert von den Konventionsstaaten im Sinne eines effektiven Menschenrechtsschutzes im Allgemeinen eine umfassende Implementierung der Urteile. Der Konventionsstaat hat dabei substantielle, sowohl langfristige als auch kurzfristige Abhilfemassnahmen zu ergreifen. Anders als a priori zu erwarten gewesen wäre, fordert das Ministerkomitee nämlich nicht nur im Nachgang an Piloturteile generelle Massnahmen. Auch bei den übrigen Urteilen, in denen das Ergreifen genereller Massnahmen vom Gerichtshof noch nicht vorgespurt wurde, verlangt das Ministerkomitee zur vollständigen Implementierung i.S.v. Art. 46 Abs. 1 EMRK generelle Abhilfemassnahmen vom Konventionsstaat. Dementsprechend lange dauert es in der Praxis auch, bis das Urteil vollständig implementiert ist und die Überwachung des Ministerkomitees abgeschlossen werden kann. Wenn diese lange Dauer nicht auf einen mangelnden Kooperationswillen zurückzuführen ist und parallel zu den langfristigen Massnahmen auch schnell wirksame ergriffen werden, ist dies aber nicht zu bemängeln. Auch für die Zukunft bleibt deshalb an die Mitgliedstaaten zu appellieren, bei der Umsetzung zu kooperieren und auch finanzielle Mittel für wirksame Abhilfemassnahmen bereitzustellen. Ist Letzteres für die Konventionsstaaten aufgrund der Kostenintensität der notwendigen Massnahmen eine unüberwindbare Herausforderung, ist es ihre Pflicht, Finanzierungshilfen zu organisieren. Gerade der HRTF hat sich diesbezüglich in der Praxis hervorgetan. Korrelierend zur Sachverhaltserstellung vor dem Gerichtshof greift auch das Ministerkomitee bei der Beurteilung der Frage, ob eine vollständige Umsetzung erfolgt ist, bzw. ob die ergriffenen Massnahmen den gewünschten Erfolg gebracht haben und sich folglich die allgemeine Situation verbessert hat, immer wieder auf Monitoringberichte anderer Gremien (insbesondere des CPT) zurück. Ihnen kommt folglich nicht nur in der Rechtsprechung selbst, sondern auch im Implementierungsverfahren ein nicht zu unterschätzender Stellenwert zu. 


\section{Literaturverzeichnis}

Abels Denis, Prisoners of the International Community: The Legal Position of Persons Detained at International Criminal Tribunals, Den Haag 2012

Aebi Marcelo F./Tiago Mélanie M., Space I - 2018, Prison Population, Strassburg, 20. Dezember 2018, aktualisiert am 11. Juni 2019, abrufbar unter $<$ https:// wp.unil.ch/space/files/2019/06/FinalReportSPACEI2018_190611-1.pdf>, zuletzt abgerufen am 06.08.2020 (zit.: Aebi/Tiago SPACE I)

Addo Michael/Grief Nicholas, Does Article 3 of The European Convention on Human Rights Enshrine Absolute Rights?, EJIL 1998, S. $510 \mathrm{ff}$.

Addo Michael/Grief Nicholas, Some Practical Issues Affecting the Notion of Absolute Right in Article 3 ECHR, European Law Review - Human Rights Survey 1998, S. 17 ff.

Aebi Marcelo F./Tiago Mélanie M., Prison and Prisoners in Europe 2018: Key Findings of the SPACE I Report, Strassburg, 15. Juni 2019, abrufbar unter $<$ http://wp.unil.ch/space/files/2020/02/Key-Findings-2018_190615.pdf >, zuletzt abgerufen am 06.01.2020 (zit.: Aebi/Tiago Key-Findings)

Aemisegger Heinz, Probleme der Umsetzung der EMRK im schweizerischen Recht, in: Jaag Tobias/Kaufmann Christine (Hrsg.), 40 Jahre Beitritt der Schweiz zur EMRK, Referate zur Jubiläumstagung vom 27. November 2014, Zürich 2015, S. $201 \mathrm{ff}$.

Alleweldt Ralf, Wirkungen von Urteilen des EGMR nach 60 Jahren: Noch klassisches Völkerrecht oder schon Teil eines „ordre constitutionnel européen“? - Kommentar, in: Zimmermann Andreas (Hrsg.), 60 Jahre Europäische Menschenrechtskonvention: Die Konvention als „living instrument“, Berlin 2014, S. $75 \mathrm{ff}$.

Alleweldt Ralf, Protection Against Expulsion Under Article 3 of the European Convention on Human Rights, EJIL 1993, S. $360 \mathrm{ff}$.

Anagnostou Dia/Mungiu-Pippidi Alina, Domestic Implementation of Human Rights Judgments in Europe: Legal Infrastructure and Government Effectiveness Matter, EJIL 2014, S. $205 \mathrm{ff}$.

Anisman Hymie, An Introduction to Stress \& Health, Los Angeles 2014

Altwicker-Hámori Szilvia/Altwicker Tilmann/Peters Anne, Measuring Violations of Human Rights: An Empirical Analysis of Awards in Respect of NonPecuniary Damage Under the European Convention on Human Rights, ZaöRV 2016, S. $1 \mathrm{ff}$.

BaAde Björnstjern, Der Europäische Gerichtshof für Menschenrechte als Diskurswächter: Zur Methodik, Legitimität und Rolle des Gerichtshofs im demokratisch-rechtsstaatlichen Entscheidungsprozess, Heidelberg 2017

Baechtold Andrea, Inventar des kantonalen Strafvollstreckungs- und Strafvollzugsrechts, SZK 1/2004, S. $61 \mathrm{ff}$. 


\section{Literaturverzeichnis}

Baechtold Andrea, Switzerland, in: van Zyl Smit Dirk/Dünkel Frieder (Hrsg.), Imprisonment Today and Tomorrow, International Perspectives on Prisoners' Rights and Prison Conditions, 2. Aufl., Den Haag 2001, S. 653 ff.

Baechtold Andrea, Straf- und Massnahmenvollzug, Bern 1990

Baechtold Andrea/Weber Jonas/Hostettler Ueli, Strafvollzug, Straf- und Massnahmenvollzug an Erwachsenen in der Schweiz, 3. Aufl., Bern 2016

Baldus Manfred, Menschenwürdegarantie und Absolutheitsthese, Zwischenbericht zu einer zukunftsweisenden Debatte, AöR 2011, S. 529 ff.

Baldus Manfred, Der Kernbereich privater Lebensgestaltung - absolut geschützt, aber abwägungsoffen, JZ 2008, S. $218 \mathrm{ff}$.

BARISCH SuSANN, Überbelegung - Alltag in deutschen Justizvollzugsanstalten oder: „Einer geht noch, einer geht noch rein?!“, KJ 2008, S. 425 ff.

Bates Ed, The Evolution of the European Convention on Human Rights, Oxford 2010

Baumann Jessica, Das Piloturteilsverfahren als Reaktion auf massenhafte Parallelverfahren: Eine Bestandesaufnahme der Rechtswirkungen der Urteile des Europäischen Gerichtshofs für Menschenrechte, Berlin 2016

Benavides Casals Maria Angélica, Die Auslegungsmethoden bei Menschenrechtsverträgen: Die Rechtsprechung des Europäischen Gerichtshofes für Menschenrechte und des Interamerikanischen Gerichtshofs für Menschenrechte, Baden-Baden 2010

Bergmann Jan Michael, Das Menschenbild der Europäischen Menschenrechtskonvention, Baden-Baden 1995

Bergsmo Morten (Hrsg.), Quality Control in Fact-Finding, 2013, abrufbar unter $<$ https://www.legal-tools.org/doc/5b59fd/pdf/>, zuletzt abgerufen am 06.08.2020 (zit.: Bergsmo (Hrsg.), Quality Control in Fact-Finding)

Bernhardt Rudolf, Thoughts on the Interpretation of Human Rights Treaties, in: Matscher Franz/Petzold Herbert (Hrsg.), Protecting Human Rights: The European Dimension, Festschrift für Gérard J. Wiarda, Köln 1988, S. 65 ff. (zit.: Bernhard FS Wiarda)

Besson Samantha, The „erga omnes“ Effect of Judgments of the European Court of Human Rights - „What's in a Name?“, in: Besson Samantha (Hrsg.), La Cour européenne des droits de l'homme après le Protocole 14 - premier bilan et perspectives, Zürich 2011, S. $125 \mathrm{ff}$.

Biaggini Giovanni/Gächter Thomas/Kiener Regina (Hrsg.), Staatsrecht, 2. Aufl., Zürich 2015 (zit.: BeArbeIter in: Biaggini/Gächter/Kiener)

Bocchi Marco, The Effectiveness of the Pilot Judgment Procedure: A Comparative Case-Study on Inhuman and Degrading Treatment against Detainees in Russia and Italy, in: Workshop on the ECtHR Tampere 2015, 2016, abrufbar unter $<$ https://blogs.uta.fi/ecthrworkshop/2016/02/08/bocchi/>, zuletzt abgerufen am 04.01.2020

Brade Alexander, Additive Grundrechtseingriffe, Ein Beitrag zur Grundrechtsdogmatik, Baden-Baden 2020 
Breuer Marten, Wirkungen von Urteilen des EGMR nach 60 Jahren: noch klassisches Völkerrecht oder schon Teil eines „ordre constitutionnel européen“?, in: Zimmermann Andreas (Hrsg.), 60 Jahre Europäische Menschenrechtskonvention: Die Konvention als „living instrument“, Berlin 2014, S. 51 ff.

Breuer Marten, Zulässigkeit und Grenzen richterlicher Rechtsfortbildung in der Rechtsprechung des EGMR, ZÖR 2013, S. $729 \mathrm{ff}$.

Breuer Marten, Zur Anordnung konkreter Abhilfemassnahmen durch den EGMR: Der Gerichtshof betritt neue Wege im Fall Asanidse gegen Georgien, EuGRZ 2004, S. 257 ff.

Brownlie Ian, Brownlie's Principles of Public International Law, 9. Aufl., Oxford 2019

Brummer Klaus, Der Europarat, Eine Einführung, Wiesbaden 2008

Buergenthal Thomas, Die Bedeutung der Allgemeinen Erklärung der Menschenrechte für den internationalen Menschenrechtsschutz, in: Klein Eckart (Hrsg.), Studien zu Grund- und Menschenrechten „Menschenrechte für alle“ - 50 Jahre Allgemeine Erklärung der Menschenrechte, Potsdam 1999, S. $19 \mathrm{ff}$.

Catak Kanber Bahar Irem, Die ausländerrechtliche Administrativhaft, Die rechtliche Umsetzung im schweizerischen Recht, Bern 2017

Céré Jean-Paul, Détention, maladie et traitement inhumain ou dégradant, Note sous l'ârret Rivière c. France du 11 juillet 2006, de la Cour européenne des droits de l'homme (2 $2^{\mathrm{e}}$ section), RTDH 2006, S. $261 \mathrm{ff}$.

Cernko Daniela, Die Umsetzung der CPT-Empfehlungen im deutschen Strafvollzug: Eine Untersuchung über den Einfluss des Europäischen Komitees zur Verhütung von Folter und unmenschlicher oder erniedrigender Behandlung oder Strafe (CPT) auf die deutsche Strafvollzugsverwaltung, Berlin 2014

Cerone John, A Taxonomy of Soft Law, Stipulating a Definition, in: Lagoutte Stéphanie/Gammeltoft-Hansen Thomas/Cerone John (Hrsg.), Tracing the Roles of Soft Law in Human Rights, Oxford 2016, S. 15 ff.

Charles Anna/Draper Heather, Equivalence of Care in Prison Medicine: Is Equivalence of Process the Right Measure of Equity, Journal of Medical Ethics 2012, S. $215 \mathrm{ff}$.

Clark Roger S., The United Nations Crime Prevention and Criminal Justice Program, Formulation of Standards and Efforts at their Implementation, Philadelphia 1995

Cornille Perrine/Devos Marie/Mahieu Alice, Étude sur l'article 3 de la CEDH, BP 2006, S. $1 \mathrm{ff}$.

Coullery Pascal, Der Grundrechtsanspruch auf medizinische Leistungen: Ein verfassungsrechtlicher Diskussionsbeitrag zur Rationalisierungsdebatte im Gesundheitswesen, AJP 2001, S. $632 \mathrm{ff}$.

Coyle Andrew/Fair Helen/Jacobson Jessica/Walmsley Roy, Imprisonment Worldwide: The Current Situation and an Alternative Future, Bristol 2016 
Cremer Hans-Joachim, Prescriptive Orders in the Operative Provisions of Judgments by the European Court of Human Rights: Beyond res judicanda?, in: Seibert-Fohr Anja/Villiger Mark, Judgments of the European Court of Human Rights - Effects and Implementation, Farnham 2014, S. 39 ff.

De Londras Fiona/Dzehtsiarou Kanstantsin, Mission Impossible? Addressing Non-Execution through Infringement Proceedings in the European Court of Human Rights, International \& Comparative Law Quarterly 2017, S. 467 ff.

Dемко Daniela, Zur „Einzelfallprüfung“ und „geltungszeitlichen Interpretation“ im Rahmen des Art. 3 EMRK, HRRS 2005, S. $94 \mathrm{ff.}$

Devresse MArie-Sophie, La gestion de la surpopulation pénitentiaire: perspectives politiques, administratives et juridictionnelles, Droit et Société 2013, S. $339 \mathrm{ff}$.

Dörr Oliver/Grote Rainer/Marauhn Thilo (Hrsg.), EMRK/GG Konkordanzkommentar zum europäischen und deutschen Grundrechtsschutz, Band I, 2. Aufl., Tübingen 2013 (zit.: Dörr/Grote/Marauhn/Bearbeiter)

Dörr Oliver/Schmalenbach Kirsten (Hrsg.), Vienna Convention on the Law of Treaties, A Commentary, 2. Aufl., Berlin 2012 (zit.: Dörr/Schmalenbach/BEARBEITER)

Drenkhahn Kirstin/Dudeck Manuela/Dünkel Frieder, Long-Term Imprisonment and Human Rights, London 2014

Drenkhahn Kirstin/Morgenstern Christine, A European Perspective on Inmates' Perceptions of Safety, in: Reeves Carla (Hrsg.), Experiencing Imprisonment: Research on the Experience of Living and Working in Carceral Institutions, London 2016, S. $137 \mathrm{ff}$.

Dummermuth Martin, Die EMRK und die Schweiz - eine Standortbestimmung nach 40 Jahren, SJZ 2014, S. $597 \mathrm{ff}$.

Dünkel Frieder/Baechtold Andrea/van Zyl Smit Dirk, Europäische Mindeststandards und Empfehlungen als Orientierungspunkte für die Gesetzgebung und Praxis dargestellt am Beispiel der Empfehlungen für inhaftierte Jugendliche und Jugendliche in ambulanten Massnahmen, in: Goerdeler Jochen/Walkenhorst Philipp (Hrsg.), Jugendstrafvollzug in Deutschland: Neue Gesetze, neue Strukturen, neue Praxis, Mönchengladbach 2007, S. 114 ff.

Dünkel Frieder/Geng Bernd, Fakten zur Überbelegung im Strafvollzug und Wege zur Reduzierung von Gefangenenraten, Neue Kriminalpolitik 2003, S. 146 ff.

Dünkel Frieder/Lappi-Sepälä Tapio/Morgenstern Christine/van Zyl Smit Dirk, Gefangenenrate und Kriminalpolitik in Europa: Zusammenfassung und Schlussfolgerungen, in: Dünkel Frieder/Lappi-Sepälä Tapio/Morgenstern Christine/van Zyl Smit Dirk (Hrsg.), Kriminalität, Kriminalpolitik, strafrechtliche Sanktionspraxis und Gefangenenraten im europäischen Vergleich, Band II, Mönchengladbach 2010, S. $1023 \mathrm{ff}$.

Dünkel Frieder/Morgenstern Christine/Zolondek, Europäische Strafvollzugsgrundsätze verabschiedet!, Neue Kriminalpolitik 2006, S. 86 ff. 
Dünkel Frieder/Morgenstern Christine, Überbelegung im Strafvollzug - Gefangenenraten im internationalen Vergleich, in: Britz Guido (Hrsg.), Grundfragen staatlichen Strafens: Festschrift für Heinz Müller-Dietz zum 70. Geburtstag, München 2001, S. 133 ff. (zit.: DünkeL/Morgenstern FS Müller-Dietz)

Dünkel Frieder/van Zyl Smit Dirk, Conclusion, in: van Zyl Smit Dirk/Dünkel Frieder (Hrsg.), Imprisonment Today and Tomorrow: International Perspectives on Prisoners" Rights and Prison Conditions, 2. Aufl., Den Haag 2001, S. 796 ff.

Ehlers Dirk, Europäische Grundrechte und Grundfreiheiten, 4. Aufl., Berlin 2014

Enengel Petra, Grundrechtsschutz im Strafvollzug - unter besonderer Berücksichtigung der Rechtsprechung des EGMR zu Artikel 3 und Artikel 8 EMRK, Wien 2014

Eschment Jörn, Musterprozesse vor dem Europäischen Gerichtshof für Menschenrechte, Probleme und Perspektiven des Piloturteilsverfahrens, Frankfurt am Main 2011

Evans Malcolm David, Getting to Grips with Torture, International and Comparative Law Quarterly 2002, S. 365 ff.

Farrington David P./Nuttall Christopher P., Prison Size, Overcrowding, Prison Violence, and Recidivism, Journal of Criminal Justice 1980, S. $221 \mathrm{ff}$.

Fassbender Bardo, Idee und Anspruch der Menschenrechte im Völkerrecht, Die Allgemeine Erklärung der Menschenrechte, APuZ 46/2008, S. 3 ff.

Favuzza Federica, Torreggiani and Prison Overcrowding in Italy, HRLR 2017, S. $153 \mathrm{ff}$.

Feldman David, Civil Liberties and Human Rights in England and Wales, 2. Aufl., Oxford 2002

Flauss Jean-François, Du droit international comparé des droits de l'homme dans la jurisprudence de la Cour européenne des droits de l'homme, in: Widmer Pierre (Hrsg.), Le rôle du droit comparé dans l'avènement du droit européen: Lausanne, 14-15 avril 2000, Zürich 2002, S. 159 ff.

Forowicz Magdalena, The Reception of International Law in the Case Law of the European Convention of Human Rights, Zürich 2010

Frowern Jochen, The Binding Force of ECHR Judgments and its Limits, in: Breitenmoser Stephan/Ehrenzeller Bernhard/Sassoli Marco/Stoffel Walter/Wagner Pfeifer Beatrice (Hrsg.), Menschenrechte, Demokratie und Rechtsstaat - Liber Amicorum Luzius Wildhaber, Zürich 2007, S. 261 ff. (zit.: Froweın FS Wildhaber)

Frowein Jochen/Peukert Wolfgang (Hrsg.), Europäische Menschenrechtskonvention: EMRK-Kommentar, 3. Aufl., Kehl 2011 (zit.: Frowein/Peukert/BeArbeITER)

Frühwald Stefan/Frottier Patrick/Eher Reinhard/Benda Norbert/Ritter Kristina, Welche Relevanz hat die dokumentierte Suizidalität in Gefangenensuiziden? Did Suicidal Behavior Have Relevance for Prison Suicide?, Psychiatrische Praxis 2001, S. $326 \mathrm{ff}$.

Furusho Carolina Yoko, Uncovering the Human Rights of the Vulnerable Subject and Correlated State Duties under Liberalism, UCL JLJ 2016, S. 175 ff. 
Gächter Thomas, Grenzen der Solidarität?, Individuelle Ansprüche auf medizinische Leistungen gegenüber der Rechts- und Versichertengemeinschaft, in: Zäch Roger/Breining-Kaufmann Christine/Ernst Wolfgang/Oberhammer Paul/ Portmann Wolfgang/Thier Andreas (Hrsg.), Individuum und Verband, Festgabe zum schweizerischen Juristentag 2006, Zürich 2006, S. $473 \mathrm{ff}$.

Gammeltoft-Hansen Thomas/Lagoutte Stéphanie/Cerone John, Introduction: Tracing the Roles of Soft Law in Human Rights, in: Lagoutte Stéphanie/Gammeltoft-Hansen Thomas/Cerone John (Hrsg.), Tracing the Roles of Soft Law in Human Rights, Oxford 2016, S. 1 ff.

Garcia-Guerrero Julio/Marco A., Sobreocupación en los Centros Penitenciarios y su impacto en la salud / Overcrowding in Prisons and its Impact on Health, Revista Española de Sanidad Penitenciaria 2012, S. $106 \mathrm{ff}$.

Garlicki LeCH, Broniowski and after: on the Dual Nature of „Pilot Judgment“, in: Caflisch Lucius/Callewaert Johan/Liddell Roderick/Mahoney Paul/Villiger Mark (Hrsg.), Liber Amicorum Luzius Wildhaber: Human Rights - Strasbourg views, Kehl 2007, S. 177 ff. (zit.: Garlicki FS Wildhaber)

Gebauer Peer, Zur Grundlage des absoluten Folterverbots, NVwZ 2004, S. 1405 ff.

Gewirth Alan, There are Absolute Rights, The Philosophical Quarterly 1982, S. $348 \mathrm{ff}$.

Gewirth Alan, Are There Any Absolute Rights?, The Philosophical Quarterly 1981, S. 1 ff.

Gilch Stefan, Die Reformen am Europäischen Gerichtshof für Menschenrechte unter besonderer Berücksichtigung des 14. Zusatzprotokolls zur EMRK: Ein internationales Gericht am Wendepunkt seiner Entwicklung, Göttingen 2009

Glas Lize, From Interlaken to Copenhagen: What Has Become of the Proposals Aiming to Reform the Functioning of the European Court of Human Rights?, HRLR 2020, S. $121 \mathrm{ff}$.

Glas Lize, The Execution Process of Pilot Judgments before the Committee of Ministers, HR\&ILD 2019, S. $73 \mathrm{ff}$.

Glas Lize, The Boundaries to Dialogue with the European Court of Human Rights, EYHR 2018, S. 287 ff.

Glas Lize, The European Court of Human Rights' Use of Non-Binding and Standard-Setting Council of Europe Documents, HRLR 2017, S. $97 \mathrm{ff}$.

Glas Lize, The Theory, Potential and Practice of Procedural Dialogue in the European Convention on Human Rights System, Cambridge 2016

Glas Lize, Changes in the Procedural Practice of the European Court of Human Rights: Consequences for the Convention System and Lessons to be Drawn, HRLR 2014, S. $671 \mathrm{ff}$.

Grabenwarter Christoph, Rechtliche Rahmenbedingungen des Verhältnisses zwischen EU und Europarat aus der Perspektive des Europarates und die Rolle der Mitgliedstaaten, ZaörV 2014, S. 419 ff.

Grabenwarter Christoph, Grundrechtsvielfalt und Grundrechtskonflikte im europäischen Mehrebenensystem - Wirkungen von EGMR-Urteilen und der Beurteilungsspielraum der Mitgliedstaaten, EuGRZ 2011, S. 229 ff. 
Grabenwarter Christoph, Wirkung eines Urteils des Europäischen Gerichtshofs für Menschenrechte - am Beispiel des Falls M. gegen Deutschland, JZ 2010, S. $857 \mathrm{ff}$.

Grabenwarter Christoph/Pabel Katharina, Europäische Menschenrechtskonvention, 6. Aufl., München 2016

Gräfenstein Evelyn, Art. 3 EMRK und die Behandlung von Strafgefangenen, ZfStrVO 2003, S. 10 ff.

Greer Steven, Is the Prohibition against Torture, Cruel, Inhuman and Degrading Treatment Really „Absolute“ in International Human Rights Law? A Reply to Graffin and Mavronicola, HRLR 2018, S. 297 ff.

Greer Steven, Is the Prohibition against Torture, Cruel, Inhuman and Degrading Treatment Really „Absolute“ in International Human Rights Law?, HRLR 2015, S. $101 \mathrm{ff}$.

Griffel Alain, Allgemeines Verwaltungsrecht im Spiegel der Rechtsprechung, Zürich 2017

Häfelin Ulrich/Müller Georg/Uhlmann Felix, Allgemeines Verwaltungsrecht, 7. Aufl., Zürich 2016

Haider Dominik, The Pilot-Judgment Procedure of the European Court of Human Rights, Leiden 2013

Haney Craig, Prison Overcrowding, in: Cutler Brian/Zapf Patricia (Hrsg.), APA Handbook of Forensic Psychology, Criminal Investigation, Adjudication, and Sentencing Outcomes, Band II, Washington 2015, S. 415 ff.

Harris David/O'Boyle Michael/Warbrick Colin, Law of the European Convention on Human Rights, 4. Aufl., Oxford 2018

Hass Solveig, Die Urteile des Europäischen Gerichtshofs für Menschenrechte: Charakter, Bindungswirkung und Durchsetzung, Frankfurt am Main 2006

Hassemer Winfried, Unverfügbares im Strafprozess, in: Kaufmann Arthur/Zacher Hans (Hrsg.), Rechtsstaat und Menschenwürde: Festschrift für Werner Maihofer zum 70. Geburtstag, Frankfurt am Main 1988, S. 183 ff. (zit.: Hassemer FS Maihofer)

Heine Henriette, Die Rechtsstellung des Beschuldigten im Rahmen der Europäisierung des Strafverfahrens, Frankfurt am Main 2009

Helgesen Jan Erik, What Are the Limits of the Evolutive Interpretation of the European Convention on Human Rights?, HRLJ 2011, S. 275 ff.

Hillenkamp Thomas, Der Arzt im Strafvollzug - Rechtliche Stellung und medizinischer Auftrag, in: Hillenkamp Thomas/Tag Brigitte (Hrsg.), Intramurale Medizin - Gesundheitsfürsorge zwischen Heilauftrag und Strafvollzug, Berlin 2005, S. $11 \mathrm{ff}$.

Hillgenberg Hartmut, A Fresh Look at Soft Law, EJIL 1999, S. 499 ff.

Hirsch Silke Marion, Die Kommunikationsmöglichkeiten des Strafgefangenen mit seiner Familie, Frankfurt am Main 2003 
Hohl-Chirazi Catherine, Mesures de substitution et conditions de détention dignes: les obligations positives du juge de la détention, forumpoenale 2018, S. $187 \mathrm{ff}$.

Holoubek Michael, Grundrechtliche Gewährleistungspflichten: Ein Beitrag zu einer allgemeinen Grundrechtsdogmatik, Wien 1997

Hungerbühler Francine, Die Kommission zur Verhütung von Folter, Zürich 2013

JaAg Tobias/Hänni Julia, Europarecht: Die europäischen Institutionen aus schweizerischer Sicht, 4. Aufl., Zürich 2015

Jacobs Francis Geoffrey/White Robin C.A./Ovey Clare, The European Convention on Human Rights, in: Rainey Bernadette/Wicks Elizabeth/Ovey Clare (Hrsg.), 7. Aufl., Oxford 2017

Jaeger Renate, Nach der Reform ist vor der Reform, in: Zimmermann Andreas (Hrsg.), 60 Jahre Europäische Menschenrechtskonvention: Die Konvention als „living instrument“, Berlin 2014, S. $125 \mathrm{ff}$.

Jahn Jannika, Ruling (In)Directly through Individual Measures? Effect and Legitimacy of the ECtHR's New Remedial Power, ZaöRV 2014, S. $1 \mathrm{ff}$.

Jotterand Fabrice/Wangmo Tenzin, The Principle of Equivalence Reconsidered: Assessing the Relevance of the Principle of Equivalence in Prison Medicine, The American Journal of Bioethics, Heft 7 2014, S. 4 ff.

Jung Heike, Die Empfehlungen des Ministerkomitees des Europarates - zugleich ein Beitrag zur europäischen Rechtsquellenlehre, in: Bröhmer Jürgen/ Bieber Roland/Callies Christian/Langenfeld Christine/Weber Stefan/Wolf Joachim (Hrsg.), Internationale Gemeinschaft und Menschenrechte: Festschrift für Georg Ress zum 70. Geburtstag am 21. Januar 2005, Köln 2005, S. 519 ff. (zit.: Jung FS Ress)

Jung Heike, Sanktionensysteme und Menschenrechte, Bern 1992

Junger-Tas Josine, Introduction: The Respect of Human Rights of Prisoners in Europe, Eur J Crim Policy Res 2006, S. $79 \mathrm{ff}$.

Kaiser Günther/Schöch Heinz, Strafvollzug, 5. Aufl., Heidelberg 2002

KäLin Walter/Künzli Jörg, Universeller Menschenrechtsschutz, Der Schutz des Individuums auf globaler und regionaler Ebene, 4. Aufl., Basel 2019

Karpenstein Ulrich/Mayer Franz C. (Hrsg.), Konvention zum Schutz der Menschenrechte und Grundfreiheiten: Kommentar, 2. Aufl., München 2015 (zit.: Karpenstein/Mayer/BeARbeIter)

Karstedt Susanne, Freiheit, Gleichheit und (Straf)Recht: Werte und Strafen in demokratischen Gesellschaften, in: Dünkel Frieder/Lappi-Seppälä Tapio/Morgenstern Christine/van Zyl Smit Dirk (Hrsg.), Kriminalität, Kriminalpolitik, strafrechtliche Sanktionspraxis und Gefangenenrate im europäischen Vergleich, Band II, Mönchengladbach 2010, S. 935 ff.

Keller Helen/Marti Cedric, Reconceptualizing Implementation: The Judicialization of the Execution of the European Court of Human Rights' Judgments, EJIL 2015, S. $829 \mathrm{ff}$. 
Keller Helen/Marti Cedric, Berücksichtigung der EGMR-Praxis durch innerstaatliche Gerichte, Justice - Justiz - Giustizia: Die Schweizer Richterzeitung Heft 1 2015, S. 1 ff.

Keller Helen/Schädler Simon, Freiheitsrechte im Strafvollzug - Plädoyer für die Abkehr vom Sonderstatus, ZSR 2013, S. $195 \mathrm{ff}$.

Kiener Regina, Die Umsetzung menschenrechtlicher Vorgaben in Polizei, Justiz und Justizvollzug: Das Beispiel der föderalistischen Schweiz, in: Fink Daniel/ Arnold Jörg/Genillod-Villard Françoise/Oberholzer Niklaus (Hrsg.), Kriminalität, Strafrecht und Föderalismus, Bern 2019, S. 3 ff.

Kiener Regina, Der Einfluss der EMRK auf die BV 1999, in: Jaag Tobias/Kaufmann Christine (Hrsg.), 40 Jahre Beitritt der Schweiz zur EMRK: Referate zur Jubiläumstagung vom 27. November 2014, Zürich 2015, S. $53 \mathrm{ff}$.

Kiener Regina/Kälin Walter/Wyttenbach Judith, Grundrechte, 3. Aufl., Bern 2018

Kindt Eline, The Pilot Judgment Procedure at the European Court of Human Rights: An Evaluation in the Light of Procedural Efficiency and Access to Justice, Ghent 2018

KLabbers Jan, The Undesirability of Soft Law, Nordic Journal of International Law 1998, S. $381 \mathrm{ff}$.

Klare Hugh J., The Work of the European Committee on Crime Problems, The British Journal of Criminology 1961, S. 377 ff.

Kley Andreas/Sigrist Martin, Der Beitritt der Schweiz zur EMRK - Vorbereitung und Umsetzung des Beitritts vor und nach 1974, in: Jaag Tobias/Kaufmann Christine (Hrsg.), 40 Jahre Beitritt der Schweiz zur EMRK, Referate zur Jubiläumstagung vom 27. November 2014, Zürich 2015, S. $17 \mathrm{ff}$.

Koeppel Thordis, Kontrolle des Strafvollzuges: Individueller Rechtsschutz und generelle Aufsicht: Ein Rechtsvergleich, Mönchengladbach 1999

Kretschmer JoAснim, Die Mehrfachbelegung von Hafträumen im Strafvollzug in ihrer tatsächlichen und rechtlichen Problematik, NStZ 2005, S. $251 \mathrm{ff}$.

Kretschmer Joachim, Die menschen(un)würdige Unterbringung von Strafgefangenen, NJW 2009, S. $2406 \mathrm{ff}$.

Kromrey Hans, Haftbedingungen als Auslieferungshindernis: Ein Beitrag zur Verwirklichung der Menschenrechte, Mönchengladbach 2017 (zit.: Kromrey H.)

Kromrey Hans/Morgenstern Christine, Die Menschenwürde und das Auslieferungsverfahren, ZIS 2017, S. $106 \mathrm{ff}$.

Kromrey Ilka, Belastungskumulation: Ein Beitrag zur Erweiterung des grundrechtlichen Eingriffsbegriffs, Tübingen 2018 (zit.: Kromrey I.)

Künzli Jörg/Achermann Alberto, Gesundheitsrelevante Rechte inhaftierter Personen im Bereich des Schutzes vor Infektionskrankheiten und Kompetenzen des Bundes zu ihrer Durchsetzung, Studie zuhanden des Bundesamtes für Gesundheit, Bern 2007, abrufbar unter $<$ https://www.bag.admin.ch/dam/bag/fr/dokume nte/e-f/evalber-mt/2001-2010/2007-rechte-inhaftlierte-infektionskrankheiten-sch lussbericht.pdf.download.pdf/2007-rechte-inhaftierte-infektkrankheiten-d.pdf>, zuletzt abgerufen am 07.08.2020 
Künzli Jörg/Eugster Anja/Spring Alexander, Die Anerkennung justiziabler Rechte im Bereich der wirtschaftlichen, sozialen und kulturellen Menschenrechte durch das Bundes- und das kantonale Recht, Studie des Schweizerischen Kompetenzzentrums für Menschenrechte zuhanden des Lenkungsausschusses EDA/EJPD, Bern 2014, abrufbar unter $<$ https:/www.skmr.ch/cms/upload/pdf/1 41112_Studie_WSK-Rechte_Publikation_Okt_2014.pdf $>$, zuletzt abgerufen am 07.08.2020

KünZli Jörg/Weber Florian, Gesundheit im Freiheitsentzug, Rechtsgutachten zur Gesundheitsversorgung von inhaftierten Personen ohne Krankenversicherung, Bern 2018, abrufbar unter < https:/www.skmr.ch/cms/upload/pdf/191120_ Gesundheitsversorgung_Inhaftierte.pdf>, zuletzt abgerufen am 07.08.2020

Lalumière Catherine, Human Rights in Europe: Challenges for the Next Millennium, in: Macdonald Ronald St. J./Matscher Franz/Petzhold Herbert (Hrsg.), The European System for the Protection of Human Rights, Dordrecht 1993, S. XV ff.

Laubenthal Klaus, Strafvollzug, 7. Aufl., Berlin 2015

Leach Philip/Hardman Helen/Stephenson Svetlana, Can the European Court's Pilot Judgment Procedure Help Resolve Systematic Human Rights Violations? Burdov and the Failure to Implement Domestic Court Decisions in Russia, HRLR 2010, S. $346 \mathrm{ff}$.

Leach Philip/Hardman Helen/Stephenson Svetlana/Blitz Brad, Responding to Systematic Human Rights Violations: An Analysis of „Pilot Judgments“ of the European Court of Human Rights and Their Impact at National Level, Antwerp 2010

Leach Philip/Paraskeva Costas/Uzelac Gordana, International Human Rights \& Fact-Finding: An Analysis of the Fact-finding Missions Conducted by the European Commission and Court of Human Rights, London 2009

Leeb David, Die innerstaatliche Umsetzung der Feststellungsurteile des Europäischen Gerichtshofes für Menschenrechte im entschiedenen Fall, Linz 2001

Levinson Jerrold, Gewirth on Absolute Rights, The Philosophical Quarterly 1982, S. $73 \mathrm{ff}$.

Ligthart Sjors/van Oploo Laura/Meijers Jesse/Meynen Gerben/Kooijmans Tijs, Prison and the Brain; Neuropsychological Research in the Light of the European Convention on Human Rights, NJECL 2019, S. 287 ff.

Lines Rich, From Equivalence of Standards to Equivalence of Objectives: The Entitlement of Prisoners to Health Care Standards Higher than Those Outside Prisons, International Journal of Prisoner Health 2006, S. 269 ff.

Livingstone Stephen, Prisoners' Rights in the Context of the European Convention on Human Rights, Punishment \& Society 2000, S. 309 ff.

Maelicke Bernd, Überbelegung = Fehlbelegung? !!! Plädoyer für grundlegende Systemverbesserung im deutschen Strafvollzug, Neue Kriminalpolitik 2003, S. $143 \mathrm{ff}$. 
Mahoney Paul, The Comparative Method in Judgments of the European Court of Human Rights; Reference Back to National Law, in: Widmer Pierre (Hrsg.), Le rôle du droit comparé dans l'avènement du droit européen, Lausanne, 14-15 avril 2000, Zürich 2002, S. 143 ff.

Mahoney Paul/Kondak Rachael, Common Ground: A Starting Point or Destination for Comparative-Law Analysis by the European Court of Human Rights?, in: Andenas Mads/Fairgrieve Duncan (Hrsg.), Courts and Comparative Law, Oxford 2015, S. 119 ff.

Mahoney Paul/Prebensen Søren, The European Court of Human Rights, in: Macdonald Ronald St. J./Matscher Franz/Petzhold Herbert (Hrsg.), The European System for the Protection of Human Rights, Dordrecht 1993, S. $621 \mathrm{ff}$.

Matscher Franz, Die Begründung der Entscheidungen des Europäischen Gerichtshofs für Menschenrechte, in: Beyerlin Ulrich/Bothe Michael/Hofmann Rainer/Petersmann Ernst-Ulrich (Hrsg.), Recht zwischen Umbruch und Bewahrung: Völkerrecht, Europarecht, Staatsrecht: Festschrift für Rudolf Bernhardt, Berlin 1995, S. 503 ff. (zit.: Matscher FS Bernhardt)

Mavronicola Natasa, Is the Prohibition Against Torture and Cruel, Inhuman and Degrading Treatment Absolute in International Human Rights Law? A Reply to Steven Greer, HRLR 2017, S. 479 ff.

Mavronicola Natasa, Crime, Punishment and Article 3 ECHR: Puzzles and Prospects of Applying an Absolute Right in a Penal Context, HRLR 2015, S. $721 \mathrm{ff}$.

Mavronicola Natasa, What Is an Absolute Right? Deciphering Absoluteness in the Context of Article 3 of the European Convention on Human Rights, HRLR 2012, S. $723 \mathrm{ff}$.

Mavronicola Natasa/Messineo Francesco, Relatively Absolute? The Undermining of Article 3 ECHR in Ahmad v UK, MLR 2013, S. $589 \mathrm{ff}$.

Meier Bernd-Dieter, Ärztliche Versorgung im Strafvollzug: Äquivalenzprinzip und Ressourcenknappheit, in: Hillenkamp Thomas/Tag Brigitte (Hrsg.), Intramurale Medizin - Gesundheitsfürsorge zwischen Heilauftrag und Strafvollzug, Berlin 2005, S. $35 \mathrm{ff}$.

Meier Bernd-Dieter, Äquivalenzprinzip, in: Gefängnismedizin; Medizinische Versorgung unter Haftbedingungen, Keppler Karlheinz/Stöver Heino (Hrsg.), Stuttgart 2009, S. $76 \mathrm{ff}$.

Melis Martina, From the Principle of Equivalence to the Practice of Care: Thoughts from the 9th ENDIPP Conference, International Journal of Prisoner Health 2009, S. $303 \mathrm{ff}$.

Meyer Frank, in: Wolter Jürgen (Hrsg.), Systematischer Kommentar zur Strafprozessordnung mit GVG und EMRK, Band X, 5. Aufl., Köln 2019 (zit.: SK-StPO/ MeYer)

Meyer Frank, Der EGMR als Tatsacheninstanz und das Recht auf Wahrheit - Morgendämmerung eines neuen Konventionsrechts?, in: Stuckenberg CarlFriedrich/Gärditz Klaus Ferdinand (Hrsg.), Strafe und Prozess im freiheitlichen Rechtsstaat: Festschrift für Hans-Ullrich Paeffgen zum 70. Geburtstag am 2. Juli 2015, Berlin 2015, S. 793 ff. (zit.: Meyer FS Paeffgen) 
Meyer Frank, Strafrechtsgenese in internationalen Organisationen, Eine Untersuchung der Strukturen und Legitimationsvoraussetzungen strafrechtlicher Normbildungsprozesse in Mehrebenensystemen, Baden-Baden 2012

Meyer Frank, Eine Geologie des Strafrechts, ZStW 123 (2011), S. 1 ff.

Meyer-Ladewig Jens/Nettesheim Martin/von Raumer Stefan (Hrsg.), Europäische Menschenrechtskonvention, Handkommentar, 4. Aufl., Baden-Baden 2017 (zit.: Meyer-Ladewig/Bearbeiter)

Milej Tomasz, Entwicklung des Völkerrechts: Der Beitrag internationaler Gerichte und Sachverständigengremien, Berlin 2014

Möhlenbeck Michaela, Das absolute Folterverbot: Seine Grundlagen und die strafrechtlichen sowie strafprozessualen Folgen seiner Verletzung, Frankfurt am Main 2008

Morgan Rod, The European Committee for the Prevention of Torture and Inhuman and Degrading Treatment or Punishment, in: van Zyl Smit Dirk/Dünkel Frieder (Hrsg.), Imprisonment Today and Tomorrow, International Perspectives on Prisoners' Rights and Prison Condition, 2. Aufl., Den Haag 2001, S. 717 ff.

Morgenstern Christine, Anrechnung von Auslandshaft nach $\mathbb{\$} 51$ Abs. 3 und 4 StGB und die Frage der Haftbedingungen, StV 2016, S. 395 ff.

Morgenstern Christine, Verfassungs- und europarechtliche Vorgaben für den Untersuchungshaftvollzug - zugleich Anmerkung zu BVerfG, Beschl. v. 17.11.2012 2 BvR 736/11, StV 2013, S. $529 \mathrm{ff}$.

Morgenstern Christine, Internationale Instrumente und Entwicklungen zur Humanisierung im Strafvollzug, in: Dünkel Frieder/Drenkhahn Kristin/Morgenstern Christine (Hrsg.), Humanisierung des Strafvollzugs - Konzepte und Praxismodelle, Mönchengladbach 2008, S. 35 ff.

Morgenstern Christine, EU-Ausländer in europäischen Gefängnissen, Neue Kriminalpolitik 2007, S. 139 ff.

Morgenstern Christine, Internationale Mindeststandards für ambulante Strafen und Massnahmen, Mönchengladbach 2002

Morgenstern Christine/van Zyl Smit Dirk, International Human Rights Standards and Community Sanctions, in: Bruinsma Gerben/Weisburd David (Hrsg.), Encyclopedia of Criminology and Criminal Justice, New York 2014, S. $2614 \mathrm{ff}$.

Mowbray Alastair, Subsidiarity and the European Convention on Human Rights, HRLR 2015, S. 313 ff.

Mowbray Alastair, Between the Will of the Contracting Parties and the Needs of Today: Extending the Scope of Convention Rights and Freedoms Beyond What Could Have Been Foreseen by the Drafters of the ECHR, in: Brems Eva/Gerards Janneke (Hrsg.), Shaping Rights in the ECHR: The Role of the European Court of Human Rights in Determining the Scope of Human Rights, Cambridge 2013, S. $17 \mathrm{ff}$.

Mowbray Alastair, The Development of Positive Obligations Under the European Convention on Human Rights by the European Court of Human Rights, Oxford 2004 
Müller Markus, Das besondere Rechtsverhältnis, Bern 2003

Murdoch Jim, The Treatment of Prisoners, European Standards, Strassburg 2006

Murdoch Jim, Tackling III-Treatment in Places of Detention: The Work of the Council of Europe's "Torture Committee“, Eur J Crim Policy Res 2006, S. $121 \mathrm{ff}$.

Nagler Katrin, Medizinische Grundversorgung im geschlossenen Strafvollzug, Vorgaben auf Bundesebene unter besonderer Berücksichtigung des Angleichungsgrundsatzes und des verfassungsrechtlichen Anspruchs auf Hilfe in Notlagen, Zürich 2012

Nawparwa Manazha, Europäische Union und Europarat: Komplementarität und Rivalität, Frankfurt am Main 2014

Neale Kenneth, The European Prison Rules, Contextual, Philosophical and Practical Aspects, in: Muncie John/Sparks Richard (Hrsg.), Imprisonment, European Perspectives, New York 1991, S. 203 ff.

Nicolau George, The New Perspective of the European Court of Human Rights on the Effectiveness of its Judgments, in: Hohmann-Dennhardt/Masuch Peter/Villiger Mark (Hrsg.), Grundrechte und Solidarität: Durchsetzung und Verfahren: Festschrift für Renate Jaeger, Kehl 2010, S. 163 ff. (zit.: Nicolau FS Jaeger)

Niveau Gérard, Relevance and Limits of the Principle of Equivalence of Care in Prison Medicine, Journal of Medical Ethics 2007, S. $610 \mathrm{ff}$.

Nowak Manfred/Ammer Margit/Birk Moritz/Monina Giuliana (Hrsg.), The United Nations Convention against Torture and its Optional Protocol: A Commentary, 2. Aufl., Oxford 2019 (zit.: Nowak/Ammer/Birk/Monina/Bearbeiter)

Oberheim Rainer, Gefängnisüberfüllung: Ursachen, Folgen und Lösungsmöglichkeiten in der Bundesrepublik Deutschland mit einem internationalen Vergleich, Frankfurt am Main 1985

Oesch Matthias, Europarecht, Band I, Grundlagen, Institutionen, Verhältnis Schweiz-EU, 2. Aufl., Bern 2019

OMlin Esther, Nationale Kommission zur Verhütung von Folter (NKVF), in: Brägger Benjamin F. (Hrsg.), Das schweizerische Vollzugslexikon: Von der vorläufigen Festnahme zur bedingten Entlassung, Basel 2014, S. $319 \mathrm{f}$.

Ostfeld Adrian M./Kasl Stanislav V./D’Atri David S./Fitzgerald Edward F., Stress, Crowding and Blood Pressure in Prison, Hillsdale New Jersey 1987

Pabel Katharina, Ministerkomitee und EMRK: Fremdkörper oder (noch) essentieller Bestandteil?, in: Zimmermann Andreas (Hrsg.), 60 Jahre Europäische Menschenrechtskonvention: Die Konvention als „living instrument“, Berlin 2014, S. $81 \mathrm{ff}$.

Pabel Katharina/Schmahl Stefanie (Hrsg.), Internationaler Kommentar zur Europäischen Menschenrechtskonvention, Loseblattsammlung, Köln 2019 (zit.: IK/BEARBEITER) 


\section{Literaturverzeichnis}

Papier Hans-Jürgen, Die Mühsal der Ebene - Zur Beschränkbarkeit von Grundund Menschenrechten, in: Breitenmoser Stephan/Ehrenzeller Bernhard/Sassoli Marco/Stoffel Walter/Wagner Pfeifer Beatrice (Hrsg.), Menschenrechte, Demokratie und Rechtsstaat: Liber Amicorum Luzius Wildhaber, Zürich 2007, S. 523 ff. (zit.: Papier FS Wildhaber)

Peters Anne/Altwicker Tilmann, Europäische Menschenrechtskonventionen: Mit rechtsvergleichenden Bezügen zum deutschen Grundgesetz, 2. Aufl., München 2012

Pitts James M.A./Griffin Hayden O./Johnson Wesley W., Contemporary Prison Overcrowding: Short-Term Fixes to a Perpetual Problem, Contemporary Justice Review 2014, S. $124 \mathrm{ff}$.

Pont Jörg, Medical Ethics in Prisons: Rules, Standards and Challenges, International Journal of Prisoner Health 2006, S. 259 ff.

Popovic Dragoljub, The Emergence of the European Human Rights Law, Den Haag 2011

Pösl Michael, Das Verbot der Folter in Art. 3 EMRK, Grundlegung und Fortwirkung auf dem Gebiet des Strafrechts, Baden-Baden 2015

Prosenjak Dominik, Der Folterbegriff nach Art. 3 EMRK, Hamburg 2011

Reynaud Alain, Les droits de l'homme dans les prisons, Strassburg 1986

Richter Claus, Aspekte der universellen Geltung der Menschenrechte und der Herausbildung von Völkergewohnheitsrecht, München 2007

Riegel Ralf/Speicher Kristina, Die Haftsituation im ersuchten Staat als Auslieferungshindernis, StV 2016, S. $250 \mathrm{ff}$.

Rohleder Kristin, Grundrechtsschutz im europäischen Mehrebenen-System: Unter besonderer Berücksichtigung des Verhältnisses zwischen Bundesverfassungsgericht und Europäischem Gerichtshof für Menschenrechte, Baden-Baden 2009

Ronc Pascal, Die Menschenwürde als Prinzip der EMRK: Eine Analyse unter besonderer Berücksichtigung der Rechtsprechung des EGMR zum Strafrecht zugleich ein Beitrag zur Methodik der Auslegung der EMRK, Berlin 2020

Ronc Pascal, Lebenslanger Freiheitsentzug, Art. 3 EMRK und die Rolle von Soft Law, ex ante 2017, S. $67 \mathrm{ff}$.

Rozakis Christos, The European Judge as Comparatist, TLR 2005, S. $257 \mathrm{ff}$.

Rudolf BeAte, Beweisprobleme in Verfahren wegen Verletzung von Art. 3 EMRK / Zugleich Anmerkung zum Urteil des EGMR vom 4.12.1995 im Fall Ribitsch gegen Österreich, EuGRZ 1996, S. 497 ff.

Ruschemeier Hannah, Der additive Grundrechtseingriff, Berlin 2019

Saliger Frank, Absolutes im Strafprozess? Über das Folterverbot, seine Verletzung und die Folgen seiner Verletzung, ZStW 116 (2004), S. 35 ff.

Schabas William A., The European Convention on Human Rights: A Commentary, Oxford 2015

Schäfer Alexander T., Rechtliche Probleme der medizinischen Versorgung im Justizvollzug, Eine Untersuchung unter besonderer Berücksichtigung extramuraler Heilbehandlungen, Hamburg 2009 
Schärer Deborah Vanessa, Konkordate, in: Brägger Benjamin F. (Hrsg.), Das schweizerische Vollzugslexikon: Von der vorläufigen Festnahme zur bedingten Entlassung, Basel 2014, S. $251 \mathrm{ff}$.

Schärer Deborah Vanessa, Konkordatliche Richtlinien: Blosse Gentlemen's Agreements oder verbindliches Strafvollzugsrecht?, SZK 2/2012, S. $51 \mathrm{ff}$.

Schilling Theodor, Internationaler Menschenrechtsschutz: Das Recht der EMRK und des IPbpR, 3. Aufl., Tübingen 2016

Schmahl Stefanie, Piloturteile des EGMR als Mittel der Verfahrensbeschleunigung, EuGRZ 2008, S. 369 ff.

Schмidт Johann, Überbelegung im Strafvollzug: Ein Versuch, juristische Kriterien für die zulässige Belegung von Strafanstalten bzw. Hafträumen zu entwickeln, Frankfurt am Main 1986

Schürer Stefan, Der Europäische Gerichtshof für Menschenrechte als Tatsacheninstanz - Zur Bedeutung divergierender Sachverhaltsfeststellungen durch den EGMR am Beispiel einiger Schweizer Fälle, EuGRZ 2014, S. 512 ff.

Sicilianos Linos-Alexander, The Role of the European Court of Human Rights in the Execution of its own Judgments: Reflections on Article 46 ECHR, in: Seibert-Fohr Anja/Villiger Mark (Hrsg.), Judgments of the European Court of Human Rights - Effects and Implementation, Farnham 2014, S. 285 ff.

Sjöнolm Maria, Gender-Sensitive Norm Interpretation by Regional Human Rights Law Systems, Leiden 2018

Skogly Sigrun, The Requirement of Using the „Maximum of Available Resources" for Human Rights Realisation: A Question of Quality as well as Quantity?, HRLR 2012, S. 393 ff.

Smet Stijn, On the Existence and Nature of Conflicts between Human Rights at the European Court of Human Rights, HRLR 2017, S. $499 \mathrm{ff}$.

Smet Stijn, Conflicts between Absolute Rights: A Reply to Steven Greer, HRLR 2013, S. $469 \mathrm{ff}$.

Smet Stijn, The Absolute Prohibition of Torture and Inhuman or Degrading Treatment in Article 3 ECHR, Truly a Question of Scope Only?, in: Brems Eva/Gerards Janneke (Hrsg.), Shaping Rights in the ECHR: The Role of the European Court of Human Rights in Determining the Scope of Human Rights, Cambridge 2013

Snacken Sonja, A Reductionist Penal Policy and European Human Rights Standards, Eur J Crim Policy Res 2006, S. 143 ff.

Starmer Keir, Positive Obligations under the Convention, in: Jowell Jeffrey/Cooper Jonathan (Hrsg.), Understanding Human Rights Principles, Oxford 2001, S. $139 \mathrm{ff}$.

Stein Alexander, Das Verbot der Folter im internationalen und nationalen Recht - unter Betrachtung seiner Durchsetzungsinstrumente und seines absoluten Charakters, Hamburg 2007

Strecker Michael B., Der Umgang des Europäischen Gerichtshofs für Menschenrechte mit systematischen Menschenrechtsverletzungen: Die Pilotverfahrenstechnik, Art. 61 EGMR-VerfO, ZEuS 2016, S. 235 ff. 
Stone Sweet Alec/Keller Helen, The Reception of the ECHR in National Legal Orders, in: Keller Helen/Stone Sweet Alec (Hrsg.), A Europe of Rights: The Impact of the ECHR on National Legal Systems, Oxford 2008, S. $3 \mathrm{ff}$.

Stöver Heino, Internationale Aspekte der Gesundheitsversorgung in Haft, in: Gefängnismedizin, Medizinische Versorgung unter Haftbedingungen, Keppler Karlheinz/Stöver Heino (Hrsg.), Stuttgart 2009, S. $301 \mathrm{ff}$.

Stöver Heino/MacDonald Morag/Atherton Susie, Harm Reduction in European Prisons: A Compilation of Models of Best Practice, Oldenburg 2007

Sudre Frédéric, L'article $3^{\text {bis }}$ de la Convention européenne des droits de l'homme: Le droit à des conditions de détention conformes au respect de la dignité humaine, in: Bruylant Emile (Hrsg.), Liberté, justice, tolérance: Mélanges en hommage au Doyen Gérard Cohen-Jonathan, Paris 2004, S. 1499 ff. (zit.: SUDRE FS Cohen-Jonathan)

Tag BrigitTe, Das Arztgeheimnis im Strafvollzug, in: Hillenkamp Thomas/Tag Brigitte (Hrsg.), Intramurale Medizin - Gesundheitsfürsorge zwischen Heilauftrag und Strafvollzug, Berlin 2005, S. 89 ff.

Thiele Carmen, Menschenrechtsschutz und europäische Integration, in: Beichelt Timm/Choluj Bozena/Rowe Gerard/Wagener Hans-Jürgen (Hrsg.), Europa-Studien: Eine Einführung, 2. Aufl., Wiesbaden 2013

Thurnherr Daniela, The Reception Process in Austria and Switzerland in: Keller Helen/Stone Sweet Alec (Hrsg.), A Europe of Rights: The Impact of the ECHR on National Legal Systems, Oxford 2008, S. 311 ff.

Thürer DANiel, „Soft Law“ - eine neue Form von Völkerrecht, ZSR 1985, S. $429 \mathrm{ff}$.

Tichy Helmut, Recommendations des Europarats, ZaöRV 2016, S. 415 ff.

Trechsel Stefan, Human Rights of Persons Deprived of their Liberty - the Rights of Prisoners, in: Engel Norbert Paul (Hrsg.), Rights of Persons Deprived of their Liberty, Equality and Non-Discrimination: Proceedings of the 7th International Colloquy on the European Convention on Human Rights Organised by the Secretariat General of the Council of Europe in Collaboration with the Danish, Finnish, Norwegian, and Swedish Institutes of Human Rights, Copenhagen, Oslo, Lund (30 May - 2 June 1990), Kehl 1994, S. 30 ff. (zit.: Trechsel, Human Rights)

Tulkens Françoise, Cellule collective et espace personnel: Un arrêt en trompel'œil, RTDH 2017, S. $989 \mathrm{ff}$.

van der Berg Else Feikje, Art. 3 EMRK: Verbot der Folter und der unmenschlichen oder erniedrigenden Behandlung oder Strafe, Baden-Baden 2019

van Zyl Smit Dirk, Regulation of Prison Conditions, Crime and Justice 2010, S. $503 \mathrm{ff}$.

van Zyl Smit Dirk, Humanising Imprisonment: A European Project?, Eur J Crim Policy Res 2006, S. $107 \mathrm{ff}$.

van Zyl Smit Dirk/Snacken Sonja, Principles of European Prison Law and Policy: Penology and Human Rights, Oxford 2009 
Vermeulen Ben/Battjes Hemme, Freedom from Torture and Other Inhuman or Degrading Treatment or Punishment, in: van Dijk Pieter/van Hoof Fried/van Rijn Arjen/Zwaak Leo (Hrsg.), Theory and Practice of the European Convention on Human Rights, 5. Aufl., Cambridge 2018, S. $381 \mathrm{ff}$.

Villeneuve Léticia, Could Progressive „Hardening“ of Human Rights Soft Law Impair its Further Expansion? - Insights from the UN-Declaration on the Rights of Indigenous Peoples, in: Lagoutte Stéphanie/Gammeltoft-Hansen Thomas/ Cerone John (Hrsg.), Tracing the Roles of Soft Law in Human Rights, Oxford 2016, S. $213 \mathrm{ff}$.

Villiger Mark, Commentary on the 1969 Vienna Convention on the Law of Treaties, Leiden 2009

Villiger Mark, Articles 31 and 32 of the Vienna Convention on the Law of Treaties in the Case-Law of the European Court of Human Rights, in: Bröhmer Jürgen/Bieber Roland/Callies Christian/Langenfeld Christine/Weber Stefan/Wolf Joachim (Hrsg.), Internationale Gemeinschaft und Menschenrechte: Festschrift für Georg Ress zum 70. Geburtstag am 21. Januar 2005, Köln 2005, S. 317 ff. (zit.: Villiger FS Ress)

Villiger Mark, Handbuch der Europäischen Menschenrechtskonvention (EMRK) - Unter besonderer Berücksichtigung der schweizerischen Rechtslage, 2. Aufl., Zürich 1999

Voeten Erik, Domestic Implementation of European Court of Human Rights Judgments: Legal Infrastructure and Government Effectiveness Matter: A Reply to Dia Anagnostou and Alina Mungiu-Pippidi, EJIL 2014, S. $229 \mathrm{ff}$.

Vóкó György, Europäisches Strafvollzugsrecht, Passau 2009

von Kielmansegg Sebastian, Grundrechte im Näheverhältnis: Eine Untersuchung zur Dogmatik des Sonderstatusverhältnisses, Tübingen 2012

von Kielmansegg Sebastian, Das Sonderstatusverhältnis, Juristische Arbeitsblätter 2012, S. $881 \mathrm{ff}$.

von Schwichow Lennart, Die Menschenwürde in der EMRK: Mögliche Grundannahmen, ideologische Aufladung und rechtspolitische Perspektiven nach der Rechtsprechung des Europäischen Gerichtshof für Menschenrechte, Tübingen 2016

Voyame Joseph, Das UNO-Übereinkommen gegen Folter und andere grausame, unmenschliche oder erniedrigende Behandlung oder Strafe, in: Matscher Franz (Hrsg.), Folterverbot sowie Religions- und Gewissensfreiheit im Rechtsvergleich, Kehl am Rhein 1990, S. 109 ff.

Waldmann Bernhard/Belser Eva Maria/Epiney Astrid (Hrsg.), Basler Kommentar zur Bundesverfassung, Basel 2015 (zit.: BSK BV/BEARBEITER)

Walmsley Roy, World Prison Population List, 12. Aufl., November 2018, abrufbar unter $<$ https://www.prisonstudies.org/sites/default/files/resources/downloads/wp pl_12.pdf $>$, zuletzt abgerufen am 09.08.2020 
Walter Franz, Regionale Ausprägung im Straf- und Massnahmenvollzug - eine subjektive Annäherung, in: Fink Daniel/Arnold Jörg/Genillod-Villard Françoise/Oberholzer Niklaus (Hrsg.), Kriminalität, Strafrecht und Föderalismus, Bern 2019, S. $241 \mathrm{ff}$.

Webster Elaine, Dignity, Degrading Treatment and Torture in Human Rights Law: The Ends of Article 3 of the European Convention on Human Rights, London 2018

Weil Prosper, Towards Relative Normativity in International Law, AJIL 1983, S. $413 \mathrm{ff}$.

Wildhaber Luzius/Hjartarson Arnaldur/Donnelly Stephen, No Consensus on Consensus? The Practice of the European Court of Human Rights, HRLJ 2013, S. 248

Wilkitzki Peter, Die Regionalisierung des internationalen Strafrechts, ZStW 105 (1993), S. $821 \mathrm{ff}$.

Wittinger Michaela, Der Europarat: Die Entwicklung seines Rechts und der „europäischen Verfassungswerte“, Baden-Baden 2005

Wittling-Vogel Almut, Ministerkomitee und EMRK: Fremdkörper oder essentieller Bestandteil?, in: Zimmermann Andreas (Hrsg.), 60 Jahre Europäische Menschenrechtskonvention: Die Konvention als „living instrument“, Berlin 2014, S. $103 \mathrm{ff}$.

Wolff Hans/Casillas Alejandra/Perneger Thomas/Heller Patrick/Golay Diane/Mouton Elisabeth/Bodenmann Patrick/Getaz Laurent, Self-Harm and Overcrowding among Prisoners in Geneva, Switzerland, International Journal of Prisoner Health 2016, S. $39 \mathrm{ff}$.

Wolff Hans/Sebo Paul/Haller Dagmar M./Eytan Ariel/Niveau Gérard/ Bertrand Dominique/Gétaz Laurent/Cerutti Bernard, Health Problems among Detainees in Switzerland: A Study Using the ICPC-2 Classification, BMC Public Health 11, 245 (2011), S. 1 ff.

Zagrebelsky Vladimiro, Questions autour de Broniowski, in: Caflisch Lucius/Callewaert Johan/Liddell Roderick/Mahoney Paul/Villiger Mark (Hrsg.), Liber Amicorum Luzius Wildhaber: Human Rights - Strasbourg views, Kehl 2007, S. 521 ff. (zit.: ZaGrebeLSKI FS Wildhaber)

Zemanek Karl, Is the Term „Soft Law“ Convenient?, in: Hafner Gerhard/Loibl Gerhard/Rest Alfred/Sucharipa-Behrmann Lilly/Zemanek Karl (Hrsg.), Liber Amicorum: Professor Ignaz Seidl-Hohenveldern in Honour of his 80th Birthday, Den Haag 1998, S. 843 ff. (zit.: Zemanex FS Seidl-Hohenveldern)

Zimmermann Ralph, Die Schrankenregelungen der Europäischen Menschenrechtskonvention, des Grundgesetzes und der Grundrechtecharta der Europäischen Union im Vergleich, in: Böllmann Felix/Hemme Susanne/Korkmaz Ömer/Kühn Friedrich/Sinn Arndt (Hrsg.), Die Menschenrechte als Grundlage für eine gesamteuropäische Rechtsentwicklung und ihr Einfluss auf das Strafrecht, das öffentliche Recht und das Zivilrecht, Berlin 2006, S. 63 ff. 


\section{Materialienverzeichnis}

\section{A. Vereinte Nationen}

\section{Empfehlungen der Generalversammlung}

A/RES/70/175, United Nations Standard Minimum Rules for the Treatment of Prisoners („The Nelson Mandela Rules“), adopted by the General Assembly on 17 December 2015 (zit.: A/RES/70/175, United Nations Standard Minimum Rules for the Treatment of Prisoners [„The Nelson Mandela Rules“])

A/RES/65/229, United Nations Rules for the Treatment of Women Prisoners and Non-Custodial Measures for Women Offenders („The Bangkok Rules“), adopted by the General Assembly on 21 December 2010 (zit.: A/RES/65/229, Concerning the United Nations Rules for the Treatment of Women Prisoners and NonCustodial Measures for Women Offenders [„The Bangkok Rules“])

A/RES/61/252, Questions Relating to the Programme Budget for the Biennium 2006-2007, adopted by the General Assembly on 22 December 2006 (zit.: A/RES/61/252, Questions Relating to the Programme Budget for the Biennium 2006-2007)

A/RES/46/152, Creation of an Effective United Nations Crime Prevention and Criminal Justice Programme, adopted by the General Assembly on 18 December 1991 (zit.: A/RES/46/152, Concerning the Creation of an Effective United Nations Crime Prevention and Criminal Justice Programme)

A/RES/45/111, The Basic Principles for the Treatment of Prisoners, adopted by the General Assembly on 14 December 1990 (zit.: A/RES/45/111, Concerning the Basic Principles for the Treatment of Prisoners)

A/RES/43/173, Body of Principles for the Protection of All Persons under Any Form of Detention or Imprisonment, adopted by the General Assembly on 9 December 1988 (zit.: A/RES/43/173, Concerning the Body of Principles for the Protection of All Persons under Any Form of Detention or Imprisonment)

A/RES/40/33, United Nations Standard Minimum Rules for the Administration of Juvenile Justice („The Beijing Rules“), adopted by the General Assembly on 29 November 1985 (zit.: A/RES/40/33, Concerning the United Nations Standard Minimum Rules for the Administration of Juvenile Justice [„The Beijing Rules"])

A/RES/217(III)[A], Universal Declaration of Human Rights, adopted by the General Assembly on 10 December 1948 (zit.: A/RES/217(III)[A], Universal Declaration of Human Rights) 


\section{Wirtschafts- und Sozialrat (ECOSOC)}

E/RES/1992/1, Establishment of the Commission on Crime Prevention and Criminal Justice, adopted by The Economic and Social Council on 6 February 1992 (zit.: E/RES/1992/1, Concerning the Establishment of the Commission on Crime Prevention and Criminal Justice)

E/RES/1884/47, Procedures for the Effective Implementation of the Standard Minimum Rules for the Treatment of Prisoners, adopted by the Economic and Social Council on 25 May 1984 (zit.: E/RES/1984/47, Procedures for the Effective Implementation of the Standard Minimum Rules for the Treatment of Prisoners)

E/RES/1884/47, Annex to the Procedures for the Effective Implementation of the Standard Minimum Rules for the Treatment of Prisoners, adopted by the Economic and Social Council on 25 May 1984 (zit.: E/RES/1984/47, Annex to the Procedures for the Effective Implementation of the Standard Minimum Rules for the Treatment of Prisoners)

\section{Weiteres}

E/C.12/2000/4, CESCR General Comment No. 14: The Right to the Highest Attainable Standard of Health (Art. 12), adopted at the 22nd Session of the Committee on Economic, Social and Cultural Rights on 11 August 2000 (zit.: WSKAusschuss, General Comment No. 14)

Short Guide to the Revised United Nations Standard Minimum Rules for the Treatment of Prisoners („The Nelson Mandela Rules“), abrufbar unter $<$ https:/ /cdn.penalreform.org/wp-content/uploads/2016/01/PRI_Nelson_Mandela_Ru les_Short_Guide_WEB.pdf> (zit.: Short Guide to the Revised United Nations Standard Minimum Rules for the Treatment of Prisoners („The Nelson Mandela Rules“), zuletzt abgerufen am 06.08.2020)

\section{B. Europarat}

\section{Europäischer Gerichtshof für Menschenrechte}

European Court of Human Rights, Rules of Court, Strasbourg January 2020, abrufbar unter $<$ https://www.echr.coe.int/Documents/Rules_Court_ENG.pdf $>$ (zit.: Rules of Court, zuletzt abgerufen am 06.08.2020)

European Court of Human Rights, Factsheet Interim Measures, Strasbourg January 2019, abrufbar unter <https://www.echr.coe.int/Documents/FS_Interim_ measures_ENG.pdf $>$ (zit.: Factsheet Interim Measures, zuletzt abgerufen am 10.11.2019) 


\section{Europäisches Komitee zur Verhütung der Folter und der unmenschlichen und erniedrigenden Behandlung oder Strafe (CPT)}

\section{Jahresberichte (General Reports)}

CPT/Inf (2020) 17, 29th General Report of the CPT, European Committee for the Prevention of Torture and Inhuman or Degrading Treatment or Punishment, Covering the Period 1 January 2019 to 31 December 2019, Strasbourg May 2020 (zit.: CPT - 29th General Report [CPT/Inf (2020) 17])

CPT/Inf (2019) 9, 28th General Report of the CPT, European Committee for the Prevention of Torture and Inhuman or Degrading Treatment or Punishment, Covering the Period 1 January 2018 to 31 December 2018, Strasbourg April 2019 (zit.: CPT - 28th General Report [CPT/Inf (2019) 9])

CPT/Inf (2018) 4, 27th General Report of the CPT, European Committee for the Prevention of Torture or Degrading Treatment or Punishment, Covering the Period 1 January 2017 to 31 December 2017, Strasbourg April 2018 (zit.: CPT 27th General Report [CPT/Inf (2018) 4])

CPT/Inf (2017) 5, 26th General Report of the CPT, European Committee for the Prevention of Torture and Inhuman or Degrading Treatment or Punishment, Covering the Period 1 January 2016 to 31 December 2016, Strasbourg April 2017 (zit.: CPT - 26th General Report [CPT/Inf (2017) 5])

CPT/Inf (2016) 10, 25th General Report of the CPT, European Committee for the Prevention of Torture and Inhuman or Degrading Treatment or Punishment, Covering the Period 1 January 2015 to 31 December 2015, Strasbourg April 2016 (zit.: CPT - 25th General Report [CPT/Inf (2016) 10])

CPT/Inf (2015) 1, 24th General Report of the CPT, European Committee for the Prevention of Torture and Inhuman or Degrading Treatment or Punishment, Covering the Period 1 August 2013 to 31 December 2014, Strasbourg January 2015 (zit.: CPT - 24th General Report [CPT/Inf (2015) 1])

CPT/Inf (2013) 29, 23rd General Report of the CPT, European Committee for the Prevention of Torture and Inhuman or Degrading Treatment or Punishment, Covering the Period 1 August 2012 to 31 July 2013, Strasbourg 6 November 2013 (zit.: CPT - 23th General Report [CPT/Inf (2013) 29])

CPT/Inf (2012) 25, 22nd General Report of the CPT, European Committee for the Prevention of Torture and Inhuman or Degrading Treatment or Punishment, Covering the Period 1 August 2011 to 31 July 2012, Strasbourg 6 November 2012 (zit.: CPT - 22nd General Report [CPT/Inf (2012) 25])

CPT/Inf (2011) 28, 21st General Report of the European Committee for the Prevention of Torture and Inhuman or Degrading Treatment or Punishment (CPT) Covering the Period 1 August 2010 to 31 July 2011, Strasbourg 10 November 2011 (zit.: CPT - 21st General Report [CPT/Inf (2011) 28])

CPT/Inf (2010) 28, 20th General Report of the European Committee for the Prevention of Torture and Inhuman or Degrading Treatment or Punishment (CPT) Covering the Period 1 August 2009 to 31 July 2010, Strasbourg 26 October 2010 (zit.: CPT - 20th General Report [CPT/Inf (2010) 28]) 
CPT/Inf (2009) 27, 20 Years of Combating Torture, 19th General Report of the European Committee for the Prevention of Torture an Inhuman or Degrading Treatment or Punishment (CPT) Covering the Period 1 August 2008 to 31 July 2009, Strasbourg 20 October 2009 (zit.: CPT - 19th General Report [CPT/Inf (2009) 27])

CPT/Inf (2008) 25, 18th General Report on the CPT's Activities Covering the Period 1 August 2007 to 31 July 2008, Strasbourg 18 September 2008 (zit.: CPT - 18th General Report [CPT/Inf (2008) 25])

CPT/Inf (2007) 39, 17th General Report on the CPT's Activities Covering the Period 1 August 2006 to 31 July 2007, Strasbourg 14 September 2007 (zit.: CPT - 17th General Report [CPT/Inf (2007) 39])

CPT/Inf (2005) 17, 15th General Report on the CPT's Activities Covering the Period 1 August 2004 to 31 July 2005, Strasbourg 22 September 2005 (zit.: CPT - 15th General Report [CPT/Inf (2005) 17])

CPT/Inf (2003) 35, 13th General Report on the CPT's Activities Covering the Period 1 January 2002 to 31 July 2003, Strasbourg 10 September 2003 (zit.: CPT - 13th General Report [CPT/Inf (2003) 35])

CPT/Inf (2001) 16, 11th General Report on the CPT's Activities Covering the Period 1 January to 31 December 2000, Strasbourg 3 September 2001 (zit.: CPT - 11th General Report [CPT/Inf (2001) 16])

CPT/Inf (98) 12, 8th General Report on the CPT's Activities Covering the Period 1 January to 31 December 1997, Strasbourg 31. August 1998 (zit.: CPT 8th General Report [CPT/Inf (98) 12])

CPT/Inf (97) 10, 7th General Report on the CPT's Activities Covering the Period 1 January to 31 December 1996, Strasbourg 22 August 1997 (zit.: CPT 7th General Report [CPT/Inf (97) 10])

CPT/Inf (93) 12, 3rd General Report on the CPT's Activities Covering the Period 1 January to 31 December 1992, Strasbourg 4 June 1993 (zit.: CPT - 3rd General Report [CPT/Inf (93) 12])

CPT/Inf (92) 3, 2nd General Report on the CPT's Activities Covering the Period 1 January to 31 December 1991, Strasbourg 13 April 1992 (zit.: CPT - 2nd General Report [CPT/Inf (92) 3])

CPT/Inf (91) 3, 1st General Report on the CPT's Activities Covering the Period November 1989 to December 1990, Strasbourg 20 February 1991 (zit.: CPT 1st General Report [CPT/Inf (91) 3])

\section{Länderspezifische Dokumente}

CPT/Inf (2020) 15, Report to the Greek Government on the Visit to Greece Carried out by the European Committee for the Prevention of Torture and Inhuman or Degrading Treatment of Punishment (CPT) from 28 March to 9 April 2019, Strasbourg 9 April 2020 (zit.: CPT - Report to the Greek Government [CPT/Inf (2020) 15]) 
CPT/Inf (2019) 16, Report to the Georgian Government on the Visit to Georgia Carried out by the European Committee for the Prevention of Torture and Inhuman or Degrading Treatment or Punishment (CPT) from 10 to 21 September 2018, Strasbourg 10 May 2019 (zit.: CPT - Report to the Georgian Government [CPT/Inf (2019) 16])

CPT/Inf (2017) 30, Report to the Government of „The Former Yugoslav Republic of Macedonia“ on the Visit to "The Former Yugoslav Republic of Macedonia" Carried out by the European Committee for the Prevention of Torture and Inhuman or Degrading Treatment or Punishment (CPT) from 6 to 9 December 2016, Strasbourg 12 October 2017 (zit.: CPT - Report to the Government of „The Former Yugoslav Republic of Macedonia“ [CPT/Inf (2017) 30])

CPT/Inf (2016) 18, Rapport au Conseil fédéral suisse relatif à la visite effectuée en Suisse par le Comité Européen pour la prévention de la torture et des peines ou traitements inhumains ou dégradants (CPT) du 13 avril 2015 au 24 avril 2015, Strasbourg 23 juin 2016 (zit.: CPT - Rapport au Conseil fédéral suisse [CPT/Inf (2016) 18])

CPT/Inf (2015) 17, European Committee for the Prevention of Torture and Inhuman or Degrading Treatment or Punishment (CPT), Public Statement Concerning Bulgaria, Strasbourg 26 March 2015 (zit.: CPT - Public Statement Concerning Bulgaria [CPT/Inf (2015) 17])

CPT/Inf (2014) 13, Report to the Hungarian Government on the Visit to Hungary Carried out by the European Committee for the Prevention of Torture and Inhuman or Degrading Treatment or Punishment (CPT) from 3 to 12 April 2013, Strasbourg 30 April 2014 (zit.: CPT - Report to the Hungarian Government [CPT/Inf (2014) 13])

CPT/Inf (2012) 21, Report to the Government of the Netherlands on the Visit to the Netherlands Carried out by the European Committee for the Prevention of Torture and Inhuman or Degrading Treatment or Punishment (CPT) from 10 to 21 October 2011, Strasbourg 9 August 2012 (zit.: CPT - Report to the Government of the Netherlands [CPT/Inf (2012) 21])

CPT/Inf (2011) 3, Report to the Government of Ireland on the Visit to Ireland Carried out by the European Committee for the Prevention of Torture and Inhuman or Degrading Treatment or Punishment (CPT) from 25 January to 5 February 2010, Strasbourg 10 February 2011 (zit.: CPT - Report to the Government of Ireland [CPT/Inf (2011) 3])

CPT/Inf (2010) 24 Rapport au gouvernement de la Belgique relatif à la visite effectuée en Belgique par le Comité européen pour la prévention de la torture et des peines ou traitements inhumains ou dégradants (CPT) du 28 septembre au 7 octobre 2009, Strasbourg 23 juillet 2010 (zit.: CPT - Rapport au gouvernement de la Belgique [CPT/Inf (2010) 24])

CPT/Inf (2010) 16, Report to the Hungarian Government on the Visit to Hungary Carried out by the European Committee for the Prevention of Torture and Inhuman or Degrading Treatment or Punishment (CPT) from 24 March 2009 to 2 April 2009, Strasbourg 8 June 2010 (zit.: CPT - Report to the Hungarian Government [CPT/Inf (2010) 16]) 
CPT/Inf (2008) 29, Report to the Croatian Government on the Visit to Croatia Carried out by the European Committee for the Prevention of Torture and Inhuman or Degrading Treatment or Punishment (CPT) from 4 May 2007 to 14 May 2007, Strasbourg 9 October 2008 (zit.: CPT - Report to the Croatian Government [CPT/Inf (2008) 29])

CPT/Inf (2008) 11, Report to the Bulgarian Government on the Visit to Bulgaria Carried out by the European Committee for the Prevention of Torture and Inhuman or Degrading Treatment or Punishment (CPT) from 10 September 2006 to 21 September 2006, Strasbourg 28 February 2008 (zit.: CPT - Report to the Bulgarian Government [CPT/Inf (2008) 11])

CPT/Inf (2008) 7, Report to the Slovenian Government on the Visit to Slovenia Carried out by the European Committee for the Prevention of Torture and Inhuman or Degrading Treatment or Punishment (CPT) from 31 January to 8 February 2006, Strasbourg 15 February 2008) (zit.: CPT - Report to the Slovenian Government [CPT/Inf (2008) 7])

CPT/Inf (2006) 24, Report to the Albanian Government on the Visit to Albania Carried out by the European Committee for the Prevention of Torture and Inhuman or Degrading Treatment or Punishment (CPT) from 23 May 2005 to 3 June 2005, Strasbourg 12 July 2006 (zit.: CPT - Report to the Albanian Government [CPT/Inf (2006) 24])

CPT/Inf (2005) 8, Report to the Latvian Government on the Visit to Latvia Carried out by the European Committee for the Prevention of Torture and Inhuman or Degrading Treatment or Punishment (CPT) from 25 September 2002 to 4 October 2002, Strasbourg 13 March 2008 (zit.: CPT - Report to the Latvian Government [CPT/Inf (2005) 8])

CPT/Inf (2004) 36, Report to the Azerbaijani Government on the Visit to Azerbaijan Carried out by the European Committee for the Prevention of Torture and Inhuman or Degrading Treatment or Punishment (CPT) from 24 November 2002 to 6 December 2002, Strasbourg 7 December 2004 (zit.: CPT - Report to the Azerbaijani Government [CPT/Inf (2004) 36])

\section{Themenspezifische Dokumente}

CPT/Inf (2017) 20, Inspection of a Prison Medical Service by a CPT Doctor, Checklist, Strasbourg 21 August 2017 (zit.: CPT - Inspection of a Prison Medical Service by a CPT Doctor [CPT/Inf (2017) 20])

CPT/Inf (2015) 44, Living Space per Prisoner in Prison Establishments: CPT Standards, Strasbourg 15 December 2015 (zit.: CPT - Living Space per Prisoner [CPT/Inf (2015) 44])

CPT/Inf (93) 12, Health Care Services in Prisons, Extract from the 3rd General Report of the CPT, Strasbourg 1993 (zit.: CPT - Health Care Services in Prisons [CPT/Inf (93) 12]) 


\section{Weiteres}

Explanatory Report to the European Convention for the Prevention of Torture and Inhuman or Degrading Treatment or Punishment, Strasbourg 26 November 1987, ETS No. 126 (zit.: Explanatory Report to the European Convention for the Prevention of Torture and Inhuman or Degrading Treatment or Punishment v. 26.11.1987)

Public Statements, abrufbar unter $<$ https://www.coe.int/de/web/cpt/public-state ments> (zit.: CPT - Public Statements, zuletzt abgerufen am 07.07.2020)

\section{Ministerkomitee}

\section{Entschliessungen und Empfehlungen (Resolutions and Recommendations)}

Recommendation CM/Rec (2014) 3 of the Committee of Ministers to Member States Concerning Dangerous Offenders, adopted by the Committee of Ministers on 19 February 2014 at the 1192nd meeting of the Ministers' Deputies (zit.: Recommendation CM/Rec (2014) 3 Concerning Dangerous Offenders)

Recommendation CM/Rec (2012) 12 of the Committee of Ministers to Member States Concerning Foreign Prisoners, adopted by the Committee of Ministers on 10 October 2012 at the 1152nd meeting of the Ministers' Deputies (zit.: Recommendation CM/Rec (2012) 12 Concerning Foreign Prisoners)

Recommendation CM/Rec (2012) 5 of the Committee of Ministers to Member States on the European Code of Ethics for Prison Staff, adopted by the Committee of Ministers on 12 April 2012 at the 1140th meeting of the Ministers' Deputies (zit.: Recommendation CM/Rec (2012) 5 on the European Code of Ethics for Prison Staff)

Recommendation CM/Rec (2010) 3 of the Committee of Ministers to Member States on Effective Remedies for Excessive Length of Proceedings, adopted by the Committee of Ministers on 24 February 2010 at the 1077th meeting of the Ministers' Deputies (zit.: Recommendation CM/Rec (2010) 3 on Effective Remedies for Excessive Length of Proceedings)

Recommendation CM/Rec (2010) 1 of the Committee of Ministers to Member States on the Council of Europe Probation Rules, adopted by the Committee of Ministers on 20 January 2010 at the 1075th meeting of Ministers' Deputies (zit.: Recommendation CM/Rec (2010) 1 on the Council of Europe Probation Rules)

Recommendation CM/Rec (2008) 11 of the Committee of Ministers to Member States on the European Rules for Juvenile Offenders Subject to Sanctions or Measures, adopted by the Committee of Ministers on 5 November 2008 at the 1140th meeting of the Ministers' Deputies (zit.: Recommendation CM/Rec (2008) 11 on the European Rules for Juvenile Offenders Subject to Sanctions or Measures) 
Recommendation CM/Rec (2008) 2 of the Committee of Ministers to Member States on Efficient Domestic Capacity for Rapid Execution of Judgments of the European Court of Human Rights, adopted by the Committee of Ministers on 6 February 2008 at the 1017th meeting of the Ministers' Deputies (zit.: Recommendation CM/Rec (2008) 2 on Efficient Domestic Capacity for Rapid Execution of Judgments of the European Court of Human Rights)

Recommendation Rec (2006) 13 of the Committee of Ministers to Member States on the Use of Remand in Custody, the Conditions in which it Takes Place and the Provision of Safeguards against Abuse, adopted by the Committee of Ministers on 27 September 2006 at the 974th meeting of the Ministers' Deputies (zit.: Recommendation Rec (2006) 13 on the Use of Remand in Custody, the Conditions in which it Takes Place and the Provision of Safeguards against Abuse)

Recommendation Rec (2006) 2 of the Committee of Ministers to Member States on the European Prison Rules, adopted by the Committee of Ministers on 11 January at the 952nd meeting of the Ministers' Deputies (zit.: Recommendation Rec (2006) 2 on the European Prison Rules 2006); Commentary to Recommendation Rec (2006) 2 of the Committee of Ministers to the Member States on the European Prison Rules, PC-CP (2018) 15 rev 3, Strasbourg 8 October 2018 (zit.: Commentary to Recommendation Rec (2006) 2 on the European Prison Rules)

Resolution Res (2004) 3 of the Committee of Ministers on Judgments Revealing an Underlying Systemic Problem, adopted by the Committee of Ministers on 12 May 2004 at its 114th session (zit.: Resolution Res (2004) 3 on Judgments Revealing an Underlying Systemic Problem)

Recommendation Rec (2003) 23 of the Committee of Ministers to Member States on the Management by Prison Administrations of Life Sentence and Other Long-Term Prisoners, adopted by the Committee of Ministers on 9 October 2003 at the 855th meeting of the Ministers' Deputies (zit.: Recommendation Rec (2003) 23 on the Management by Prison Administrations of Life Sentence and Other Long-Term Prisoners)

Recommendation No. R (99) 22 of the Committee of Ministers to Member States Concerning Prison Overcrowding and Prison Population Inflation, adopted by the Committee of Ministers on 30 September 1999 at the 681st meeting of the Minsiters' Deputies (zit.: Recommendation R (99) 22 Concerning Prison Overcrowding and Prison Population Inflation)

Recommendation No. R (98) 7 of the Committee of Ministers to Member States Concerning the Ethical and Organisational Aspect of Health Care in Prison, adopted by the Committee of Ministers on 8 April 1998 at the 627th meeting of Ministers' Deputies (zit.: Recommendation R (98) 7 Concerning the Ethical and Organisational Aspect of Health Care in Prison)

Recommendation No. R (97) 12 of the Committee of Ministers to Member States on Staff Concerned with the Implementation of Sanctions and Measures, adopted by the Committee of Ministers on 10 September 1997 at the 600th meeting of Ministers' Deputies (zit.: Recommendation R (97) 12 on Staff Concerned with the Implementation of Sanctions and Measures) 
Recommendation No. R (93) 6 of the Committee of Ministers to Member States Concerning Prison and Criminological Aspects of the Control of Transmissible Diseases Including AIDS and Related Health Problems in Prisons, adopted by the Committee of Ministers on 18 October 1993 at the 500th meeting of Ministers' Deputies (zit.: Recommendation R (93) 6 Concerning Prison and Criminological Aspects of the Control of Transmissible Diseases Including AIDS and Related Health Problems in Prisons)

Recommendation No. R (92) 18 of the Committee of Ministers to Member States Concerning the Practical Application of the Convention on the Transfer of Sentenced Persons, adopted by the Committee of Ministers on 19 October 1992 at the 482th meeting of Ministers' Deputies (zit.: Recommendation R (92) 18 Concerning the Practical Application of the Convention on the Transfer of Sentenced Persons)

Recommendation No. R (89) 12 of the Committee of Ministers to Member States on Education in Prison, adopted by the Committee of Ministers on 13 October 1989 at the 429th meeting of the Ministers' Deputies (zit.: Recommendation $\mathrm{R}$ (89) 12 on Education in Prison)

Recommendation No. R (87) 3 of the Committee of Ministers to Member States on the European Prison Rules, adopted by the Committee of Ministers on 12 February 1987 at the 404th meeting of the Ministers' Deputies (zit.: Recommendation R (87) 3 on the European Prison Rules 1987)

Recommendation No. R (84) 12 of the Committee of Ministers to Member States Concerning Foreign Prisoners, adopted by the Committee of Ministers on 21 June 1984 at the 374th meeting of the Ministers' Deputies (zit.: Recommendation R (84) 12 Concerning Foreign Prisoners)

Recommendation No. R (82) 17 of the Committee of Ministers to Member States Concerning Custody and Treatment of Dangerous Prisoners, adopted by the Committee of Ministers on 24 September 1982 at the 350th meeting of Ministers' Deputies (zit.: Recommendation R (82) 17 Concerning Custody and Treatment of Dangerous Prisoners)

Resolution (73) 5 of the Committee of Ministers, Standard Minimum Rules for the Treatment of Prisoners, adopted by the Committee of Ministers on 19 January 1973 at the 217th meeting of the Ministers' Deputies (zit.: Resolution (73) 5, Standard Minimum Rules for the Treatment of Prisoners)

\section{Implementierungsverfahren}

\subsection{Allgemeine Dokumente}

\section{a. Deklarationen (Declarations)}

Copenhagen Declaration, High Level Conference on the Future of the European Court of Human Rights, at the initiative of the Danish Chairmanship of the Committee of Ministers of the Council of Europe, Copenhagen 12-13 April 2018 (zit.: Copenhagen Declaration v. 13.04.2018) 


\section{Materialienverzeichnis}

Brussels Declaration, High Level Conference on the Future of the European Court of Human Rights, Our Shared Responsibility, at the initiative of the Belgian Chairmanship of the Committee of Ministers of the Council of Europe, Brussels 26-27 March 2015 (zit.: Brussels Declaration v. 27.03.2015)

Brighton Declaration, High Level Conference on the Future of the European Court of Human Rights, at the initiative of the United Kingdom Chairmanship of the Committee of Ministers of the Council of Europe, Brighton 19-20 April 2012 (zit.: Brighton Declaration v. 20.04.2012)

Izmir Declaration, High Level Conference on the Future of the European Court of Human Rights, organised within the framework of the Turkish Chairmanship of the Committee of Ministers of the Council of Europe, Izmir 26-27 April 2011 (zit.: Izmir Declaration v. 27.04.2011)

Interlaken Declaration, High Level Conference on the Future of the European Court of Human Rights, at the initiative of the Swiss Chairmanship of the Committee of Ministers of the Council of Europe, Interlaken 18-19 February 2010 (zit.: Interlaken Declaration v. 19.02.2010)

\section{b. Jahresberichte zur Überwachung der Umsetzung der Urteile des Gerichtshofs (Annual} Reports)

Council of Europe Committee of Ministers, Supervision of the Execution of Judgments and Decisions of the European Court of Human Rights, 13th Annual Report of the Committee of Ministers 2019, Strasbourg June 2020 (zit.: Annual Report 2019 Concerning Supervision of the Execution of Judgments and Decisions)

Council of Europe Committee of Ministers, Supervision of the Execution of Judgments and Decisions of the European Court of Human Rights, 11th Annual Report of the Committee of Ministers 2017, Strasbourg March 2018 (zit.: Annual Report 2017 Concerning Supervision of the Execution of Judgments and Decisions)

Council of Europe Committee of Ministers, Supervision of the Execution of Judgments and Decisions of the European Court of Human Rights, 10th Annual Report of the Committee of Ministers 2016, Strasbourg March 2017 (zit.: Annual Report 2016 Concerning Supervision of the Execution of Judgments and Decisions)

Council of Europe Committee of Ministers, Supervision of the Execution of Judgments and Decisions of the Court of Human Rights, Annual Report 2011, Strasbourg April 2012 (zit.: Annual Report 2011 Concerning Supervision of the Execution of Judgments and Decisions) 
c. Regeln zum Implementierungsverfahren (Rules)

Rules of the Committee of Ministers for the Supervision of the Execution of Judgments and of the Terms of Friendly Settlements, adopted by the Committee of Ministers on 10 May 2006 at the 964th meeting of the Ministers' Deputies and amended on 18 January 2017 at the 1275th meeting of the Ministers' Deputies (zit.: Rules of the Committee of Ministers for the Supervision of the Execution of Judgments and of the Terms of Friendly Settlements)

\section{d. Weiteres}

iGuide, Committee of Ministers Procedures and Working Methods, 17 October 2017, abrufbar unter $<$ https://search.coe.int/cm/Pages/result_details.aspx?Objec tID=090000168058d922> (zit.: iGuide, Committee of Ministers Procedures and Working Methods, zuletzt abgerufen am 01.02.2020)

\subsection{Dokumente betr. spezifischer Umsetzungsverfahren} a. Aktionspläne und Aktionsberichte der Konventionsstaaten (Action Plans / Action
Reports)

aa. Implementierungsverfahren Ananyev u.a./RUS

Action Plan v. 29.04.2019 - Communication from the Russian Federation Concerning the Kalashnikov Group of Cases/RUS u. Ananyev u.a./RUS, DH-DD (2019) 473 (zit.: Action Plan v. 29.04.2019, DH-DD (2019) 473)

Action Plan v. 26.04.2017 - Communication from the Russian Federation Concerning the Case of Ananyev u.a./RUS, DH-DD (2017) 560 (zit.: Action Plan v. 26.04.2017, DH-DD (2017) 560)

Action Plan v. 19.04.2017 - Communication from the Russian Federation Concerning the Cases of Kalashnikov/RUS u. Ananyev u.a./RUS, DH-DD (2017) 467 (zit.: Action Plan v. 19.04.2017, DH-DD (2017) 467)

Action Plan v. 10.08.2015 - Communication from the Russian Federation Concerning the Case of Ananyev u.a./RUS, DH-DD (2015) 862 (zit.: Action Plan v. 10.08.2015, DH-DD (2015) 862)

Action Plan v. 30.04.2014 - Communication from the Russian Federation Concerning the Case of Ananyev u.a./RUS, DH-DD (2014) 580 (zit.: Action Plan v. 30.04.2014, DH-DD (2014) 580)

Action Plan v. 14.08.2013 - Communication from the Russian Federation Concerning the Case of Ananyev u.a./RUS, DH-DD (2013) 936 (zit.: Action Plan v. 14.08.2013, DH-DD (2013) 936)

Action Plan v. 10.10.2012 - Communication from the Russian Federation Concerning the Case of Ananyev u.a./RUS, DH-DD (2012) 1009 (zit.: Action Plan v. 10.10.2012, DH-DD (2012) 1009) 


\section{bb. Implementierungsverfahren Grori/ALB und Dybeku/ALB}

Revised Action Report v. 03.06.2016 - Communication from Albania in the Cases of Dybeku/ALB u. Grori/ALB, DH-DD (2016) 739 (zit.: Revised Action Report v. 03.06.2016, DH-DD (2016) 739)

Revised Action Report v. 09.07.2015 - Communication from Albania Concerning the Cases of Dybeku/ALB u. Grori/ALB, DH-DD (2015) 768 (zit.: Revised Action Report v. 09.07.2015, DH-DD (2015) 768)

Revised Action Report v. 30.07.2014 - Communication from Albania Concerning the Cases of Dybeku/ALB u. Grori/ALB, DH-DD (2014) 974 (zit.: Revised Action Report v. 30.07.2014, DH-DD (2014) 974)

Action Report v. 03.07.2014 - Communication from Albania Concerning the Cases of Dybeku/ALB u. Grori/ALB, DH-DD (2014) 893 (zit.: Action Report v. 03.07.2014, DH-DD (2014) 893)

Action Plan v. 10.04.2013 - Communication from Albania Concerning the Cases of Dybeku/ALB u. Grori/ALB, DH-DD (2013) 428 (zit.: Action Plan v. 10.04.2013, DH-DD (2013) 428)

Action Plan / Action Report v. 09.11.2011 - Communication from Albania Concerning the Cases of Grori/ALB u. Dybeku/ALB, DH-DD (2011) 1041 (zit.: Action Plan / Action Report v. 09.11.2011, DH-DD (2011) 1041)

cc. Implementierungsverfabren Neshkov u.a./BUL

Addendum to the Action Plan v. 19.01.2018 - Communication from Bulgaria Concerning the Cases of Iordan Petrov/BUL, Kehayov/BUL u. Neshkov u.a./BUL, DH-DD (2018) 13-add (zit.: Addendum to the Action Plan v. 19.01.2018, DHDD (2018) 13-add)

Action Plan v. 21.12.2017 - Communication from Bulgaria Concerning the Cases of Kehayov/BUL u. Neshkov u.a./BUL, DH-DD (2018) 13 (zit.: Action Plan v. 21.12.2017, DH-DD (2018) 13)

Addendum v. 26.01.2017 to Updated Action Plan v. 16.12.2016 - Communication from Bulgaria Concerning the Kehayov Group of Cases/BUL u. Neshkov u.a./BUL, DH-DD (2017) 5-add (zit.: Addendum v. 26.01.2017 to Updated Action Plan v. 16.12.2016, DH-DD (2017) 5-add)

Updated Action Plan v. 16.12.2016 - Communication from Bulgaria Concerning the Kehayov Group of Cases/BUL u. Neshkov u.a./BUL, DH-DD (2017) 5 (zit.: Updated Action Plan v. 16.12.2016, DH-DD (2017) 5)

Addendum to the Revised Action Plan (DH-DD (2016) 25, DH-DD (2016) 25-add) v. 29.04.2016 - Communication from Bulgaria Concerning the Cases of Kehayov Group/BUL, Neshkov u.a./BUL u. Harakchiev u. Tolumov/BUL, DH-DD (2016) 587 (zit.: Addendum to the Revised Action Plan (DH-DD (2016) 25, DH-DD (2016) 25-add) v. 29.04.2016, DH-DD (2016) 587)

Addendum to the Revised Action Plan v. 16.02.2016 - Communication from Bulgaria Concerning Cases of Kehayov Group/BUL, Neshkov u.a./BUL u. Harakchiev u. Tolumov/BUL, DH-DD (2016) 25-add (zit.: Addendum to the Revised Action Plan v. 16.02.2016, DH-DD (2016) 25-add) 
Revised Action Plan v. 04.01.2016 - Communication from Bulgaria Concerning the Cases of Kehayov Group/BUL, Neshkov u.a./BUL u. Harakchiev u. Tolumov/ BUL, DH-DD (2016) 25 (zit.: Revised Action Plan v. 04.01.2016, DH-DD (2016) 25)

Revised Action Plan v. 31.08.2015 - Communication from Bulgaria Concerning the Case of Neshkov u.a./BUL u. Kehayov Group of Cases/BUL, DH-DD (2015) 755-rev (zit.: Revised Action Plan v. 31.08.2015, DH-DD (2015) 755-rev)

Action Plan v. 06.07.2015 - Communication from Bulgaria Concerning the Case of Neshkov u.a./BUL u. Kehayov Group of Cases/BUL, DH-DD (2015) 755 (zit.: Action Plan v. 06.07.2015, DH-DD (2015) 755)

dd. Implementierungsverfabren Orchowski/POL und Norbert Sikorski/POL

Consolidated Action Report v. 21.06.2016 - Communication from Poland in the Orchowski Group of Cases/POL, DH-DD (2016) 791 (zit.: Consolidated Action Report v. 21.06.2016, DH-DD (2016) 791)

Updated Action Report v. 28.04.2016 - Communication from Poland Concerning the Orchowski Group of Cases/POL, DH-DD (2016) 560 (zit.: Updated Action Report v. 28.04.2016, DH-DD (2016) 560)

Consolidated Action Report v. 08.07.2014 - Communication from Poland Concerning the Orchowski Group of Cases/POL, DH-DD (2014) 950 (zit.: Consolidated Action Report v. 08.07.2014, DH-DD (2014) 950)

Action Plan / Action Report v. 12.09.2011 - Communication from Poland Concerning the Cases of Orchowski/POL u. Norbert Sikorski/POL, DH-DD (2011) 709 (zit.: Action Plan / Action Report v. 12.09.2011, DH-DD (2011) 709)

Action Plan / Action Report v. 17.03.2010 - Communication from Poland Concerning the Case of Orchowski/POL, DH-DD (2011) 627 (zit.: Action Plan / Action Report v. 17.03.2010, DH-DD (2011) 627)

ee. Implementierungsverfabren Rezmiveşu.a./ROM

Communication from the Authorities v. 14.02.2020, DH-DD (2020) 143 (zit.: Communication from the Authorities v. 14.02.2020, DH-DD (2020) 143)

Communication from the Authorities v. 04.11.2019 on the General Measures (Update to the Action Plan v. 25.01.2018) in the Cases of Rezmiveş u.a./ROM u. Bragadireanu/ROM, DH-DD (2019) 1273 (zit.: Update v. 04.11.2019 to the Action Plan v. 25.01.2018, DH-DD (2019) 1273)

Communication from the Authorities v. 23.04.2019 on the General Measures (Update to the Action Plan v. 25.01.2018) in the Cases of Bragadireanu/ROM u. Rezmiveş u.a./ROM, DH-DD (2019) 440 (zit.: Update v. 23.04.2019 to the Action Plan v. 25.01.2018)

Communication from the Authorities v. 31.10.2018 on the General Measures (Update to the Action Plan v. 25.01.2018) Concerning the Cases of Rezmives u.a./ROM u. Bragadireanu/ROM, DH-DD (2018) 1069 (zit.: Update v. 31.10.2018 to the Action Plan v. 25.01.2018, DH-DD (2018) 1069) 
Communication from the Authorities v. 22.03.2018 on the General Measures (Update to the Action Plan v. 25.01.2018) Concerning the Cases of Bragadireanu/ROM u. Rezmiveş u.a./ROM, DH-DD (2018) 330 (zit.: Update v. 22.03.2018 to the Action Plan v. 25.01.2018, DH-DD (2018) 330)

Action Plan v. 25.01.2018 - Communication from Romania Concerning the Cases of Rezmiveş u.a./ROM u. Bragadireanu/ROM, DH-DD (2018) 90 (zit.: Action Plan v. 25.01.2018, DH-DD (2018) 90)

\section{ff. Implementierungsverfahren Sukachov/UKR}

Action Plan v. 06.07.2020 - Communication from Ukraine Concerning the Case of Logvinenko/UKR, Nevmerzhitsky/UKR, Yakovenko/UKR, Isayev/UKR, Melnik/UKR u. Sukachov/UKR, (zit.: Action Plan v. 06.07.2020, DH-DD (2020) 625)

gg. Implementierungsverfahren Torreggiani u.a./ITA

Action Report v. 20.11.2015 - Communication from Italy Concerning the Case of Torregiani u.a./ITA, DH-DD (2015) 1251 (zit.: Action Report v. 20.11.2015, DH-DD (2015) 1251)

Updated Action Plan v. 15.09.2014 - Communication from Italy Concerning the Case of Torreggiani u.a./ITA, DH-DD (2014) 1143 (zit.: Updated Action Plan v. 15.09.2014, DH-DD (2014) 1143)

Action Report v. 29.11.2013 - Communication from Italy Concerning the Case of Torreggiani u.a./ITA, DH-DD (2013) 1368 (zit.: Action Report v. 29.11.2013, DH-DD (2013) 1368)

\section{hh. Implementierungsverfahren Tunis/EST}

Revised Action Report v. 29.09.2015 - Communication from Estonia Concerning the Case of Tunis/EST, DH-DD (2015) 1002 (Revised Action Report v. 29.09.2015, DH-DD (2015) 1002)

Action Report v. 12.09.2014 - Communication from Estonia Concerning the Case of Tunis/EST, DH-DD (2014) 1146 (zit.: Action Report v. 12.09.2014, DH-DD (2014) 1146)

\section{ii. Implementierungsverfahren Varga u.a./HUN}

Action Plan v. 08.03.2019 - Communication from Hungary Concerning the István Gábor Kovács Group of Cases/HUN u. Varga u.a./HUN, DH-DD (2019) 311 (zit.: Action Plan v. 08.03.2019, DH-DD (2019) 311)

Action Plan v. 15.06.2018 - Communication from Hungary Concerning the Cases of Varga u.a./HUN u. István Gábor Kovács Group/HUN, DH-DD (2018) 690 (zit.: Action Plan v. 15.06.2018, DH-DD (2018) 690)

Action Plan v. 15.09.2017 - Communication from Hungary Concerning the Cases of Varga u.a./HUN u. István Gábor Kovács/HUN, DH-DD (2017) 1012 (zit.: Action Plan v. 15.09.2017, DH-DD (2017) 1012) 
Action Plan v. 03.03.2017 - Communication from Hungary Concerning the Cases of István Gábor Kovács/HUN u. Varga u.a./HUN, DH-DD (2017) 313 (zit.: Action Plan v. 03.03.2017, DH-DD (2017) 313)

Updated Action Plan v. 14.10.2016 - Communication from Hungary Concerning the István Gábor Kovács Group of Cases/HUN u. Varga u.a./HUN, DH-DD (2016) 1223 (zit.: Updated Action Plan v. 14.10.2016, DH-DD (2016) 1223)

Revised Action Plan v. 01.07.2016 - Communication from Hungary in the István Gábor Kovács Group of Cases/HUN u. Varga u.a./HUN, DH-DD (2016) 855 (zit.: Revised Action Plan v. 01.07.2016, DH-DD (2016) 855)

Updated Action Plan v. 14.12.2015 - Communication from Hungary Concerning the Case of István Gábor Kovács Group/HUN u. Varga u.a./HUN, DH-DD (2015) 1373 (zit.: Updated Action Plan v. 14.12.2015, DH-DD (2015) 1373)

\section{jj. Implementierungsverfahren W.D./BEL}

Plan d'action révisé v. 21.06.2018 - Communication de la Belgique concernant l'affaire L.B./BEL, DH-DD (2018) 649 (zit.: Plan d'action révisé v. 21.06.2018, DH-DD (2018) 649)

Plan d'action révisé v. 07.11.2017 - Communication de la Belgique concernant le groupe d'affaires L.B./BEL, DH-DD (2017) 186-rev (zit.: Plan d'action révisé v. 07.11.2017, DH-DD (2017) 186-rev)

Plan d'action v. 15.02.2017 - Communication de la Belgique concernant l'affaire L.B./BEL, DH-DD (2017) 186 (zit.: Plan d'action v. 15.02.2017, DH-DD (2017) 186)

\section{b. Beschlüsse des Ministerkomitees (Decisions)}

\section{aa. Implementierungsverfahren Ananyev u.a./RUS}

Decision Case H46-23 v. 06.06.2019, Ananyev u.a./RUS u. Kalashnikov Group/RUS, CM/Del/Dec (2019) 1348/H46-23 (zit.: Decision v. 06.06.2019, CM/Del/Dec (2019) 1348/H46-23)

Decision Case H46-24 v. 07.06.2017, Ananyev u.a./RUS u. Kalashnikov Group/RUS, CM/Del/Dec (2017) 1288/H46-24 (zit.: Decision v. 07.06.2017, CM/Del/Dec (2017) 1288/H46-24)

Decision Case No. 15 v. 05.06.2014, Ananyev u.a./RUS, CM/Del/Dec (2014) 1201/15 (zit.: Decision v. 05.06.2014, CM/Del/Dec (2014) 1201/15)

Decision Case No. 24 v. 07.03.2013, Ananyev u.a./RUS, CM/Del/Dec (2013) 1164/24 (zit.: Decision v. 07.03.2013, CM/Del/Dec (2013) 1164/24)

Decision Case No. 20 v. 06.12.2012, Ananyev u.a./RUS, CM/Del/Dec (2012) 1157/20 (zit.: Decision v. 06.12.2012, CM/Del/Dec (2012) 1157/20)

Decision Case No. 17 v. 26.09.2012, Ananyev u.a./RUS, CM/Del/Dec (2012) 1150/17 (zit.: Decision v. 26.09.2012, CM/Del/Dec (2012) 1150/17)

Decision Case No. 17 v. 06.06.2012, Ananyev u.a./RUS, CM/Del/Dec (2012) 1144/17 (zit.: Decision v. 06.06.2012, CM/Del/Dec (2012) 1144/17) 
bb. Implementierungsverfahren Neshkov u.a./BUL

Decision Cases H46-4 v. 15.03.2018, Kehayov Group/BUL u. Neshkov u.a./BUL, CM/Del/Dec (2018) 1310/H46-4 (zit.: Decision v. 15.03.2018, CM/Del/Dec (2018) 1310/H46-4)

Decision Cases H46-9 v. 10.03.2017, Kehayov Group/BUL u. Neshkov u.a./BUL, CM/Del/Dec (2017) 1280/H46-9 (zit.: Decision v. 10.03.2017, CM/Del/Dec (2017) 1280/H46-9)

Decision Cases H46-6 v. 08.-10.03.2016, Kehayov Group/BUL u. Neshkov u.a./BUL, CM/Del/Dec (2016) 1250/H46-6 (zit.: Decision v. 08.-10.03.2016, CM/Del/Dec (2016) 1250/H46-6)

Decision Cases No. 6 v. 24.09.2015, Kehayov Group/BUL u. Neshkov u.a./BUL, CM/Del/Dec (2016) 1250/H46-6 (zit.: Decision v. 24.09.2015, CM/Del/Dec (2016) 1250/H46-6)

cc. Implementierungsverfahren Orchowski/POL und Norbert Sikorski/POL

Decision Cases No. 17 v. 07.03.2013, Orchowski Group/POL, CM/Del/Dec (2013) 1164/17 (zit.: Decision v. 07.03.2013, CM/Del/Dec (2013) 1164/17)

Decision Cases No. 7 v. 14.09.2011, Orchowski/POL u. Norbert Sikorski/POL, CM/Del/Dec (2011) 1120/7 (zit.: Decision v. 14.09.2011, CM/Del/Dec (2011) $1120 / 7)$

dd. Implementierungsverfahren Rezmiveş u.a./ROM

Decision Case H46-23 v. 05.03.2020, Rezmiveș u.a./ROM u. Bragadireanu Group/ROM, CM/Del/Dec (2020) 1369/H46-23 (zit.: Decision v. 05.03.2020, CM/Del/Dec (2020) 1369/H46-23)

Decision Case H46-19 v. 05.12.2019, Rezmiveş u.a./ROM u. Bragadireanu Group/ROM, CM/Del/Dec (2019) 1362/H46-19 (zit.: Decision v. 05.12.2019, CM/Del/Dec (2019) 1362/H46-19)

Decision Case H46-21 v. 06.06.2019, Rezmiveş u.a./ROM u. Bragadireanu Group/ROM, CM/Del/Dec (2019) 1348/H46-21 (zit.: Decision v. 06.06.2019, CM/Del/Dec (2019) 1348/H46-21)

Decision Case H46-23 v. 06.12.2018, Rezmiveș u.a./ROM u. Bragadireanu Group/ROM, CM/Del/Dec (2018) 1331/H46-23 (zit.: Decision v. 06.12.2018, CM/Del/Dec (2018) 1331/H46-23)

Decision Case H46-13 v. 15.03.2018, Bragadireanu Group/ROM u. Rezmiveş u.a./ROM，CM/Del/Dec (2018) 1310/H46-13 (zit.: Decision v. 15.03.2018, $\mathrm{CM} / \mathrm{Del} / \mathrm{Dec}$ (2018) 1310/H46-13)

ee. Implementierungsverfahren Torreggiani u.a./ITA

Decision Cases No. 11 v. 04.12.2014, Torreggiani u.a./ITA u. Sulejmanovic/ITA, CM/Del/Dec (2014) 1214/11 (zit.: Decision v. 04.12.2014, CM/Del/Dec (2014) $1214 / 11)$ 
Decision Cases No. 10 v. 05.06.2014, Torreggiani u.a./ITA u. Sulejmanovic/ITA, CM/Del/Dec (2014) 1201/10 (zit.: Decision v. 05.06.2014, CM/Del/Dec (2014) 1201/10)

Decision Cases No. 11 v. 06.03.2014, Torreggiani u.a./ITA u. Sulejmanovic/ITA, CM/Del/Dec (2014) 1193/11 (zit.: Decision v. 06.03.2014, CM/Del/Dec (2014) 1193/11)

\section{ff. Implementierungsverfabren Varga u.a./HUN}

Decision Case H46-8 v. 15.03.2018, Varga u.a./HUN u. István Gábor Kovács Group/HUN, CM/Del/Dec (2018) 1310/H46-8 (zit.: Decision v. 15.03.2018, $\mathrm{CM} / \mathrm{Del} / \mathrm{Dec}(2018)$ 1310/H46-8)

Decision Case H46-16 v. 07.06.2017, Varga u.a./HUN u. István Gábor Kovács Group/HUN, CM/Del/Dec (2017) 1288/H46-16 (zit.: Decision v. 07.06.2017, CM/Del/Dec (2017) 1288/H46-16)

Decision Case H46-11 v. 08.-10.03.2016, Varga u.a./HUN u. István Gábor Kovács Group/HUN, CM/Del/Dec (2016) 1250/H46-11 (zit.: Decision v. 08.-10.03.2016, $\mathrm{CM} / \mathrm{Del} / \mathrm{Dec}$ (2016) 1250/H46-11)

\section{gg. Implementierungsverfahren W.D./BEL}

Décision H46-3 v. 20.09.2018, L.B. groupe/BEL u. W.D./BEL, CM/Del/Dec (2018) 1324/H46-3 (zit.: Décision v. 20.09.2018, CM/Del/Dec (2018) 1324/H46-3)

Décision H46-5 v. 07.12.2017, L.B. groupe/BEL u. W.D./BEL, CM/Del/Dec (2017) 1302/H46-5 (zit.: Décision v. 07.12.2017, CM/Del/Dec (2017) 1302/H46-5)

Décision H46-6 v. 10.03.2017, L.B. groupe/BEL u. W.D/BEL, CM/Del/Dec (2017) 1280/H46-6 (zit.: Décision v. 10.03.2017, CM/Del/Dec (2017) 1280/H46-6)

\section{c. Mitteilungen im Implementierungsverfahren (Communications)}

\section{aa. Implementierungsverfahren Ananyev u.a./RUS}

Communication from the Public Verdict Foundation v. 30.04.2019 in the Case of Ananyev u.a./RUS, DH-DD (2019) 517 (zit.: Communication from the Public Verdict Foundation v. 30.04.2019, DH-DD (2019) 517)

Communication from the Public Verdict Foundation v. 26.05.2017 in the Cases of Kalasnikov/RUS u. Ananyev u.a./RUS, DH-DD (2017) 668 (zit.: Communication from the Public Verdict Foundation v. 26.05.2017, DH-DD (2017) 668)

Communication from the Public Verdict Foundation v. 07.10.2013 in the Case of Ananyev u.a./RUS, DH-DD (2014) 44 (zit.: Communication from the Public Verdict Foundation v. 07.10.2013, DH-DD (2014) 44)

Communication from the Association of Russian Lawyers for Human Rights v. 29.11.12 in the Cases of Mikheyev Group/RUS, Dedovsky u.a./RUS, Fedotov/ RUS, Kalasnikov/RUS, Ananyev u.a./RUS, DH-DD (2013) 92 (zit.: Communication from the Association of Russian Lawyers for Human Rights v. 29.11.12, DH-DD (2013) 92) 
Communication from the Penal Reform International PRI v. 27.09.2012 in the Case of Ananyev u.a./RUS, DH-DD (2012) 1026 (zit.: Communication from the Penal Reform International PRI) v. 27.09.2012 in the Case of Ananyev u.a./RUS, DH-DD (2012) 1026)

\section{bb. Implementierungsverfahren Neshkov u.a./BUL}

Communication from the Bulgarian Prisoners' Association v. 16.03.2018 in the Case Neshkov u.a./BUL and a Part of Kehayov Group/BUL and Reply from the Bulgarian Authorities, DH-DD (2018) 301 (zit.: Communication from the Bulgarian Prisoners' Association v. 16.03.2018 and Reply from the Bulgarian Authorities, DH-DD (2018) 301)

Communication from the Applicant v. 17.05.2017 in the Case of Neshkov u.a./BUL, DH-DD (2017) 593 (zit.: Communication from the Applicant v. 17.05.2017, DH-DD (2017) 593)

Communication from the Bulgarian Helsinki Committee v. 16.09.2016 in the Case of Neshkov u.a./BUL and a Part of Kehayov Group/BUL, DH-DD (2016) 1098 (zit.: Communication from the Bulgarian Helsinki Committee v. 16.09.2016, DH-DD (2016) 1098)

\section{cc. Implementierungsverfahren Orchowski/POL und Norbert Sikorski/POL}

Communication from Poland v. 16.07.2013 in Reply to the Communication of a NGO v. 21.06.2013 Concerning the Cases of Orchowski/POL u. Norbert Sikorskil POL, DH-DD (2013) 86 (zit.: Communication from Poland v. 16.07.2013 in Reply to the Communication v. 21.06.2013, DH-DD (2013) 86)

Communication from the Helsinki Foundation for Human Rights and the Association for Legal Intervention in the Cases of Orchowski/POL u. Norbert Sikorski/POL v. 21.06.2013, DH-DD (2013) 798 (zit.: Communication from the Helsinki Foundation for Human Rights and the Association for Legal Intervention v. 21.06.2013, DH-DD (2013) 798)

Communication from the Office of the Human Rights Defender in the Cases of Orchowski/POL u. Norbert Sikorski/POL and Reply of the Government v. 16.11.2011, DH-DD (2011) 1108 (zit.: Communication from the Office of the Human Rights Defender and Reply of the Government v. 16.11.2011, DH-DD (2011) 1108)

\section{dd. Implementierungsverfahren Rezmiveş u.a./ROM}

Letter from the Registrar v. 12.02.2020 in the Cases of Bragadireanu/ROM u. Rezmiveş u.a./ROM, DH-DD (2020) 141 (zit.: Letter from the Registrar v. 12.02.2020, DH-DD (2020) 141)

Communication from the Association for the Defence of Human Rights in Romania - Helsinki Committee v. 21.10.2019 in the Cases of Rezmiveş u.a./ROM u. Bragadireanu Group/ROM, DH-DD (2019) 1263 (zit.: Communication from the Association for the Defence of Human Rights in Romania - Helsinki Committee v. 21.10.2019, DH-DD (2019) 1263) 
ee. Implementierungsverfahren Torreggiani u.a./ITA

Communication des autorités v. 25.01.2016 concernant l'affaire Torreggiani u.a./ ITA, DH-DD (2016) 106 (zit.: Communication des autorités v. 25.01.2016, DHDD (2016) 106)

Communication from L'Altro Diritto v. 02.12.2015 in the Case of Torregiani u.a./ITA, DH-DD (2015) 1371 (zit.: Communication from L'Altro Diritto v. 02.12.2015, DH-DD (2015) 1371)

Communication from the Radicali Italiani v. 05.05.2015 in the Case of Torreggia$n i$ u.a./ITA, DH-DD (2015) 562 (zit.: Communication from the Radicali Italiani v. 05.05.2015, DH-DD (2015) 562)

Communication from the Radicali Italiani v. 24.10.2014 in the Case of Torreggiani u.a./ITA, DH-DD (2014) 1325 (zit.: Communication from the Radicali Italiani v. 24.10.2014, DH-DD (2014) 1325)

Communication from the Radicali Italiani v. 22.09.2014 in the Case of Torreggiani u.a./ITA, DH-DD (2014) 1131 (zit.: Communication from the Radicali Italiani v. 22.09.2014, DH-DD (2014) 1131)

Communication from the Radicali Italiani v. 23.05.2014 in the Case of Torreggiani u.a./ITA, DH-DD (2014) 740 (zit.: Communication from the Radicali Italiani v. 23.05.2014, DH-DD (2014) 740)

Communication from the Nonviolent Radical Party Transnational and Transparty v. 10.04.2014 in the Case of Torreggiani u.a./ITA, DH-DD (2014) 585 (zit.: Communication from the Nonviolent Radical Party Transnational and Transparty v. 10.04.2014 DH-DD (2014) 585)

Communication from Italy (Information on the Implementation of the Action plan) v. 03.04.2014 Concerning the Case of Torreggiani u.a./ITA, DH-DD (2014) 471 (zit.: Communication from Italy v. 03.04.2014, DH-DD (2014) 471)

Communication de l'Italie v. 07.10.2013 relative à l'affaire Torreggiani u.a./ITA, DH-DD (2013) 1119 (zit.: Communication de l'Italie v. 07.10.2013, DH-DD (2013) 1119)

\section{ff. Implementierungsverfabren Varga u.a./HUN}

Communication from the Hungarian Helsinki Committee v. 30.06.2020 Concerning the Cases of István Gábor Kovács/HUN u. Varga u.a./HUN, DH-DD (2020) 624 (zit.: Communication from the Hungarian Helsinki Committee v. 30.06.2020, DH-DD (2020) 624)

Communication from the Hungarian Helsinki Committee v. 21.04.2020 in the Cases of István Gábor Kovács/HUN u. Varga u.a./HUN, DH-DD (2020) 396 (zit.: Communication from the Hungarian Helsinki Committee v. 21.04.2020, DHDD (2020) 396)

Reply from the Authorities v. 04.02.2020 to Communications from NGOs (DHDD (2020) 76, DH-DD (2020) 96) in the Cases of István Gábor Kovács/HUN u. Varga u.a./HUN, DH-DD (2020) 107 (zit.: Reply from the Authorities v. 04.02.2020 to Communications from NGOs (DH-DD (2020) 76, DH-DD (2020) 96), DH-DD (2020) 107) 
Communication from the Hungarian Helsinki Committee v. 21.01.2020 in the Cases of István Gábor Kovács/HUN u. Varga u.a./HUN, DH-DD (2020) 96 (zit.: Communication from the Hungarian Helsinki Committee v. 21.01.2020, DHDD (2020) 96)

Communication from the Hungarian Helsinki Committee v. 20.01.2020 in the Cases of István Gábor Kovács/HUN u. Varga u.a./HUN, DH-DD (2020) 76 (zit.: Communication from the Hungarian Helsinki Committee v. 20.01.2020, DHDD (2020) 76)

Communication from the Helsinki Committee v. 28.08.2017 in the Cases of Varga u.a./HUN u. István Gábor Kovács/HUN, DH-DD (2017) 1003 (zit.: Communication from the Helsinki Committee v. 28.08.2017, DH-DD (2017) 1003)

\section{d. Resolutionen im Implementierungsverfahren (Resolutions)}

\section{aa. Schlussresolutionen des Ministerkomitees (Final Resolutions)}

Resolution CM/ResDH (2018) 455, Execution of the Judgments of the European Court of Human Rights, 136 Cases/RUS (from the Kalashnikov Group), adopted by the Committee of Ministers on 12 December 2018 at the 1332nd meeting of the Ministers' Deputies (zit.: Resolution CM/ResDH (2018) 455, Execution of the Judgments of the European Court of Human Rights, 136 Cases/RUS [from the Kalashnikov Group])

Resolution CM/ResDH (2017) 35, Execution of the Judgment of the European Court of Human Rights Bulatović/MNE, adopted by the Committee of Ministers on 1 February 2017 at the 1276th meeting of the Ministers' Deputies (zit.: Resolution CM/ResDH (2017) 35, Execution of the Judgment Bulatović/MNE)

Résolution CM/ResDH (2017) 34, Exécution de l'arrêt de la Cour européenne des droits de l'homme Kasperovičius/LTU, adoptée par le Comité de Ministres le $1^{\text {er }}$ février 2017, lors de la $1276^{\text {e }}$ réunion des Délégués des Ministres (zit.: Résolution CM/ResDH (2017) 34, Exécution de l'arrêt Kasperovičius/LTU)

Resolution CM/ResDH (2016) 278, Execution of the Judgments of the European Court of Human Rights, Eight Cases/POL: Kaprykowski u.a./POL, adopted by the Committee of Ministers on 21 September 2016 at the 1265th meeting of the Ministers' Deputies (zit.: Resolution CM/ResDH (2016) 278, Execution of the Judgments Kaprykowski u.a./POL)

Resolution CM/ResDH (2016) 273, Execution of the Judgments of the European Court of Human Rights, Dybeku/ALB u. Grori/ALB, adopted by the Committee of Ministers on 21 September 2016 at the 1265th meeting of the Ministers' Deputies (zit.: Resolution CM/ResDH (2016) 273, Execution of the Judgments Dybeku/ALB u. Grori/ALB)

Resolution CM/ResDH (2016) 254, Execution of the Judgments of the European Court of Human Rights, Seven Cases/POL: Orchowski u.a./POL, adopted by the Committee of Ministers on 21 September 2016 at the 1265th meeting of the Ministers' Deputies (zit.: Resolution CM/ResDH (2016) 254, Execution of the Judgments Orchowski u.a./POL) 
Resolution CM/ResDH (2016) 126, Execution of the Judgment of the European Court of Human Rights Mathew/NED, adopted by the Committee of Ministers on 7 June 2016 at the 1259th meeting of the Ministers' Deputies (zit.: Resolution CM/ResDH (2016) 12, Execution of the Judgment Mathew/NED)

Resolution CM/ResDH (2016) 122, Execution of the Judgments of the European Court of Human Rights, Seven Cases/LAT: Kadikis u.a./LAT, adopted by the Committee of Ministers on 7 June 2016 at the 1259th meeting of the Ministers' Deputies (zit.: Resolution CM/ResDH (2016) 122, Execution of the Judgments Kadikis u.a./LAT)

Resolution CM/ResDH (2016) 28, Execution of the Judgments of the European Court of Human Rights, Torreggiani u.a./ITA u. Sulejmanovic/ITA, adopted by the Committee of Ministers on 8 March 2016 at the 1250th meeting of the Ministers' Deputies (zit.: Resolution CM/ResDH (2016) 28, Execution of the Judgments Torreggiani u.a./ITA u. Sulejmanovic/ITA)

Resolution CM/ResDH (2016) 22, Execution of the Judgment of the European Court of Human Rights, Tunis/EST, adopted by the Committee of Ministers on 8 March 2016 at the 1250th meeting of the Ministers' Deputies (zit.: Resolution CM/ResDH (2016) 22, Execution of the Judgment Tunis/EST)

Resolution CM/ResDH (2014) 209, Execution of the Judgments of the European Court of Human Rights, Five Cases/GEO: Ghavtadze u.a./GEO, adopted by the Committee of Ministers on 12 November 2014 at the 1211th meeting of the Ministers' Deputies (zit.: Resolution CM/ResDH (2014) 209, Execution of the Judgments Ghavtadze u.a./GEO)

Resolution CM/ResDH (2014) 162, Execution of the Judgment of the European Court of Human Rights, Jashi/GEO, adopted by the Committee of Ministers on 25 September 2014 at the 128th meeting of the Ministers' Deputies (zit.: Resolution CM/ResDH (2014) 162, Execution of the Judgment Jashi/GEO)

Summary of Final Resolutions adopted by the Committee of Ministers in 2016, with Exception of those Concerning Friendly Settlements, abrufbar unter $<$ https://rm.coe.int/final-resolutions-summary-2016-en-final/16807178d3> (zit.: Summary of Final Resolutions 2016, zuletzt abgerufen am 10.04.2018)

\section{bb. Zwischenresolutionen des Ministerkomitees (Interim Resolutions)}

Interim Resolution CM/ResDH (2018) 472, Execution of the Judgments of the European Court of Human Rights Nevmerzhitsky/UKR, Yakovenko/UKR, Logninenko/UKR, Isayev/UKR u. Melnik Groups/UKR, adopted by the Committee of Ministers on 6 December 2018 at the 1331st meeting of the Ministers' Deputies (zit.: Interim Resolution CM/ResDH (2018) 472, Execution of the Judgments of the European Court of Human Rights Nevmerzhitsky/UKR, Yakovenko/UKR, Logninenko/UKR, Isayev/UKR u. Melnik Groups/UKR) 
Interim Resolution CM/ResDH (2010) 35, Execution of the Judgments of the European Court of Human Rights in 31 Cases/RUS Mainly Concerning Conditions of Detention in Remand Prisons (Kalashnikov Group), adopted by the Committee of Ministers on 4 March 2010 at the 1078th meeting of the Ministers' Deputies (zit.: Interim Resolution CM/ResDH (2010) 35, Execution of the Judgments of the European Court of Human Rights in 31 Cases/RUS Mainly Concerning Conditions of Detention in Remand Prisons (Kalashnikov Group))

Interim Resolution CM/ResDH (2003) 123, Execution of the Judgment of the European Court of Human Rights of 15 July 2002, Final on 15 October 2002 in the Case of Kalashnikov/RUS, adopted by the Committee of Ministers on 4 June 2003 at the 841st meeting of the Ministers' Deputies (zit.: Interim Resolution $\mathrm{CM} / \mathrm{ResDH}$ (2003) 123, Execution of the Judgment of the European Court of Human Rights in the Case of Kalashnikov/RUS)

\section{e. Status der Implementierungsverfahren (Status of Execution)}

Status of Execution, Concerning the Cases Ananyev u.a./RUS, abrufbar unter $<$ http://hudoc.exec.coe.int/ENG?i=004-14142> (zit.: HUDOC, Status of Execution: EGMR - Ananyev u.a./RUS, Urt. v. 10.01.2012, 42525/07 u. 60800/08, zuletzt abgerufen am 25.04.2020)

Status of Execution, Concerning the Bragadireanu Group of Cases/ROM: Rezmives u.a./ROM, abrufbar unter <http://hudoc.exec.coe.int/eng?i=004-12985> (zit.: HUDOC, Status of Execution [Bragadireanu Group of Cases/ROM]: EGMR Rezmiveş u.a./ROM, Urt. v. 25.04.2017, 61467/12 u.a., zuletzt abgerufen am 25.04.2020)

Status of Execution, Concerning the Istvan Gabor Kovacs Group of Cases/HUN: Varga u.a./HUN, abrufbar unter <http://hudoc.exec.coe.int/eng?i=004-10809> (zit.: HUDOC, Status of Execution [Istvan Gabor Kovacs Group of Cases/HUN]: EGMR - Varga u.a./HUN, Urt. v. 10.03.2015, 14097/12 u.a., zuletzt abgerufen am 25.04.2020)

Status of Execution, Concerning the Kehayov Group of Cases/BUL: Neshkov u.a./ BUL, abrufbar unter <http://hudoc.exec.coe.int/ENG?i=004-3589> (zit.: HUDOC, Status of Execution [Kehayov Group of Cases/BUL]: EGMR - Neshkov u.a./ BUL, Urt. v. 27.01.2015, 36925/10 u.a., zuletzt abgerufen am 25.04.2020)

Status of Execution, Concerning the L.B. Group of Cases/BEL: W.D./BEL, abrufbar unter $<$ http://hudoc.exec.coe.int/eng?i=004-1213> (zit.: HUDOC, Status of Execution [L.B. Group of Cases/BEL]: EGMR - W.D./BEL, Urt. v. 06.09.2016, 73548/13, zuletzt abgerufen am 25.04.2020)

\section{Weiteres}

Department for the Execution of Judgments of the European Court of Human Rights, Human Rights Trust Fund, abrufbar unter $<$ https://www.coe.int/en/web /execution/human-rights-trust-fund?inheritRedirect=true $>$ (zit.: Department for the Execution of Judgments of the European Court of Human Rights, Human Rights Trust Fund, zuletzt abgerufen am 24.04.2020) 
Directorate General Human Rights and Rule of Law, Practical Impact of the Council of Europe Monitoring Mechanisms in Improving Respect for Human Rights and the Rule of Law in Member States, Strasbourg 2014, abrufbar unter $<$ https:/ /rm.coe.int/CoERMPublicCommonSearchServices/DisplayDCTMContent?docu mentId=09000016806d22c8> (zit.: Directorate General Human Rights and Rule of Law, Practical Impact of the Council of Europe Monitoring Mechanisms, zuletzt abgerufen am 23.07.2020)

Office of the Directorate General of Programmes, Human Rights Trust Fund, abrufbar unter $<$ https://www.coe.int/en/web/programmes/human-rights-trust -fund> (zit.: Office of the Directorate General of Programmes, Human Rights Trust Fund, zuletzt abgerufen am 24.04.2020)

Office of the Directorate General of Programmes, Projects Funded, abrufbar unter $<$ https://www.coe.int/en/web/programmes/projects-funded $>$ (zit.: Office of the Directorate General of Programmes, Projects Funded, zuletzt abgerufen am 24.04.2020)

Support for the Implementation of the European Court of Human Rights Judgments and European Committee for the Prevention of Torture and Inhuman or Degrading Treatment of Punishment Standards and Recommendations in Bulgaria, Council of Europe, abrufbar unter $<$ https://rm.coe.int/16806b340 5> (zit.: Support for the Implementation of the European Court of Human Rights Judgments and European Committee for the Prevention of Torture and Inhuman or Degrading Treatment of Punishment Standards and Recommendations in Bulgaria (Prison Reform), zuletzt abgerufen am 25.04.2020)

White Paper on Prison Overcrowding CM (2016) 121-add3, abrufbar unter <https: //rm.coe.int/white-paper-on-prison-overcrowding-cm-2016-121-add3-e/168 $07 \mathrm{c} 886 \mathrm{~b}>$ (zit.: White Paper on Prison Overcrowding, zuletzt abgerufen am 15.01.2020)

\section{Schweiz}

\section{Konferenz der Kantonalen Justiz- und Polizeidirektorinnen und -direktoren (KKJPD)}

Grundlagen für den strafrechtlichen Sanktionenvollzug in der Schweiz, genehmigt von der KKJPD am 13. November 2014, abrufbar unter $<$ https://www.kkjpd.ch/ files/Dokumente/Themen/Strafvollzug/1417077049-141113_Grundlagen_zum_ schweizerischen_Sanktionenvollzug_d.pdf> (zit.: Grundlagen für den strafrechtlichen Sanktionenvollzug in der Schweiz, zuletzt abgerufen am 15.01.2020)

Konferenz der Kantonalen Justiz- und Polizeidirektorinnen und -direktoren, Strafund Massnahmenvollzug, abrufbar unter <https://www.kkjpd.ch/themen.html $>$ (zit.: Konferenz der Kantonalen Justiz- und Polizeidirektorinnen und -direktoren, Straf- und Massnahmenvollzug, zuletzt abgerufen am 13.02.2020) 


\section{Nationale Kommission zur Verhütung von Folter}

Gesamtbericht über die schweizweite Überprüfung der Gesundheitsversorgung im Freiheitsentzug durch die Nationale Kommission zur Verhütung von Folter (2018-2019), Bern 14. November 2019, abrufbar unter <https:/www.nkvf.adm in.ch/dam/data/nkvf/Berichte/2019/gesundheitsversorgung/bericht.pdf $>$ (zit.: NKVF - Gesamtbericht über die schweizweite Überprüfung der Gesundheitsversorgung im Freiheitsentzug durch die Nationale Kommission zur Verhütung von Folter (2018-2019), zuletzt abgerufen am 31.12.2019)

\section{Ostschweizer Strafvollzugskonkordat}

Merkblatt betreffend Einweisungen von Gefangenen aus Vollzugseinrichtungen des OSK in ein Spital oder eine psychiatrische Klinik v. Juli 2019 (zit.: OSK Merkblatt betreffend Einweisungen von Gefangenen aus Vollzugseinrichtungen des OSK in ein Spital oder eine psychiatrische Klinik v. Juli 2019)

Merkblatt Gesundheitskosten im Straf- und Massnahmenvollzug, verabschiedet an der Konkordatskonferenz v. 24. Oktober 2008 (zit.: OSK - Merkblatt Gesundheitskosten im Straf- und Massnahmenvollzug v. 24.10.2008)

Reglement über die Organisation des Ostschweizer Konkordats (Organisationsreglement) v. 29. März 2019 (zit.: OSK - Organisationsreglement v. 29.03.2019)

Richtlinie über die Laufakte v. 19. April 2012 (zit.: OSK - Richtlinie über die Laufakte v. 19.04.2012)

\section{Schweizerische Akademie der Medizinischen Wissenschaften}

Medizin-ethische Richtlinien, Ausübung der ärztlichen Tätigkeit bei inhaftierten Personen, 4. Auflage, Basel Januar 2019, abrufbar unter $<$ https://www.samw.ch /de/Ethik/Themen-A-bis-Z/Medizin-im-Straf-und-Massnahmenvollzug.html> (zit.: SAMW - Medizin-ethische Richtlinien, Ausübung der ärztlichen Tätigkeit bei inhaftierten Personen)

Stellungnahme der Zentralen Ethikkommission (ZEK) der Schweizerischen Akademie der Medizinischen Wissenschaften (SAMW) betreffend Finanzierung medizinischer Leistungen im Gefängnis, Bern Februar 2019 abrufbar unter $<$ https: //www.samw.ch/de/Publikationen/Stellungnahmen.html> (zit.: SAMW - Stellungnahme der Zentralen Ethikkommission (ZEK) der Schweizerischen Akademie der Medizinischen Wissenschaften (SAMW) betreffend Finanzierung medizinischer Leistungen im Gefängnis)

\section{Schweizerisches Kompetenzzentrum für den Justizvollzug}

Schweizerisches Kompetenzzentrum für den Justizvollzug, Gesundheit, abrufbar unter $<$ https://www.skjv.ch/de/praxis/aktuell/gesundheit $>$ (zit.: Schweizerisches Kompetenzzentrum für den Justizvollzug - Gesundheit, zuletzt abgerufen am 23.12.2019) 
Schweizerisches Kompetenzzentrum für den Justizvollzug, Gesundheit im Justizvollzug, Chronologie abrufbar unter $<$ https://www.skjv.ch/sites/default/files/ documents/3.1.2._Chronik_Gesundheit_im_JV_0.pdf $>$ (zit.: Schweizerisches Kompetenzzentrum für den Justizvollzug - Gesundheit im Justizvollzug, Chronologie, zuletzt abgerufen am 23.12.2019)

Schweizerisches Kompetenzzentrum für den Justizvollzug, Ziele und Aufgaben, abrufbar unter $<$ https://www.skjv.ch/de/ueber-uns/ziele-aufgaben $>$ (zit.: Schweizerisches Kompetenzzentrum für den Justizvollzug - Ziele und Aufgaben, zuletzt abgerufen am 06.08.2020)

\section{Weiteres}

Informationen zum Straf- und Massnahmenvollzug, info bulletin 1/2015, Fokus: Gesundheitsversorgung, abrufbar unter $<$ https:/www.bj.admin.ch/dam/data/bj/ sicherheit/smv/smvbulletin/2015/ib-1501-d.pdf> (zit.: Informationen zum Strafund Massnahmenvollzug, info bulletin 1/2015, Fokus: Gesundheitsversorgung, zuletzt abgerufen am 23.12.2019)

Vademekum: Übertragbare Krankheiten und Abhängigkeiten im Gefängnis vom 1. Oktober 2012, abrufbar unter $<$ https:/www.skjv.ch/sites/default/files/docum ents/\%C3\%9Cbertragbare_Krankheiten_und_Abh\%C3\%A4ngigkeiten $\% 20 \mathrm{im} \%$ 20Gef\%C3\%A4ngnis_DEU.pdf> (zit.: $\bar{V}$ ademekum: Übertragbare Krankheiten und Abhängigkeiten im Gefängnis, zuletzt abgerufen am 16.02.2020)

\section{Varia}

Erklärung des Deutschen Bundestags „50 Jahre Europarat: 50 Jahre europäischer Menschenrechtsschutz“, BT-Drs 14/1568 v. 9. September 1999 (zit.: Erklärung des Deutschen Bundestags „50 Jahre Europarat: 50 Jahre europäischer Menschenrechtsschutz“, BT-Drs 14/1568 v. 09.09.1999) 
